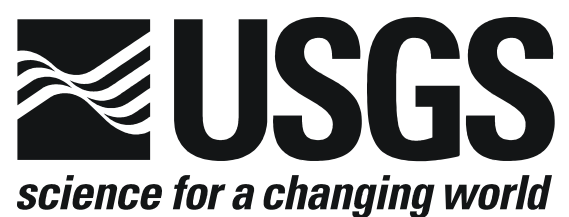

\title{
Magnetotelluric Data In the Española Basin, West of Santa Fe, New Mexico
}

By Jackie M. Williams and Brian D. Rodriguez

Open-File Report 2005-1037

U.S. DEPARTMENT OF THE INTERIOR

U.S. GEOLOGICAL SURVEY 


\section{Contents}

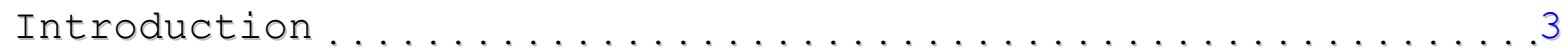

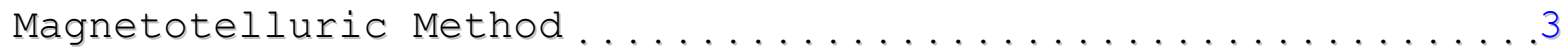

Magnetotelluric Survey ......................5

Magnetotelluric Data .......................6

Reference Cited .........................8

Appendix Magnetotelluric Data Plots .................10 


\section{Introduction}

The Santa Fe region is rapidly growing. The Santa Fe Group aquifer in the Española Basin is the main source of municipal water for the region (fig. 1), and water shortfalls could have serious consequences. Future growth and land management in the region depend on accurate assessment and protection of the region's ground-water resources. An important issue in managing the ground-water resources is a better understanding of the hydrogeology of the Santa Fe Group, the sedimentary deposits that fill the Rio Grande rift and contain the principal ground-water aquifers.

The U.S. Geological Survey (USGS) is doing a series of studies of the Española Basin in northern New Mexico. One objective of these studies is to understand the hydrogeologic framework of the Española Basin to help land managers plan and develop water supplies. These studies involve a multidisciplinary approach to better understand the critical aquifers in the intracontinental rift environment. Detailed geologic mapping, high-resolution airborne magnetic surveys, electromagnetic surveys, and hydrologic, lithologic, and hydrogeochemical data are being used to better understand the aquifer systems in the Española Basin. A magnetotelluric (MT) survey was done as part of these studies. The primary purpose of the MT survey was to map changes in electrical resistivity with depth that are related to lithologic variations important to the critical aquifers. The purpose of this report is to release the MT sounding data; no interpretation of the data is included.

\section{Magnetotelluric Method}

The MT method is a passive surface geophysical technique that uses the Earth's natural electromagnetic fields to investigate the electrical resistivity structure of the subsurface. The resistivity of geologic units is largely dependent upon their fluid content, porosity, degree of fracturing, temperature, and conductive mineral content (Keller, 1989). Saline fluids within the pore spaces and fracture openings can reduce resistivities in a resistive rock matrix. Resistivity also can be lowered by the presence of conductive clay minerals, carbon, and metallic mineralization. It is common for altered volcanic rocks to contain authigenic minerals that have resistivities 10 times less than those of the surrounding rocks (Nelson and Anderson, 1992). Increased temperatures cause higher ionic mobility and mineral activation energy, reducing rock resistivities significantly. Unaltered, unfractured igneous rocks are moderately to highly resistive (hundreds to thousands of ohm-m), whereas fault zones will show low resistivity (less than 100 ohm-m) when they are composed of rocks that are fractured enough to have hosted fluid transport and consequent mineralogical alteration (Eberhart-Phillips and others, 1995). Carbonate rocks are moderately to highly resistive (hundreds to 
thousands of ohm-m) depending upon their fluid content, porosity, fracturing, and impurities. Marine shales, mudstones, and clayrich alluvium are normally conductive (a few ohm-m to tens of ohm-m). Unaltered metamorphic rocks (non-graphitic) are moderately to highly resistive (hundreds to thousands of ohmmeters[ohm-m]). Tables of electrical resistivity for a variety of rocks, minerals, and geological environments are included in Keller (1987) and Palacky (1987).

The MT method can be used to probe the crust from depths of tens of meters to depths of tens of kilometers (Vozoff, 1991). Natural variations of the Earth's magnetic and electric field are measured and recorded at each MT station. The primary frequency bands used by the MT method are 10,000 to 1 hertz (Hz) from worldwide lightning activity and 1 to $0.0001 \mathrm{~Hz}$ from geomagnetic micro-pulsations. The natural electric and magnetic fields propagate vertically in the Earth because the large resistivity contrast between the air and the Earth causes a vertical refraction of both fields transmitted into the Earth (Vozoff, 1972).

The natural electric and magnetic fields are recorded in two orthogonal, horizontal directions. The vertical magnetic field ("tipper") also is recorded. The resulting time-series signals are used to derive the tensor apparent resistivities and phases. First, the signals are converted to complex cross-spectra using Fast-Fourier-transform (FFT) techniques. Then, least-squares, cross-spectral analysis (Bendat and Piersol, 1971) is used to solve for a transfer function that relates the observed electric fields to the magnetic fields with the assumption that the Earth consists of a two-input, two-output linear system, with the magnetic fields as input and the electric fields as output. Prior to conversion to apparent resistivity and phase, the tensor is normally rotated into principal directions that correspond to the direction of maximum and minimum apparent resistivity. For a two-dimensional (2-D) Earth, the MT fields can be de-coupled into transverse electric (TE) and transverse magnetic (TM) modes; 2-D modeling generally is done to fit both modes. When the geology satisfies the 2-D assumption, the MT data for the TE mode are for the electric field parallel to geologic strike, and the data for the TM mode are for the electric field across strike. The MT method is well suited for studying complicated geological environments because the electric and magnetic relations are sensitive to vertical and horizontal variations in resistivity. The MT method is capable of establishing whether the electromagnetic fields are responding to subsurface terranes of effectively one, two, or three dimensions. An introduction to the MT method and references for a more advanced understanding are in Dobrin and Savit (1988) and Vozoff (1991). 
Magnetotelluric Survey

Data were collected at 18 stations in 2002 and 2003 to represent the area of this study. The station locations were chosen for constraining gravity modeling across the fault, and for proximity to roads, and for avoidance of electrical noise such as power lines. All data at the stations were collected with a portable EMI MT-1 system (EMI, 1996). Horizontal electric fields were sensed using copper sulfate porous pots placed in an L-shaped, three-electrode array with dipole lengths of 30 meters $(\mathrm{m})$. The orthogonal, horizontal magnetic fields in the direction of the electric-field measurement array were sensed using permalloy-cored induction coils. Frequencies sampled ranged from 4 to $23,000 \mathrm{~Hz}(4.3945,7.3242,12.2070,19.0430,28.3203$, $41.5039,60.0586,79.0000,85.9375,100.0000,122.0703,150.0000$, $172.3633,210.0000,270.0000,340.0000,460.0000,580.0000$, $720.0000,885.0000,1170.0000,1500.0000,1870.0000,2200.0000$, $2730.0000,3550.0000,4900.0000,6500.0000,9000.0000$, $11590.0000,15290.0000,19500.0000,23370.0000)$ and 0.009 to $70 \mathrm{~Hz}$ $(0.0088,0.0146,0.0244,0.0381,0.0566,0.0830, .0879,0.1201$, $0.1465,0.1719,0.2441,0.3447,0.3809,0.5664,0.8301,1.2012$, $1.7578,2.4414,2.9297,3.4473,4.8828,7.6172,11.3281,16.6016$, $24.0234,34.3750,48.8281,68.9453)$ using single-station recordings of the orthogonal, horizontal components of the electric and magnetic fields and the vertical magnetic field.

The following table lists the 18 MT station locations as recorded using a global positioning system during field acquisition. Coordinates are referenced to the 1866 Clarke spheroid and North American 1927 Western United States datum. Longitude and latitude format is degrees:minutes:seconds. Universal Transverse Mercator (UTM) units are in meters. Station elevation is given in meters. The accuracy of the $x, y, z$ component is $\pm 5 \mathrm{~m}$.

\begin{tabular}{|c|c|c|c|c|c|}
\hline Station & Longitude & Latitude & $\begin{array}{c}\text { UTM } \\
\text { North }(\mathrm{m})\end{array}$ & $\begin{array}{c}\text { UTM } \\
\text { East }(m)\end{array}$ & Elevation (m) \\
\hline 8 & $-106: 12: \odot 8$ & $35: 37: 25$ & $3,942,655$ & $13,391,127$ & 2017 \\
\hline 9 & $-106: 09: 31$ & $35: 44: 24$ & $3,955,517$ & $13,395,221$ & 2030 \\
\hline 10 & $-106: 10: \odot 5$ & $35: 41: 53$ & $3,950,901$ & $13,394,319$ & 2170 \\
\hline 11 & $-106: 11: 19$ & $35: 38: 47$ & $3,945,184$ & $13,392,391$ & 2025 \\
\hline 12 & $-106: 23: 20$ & $35: 46: \odot 3$ & $3,958,861$ & $13,374,449$ & 2381 \\
\hline 13 & $-106: 07: 58$ & $35: 39: 16$ & $3,946,026$ & $13,397,459$ & 1976 \\
\hline 16 & $-106: 06: 50$ & $35: 42: 27$ & $3,951,868$ & $13,399,230$ & 2002 \\
\hline 17 & $-106: 21: 42$ & $35: 41: 09$ & $3,949,770$ & $13,376,785$ & 1760 \\
\hline $18 \mathrm{~m}$ & $-106: 16: 37$ & $35: 23: 32$ & $3,917,087$ & $13,384,020$ & 1845 \\
\hline $19 \mathrm{~m}$ & $-106: 10: 09$ & $35: 34: 20$ & $3,936,915$ & $13,394,057$ & 1886 \\
\hline $20 \mathrm{~m}$ & $-106: 11: 36$ & $35: 36: 16$ & $3,940,539$ & $13,391,897$ & 1986 \\
\hline $21 \mathrm{~m}$ & $-106: 11: 52$ & $35: 38: 16$ & $3,944,249$ & $13,391,560$ & 2010 \\
\hline $22 \mathrm{~m}$ & $-106: 09: 31$ & $35: 43: 35$ & $3,954,039$ & $13,395,225$ & 2042 \\
\hline $23 m$ & $-106: 09: 58$ & $35: 42: 48$ & $3,952,588$ & $13,394,527$ & 2100 \\
\hline $24 \mathrm{~m}$ & $-105: 56: 01$ & $35: 24: 25$ & $3,918,370$ & $13,415,194$ & 1860 \\
\hline $25 \mathrm{~m}$ & $-106: 10: \odot 9$ & $35: 40: 42$ & $3,948,914$ & $13,394,185$ & 2100 \\
\hline $26 \mathrm{~m}$ & $-106: 07: 07$ & $35: 40: 08$ & $3,948,043$ & $13,398,749$ & 1973 \\
\hline $27 \mathrm{~m}$ & $-106: 09: 22$ & $35: 47: 06$ & $3,960,730$ & $13,395,460$ & 2010 \\
\hline
\end{tabular}




\section{Magnetotelluric Data}

The recorded time-series data were transformed to the frequency domain and processed to determine a 2-D apparent resistivity and phase tensor at each site. Rotation of the impedance tensor to maximum and minimum directions allows for decoupling into the TE and TM modes.

Although true remote reference techniques were not used in the survey, cross-power files were sorted to select optimal signal-to-noise time-series data sets (see Appendix at the back of the report).

The effects of near-surface resistivity anomalies caused "static shifts" in the data (Sternberg and others, 1988). Static shifts were significant at stations 9, 13, 16, 20, 22, 24, and 27. Cultural features can affect the response of the MT system. Fences, pipelines, communication lines, railways, and other manmade conductors can contaminate the responses.

The figures in the Appendix represent the field-processed MT data for each station after the time-series data were converted to the frequency domain, and the tensor-transfer function was rotated into principal directions as described in the "Magnetotelluric Method" section.

For each station, nine separate plots are given:

1. Apparent Resistivity( $x$ and $y$ symbols are $x y$ and $y x$ components)

2. Impedance Phase ( $x$ and $y$ symbols are $x y$ and $y x$ components)

3. Rotation Angle

4. Impedance Skew

5. Multiple Coherency ( $x$ and $y$ symbols are $x y$ and $y x$ components)

6. Impedance Polar Plots

7. Tipper Magnitude

8. Tipper Strike

9. $\mathrm{HzHx}$ (x symbol) and HzHy (o symbol) Coherency

Error bars (], [) on the Apparent Resistivity, Impedance Phase, Skew, Tipper Magnitude, and Tipper Strike plots represent probable errors within one standard deviation of the sample variance (Gamble and others, 1979).

Apparent resistivity is the ratio of the electric field strength magnitude over the magnetic-field strength magnitude for a given frequency. The impedance phase is proportional to the slope of the apparent resistivity curve on a log-log plot, but from a baseline at -45 degrees (Vozoff, 1991). A measure of the dimensionality for MT data is provided by the impedance skew of the impedance tensor (Vozoff, 1972). If the effective, measured resistivity response to the geology beneath a MT station is truly one or two dimensional, then the skew will be zero. Both instrument and environmental sources of noise contribute to non- 
zero skew values but are typically small (about 0.1) for relatively low-noise-level recordings. Higher skews (above 0.2) are an indication of either the resistivity response to 3-D geology or higher levels of noise. Manmade electrical noise, such as power lines, power generators, and moving vehicles and trains, can have a negative effect on MT data quality. All of these local disturbances produce an incoherent noise mainly affecting frequencies above $1 \mathrm{~Hz}$. Other manmade electrical noise, such as direct current electric trains and active cathodic protection of pipelines, produce coherent electromagnetic signals mainly affecting frequencies below $1 \mathrm{~Hz}$.

In the survey area, noise from a number of small power lines and small moving vehicles was negligible at distances of $0.4 \mathrm{~km}$ and greater from the noise source. Power-line signal levels were measured at each site and were typically less than 20 percent of the maximum recordable signals. Noise from larger power lines, power generators, pipelines, and trains was negligible at distances greater than $5 \mathrm{~km}$. Local lightning, wind, and rainstorms also can degrade data quality, but these were avoided by not recording during active thunderstorm periods. Burying the magnetic induction coils and keeping the electric dipole wires flat on the ground surface minimized wind noise.

Predicted values of the electric field can be computed from the measured values of the magnetic field (Vozoff, 1991). The coherence of the predicted electric field with the measured electric field is a measure of the signal-to-noise ratio provided in the multiple coherency plots. Values are normalized between 0 and 1, where values at 0.5 signify signal levels equal to noise levels. For this data set, coherencies were generally at an acceptable level, except at times in the frequency range "dead band" $(0.01$ to $5 \mathrm{HZ})$.

The figures in the Appendix represent the field-processed MT data at each station, which includes some data scatter and poor signal-to-noise ratios. The only effort at removing noisy data points was to visually inspect and select the best signal-tonoise field data to combine into the final data plots.

The impedance polar plots provide a measure of the MT data dimensionality (Reddy and others, 1977). For 1-D resistivity structures, the principal impedance polar diagram (dashed line) is a circle. For 2-D or 3-D resistivity structures, the principal impedance polar diagram (dashed line) elongates either parallel or perpendicular to strike direction. Over resistors, the principal impedance polar diagram elongates perpendicular to strike direction, and over conductors, the principal impedance polar diagram elongates parallel to strike direction. For 2-D resistivity structures, the additional impedance polar diagram (solid line) attains the shape of a symmetric clover leaf. For 3-D resistivity structures, the additional impedance polar diagram (solid line) elongates in one direction, and its amplitude is comparable to that of the principal impedance polar 
diagram (dashed line). Station 27 indicates a 3-D response over all frequencies measured.

The tipper can be calculated when the vertical component of the magnetic field is measured. The tipper magnitude is a measure of the tipping of the magnetic field out of the horizontal plane (Vozoff, 1991). The magnitude is zero for the $1-D$ case and typically increases between 0.1 to 0.5 , and rarely as great as 1 , as it responds to vertical and subvertical structures. The tipper strike typically is used to help resolve the 90-degree ambiguity in the impedance rotation angle. The tipper magnitude of these stations typically was 0.1 to 0.6 over the lower frequencies, indicating some vertical structure at depth. The HzHx and HzHy coherency is a measure of the signalto-noise ratio of the vertical magnetic field with respect to each of the orthogonal, horizontal magnetic-field directions. Values are normalized between 0 and 1 , where values at 0.5 signify signal levels equal to noise levels. These threecomponent magnetic-field coherencies provide a check on the signal-to-noise ratio of the measured values in the tipper magnitude and tipper strike plots.

\section{References Cited}

Bendat, J.S., and Piersol, A.G., 1971, Random data-analysis and measurement procedures: New York, Wiley Interscience, $407 p$.

Dobrin, M.D., and Savit, C.H., 1988, Introduction to Geophysical prospecting (4th ed.): New York, McGraw-Hill, $867 \mathrm{p}$.

Eberhart-Phillips, Donna, Stanley, W.D., Rodriguez, B.D., and Lutter, W.J., 1995, Surface seismic and electrical methods to detect fluids related to faulting: Journal of Geophysical Research, v. 100, no. B7, p. 12919-12936.

EMI, 1996, MT-1 magnetotelluric system operation manual, version 3.2: Richmond, California, ElectroMagnetic Instruments, Inc., $220 \#$ p.

Gamble, T.D., Goubau, W.M., and Clarke, J., 1979, Error analysis for remote reference magnetotellurics: Geophysics, v. 44, no. 5, p. 959-968.

Keller, G.V., 1987, Rock and mineral properties, in Nabighian, M.N., ed., Electromagnetic methods in applied geophysics theory: Tulsa, Oklahoma, Society of Exploration Geophysicists, v. 1, p. 13-51. 
Keller, G.V., 1989, Electrical properties, in Carmichael, R.S., ed., Practical handbook of physical properties of rocks and minerals: Boca Raton, Florida, CRC Press, p. 359-427.

Nelson, P.H., and Anderson, L.A., 1992, Physical properties of ash flow tuff from Yucca Mountain, Nevada: Journal of Geophysical Research, v. 97, no. B5, p. 6823-6841.

Palacky, G.J., 1987, Resistivity characteristics of geologic targets, in Nabighian, M.N., ed., Electromagnetic methods in applied geophysics theory: Tulsa, Oklahoma, Society of Exploration Geophysicists, v. 1, p. 53-129.

Reddy, I.K., Rankin, David, and Phillips, R.J., 1977, Threedimensional modelling in magnetotelluric and magnetic variational sounding: Geophysics Journal of the Royal Astronomical Society, v. 51, p. 313-325.

Sternberg, B.K., Washburne, J.C., and Pellerin, Louise, 1988, Correction for the static shift in magnetotellurics using transient electromagnetic soundings: Geophysics, v. 53, p. 1459-1468.

Vozoff, Keeva, 1972, The magnetotelluric method in the Exploration of sedimentary basins: Geophysics, v. 37, p. $980-141$.

Vozoff, Keeva, 1991, The magnetotelluric method, in Nabighian, M.N., Electromagnetic methods in applied geophysics: Tulsa, Oklahoma, Society of Exploration Geophysicists, v. 2, pt. B, p. 641-711. 


\section{Appendix \\ Magnetotelluiric Data Plots}

There are nine separate plots for each station:

1. Apparent Resistivity for the rotated maximum ( $x$ symbol) and minimum (o symbol) modes

2. Impedance Phase for the rotated maximum ( $x$ symbol) and minimum (o symbol) modes

3. Rotation Angle for the impedance tensor (corresponds to the direction of maximum apparent resistivity)

4. Impedance skew for the impedance tensor

5. Multiple Coherency for the rotated maximum ( $x$ symbol) and minimum (o symbol) modes of the electric field

6. Impedance Polar Plots (at 12 selected frequencies)

7. Tipper Magnitude for the vertical magnetic field

8. Tipper Strike for the vertical magnetic field

9. $\mathrm{HzHx}$ (x symbol) and HzHy (o symbol) Coherency

Refer to the "Magnetotelluric Data" section in this report for an explanation of these plots. 


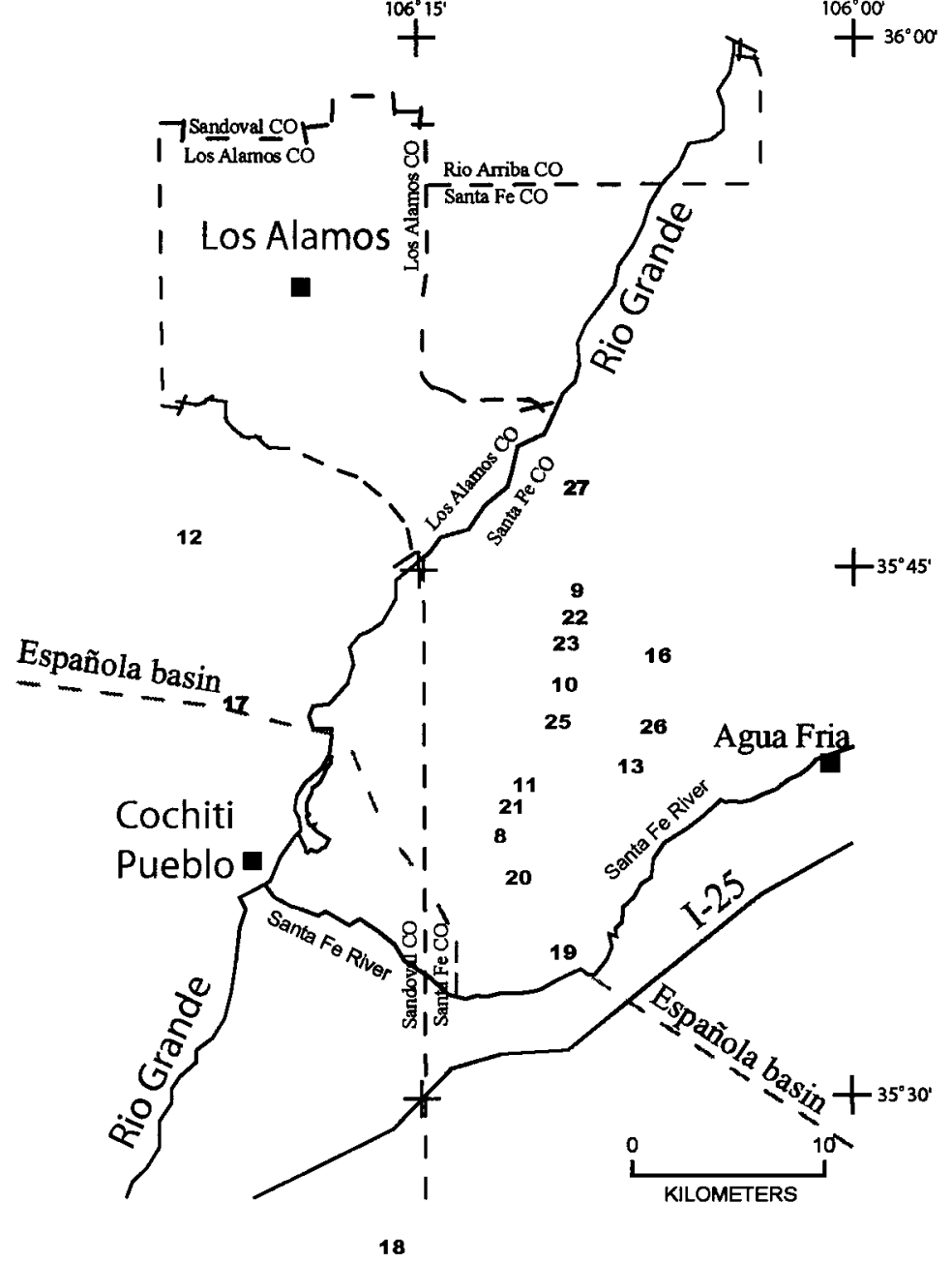

Figure 1. Index map of magnetotelluric (MT) survey area, west of Santa $\mathrm{Fe}$, in Española basin, northern New Mexico. Numbered labels are MT stations acquired in 2002 and 2003. Base map modified from USGS, Albuquerque, New Mexico 250,000 scale topographic map. 


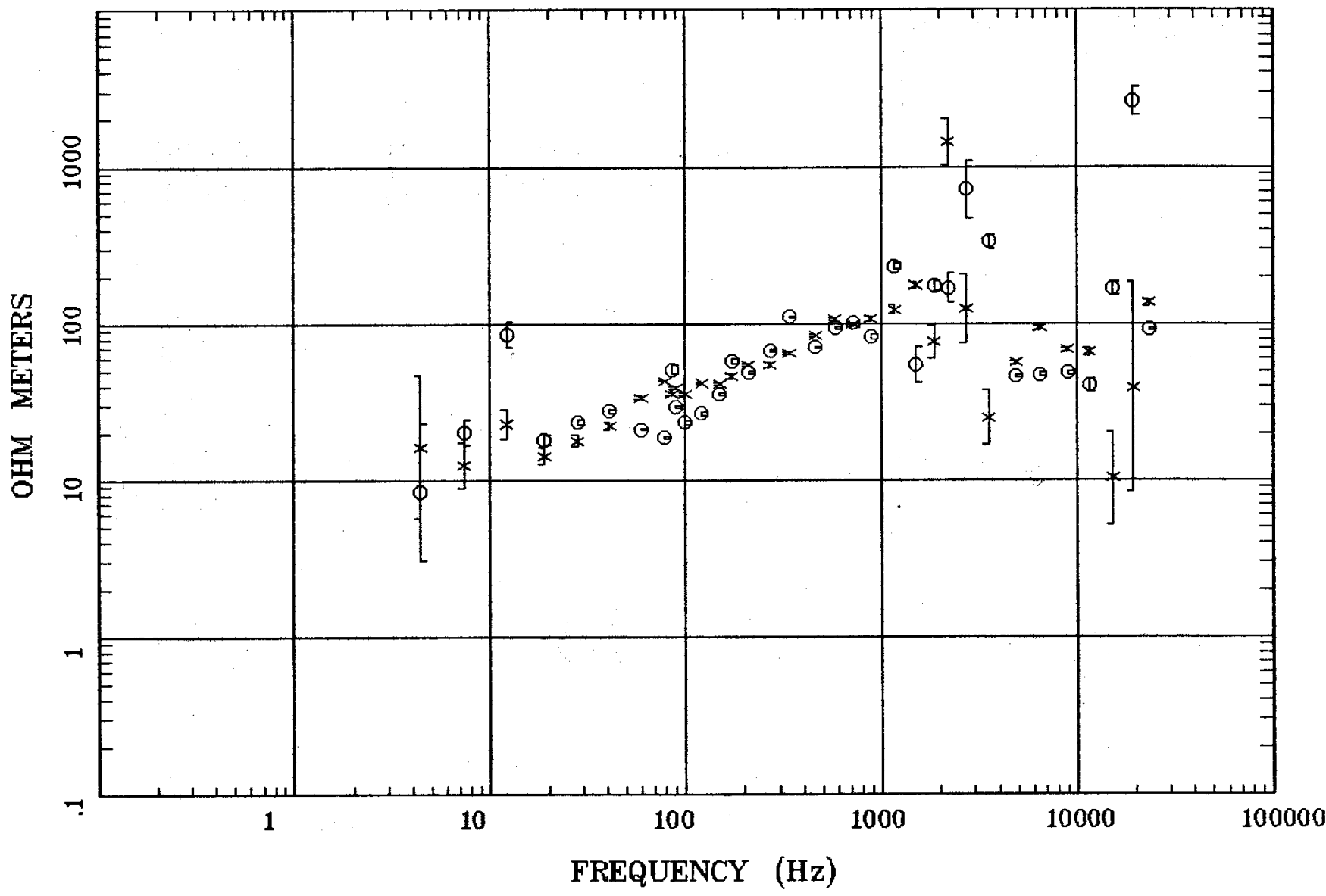

Client:

Remote: e-fld $95 \mathrm{~m}$ east Acquired: 12:3 Jul 24, 2002 Survey Co:USGS
Rotation:

Filename: cpob.avg

Channels: Ch1 Ch2 Ch3 ch4 Ch5 ch6 Ch7

Plotted: 08:31 Jul 06, 2004

< EMI - ElectroMagnetic Instruments > 


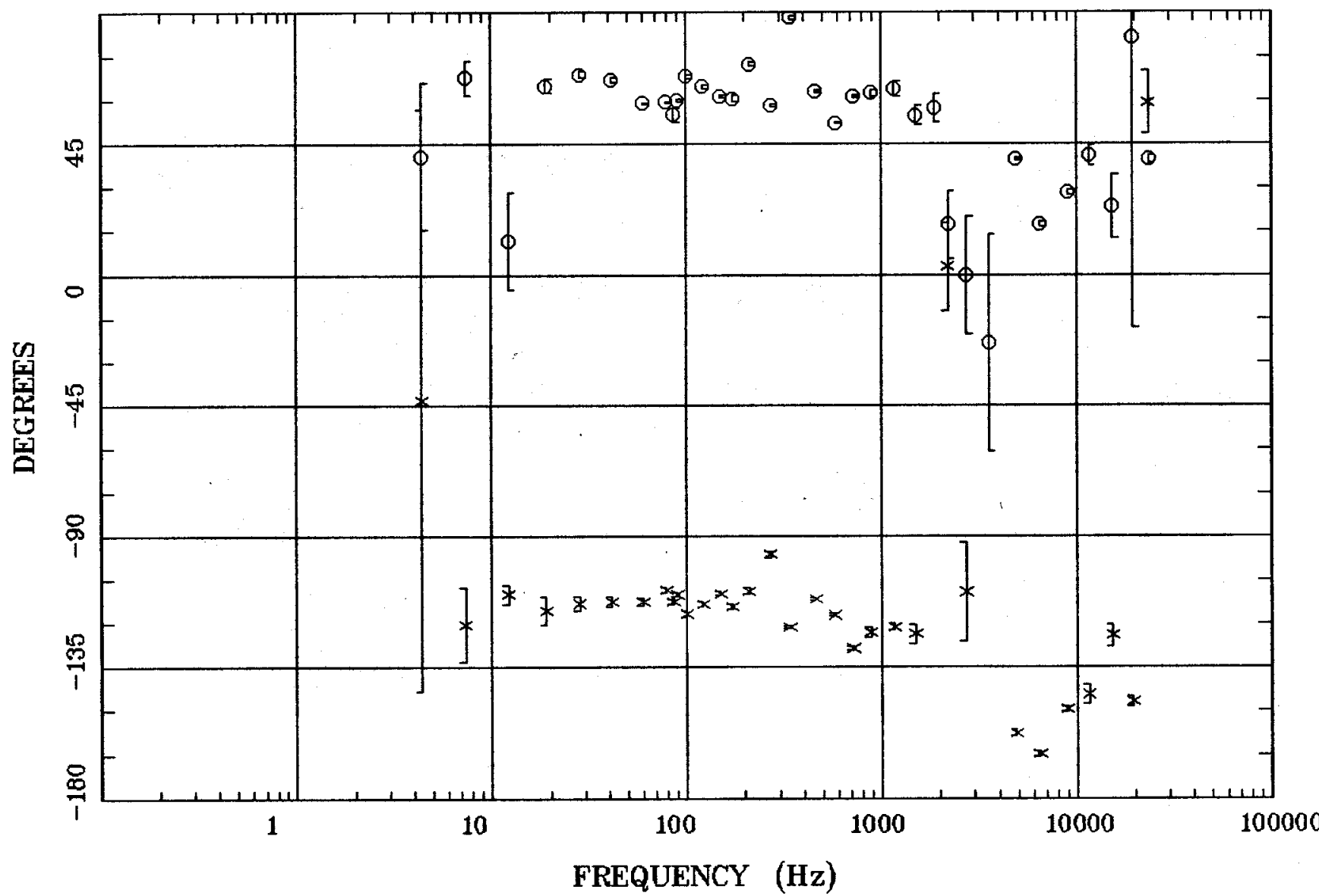

Client:

Remote: e-fld $95 \mathrm{~m}$ east Acquired: 12:3 Jul 24, 2002 Survey Ca:USGS
Rotation:

Filename: cp08.avg

Channels: Ch1 Ch2 Ch3 Ch4 Ch5 Ch6 Ch7 Plotted: 08:31 Jul 06, 2004

< EMI - ElectroMagnetic Instruments > 
North of Tetilla Peak Station 8

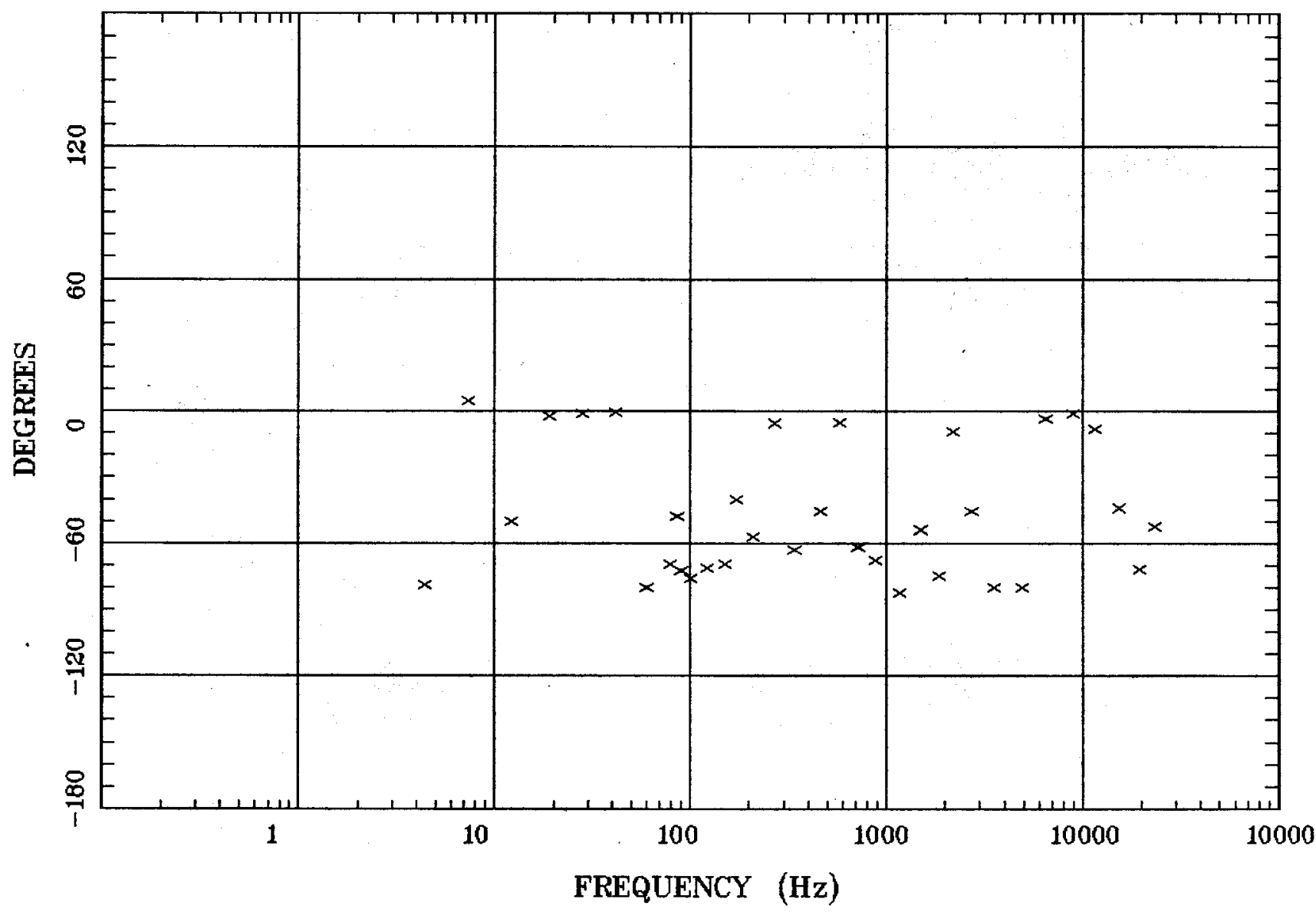

Client:

Remote: e-fld $95 \mathrm{~m}$ east Acquired: 12:3 Jul 24, 2002 Survey Co:USGS
Rotation:

Filename: cp08.avg

Channels: Ch1 Ch2 Ch3 Ch4 Ch5 Ch6 Ch7

Platted: 12:45 Jul 07, 2004

< EMI - ElectroMagnetic Instruments 


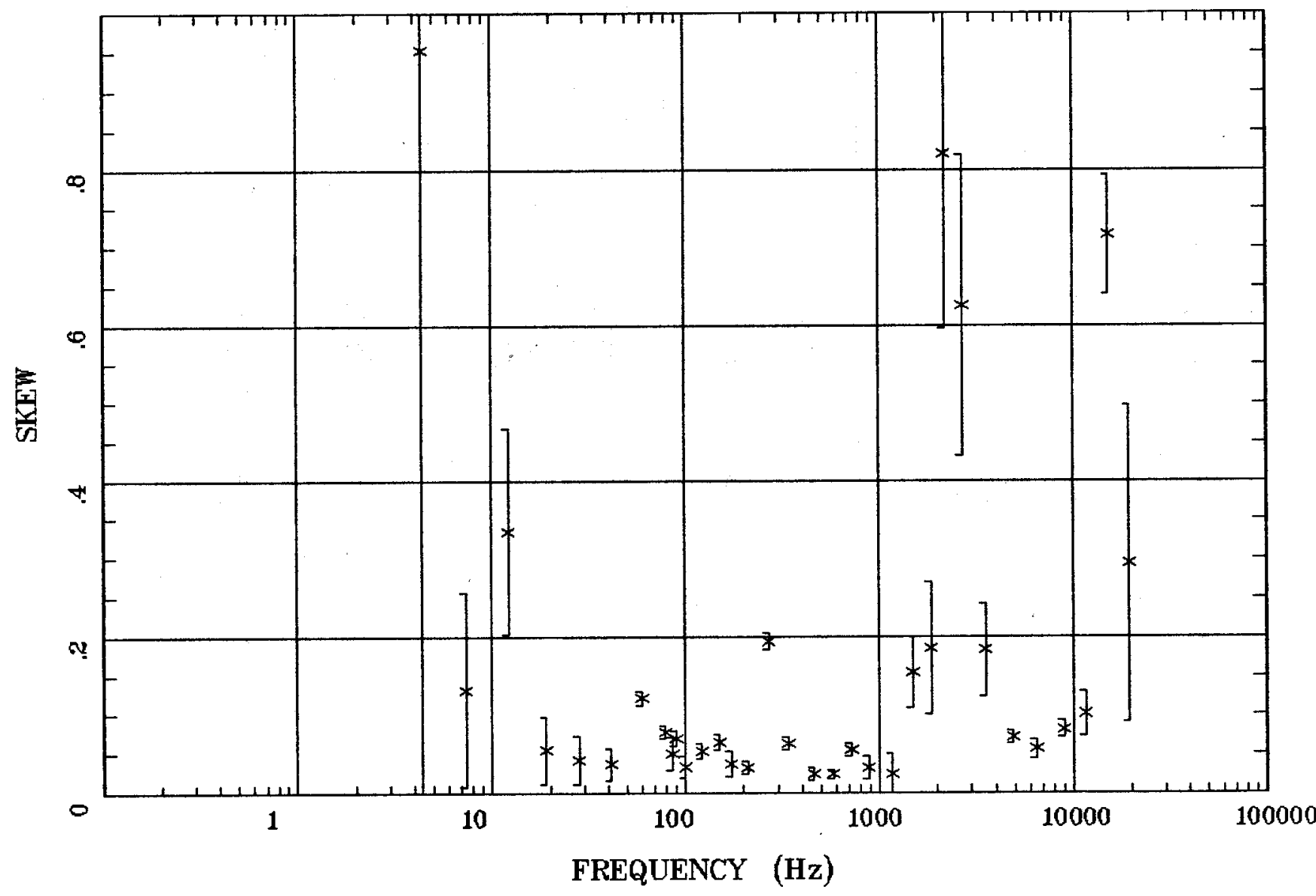

Client:

Remote: e-fld $95 \mathrm{~m}$ east Acquired: $12: 3$ Jul 24, 2002 Survey Co:USGS
Rotation:

Filename: cp08.avg

Channels: Ch1 Ch2 Ch3 ch4 Ch5 Ch6 Ch7 Plotted: 08:32 Jul 06, 2004

< EMI - ElectroMagnetic Instruments 
E MULT Coh.

North of Tetilla Peak Station 8

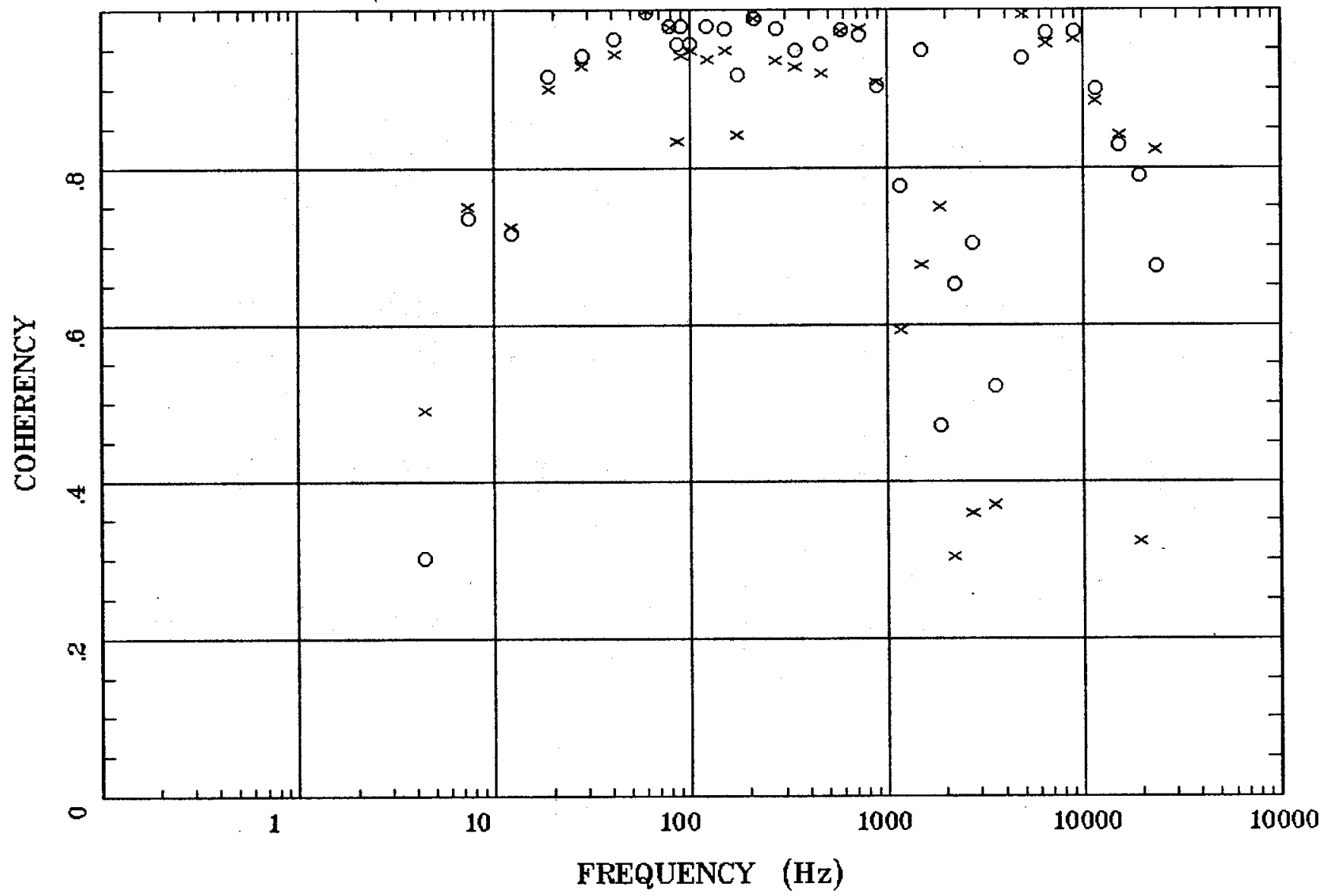

Client:

Remote: e-fld $95 \mathrm{~m}$ east Acquired: 12:3 Jul 24, 2002 Survey Co:USGS
Rotation:

Filename: cp08.avg

Channels: Ch1 Ch2 Ch3 Ch4 Ch5 Ch6 Ch7 Plotted: 08:32 Jul 06, 2004

< EMI - ElectroMagnetic Instruments 


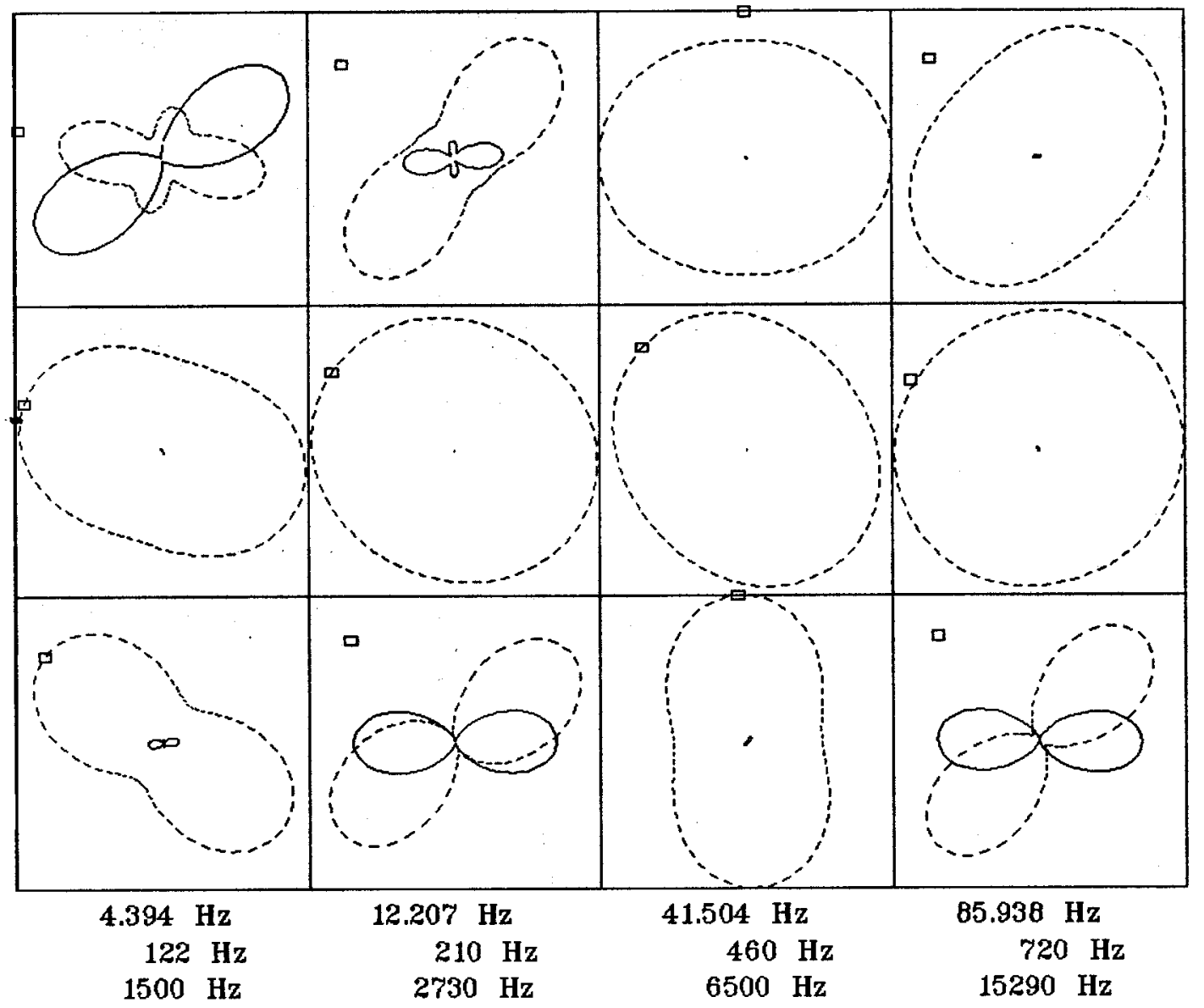

Client:

Remote: e-fld $95 \mathrm{~m}$ east Acquired: 12:3 Jul 24, 2002 Survey Co:USGS
Rotation:

Filename: cp08.avg

Channels: Ch1 Ch2 Ch3 Ch4 Ch5 Ch6 Ch7 Plotted: 08:32 Jul 06, 2004

< EMI - ElectroMagnetic Instruments > 


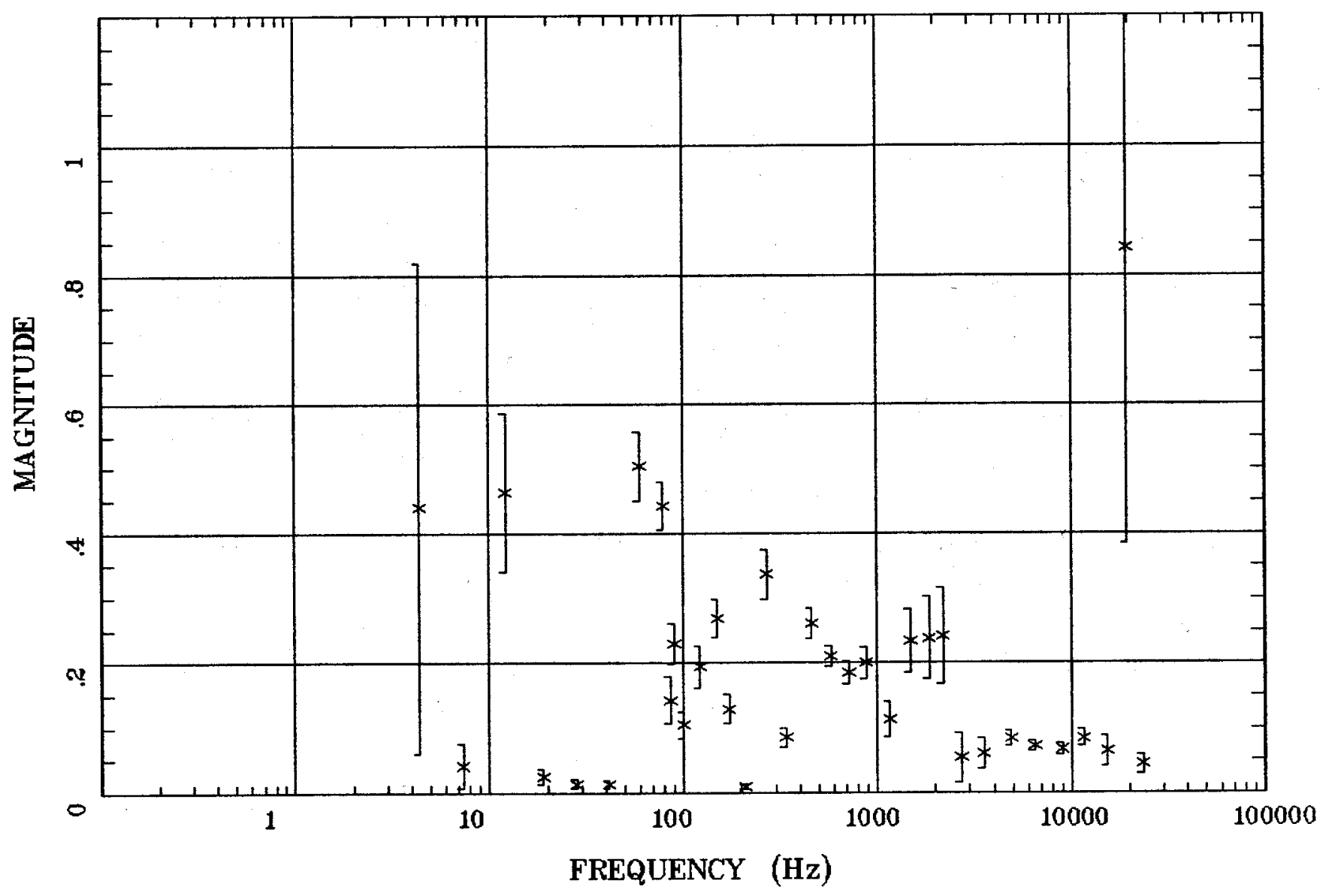

Client:

Remote: e-fld $95 \mathrm{~m}$ east

Acquired: 12:3 Jul 24, 2002

Survey Co:USGS

Rotation:

Filename: cp08.avg

Channels: Ch1 Ch2 Ch3 Ch4 Ch5 Ch6 Ch7

Plotted: 08:33 Jul 06, 2004

< EMI - ElectroMagnetic Instruments > 


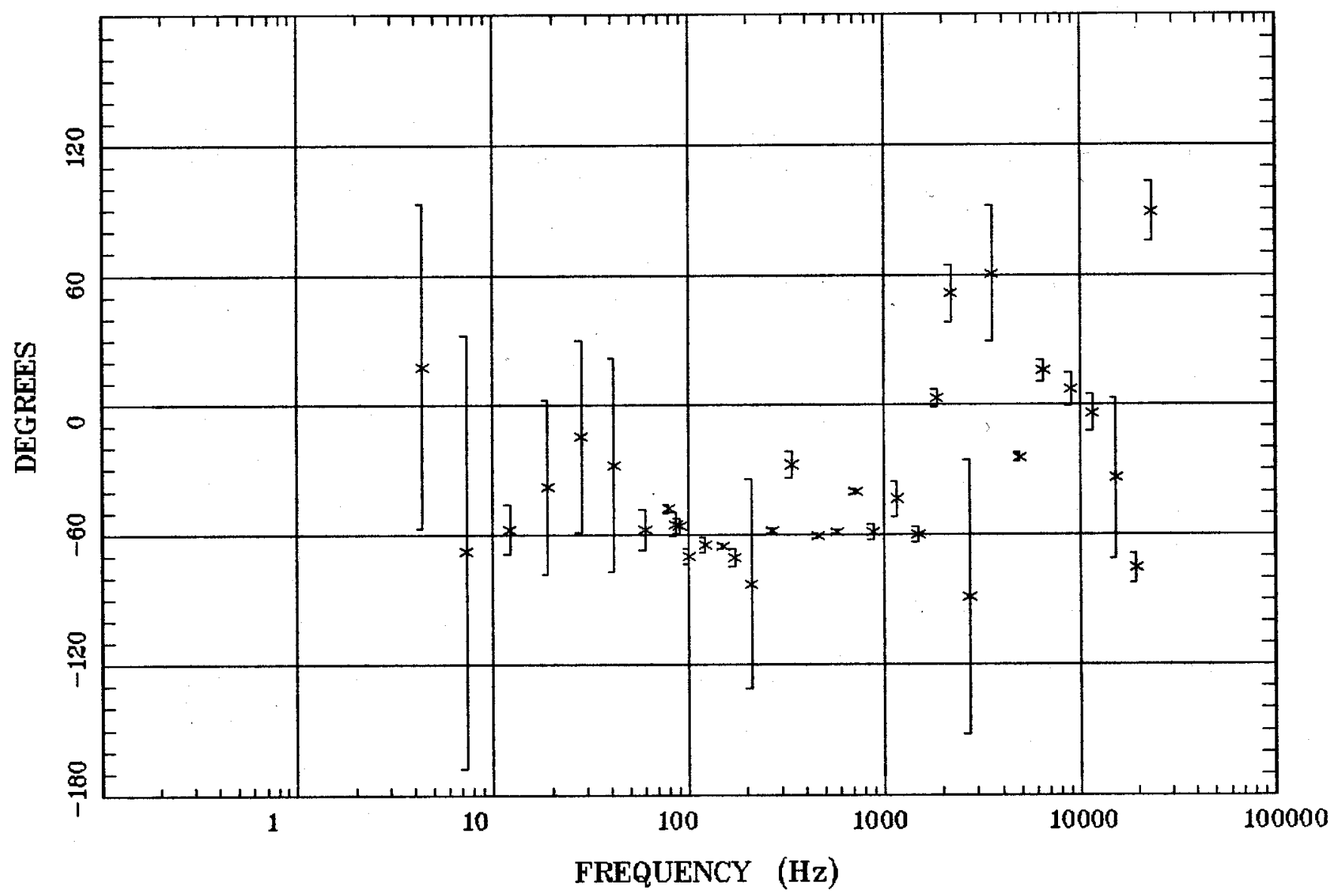

Client:

Remote: e-fld $95 \mathrm{~m}$ east Acquired: 12:3 Jul 24, 2002 Survey Co:USGS
Rotation:

Filename: cp08.avg

Channels: Ch1 Ch2 Ch3 Ch4 Ch5 Ch6 Ch7

Plotted: 08:33 Jul 06, 2004

< EMI - ElectroMagnetic Instruments > 
HzHx.x Coh HzHy.o

North of Tetilla Peak

Station 8

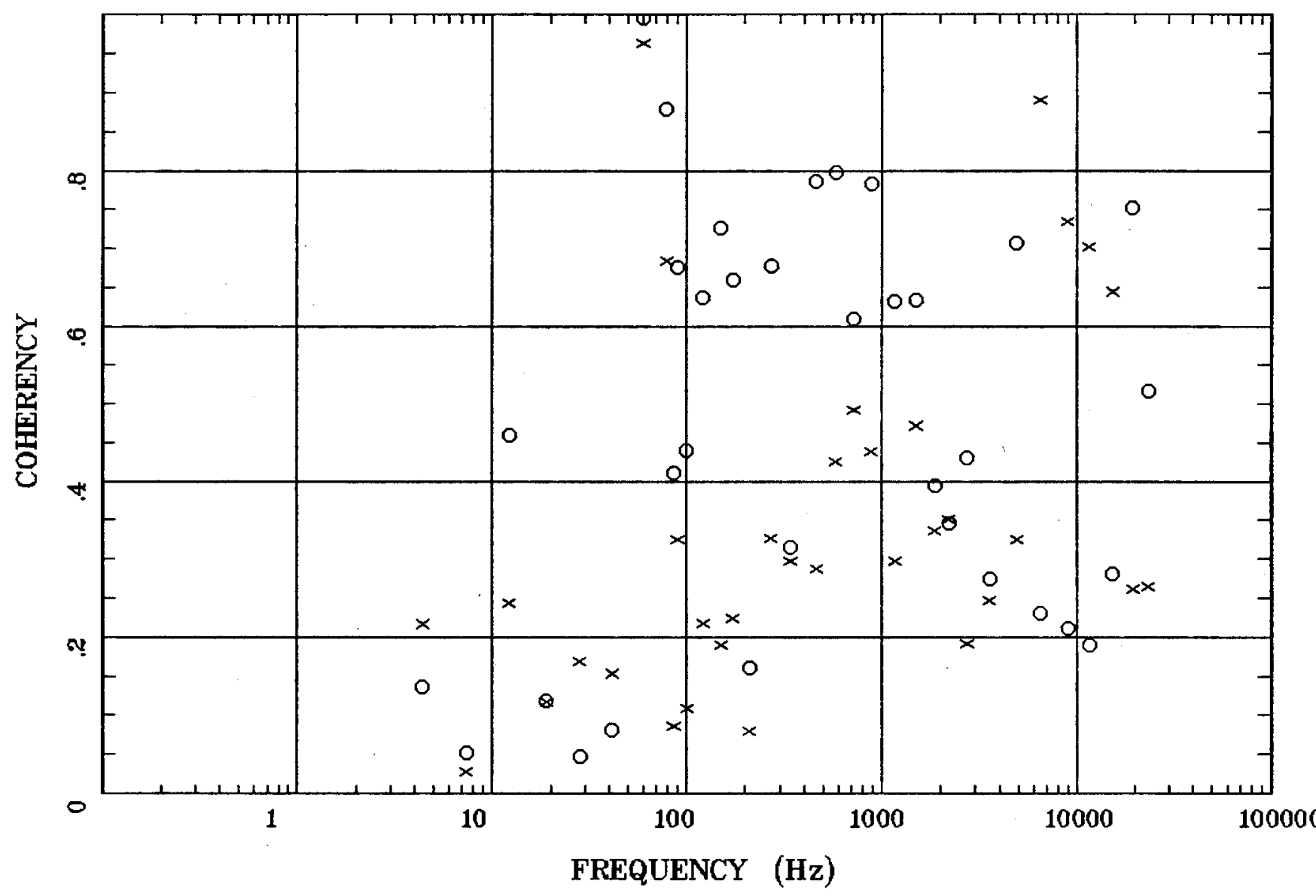

Client:

Remote: e-fld $95 \mathrm{~m}$ east Acquired: $12: 3$ Jul 24, 2002 Survey Co:USGS
Rotation:

Filename: cp08.avg

Channels: Ch1 Ch2 Ch3 Ch4 Ch5 Ch6 Ch7 Plotted: 08:33 Jul 06, 2004

< EMI - ElectroMagnetic Instruments > 


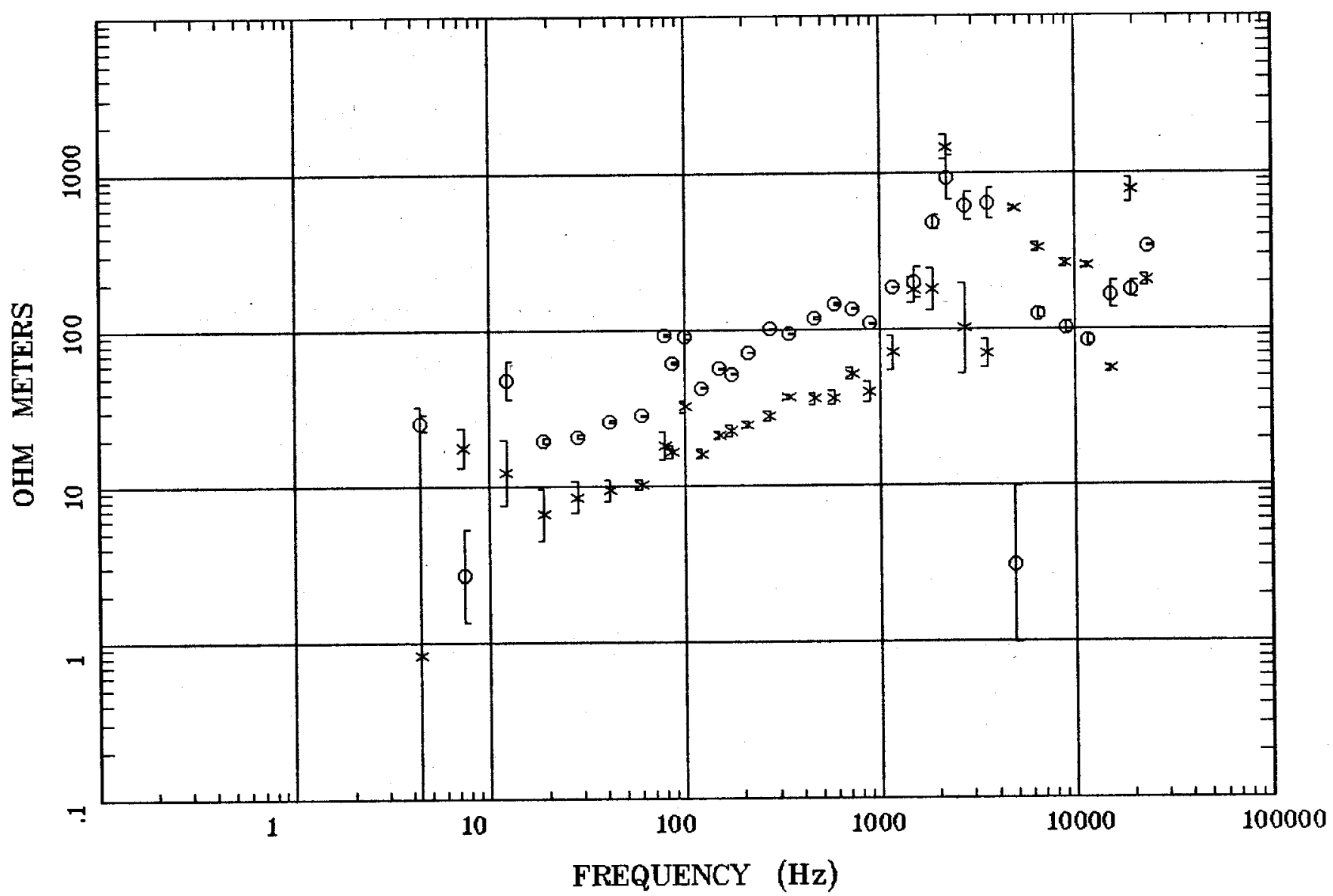

Client:

Remote: e-fld $95 \mathrm{~m}$ north Acquired: 13:4 Jul 25, 2002 Survey Co:USGS
Rotation:

Filename: cp09.avg

Channels: Ch1 Ch2 Ch3 Ch4 Ch5 Ch6 Ch7

Plotted: 09:14 Jul 06, 2004

$<$ EMI - ElectroMagnetic Instruments 
South of Ortiz Mtn, NMStation 9

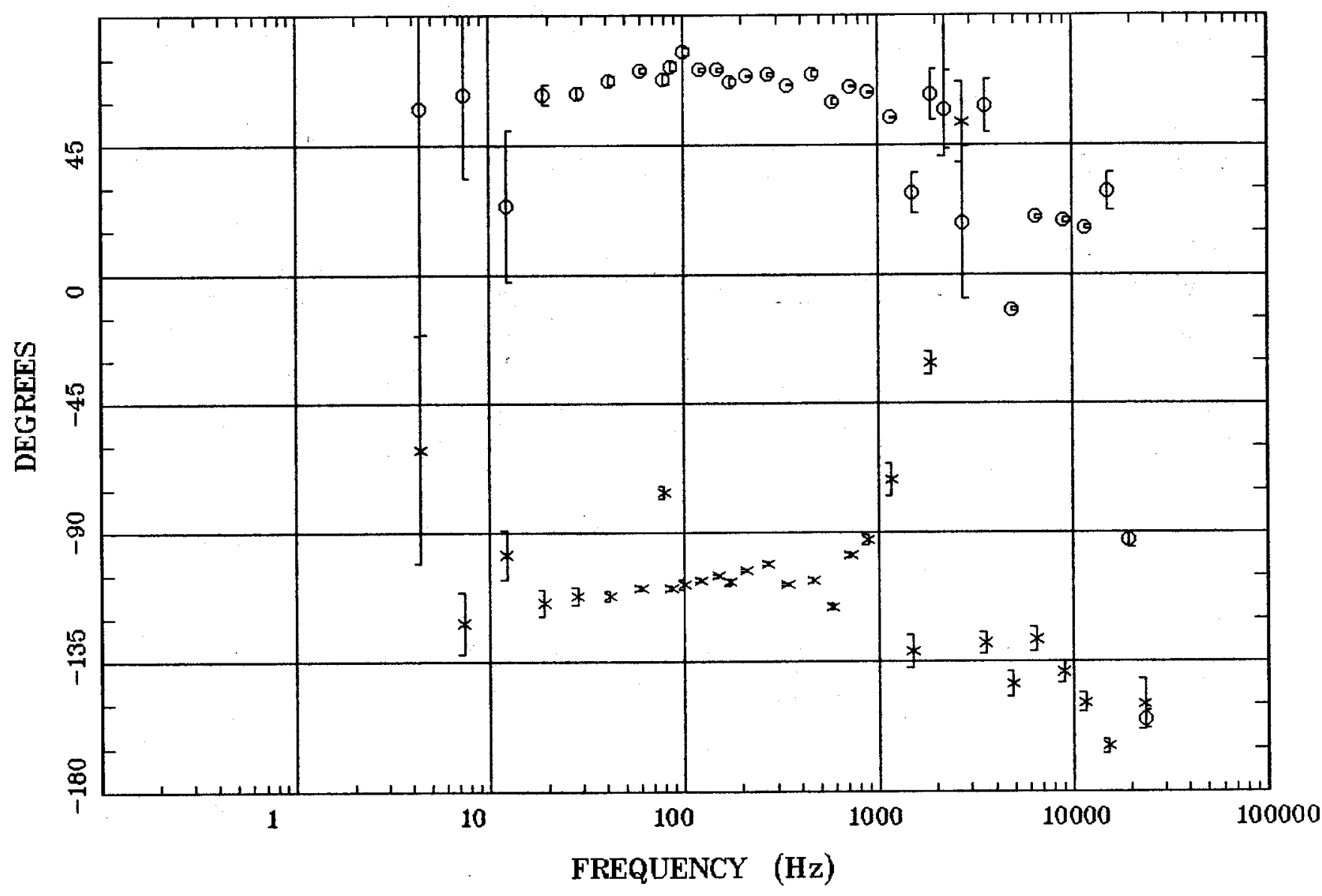

Client:

Remote: e-fld $95 \mathrm{~m}$ north Acquired: 13:4 Jul 25, 2002 Survey Co:USGS
Rotation:

Filename: cp09.avg

Channels: Ch1 Ch2 Ch3 Ch4 Ch5 Ch6 Ch7 Plotted: 09:14 Jul 06, 2004

< EMI - ElectroMagnetic Instruments 


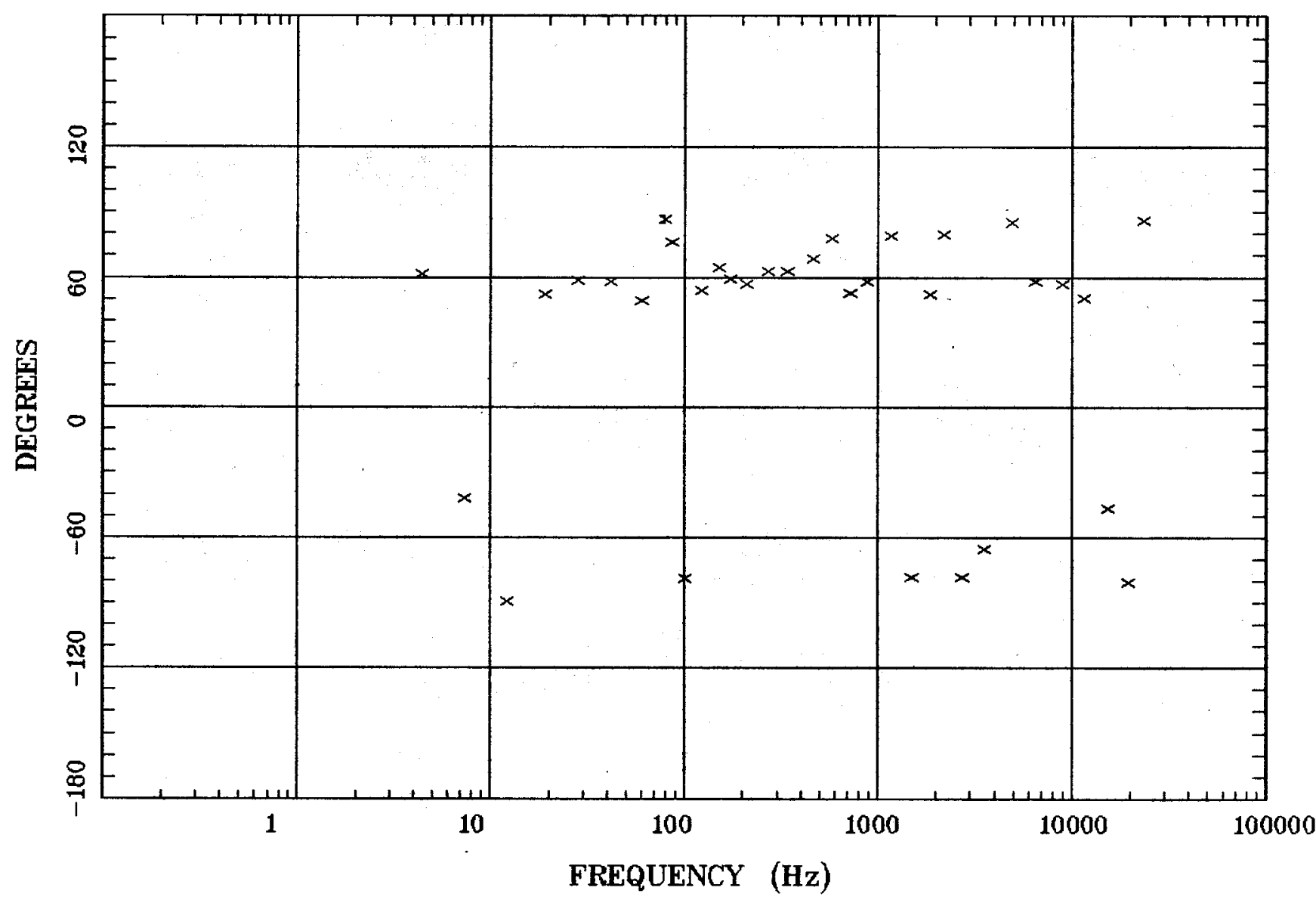

Client:

Remote: e-fld $95 \mathrm{~m}$ north Acquired: 13:4 Jul 25, 2002 Survey Co:USGS
Rotation:

Filename: cp09.avg

Channels: Ch1 Ch2 Ch3 Ch4 Ch5 Ch6 Ch7 Plotted: 12:46 Jul 07, 2004

< EMI - ElectroMagnetic Instruments 
South of Ortiz Mtn, NMStation 9

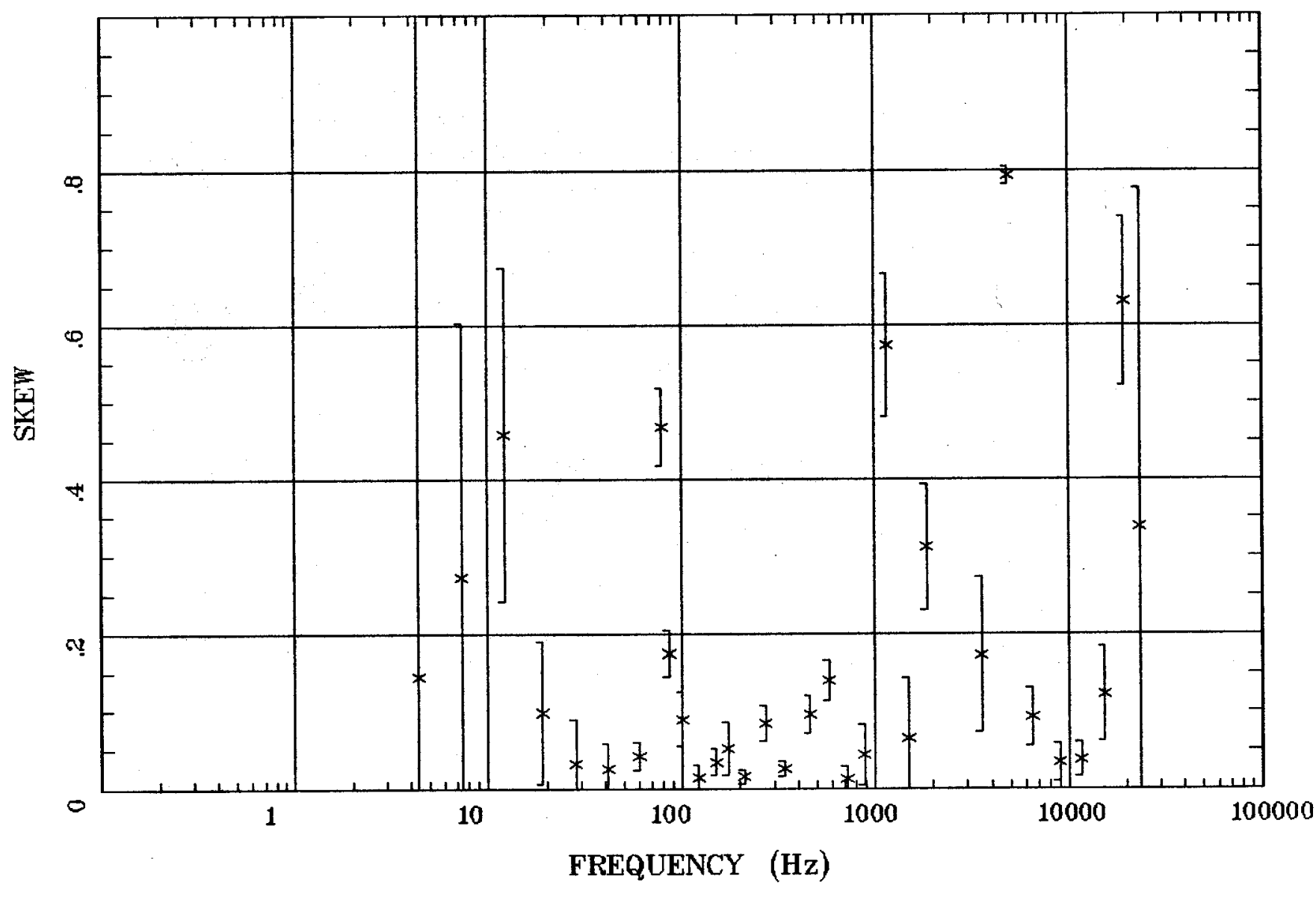

Client:

Remote: e-fld $95 \mathrm{~m}$ north Acquired: 13:4 Jul 25, 2002 Survey Co:USGS
Rotation:

Filename: cp09.avg

Channels: Ch1 Ch2 Ch3 Ch4 Ch5 Ch6 Ch'7 Plotted: 09:14 Jul 06, 2004

< EMI - ElectroMagnetic Instruments > 


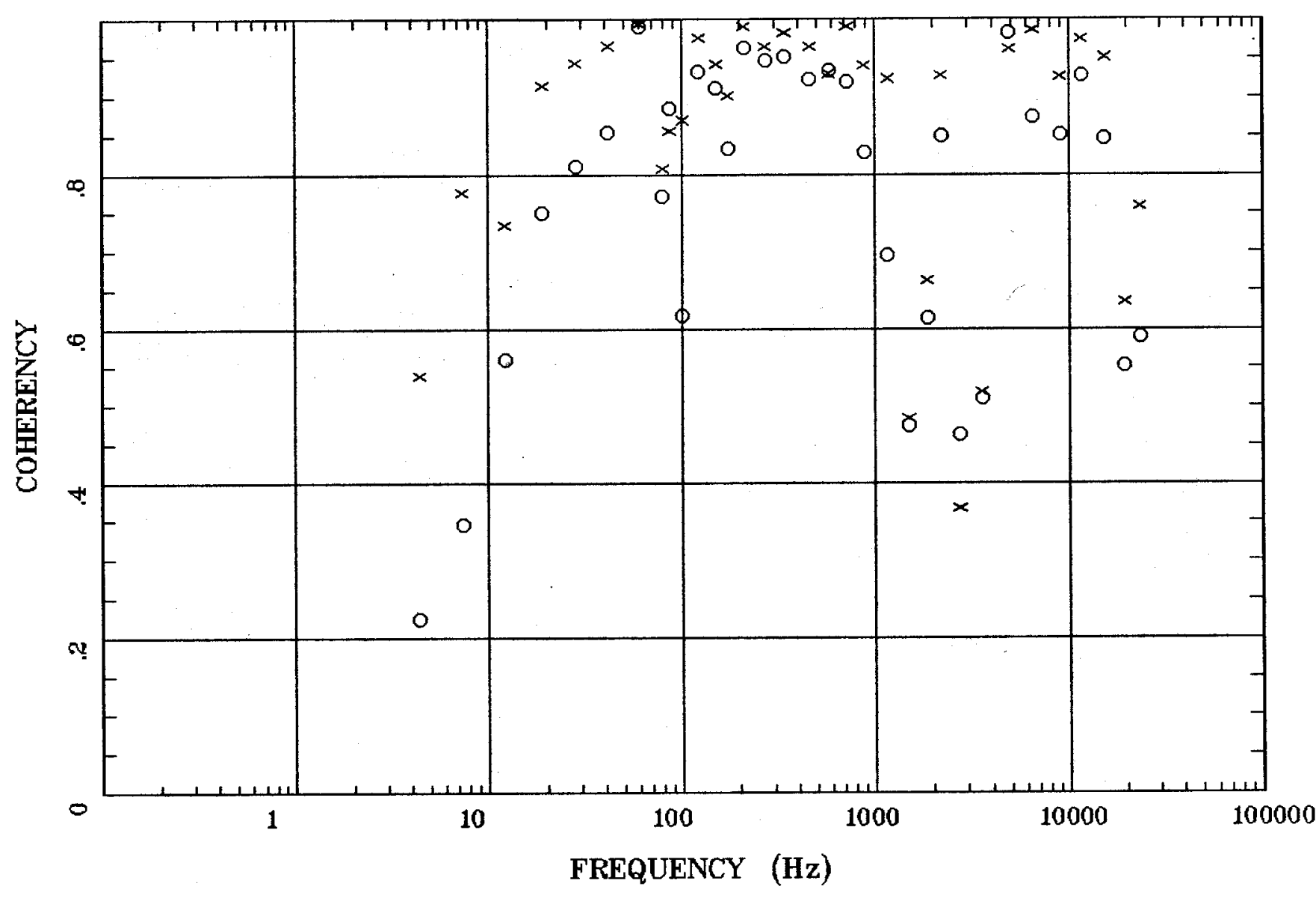

Client:

Remote: e-fld $95 \mathrm{~m}$ north Acquired: 13:4 Jul 25, 2002 Survey Co:USGS
Rotation:

Filename: cp09.avg

Channels: Ch1 Ch2 Ch3 Ch4 Ch5 Ch6 Ch7 Plotted: 09:15 Jul 06, 2004

$<$ EMI - ElectroMagnetic Instruments 


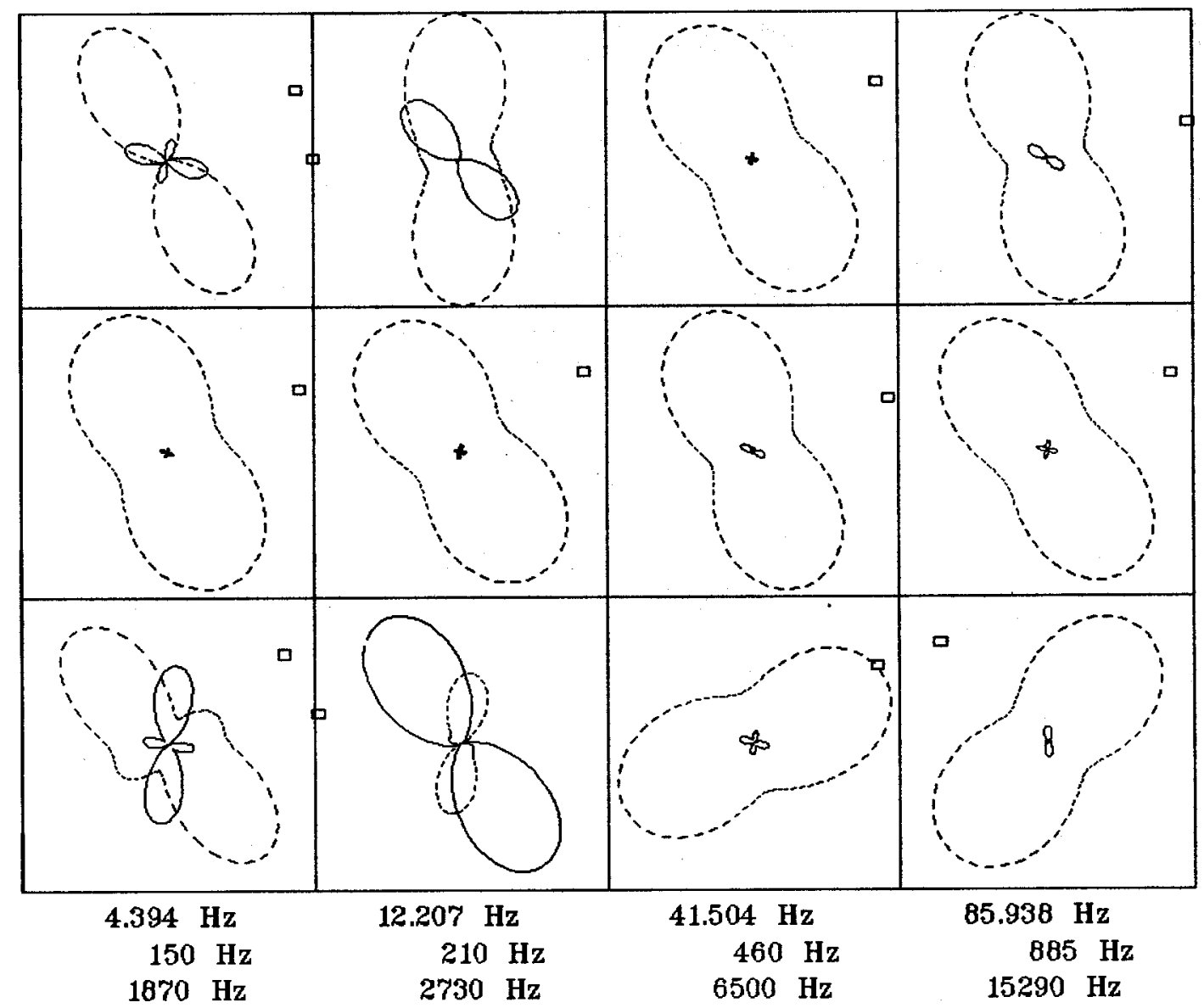

Client:

Remote: e-fld $95 \mathrm{~m}$ north Acquired: 13:4 Jul 25, 2002 Survey Co:USGS

\section{Rotation:}

Filename: cp09.avg

Channels: Ch1 Ch2 Ch3 Ch4 Ch5 Ch6 Ch7 Plotted: 09:15 Jul 06, 2004

< EMI - ElectroMagnetic Instruments > 


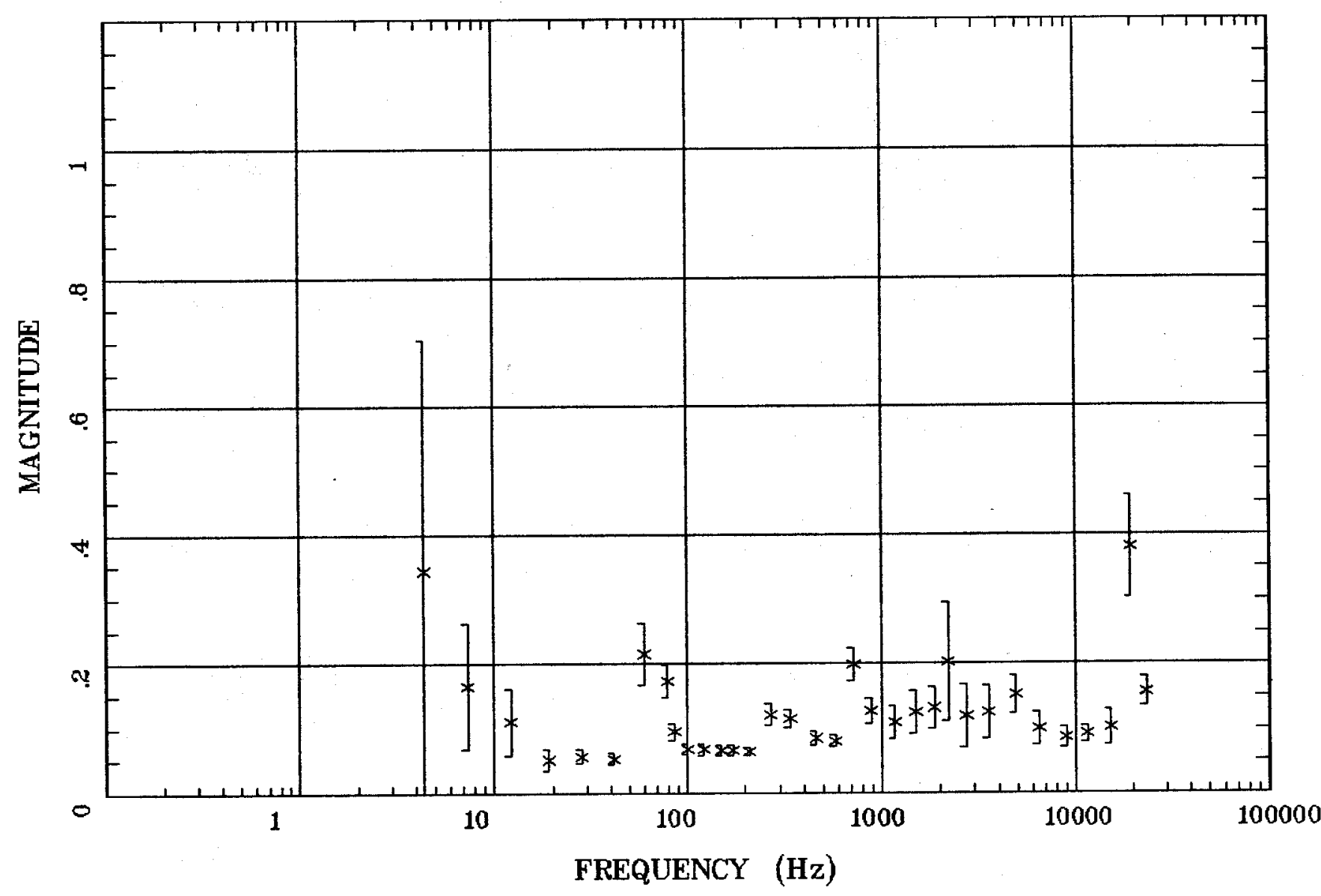

Client:

Remote: e-fld $95 \mathrm{~m}$ north Acquired: 13:4 Jul 25, 2002 Survey Co:USGS
Rotation:

Filename: cp09.avg

Channels: Ch1 Ch2 Ch3 Ch4 Ch5 Ch6 Ch7 Plotted: 09:15 Jul 06, 2004

< EMI - ElectroMagnetic Instruments > 
South of Ortiz Mtn, NMStation 9

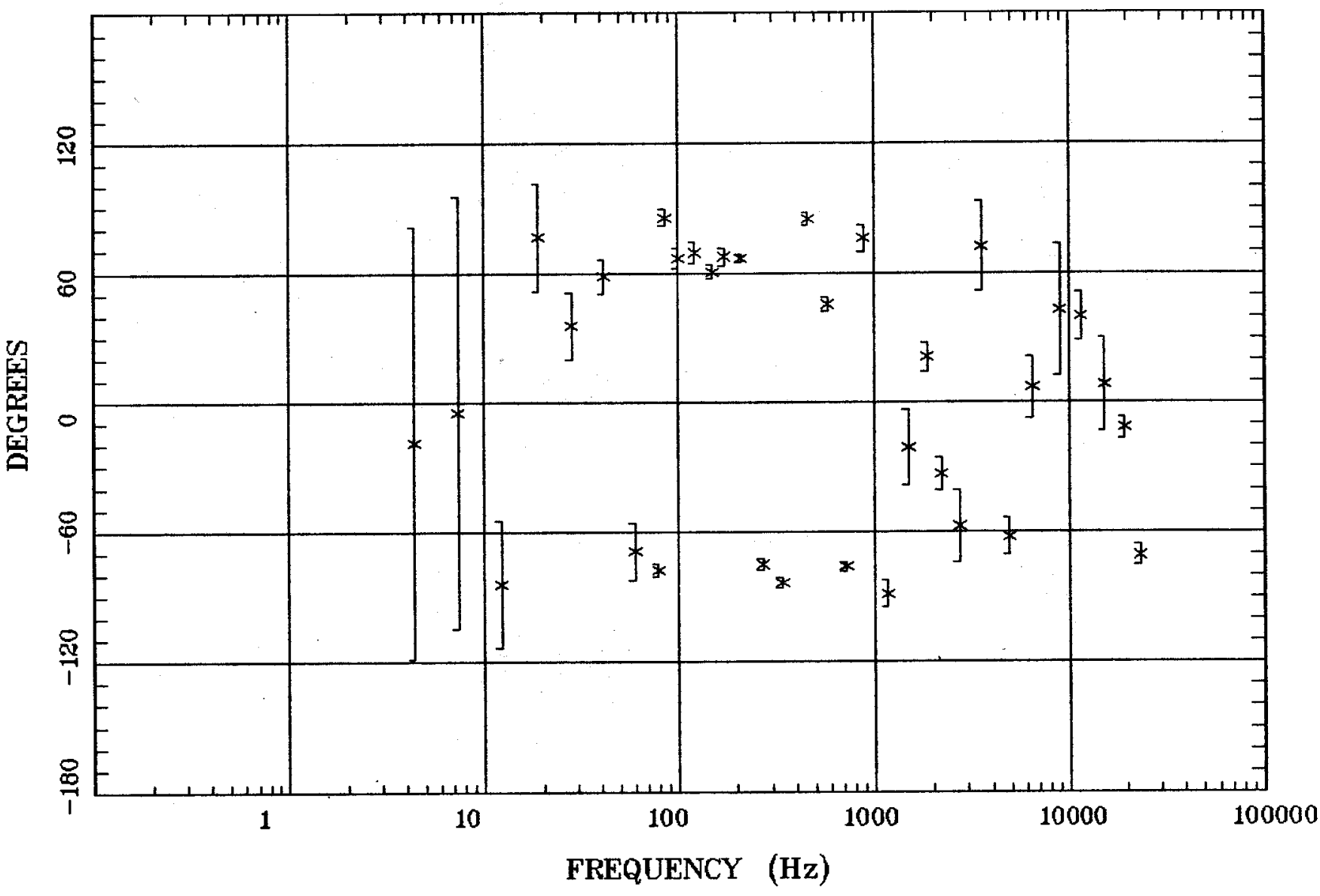

Client:

Remote: e-fld $95 \mathrm{~m}$ north Acquired: 13:4 Jul 25, 2002 Survey Co:USGS
Rotation:

Filename: cp09.avg Channels: Ch1 Ch2 Ch3 Ch4 Ch5 Ch6 Ch7 Plotted: 09:15 Jul 06, 2004

$<$ EMI - ElectroMagnetic Instruments 
HzHx.x Coh HzHy.o

South of Ortiz Mtn, NMStation 9

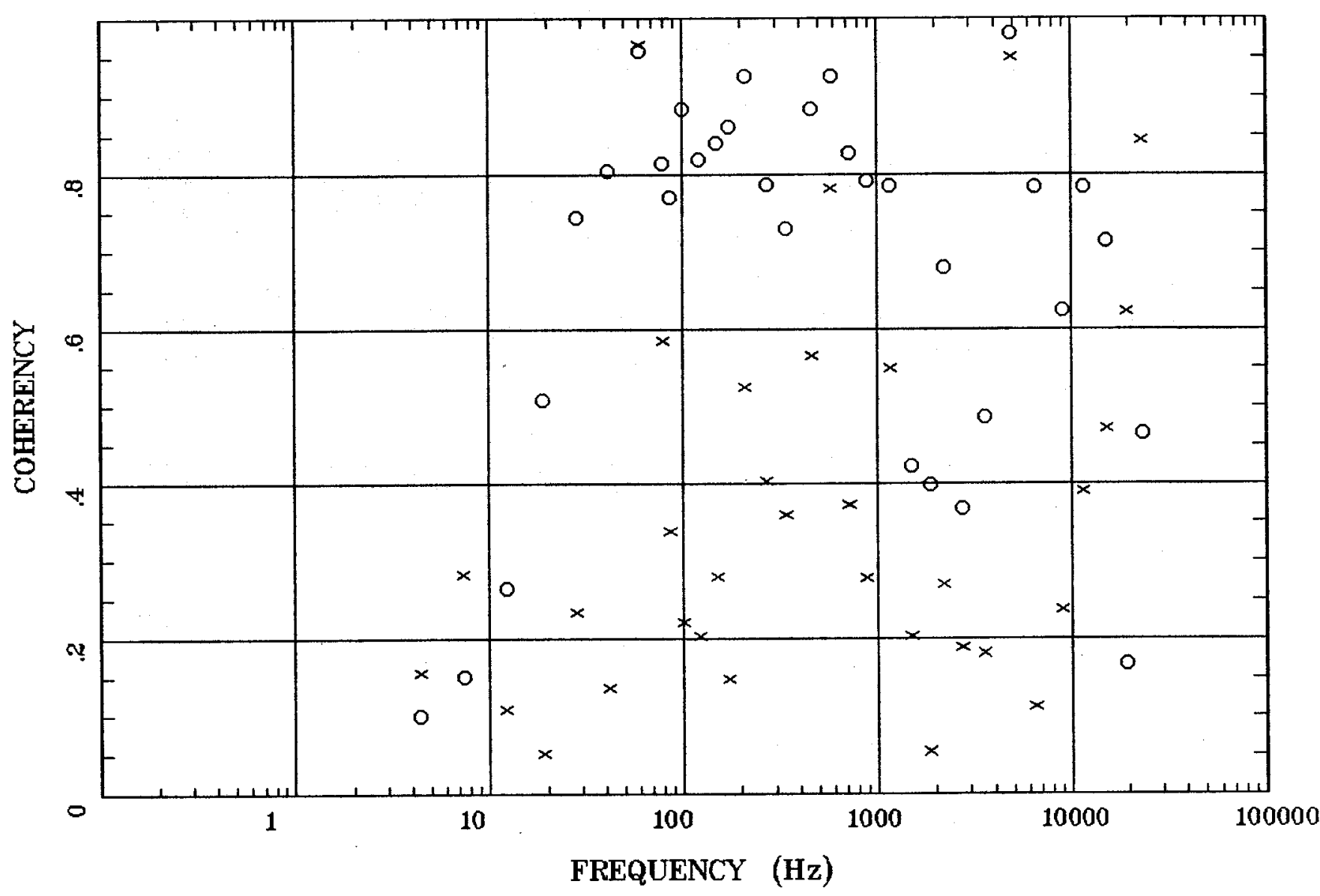

Client:

Remote: e-fld $95 \mathrm{~m}$ north

Acquired: 13:4 Jul 25, 2002 Survey Co:USGS
Rotation:

Filename: cp09.avg

Channels: Ch1 Ch2 Ch3 Ch4 Ch5 Ch6 Ch7 Plotted: 09:15 Jul 06, 2004

< EMI - ElectroMagnetic Instruments > 


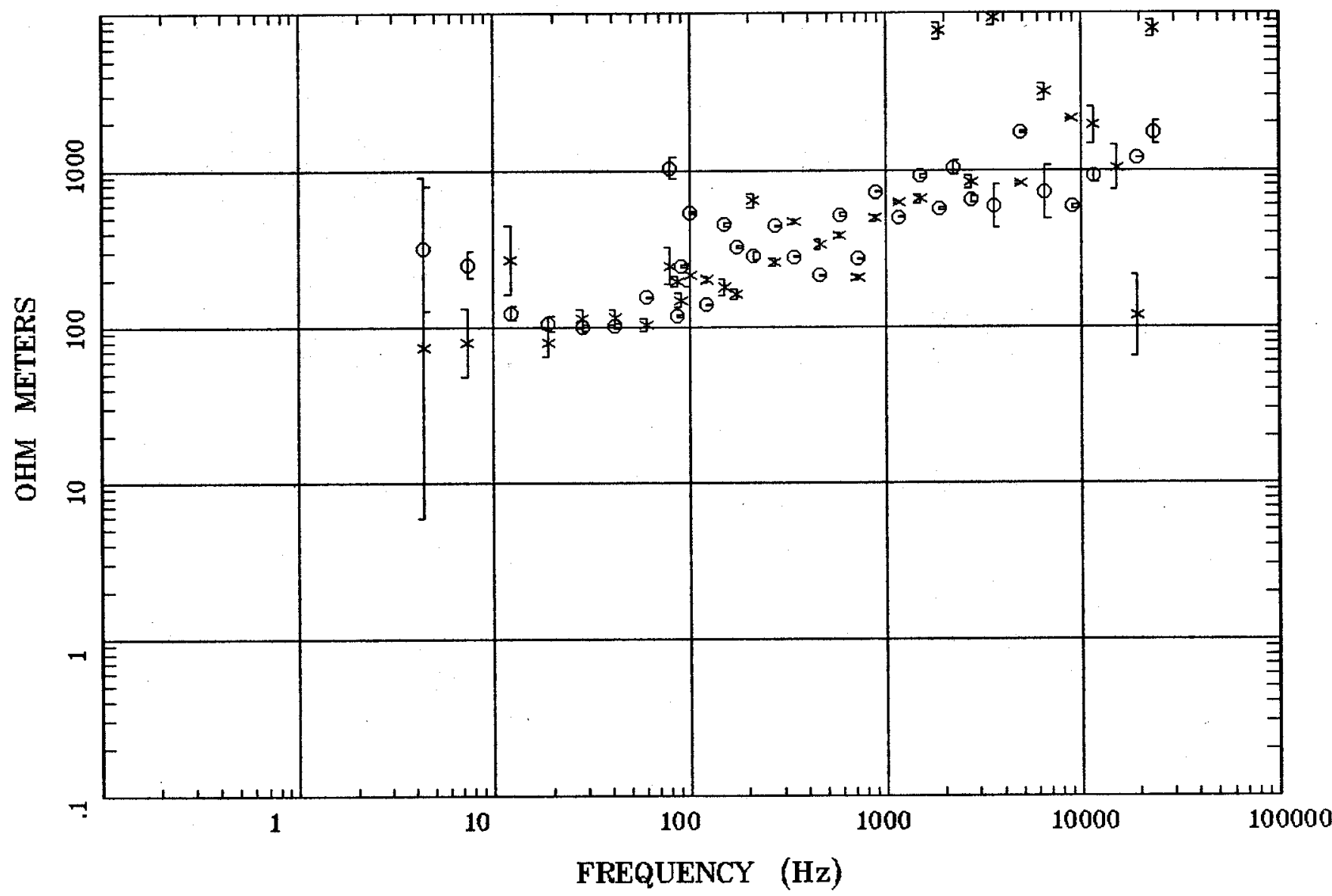

Client:

Remote: e-fld $95 \mathrm{~m}$ west

Acquired: 14:3 Jul 26, 2002 Survey Co:USGS
Rotation:

Filename: cp10.avg

Channels: Ch1 Ch2 Ch3 Ch4 Ch5 Ch6 Ch7 Plotted: 09:17 Jul 06, 2004

< EMI - ElectroMagnetic Instruments > 


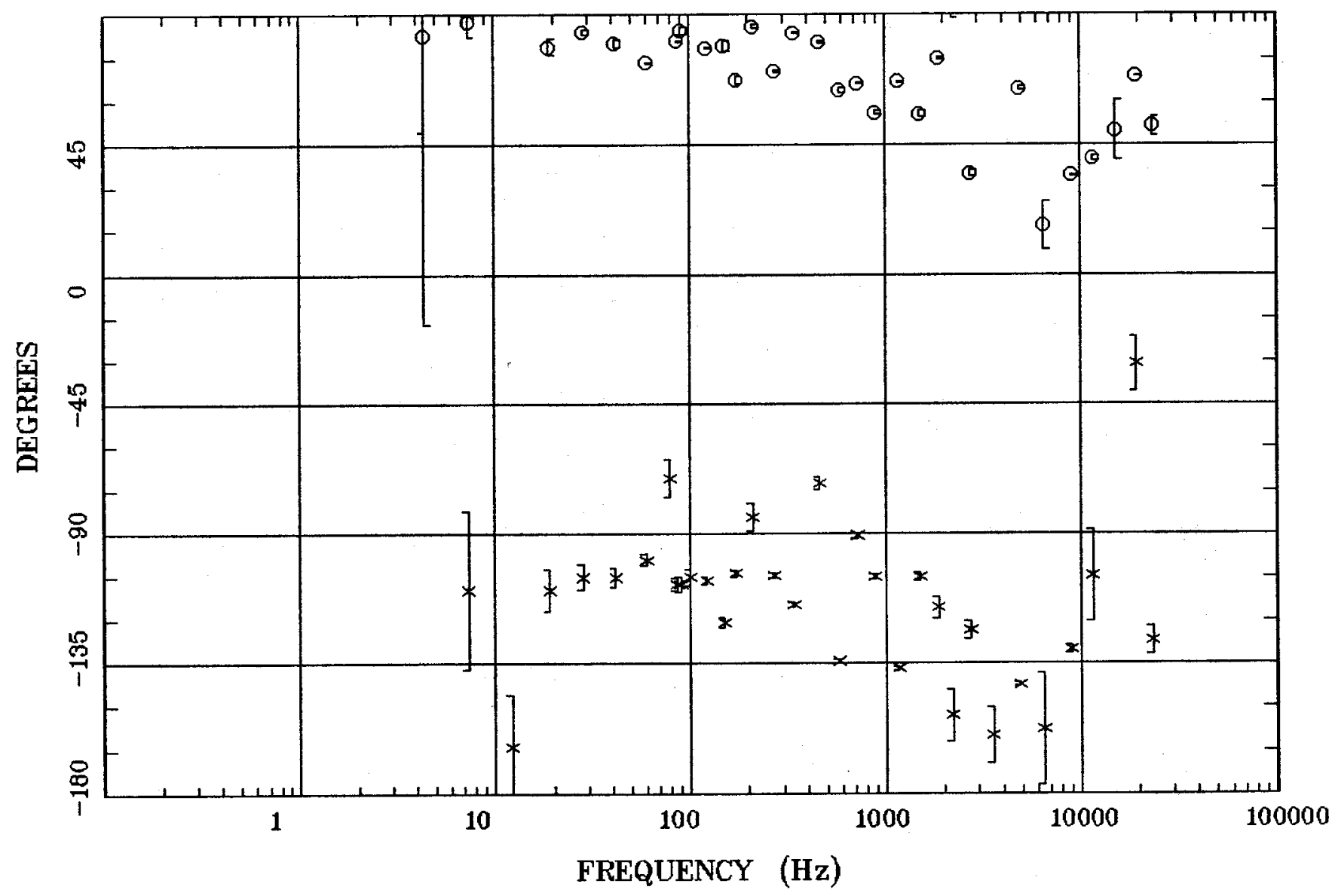

Client:

Remote: e-fld $95 \mathrm{~m}$ west Acquired: 14:3 Jul 26, 2002 Survey Ca:USGS
Rotation:

Filename: cp 10.avg

Channels: Ch1 Ch2 Ch3 Ch4 Ch5 Ch6 Ch7 Plotted: 09:17 Jul 06, 2004

< EMI - ElectroMagnetic Instruments > 


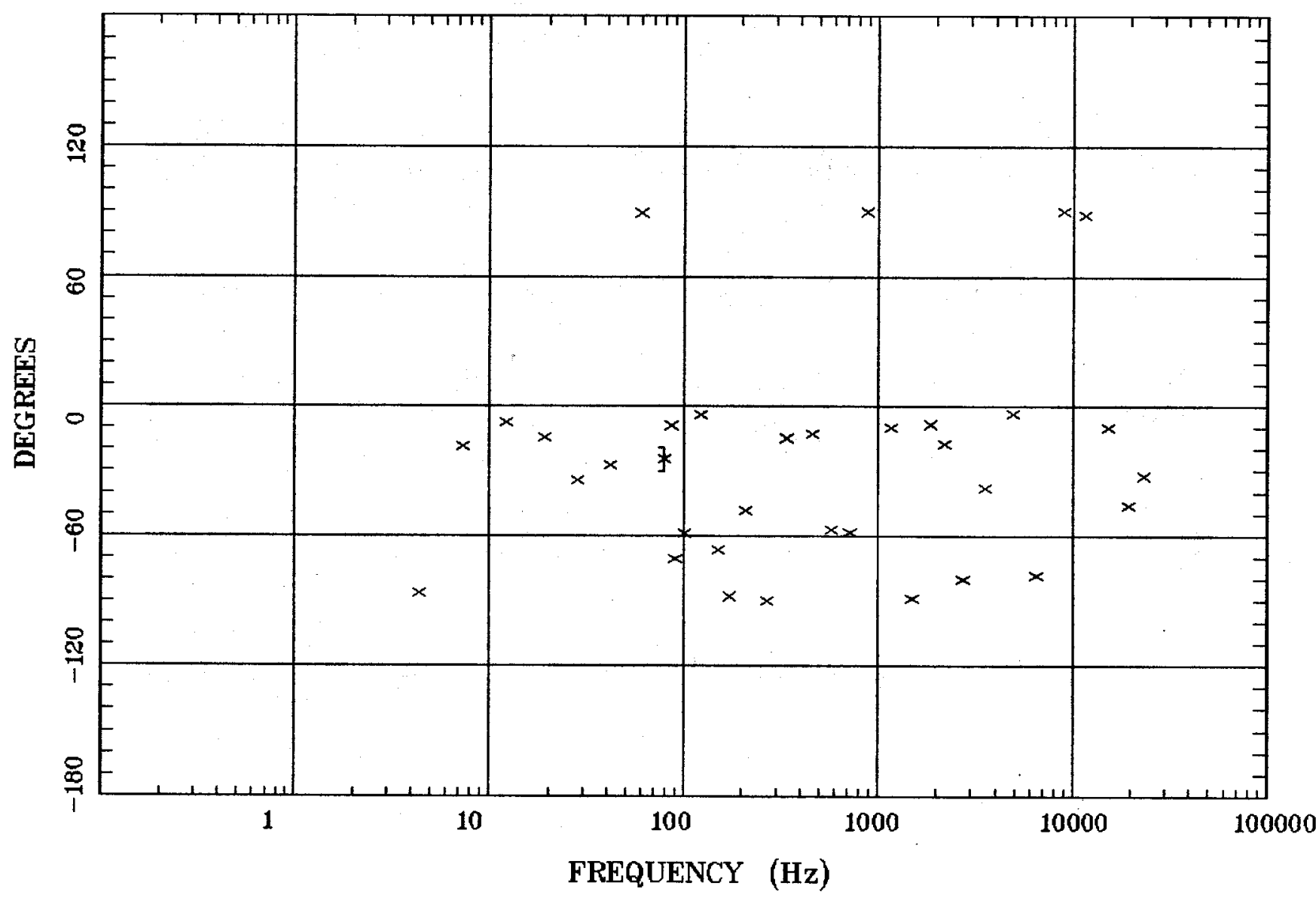

Client:

Remote: e-fld $95 \mathrm{~m}$ west Acquired: 14:3 Jul 26, 2002 Survey Co:USGS
Rotation:

Filename: cp 10.avg

Chennels: Ch1 Ch2 Ch3 Ch4 Ch5 Ch6 Ch7 Plotted: 12:46 Jul 07, 2004

$<$ EMI - ElectroMagnetic Instruments 


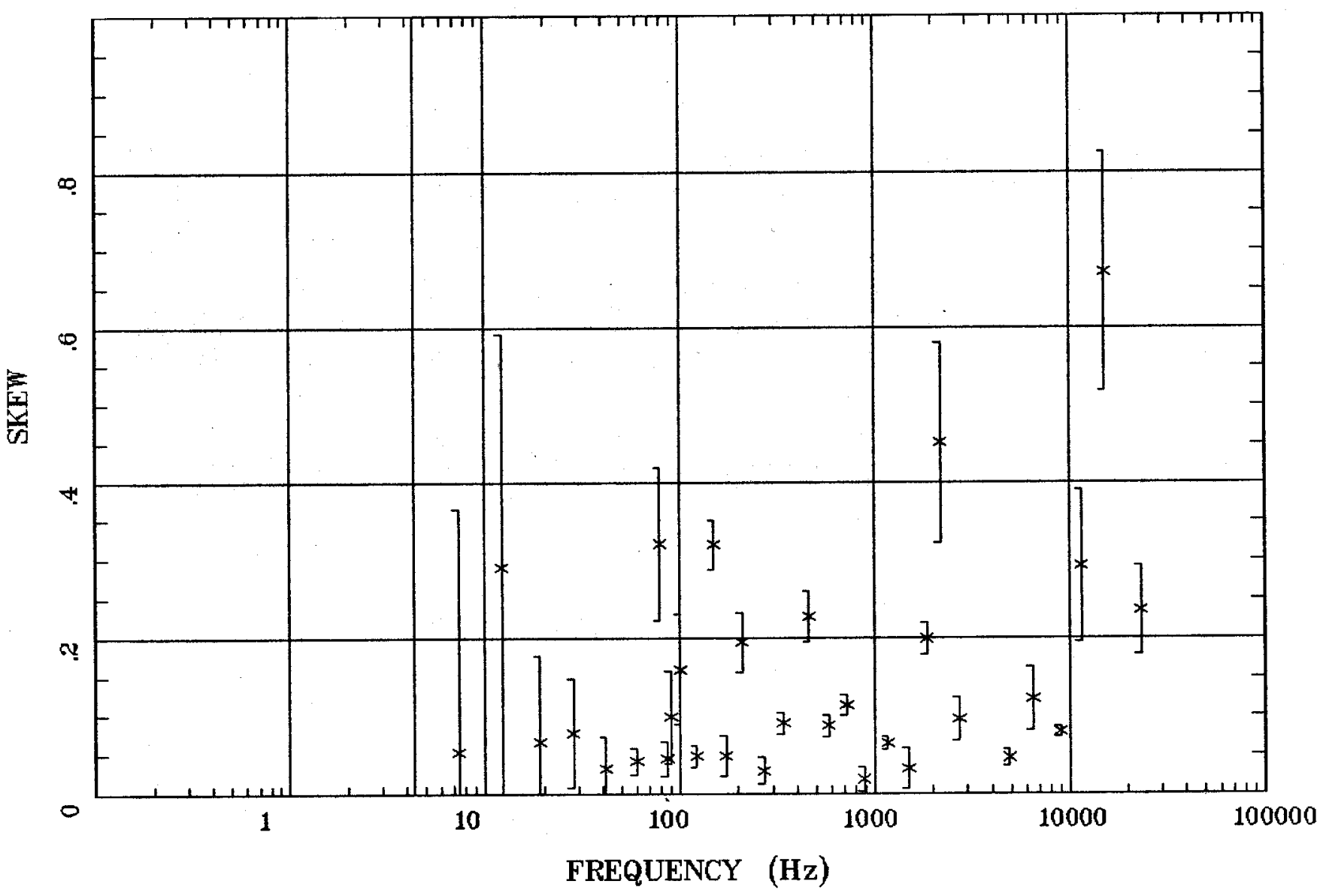

Client:

Remote: e-fld $95 \mathrm{~m}$ west Acquired: 14:3 Jul 26, 2002 Survey Co:USGS
Rotation:

Filename: cp 10.avg

Channels: Ch1 Ch2 Ch3 Ch4 Ch5 Ch6 Ch'7

Plotted: 09:17 Jul 06, 2004

< EMI - ElectroMagnetic Instruments > 


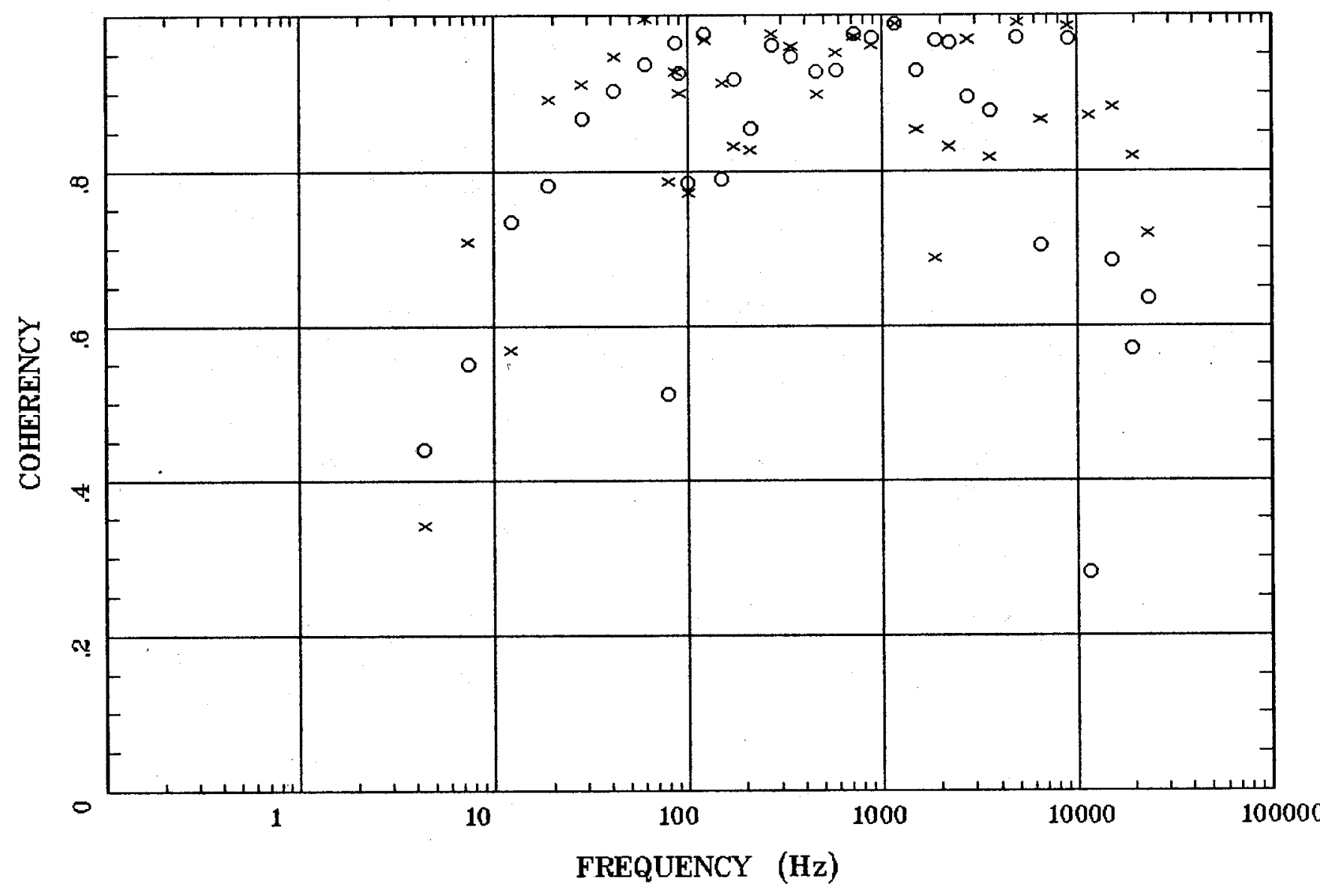

Client:

Remote: e-fld $95 \mathrm{~m}$ rest Acquired: 14:3 Jul 26, 2002 Survey Co:USGS
Rotation:

Filename: cp10.avg

Channels: Ch1 Ch2 Ch3 Ch4 Ch5 Ch6 Ch7

Plotted: 09:17 Jul 06, 2004

< EMI - ElectroMagnetic Instruments > 


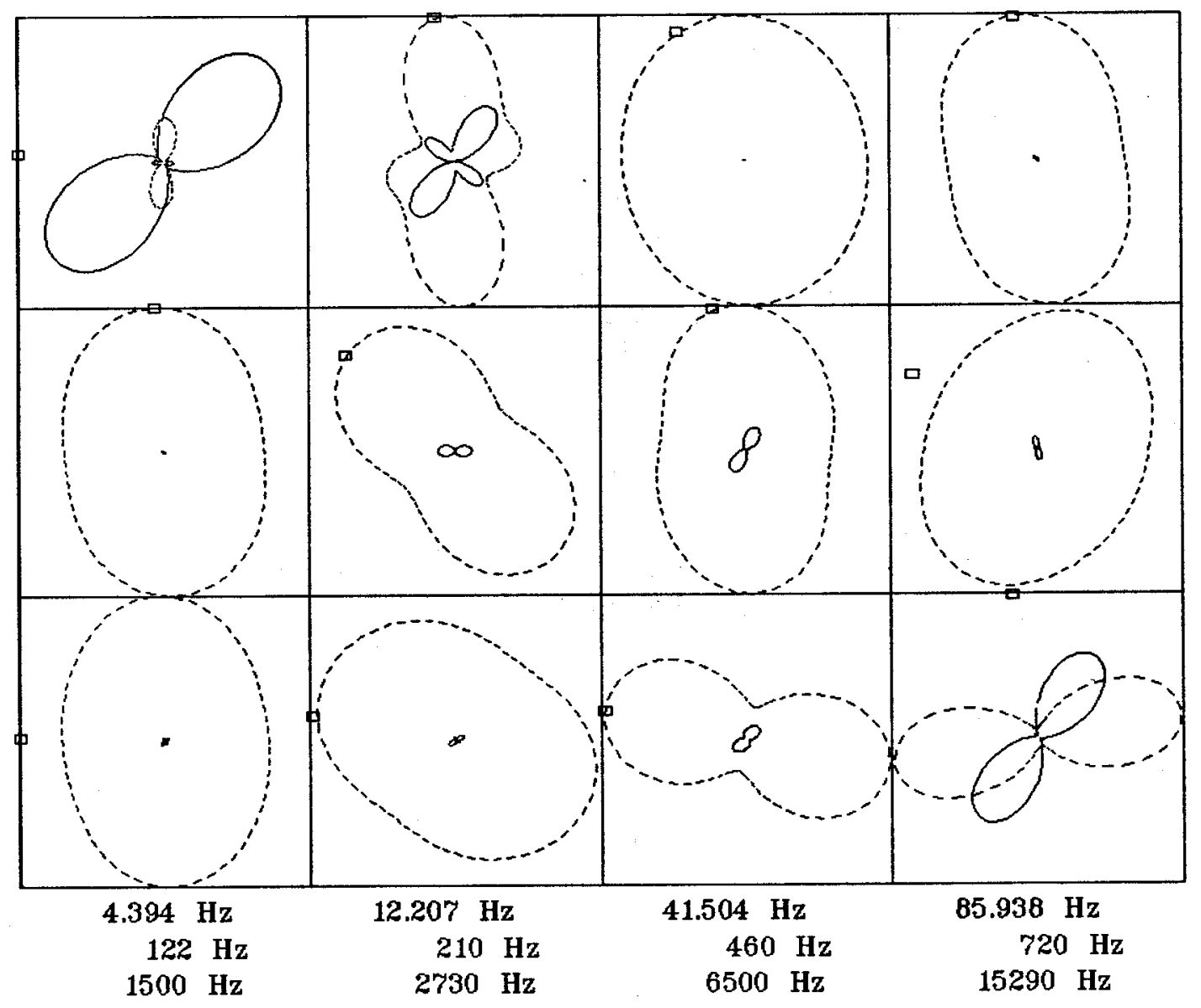

Rotation:

Client:

Remote: e-fld $95 \mathrm{~m}$ west Acquired: 14:3 Jul 26, 2002 Survey Co:USGS
Filename: cp 10.avg

Channels: Ch1 Ch2 Ch3 Ch4 Ch5 Ch6 Ch7 Plotted: 09:17 Jul 06, 2004

< EMI - ElectroMagnetic Instruments 


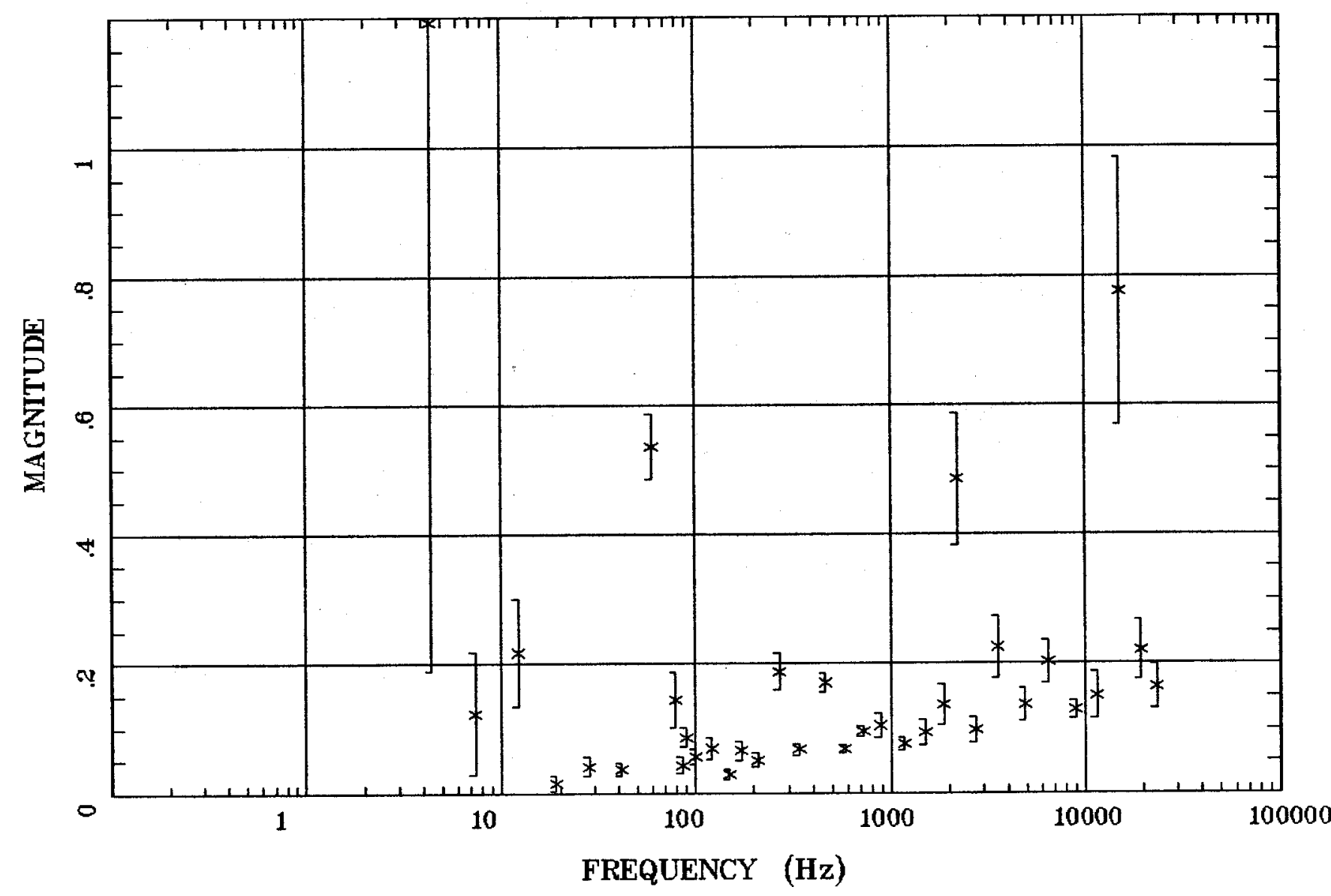

Client:

Remote: e-fld $95 \mathrm{~m}$ rest Acquired: 14:3 Jul 26, 2002 Survey Co:USGS
Rotation:

Filename: cp 10.avg

Channels: Ch1 Ch2 Ch3 Ch4 Ch5 Ch6 Ch7 Plotted: 09:17 Jul 06, 2004

< EMI - ElectroMagnetic Instruments > 


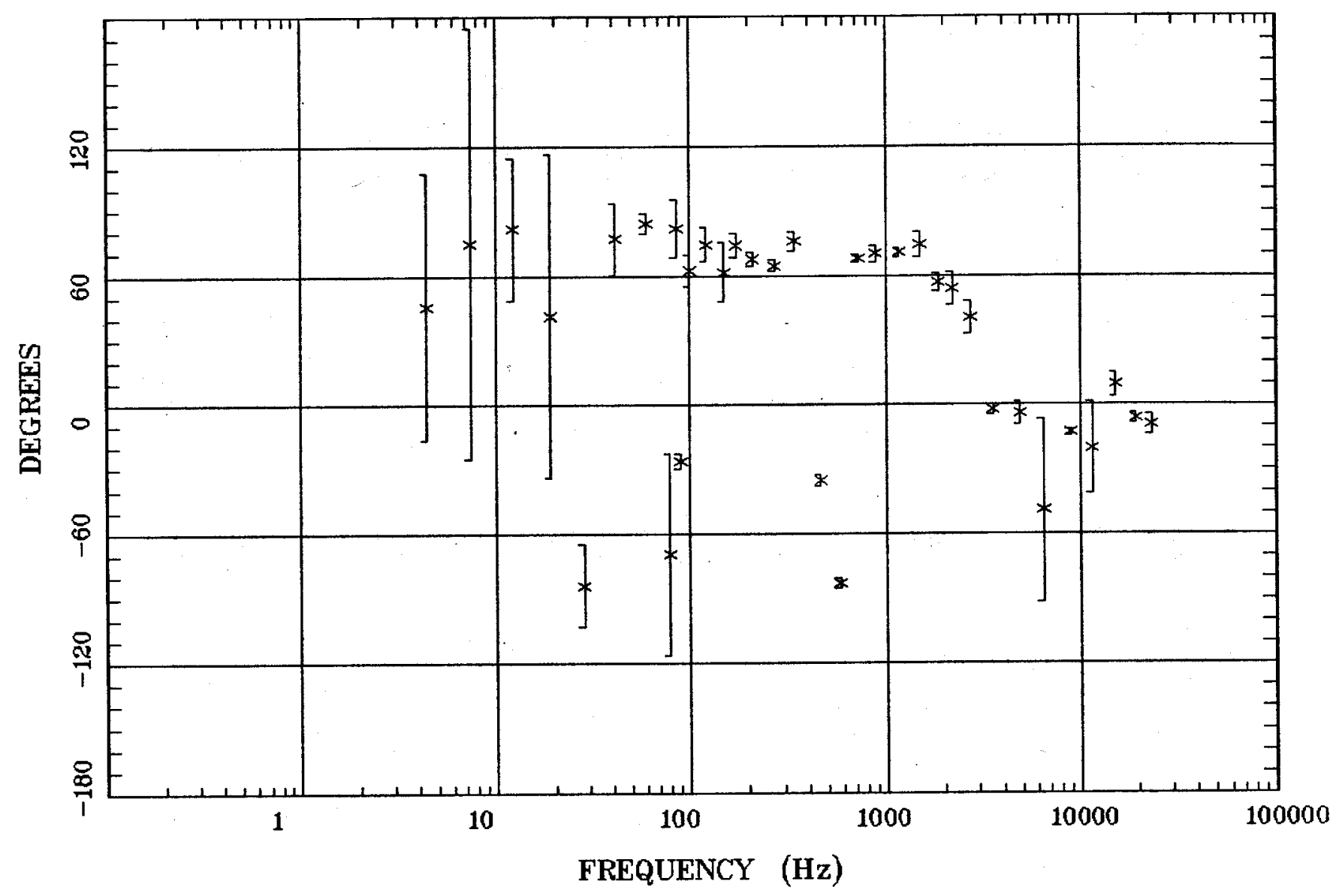

Client:

Remote: e-fld $95 \mathrm{~m}$ west Acquired: $14: 3$ Jul 26, 2002 Survey Co:USGS
Rotation:

Filename: cp 10.avg

Channels: Ch1 Ch2 Ch3 Ch4 Ch5 Ch6 Ch7

Plotted: 09:17 Jul 06, 2004

$<$ EMI - ElectroMagnetic Instruments > 


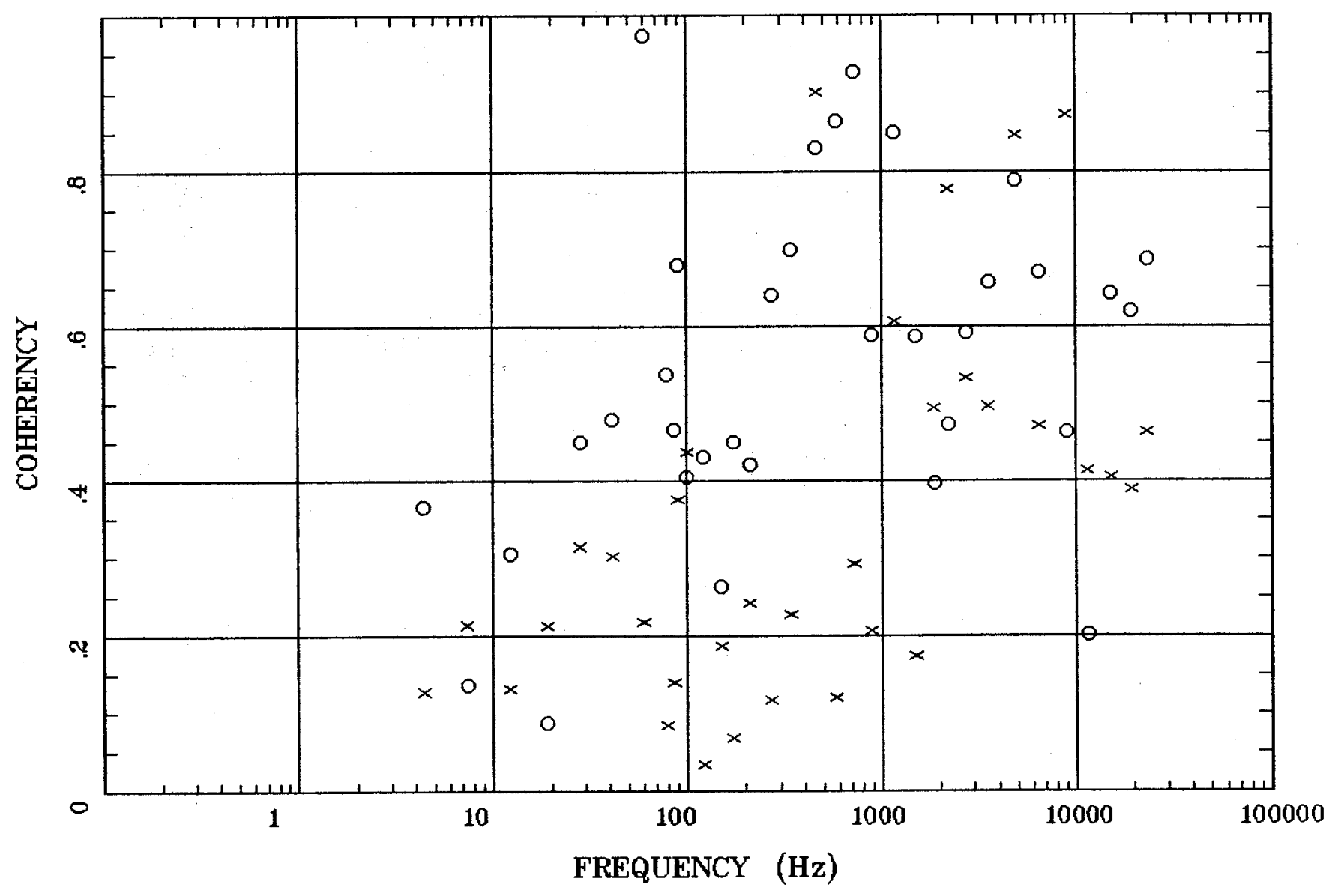

Client:

Remote: e-fld $95 \mathrm{~m}$ west Acquired: 14:3 Jul 26, 2002 Survey Co:USGS
Rotation:

Filename: cp10.avg

Channels: Ch1 Ch2 Ch3 Ch4 Ch5 Ch6 Ch7 Plotted: 09:18 Jul 06, 2004

$<$ EMI - ElectroMagnetic Instruments > 


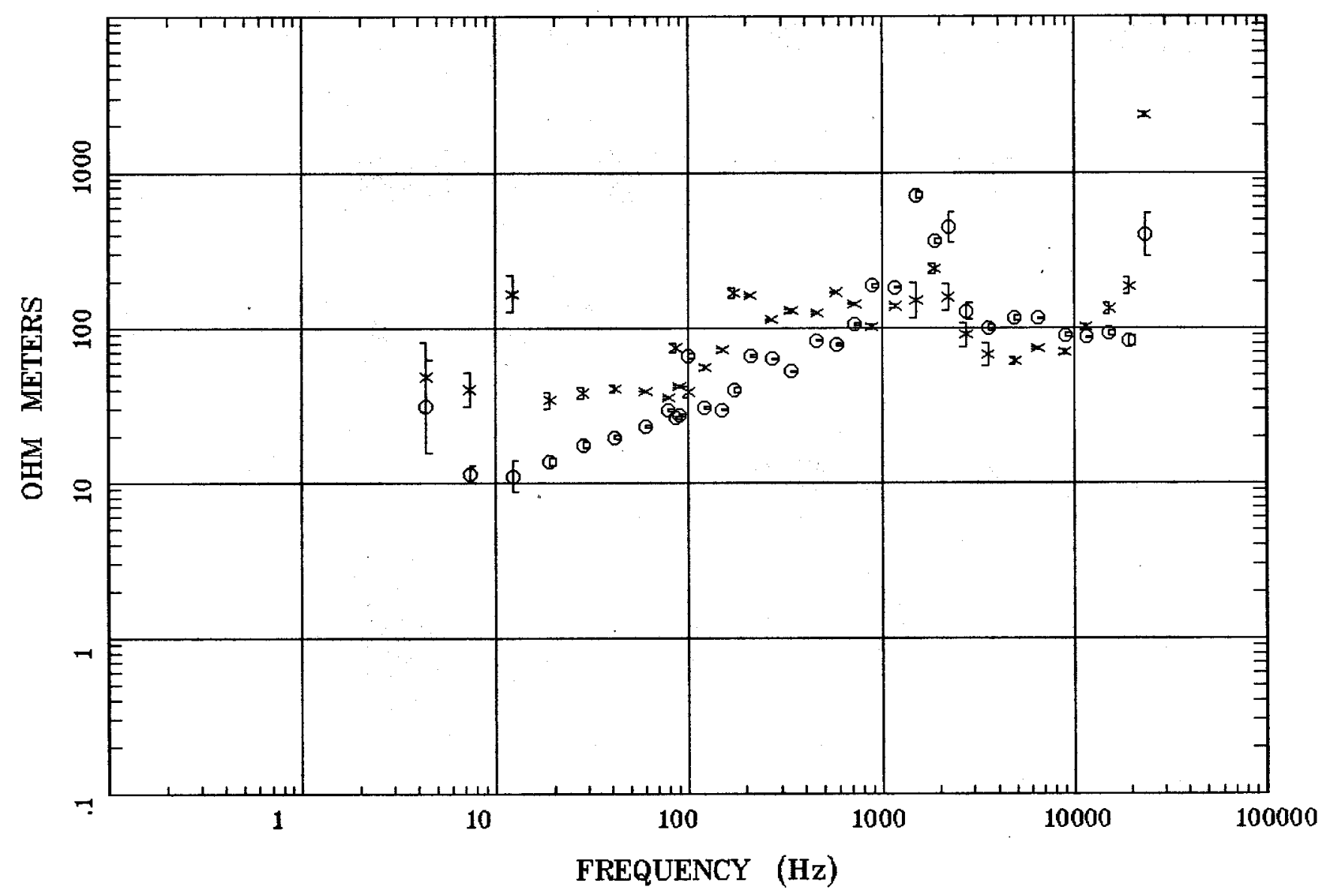

Client:

Remote: e-fld $95 \mathrm{~m}$ north Acquired: 13:2 Jul 27, 2002 Survey Co:USGS
Rotation:

Filename: cp11.avg

Channels: Ch1 Ch2 Ch3 Ch4 Ch5 Ch6 Chr Plotted: 14:30 Jul 06, 2004

< EMI - ElectroMagnetic Instruments > 


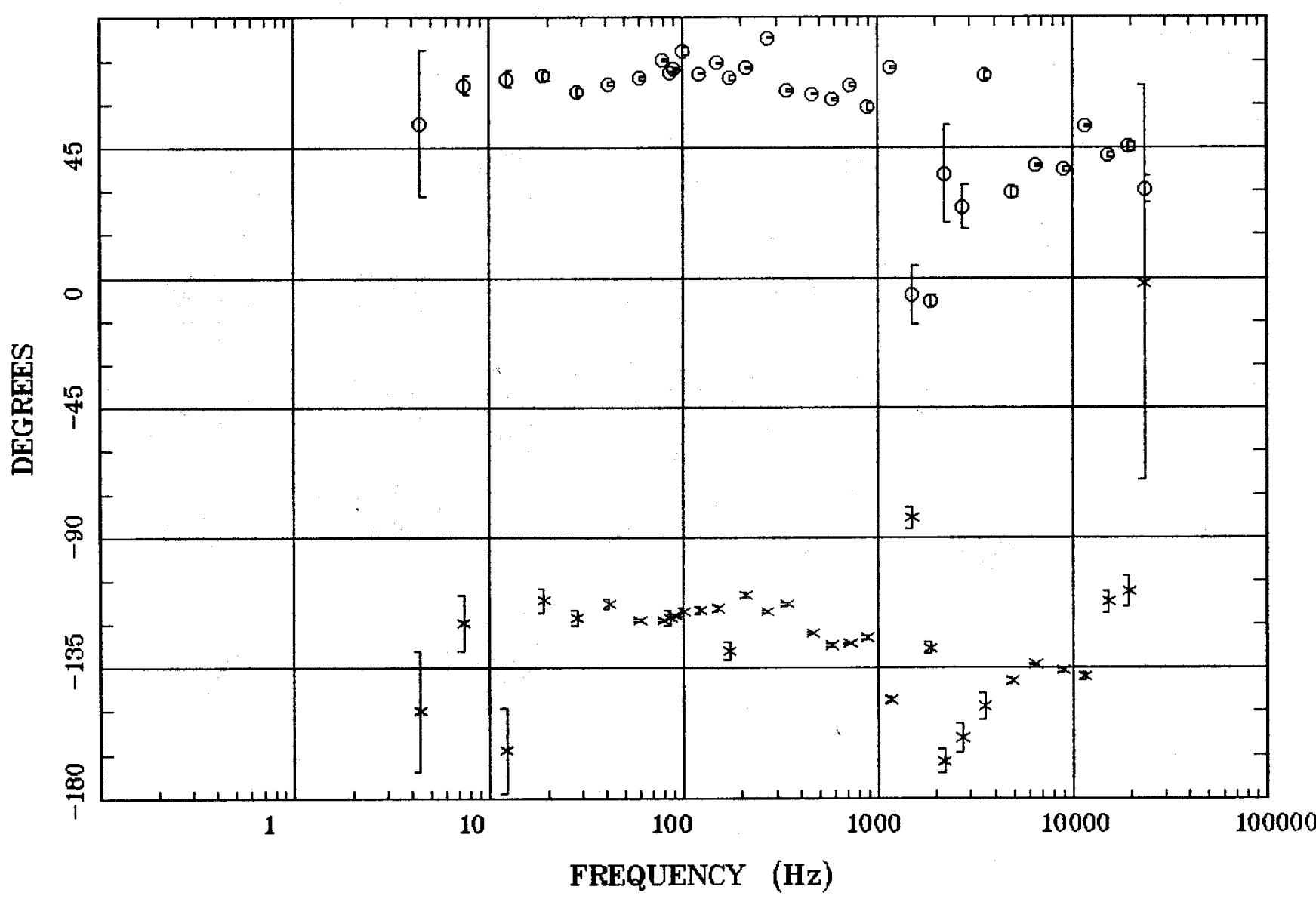

Client:

Remote: e-fld $95 \mathrm{~m}$ north Acquired: 13:2 Jul 27, 2002 Survey Co:USGS
Rotation:

Filename: cp 11.avg

Channels: Ch1 Ch2 Ch3 Ch4 Ch5 Ch6 Ch7

Plotted: 14:30 Jul 06, 2004

< EMI - ElectroMagnetic Instruments > 


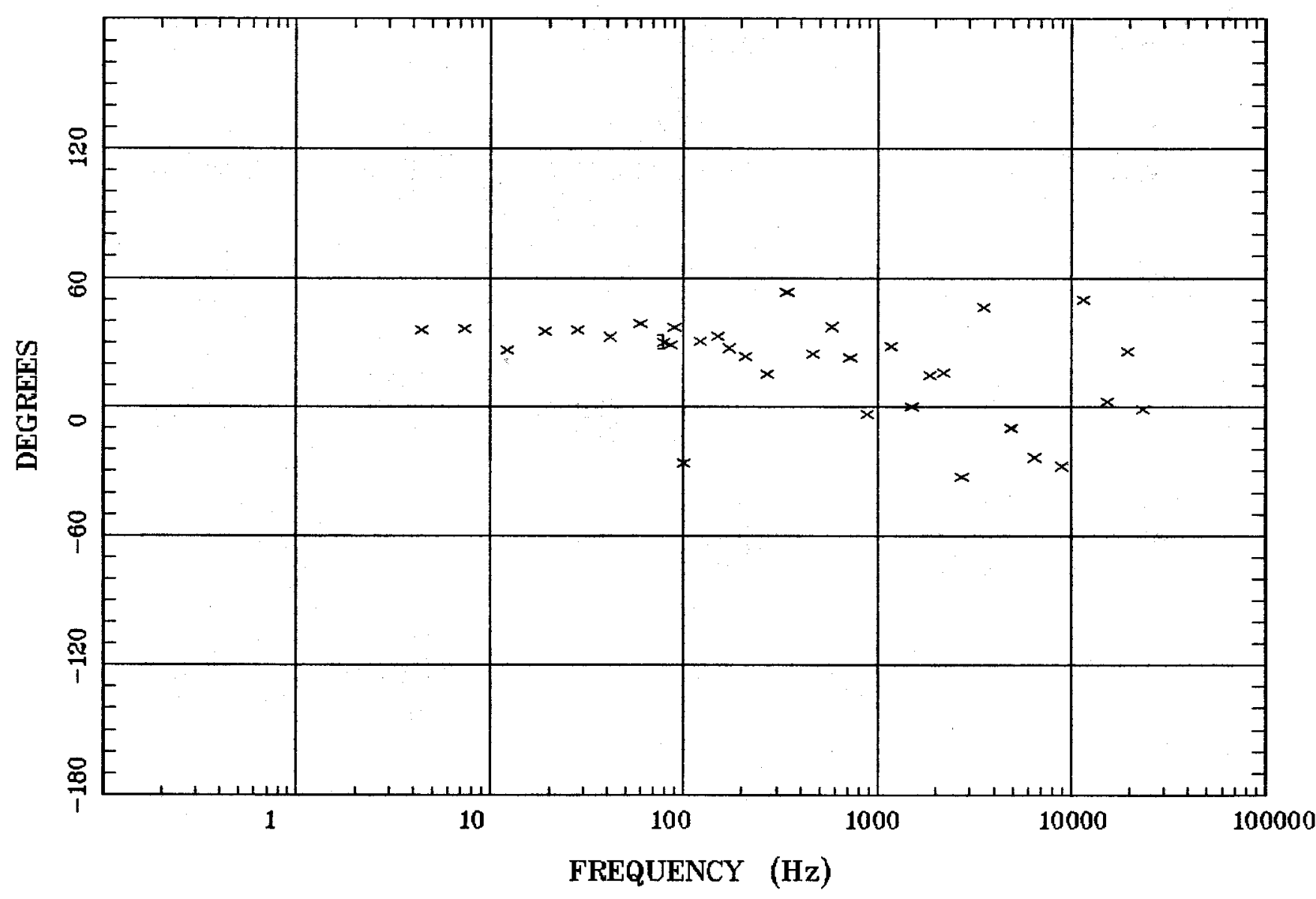

Client:

Remote: e-fld $95 \mathrm{~m}$ north Acquired: 13:2 Jul 27, 2002 Survey Co:USGS
Rotation:

Filename: cp11.avg

Channels: Ch1 Ch2 Ch3 Ch4 Ch5 Ch6 Chr Plotted: 12:47 Jul 07, 2004

$<$ EMI - ElectroMagnetic Instruments 


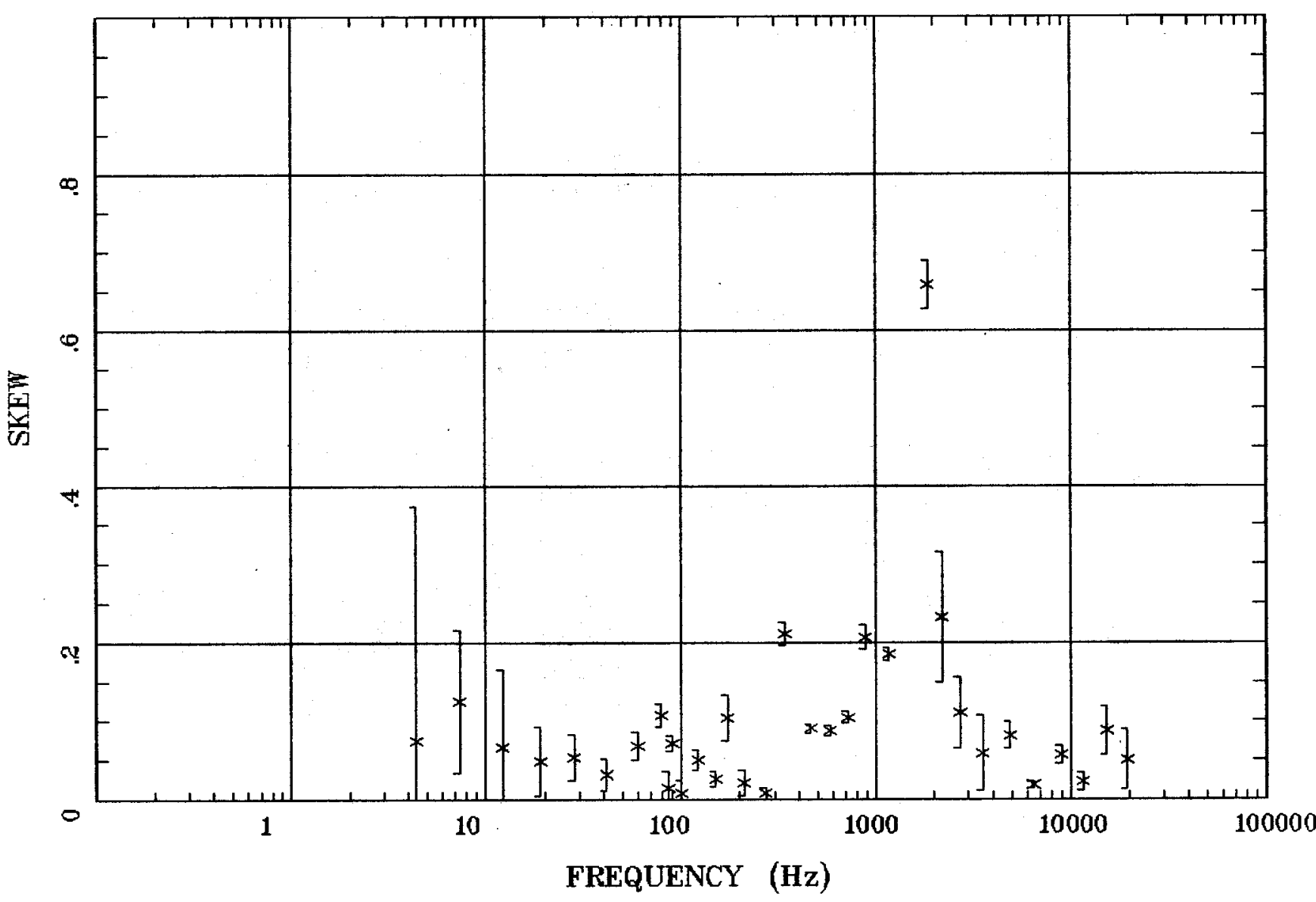

Client:

Remote: e-fld $95 \mathrm{~m}$ north Acquired: 13:2 Jul 27, 2002 Survey Co:USGS
Rotation:

Filename: cp11.avg

Channels: Ch1 Ch2 Ch3 ch4 Ch5 ch6 Ch7 Plotted: 14:30 Jul 06, 2004

< EMI - ElectroMagnetic Instruments > 


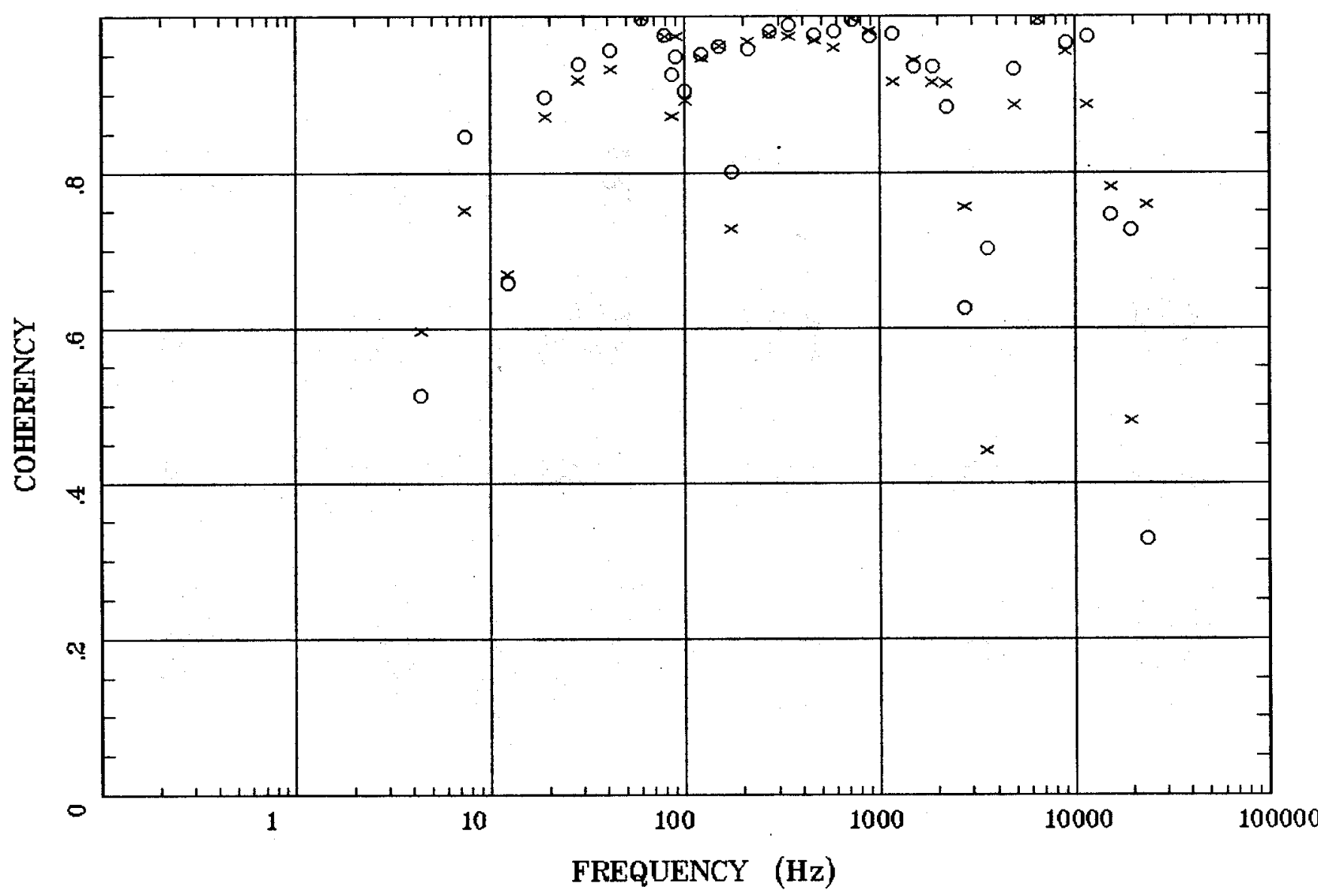

Client:

Remote: e-fld $95 \mathrm{~m}$ north Acquired: 13:2 Jul 27, 2002 Survey Co:USGS
Rotation:

Filename: cp 11.avg

Channels: Ch1 Ch2 Ch3 Ch4 Ch5 Ch6 Ch7 Plotted: 14:30 Jul 06, 2004

< EMI - ElectroMagnetic Instruments 


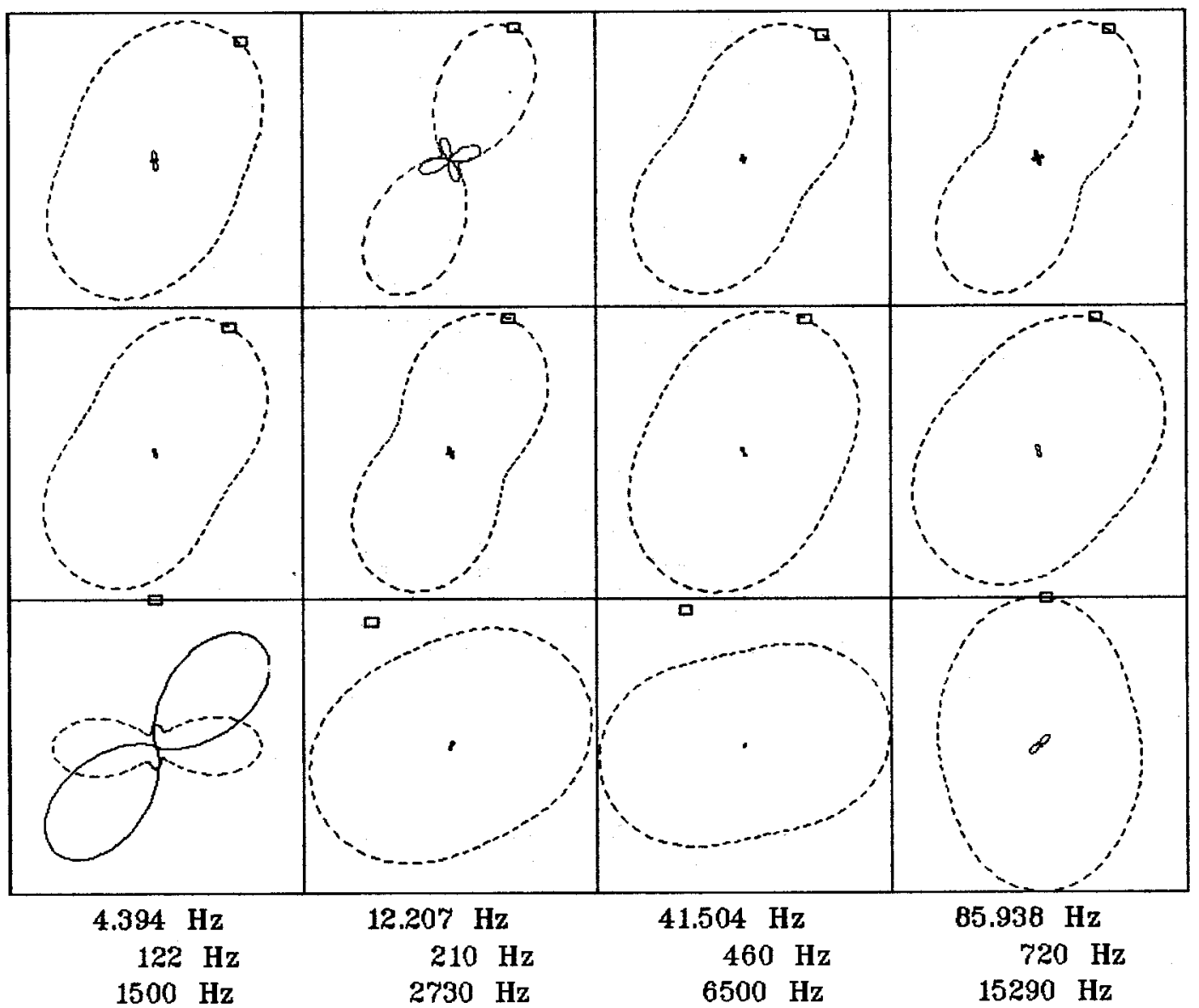

Client:

Remote: e-fld $95 \mathrm{~m}$ north Acquired: 13:2 Jul 27, 2002 Survey Co:USGS

\section{Rotation:}

Filename: cp11.avg

Channels: Ch1 Ch2 Ch3 Ch4 Ch5 Ch6 Ch7 Plotted: 14:30 Jul 06, 2004

< EMI - ElectroMagnetic Instruments > 


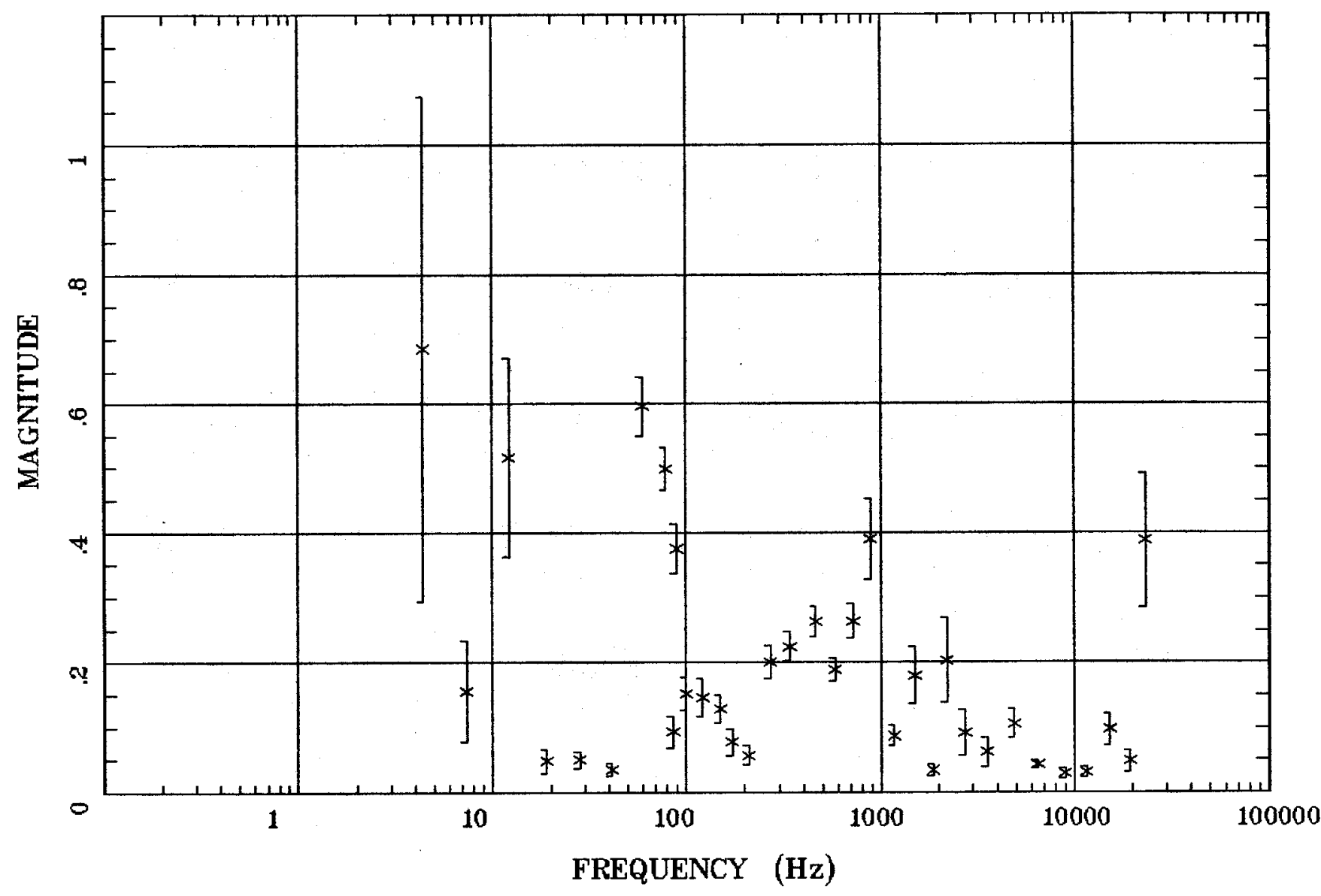

Client:

Remote: e-fld $95 \mathrm{~m}$ north Acquired: 13:2 Jul 27, 2002 Survey Co:USGS
Rotation:

Filename: cp11.avg

Channels: Ch1 Ch2 Ch3 Ch4 Ch5 Ch6 Ch7

Plotted: 14:30 Jul 06, 2004

< EMI - ElectroMagnetic Instruments > 


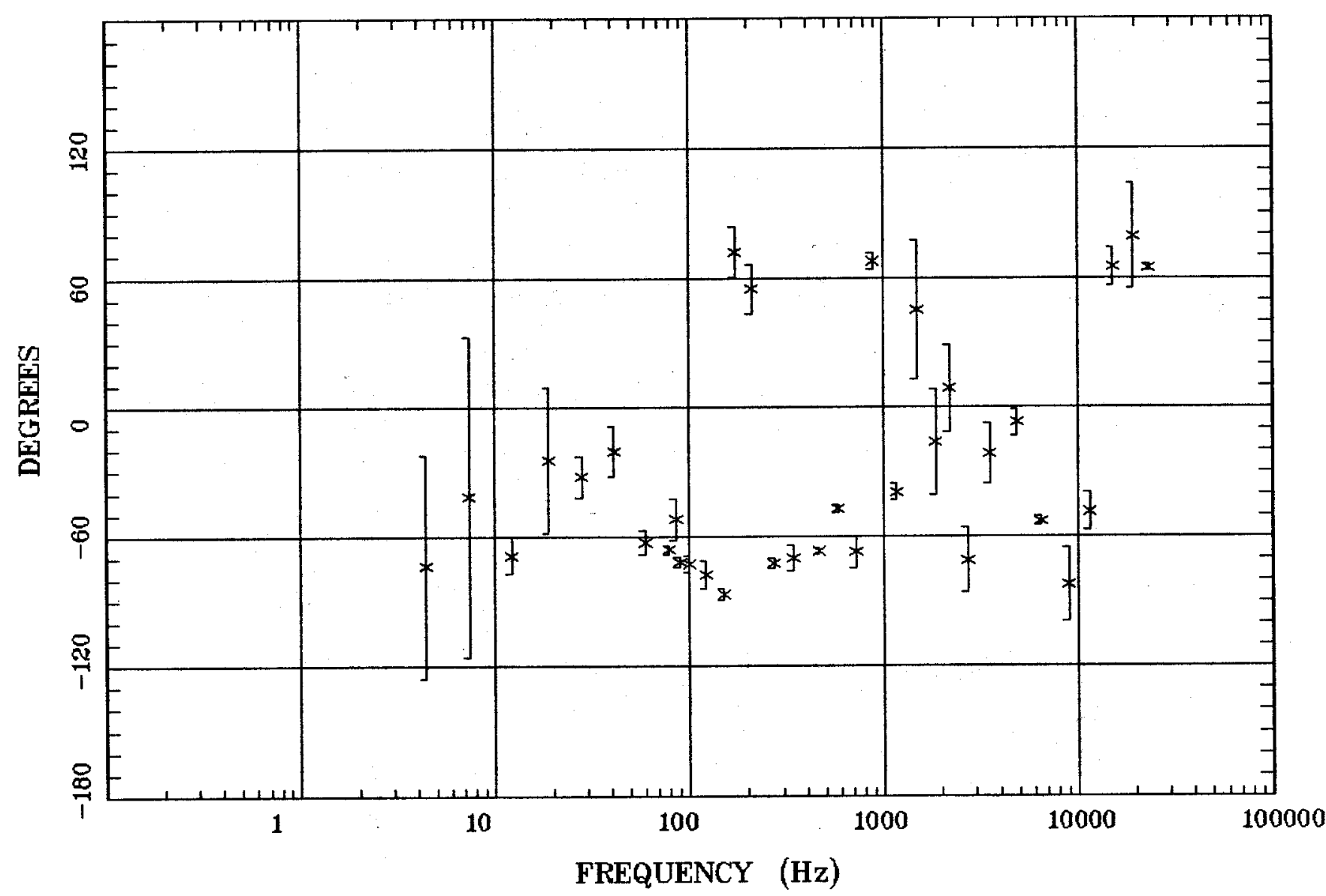

Client:

Remote: e-fld $95 \mathrm{~m}$ north Acquired: 13:2 Jul 27, 2002 Survey Co:USGS
Rotation:

Filename: cp11.avg

Channels: Ch1 Ch2 Ch3 ch4 Ch5 Ch6 Ch7 Plotted: $14: 30$ Jul 06, 2004

$<$ EMI - ElectroMagnetic Instruments > 


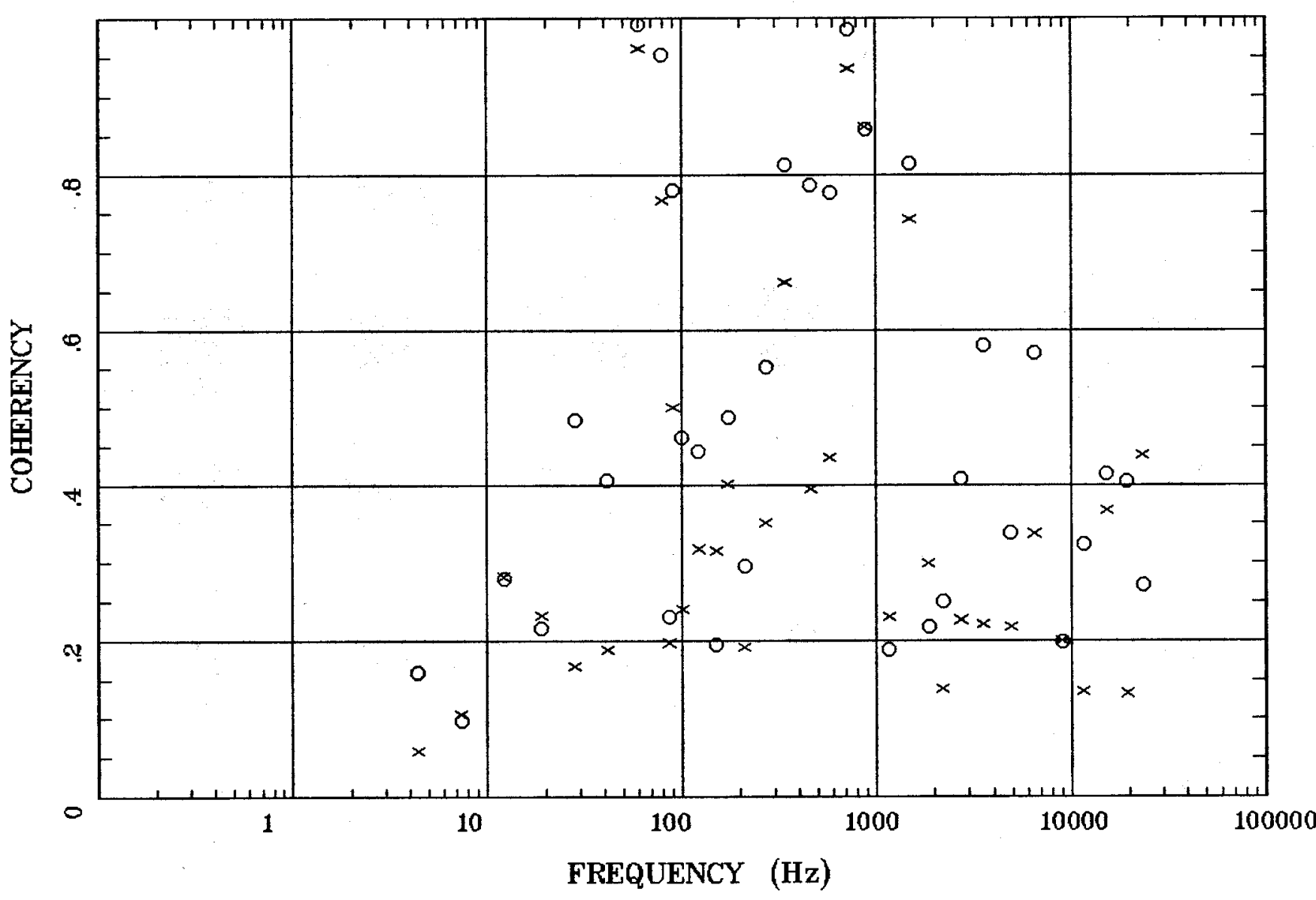

Client:

Remote: e-fld $95 \mathrm{~m}$ north

Acquired: 13:2 Jul 27, 2002 Survey Co:USGS
Rotation:

Filename: cp11.avg

Channels: Ch1 Ch2 Ch3 Ch4 Ch5 Ch6 Chy Plotted: 14:30 Jul 06, 2004

< EMI - ElectroMagnetic Instruments > 


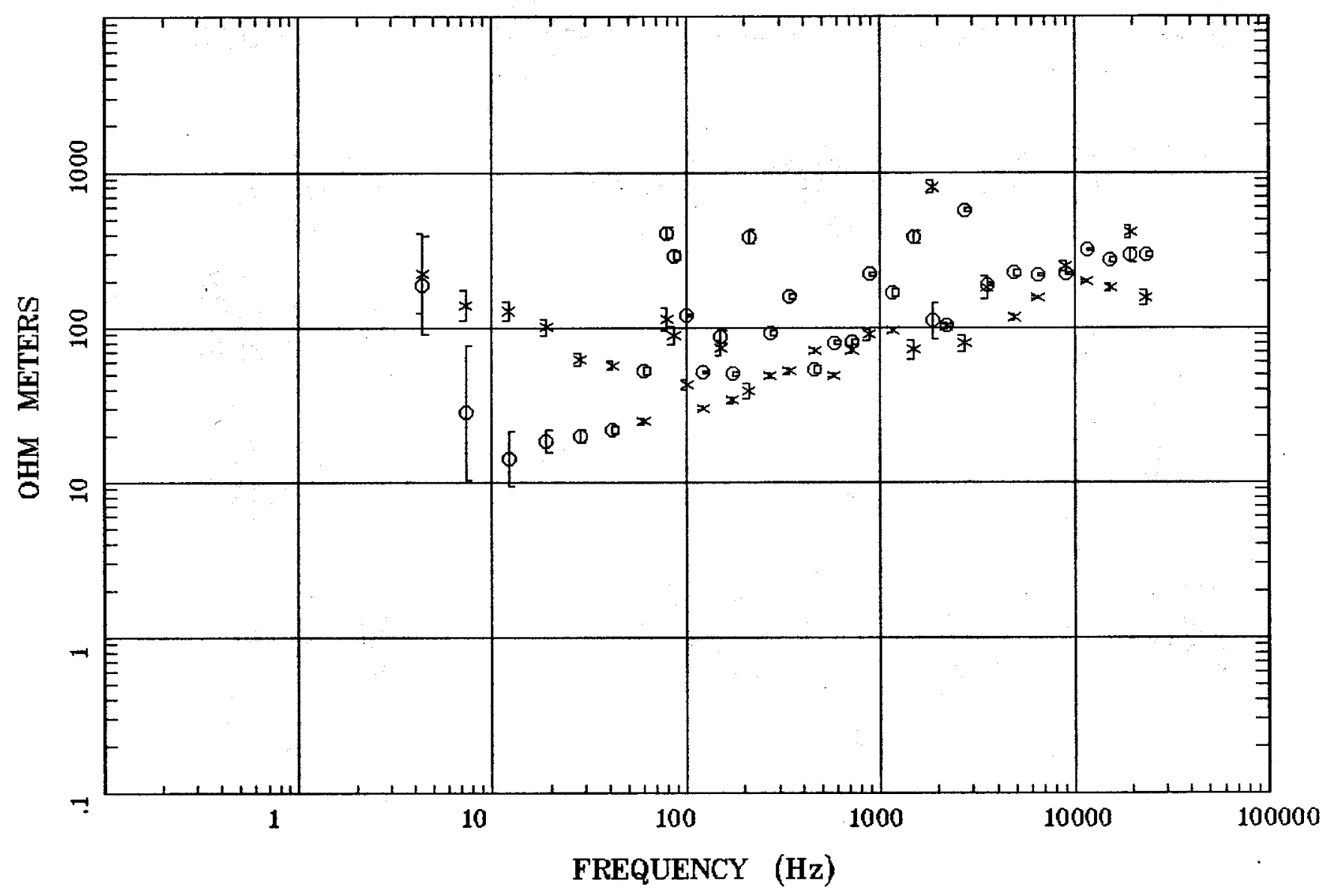

Client:

Remote: e-fld $95 \mathrm{~m}$ west Acquired: 15:1 Jul 29, 2002 Survey Co:USGS
Rotation:

Filename: cp12a.avg Channels: Ch1 Ch2 Ch3 ch4 Ch5 Ch6 Ch7 Plotted: 14:31 Jul 06, 2004

$<$ EMI - ElectroMagnetic Instruments 
Station 12

IMPEDANCE PHASE

West of St. Peters Dome

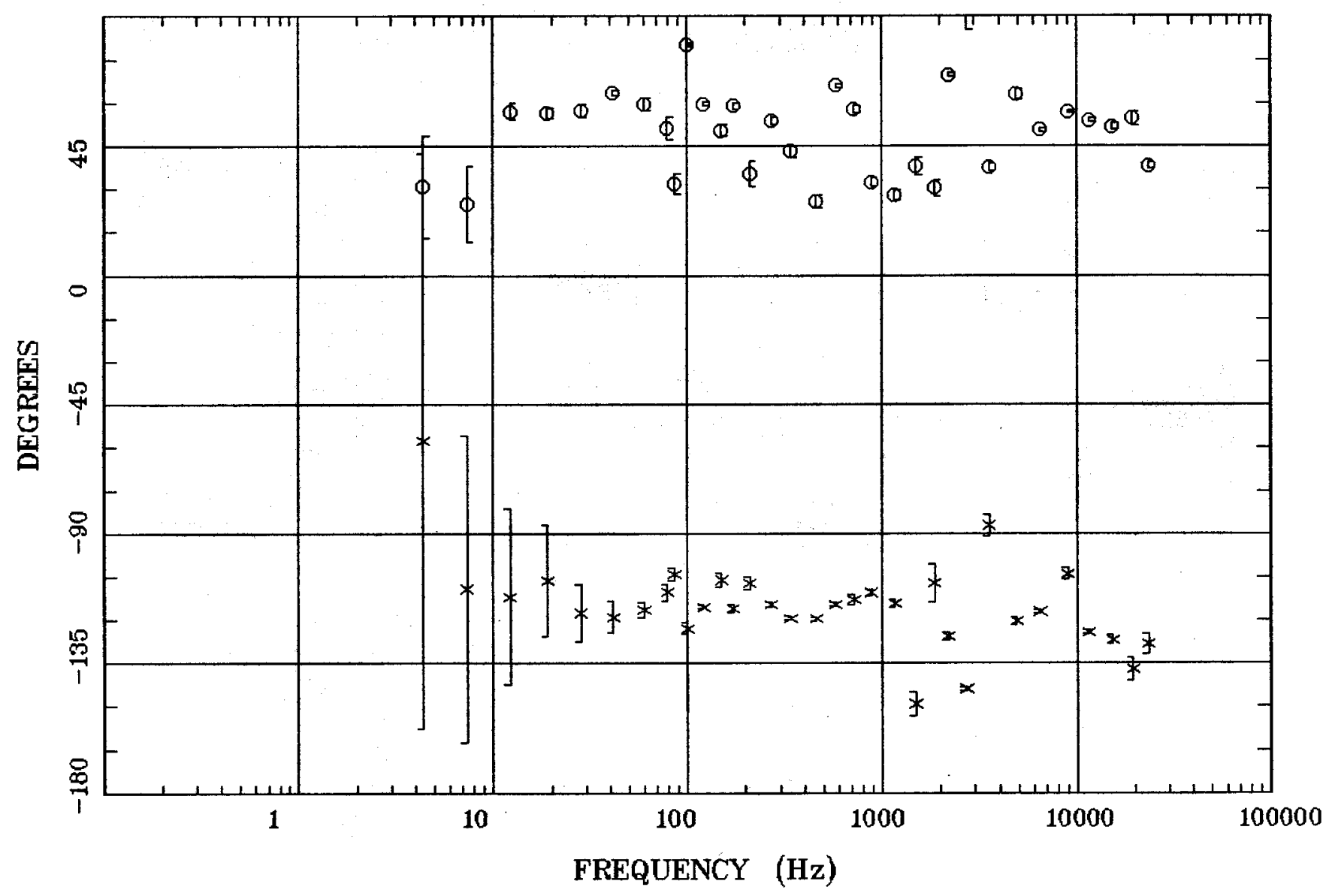

Client:

Remote: e-fld $95 \mathrm{~m}$ west Acquired: $15: 1$ Jul 29, 2002 Survey Co:USGS
Rotation:

Filename: cp 12a.avg Channels: Ch1 Ch2 Ch3 Ch4 Ch5 Ch6 Chr Plotted: 14:31 Jul 06, 2004

$<$ EMI - ElectroMagnetic Instruments 
West of St. Peters Dome

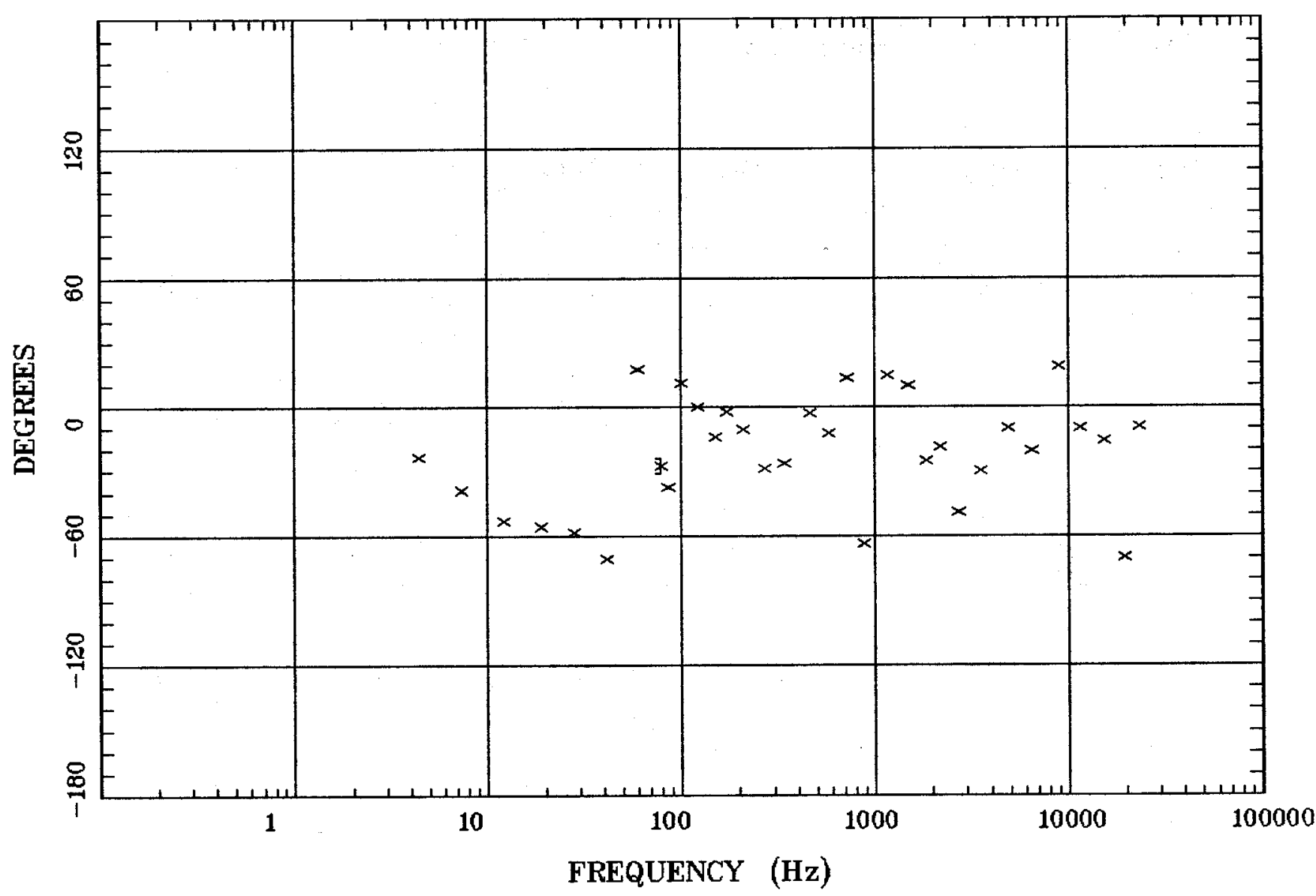

Client:

Remote: e-fld $95 \mathrm{~m}$ west

Acquired: 15:1 Jul 29, 2002 Survey Co:USGS
Rotation:

Filename: cp12a.avg

Channels: Ch1 Ch2 Ch3 ch4 Ch5 Ch6 Ch7 Plotted: 12:48 Jul 07, 2004

$<$ EMI - ElectroMagnetic Instruments > 
IMPEDANCE SKEW West of St. Peters Dome

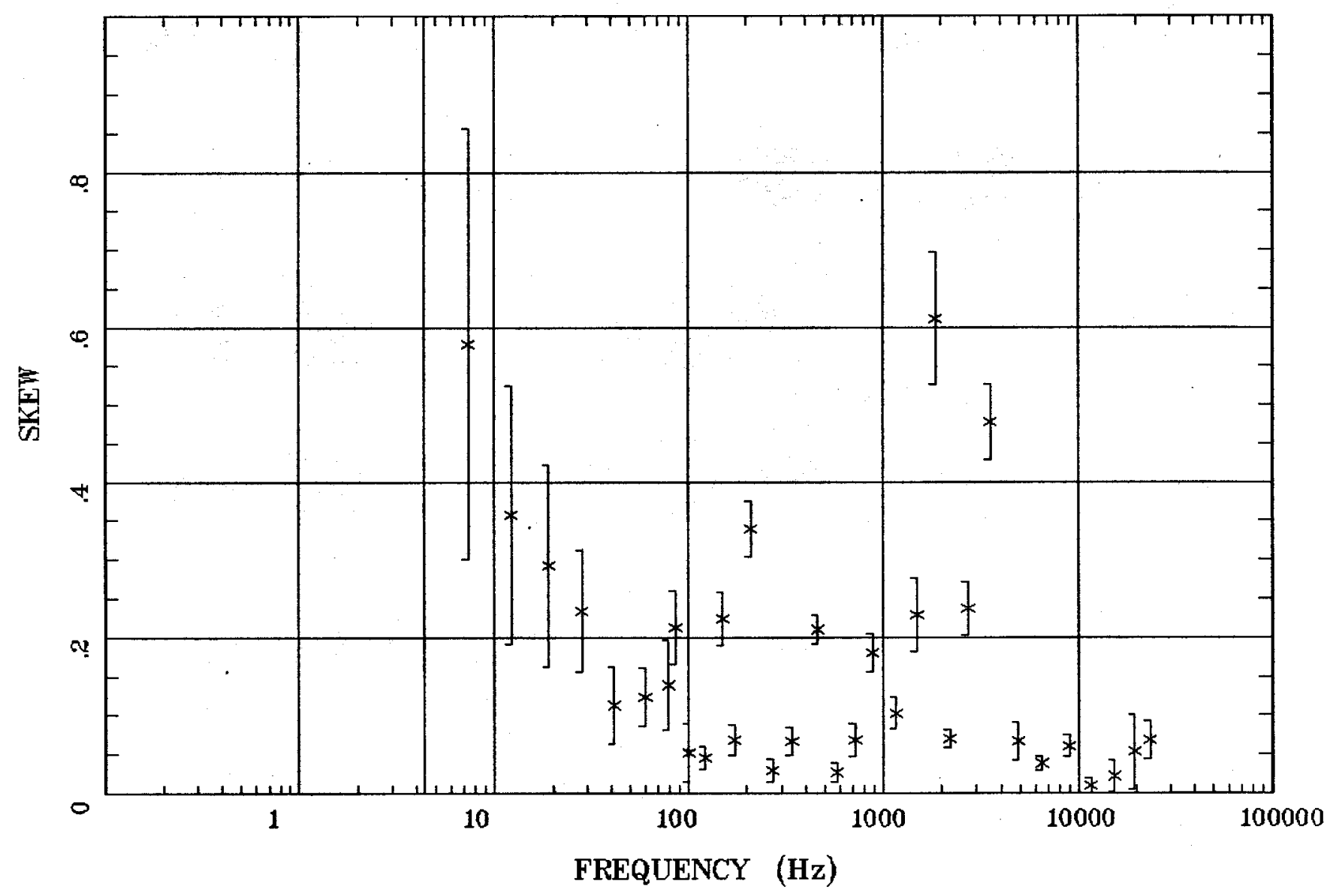

Client:

Remote: e-fld $95 \mathrm{~m}$ west

Acquired: 15:1 Jul 29, 2002 Survey Co:USGS
Rotation:

Filename: cp12a.avg

Channels: Ch1 Ch2 Ch3 Ch4 Ch5 Ch6 Ch7 Plotted: 14:31 Jul 06, 2004

< EMI - ElectroMagnetic Instruments 
E MULT Coh.

West of St. Peters Dome

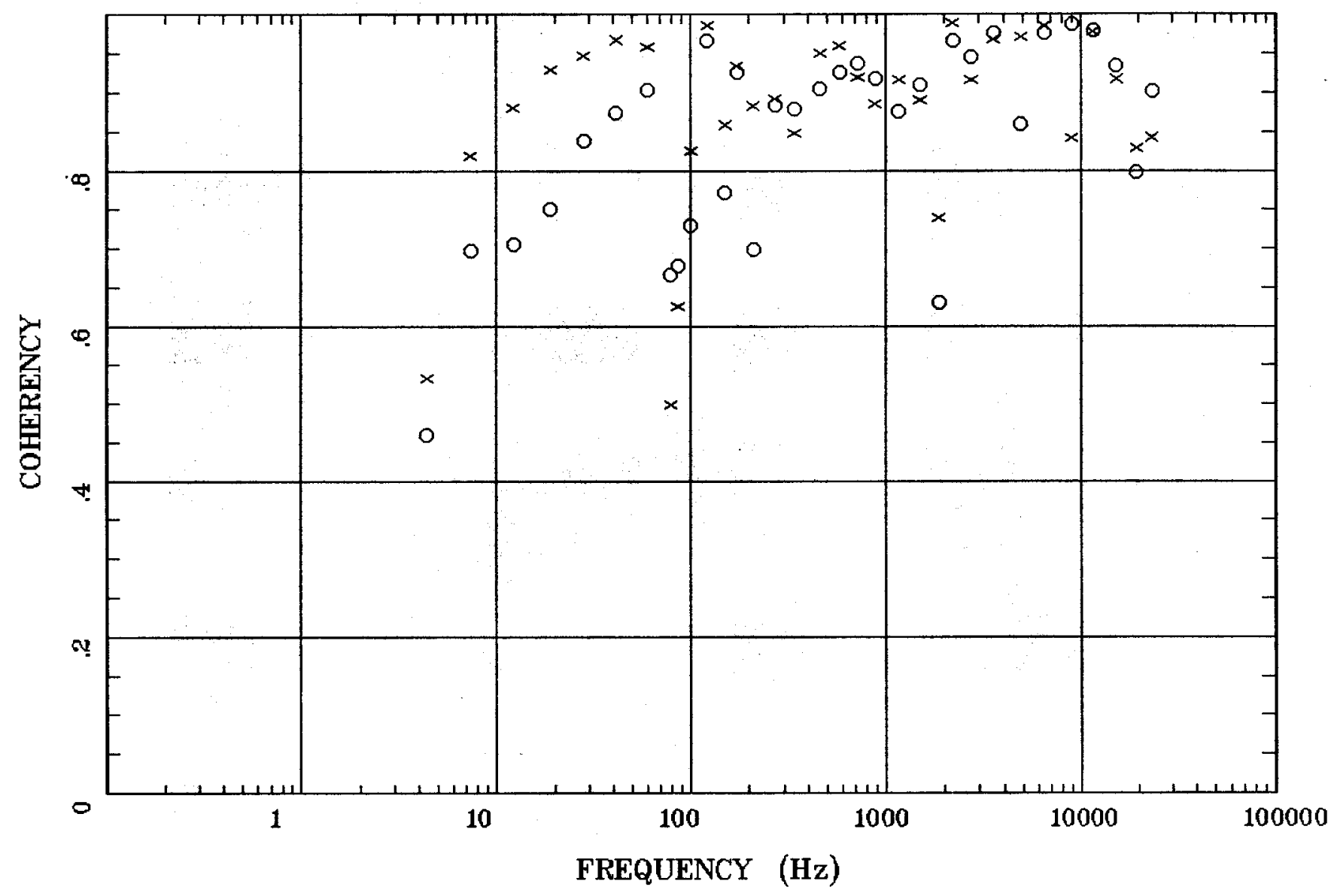

Client:

Remote: e-fld $95 \mathrm{~m}$ west

Acquired: $15: 1$ Jul 29, 2002 Survey Co:USGS
Rotation:

Filename: cp 12a.avg Channels: Ch1 Ch2 Ch3 Ch4 Ch5 Ch6 Ch7 Plotted: 14:31 Jul 06, 2004

< EMI - ElectroMagnetic Instruments 
POLAR PLOTS

West of St. Peters Dome

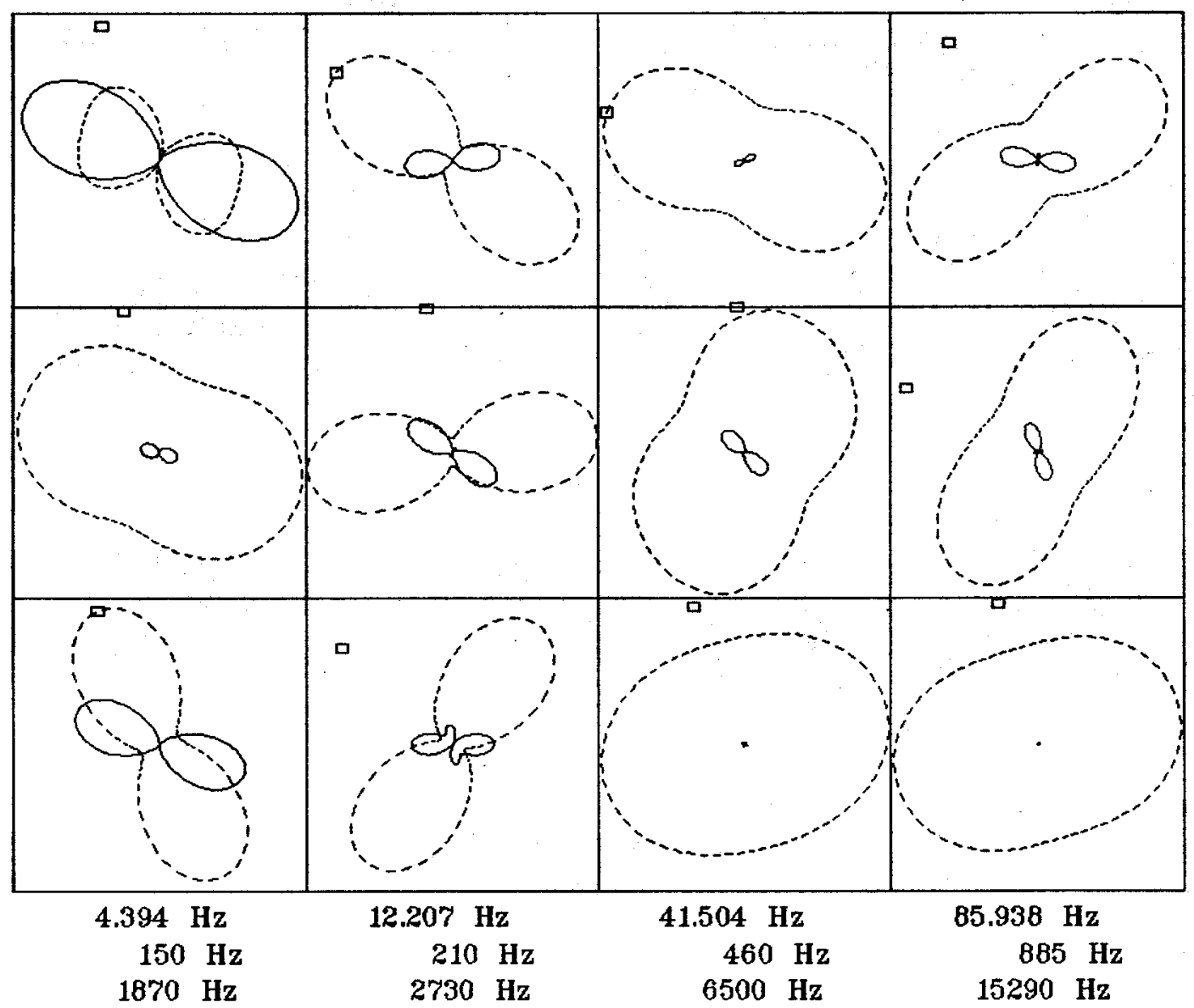

Client:

Remote: e-fld $95 \mathrm{~m}$ west Acquired: 15:1 Jul 29, 2002 Survey Co:USGS
Rotation:

Filename: cp12a.avg

Channels: Ch1 Ch2 Ch3 Ch4 Ch5 Ch6 Ch7 Plotted: 14:31 Jul 06, 2004

< EMI - ElectroMagnetic Instruments > 
TIPPER MAGNITUDE

West of St. Peters Dome

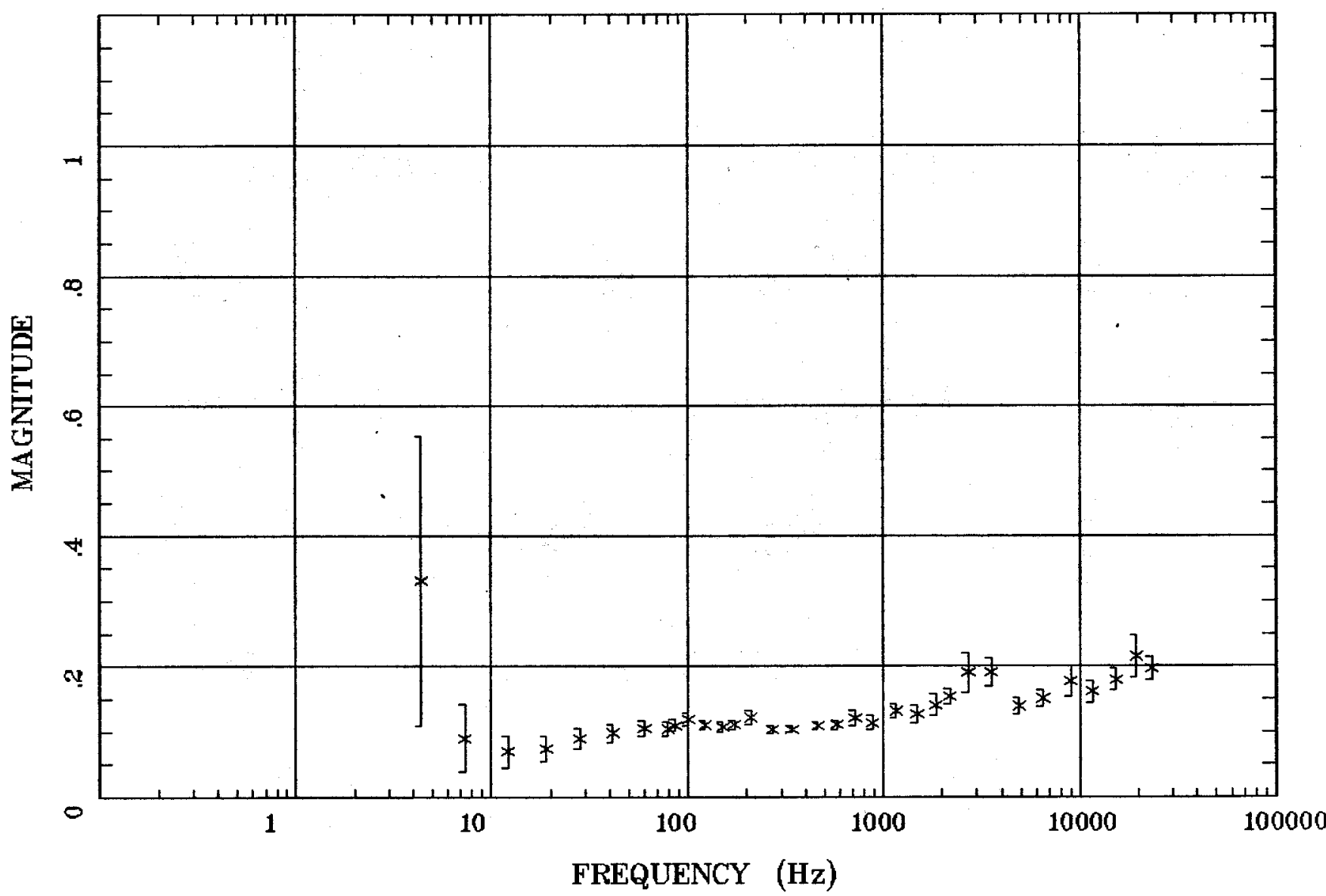

Client:

Remote: e-fld $95 \mathrm{~m}$ rest

Acquired: 15:1 Jul 29, 2002

Survey Co:USGS
Rotation:

Filename: cp12a.avg

Channels: Ch1 Ch2 Ch3 Ch4 Ch5 ch6 Ch7 Plotted: 14:31 Jul 06, 2004

< EMI - ElectroMagnetic Instruments > 
TIPPER STRIKE

West of St. Peters Dome

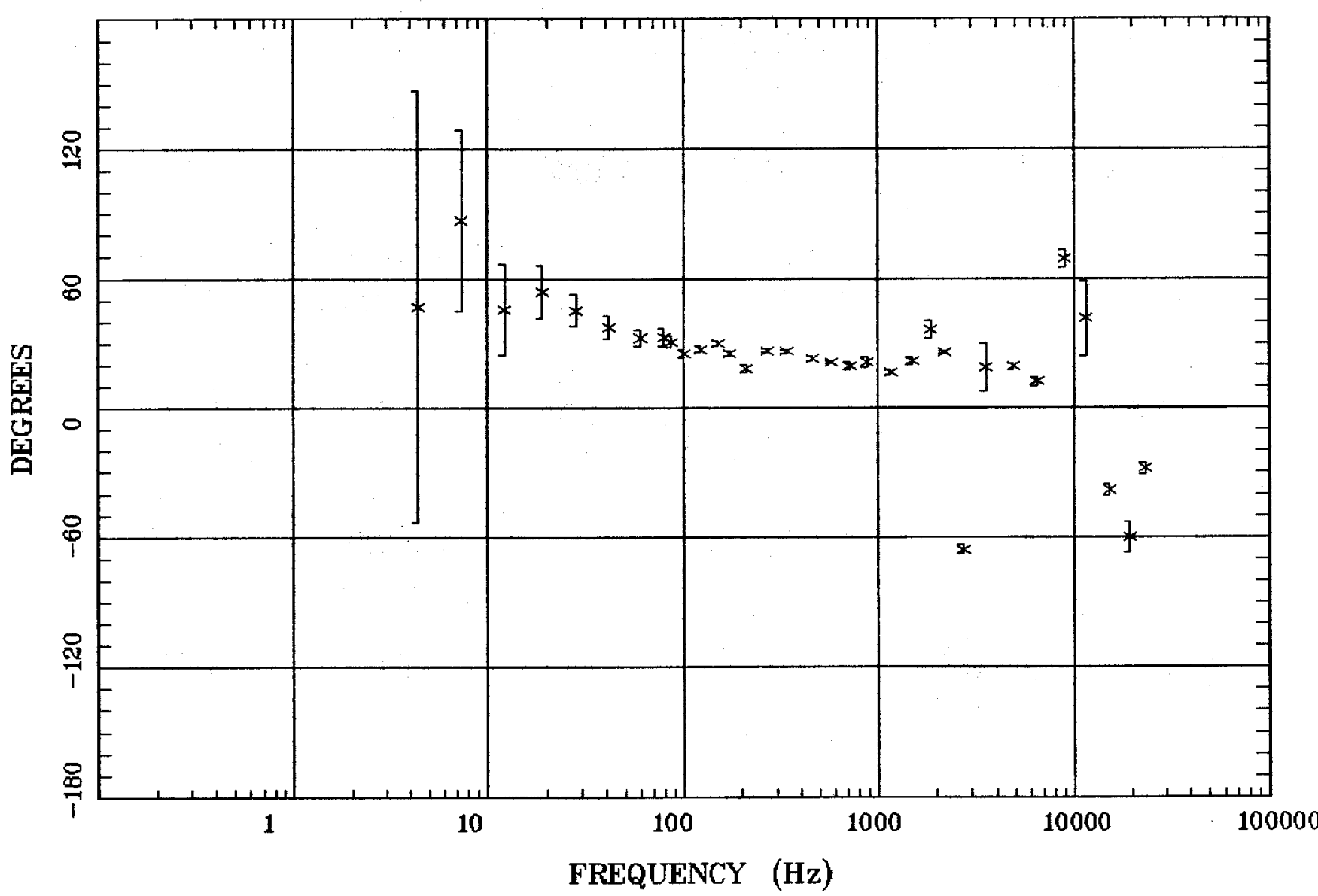

Client:

Remate: e-fld $95 \mathrm{~m}$ west

Acquired: 15:1 Jul 29, 2002 Survey Co:USGS
Rotation:

Filename: cp12a.avg

Channels: Ch1 Ch2 Ch3 Ch4 Ch5 Ch6 Ch7 Plotted: 14:32 Jul 06, 2004

< EMI - ElectroMagnetic Instruments > 
HzHx.x Coh HzHy.o

West of St. Peters Dome

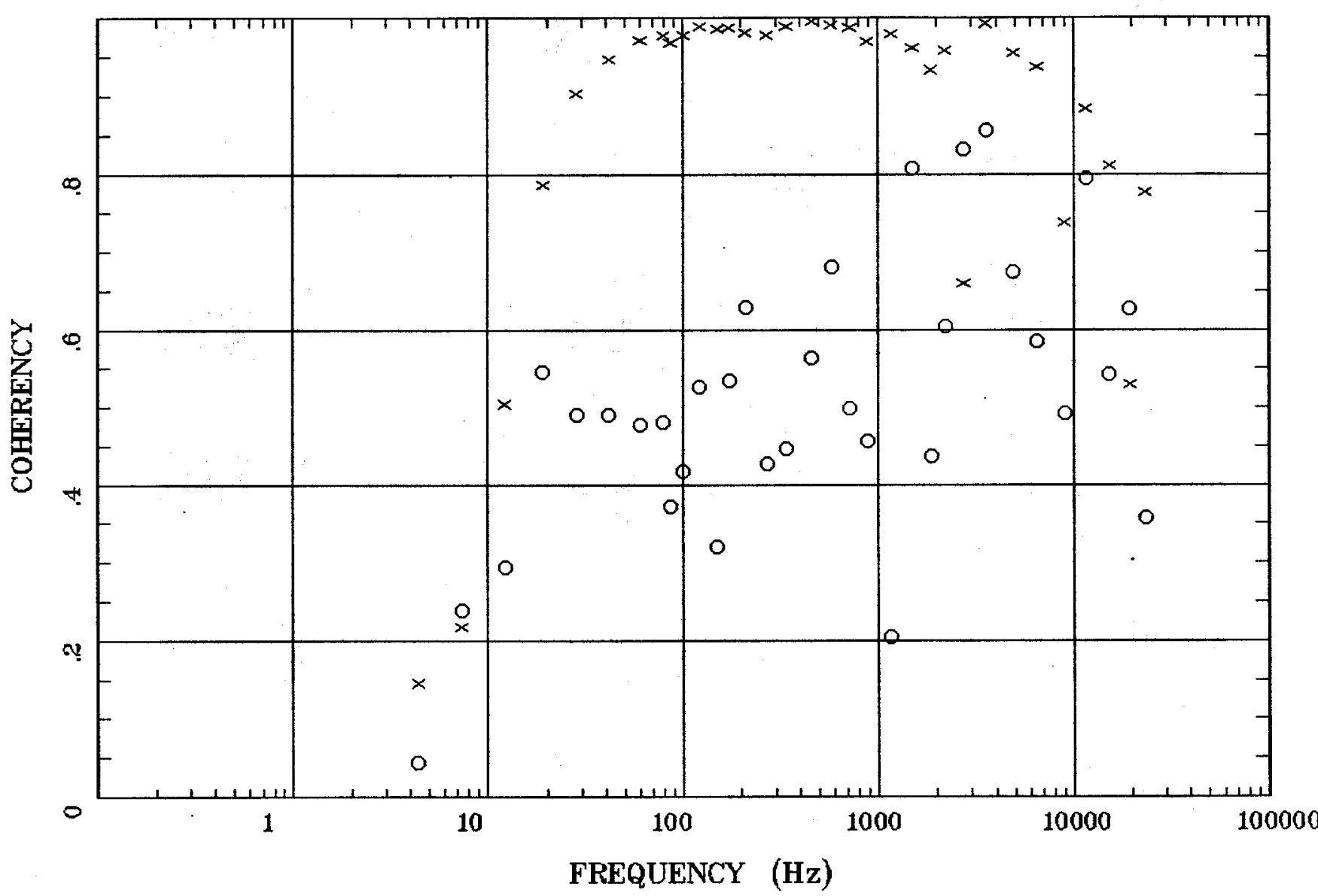

Client:

Remote: e-fld $95 \mathrm{~m}$ west

Acquired: 15:1 Jul 29, 2002 Survey Co:USGS
Rotation:

Filename: cp 12a.avg

Channels: Ch1 Ch2 Ch3 Ch4 Ch5 Ch6 Chr Plotted: 14:32 Jul 06, 2004

$<$ EMI - ElectroMagnetic Instruments > 


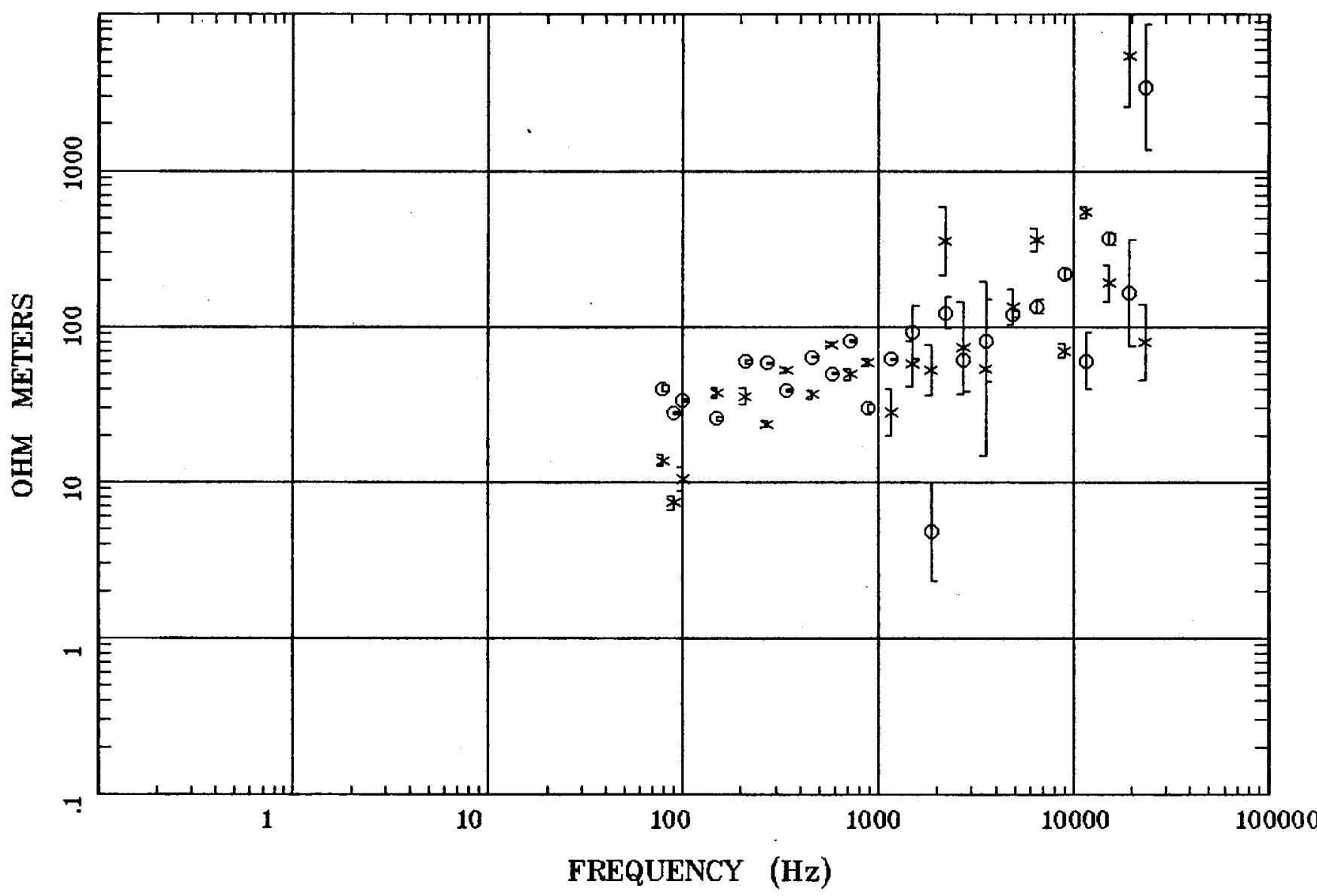

Client:

Remote: e-fld $90 \mathrm{~m}$ west

Acquired: $13: 0$ Jul 30,2002 Survey Co:USGS
Rotation:

Filename: cp 13a.avg

Channels: Ch1 Ch2 Ch3 Ch4 Ch5 Ch6 Chr Plotted: 14:33 Jul 06, 2004

< EMI - ElectroMagnetic Instruments > 


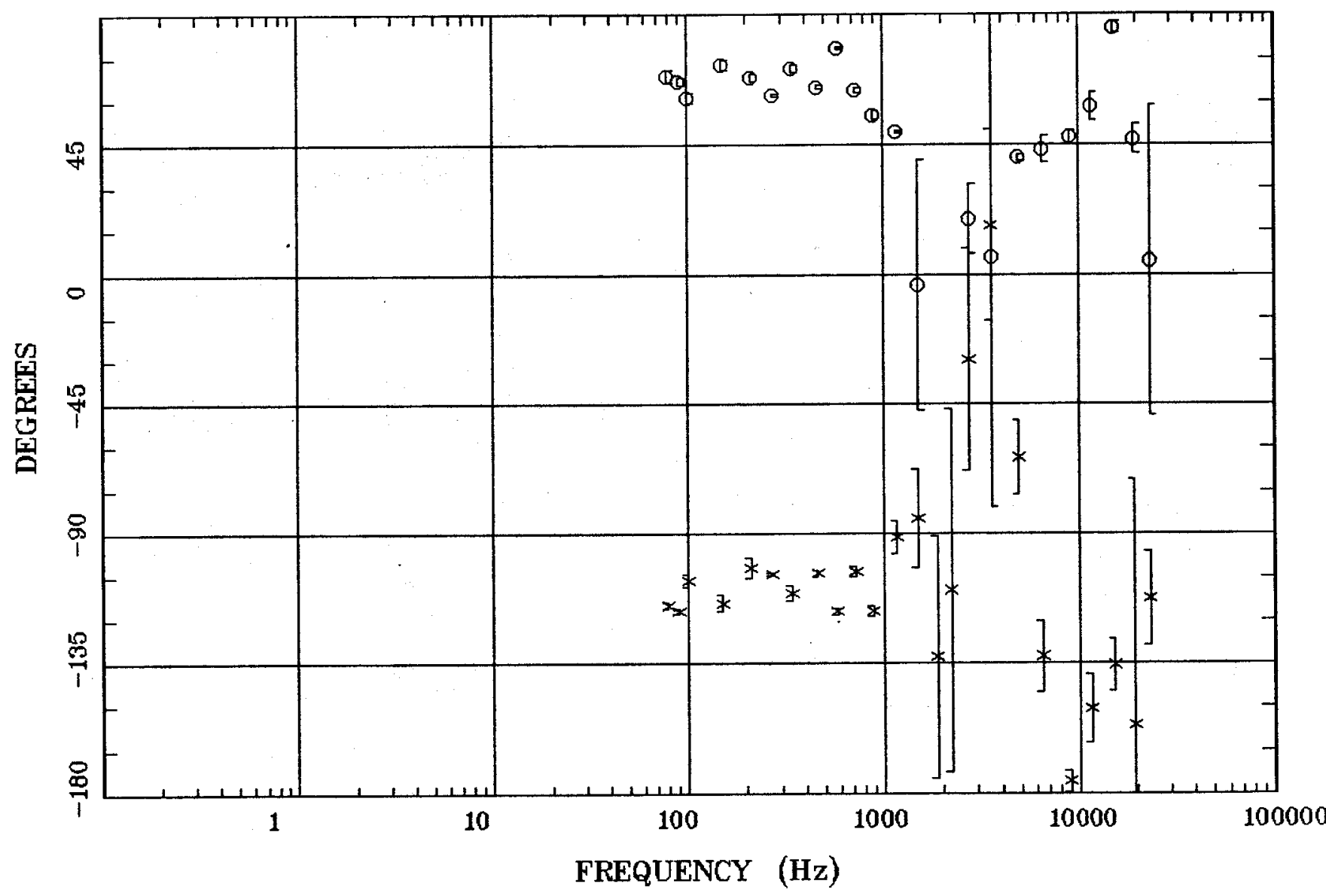

Client:

Remote: e-fld 90m west

Acquired: $13: 0$ Jul 30, 2002 Survey Co:USGS
Rotation:

Filename: cp13a.avg

Channels: Ch1 Ch2 Ch3 ch4 Ch5 Ch6 Ch7

Plotted: 14:33 Jul 06, 2004

< EMI - ElectroMagnetic Instruments > 


\section{ROTATION ANGLE}

East of Cerrito Pelado

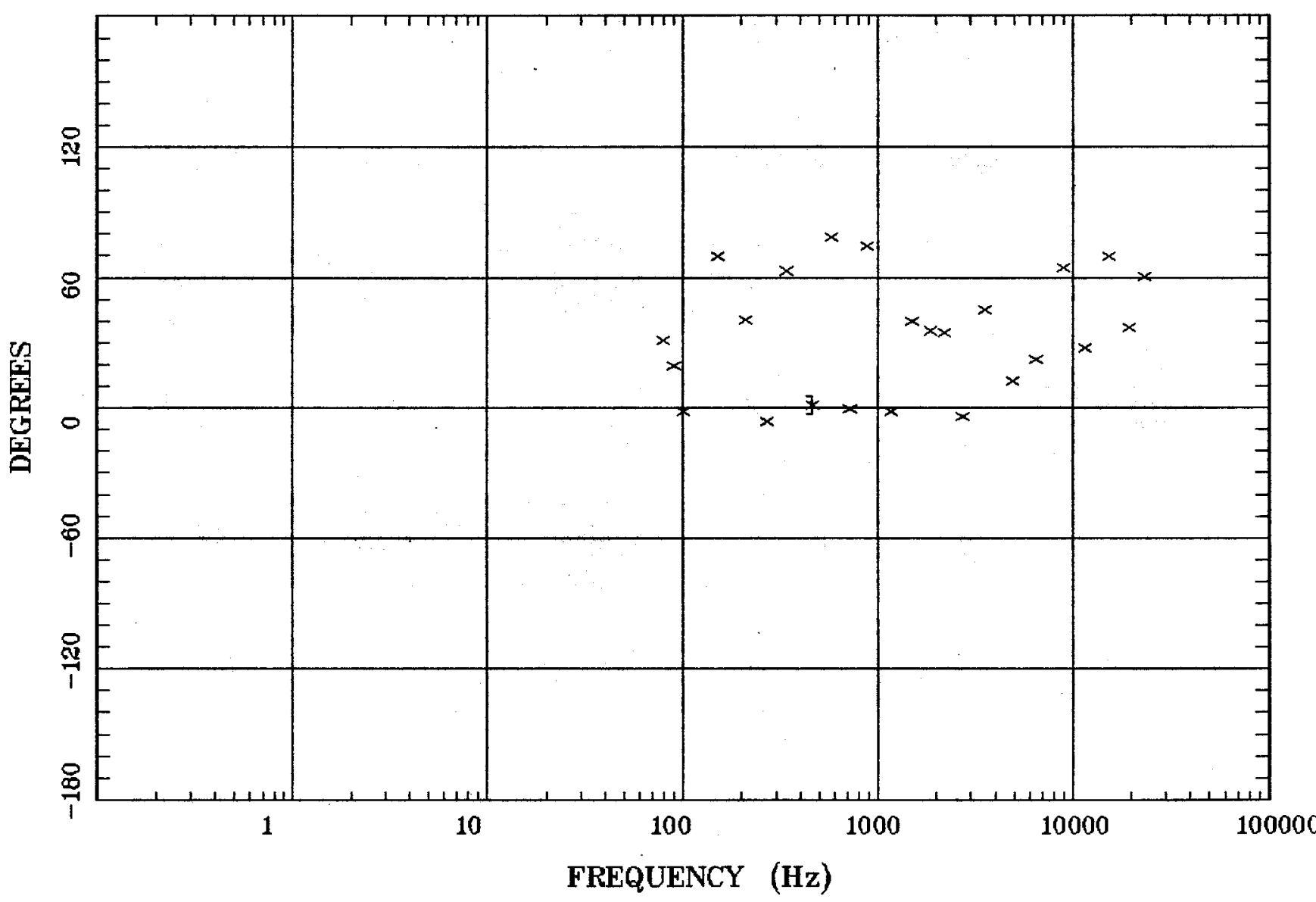

Client:

Remote: e-fld $90 \mathrm{~m}$ west

Acquired: 13:0 Jul 30, 2002 Survey Co:USGS

\section{Rotation:}

Filename: cp 13a.avg

Channels: Ch1 Ch2 Ch3 Ch4 Ch5 Ch6 Ch7 Plotted: 12:49 Jul 07, 2004

< EMI - ElectroMagnetic Instruments > 


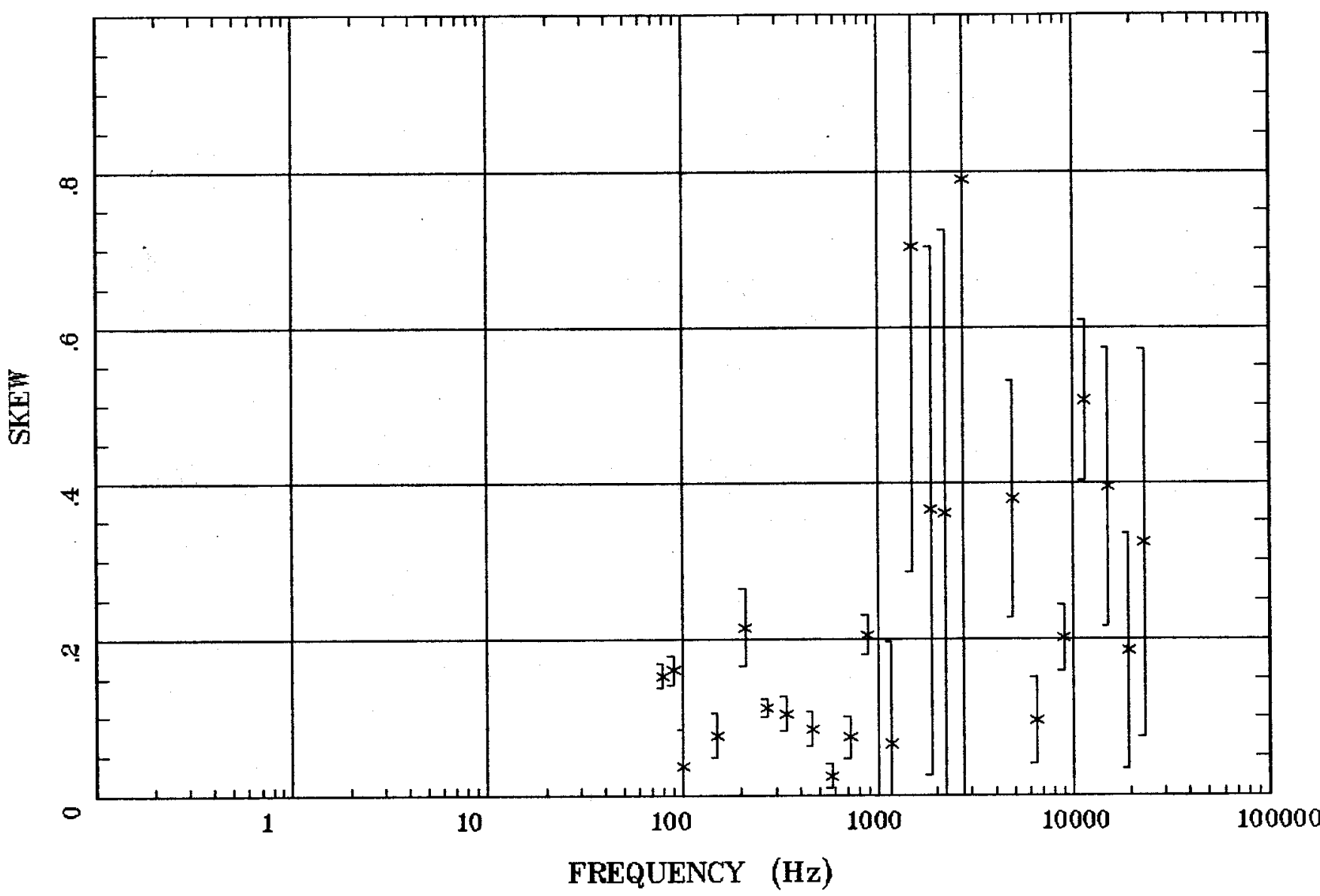

Client:

Remote: e-fld $90 \mathrm{~m}$ west

Acquired: 13:0 Jul 30, 2002 Survey Co:USGS
Rotation:

Filename: cp 13a.avg

Channels: Ch1 Ch2 Ch3 Ch4 Ch5 Ch6 Ch7 Plotted: 14:33 Jul 06, 2004

< EMI - ElectroMagnetic Instruments > 


\section{Station 13}

E MULT Coh.

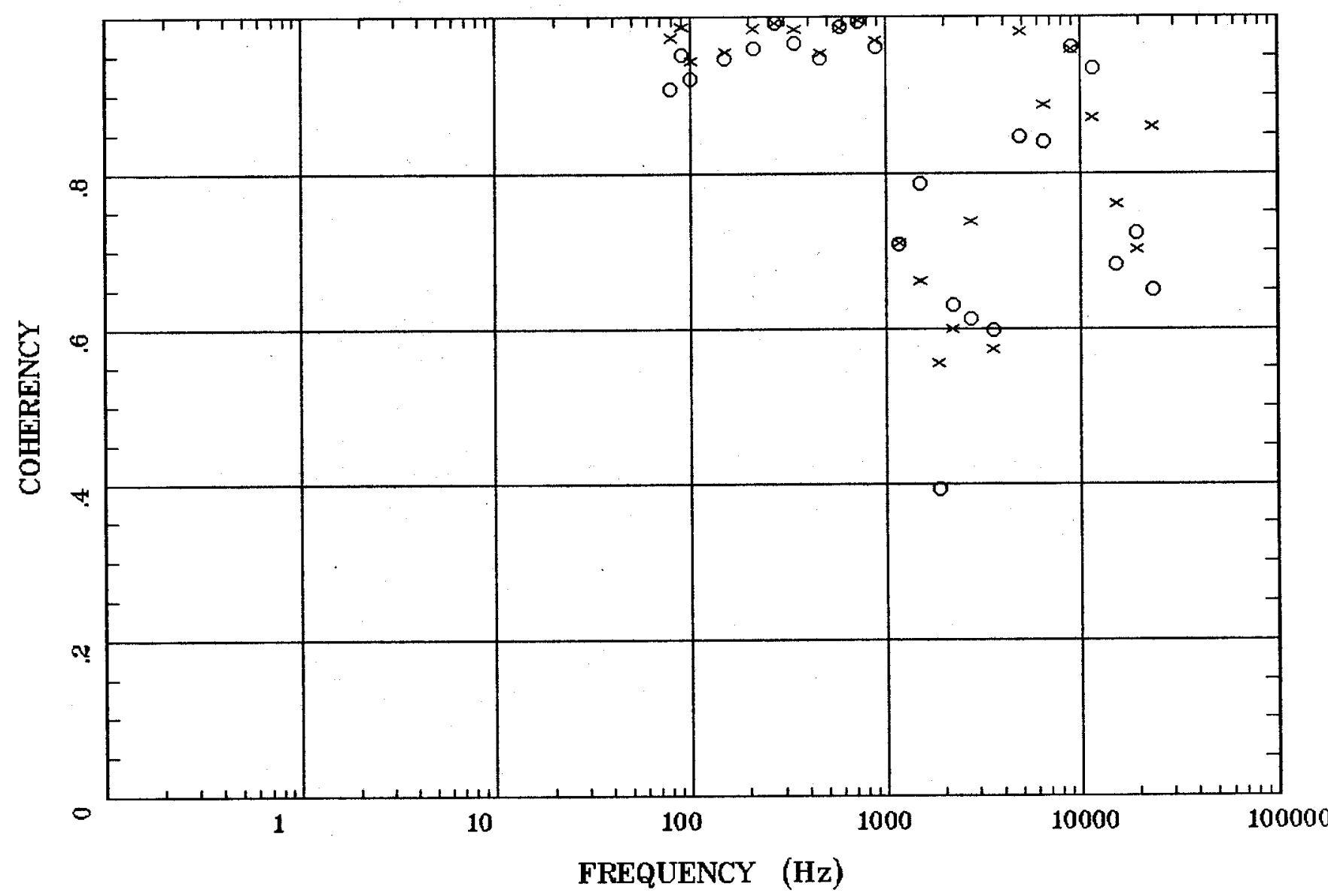

Client:

Remote: e-fld $90 \mathrm{~m}$ west

Acquired: 13:0 Jul 30, 2002

Survey Co:USGS
Rotation:

Filename: cp 13a.avg

Channels: Ch1 Ch2 Ch3 Ch4 Ch5 Ch6 Ch7

Plotted: 14:34 Jul 06, 2004

< EMI - ElectroMagnetic Instruments > 


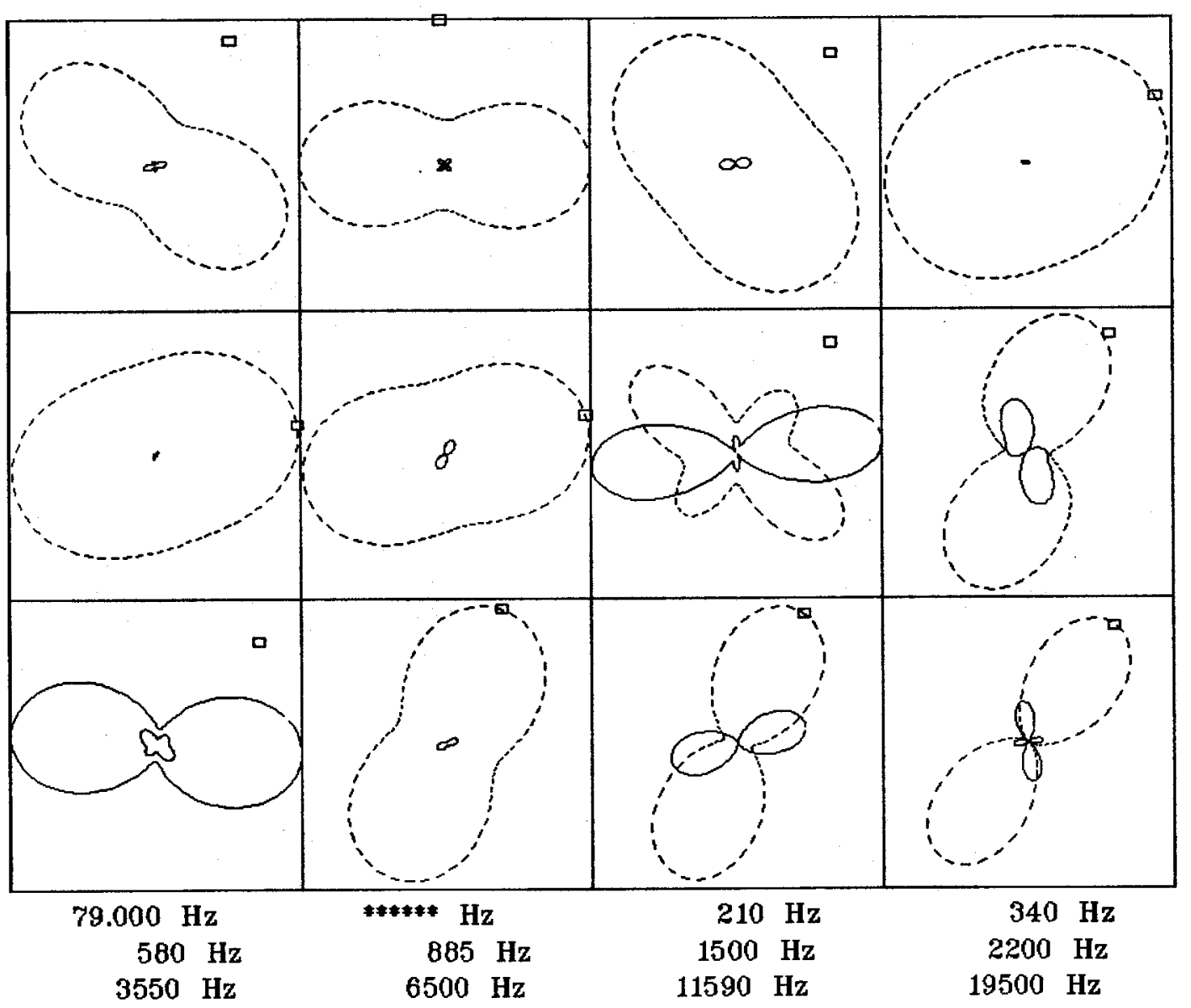

Client:

Remote: e-fld $90 \mathrm{~m}$ west Acquired: 13:0 Jul 30, 2002 Survey Co:USGS
Rotation:

Filename: cp 13a.avg

Channels: Ch1 Ch2 Ch3 Ch4 Ch5 Ch6 Ch7 Plotted: 14:34 Jul 06, 2004

< EMI - ElectroMagnetic Instruments > 


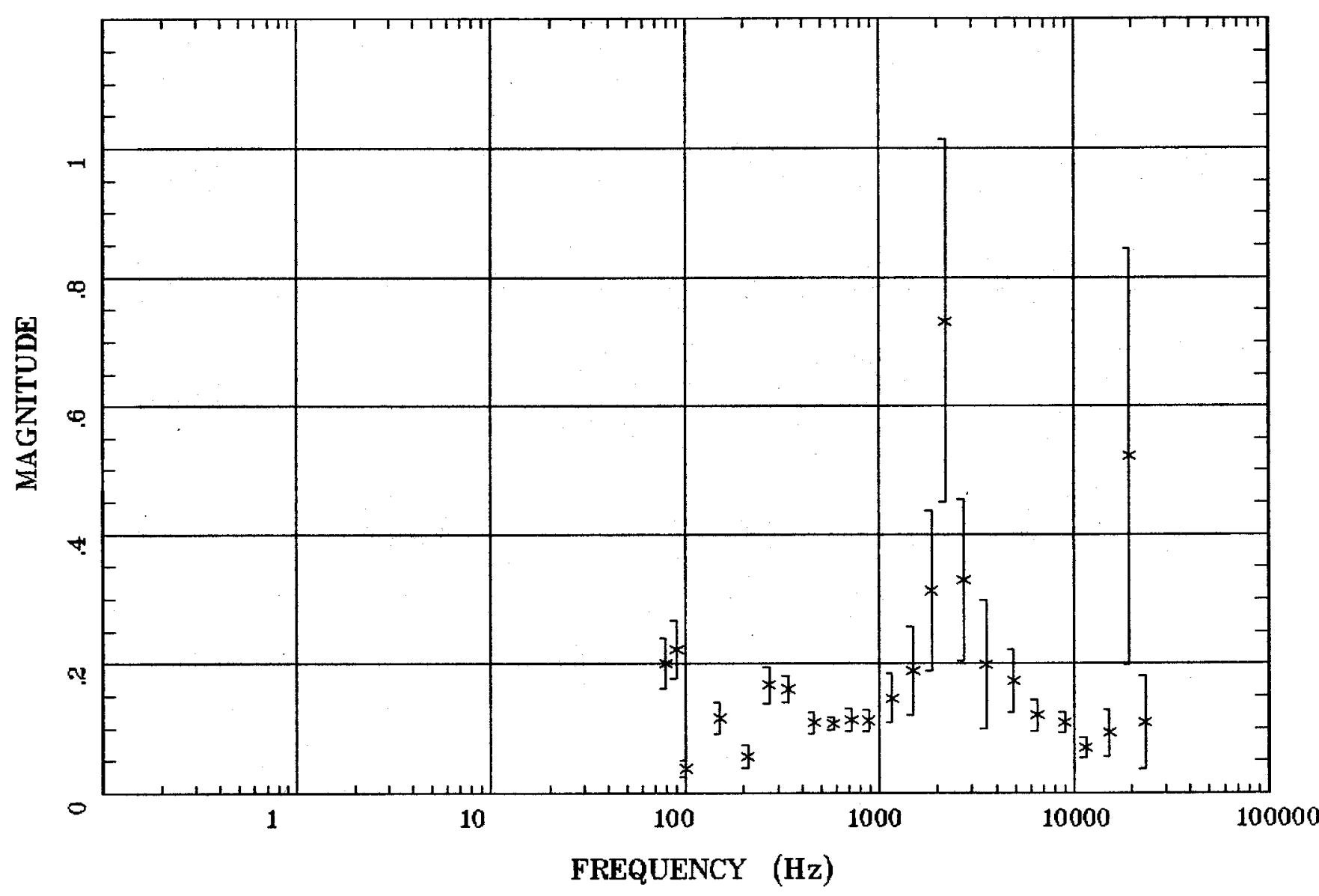

Client:

Remote: e-fld $90 \mathrm{~m}$ west Acquired: 13:0 Jul 30, 2002 Survey Co:USGS
Rotation:

Filename: cp 13a.avg

Channels: Ch1 Ch2 Ch3 Ch4 Ch5 Ch6 Ch7

Plotted: 14:34 Jul 06, 2004

< EMI - ElectroMagnetic Instruments 


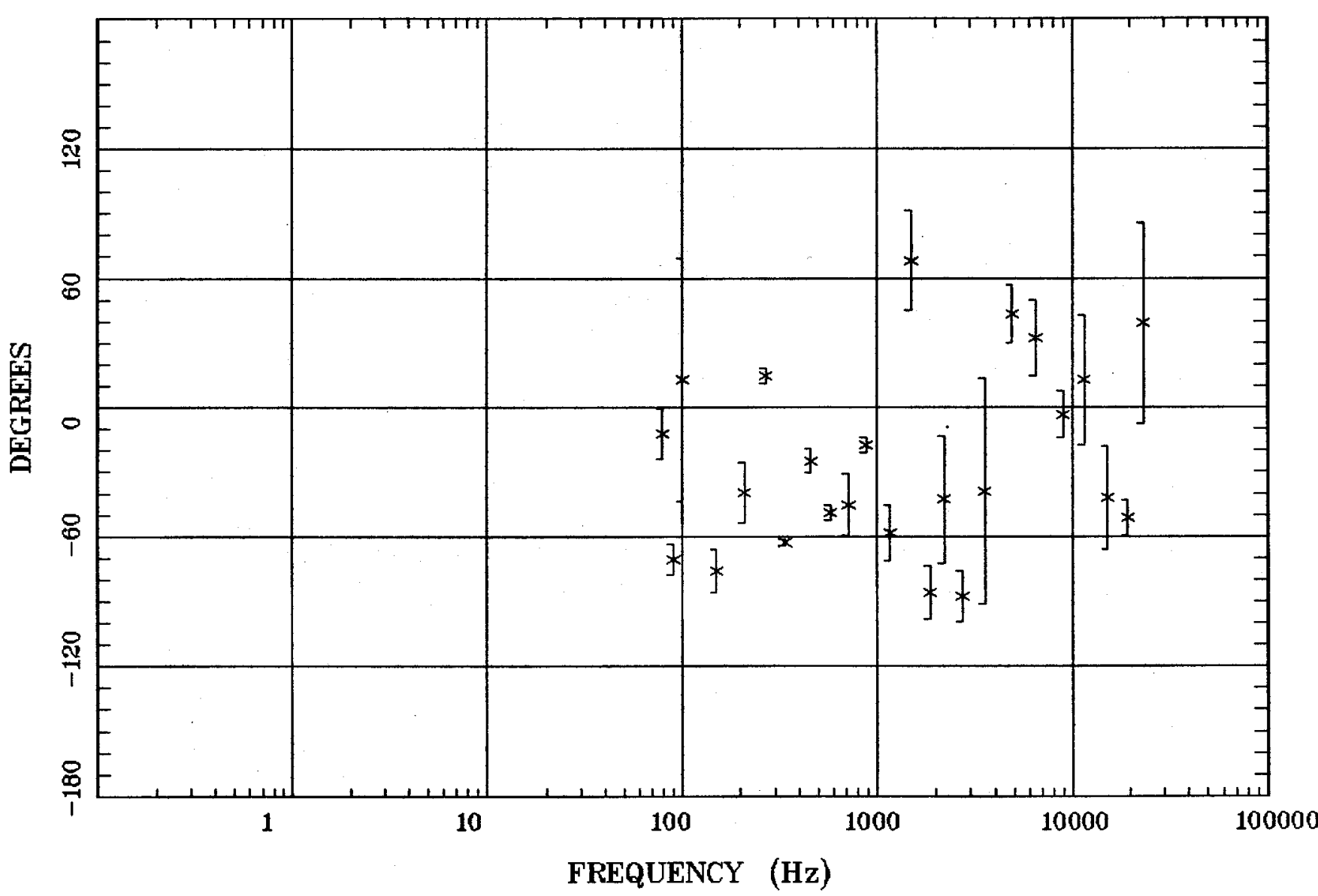

Client:

Remote: e-fld $90 \mathrm{~m}$ west

Acquired: 13:0 Jul 30, 2002 Survey Co:USGS
Rotation:

Filename: cp13a.avg

Channels: Ch1 Ch2 Ch3 Ch4 Ch5 Ch6 Ch7 Plotted: 14:34 Jul 06, 2004

< EMI - ElectroMagnetic Instruments > 


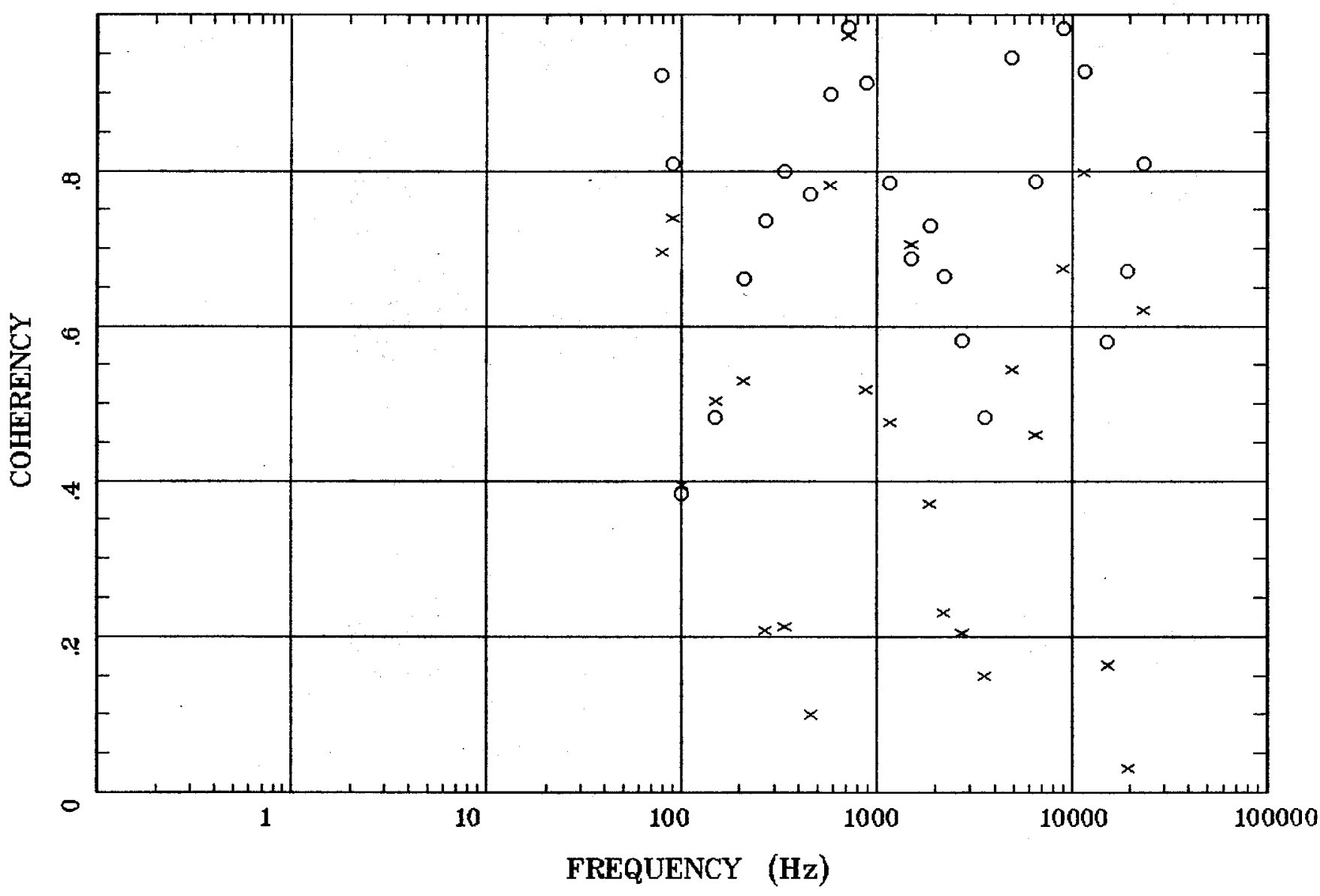

Client:

Remote: e-fld $90 \mathrm{~m}$ west Acquired: 13:0 Jul 30, 2002 Survey Co:USGS
Rotation:

Filename: cp13a.avg

Channels: Ch1 Ch2 Ch3 Ch4 Ch5 Ch6 Ch7 Plotted: 14:34 Jul 06, 2004

< EMI - ElectroMagnetic Instruments 


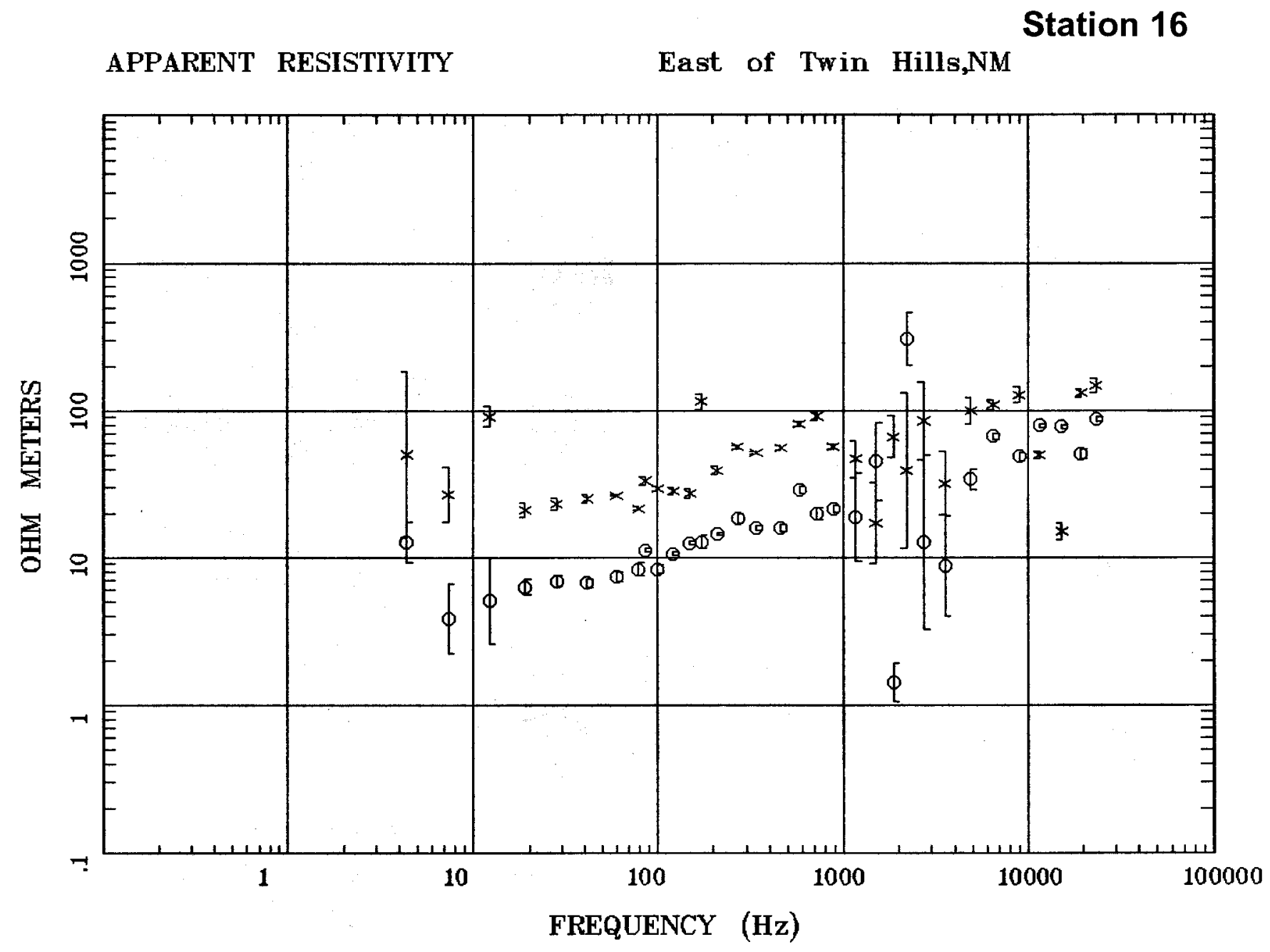

Client:

Remote: e-fld $95 \mathrm{~m}$ west

Acquired: 11:5 Aug 01, 2002 Survey Co:USGS
Rotation:

Filename: cp 16a.avg

Channels: Ch1 Ch2 Ch3 Ch4 Ch5 Ch6 Ch7

Plotted: 14:36 Jul 06, 2004

< EMI - ElectroMagnetic Instruments > 
Station 16

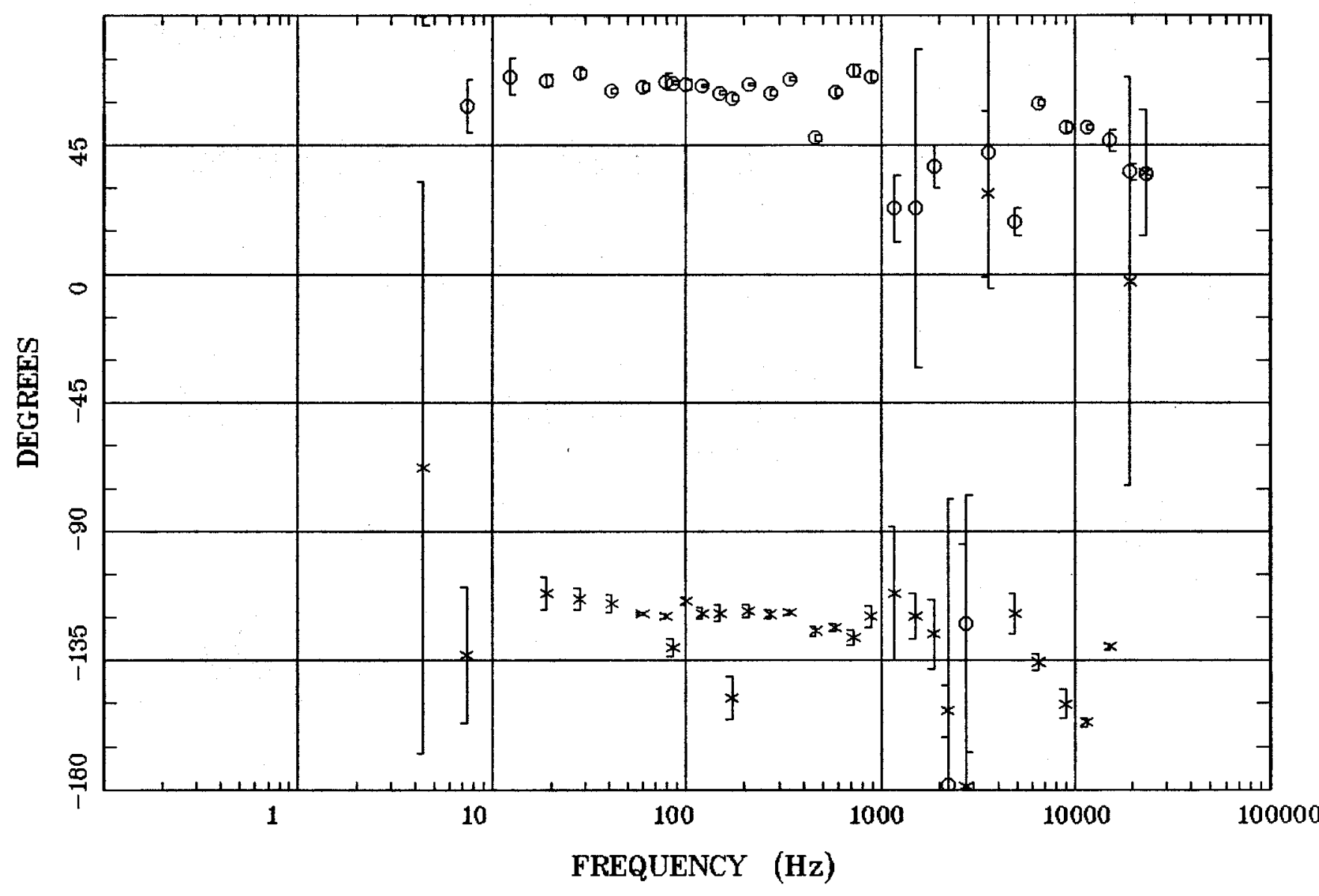

Client:

Remote: e-fld 95m west

Acquired: 11:5 Aug 01, 2002 Survey Co:USGS
Rotation:

Filename: cp 16a.avg

Channels: Ch1 Ch2 Ch3 Ch4 Ch5 Ch6 Ch7

Plotted: 14:36 Jul 06, 2004

< EMI - ElectroMagnetic Instruments 
Station 16

ROTATION ANGLE

East of Twin Hills,NM

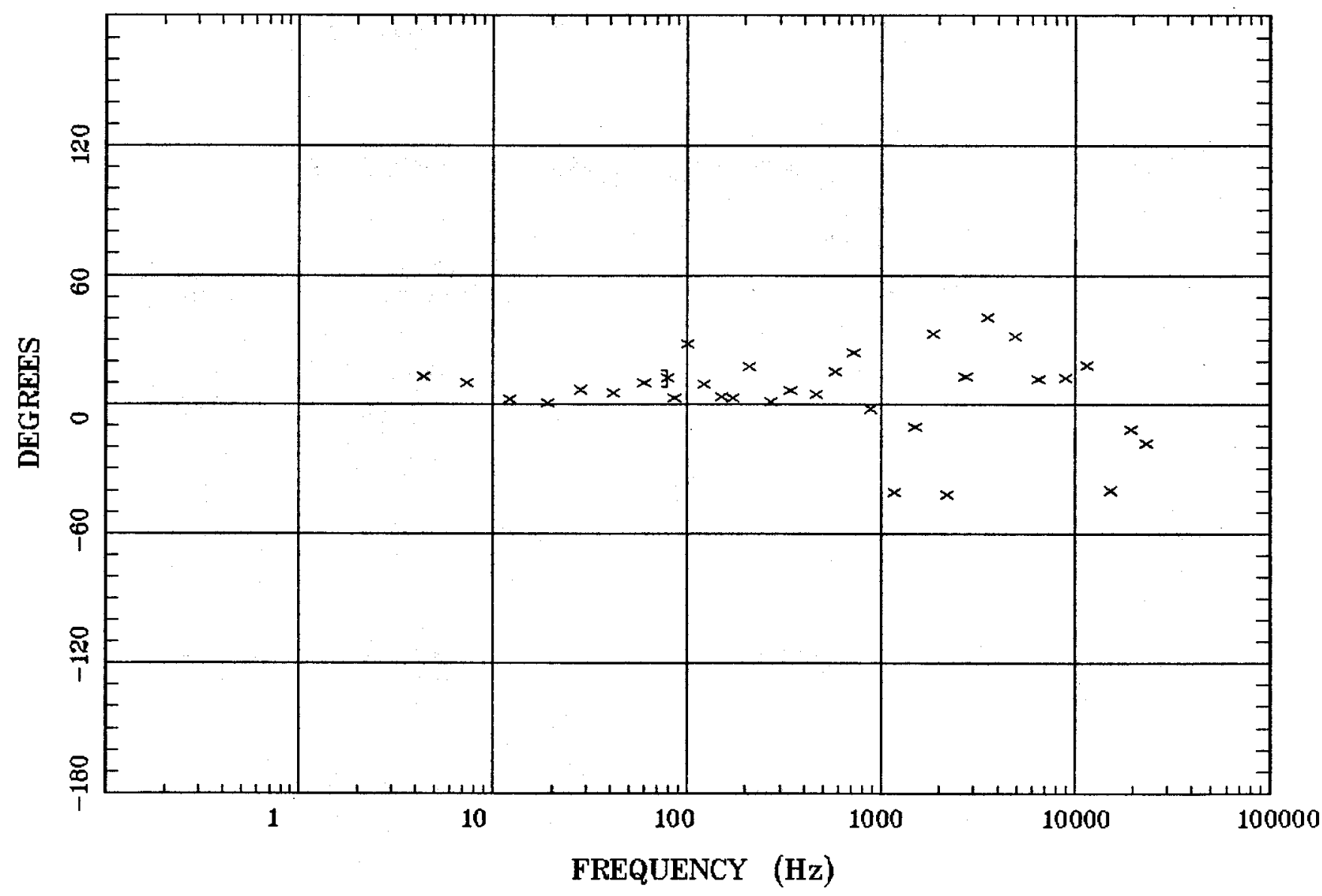

Client:

Remote: e-fld 95m west

Acquired: 11:5 Aug 01, 2002 Survey Co:USGS
Rotation:

Filename: cp16a.avg

Channels: Ch1 Ch2 Ch3 Ch4 Ch5 Ch6 Ch7

Plotted: 12:50 Jul 07, 2004

< EMI - ElectroMagnetic Instruments > 


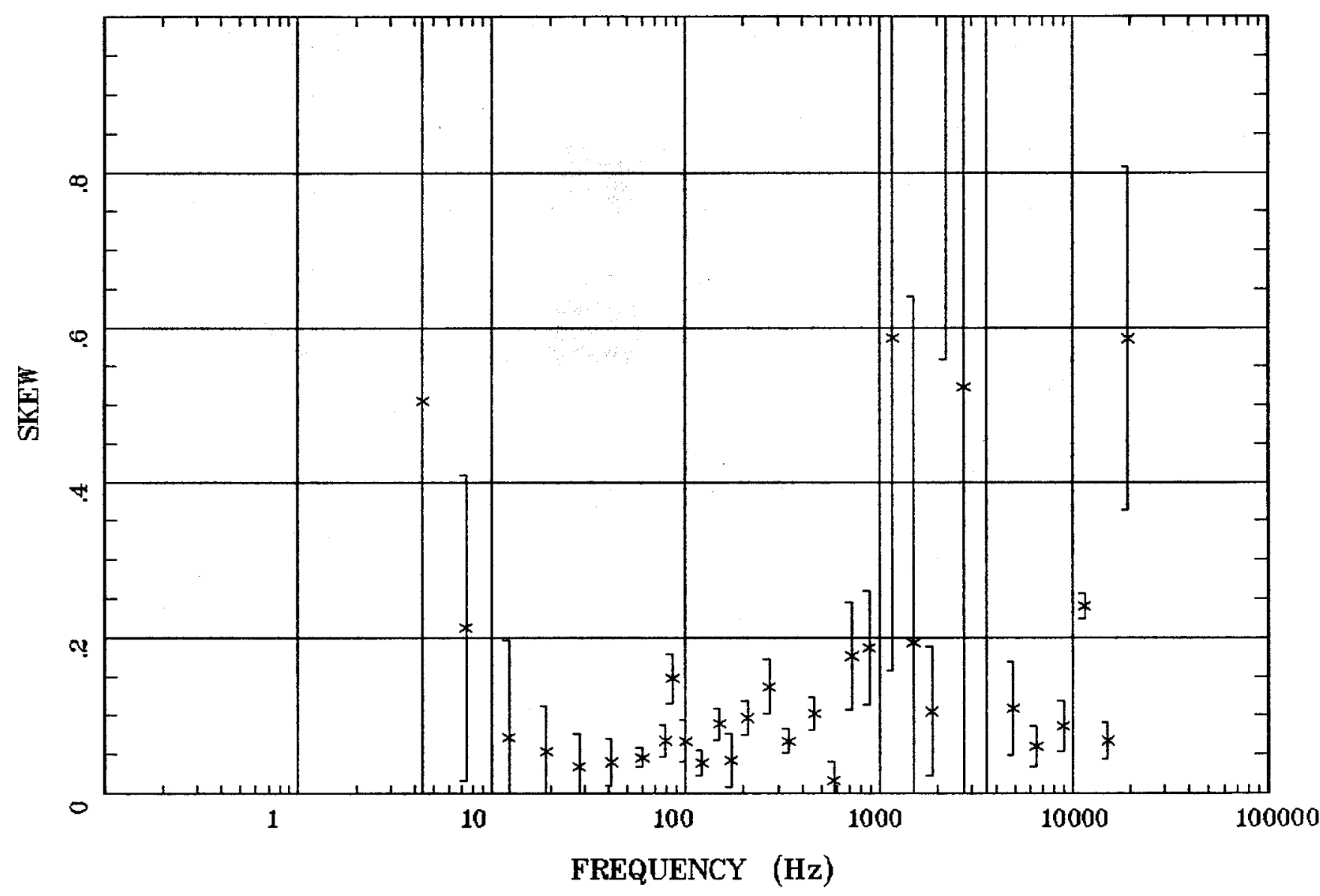

Client:

Remote: e-fld $95 \mathrm{~m}$ west

Acquired: 11:5 Aug 01, 2002 Survey Co:USGS
Rotation:

Filename: cp 16a.avg

Channels: Ch1 Ch2 Ch3 Ch4 Ch5 Ch6 Ch7 Plotted: 14:36 Jul 06, 2004

$<$ EMI - ElectroMagnetic Instruments 


\section{Station 16}

E MULT Coh.

East of Twin Hills,NM

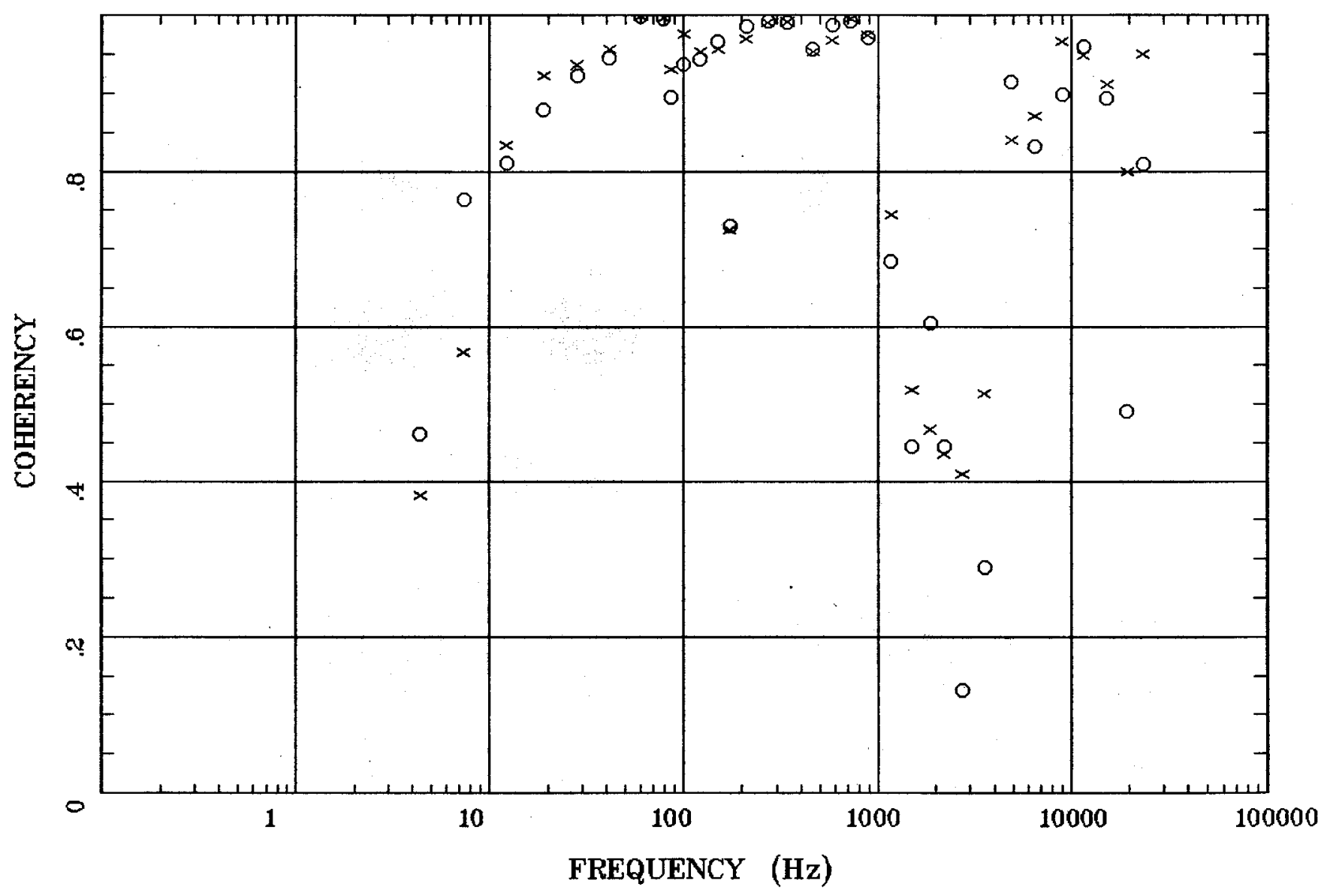

Client:

Remote: e-fld $95 \mathrm{~m}$ west Acquired: 11:5 Aug 01, 2002 Survey Co:USGS
Rotation:

Filename: cp 16a.avg

Channels: Ch1 Ch2 Ch3 Ch4 Ch5 Ch6 Ch7 Plotted: 14:36 Jul 06, 2004

< EMI - ElectroMagnetic Instruments > 


\section{POLAR PLOTS}

\section{East of Twin Hills,NM}

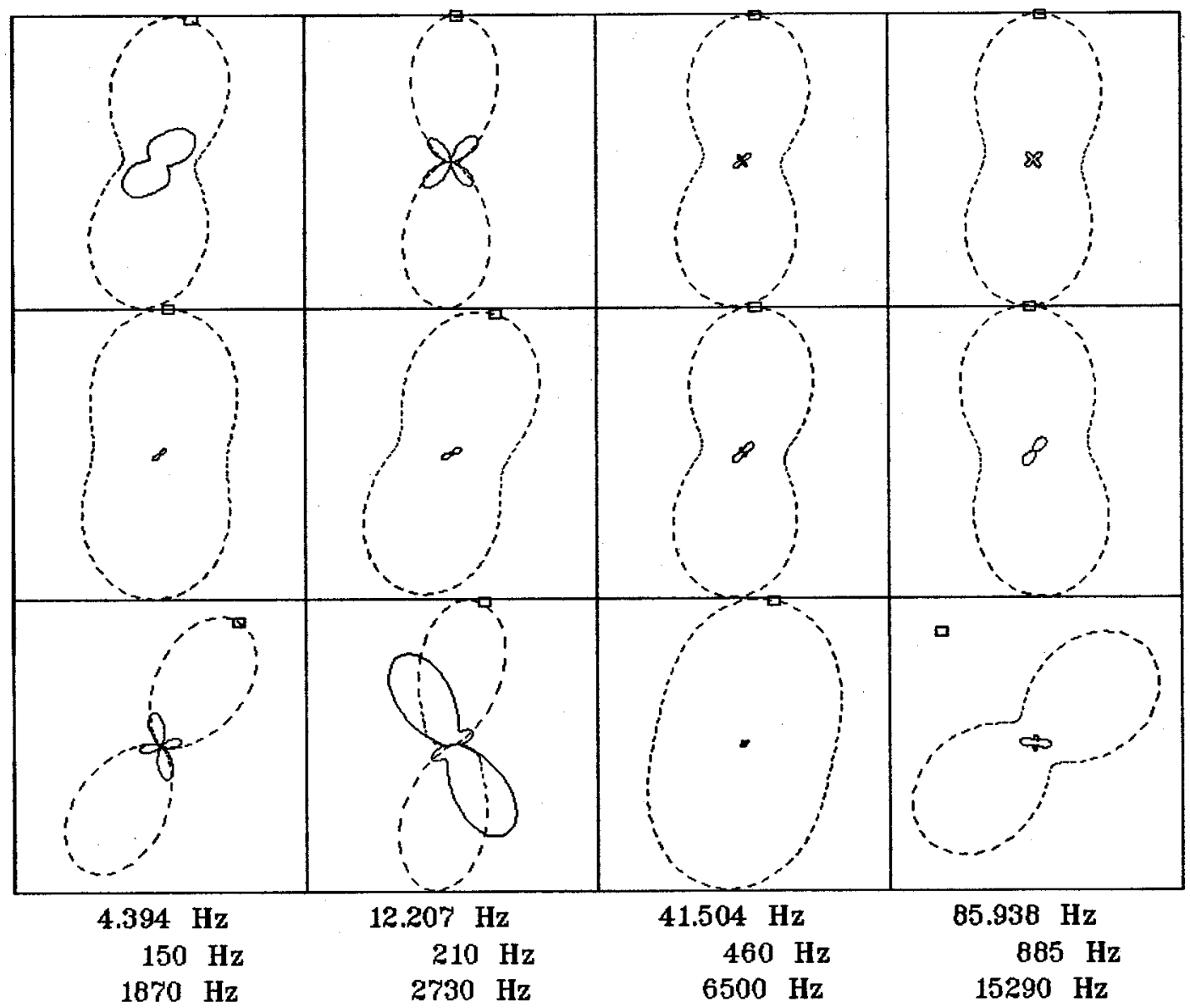

Client:

Remote: e-fld $95 \mathrm{~m}$ west

Acquired: 11:5 Aug 01, 2002

Rotation:

Filename: cp 16a.avg

Channels: Ch1 Ch2 Ch3 Ch4 Ch5 Ch6 Ch7

Plotted: 14:36 Jul 06, 2004

Survey Co:USGS

< EMI - ElectroMagnetic Instruments > 
East of Twin Hills,NM

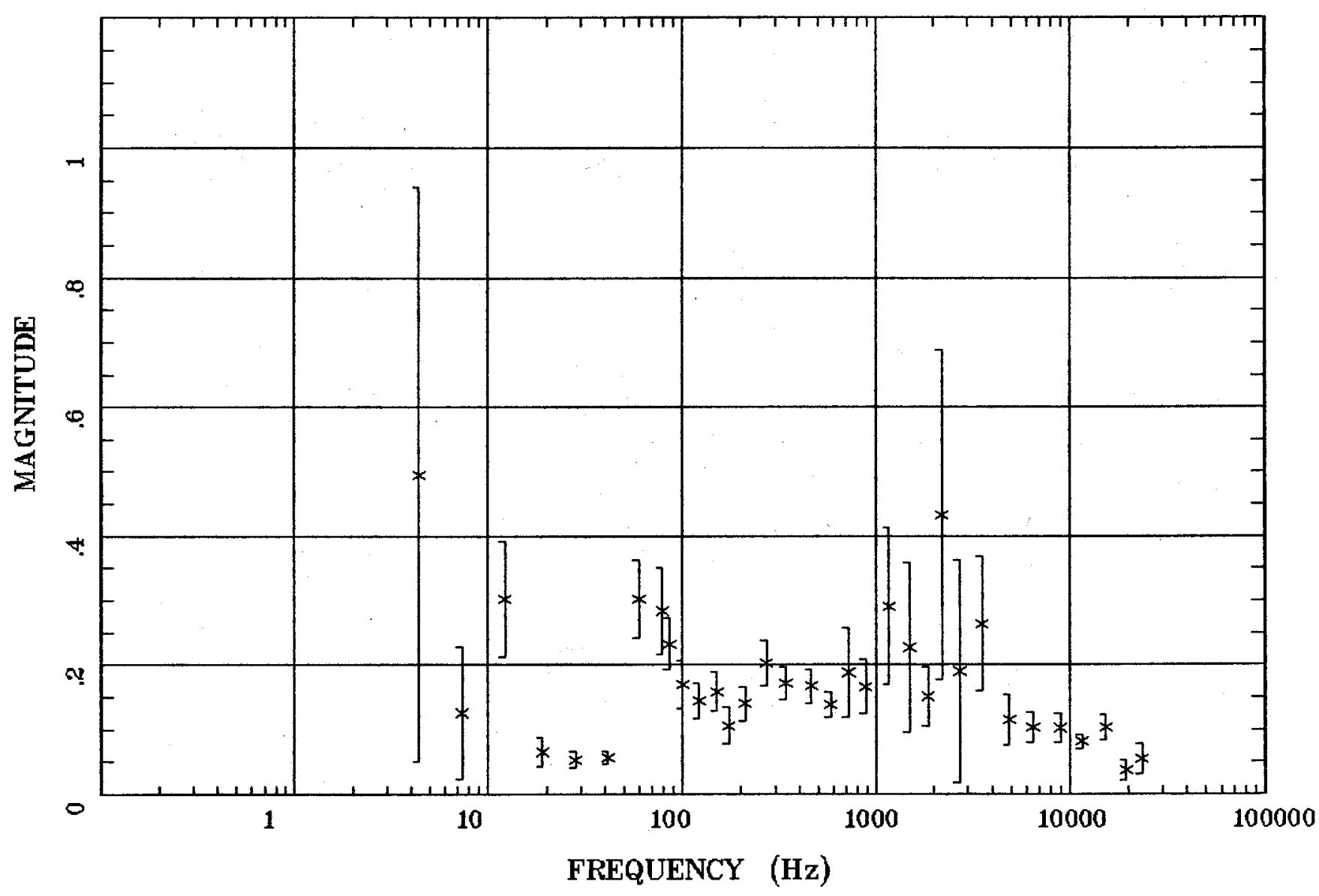

Client:

Remote: e-fld $95 \mathrm{~m}$ west Acquired: 11:5 Aug 01, 2002 Survey Co:USGS

\section{Rotation:}

Filename: cp16a.avg

Channels: Ch1 Ch2 Ch3 Ch4 Ch5 Ch6 Ch7

Plotted: 14:36 Jul 06, 2004

$<$ EMI - ElectroMagnetic Instruments 
Station 16

TIPPER STRIKE

East of Twin Hills,NM

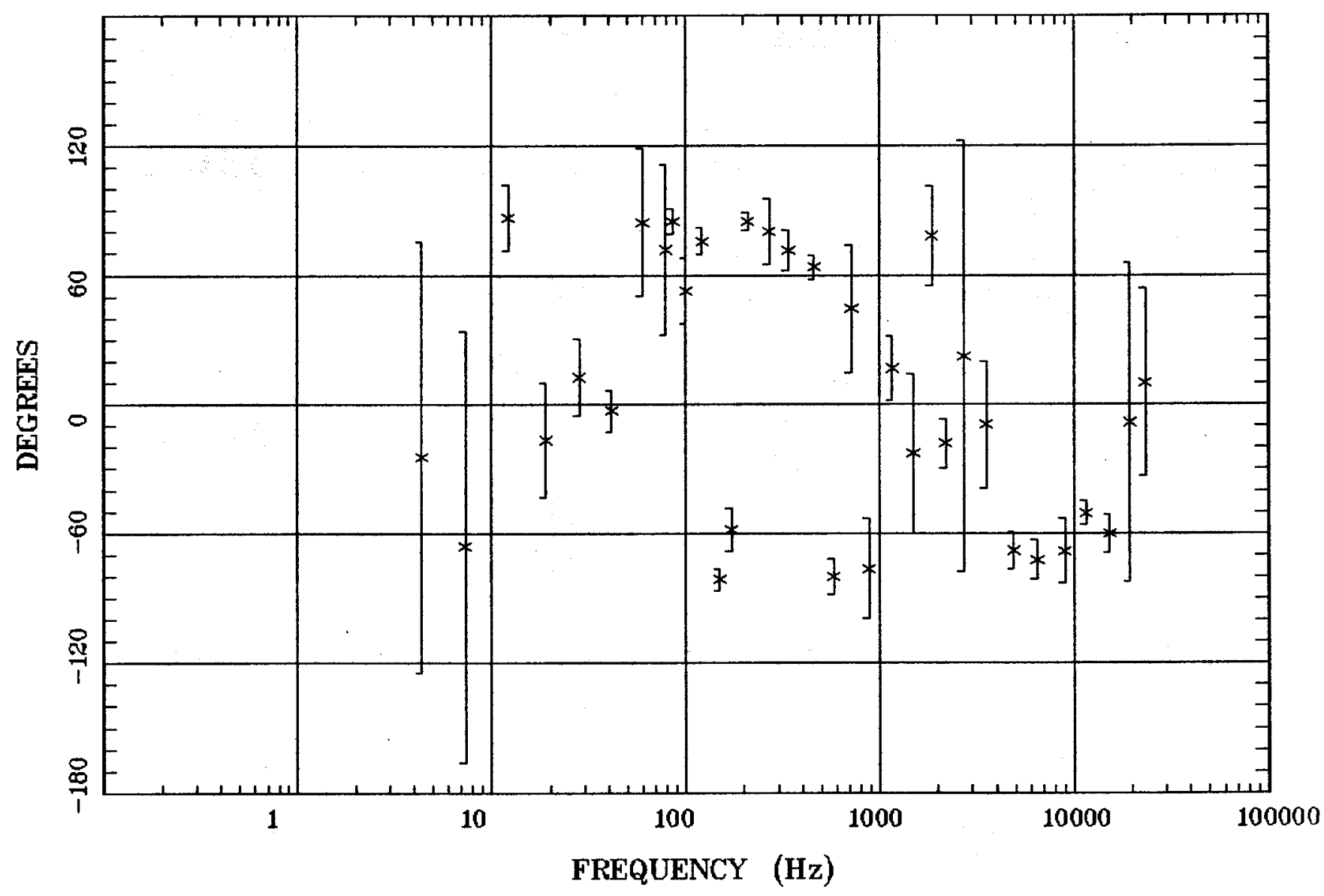

Client:

Remote: e-fld $95 \mathrm{~m}$ west

Acquired: 11:5 Aug 01, 2002

Survey Co:USGS
Rotation:

Filename: cp 16a.avg

Channels: Ch1 Ch2 Ch3 Ch4 Ch5 Ch6 Chr Plotted: 14:36 Jul 06, 2004

< EMI - ElectroMagnetic Instruments > 
Station 16

HzHx.x Coh HzHy.o

East of Twin Hills,NM

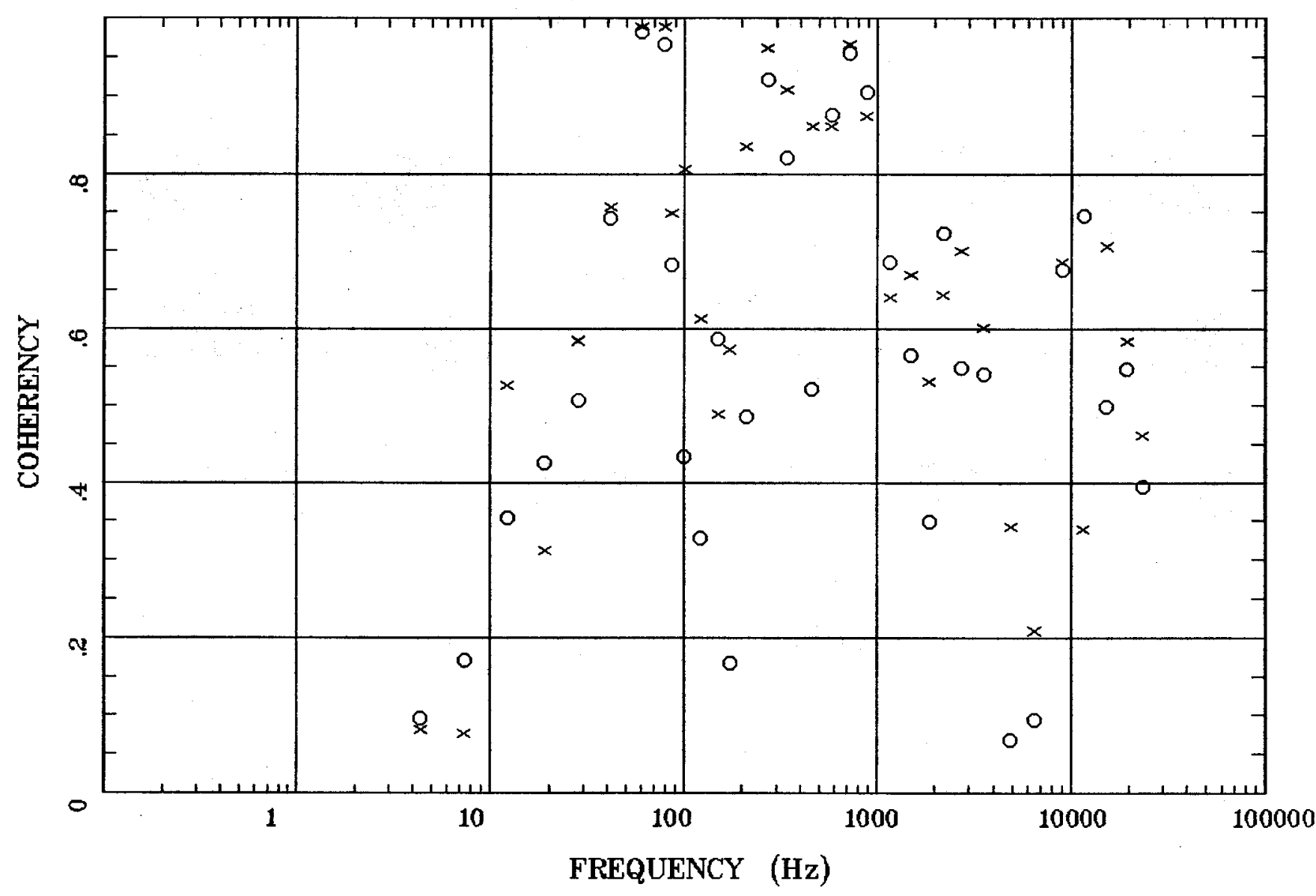

Client:

Remote: e-fld 95m west

Acquired: 11:5 Aug 01, 2002

Survey Co:USGS
Rotation:

Filename: cp16a.avg

Channels: Ch1 Ch2 Ch3 Ch4 Ch5 Ch6 Ch7

Plotted: 14:36 Jul 06, 2004

< EMI - ElectroMagnetic Instruments > 
Station 17

APPARENT RESISTIVITY

Canada de Cochiti, NM

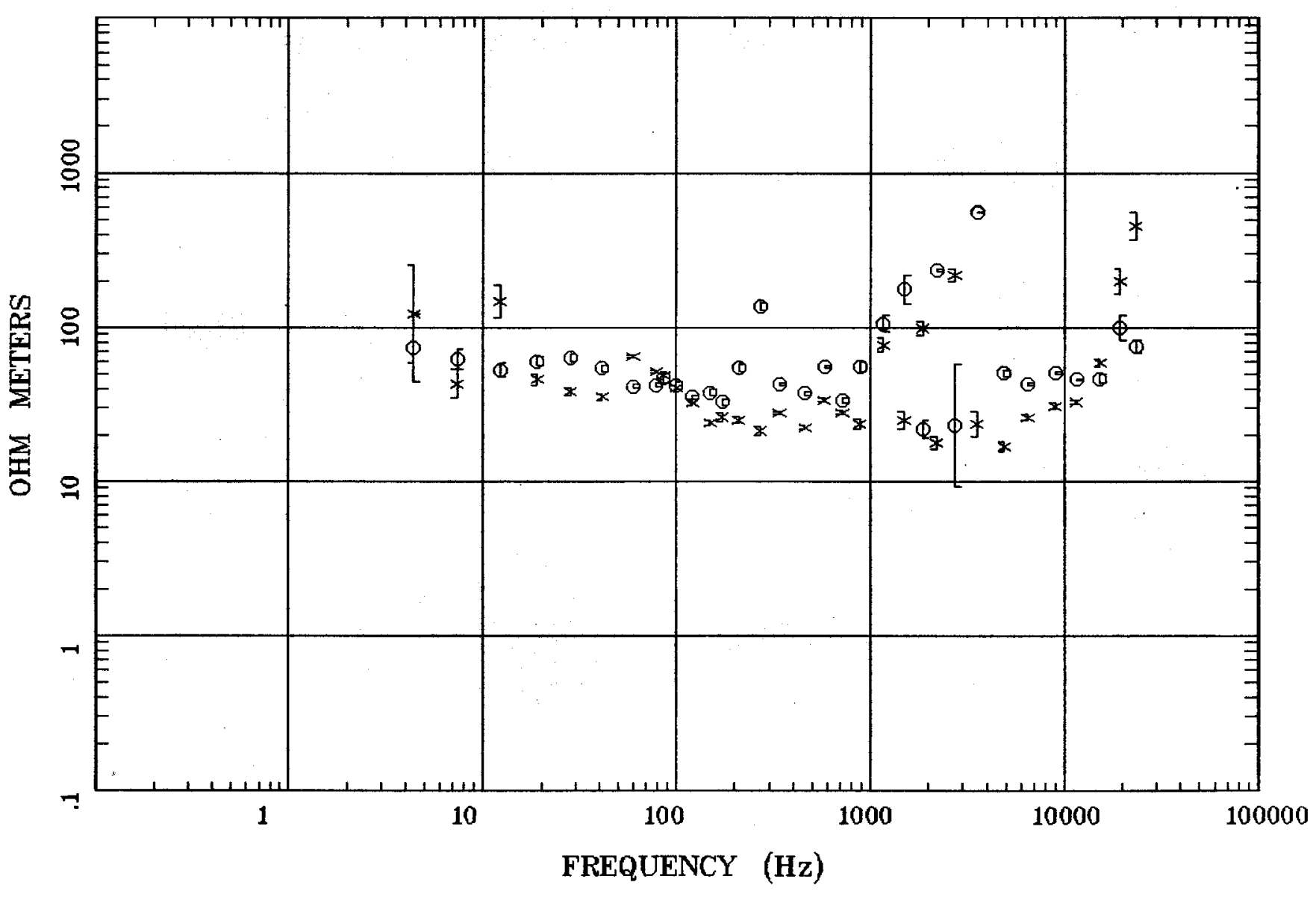

Client:

Remote: e-fld $90 \mathrm{~m}$ west

Acquired: 12:4 Aug 02, 2002

Survey Co:USGS
Rotation:

Filename: cp 17a.avg

Channels: Ch1 Ch2 Ch3 Ch4 Ch5 Ch6 Ch7

Plotted: 14:38 Jul 06, 2004

< EMI - ElectroMagnetic Instruments > 


\section{Station 17}

IMPEDANCE PHASE

Canada de Cochiti, NM

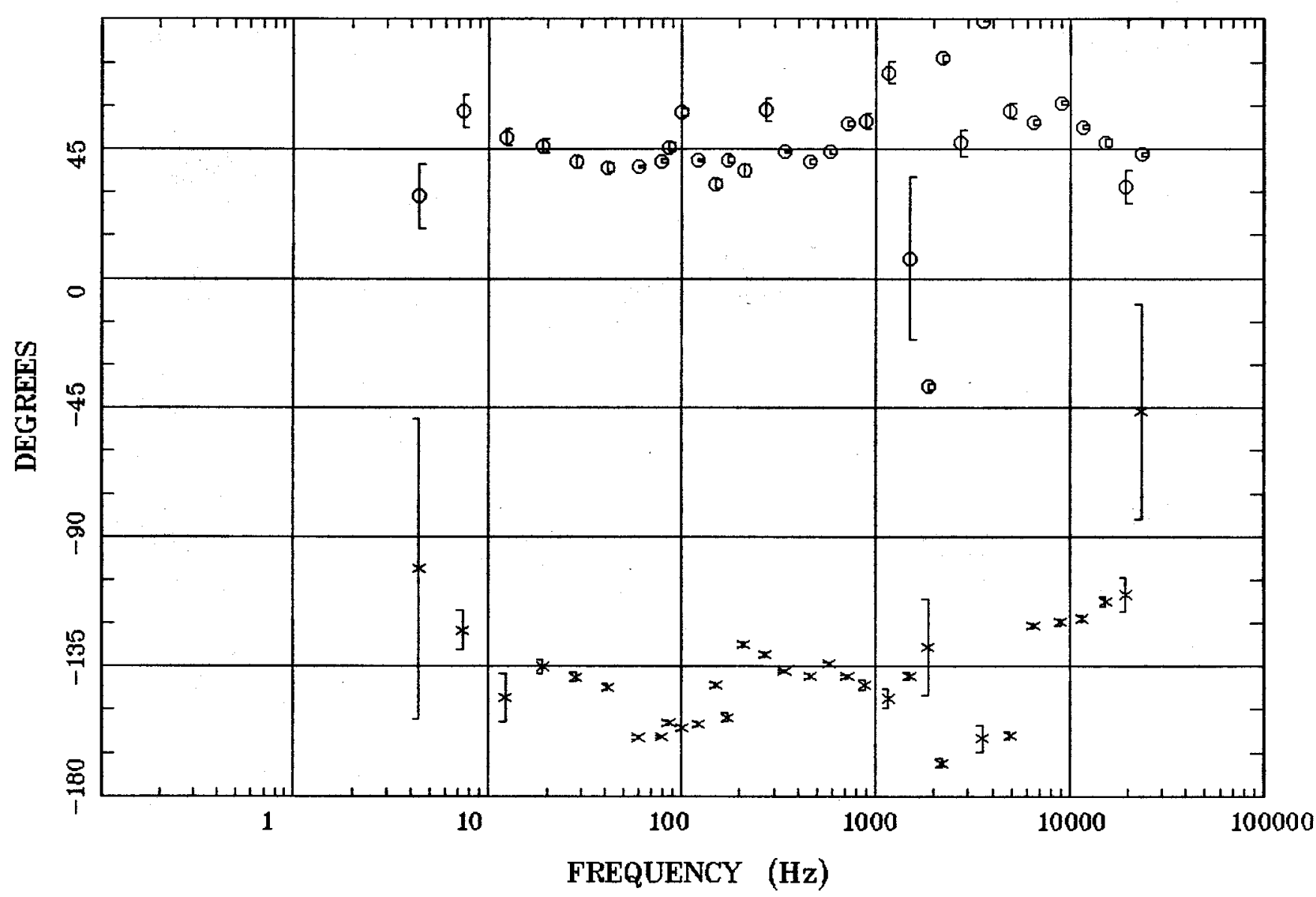

Client:

Remote: e-fld $90 \mathrm{~m}$ west

Acquired: 12:4 Aug 02, 2002 Survey Co:USGS
Rotation:

Filename: cp17a.avg Channels: Ch1 Ch2 Ch3 Ch4 Ch5 Ch6 Ch7 Plotted: 14:38 Jul 06, 2004

$<$ EMI - ElectroMagnetic Instruments 


\section{ROTATION ANGLE}

Canada de Cochiti, NM

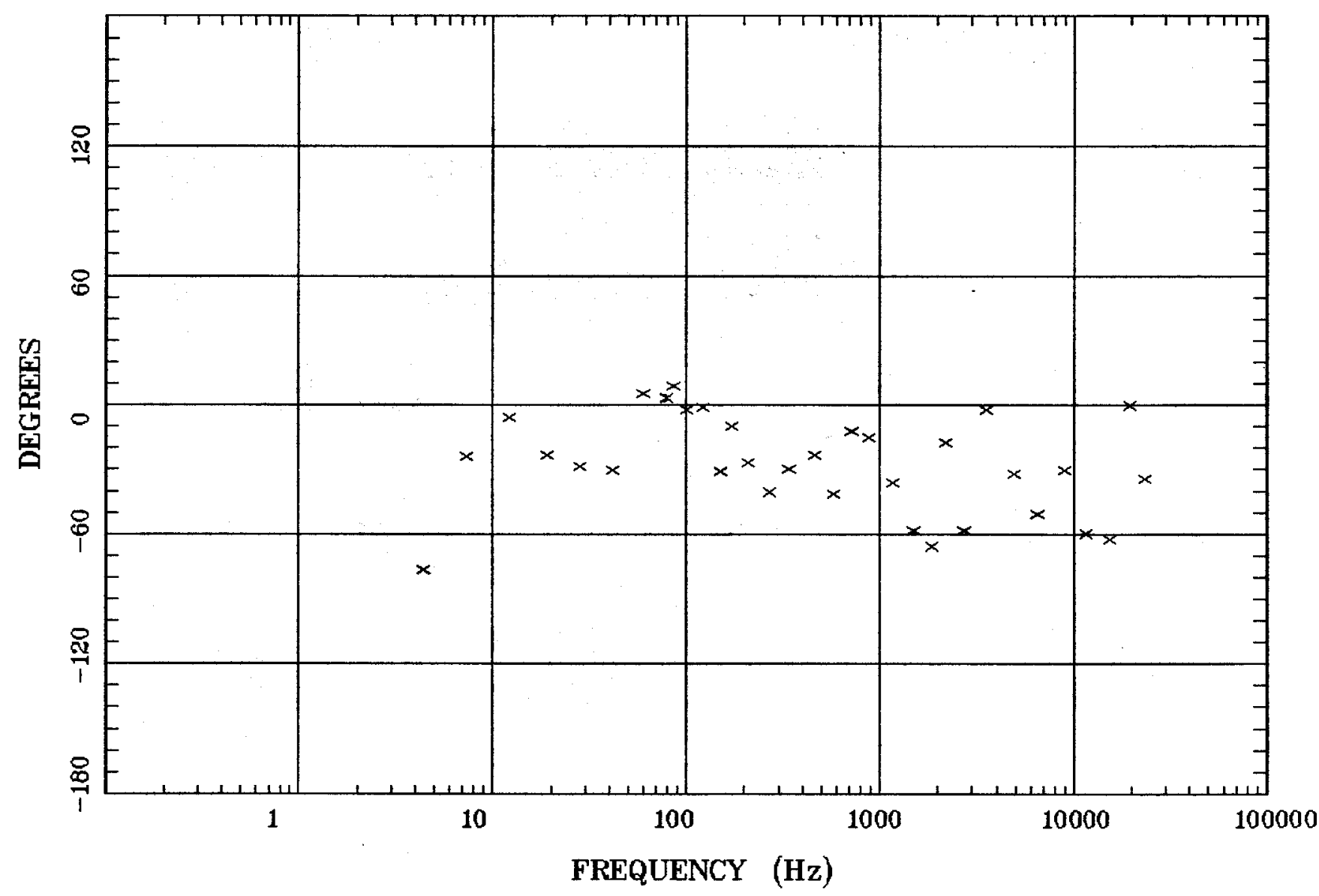

Client:

Remote: e-fld $90 \mathrm{~m}$ west Acquired: 12:4 Aug 02, 2002 Survey Co:USGS
Rotation:

Filename: cp 17a.avg

Channels: Ch1 Ch2 Ch3 Ch4 Ch5 Ch6 Ch7 Plotted: 12:51 Jul 07, 2004

< EMI - ElectroMagnetic Instruments 


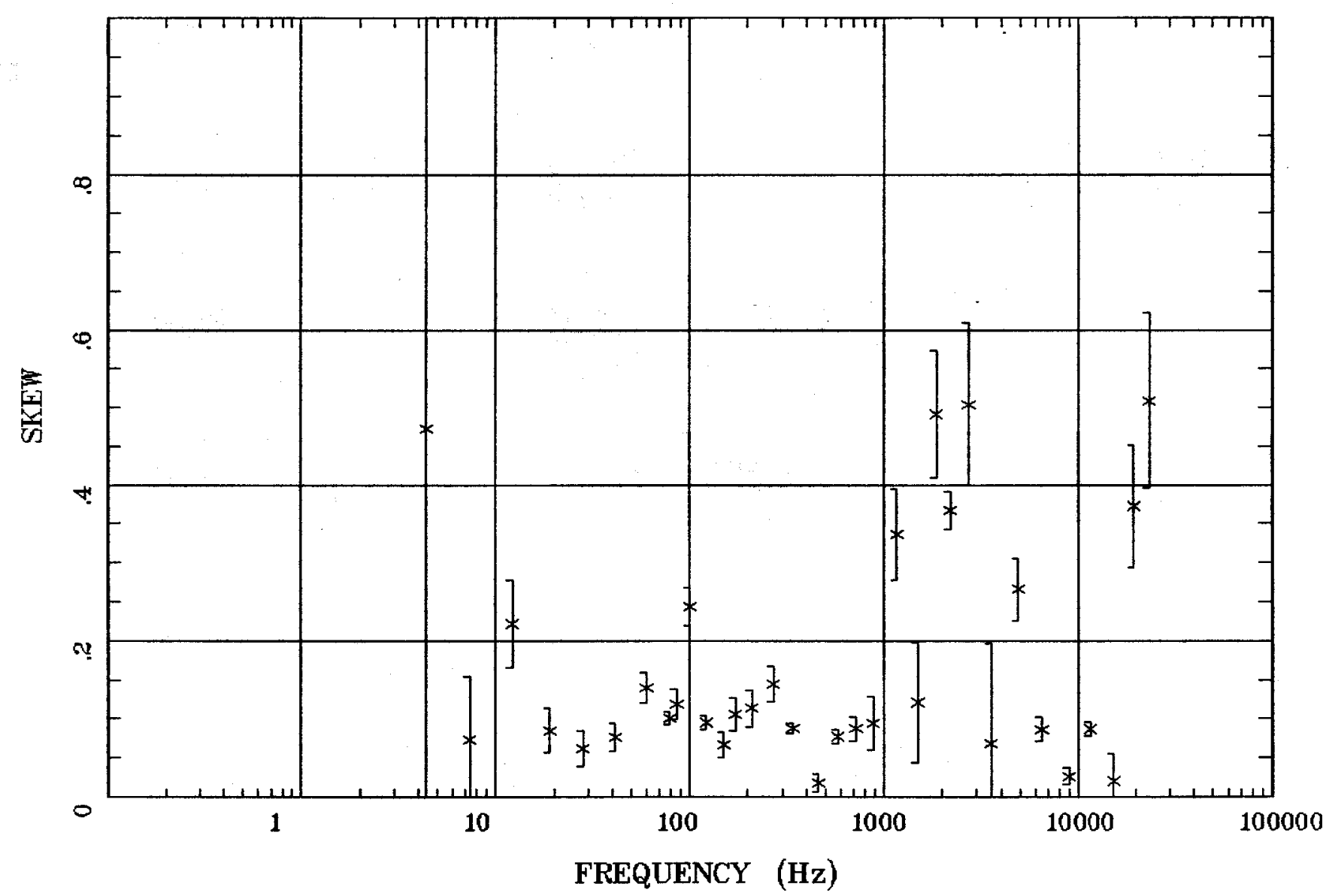

Client:

Remote: e-fld $90 \mathrm{~m}$ west Acquired: 12:4 Aug 02, 2002 Survey Co:USGS
Rotation:

Filename: cp17a.avg

Channels: Ch1 Ch2 Ch3 Ch4 Ch5 Ch6 Ch7

Plotted: 14:38 Jul 06, 2004

< EMI - ElectroMagnetic Instruments > 
E MULT Coh.

Canada de Cochiti, NM

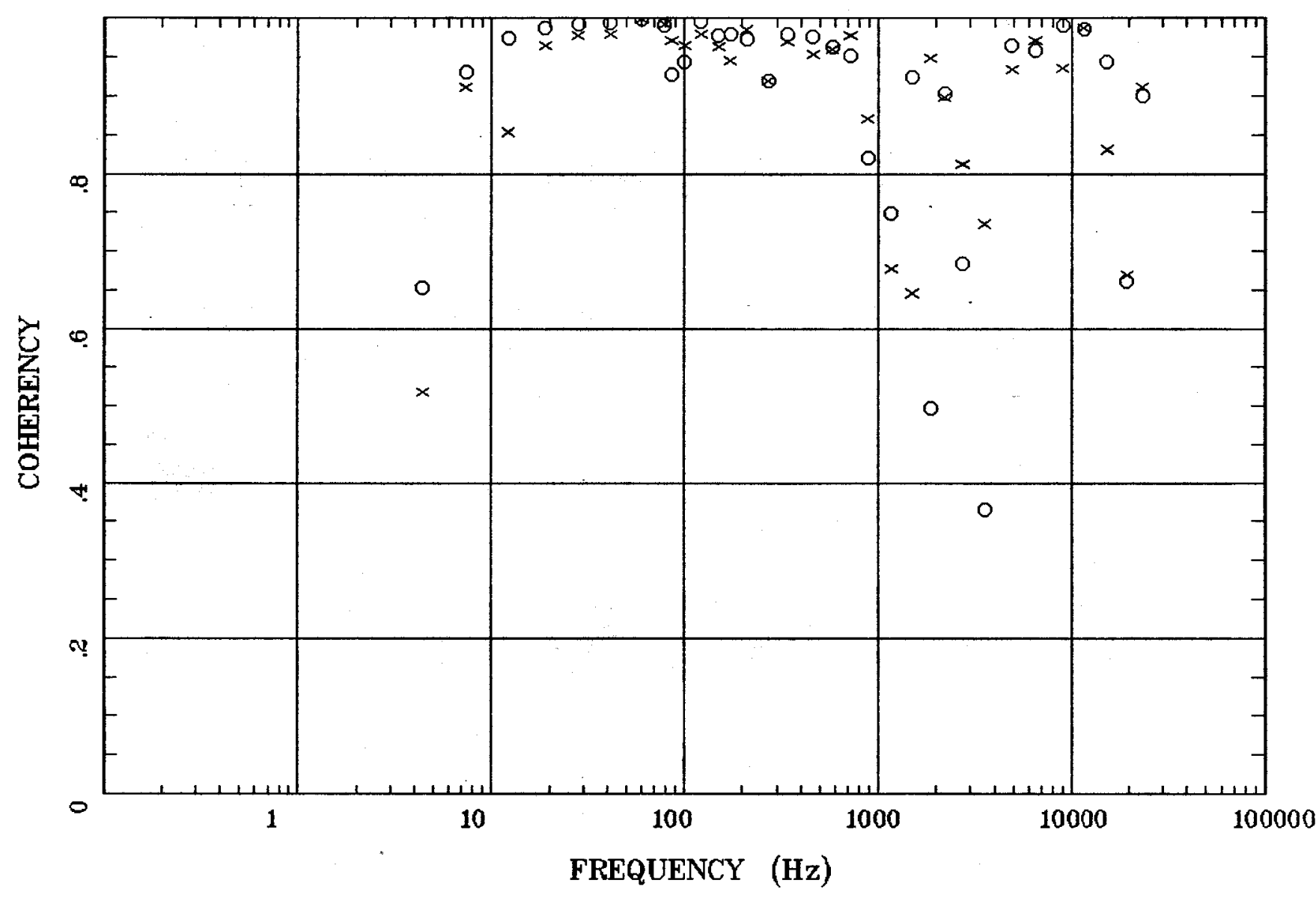

Client:

Remote: e-fld $90 \mathrm{~m}$ west

Acquired: 12:4 Aug 02, 2002

Survey Co:USGS
Rotation:

Filename: cp 17a.avg

Channels: Ch1 Ch2 Ch3 Ch4 Ch5 Ch6 Ch7

Plotted: 14:38 Jul 06, 2004

< EMI - ElectroMagnetic Instruments > 
Station 17

\section{POLAR PLOTS}

Canada de Cochiti, NM

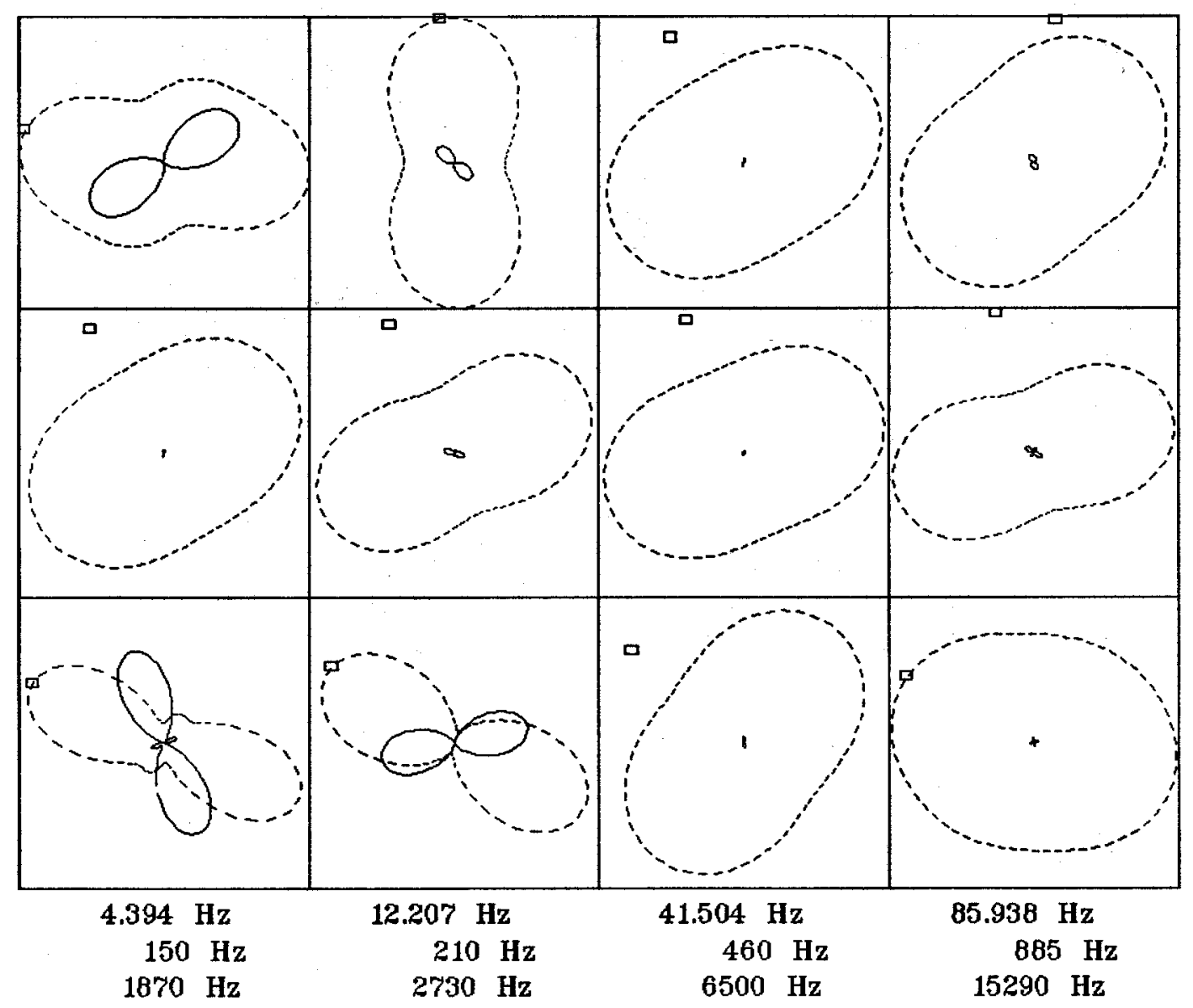

Client:

Remote: e-fld $90 \mathrm{~m}$ west

Acquired: 12:4 Aug 02, 2002 Survey Co:USGS
Rotation:

Filename: cp 17a.avg

Channels: Ch1 Ch2 Ch3 Ch4 Ch5 Ch6 Ch7 Plotted: 14:38 Jul 06, 2004

< EMI - ElectroMagnetic Instruments 
Station 17

TIPPER MAGNITUDE

Canada de Cochiti, NM

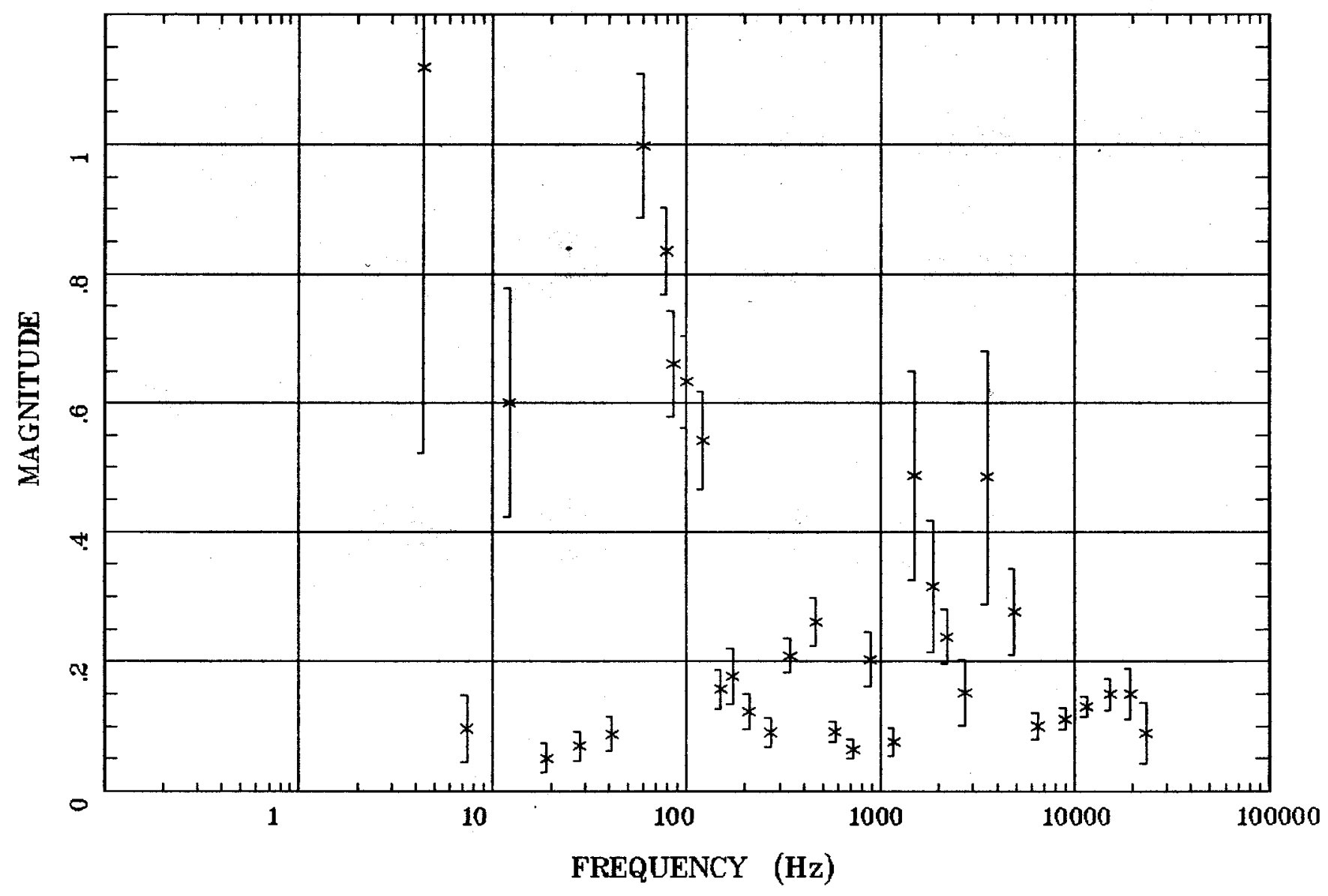

Client:

Remote: e-fld $90 \mathrm{~m}$ west

Acquired: 12:4 Aug 02, 2002

Survey Co:USGS

Rotation:

Filename: cp17a.avg

Channels: Ch1 Ch2 Ch3 Ch4 Ch5 Ch6 Ch7

Plotted: 14:38 Jul 06, 2004

< EMI - ElectroMagnetic Instruments > 


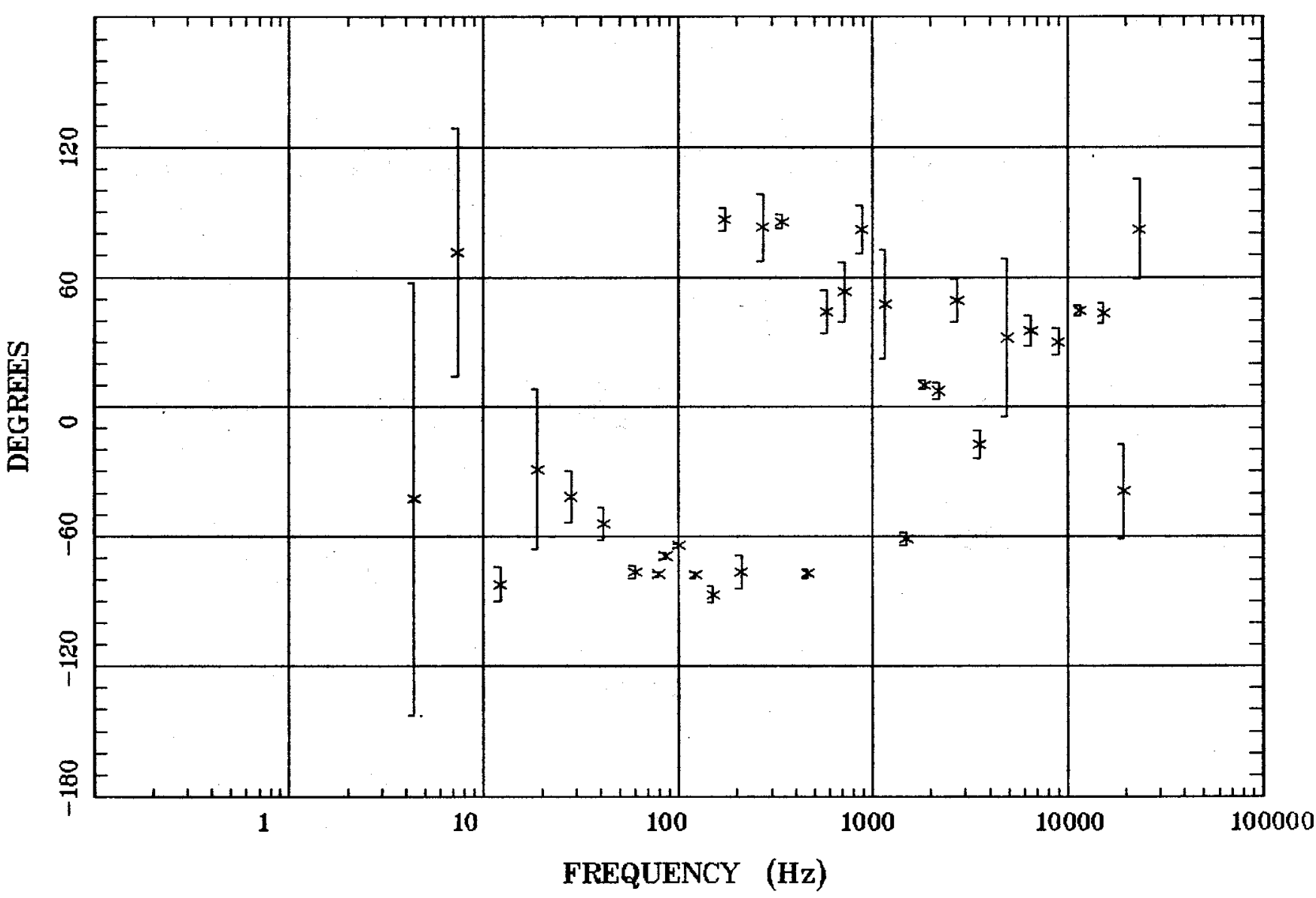

Client:

Remote: e-fld $90 \mathrm{~m}$ west

Acquired: 12:4 Aug 02, 2002 Survey Co:USGS
Rotation:

Filename: cp17a.avg

Channels: Ch1 Ch2 Ch3 Ch4 Ch5 Ch6 Ch7 Plotted: 14:38 Jul 06, 2004

< EMI - ElectroMagnetic Instruments > 


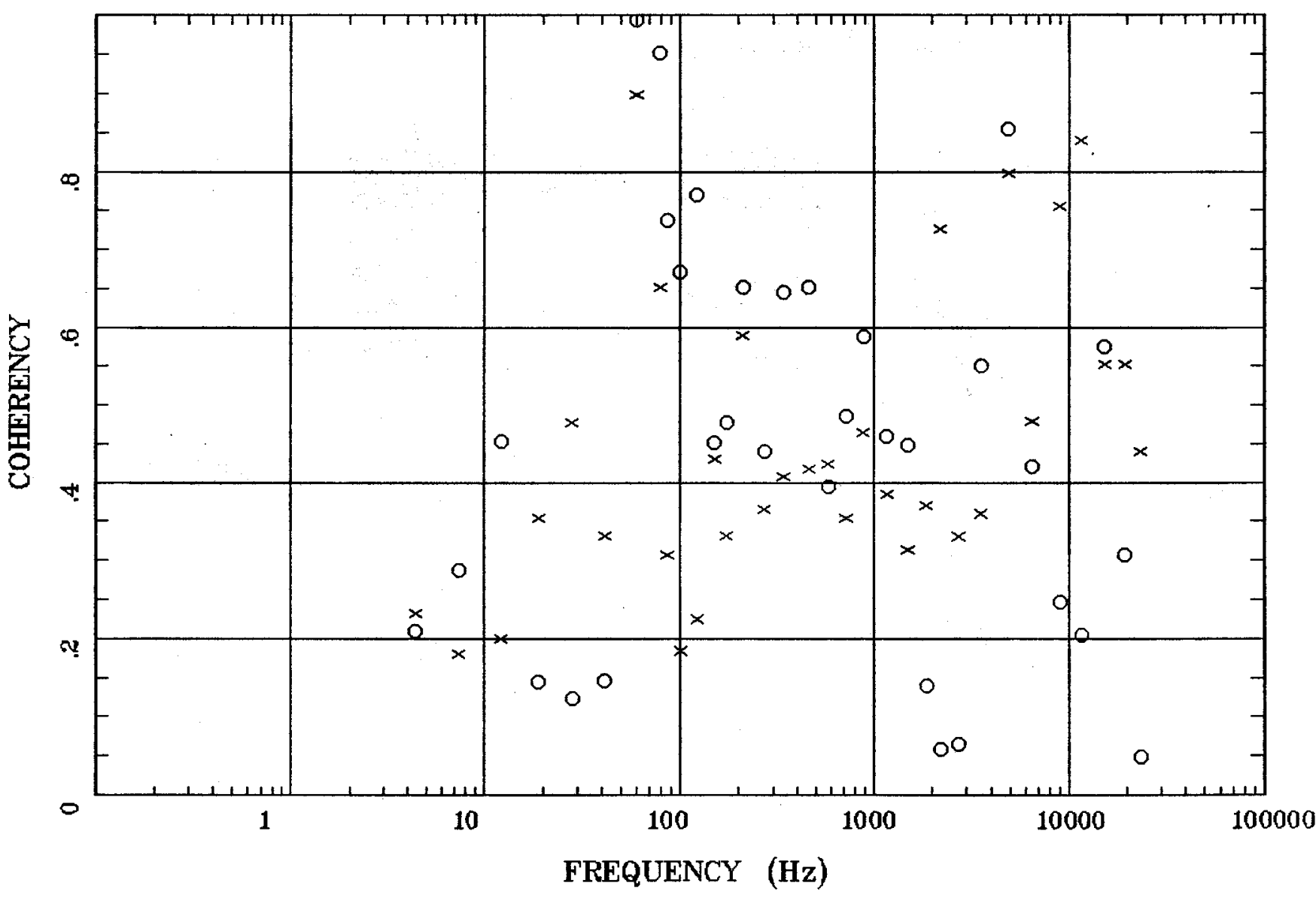

Client:

Remote: e-fld 90m west

Acquired: 12:4 Aug 02, 2002 Survey Co:USGS
Rotation:

Filename: cp17a.avg

Channels: Ch1 Ch2 Ch3 Ch4 Ch5 Ch6 Ch7 Plotted: 14:38 Jul 06, 2004

< EMI - ElectroMagnetic Instruments 


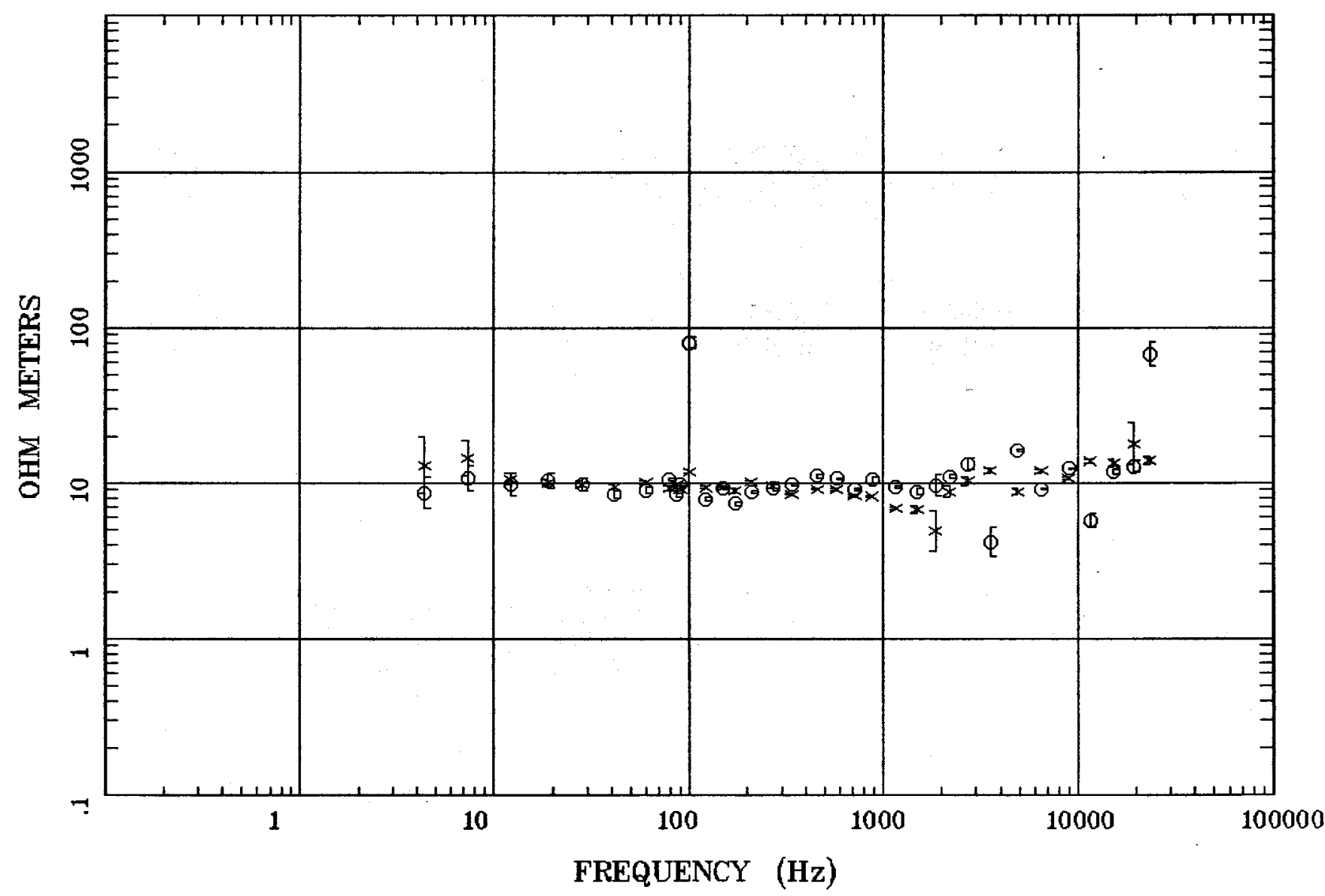

Client:

Remote: e-fld $95 \mathrm{~m}$ south

Acquired: 14:4 Jun 17, 2003 Survey Co:

Rotation:

Filename: cp 18aall.avg

Channels: Ch1 Ch2 Ch3 Ch4 Ch5 Ch6 Ch7

Plotted: 14:39 Jul 06, 2004

< EMI - ElectroMagnetic Instruments > 


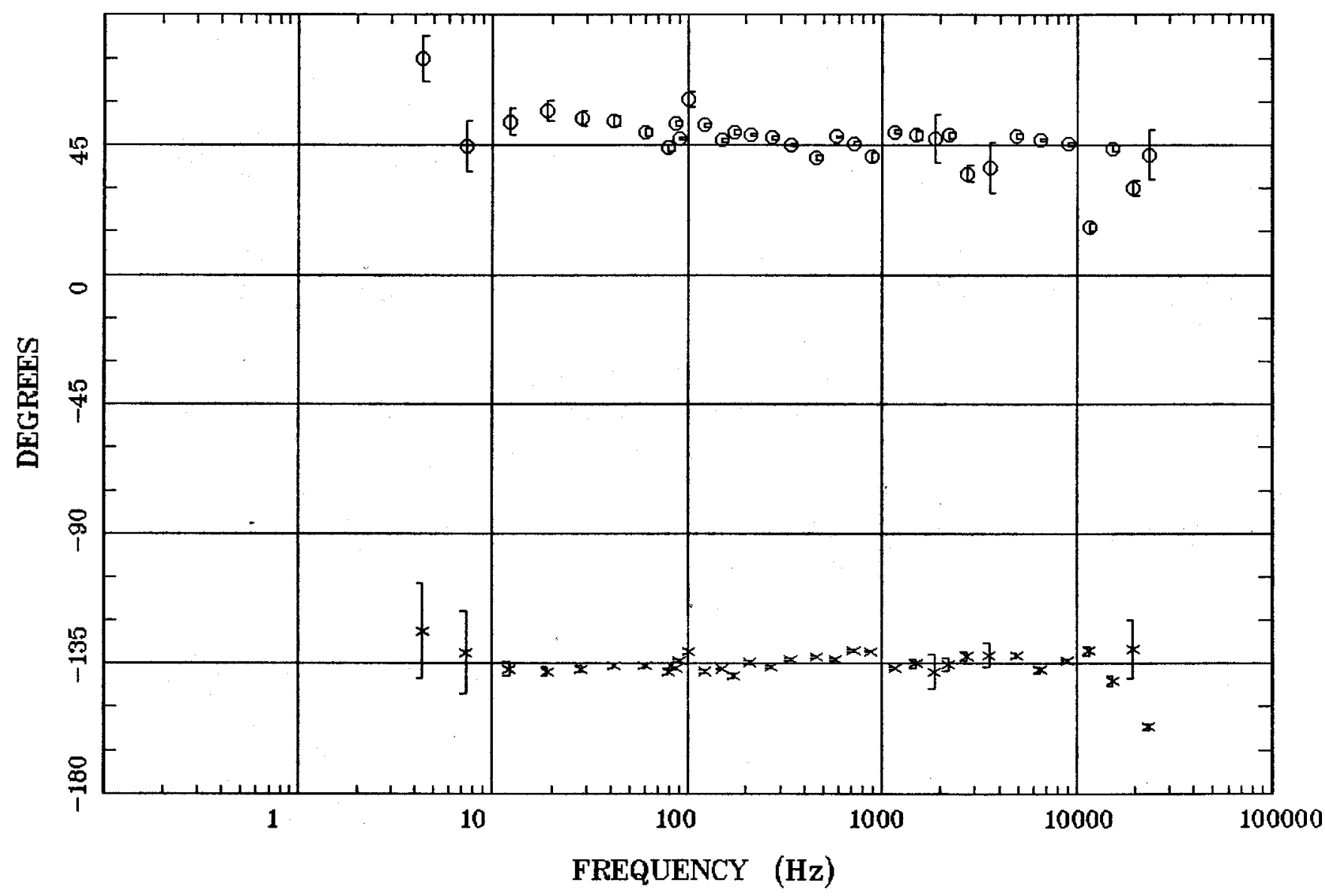

Client:

Remote: e-fld $95 \mathrm{~m}$ south Acquired: 14:4 Jun 17, 2003 Survey Co:
Rotation:

Filename: cp18aall.avg

Channels: Ch1 Ch2 Ch3 Ch4 Ch5 Ch6 Ch7 Plotted: 14:39 Jul 06, 2004

$<$ EMI - ElectroMagnetic Instruments 


\section{ROTATION ANGLE}

Santa Fe, NM

Station 18

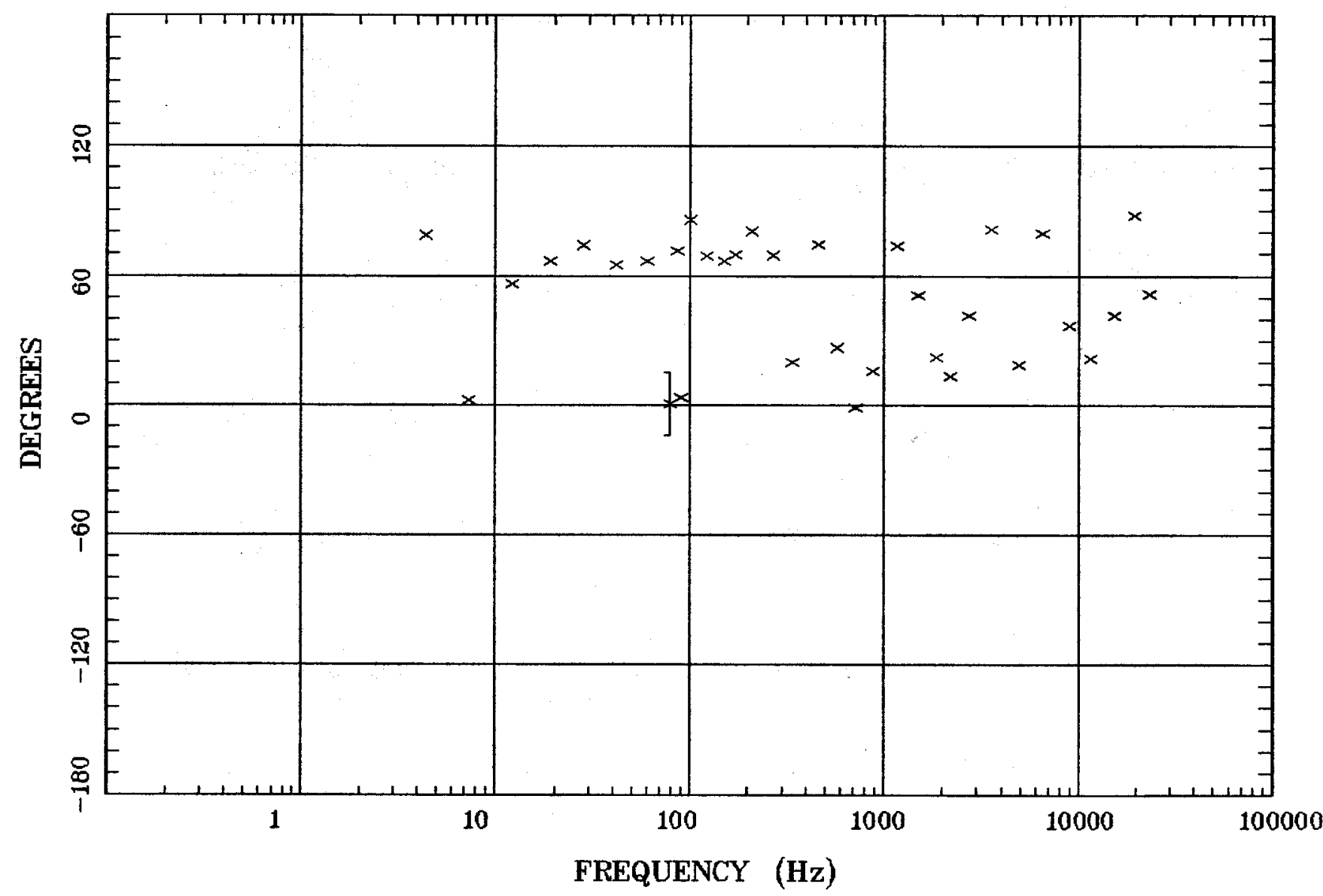

Client:

Remote: e-fld $95 \mathrm{~m}$ south Acquired: 14:4 Jun 17, 2003 Survey Co:
Rotation:

Filename: cp 18aall.avg

Channels: Ch1 Ch2 Ch3 Ch4 Ch5 Ch6 Ch7 Plotted: 12:52 Jul 07, 2004

< EMI - ElectroMagnetic Instruments 


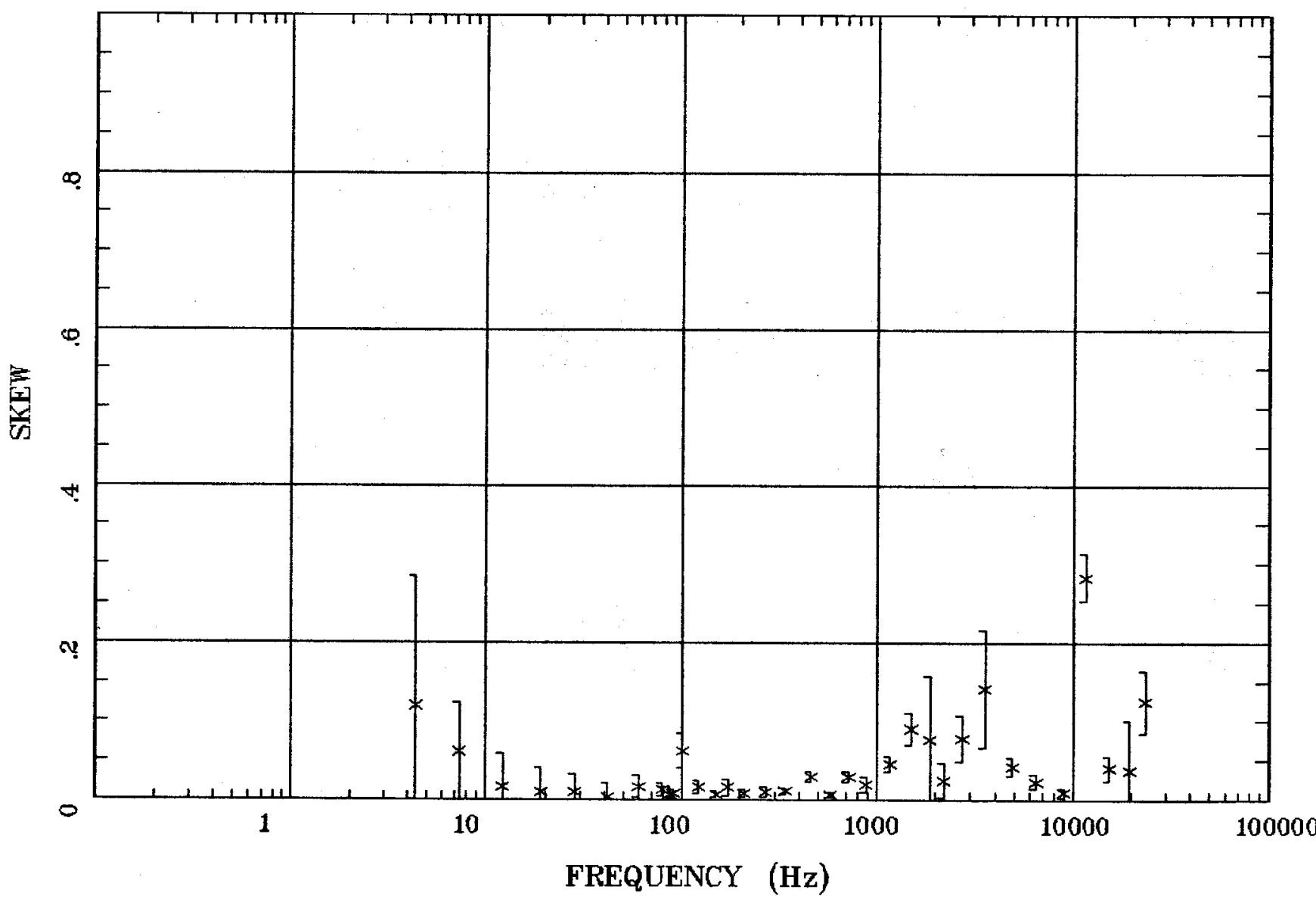

Client:

Remote: e-fld $95 \mathrm{~m}$ south Acquired: 14:4 Jun 17, 2003 Survey Co:
Rotation:

Filename: cp18aall.avg

Channels: Ch1 Ch2 Ch3 Ch4 Ch5 Ch6 Ch7 Plotted: 14:39 Jul 06, 2004

$<$ EMI - ElectroMagnetic Instruments 


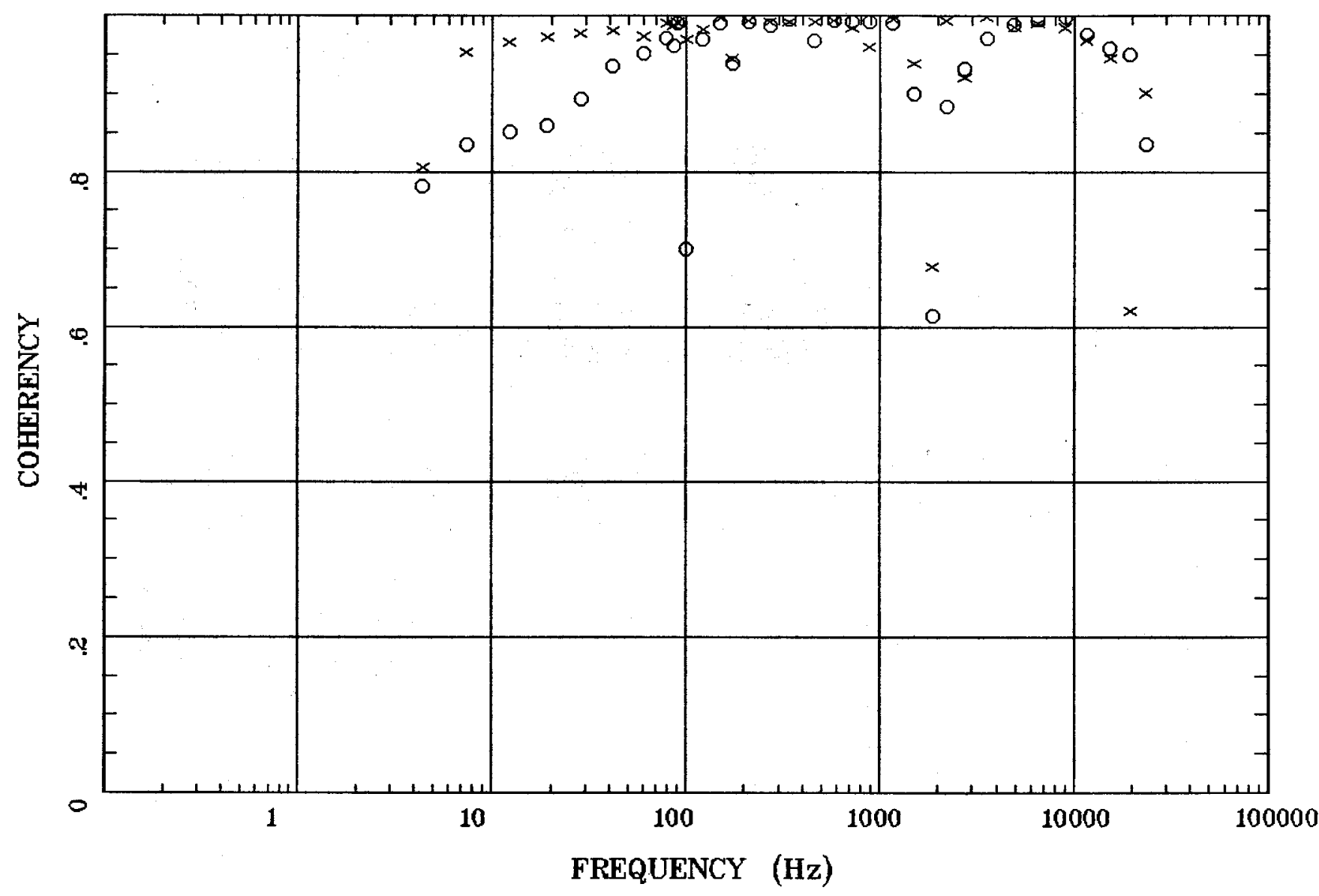

Client:

Remote: e-fld $95 \mathrm{~m}$ south Acquired: 14:4 Jun 17, 2003 Survey Co:
Rotation:

Filename: cp 18aallavg

Channels: Ch1 Ch2 Ch3 Ch4 Ch5 Ch6 Chy Plotted: 14:39 Jul 06, 2004

< EMI - ElectroMagnetic Instruments > 


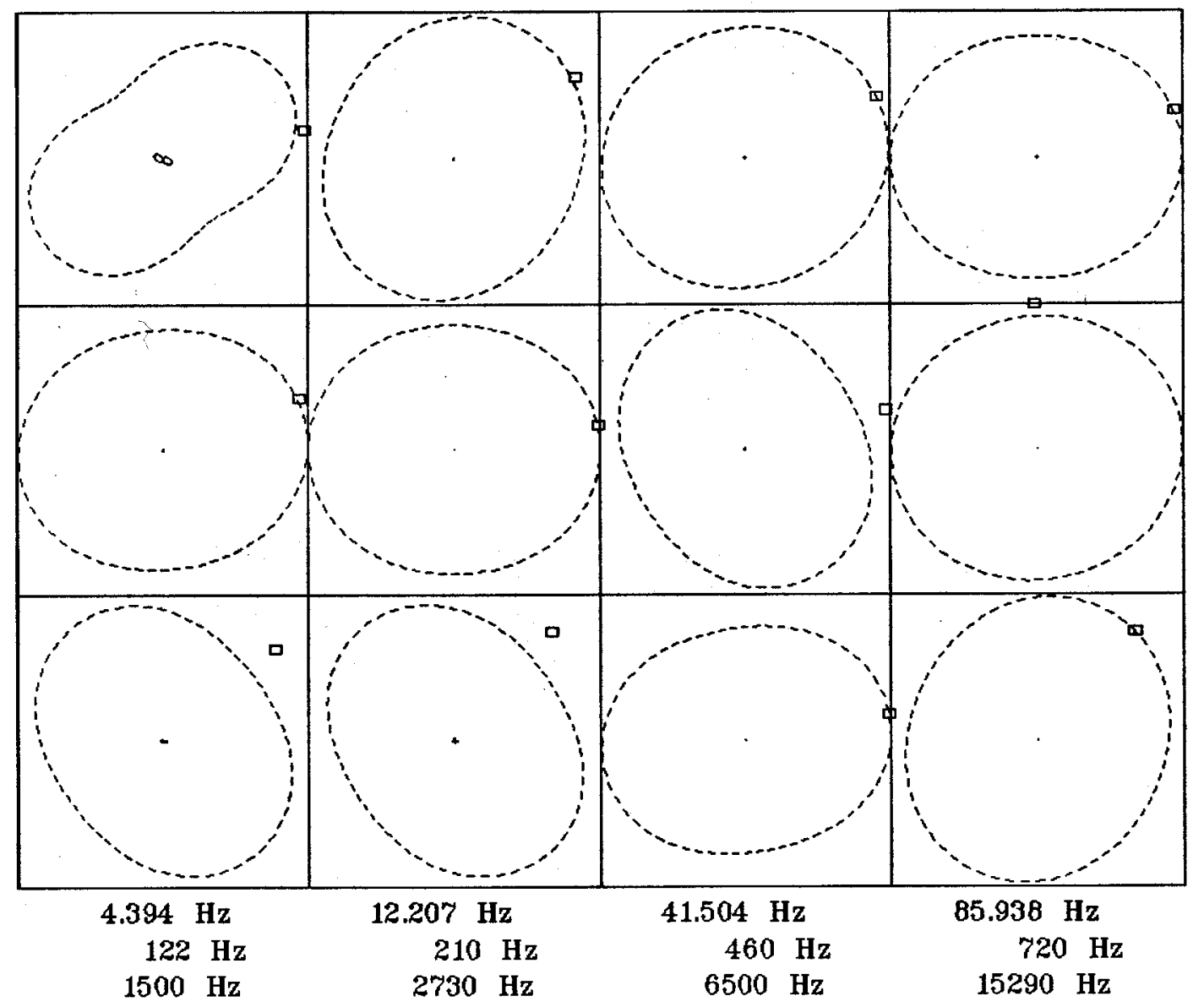

Client:

Remote: e-fld $95 \mathrm{~m}$ south Acquired: 14:4 Jun 17, 2003 Survey Co:
Rotation:

Filename: cp 18aall.avg

Channels: Ch1 Ch2 Ch3 Ch4 Ch5 Ch6 Chr Platted: 14:39 Jul 06, 2004

< EMI - ElectroMagnetic Instruments > 


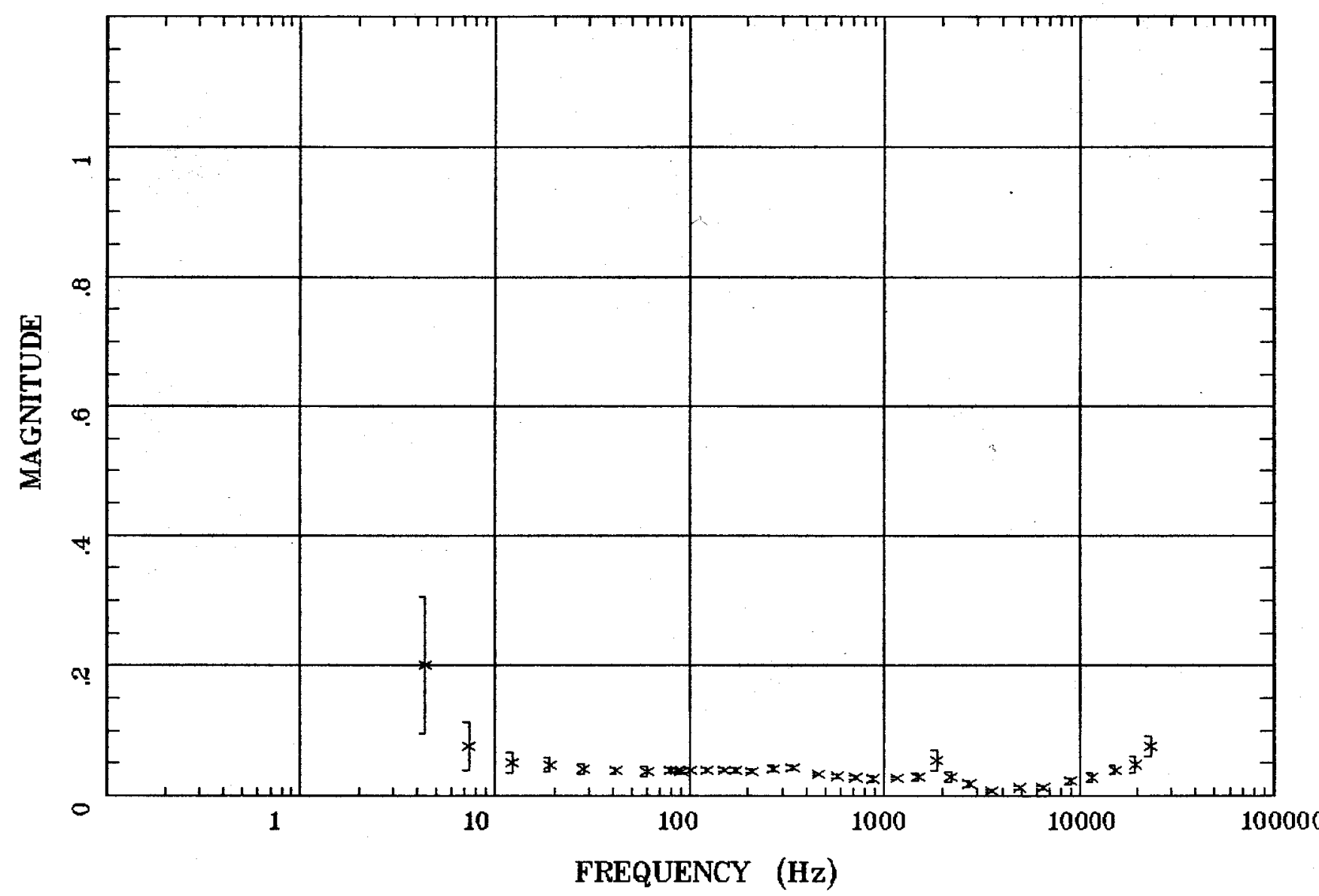

Client:

Remote: e-fld $95 \mathrm{~m}$ south Acquired: 14:4 Jun 17, 2003 Survey Co:
Rotation:

Filename: cp 1Baall.avg

Channels: Ch1 Ch2 Ch3 Ch4 Ch5 Ch6 Ch7

Plotted: 14:39 Jul 06, 2004

< EMI - ElectroMagnetic Instruments > 


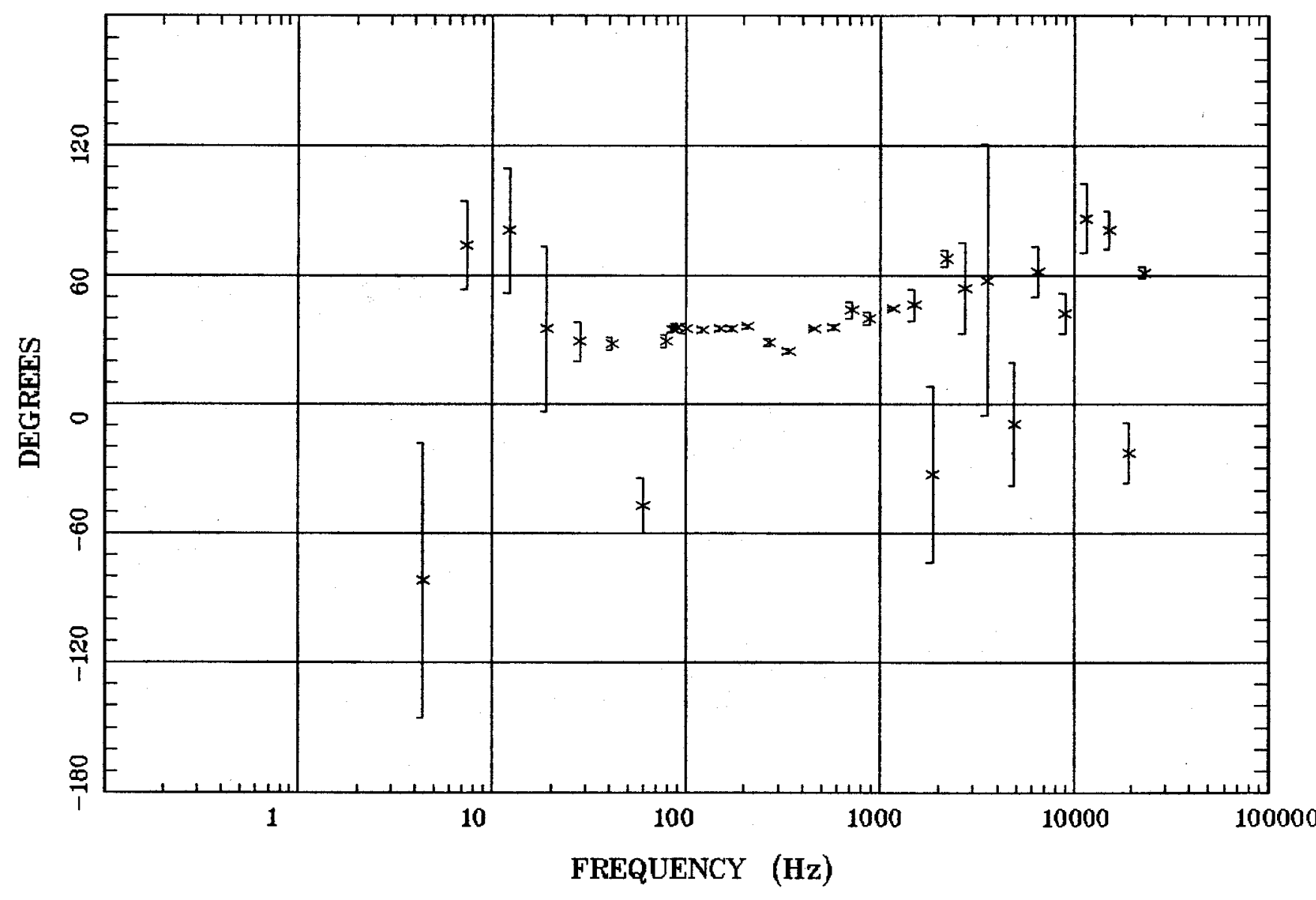

Client:

Remote: e-fld $95 \mathrm{~m}$ south Acquired: 14:4 Jun 17, 2003 Survey Co:
Rotation:

Filename: cp18aall.avg

Channels: Ch1 Ch2 Ch3 Ch4 Ch5 Ch6 Ch7

Plotted: 14:39 Jul 06, 2004

$<$ EMI - ElectroMagnetic Instruments 


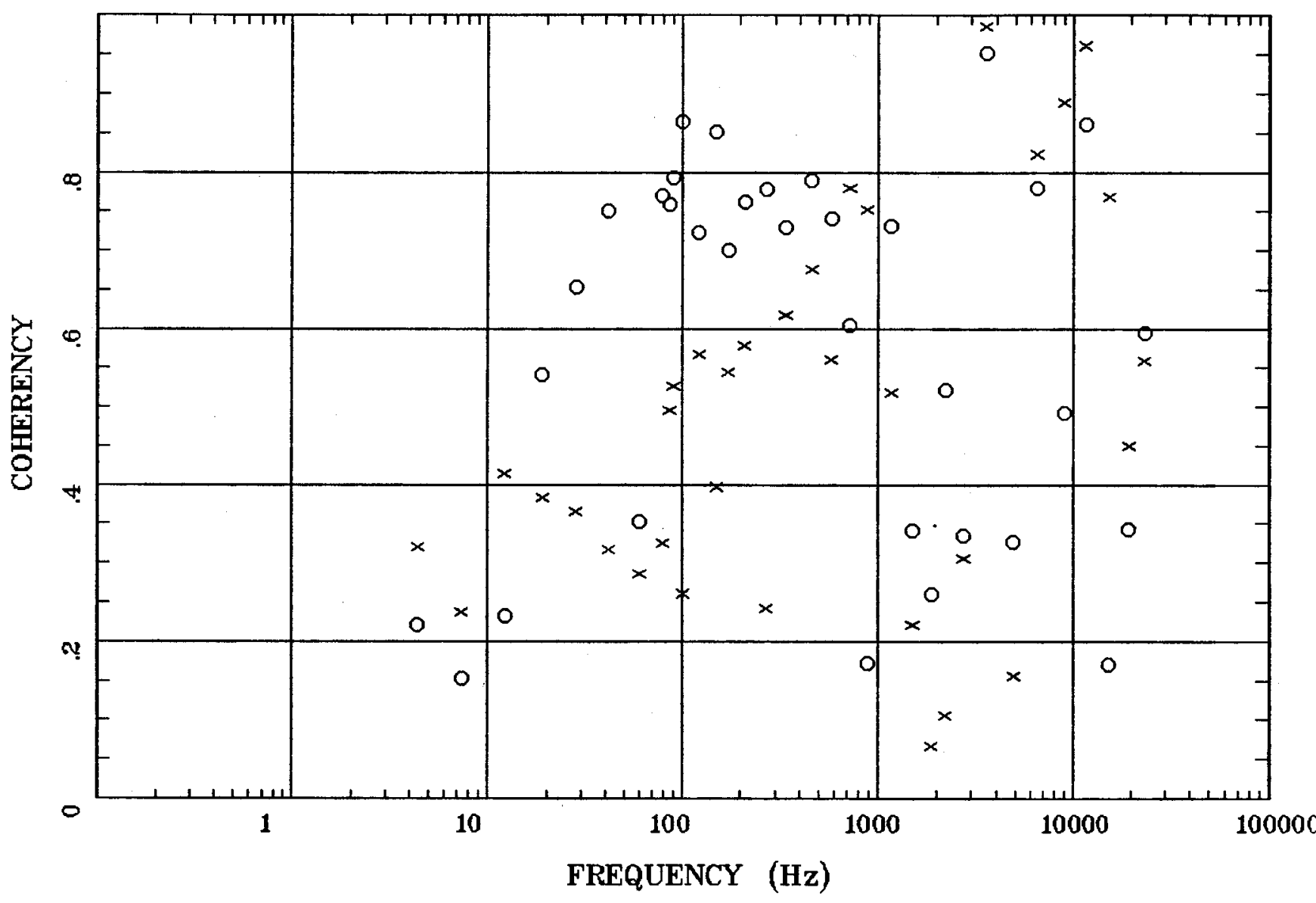

Client:

Remote: e-fld $95 \mathrm{~m}$ south Acquired: 14:4 Jun 17, 2003 Survey Co:
Rotation:

Filename: cp 18aall.avg

Channels: Ch1 Ch2 Ch3 Ch4 Ch5 Ch6 Ch7 Plotted: 14:39 Jul 06, 2004

< EMI - ElectroMagnetic Instruments > 


\section{APPARENT RESISTIVITY}

Santa Fe, NM

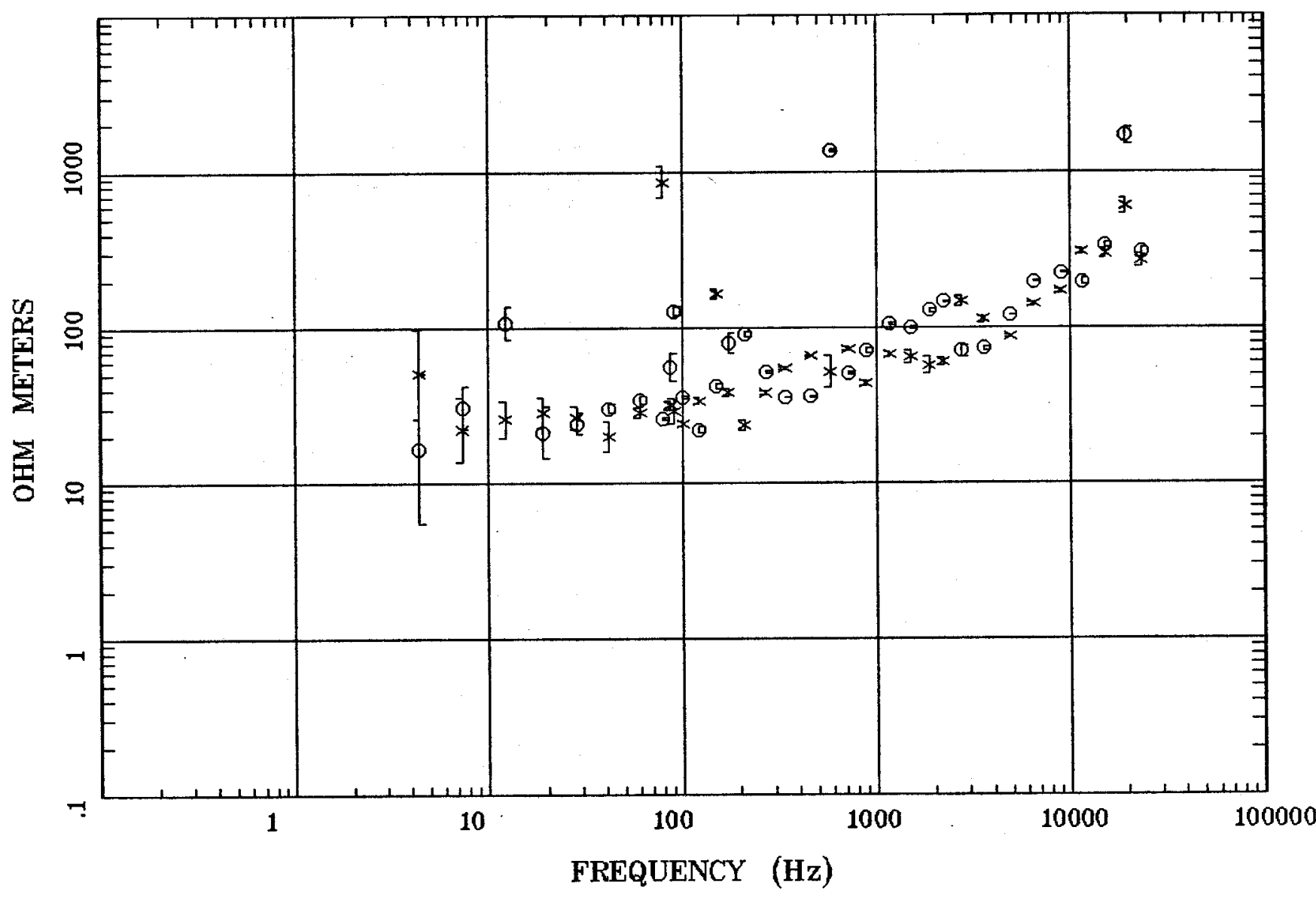

Client:

Remote: e-fld $95 \mathrm{~m}$ east Acquired: 14:2 Jun 18, 2003 Survey Co:
Rotation:

Filename: cp 19.avg

Channels: Ch1 Ch2 Ch3 Ch4 Ch5 Ch6 Ch7

Plotted: 09:48 Jul 06, 2004

< EMI - ElectroMagnetic Instruments > 


\section{IMPEDANCE PHASE}

Santa Fe, NM

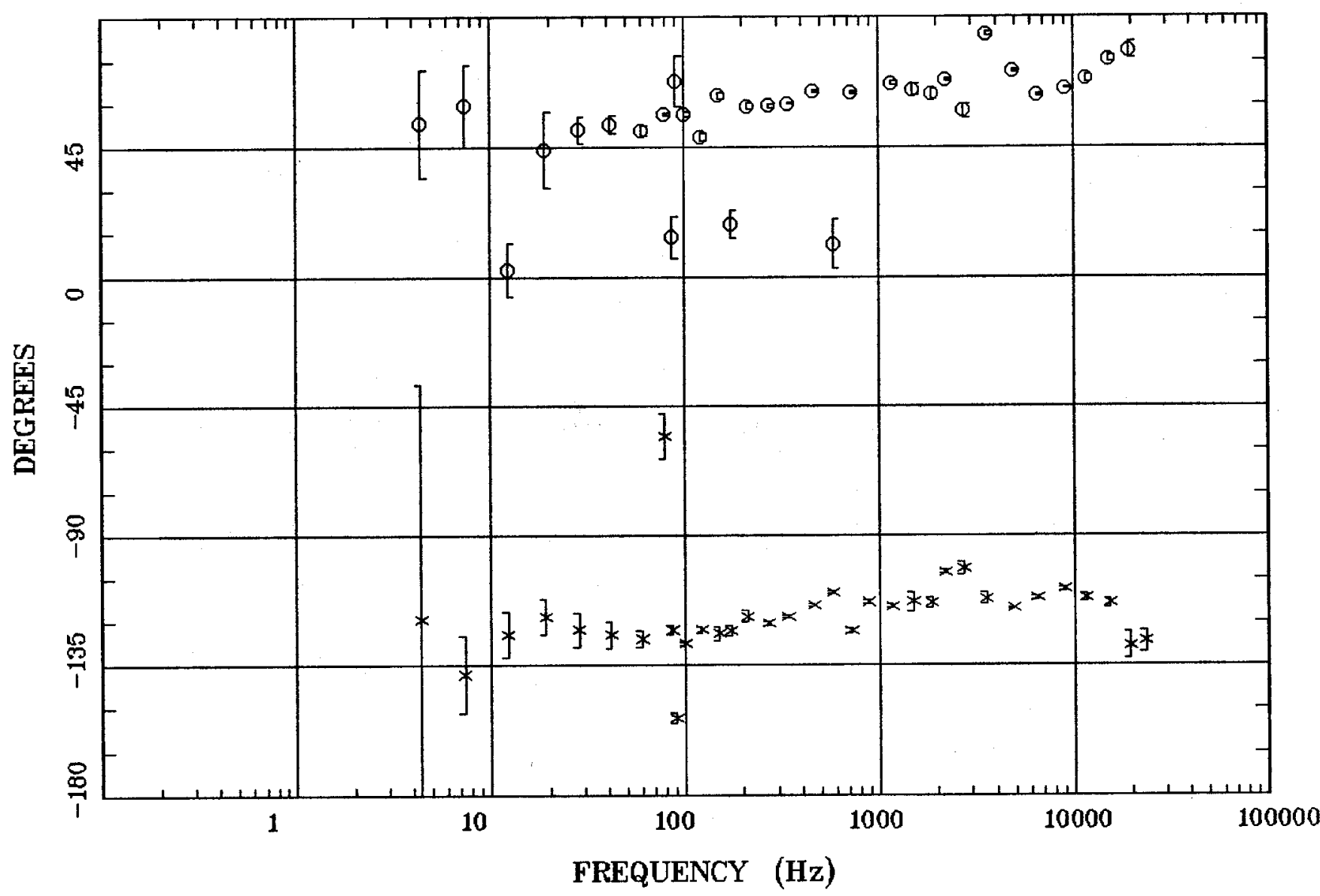

Client:

Remate: e-fld $95 \mathrm{~m}$ east Acquired: 14:2 Jun 18, 2003 Survey Co:
Rotation:

Filename: cp19.avg

Channels: Ch1 Ch2 Ch3 Ch4 Ch5 Ch6 Ch7

Plotted: 09:48 Jul 06, 2004

< EMI - ElectroMagnetic Instruments > 


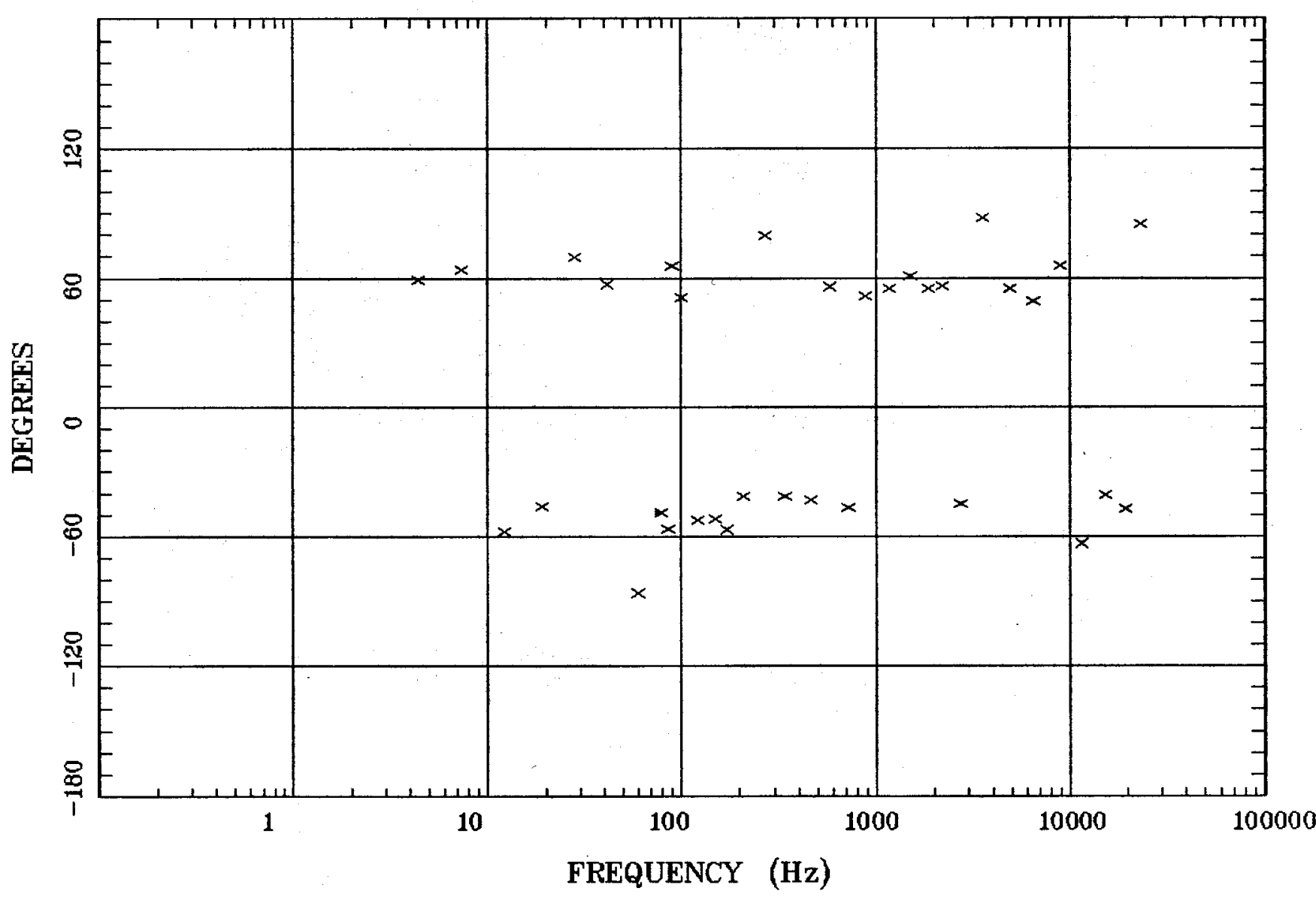

Client:

Remote: e-fld $95 \mathrm{~m}$ east Acquired: 14:2 Jun 18, 2003 Survey Co:
Rotation:

Filename: cp 19.avg

Channels: Ch1 Ch2 Ch3 ch4 Ch5 Ch6 Ch7 Plotted: 12:52 Jul 0\%, 2004

$<$ EMI - ElectroMagnetic Instruments 


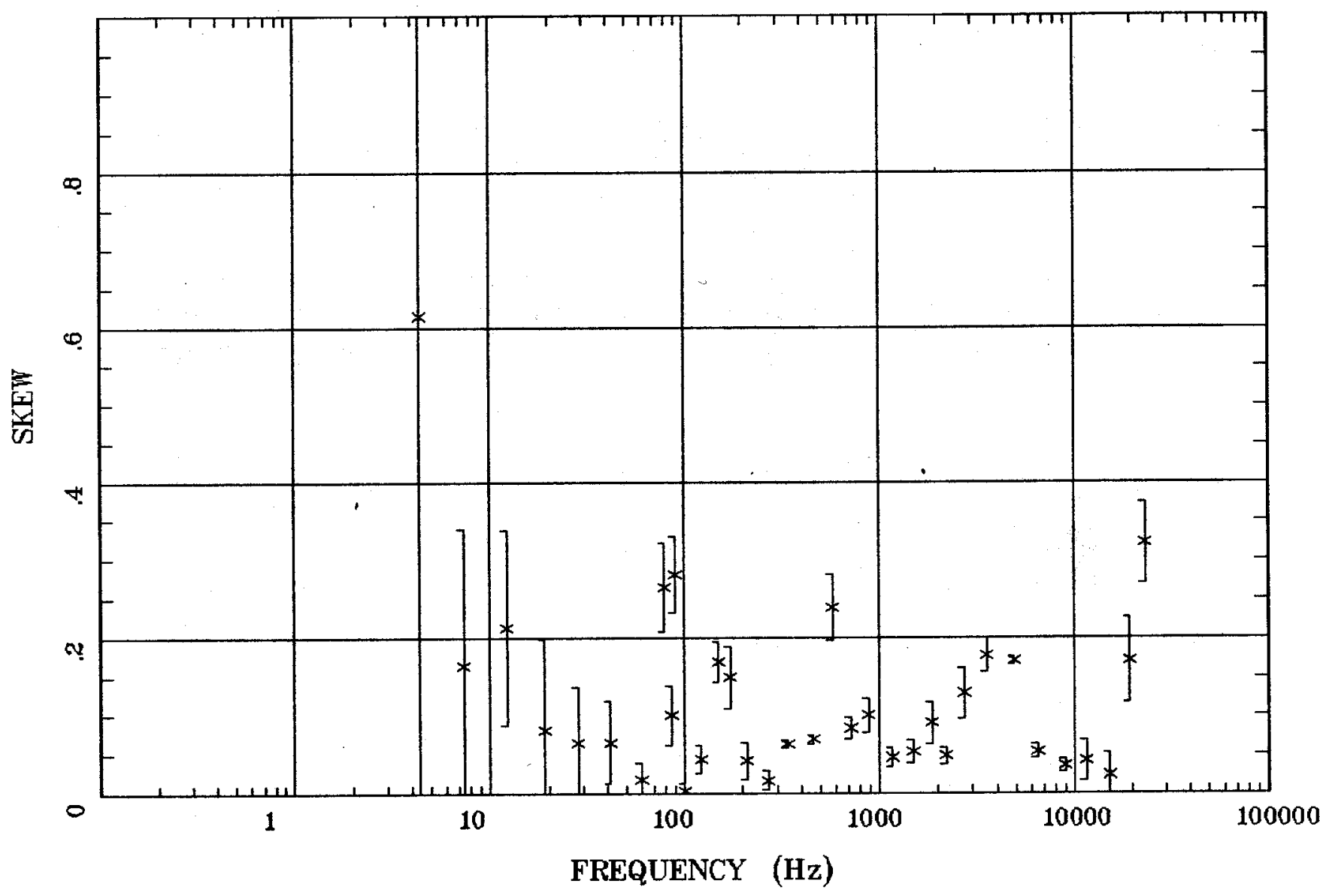

Client:

Remote: e-fld $95 \mathrm{~m}$ east

Acquired: 14:2 Jun 18, 2003 Survey Co:
Rotation:

Filename: cp19.avg

Channels: Ch1 Ch2 Ch3 Ch4 Ch5 Ch6 Ch7 Plotted: 09:48 Jul 06, 2004

< EMI - ElectroMagnetic Instruments 
Station 19

E MULT Coh. Santa Fe, NM

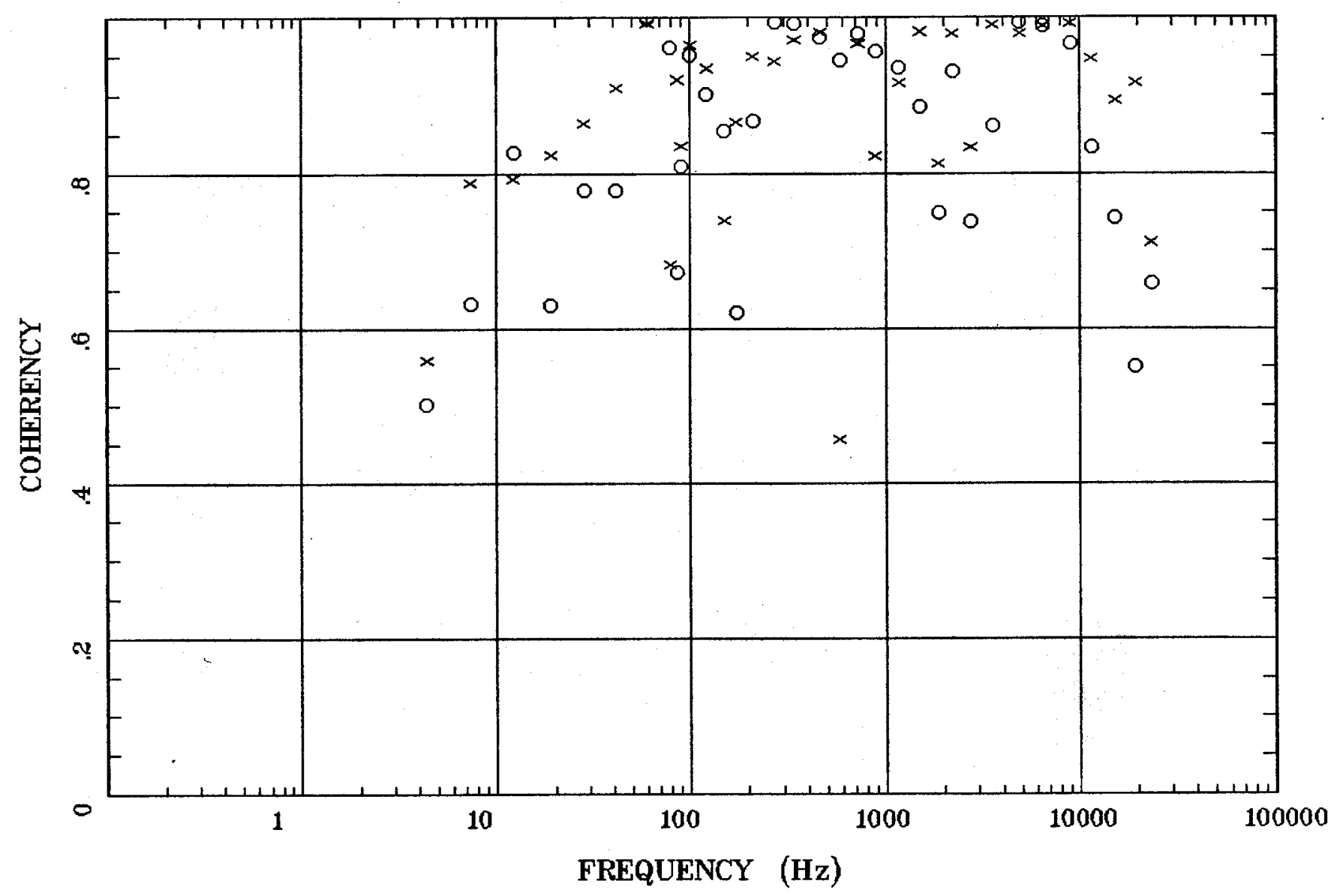

Client:

Remate: $e$-fld 95 m east Acquired: 14:2 Jun 18, 2003 Survey Co:
Rotation:

Filename: cp19.avg

Channels: Ch1 Ch2 Ch3 Ch4 Ch5 Ch6 Ch7 Plotted: 09:48 Jul 06, 2004

$<$ EMI - ElectroMagnetic Instruments > 


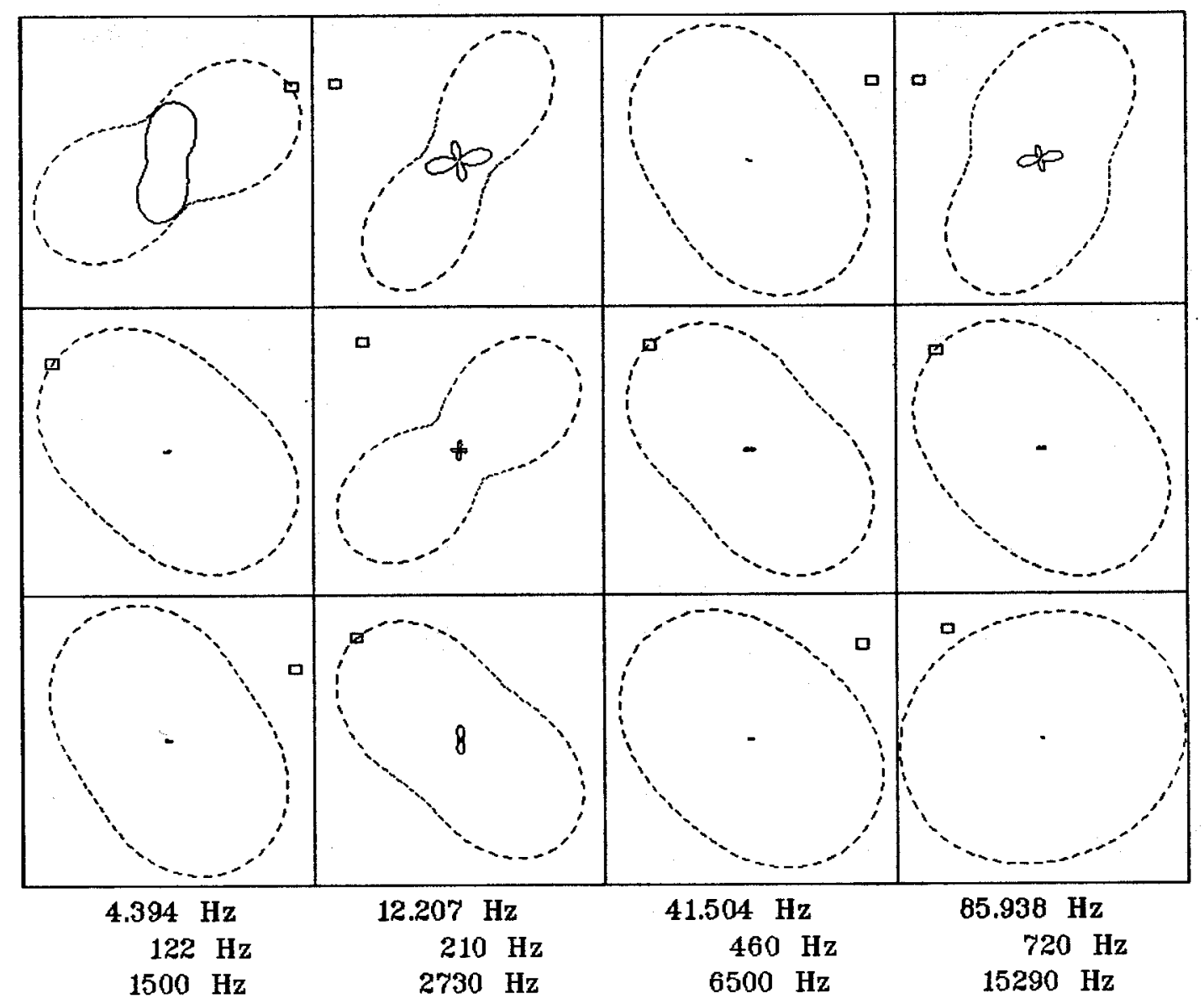

Client:

Remote: e-fld $95 \mathrm{~m}$ east Acquired: 14:2 Jun 18, 2003 Survey Co:

\section{Rotation:}

Filename: cp 19.avg Channels: Ch1 Ch2 Ch3 Ch4 Ch5 Ch6 Ch7 Plotted: 09:48 Jul 06, 2004

< EMI - ElectroMagnetic Instruments > 


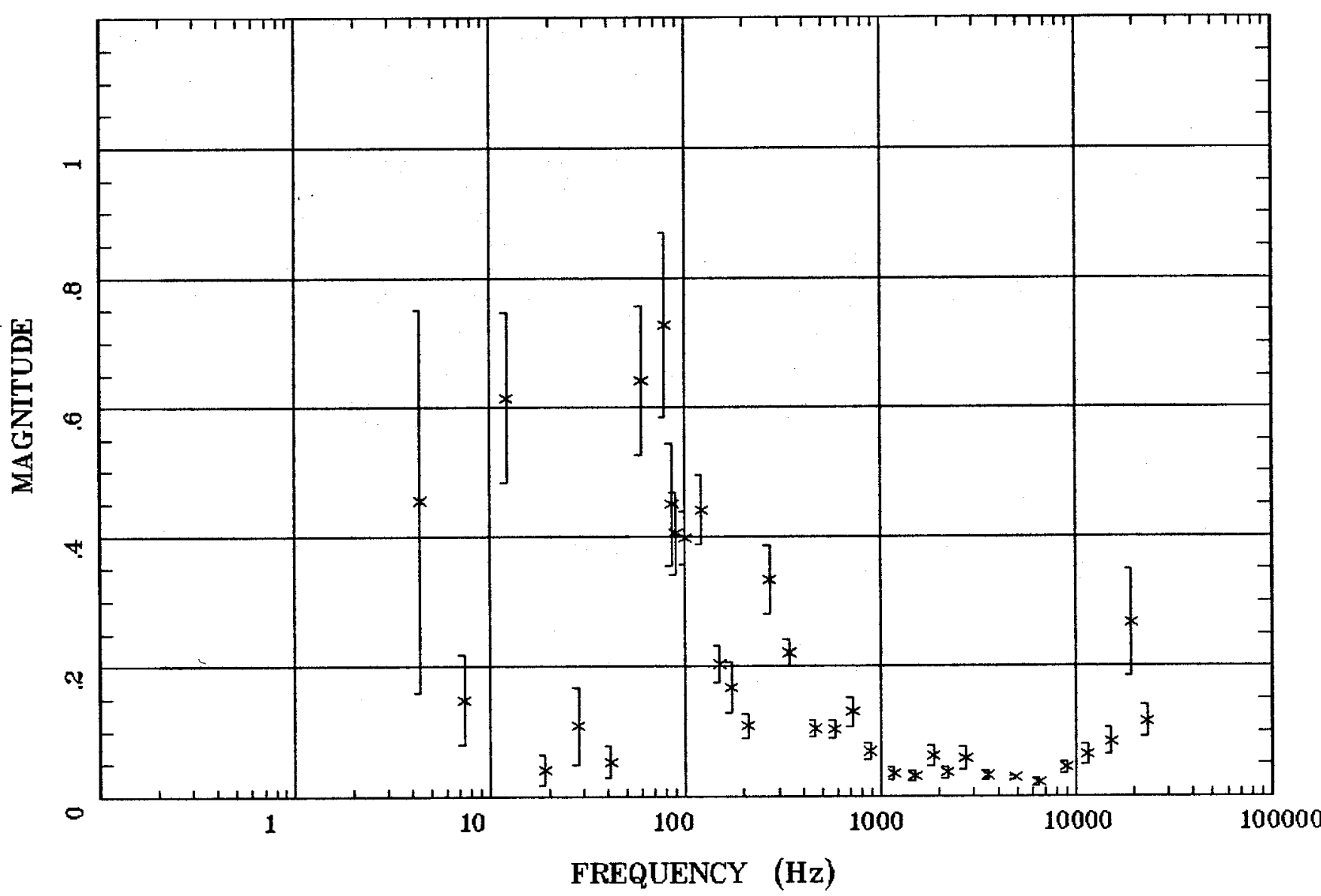

Client:

Remote: e-fld $95 \mathrm{~m}$ east

Acquired: 14:2 Jun 18, 2003 Survey Co:
Rotation:

Filename: cp19.avg

Channels: Ch1 Ch2 Ch3 Ch4 Ch5 Ch6 Ch7 Plotted: 09:48 Jul 06, 2004

$<$ EMI - ElectroMagnetic Instruments 
Santa Fe, NM

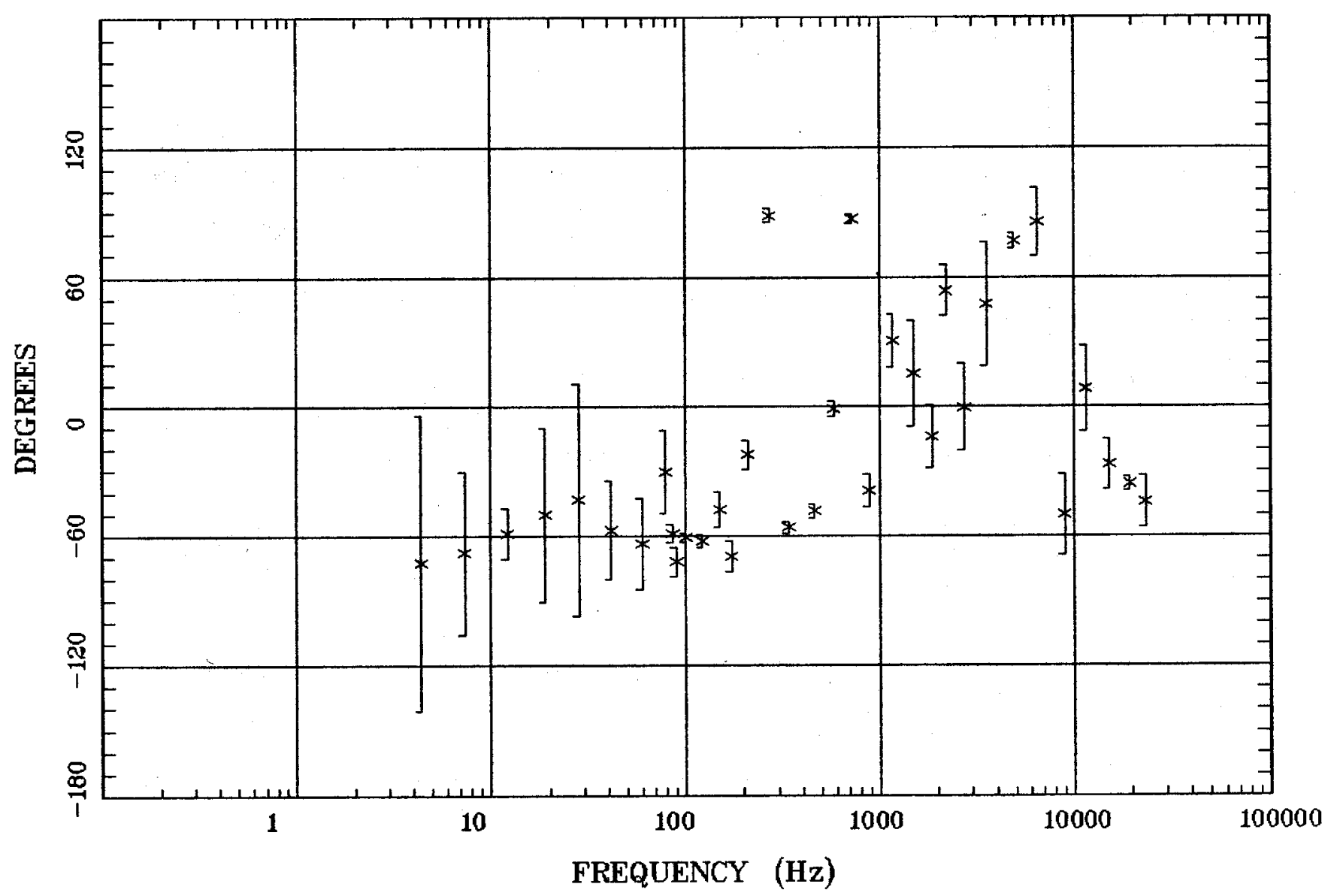

Client:

Remote: e-fld 95 m east

Acquired: 14:2 Jun 18, 2003

Survey Co:
Rotation:

Filename: cp 19.avg

Channels: Ch1 Ch2 Ch3 Ch4 Ch5 Ch6 Ch7

Plotted: 09:48 Jul 06, 2004

< EMI - ElectroMagnetic Instruments > 
HzHx.x Coh HzHy.o

Santa Fe, NM

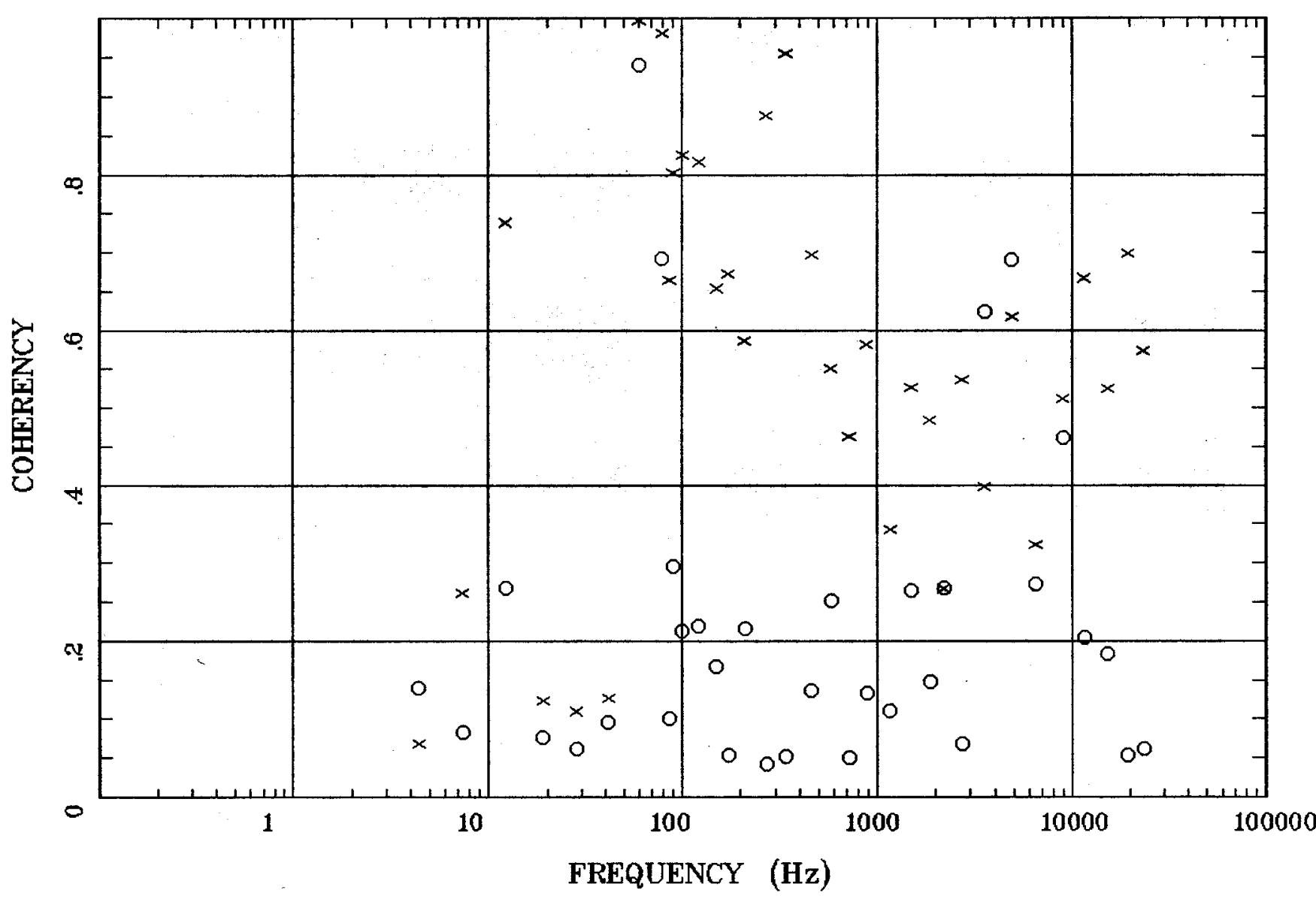

Client:

Remote: e-fld $95 \mathrm{~m}$ east Acquired: 14:2 Jun 18, 2003 Survey Co:
Rotation:

Fileneme: cp 19.avg

Channels: Ch1 Ch2 Ch3 Ch4 Ch5 Ch6 Ch7

Plotted: 09:48 Jul 06, 2004

< EMI - ElectroMagnetic Instruments 


\section{APPARENT RESISTIVITY}

Santa Fe, NM

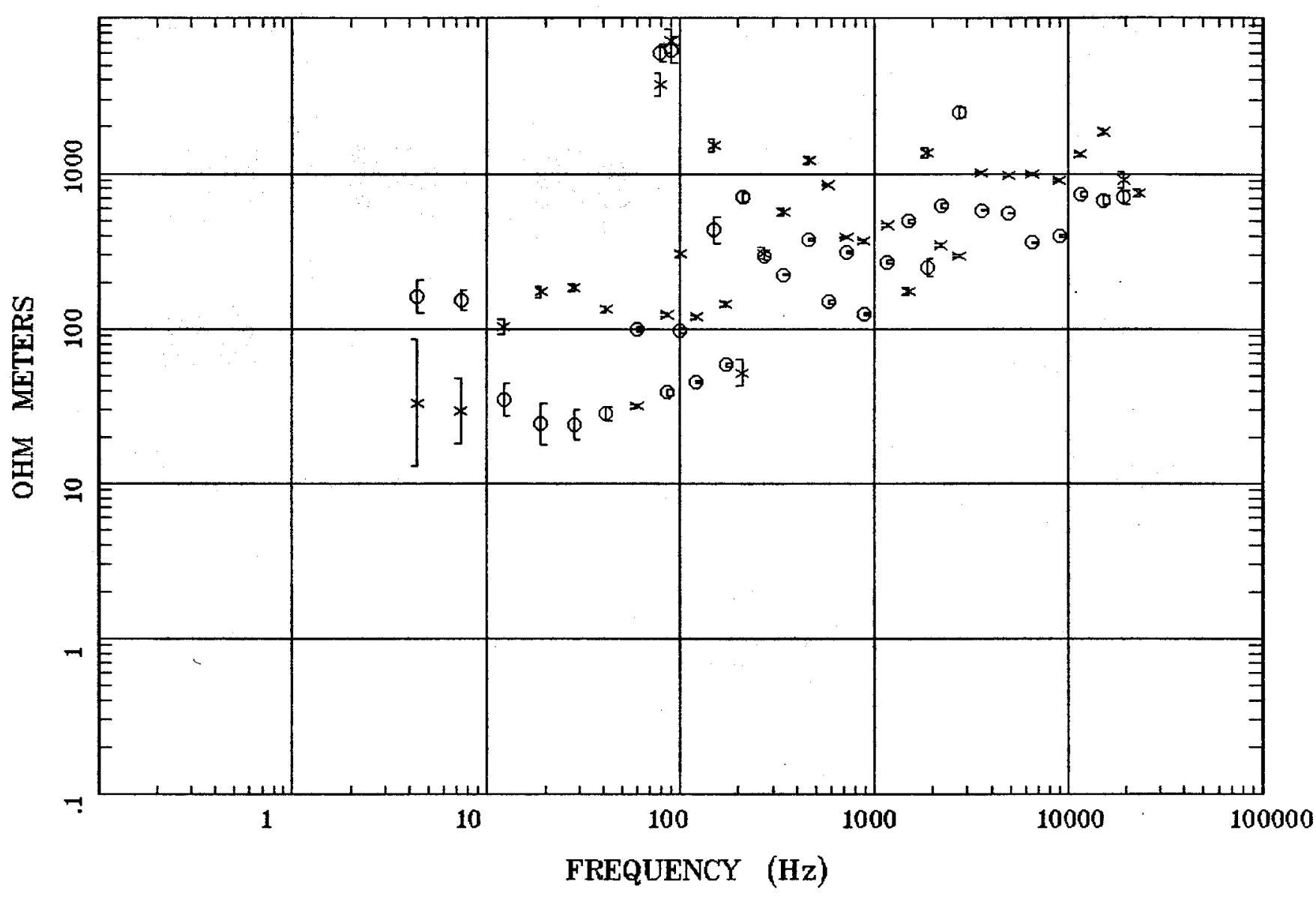

Client:

Remote: e-fld $95 \mathrm{~m}$ north Acquired: 14:0 Jun 19, 2003 Survey Co:USGS
Rotation:

Filename: cp20.avg

Channels: Ch1 Ch2 Ch3 Ch4 Ch5 Ch6 Chr Plotted: 09:50 Jul 06, 2004

$<$ EMI - ElectroMagnetic Instruments > 


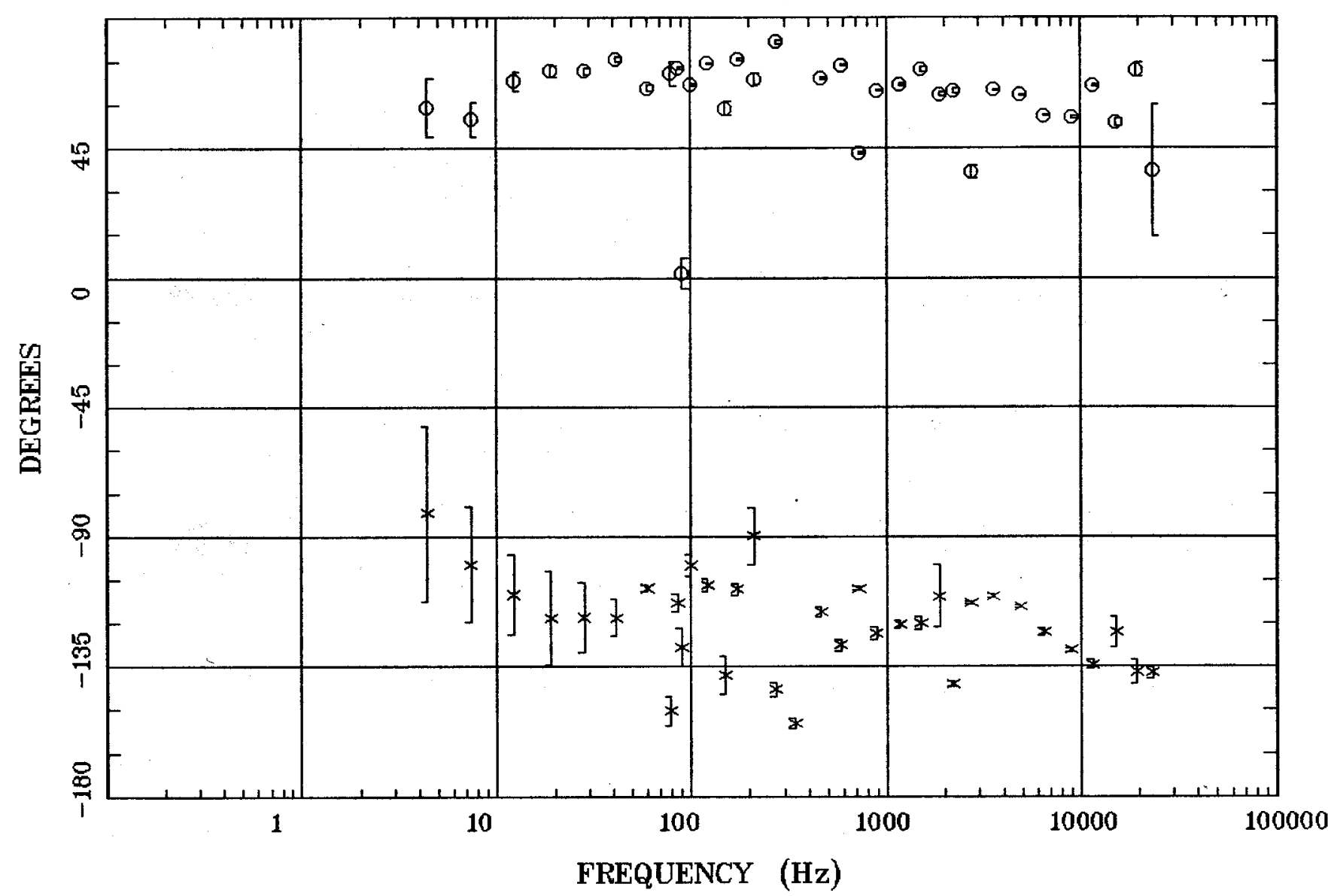

Client:

Remote: e-fld $95 \mathrm{~m}$ north Acquired: 14:0 Jun 19, 2003 Survey Co:USGS
Rotation:

Filename: cp20.evg

Channels: Ch1 ch2 ch3 ch4 Ch5 ch6 chr Plotted: 09:50 Jul 06, 2004

< EMI - ElectroMagnetic Instruments > 


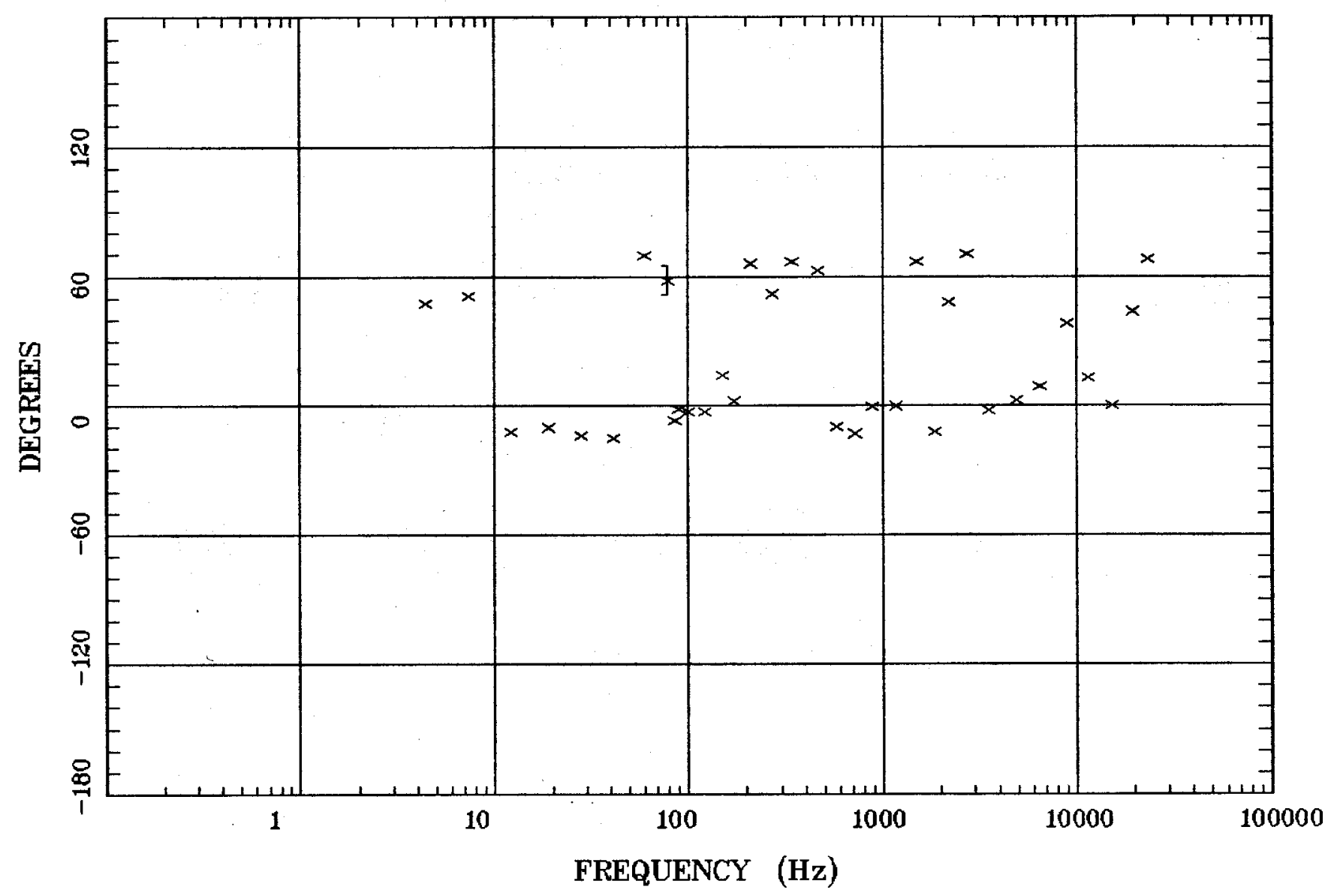

Client:

Remote: e-fld $95 \mathrm{~m}$ north

Acquired: 14:0 Jun 19, 2003 Survey Co:USGS
Rotation:

Filename: cp20.avg

Channels: Ch1 Ch2 Ch3 Ch4 Ch5 Ch6 Ch7 Plotted: 12:53 Jul 07, 2004

$<$ EMI - ElectroMagnetic Instruments > 


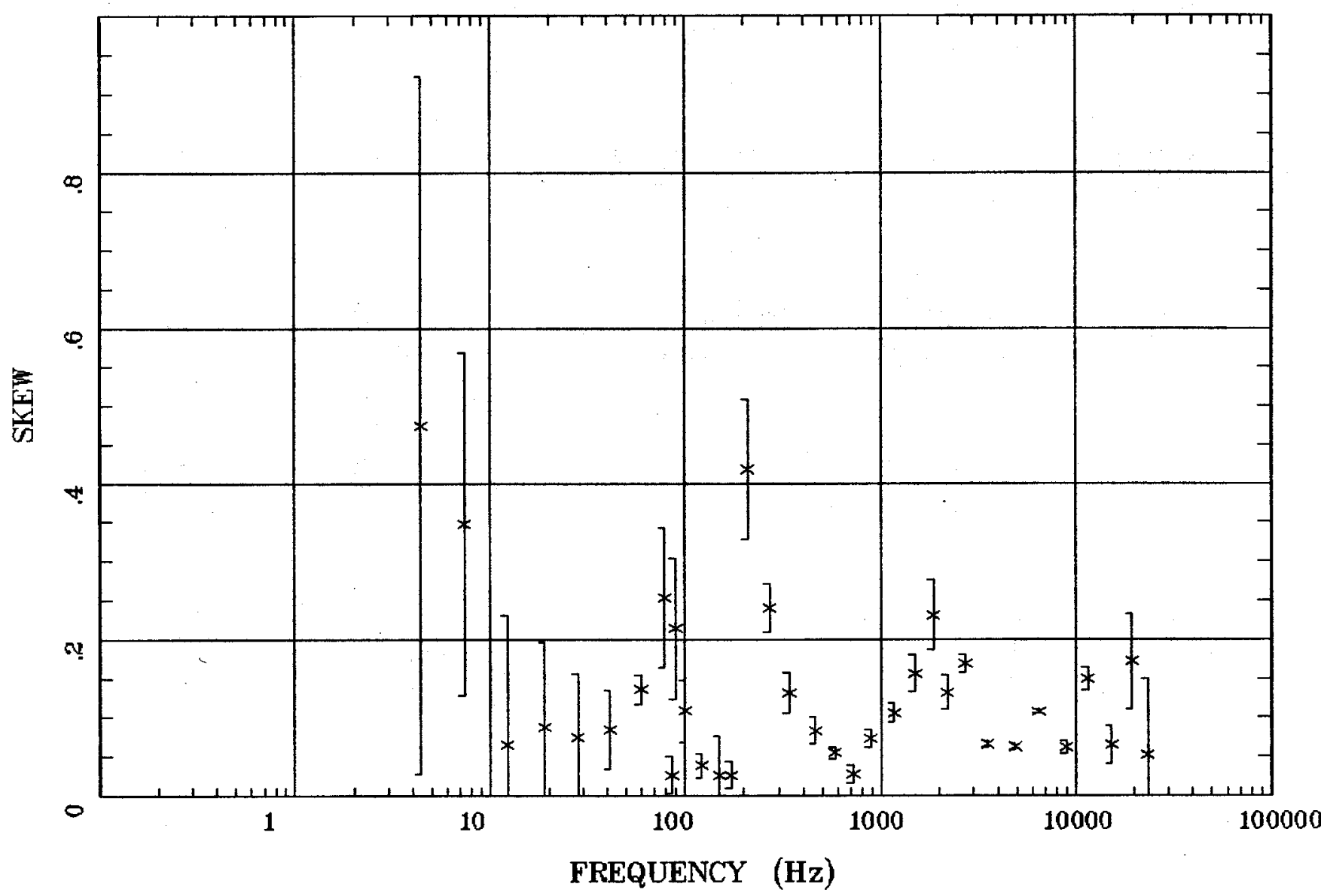

Client:

Remote: e-fld $95 \mathrm{~m}$ north Acquired: 14:0 Jun 19, 2003 Survey Co:USGS
Rotation:

Filename: cp20.avg

Channels: Ch1 Ch2 Ch3 Ch4 Ch5 ch6 Chr Plotted: 09:50 Jul 06, 2004

$<$ EMI - ElectroMagnetic Instruments > 


\section{E MULT Coh.}

Santa Fe, NM

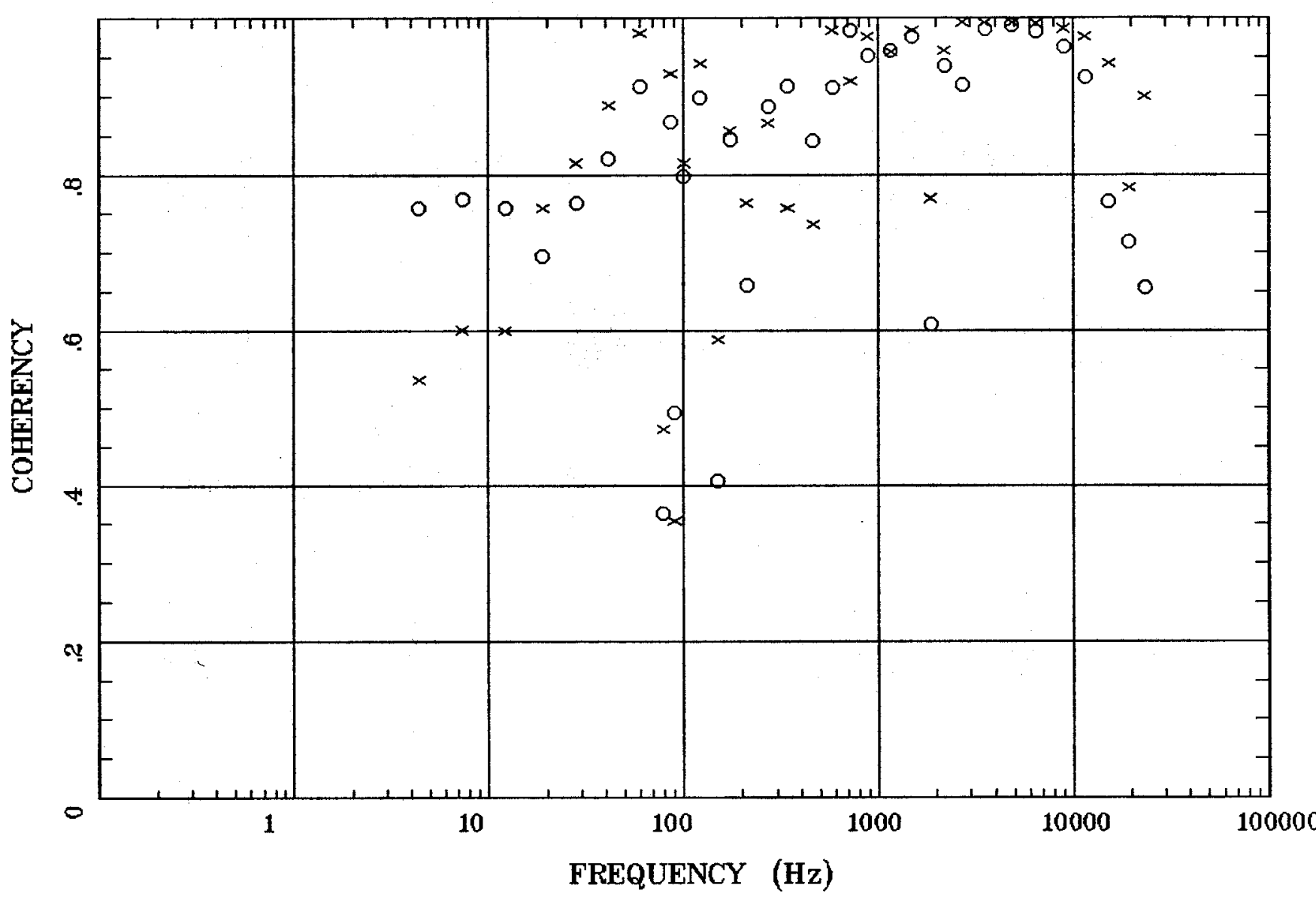

Client:

Remote: e-fld $95 \mathrm{~m}$ north Acquired: 14:0 Jun 19, 2003 Survey Co:USGS
Rotation:

Filename: cp20.avg

Channels: Ch1 Ch2 Ch3 Ch4 Ch5 Ch6 Ch7

Plotted: 09:50 Jul 06, 2004

$<$ EMI - ElectroMagnetic Instruments > 


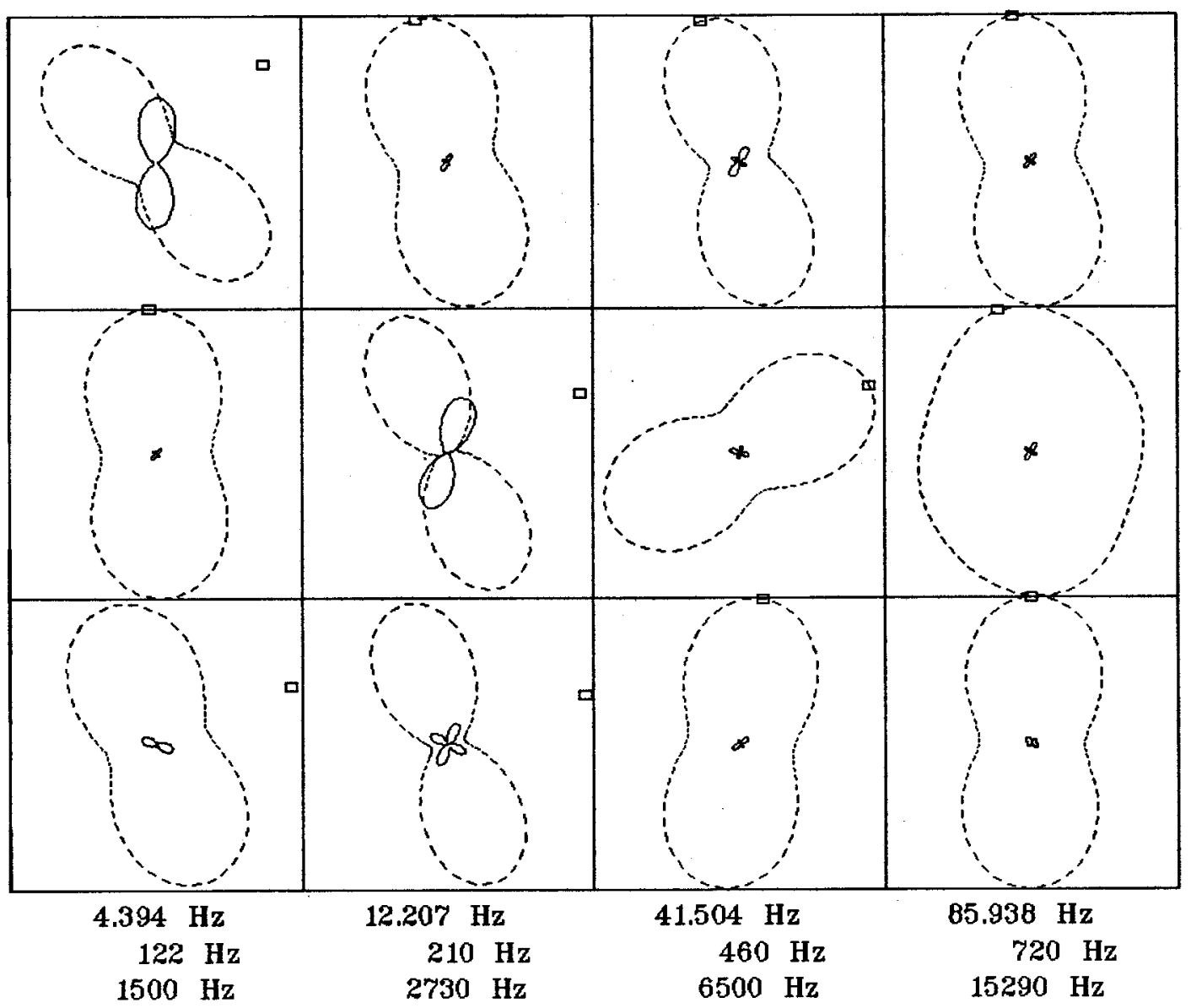

Rotation:

Client:

Remote: e-fld $95 \mathrm{~m}$ north Acquired: 14:0 Jun 19, 2003 Survey Co:USGS
Filename: cp20.avg

Channels: Ch1 Ch2 Ch3 Ch4 Ch5 Ch6 Ch7 Plotted: 09:50 Jul 06, 2004

\& EMI - ElectroMagnetic Instruments 


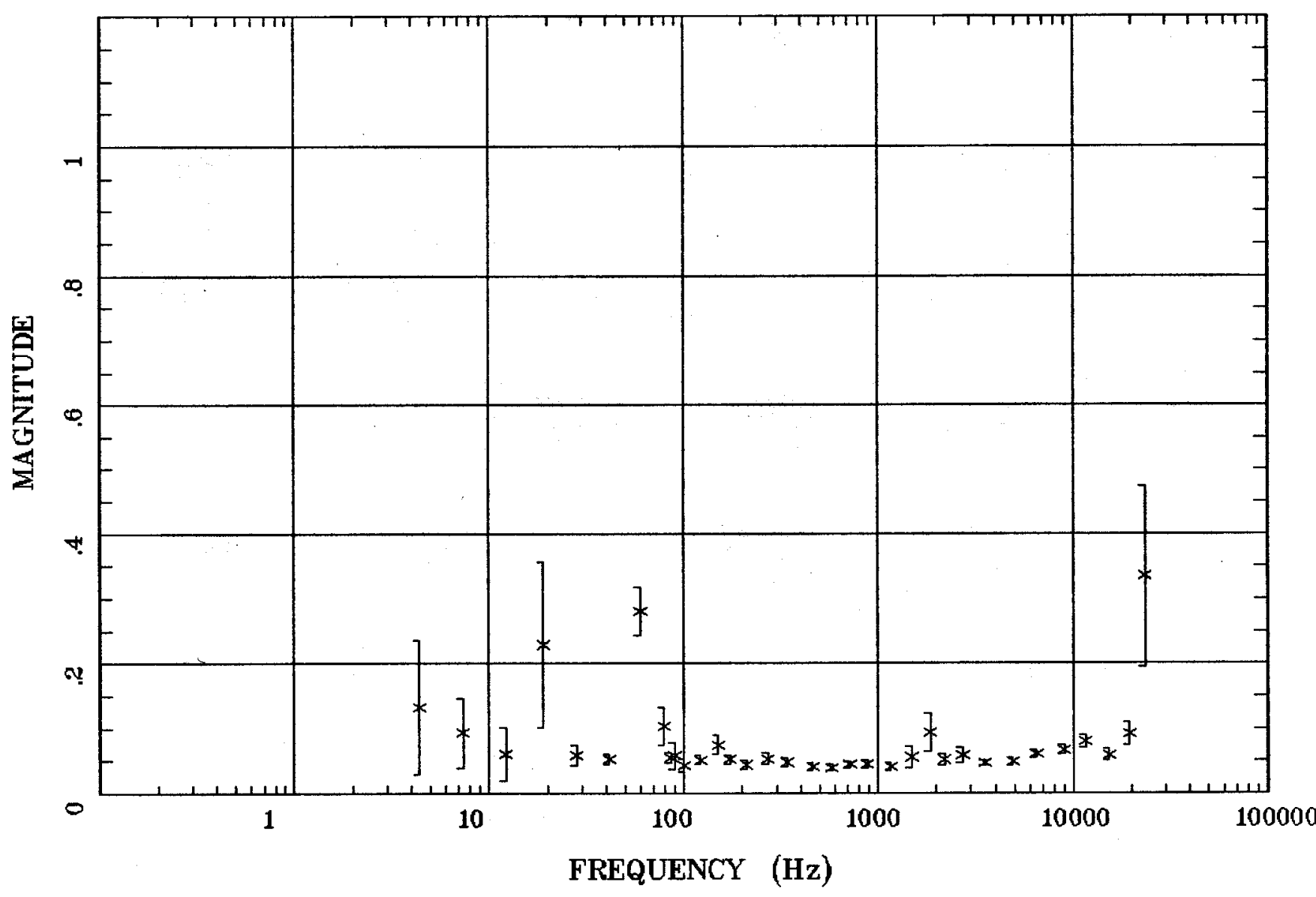

Client:

Remote: e-fld $95 \mathrm{~m}$ north Acquired: 14:0 Jun 19, 2003 Survey Co:USGS
Rotation:

Filename: cp20.avg Channels: Ch1 Ch2 Ch3 Ch4 Ch5 Ch6 Ch7 Plotted: 09:50 Jul 06, 2004

< EMI - ElectroMagnetic Instruments > 


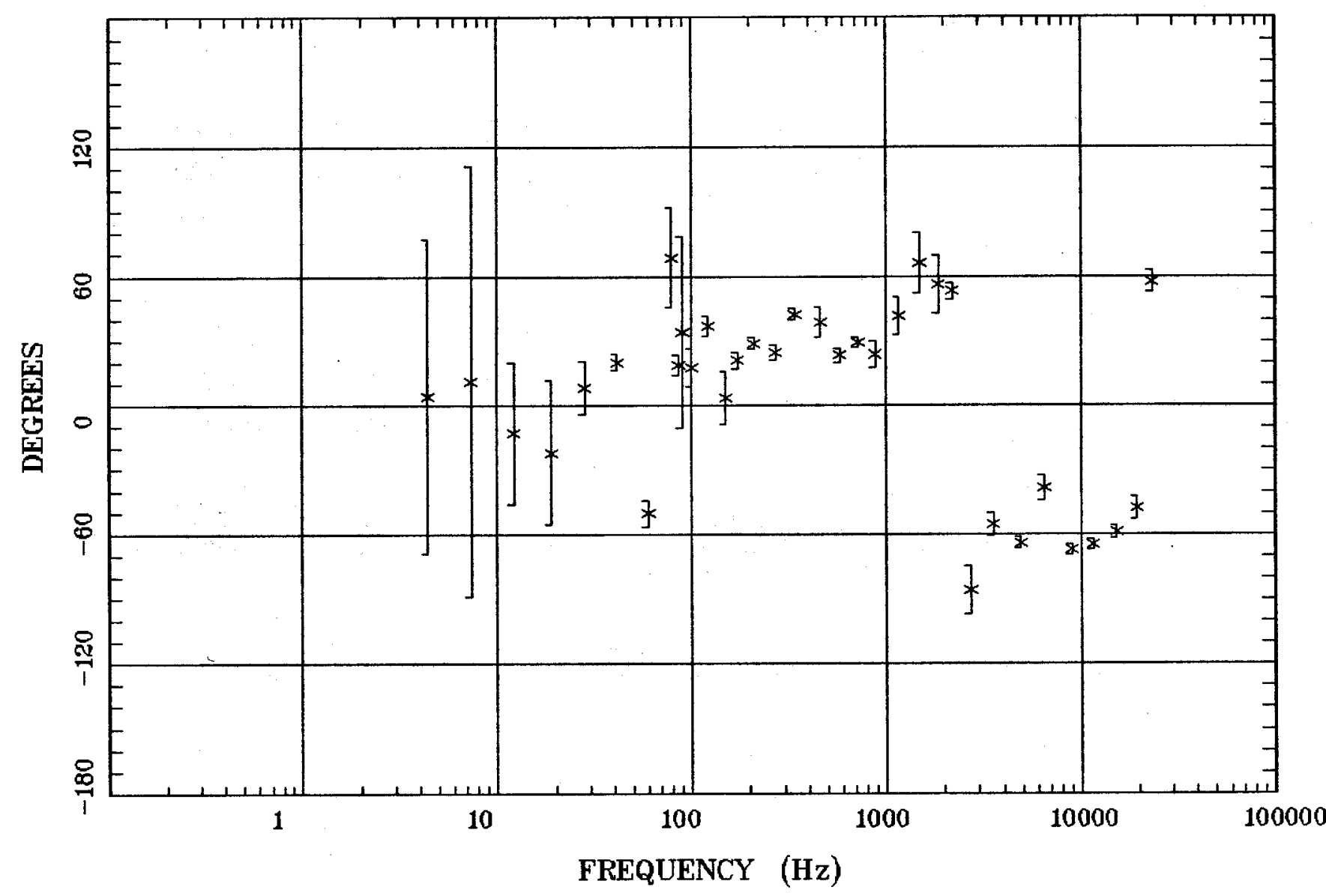

Client:

Remate: e-fld $95 \mathrm{~m}$ north Acquired: 14:0 Jun 19, 2003 Survey Co:USGS
Rotation:

Filename: cp20.avg

Channels: Ch1 Ch2 Ch3 Ch4 Ch5 Ch6 Ch7

Plotted: 09:50 Jul 06, 2004

$<$ EMI - ElectroMagnetic Instruments > 


\section{HzHx.x Coh HzHy.o}

Santa $\mathrm{Fe}, \mathrm{NM}$

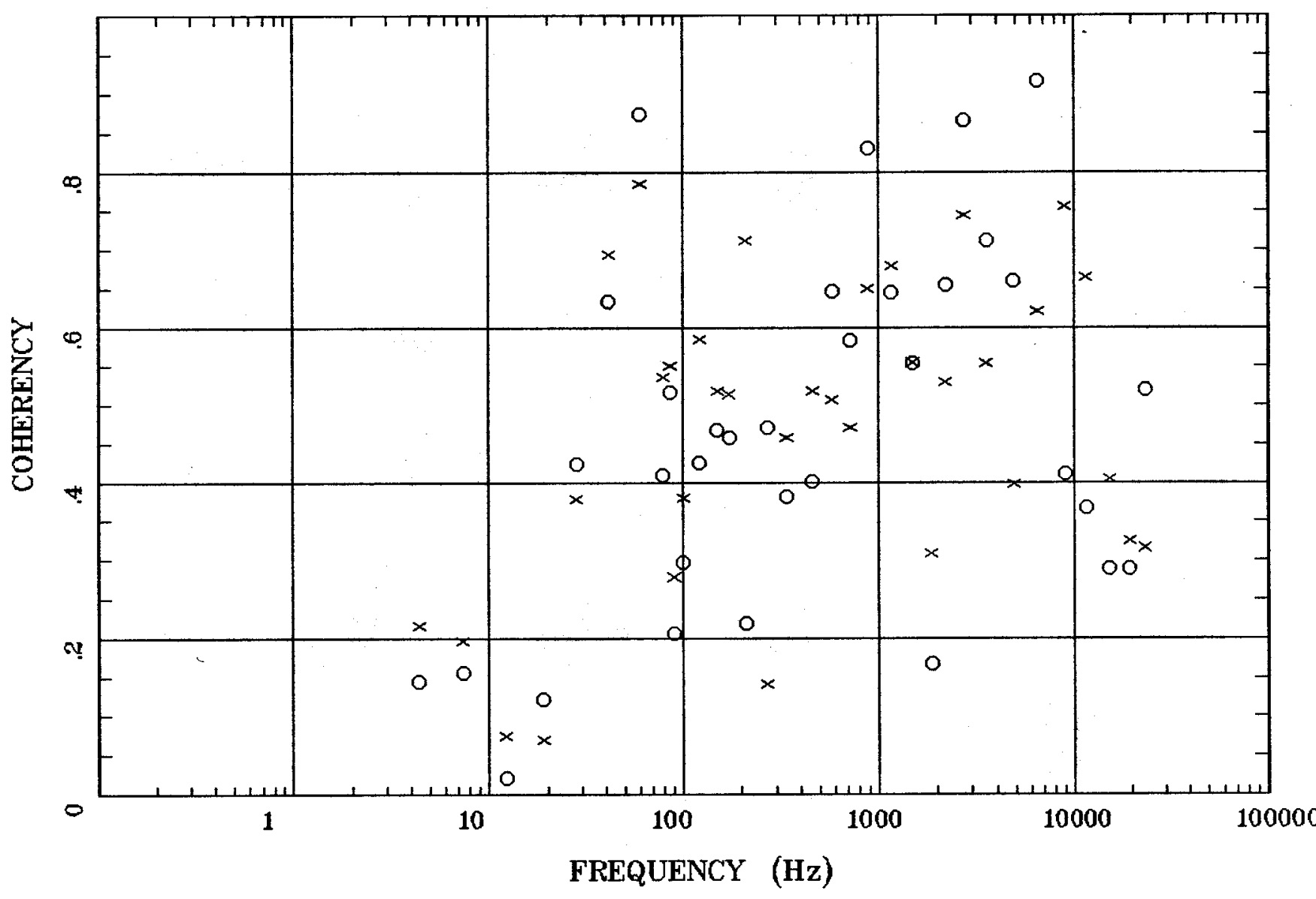

Client:

Remote: e-fld $95 \mathrm{~m}$ north Acquired: 14:0 Jun 19, 2003 Survey Co:USGS
Rotation:

Filename: cp20.avg

Channels: Ch1 Ch2 Ch3 Ch4 Ch5 Ch6 Ch7

Plotted: 09:50 Jul 06, 2004

< EMI - ElectroMagnetic Instruments > 


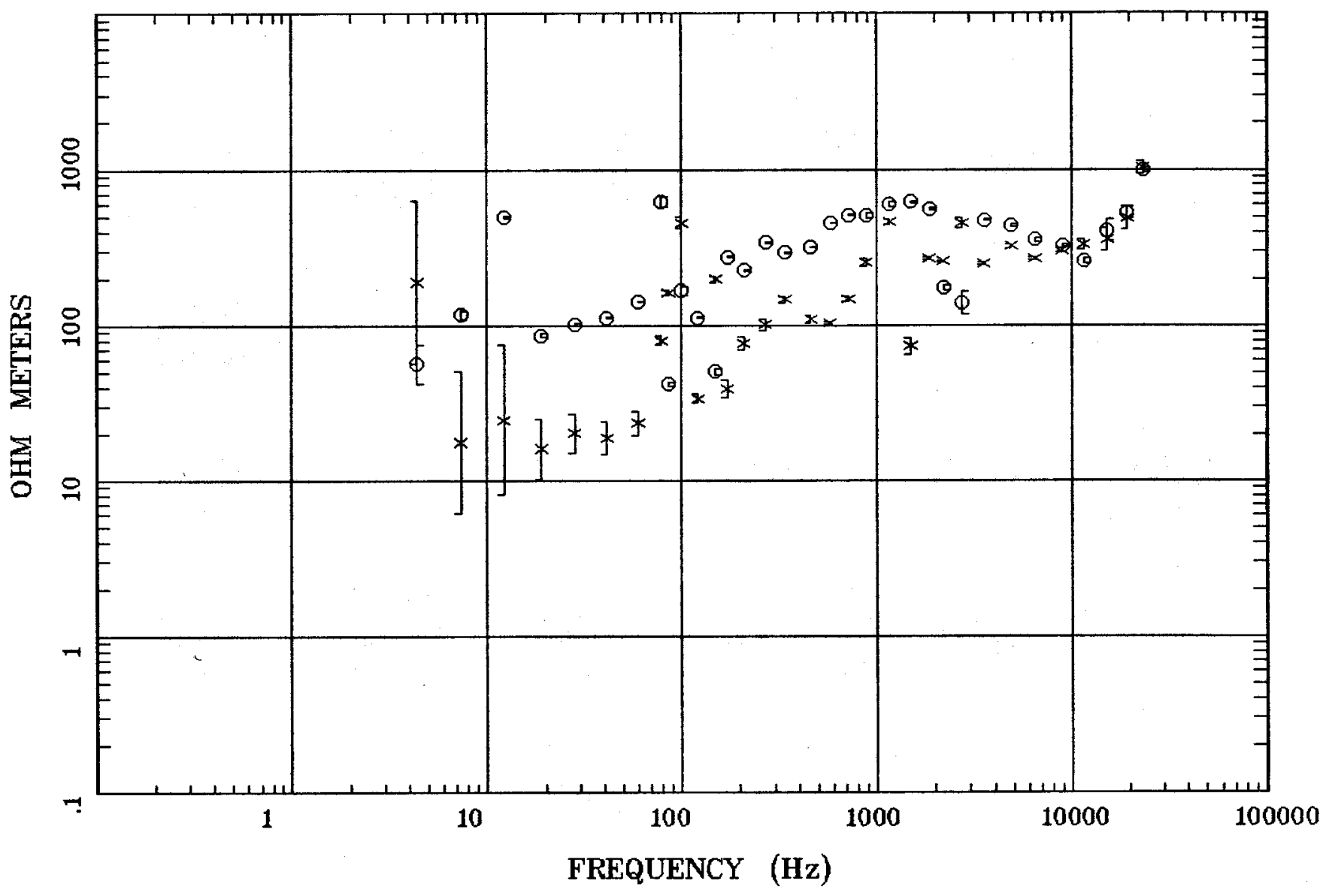

Client:

Remote: e-fld $95 \mathrm{~m}$ north Acquired: 14:5 Jun 20, 2003 Survey Co:USGS
Rotation:

Filename: cp21.avg

Channels: Ch1 Ch2 Ch3 Ch4 Ch5 Ch6 Ch7 Plotted: 09:52 Jul 06, 2004

< EMI - ElectroMagnetic Instruments 


\section{IMPEDANCE PHASE}

Santa Fe, NM

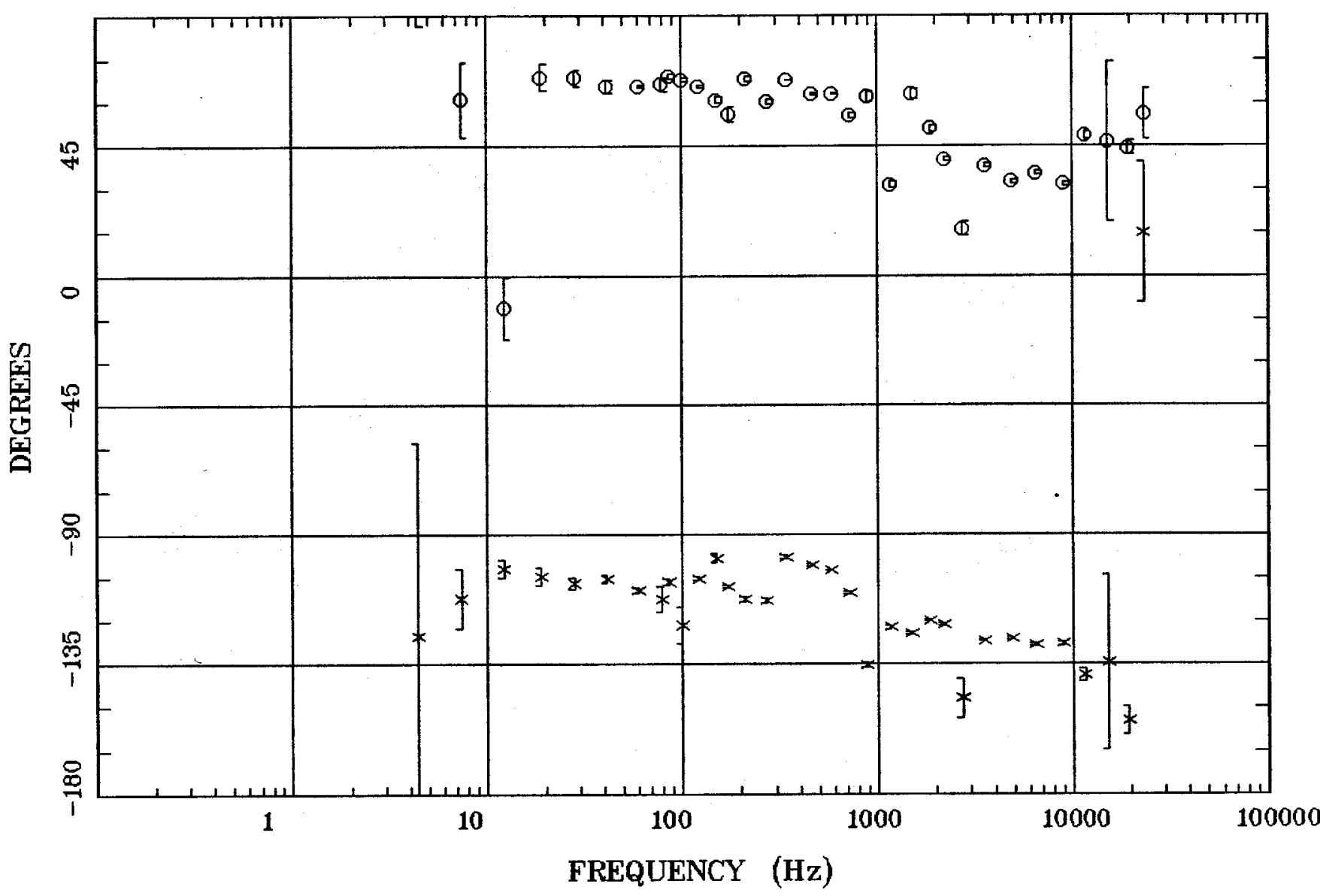

Client:

Remote: e-fld $95 \mathrm{~m}$ north Acquired: 14:5 Jun 20, 2003 Survey Co:USGS
Rotation:

Filename: cp21.avg

Channels: Ch1 Ch2 Ch3 Ch4 Ch5 Ch6 Ch7 Plotted: 09:52 Jul 06, 2004

< EMI - ElectroMagnetic Instruments > 


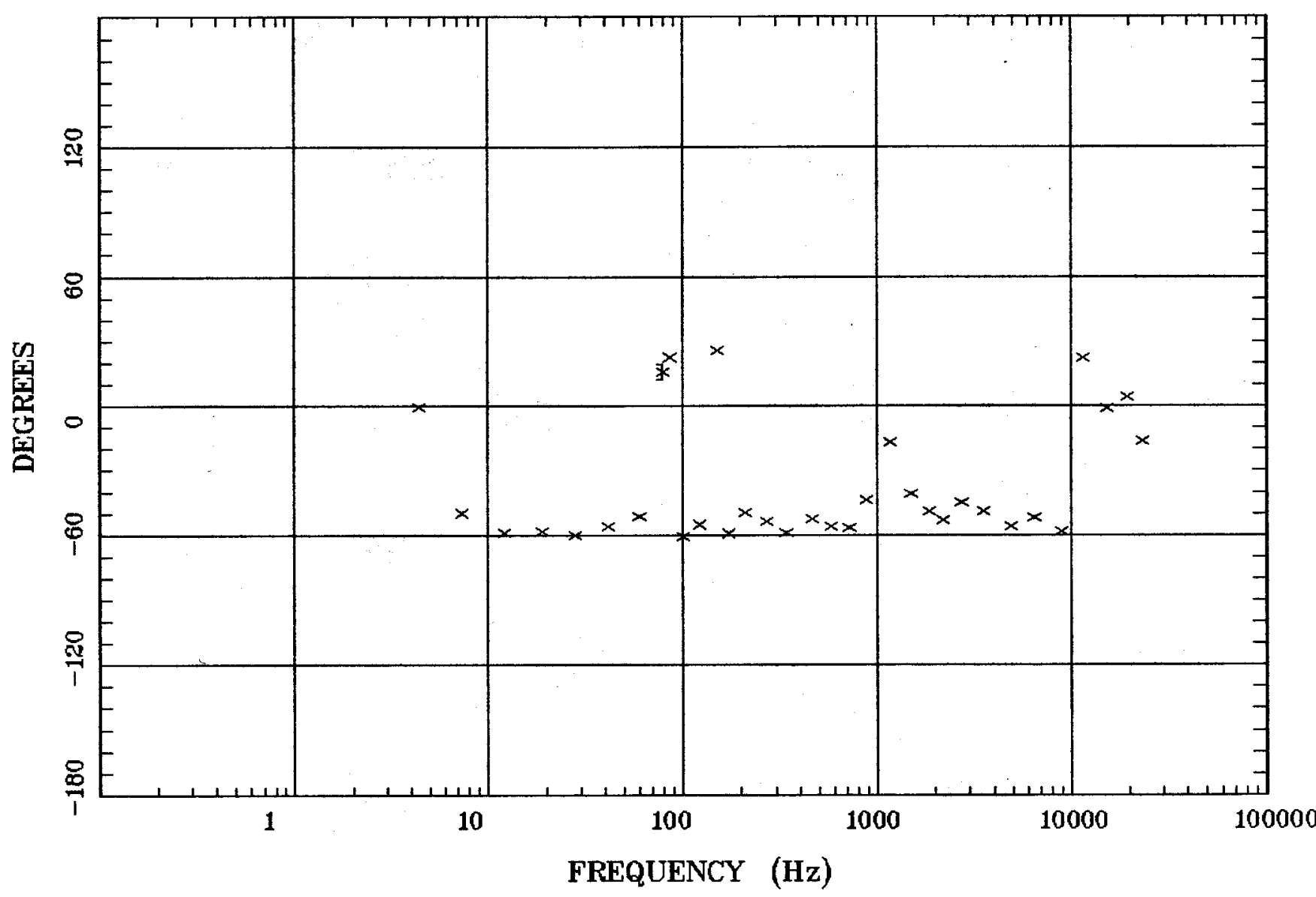

Client:

Remate: e-fld $95 \mathrm{~m}$ north Acquired: 14:5 Jun 20, 2003 Survey Co:USGS
Rotation:

Filename: cp21.avg

Channels: Ch1 Ch2 Ch3 Ch4 Ch5 Ch6 Chy Platted: 12:53 Jul 07, 2004

< EMI - ElectroMagnetic Instruments > 


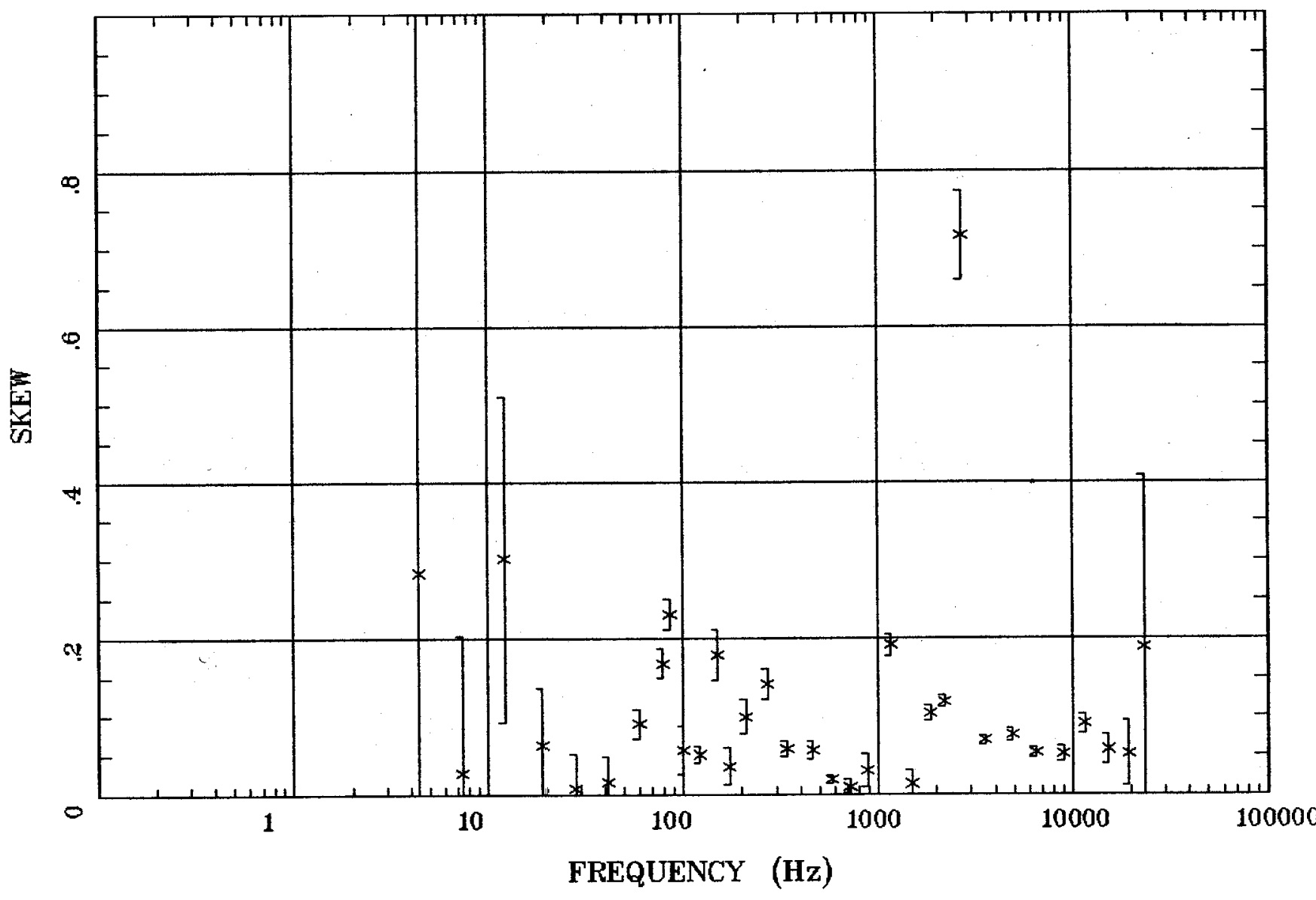

Client:

Remote: e-fld $95 \mathrm{~m}$ north Acquired: 14:5 Jun 20, 2003 Survey Co:USGS
Rotation:

Filename: cp21.avg

Channels: Ch1 Ch2 Ch3 Ch4 Ch5 Ch6 Ch7

Plotted: 09:52 Jul 06, 2004

< EMI - ElectroMagnetic Instruments > 
E MULT Coh.

Santa Fe, NM

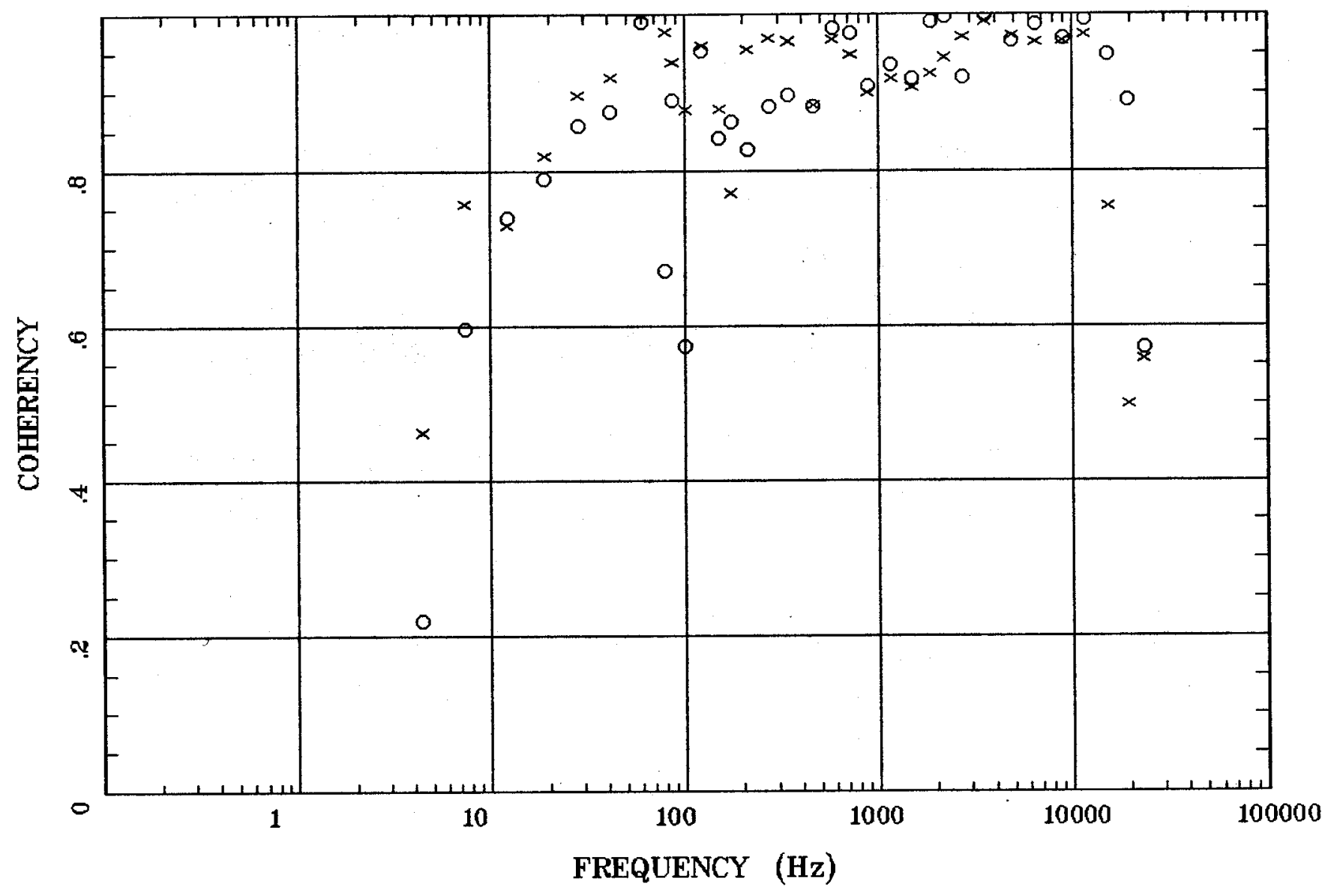

Client:

Remote: e-fld $95 \mathrm{~m}$ north Acquired: 14:5 Jun 20, 2003 Survey Co:USGS
Rotation:

Filename: cp21.avg

Channels: Ch1 Ch2 Ch3 Ch4 Ch5 Ch6 Ch7

Plotted: 09:52 Jul 06, 2004

< EMI - ElectroMagnetic Instruments > 


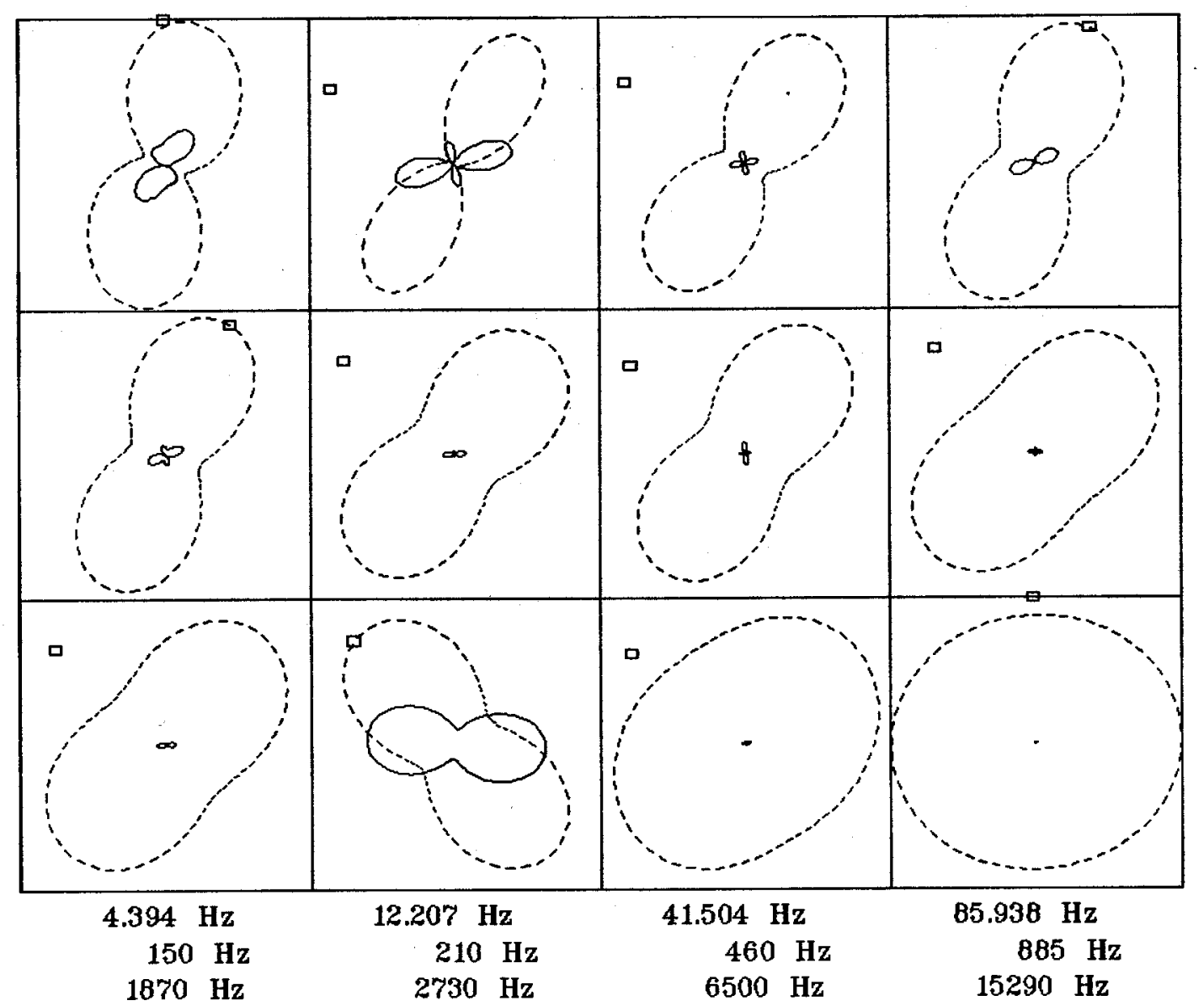

Client:

Remate: e-fld $95 \mathrm{~m}$ north Acquired: 14:5 Jun 20, 2003 Survey Co:USGS
Rotation:

Filename: cp21.avg Channels: Ch1 Ch2 Ch3 Ch4 Ch5 Ch6 Ch7 Plotted: 09:52 Jul 06, 2004

< EMI - ElectroMagnetic Instruments 
Santa Fe, NM

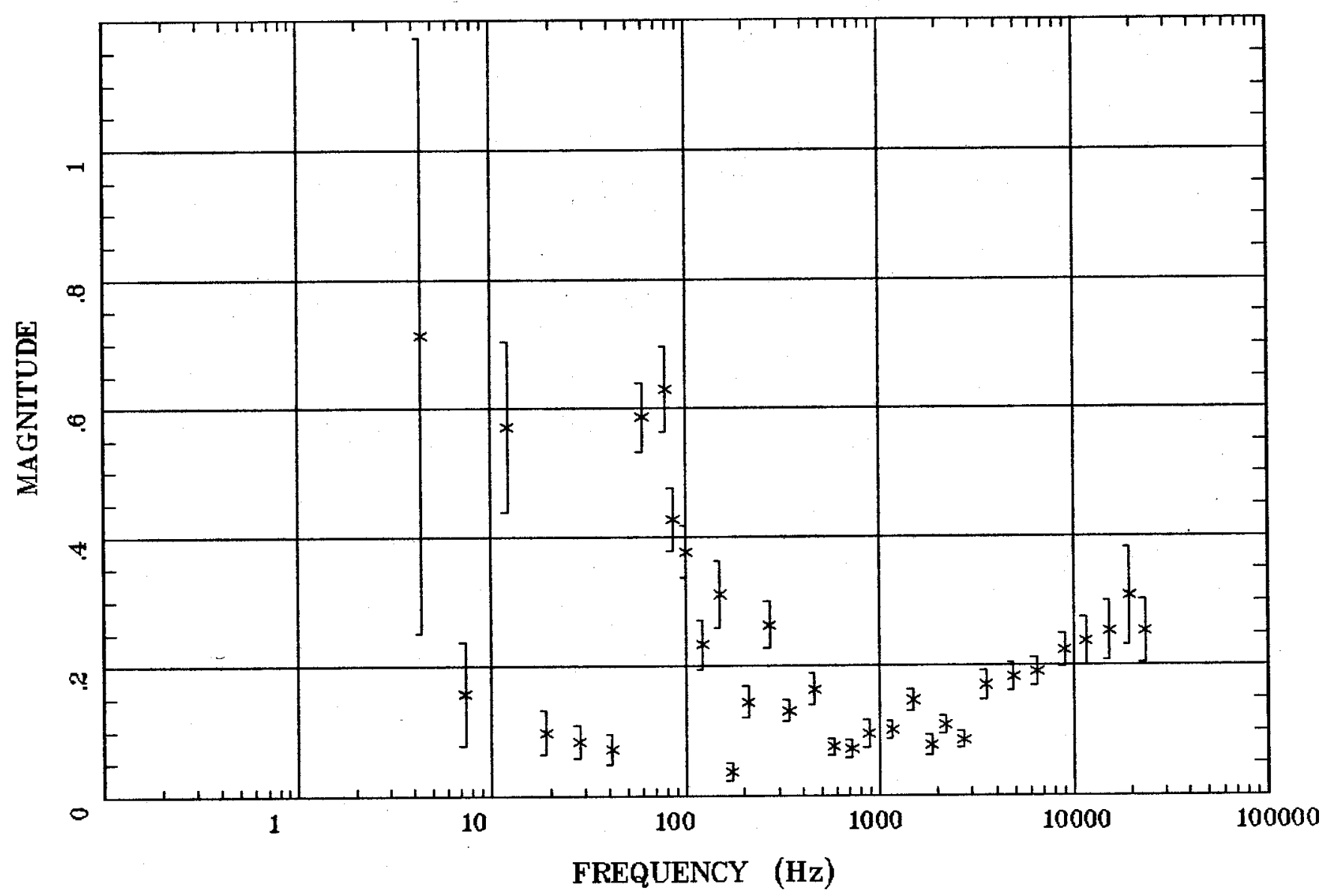

Client:

Remote: e-fld $95 \mathrm{~m}$ north Acquired: 14:5 Jun 20, 2003 Survey Co:USGS
Rotation:

Filename: cp21.avg

Channels: Ch1 Ch2 Ch3 Ch4 Ch5 Ch6 Ch7 Plotted: 09:52 Jul 06, 2004

< EMI - ElectroMagnetic Instruments > 


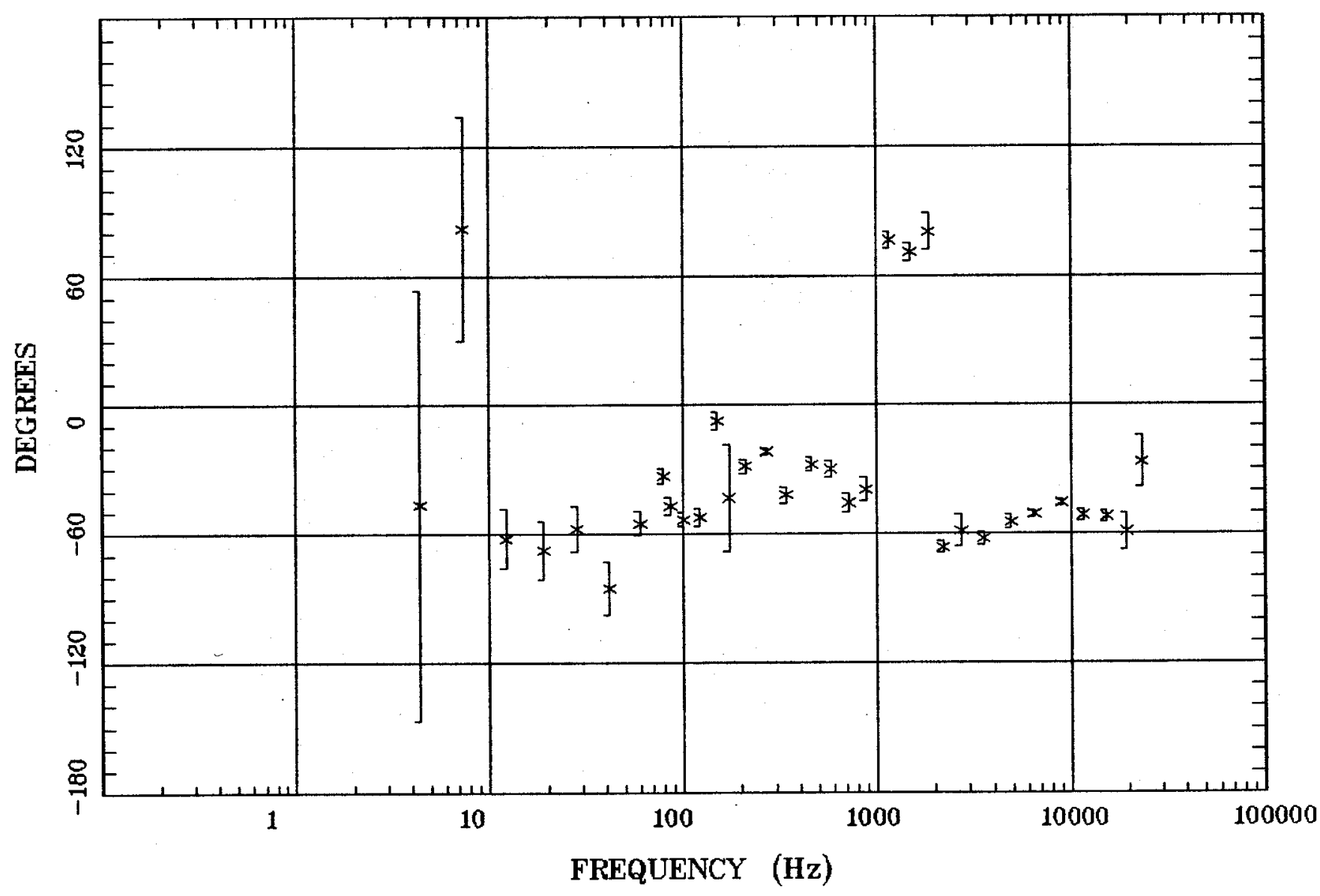

Client:

Remote: e-fld $95 \mathrm{~m}$ north Acquired: 14:5 Jun 20, 2003 Survey Co:USGS
Rotation:

Filename: cp21.avg

Channels: Ch1 Ch2 Ch3 Ch4 Ch5 Ch6 Ch7

Plotted: 09:52 Jul 06, 2004

<EMI - ElectroMagnetic Instruments > 
HzHx.x Coh HzHy.o

Santa Fe, NM

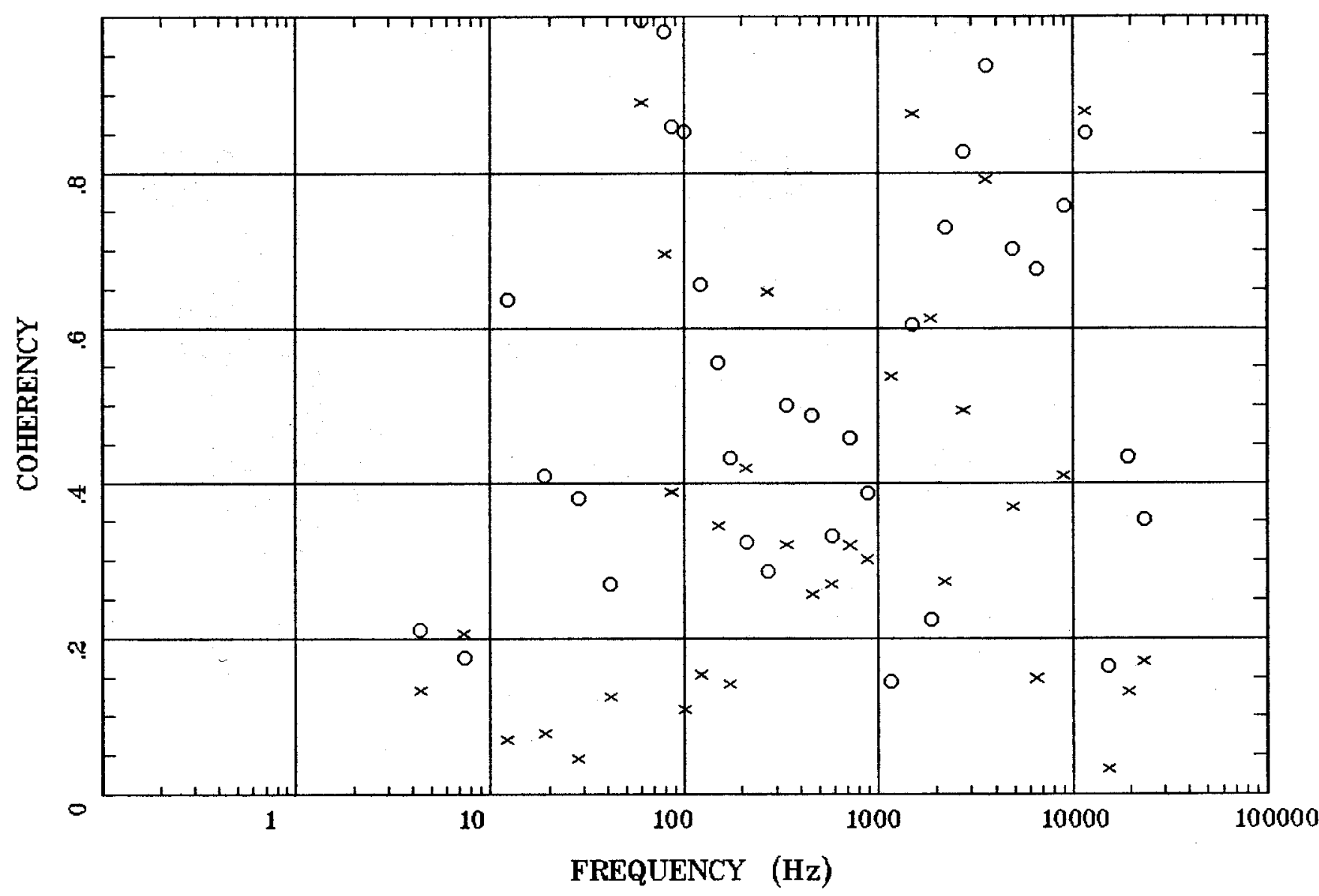

Client:

Remote: e-fld $95 \mathrm{~m}$ north Acquired: 14:5 Jun 20, 2003 Survey Co:USGS
Rotation:

Filename: cp21.avg

Channels: Ch1 Ch2 Ch3 Ch4 Ch5 Ch6 Ch7 Plotted: 15:33 Jul 06, 2004

< EMI - ElectroMagnetic Instruments 


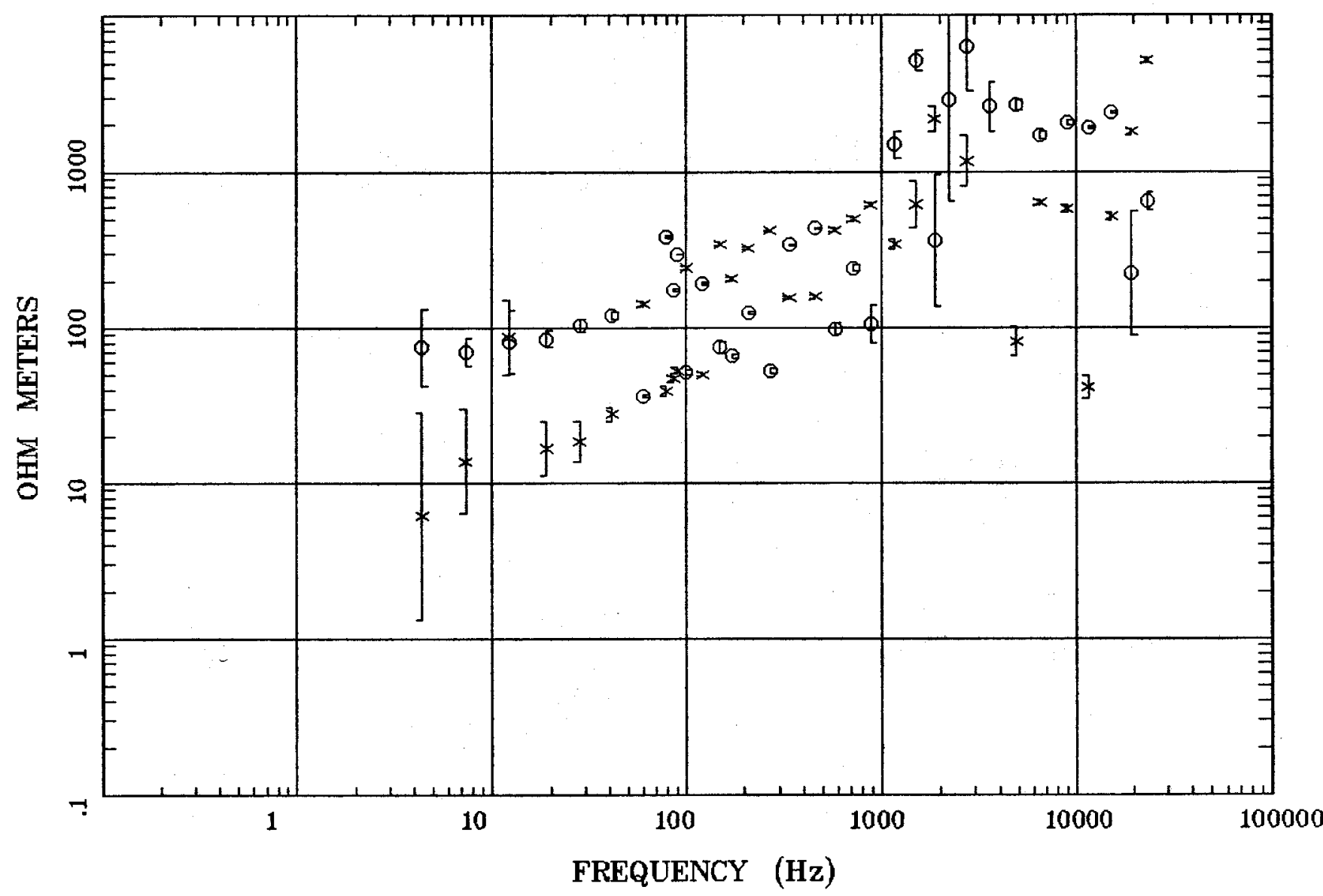

Client:

Remote: e-fld $95 \mathrm{~m}$ north Acquired: 10:5 Jan 08, 2004 Survey Co:USGS
Rotation:

Filename: cp22.avg

Channels: Ch1 Ch2 Ch3 Ch4 Ch5 Ch6 Ch7 Plotted: 15:34 Jul 06, 2004

< EMI - ElectroMagnetic Instruments 


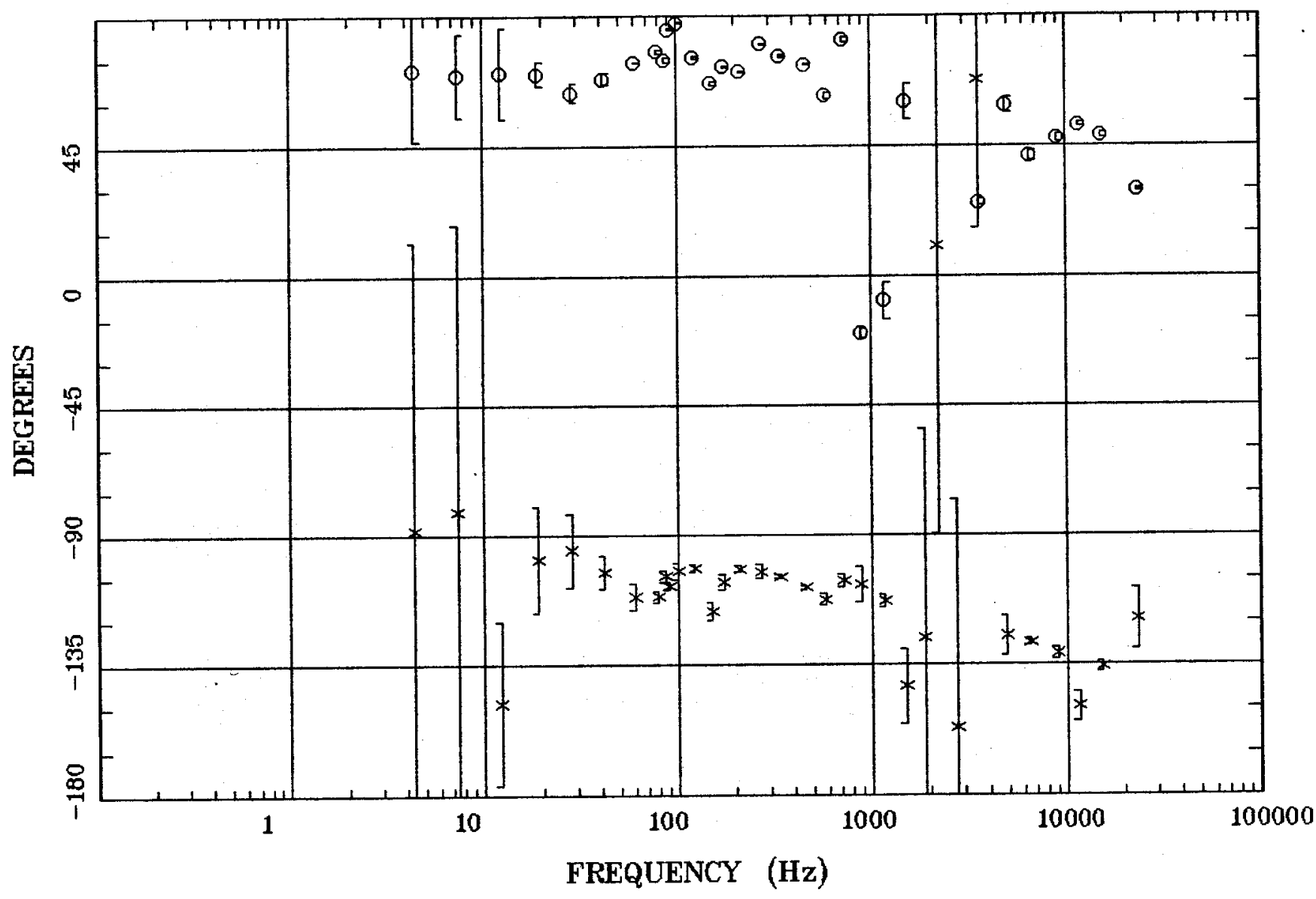

Client:

Remote: e-fld $95 \mathrm{~m}$ north Acquired: 10:5 Jan 08, 2004 Survey Co:USGS
Rotation:

Filename: cp22.avg

Channels: Ch1 Ch2 Ch3 Ch4 Ch5 Ch6 Ch7 Plotted: 15:34 Jul 06, 2004

< EMI - ElectroMagnetic Instruments 


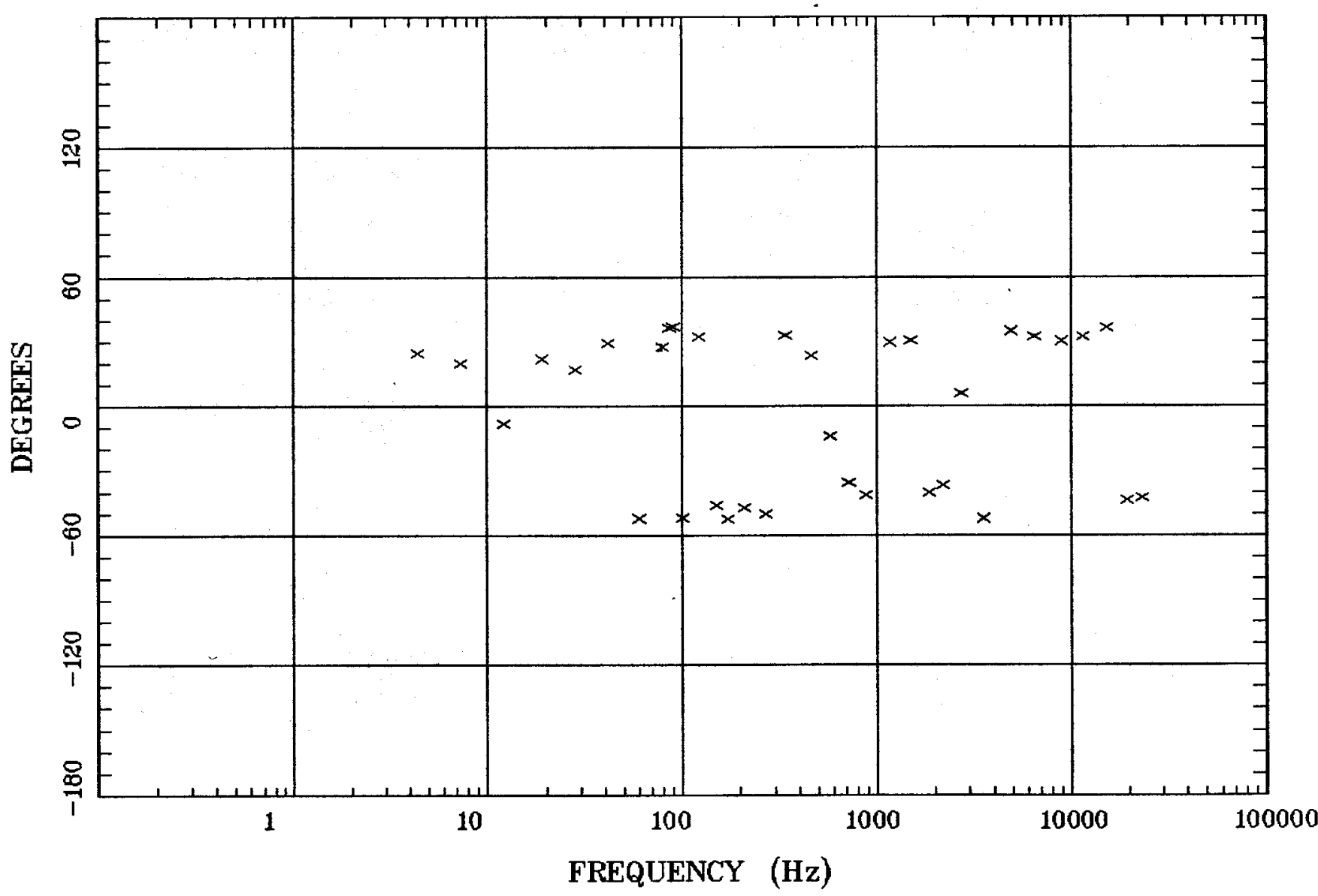

Client:

Remote: e-fld $95 \mathrm{~m}$ north Acquired: 10:5 Jan 08, 2004 Survey Co:USGS
Rotation:

Filename: cp22.avg

Channels: Ch1 Ch2 Ch3 Ch4 Ch5 Ch6 Chy Plotted: 12:55 Jul 07, 2004

$<$ EMI - ElectroMagnetic Instruments 
IMPEDANCE SKEW

Santa Fe, NM

Station 22

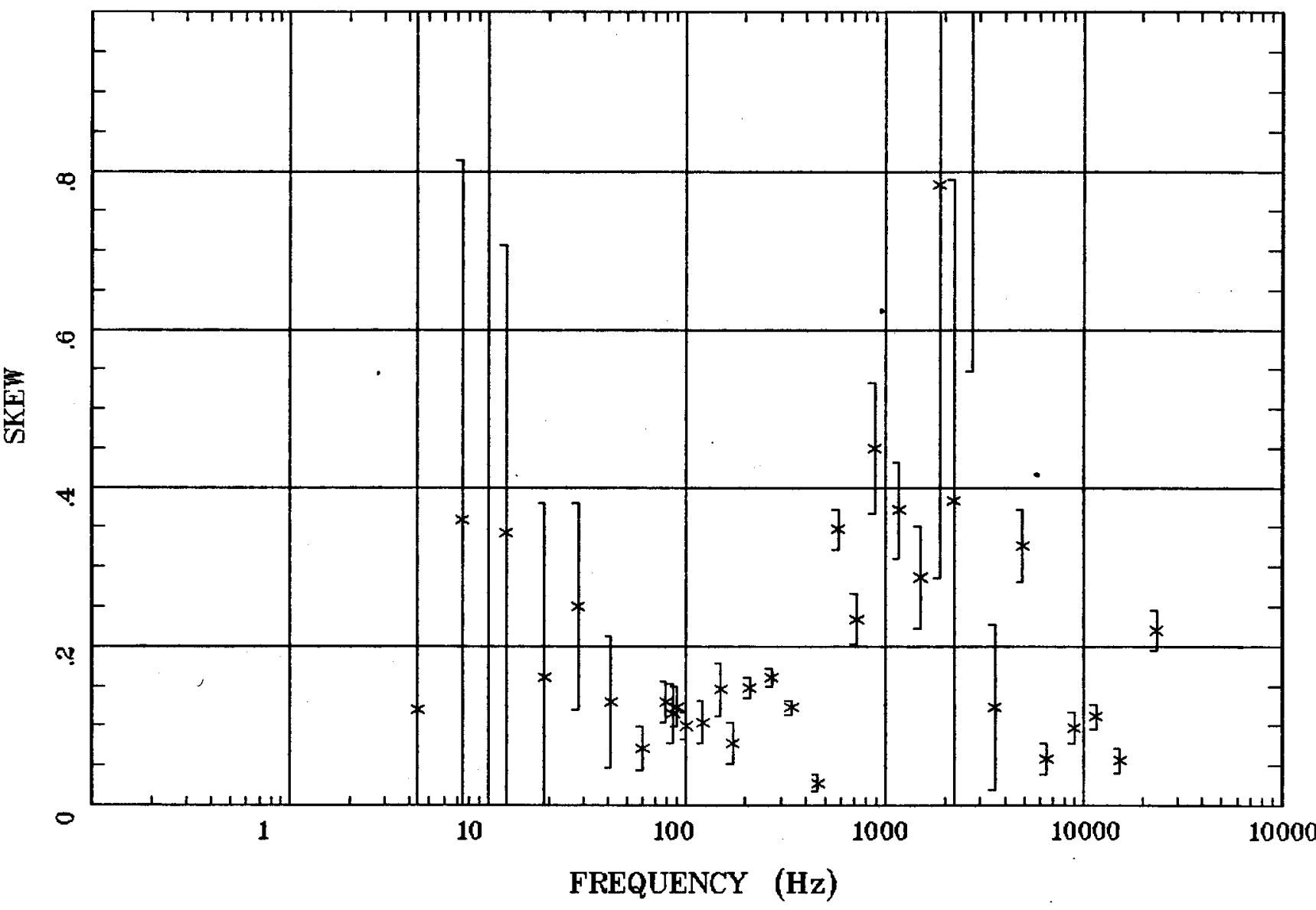

Client:

Remote: e-fld $95 \mathrm{~m}$ north Acquired: 10:5 Jan 08, 2004 Survey Co:USGS
Rotation:

Filename: cp22,avg

Channels: Ch1 Ch2 Ch3 Ch4 Ch5 Ch6 Ch7 Plotted: 15:34 Jul 06, 2004

< EMI - ElectroMagnetic Instruments > 
E MULT Coh.

Santa Fe, NM

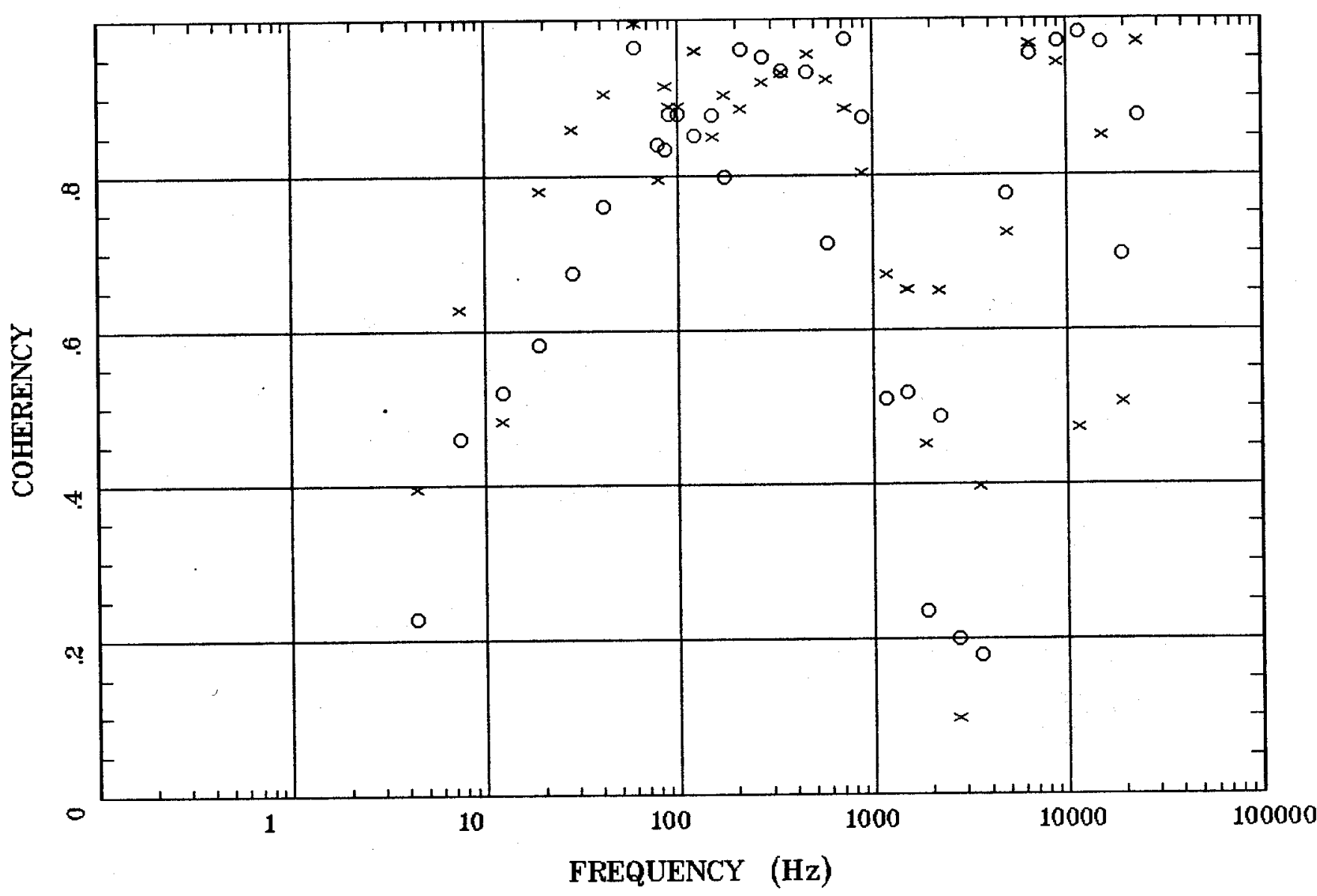

Client:

Remote: e-fld $95 \mathrm{~m}$ north Acquired: 10:5 Jan 08, 2004 Survey Co:USGS
Rotation:

Filename: cp22.avg

Channels: Ch1 Ch2 Ch3 Ch4 Ch5 Ch6 Ch'

Platted: 15:34 Jul 06, 2004

< EMI - ElectroMagnetic Instruments > 


\section{POLAR PLOTS}

\section{Santa Fe, NM}

Station 22

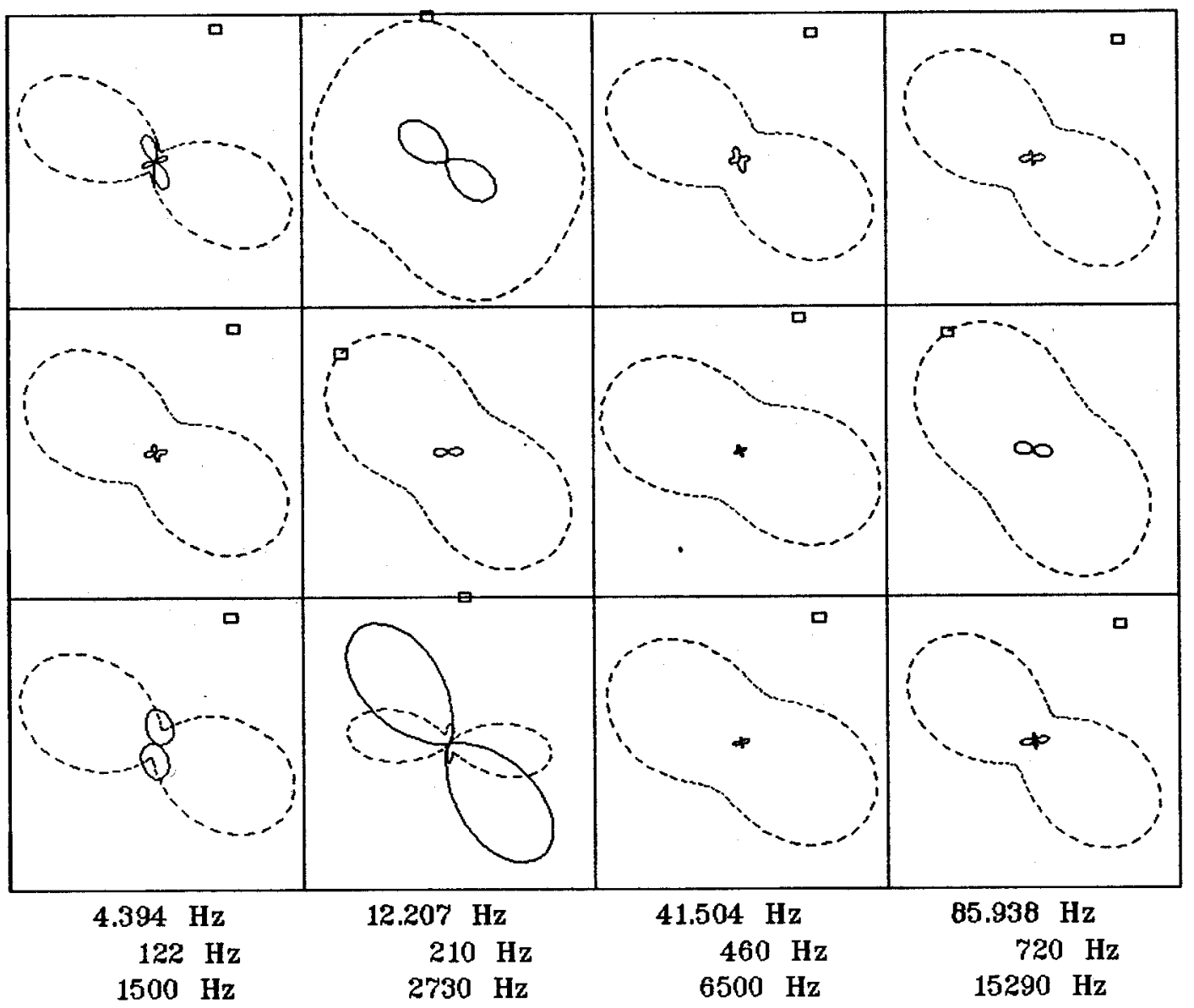

Rotation:

Client:

Remote: e-fld $95 \mathrm{~m}$ north Acquired: 10:5 Jan 08, 2004 Survey Co:USGS
Filename: cp22.avg

Channels: Ch1 Ch2 Ch3 Ch4 Ch5 Ch6 Ch7 Plotted: 15:34 Jul 06, 2004

< EMI - ElectroMagnetic Instruments > 


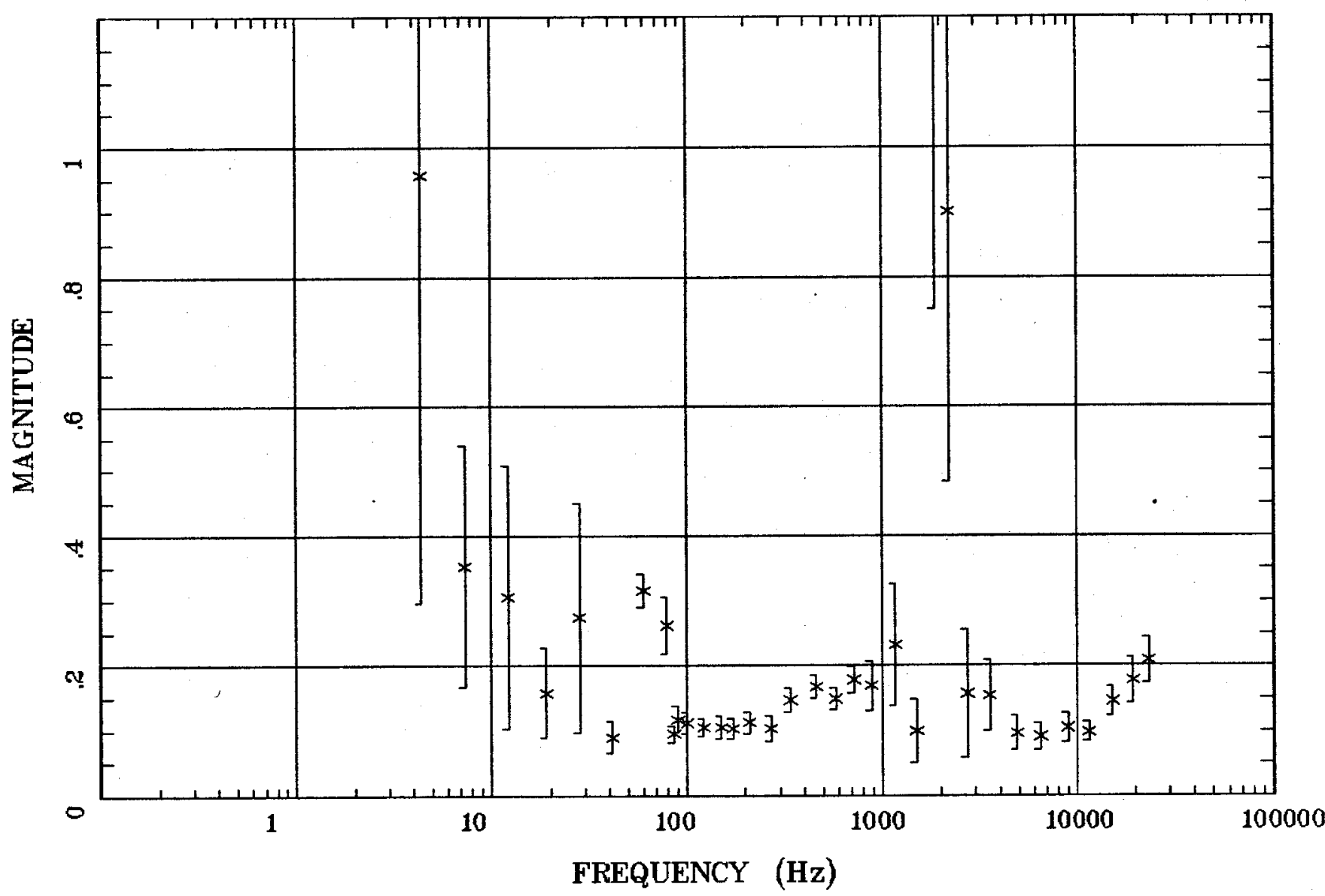

Client:

Remote: e-fld $95 \mathrm{~m}$ north Acquired: 10:5 Jan 08, 2004 Survey Co:USGS
Rotation:

Filename: cp22.avg

Channels: Ch1 Ch2 Ch3 ch4 Ch5 ch6 Ch7 Plotted: 15:34 Jul 06, 2004

$<$ EMI - ElectroMagnetic Instruments 


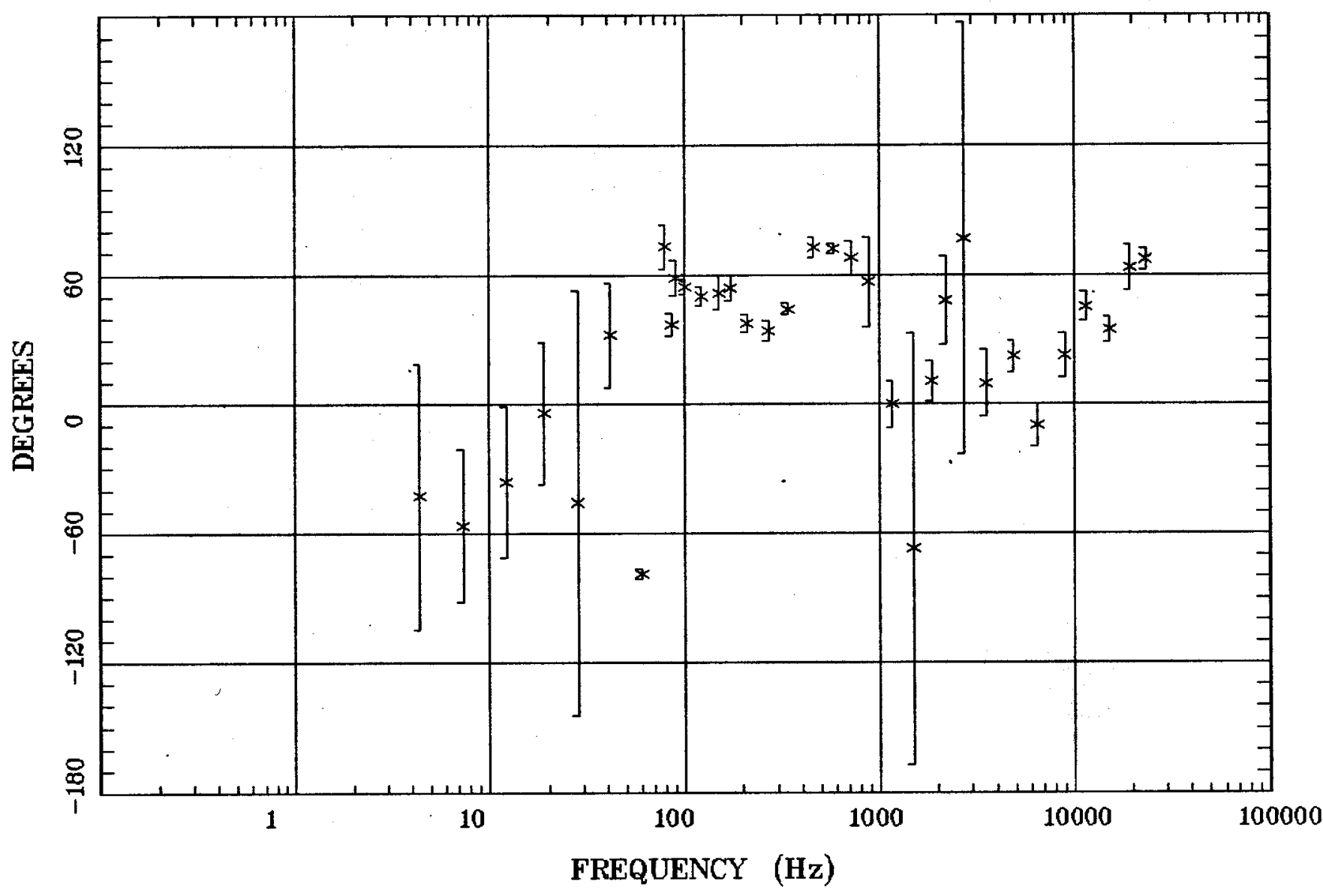

Client:

Remote: e-fld $95 \mathrm{~m}$ north Acquired: 10:5 Jan 08, 2004 Survey Co:USGS
Rotation:

Filename: cp22.avg

Channels: Ch1 Ch2 Ch3 ch4 Ch5 ch6 Ch7

Plotted: 15:34 Jul 06, 2004

< EMI - ElectroMagnetic Instruments > 


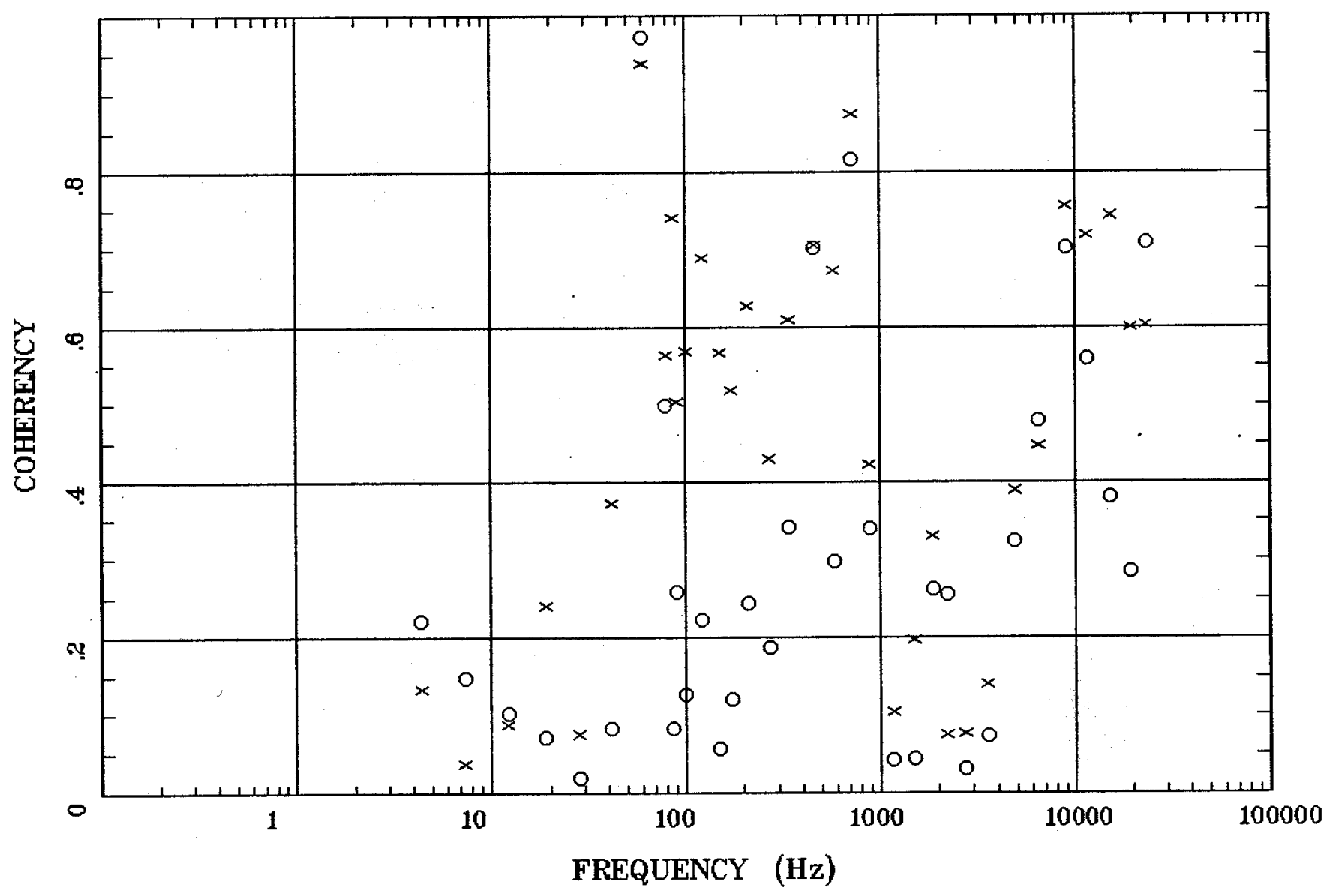

Client:

Remote: e-fld $95 \mathrm{~m}$ north Acquired: 10:5 Jan 08, 2004 Survey Co:USGS
Rotation:

Filename: cp22.avg

Channels: Ch1 Ch2 Ch3 Ch4 Ch5 Ch6 Ch7

Plotted: 15:34 Jul 06, 2004

$<$ EMI - ElectroMagnetic Instruments 


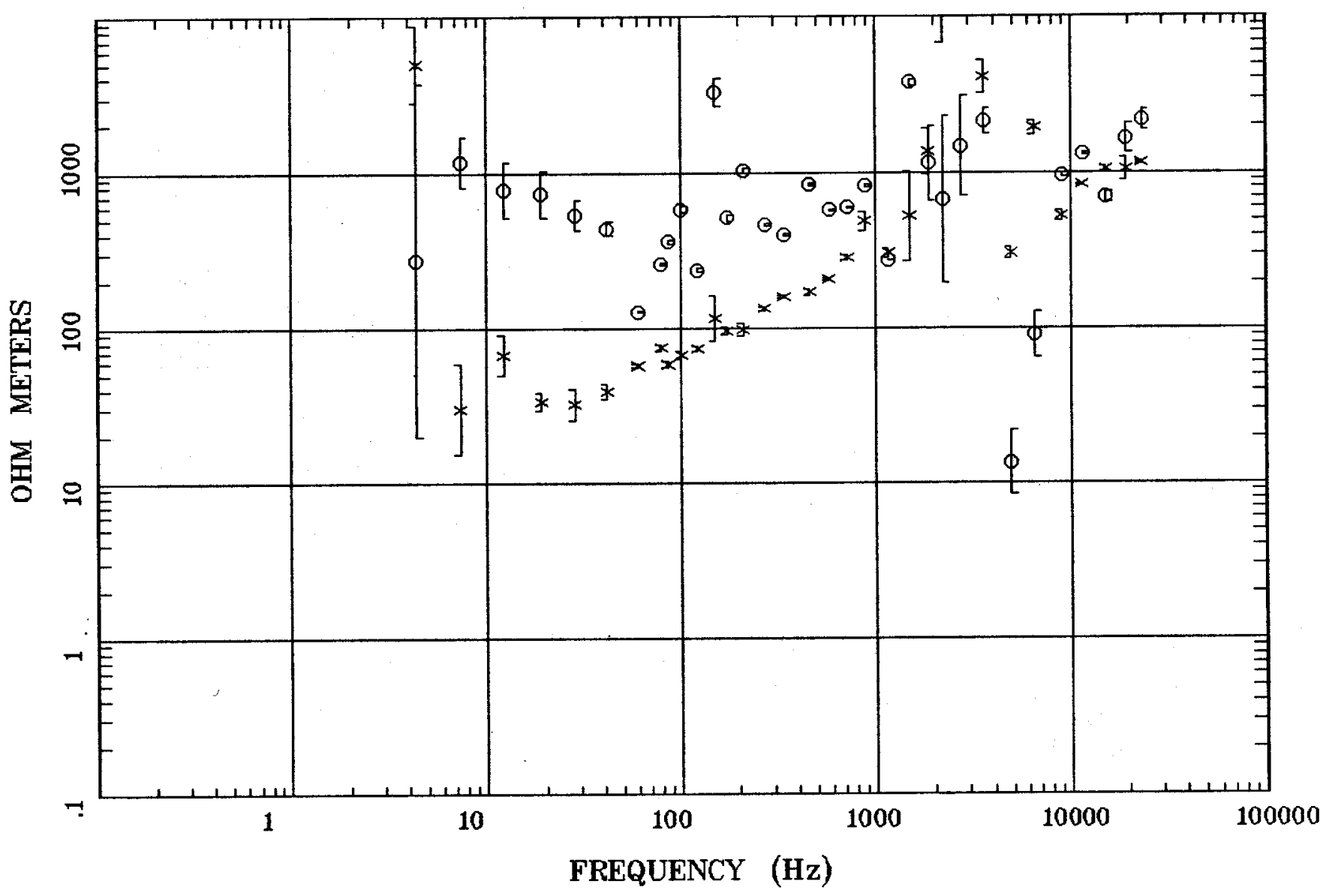

Client:

Remote: e-fld 70m north

Acquired: 14:2 Jun 22, 2003 Survey Co:USGS
Rotation:

Filename: cp23.avg

Channels: Ch1 Ch2 Ch3 Ch4 Ch5 Ch6 Ch7 Plotted: 09:55 Jul 06, 2004

$<$ EMI - ElectroMagnetic Instruments 


\section{IMPEDANCE PHASE}

Santa Fe, NM

Station 23

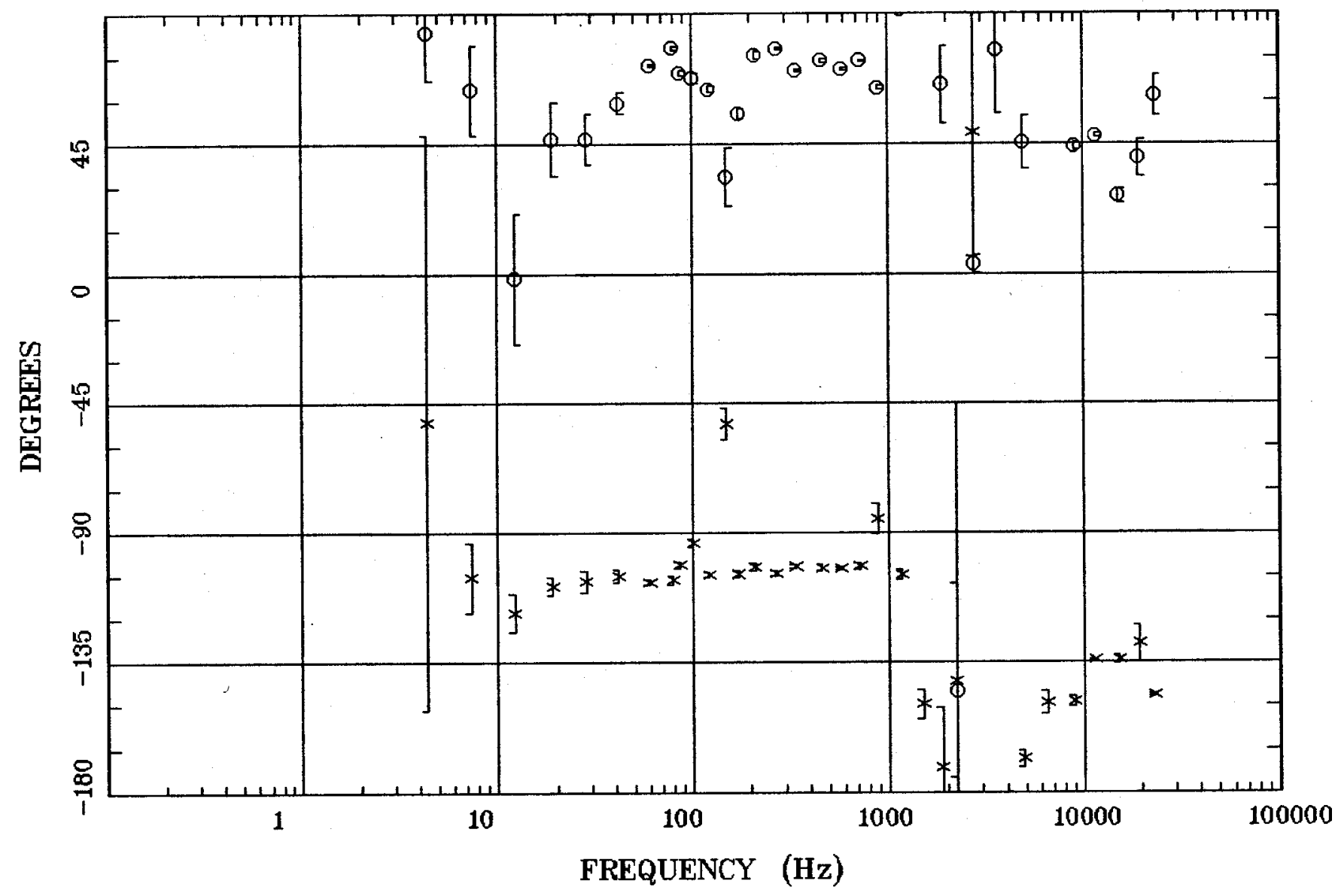

Client:

Remote: e-fld $70 m$ north

Acquired: 14:2 Jun 22, 2003 Survey Co:USGS
Rotation:

Filename: cp23.avg

Channels: Ch1 Ch2 Ch3 Ch4 Ch5 Ch6 Ch7 Plotted: 09:56 Jul 06, 2004

< EMI - ElectroMagnetic Instruments > 


\section{ROTATION ANGLE}

Santa Fe, NM

Station 23

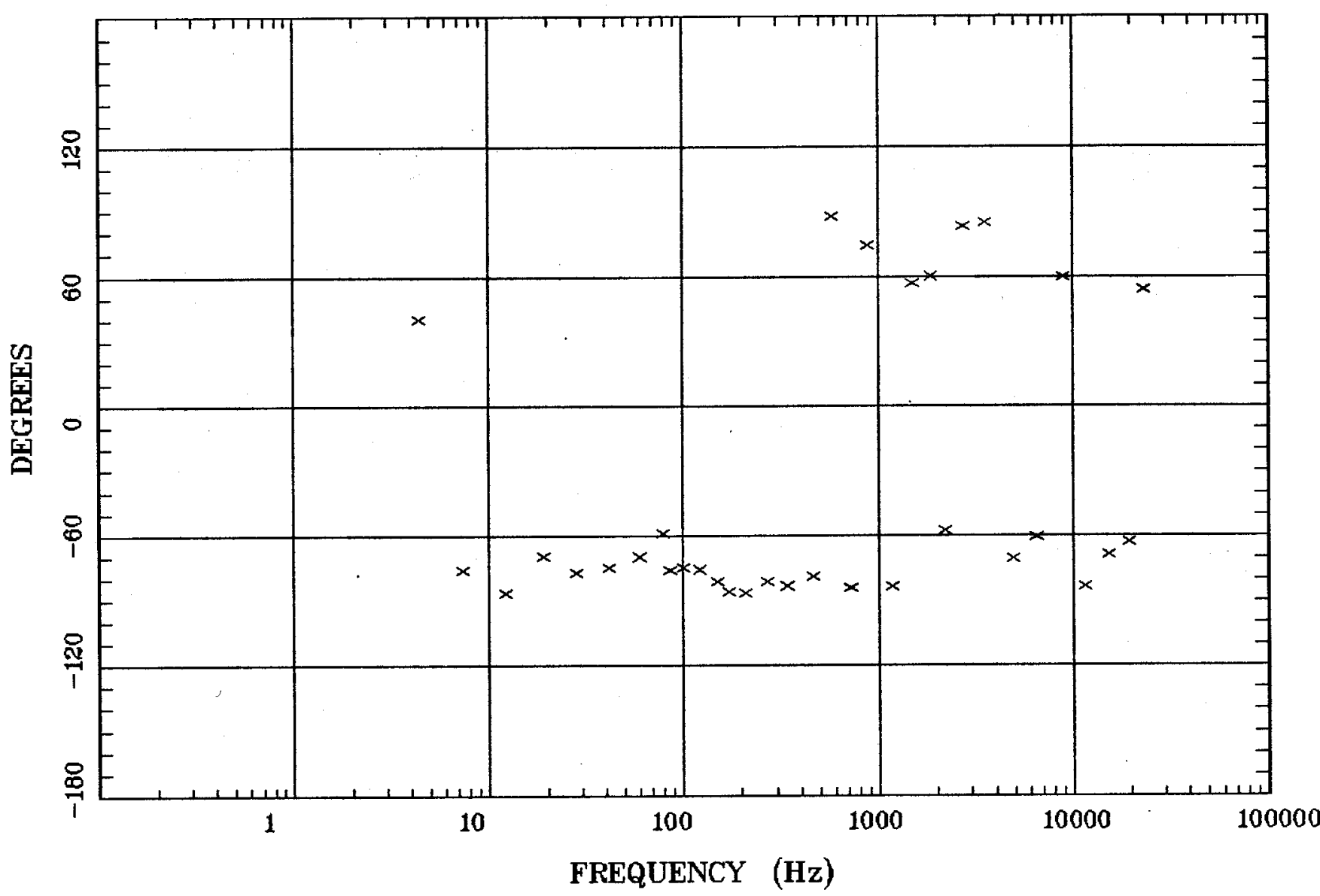

Client:

Remote: e-fld 70m north

Acquired: 14:2 Jun 22, 2003 Survey Co:USGS
Rotation:

Filename: cp23.avg

Channels: Ch1 Ch2 Ch3 Ch4 Ch5 ch6 Chr Plotted: 13:18 Jul 07, 2004

< EMI - ElectroMagnetic Instruments > 


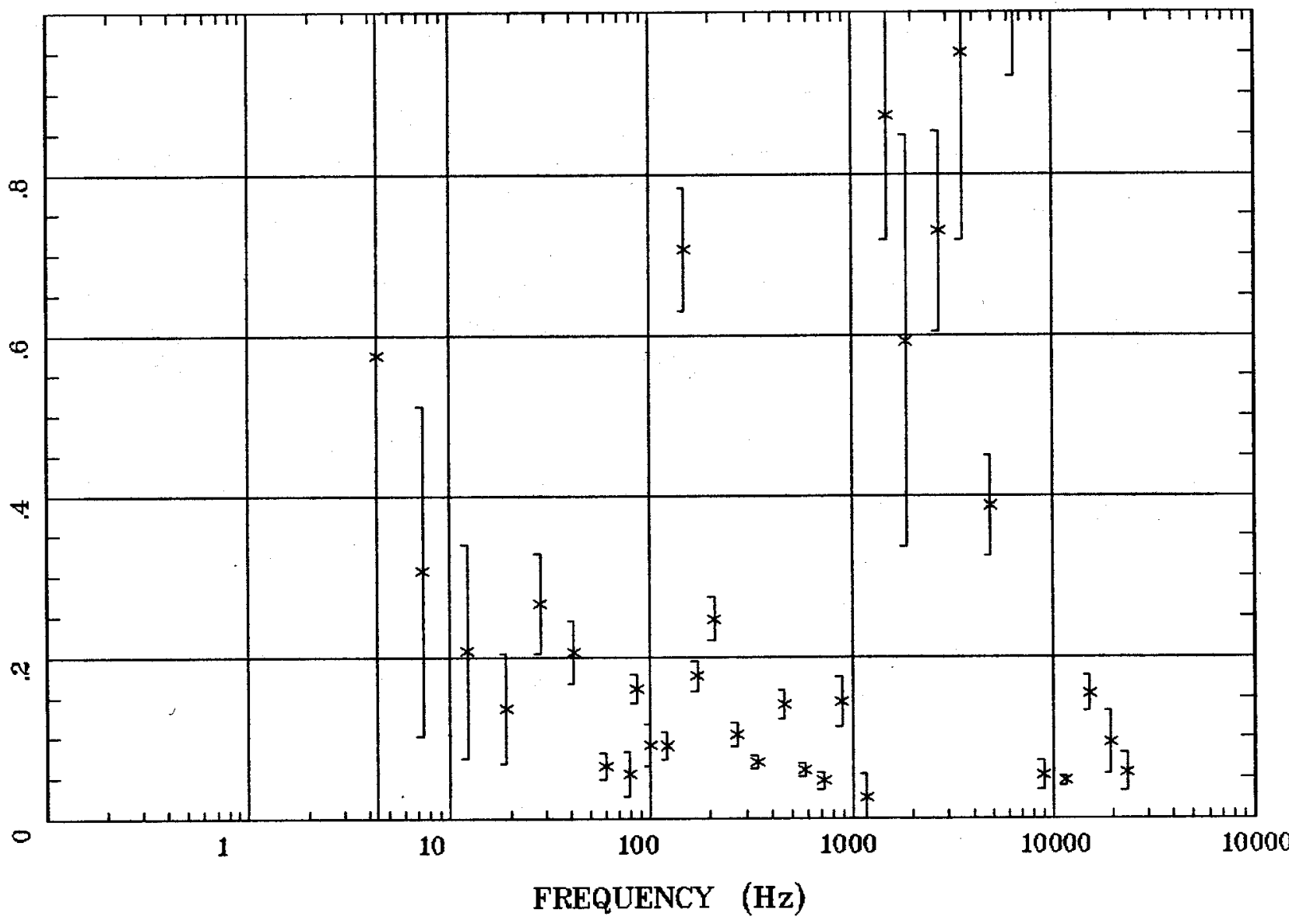

Client:

Remote: e-fld $70 \mathrm{~m}$ north Acquired: 14:2 Jun 22, 2003 Survey Co:USGS
Rotation:

Filename: cp23.avg

Channels: Ch1 Ch2 Ch3 Ch4 Ch5 Ch6 Ch7 Plotted: 09:56 Jul 06, 2004

< EMI - ElectroMagnetic Instruments > 


\section{E MULT Coh.}

Station 23

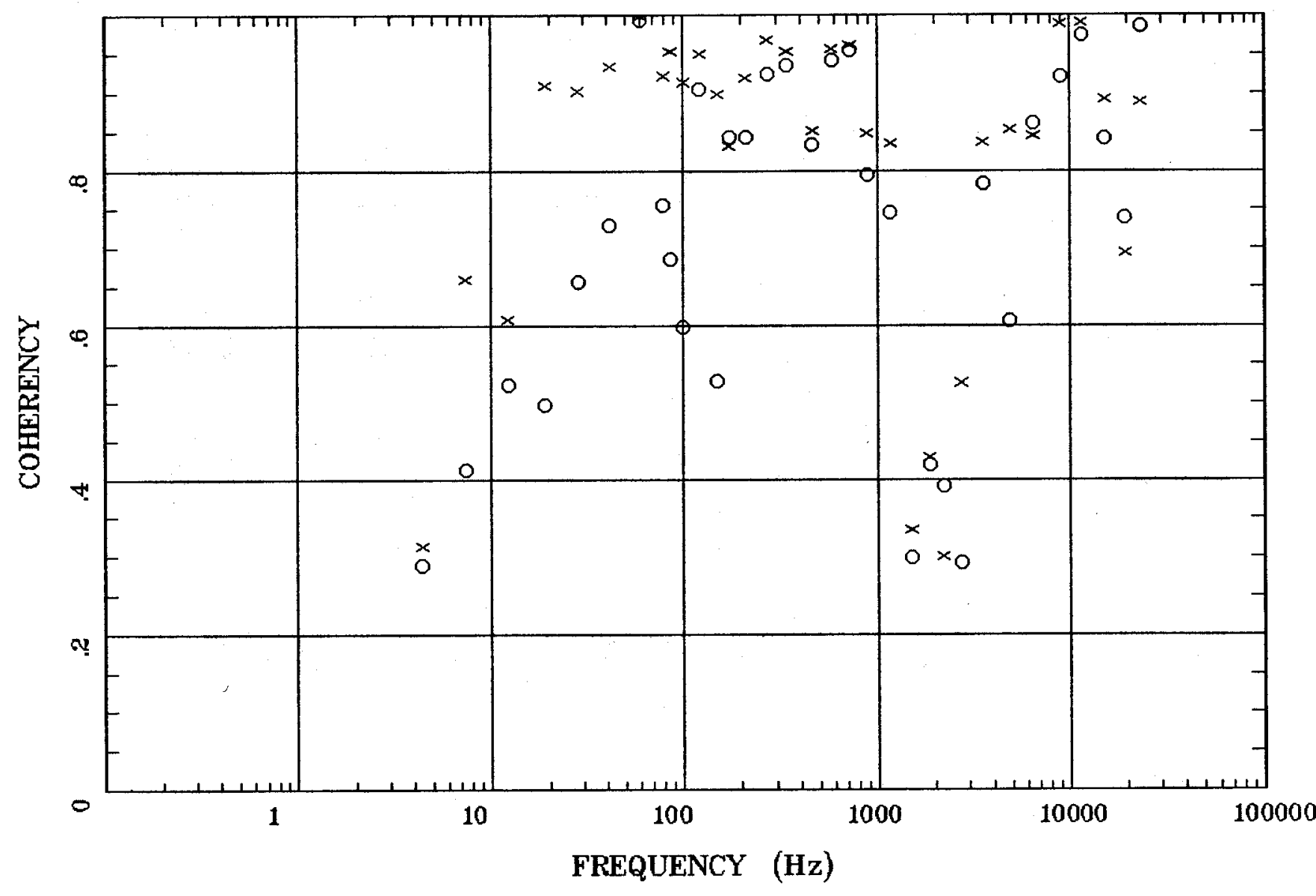

Client:

Remote: e-fld $70 \mathrm{~m}$ north Acquired: 14:2 Jun 22, 2003 Survey Co:USGS
Rotation:

Filename: cp23.avg

Channels: Ch1 Ch2 Ch3 Ch4 Ch5 Ch6 Ch7 Plotted: 09:56 Jul 06, 2004

$<$ EMI - ElectroMagnetic Instruments > 


\section{POLAR PLOTS}

Santa Fe, NM

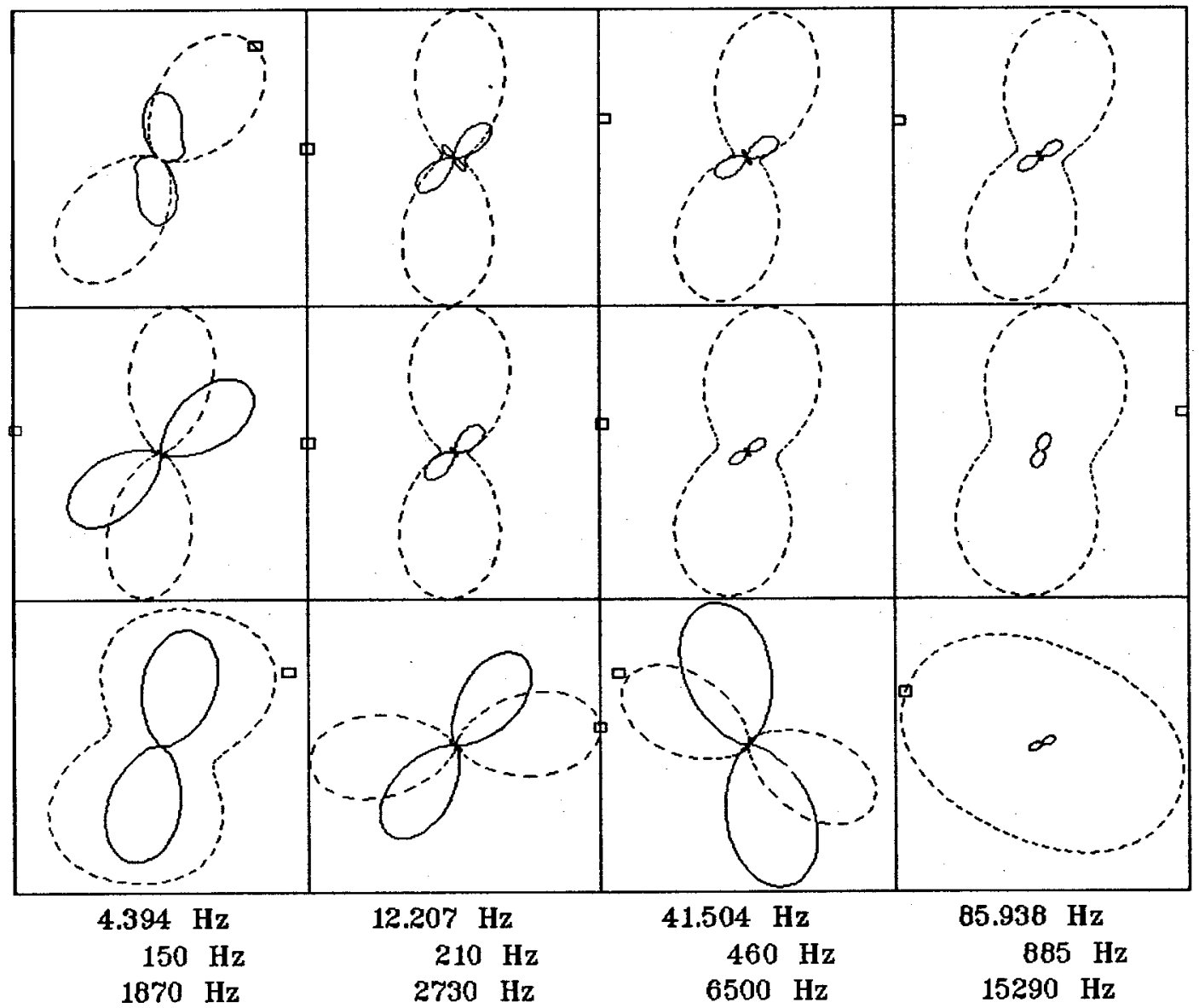

Rotation:

Client:

Remote: e-fld 70m north

Acquired: 14:2 Jun 22, 2003 Survey Co:USGS

Filename: cp23.avg

Channels: Ch1 Ch2 Ch3 Ch4 Ch5 Ch6 Ch7

Plotted: 09:56 Jul 06, 2004

< EMI - ElectroMagnetic Instruments > 


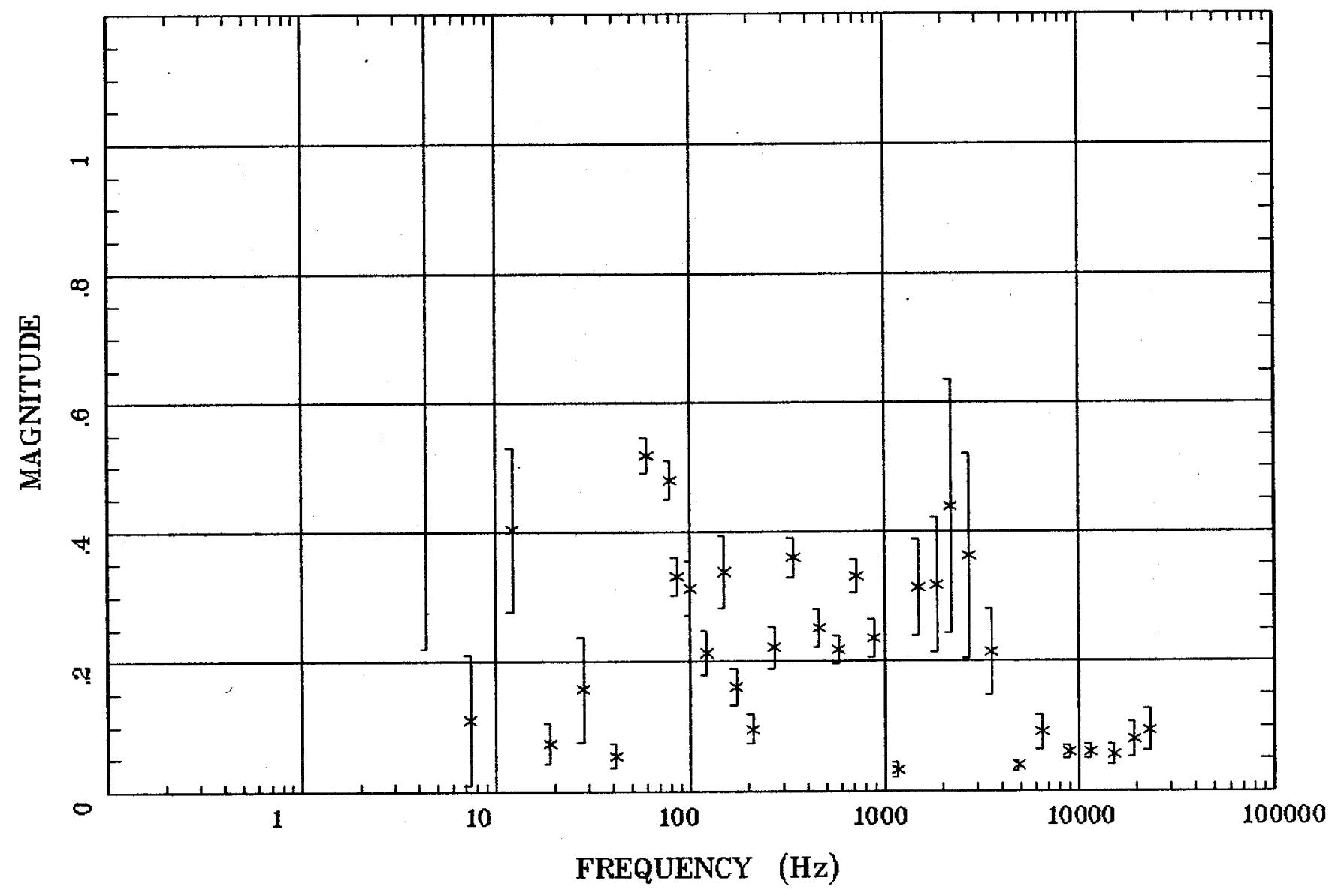

Client:

Remote: e-fld $70 \mathrm{~m}$ north Acquired: 14:2 Jun 22, 2003 Survey Co:USGS
Rotation:

Filename: cp23.avg

Channels: Ch1 Ch2 Ch3 Ch4 Ch5 Ch6 Ch7

Plotted: 09:56 Jul 06, 2004

< EMI - ElectroMagnetic Instruments > 


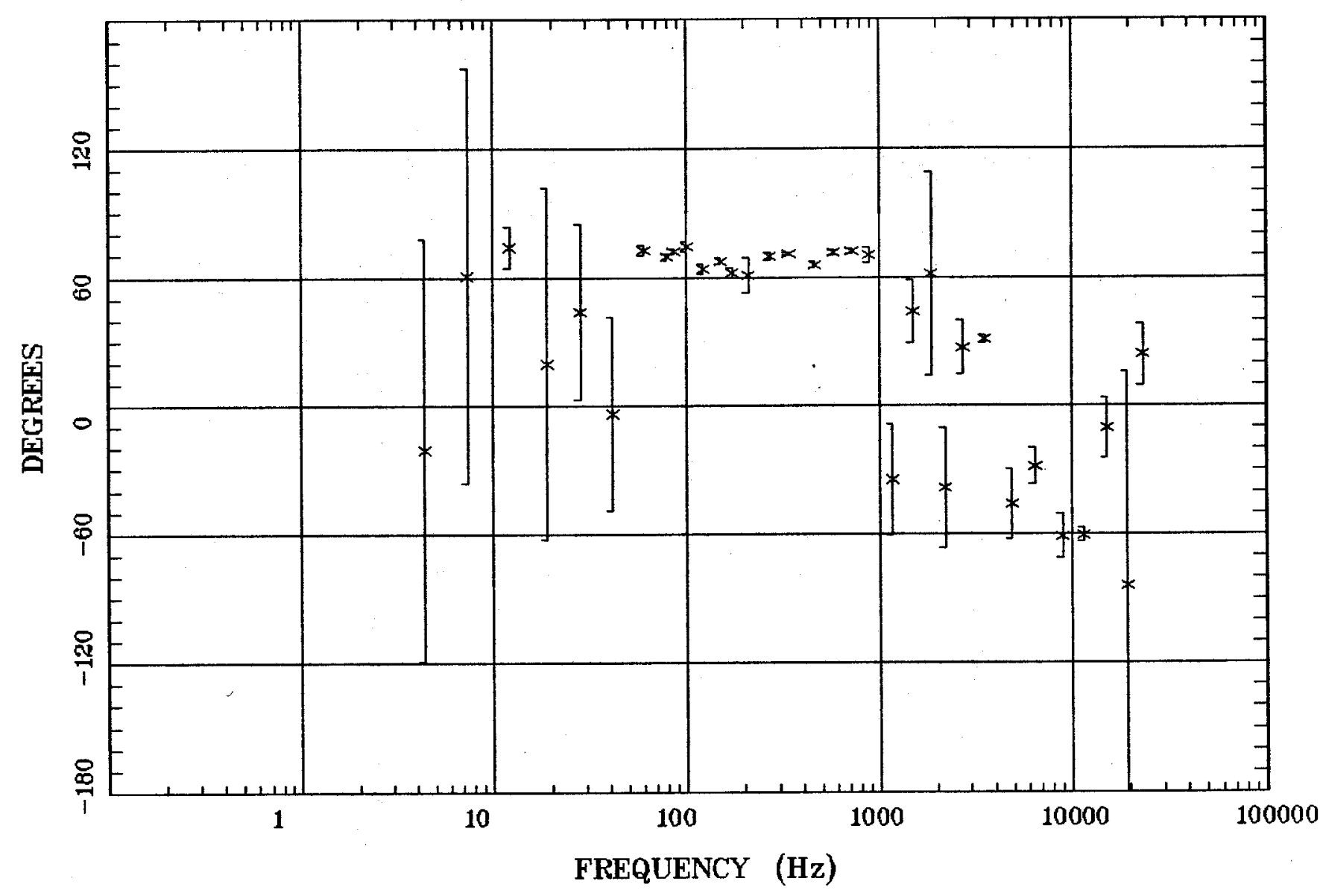

Client:

Remote: e-fld 70m north Acquired: 14:2 Jun 22, 2003 Survey Co:USGS
Rotation:

Filename: cp23.avg

Channels: Ch1 Ch2 Ch3 Ch4 Ch5 Ch6 Ch7

Platted: 09:56 Jul 06, 2004

< EMI - ElectroMagnetic Instruments > 


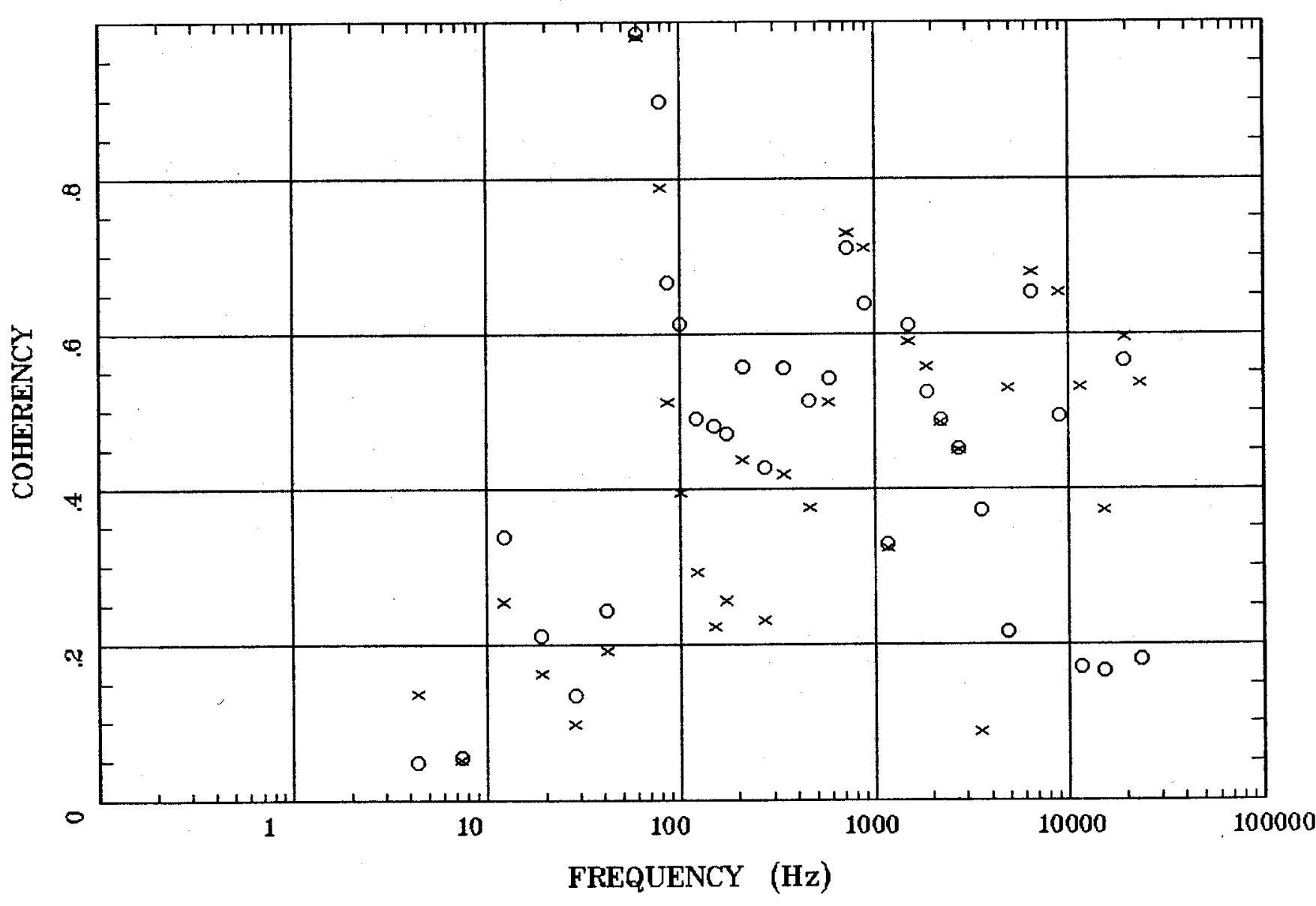

Client:

Remote: e-fld 70m north Acquired: 14:2 Jun 22, 2003 Survey Co:USGS
Rotation:

Filename: cp23.avg

Channels: Ch1 Ch2 Ch3 Ch4 Ch5 Ch6 Ch7 Plotted: 09:56 Jul 06, 2004

< EMI - ElectroMagnetic Instruments > 


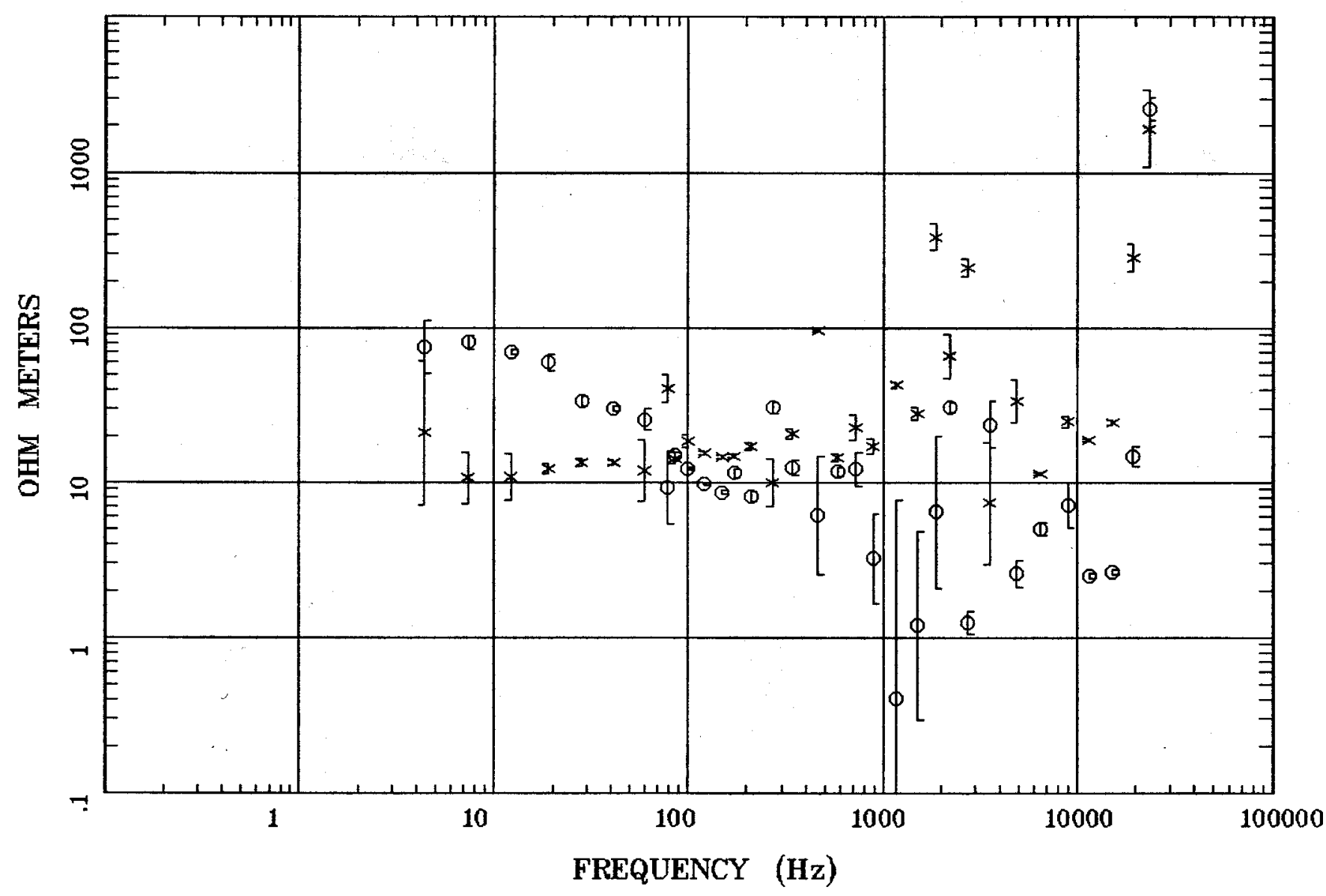

Client:

Remote: e-fld $80 \mathrm{~m}$ east

Acquired: 12:5 Jun 23, 2003 Survey Co:USGS
Rotation:

Filename: cp24a.avg

Channels: Ch1 Ch2 Ch3 Ch4 Ch5 Ch6 Ch7 Plotted: 14:41 Jul 06, 2004

< EMI - ElectroMagnetic Instruments 


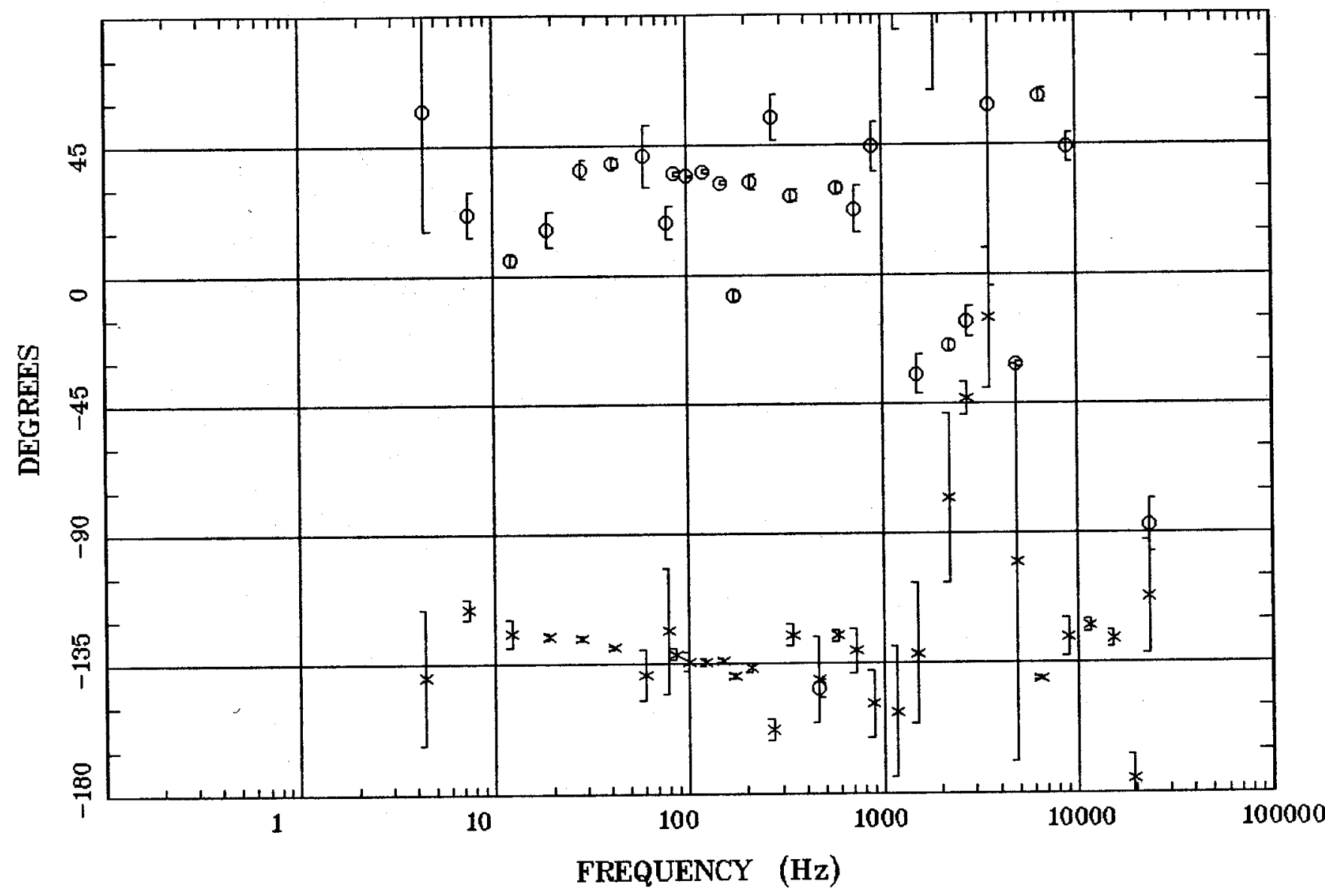

Client:

Remote: e-fld $80 \mathrm{~m}$ east Acquired: 12:5 Jun 23, 2003 Survey Co:USGS
Rotation:

Filename: cp24a.avg

Channels: Ch1 Ch2 Ch3 Ch4 Ch5 Ch6 Ch7

Plotted: 14:41 Jul 06, 2004

$<$ EMI - ElectroMagnetic Instruments > 


\section{ROTATION ANGLE}

Santa Fe, NM

Station 24

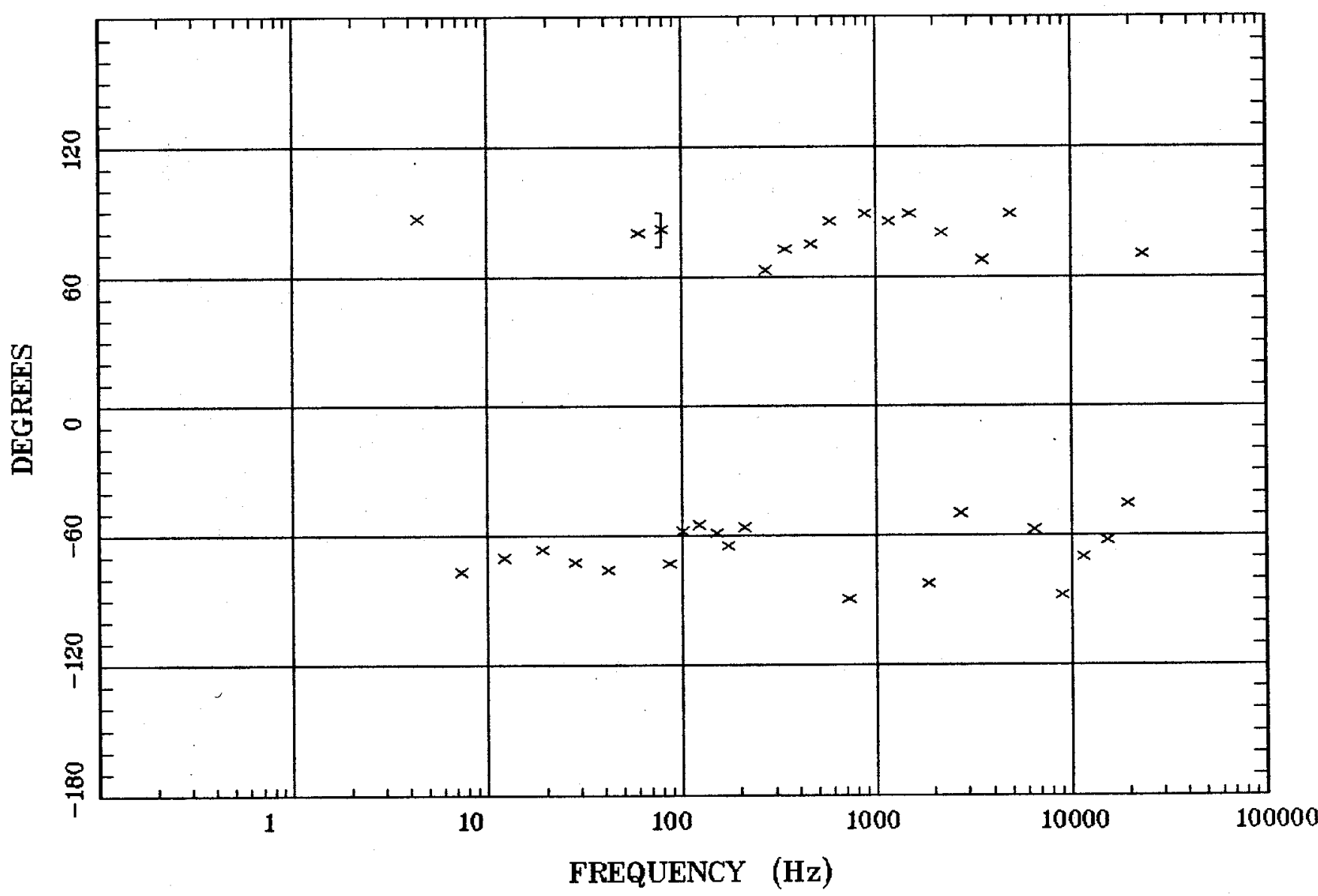

Client:

Remote: e-fld $60 \mathrm{~m}$ east Acquired: 12:5 Jun 23, 2003

Survey Co:USGS
Rotation:

Filename: cp24a.avg

Channels: Ch1 Ch2 Ch3 ch4 ch5 ch6 Chy

Plotted: 12:55 Jul 07, 2004

< EMI - ElectroMagnetic Instruments > 


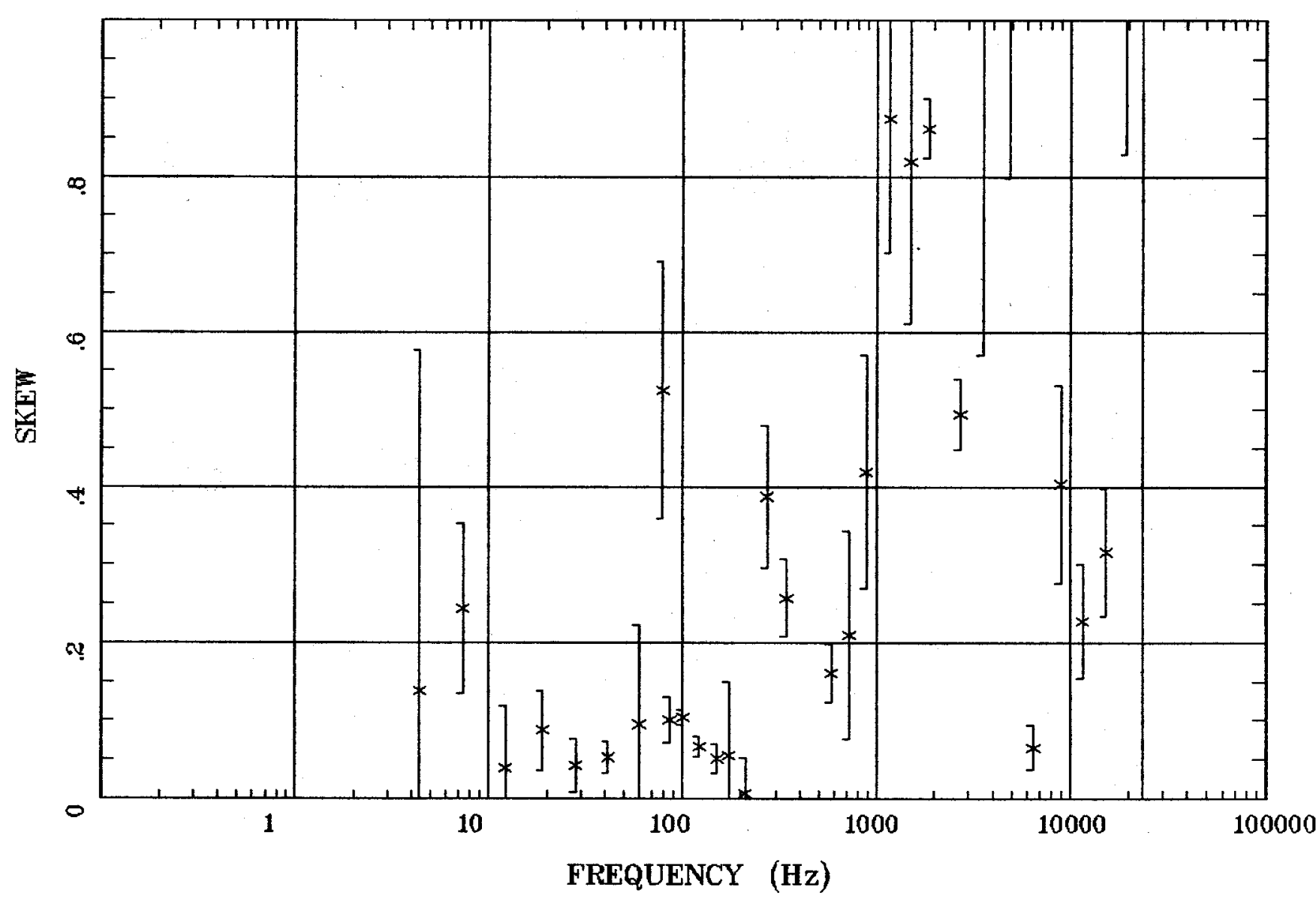

Client:

Remote: e-fld $80 \mathrm{~m}$ east Acquired: 12:5 Jun 23, 2003 Survey Co:USGS
Rotation:

Filename: cp24a.avg

Channels: Ch1 Ch2 Ch3 Ch4 Ch5 Ch6 Ch7 Plotted: 14:41 Jul 06, 2004

< EMI - ElectroMagnetic Instruments 
E MULT Coh.

Santa Fe, NM

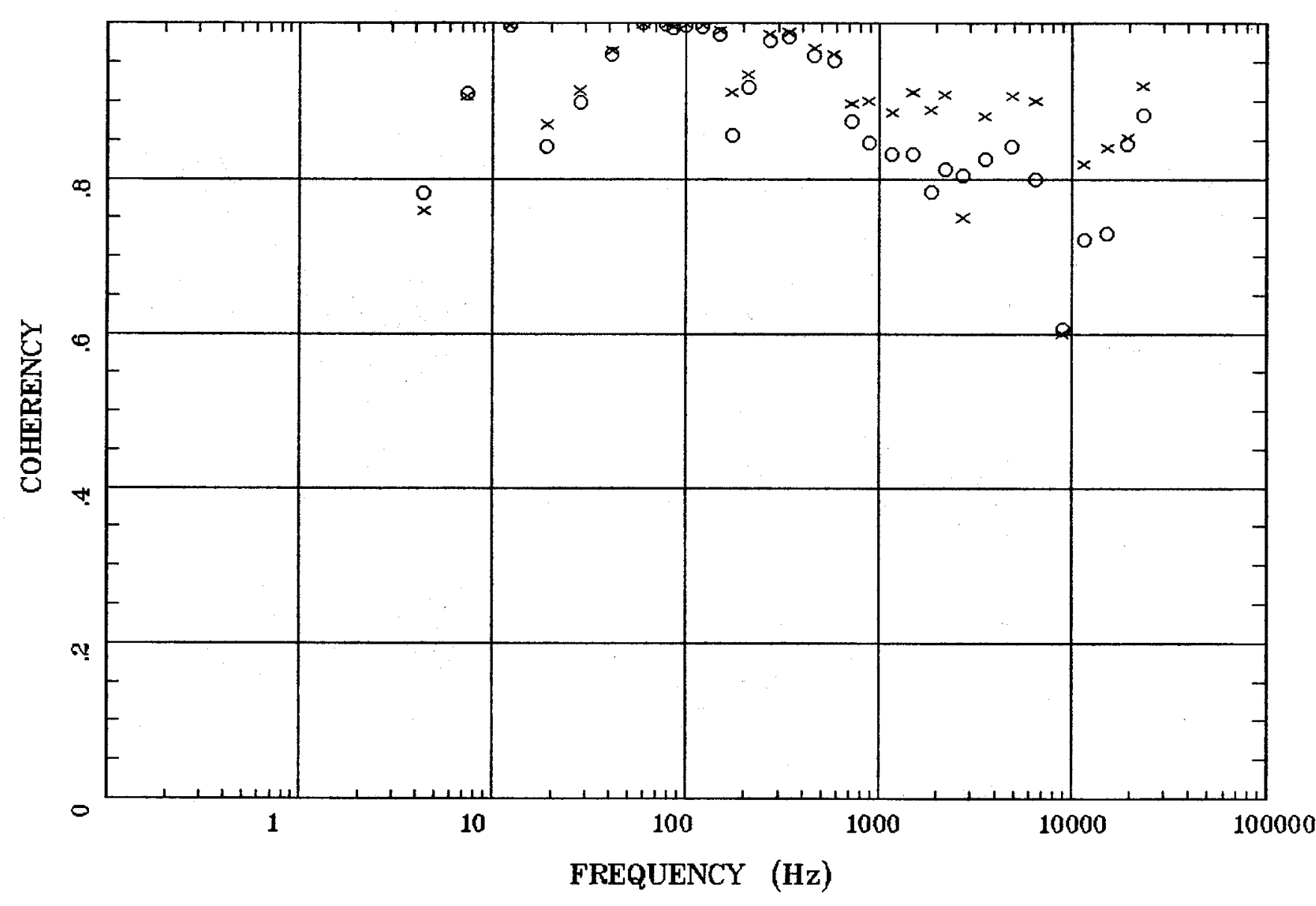

Client:

Remote: e-fld $80 \mathrm{~m}$ east

Acquired: 12:5 Jun 23, 2003

Survey Co:USGS

\section{Rotation:}

Filename: cp24a.avg

Channels: Ch1 Ch2 Ch3 Ch4 Ch5 Ch6 Ch7

Platted: 14:41 Jul 06, 2004

$<$ EMI - ElectroMagnetic Instruments > 


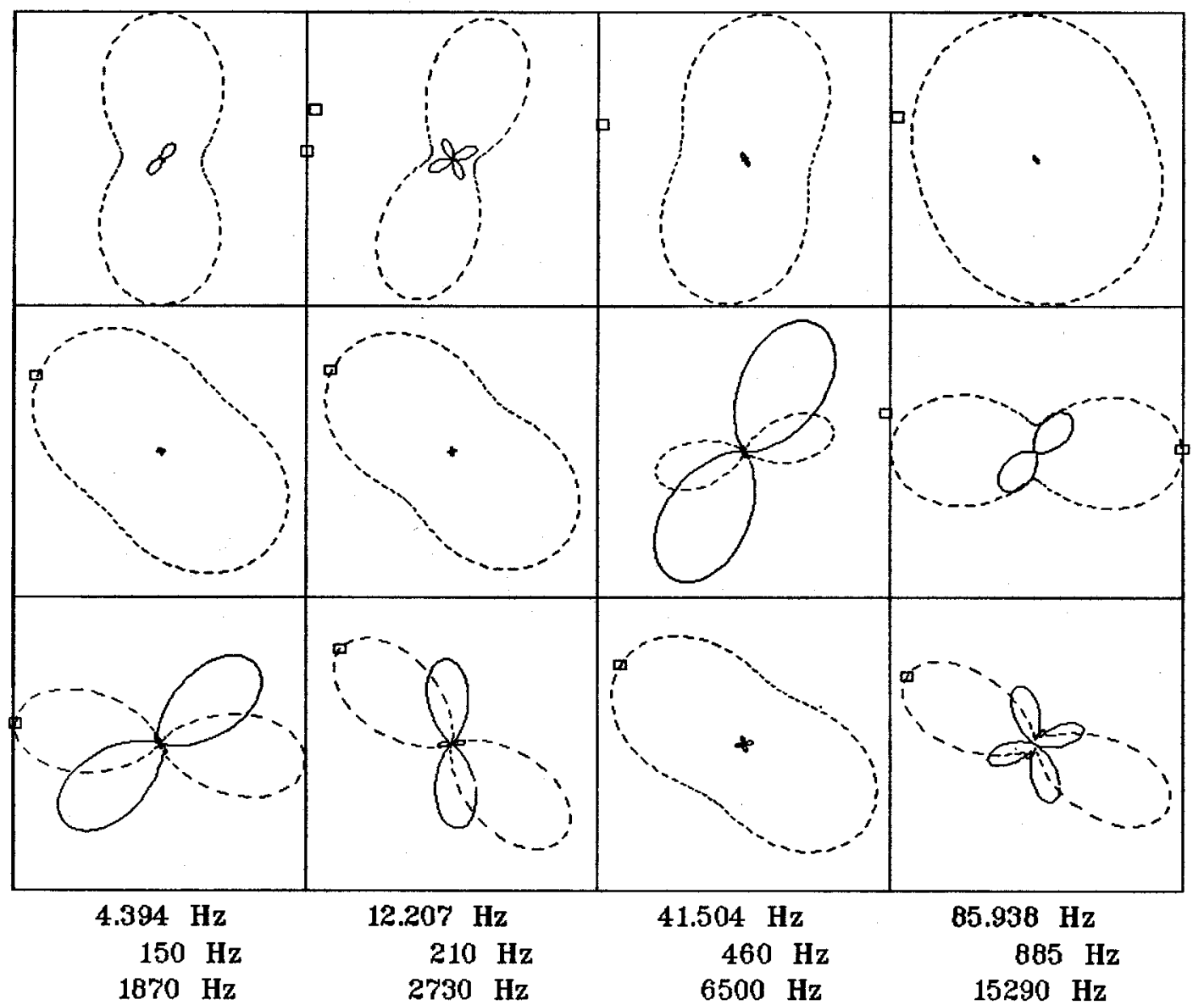

Client:

Remote: e-fld $80 \mathrm{~m}$ east Acquired: 12:5 Jun 23, 2003 Survey Co:USGS
Rotation:

Filename: cp24a.avg

Channels: Ch1 Ch2 Ch3 Ch4 Ch5 Ch6 Chr Plotted: 14:41 Jul 06, 2004

$<$ EMI - ElectroMagnetic Instruments > 


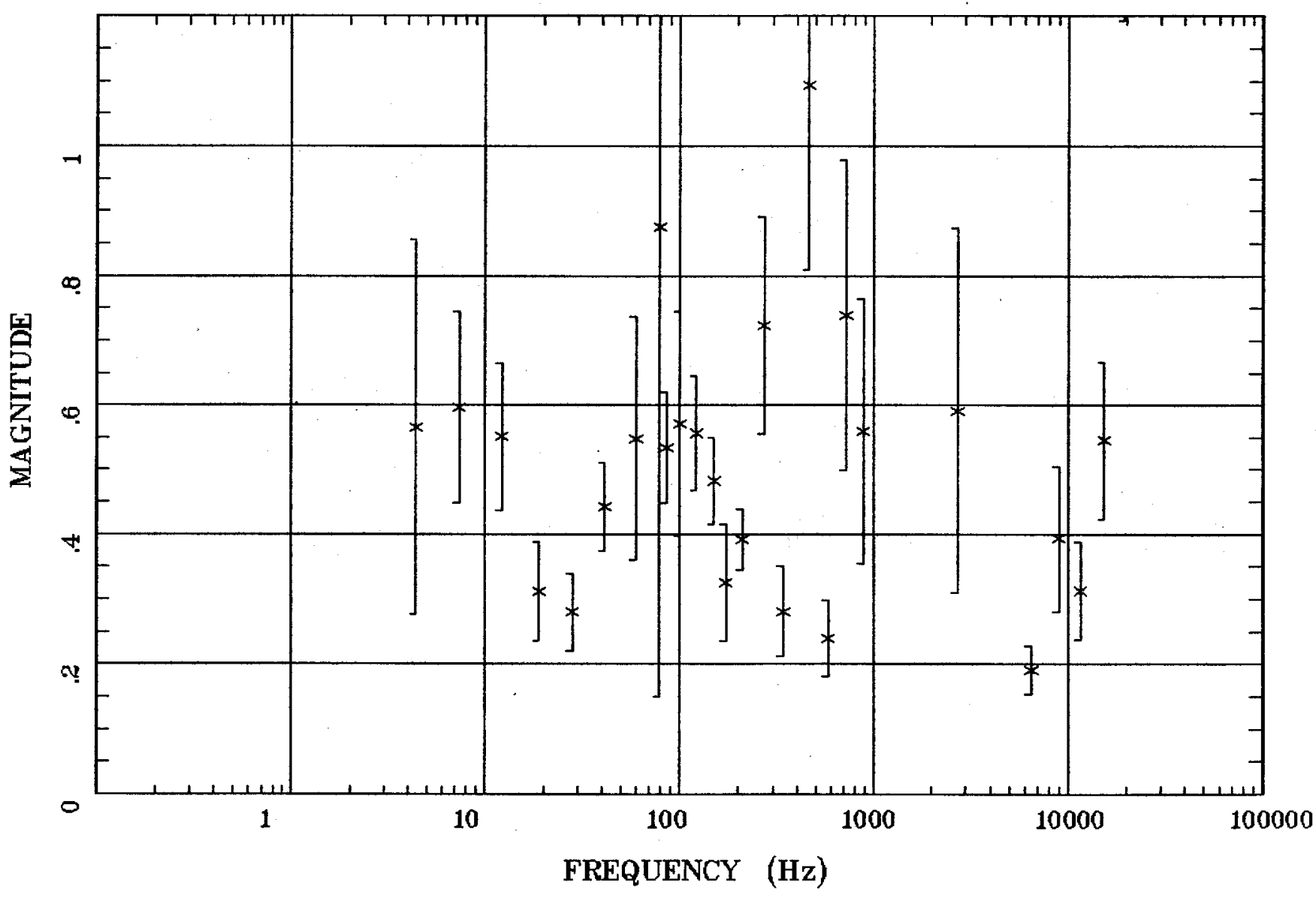

Client:

Remote: e-fld $60 \mathrm{~m}$ east

Acquired: 12:5 Jun 23, 2003

Survey Co:USGS
Rotation:

Filename: cp24a.avg

Channels: Ch1 Ch2 Ch3 Ch4 Ch5 Ch6 Chr

Plotted: 14:41 Jul 06, 2004

$<$ EMI - ElectroMagnetic Instruments > 


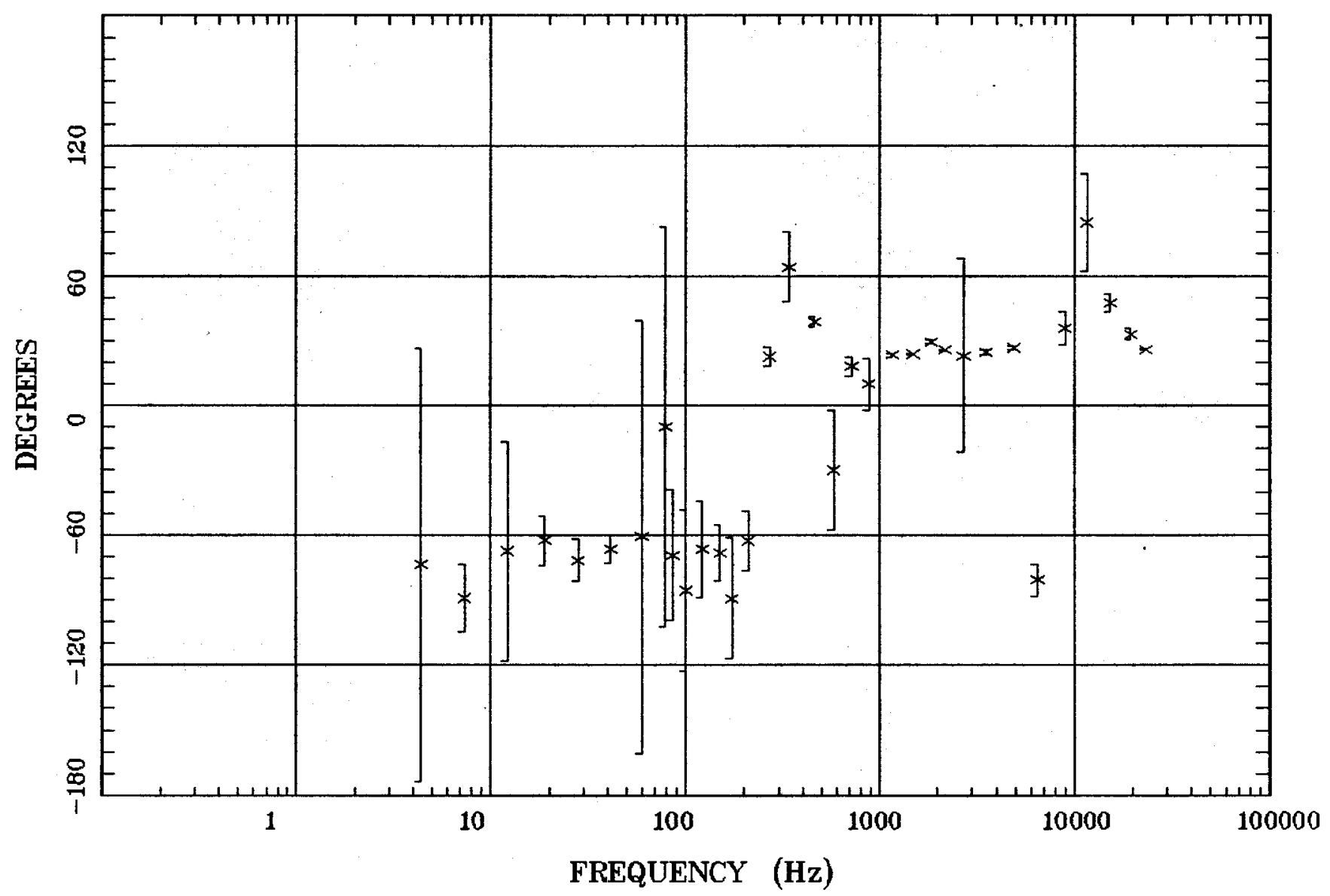

Client:

Remote: e-fld $80 \mathrm{~m}$ east Acquired: 12:5 Jun 23, 2003 Survey Co:USGS
Rotation:

Filename: cp24a.avg

Channels: Ch1 Ch2 Ch3 Ch4 Ch5 Ch6 Ch7 Plotted: 14:41 Jul 06, 2004

$<$ EMI - ElectroMagnetic Instruments > 


\section{HzHx.x Coh HzHy.o}

Santa Fe, NM

Station 24

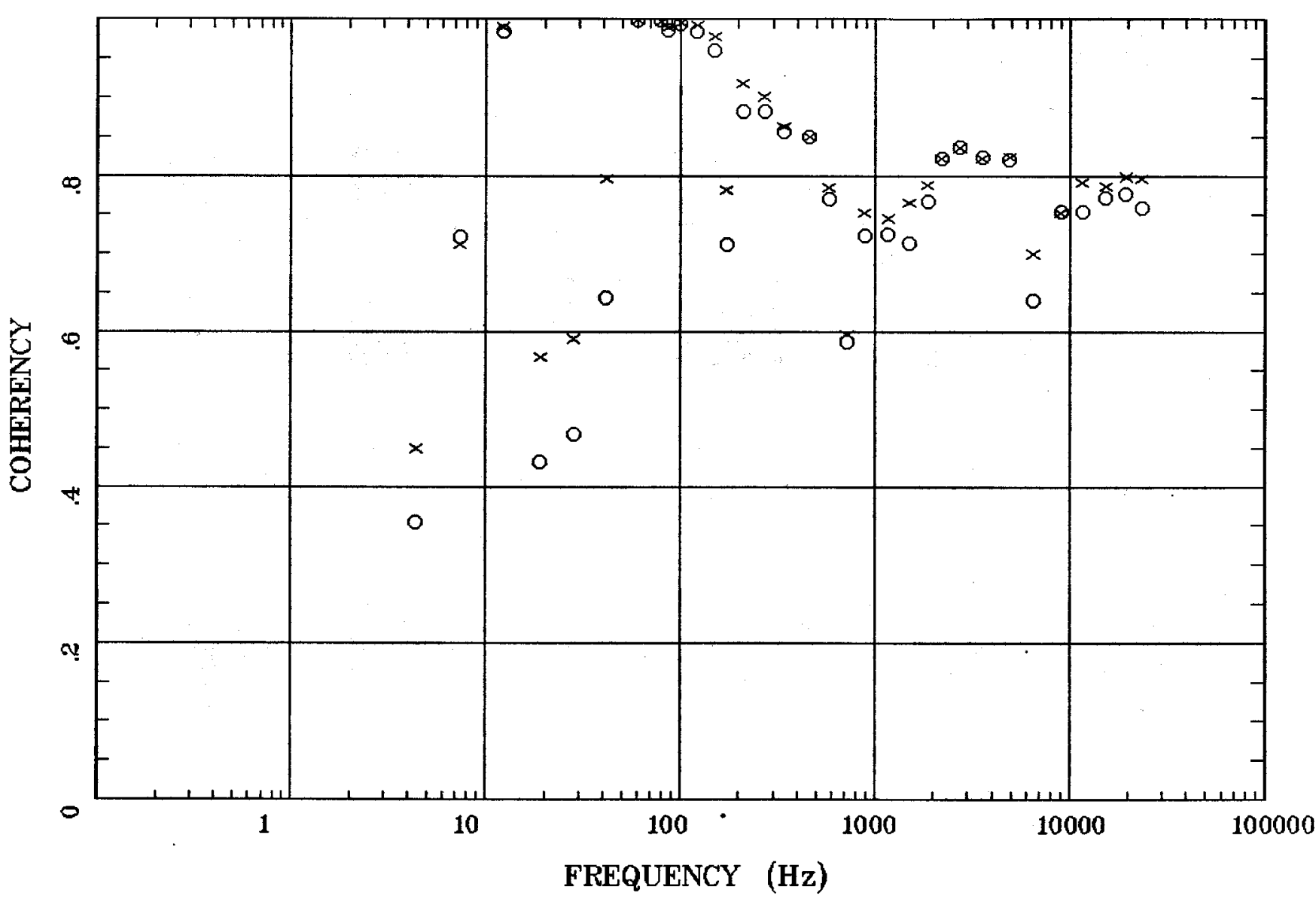

Client:

Remote: e-fld $80 \mathrm{~m}$ east

Acquired: 12:5 Jun 23, 2003

Survey Co:USGS
Rotation:

Filename: cp24a.avg

Channels: Ch1 Ch2 Ch3 Ch4 Ch5 Ch6 Ch7

Plotted: 14:41 Jul 06, 2004

$<$ EMI - ElectroMagnetic Instruments > 


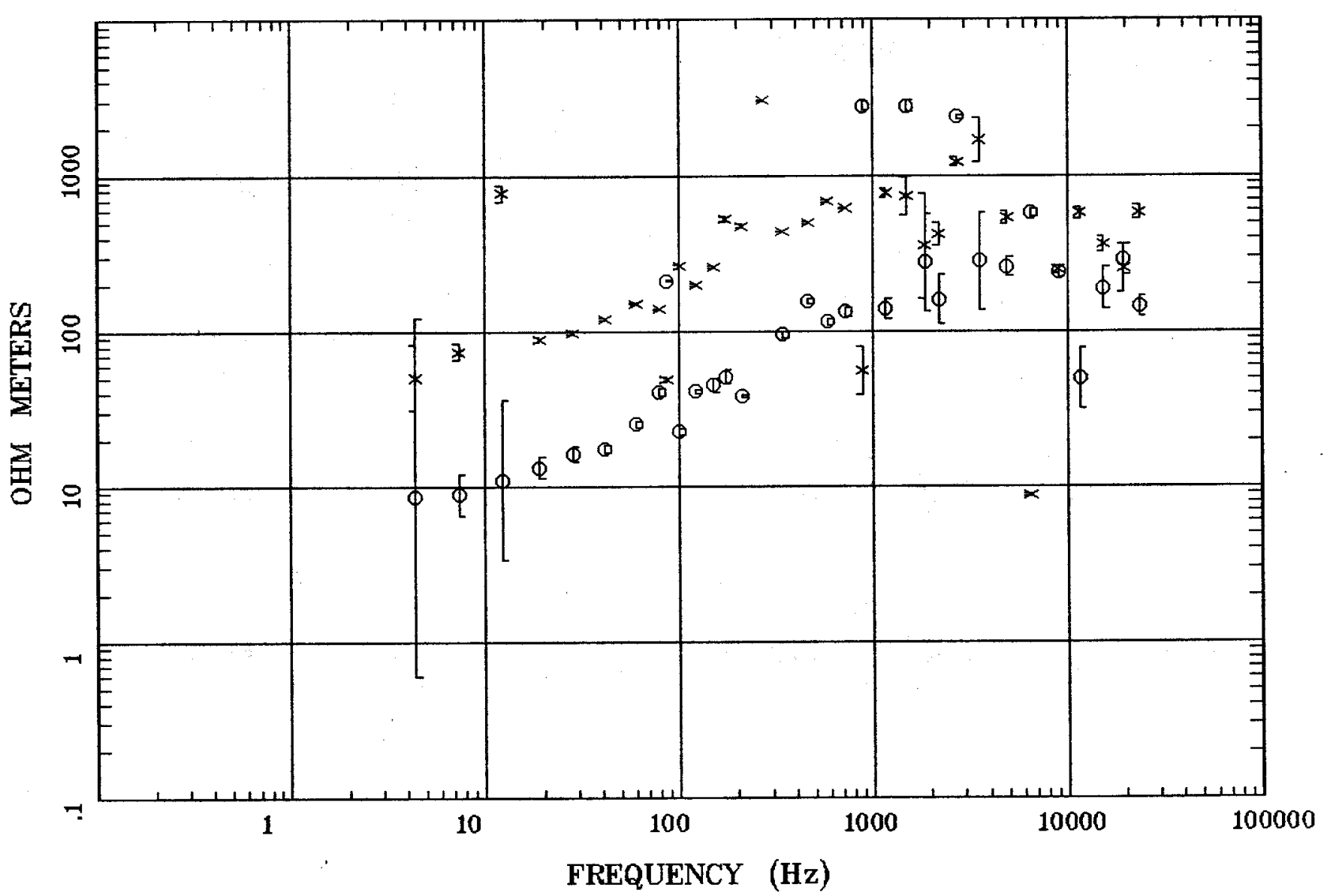

Client:

Remote: e-fld $90 \mathrm{~m}$ east

Acquired: 13:3 Jun 24, 2003

Survey Co:USGS
Rotation:

Filename: cp25.avg

Channels: Ch1 Ch2 Ch3 Ch4 Ch5 Ch6 Ch7

Plotted: 09:57 Jul 06, 2004

< EMI - ElectroMagnetic Instruments > 


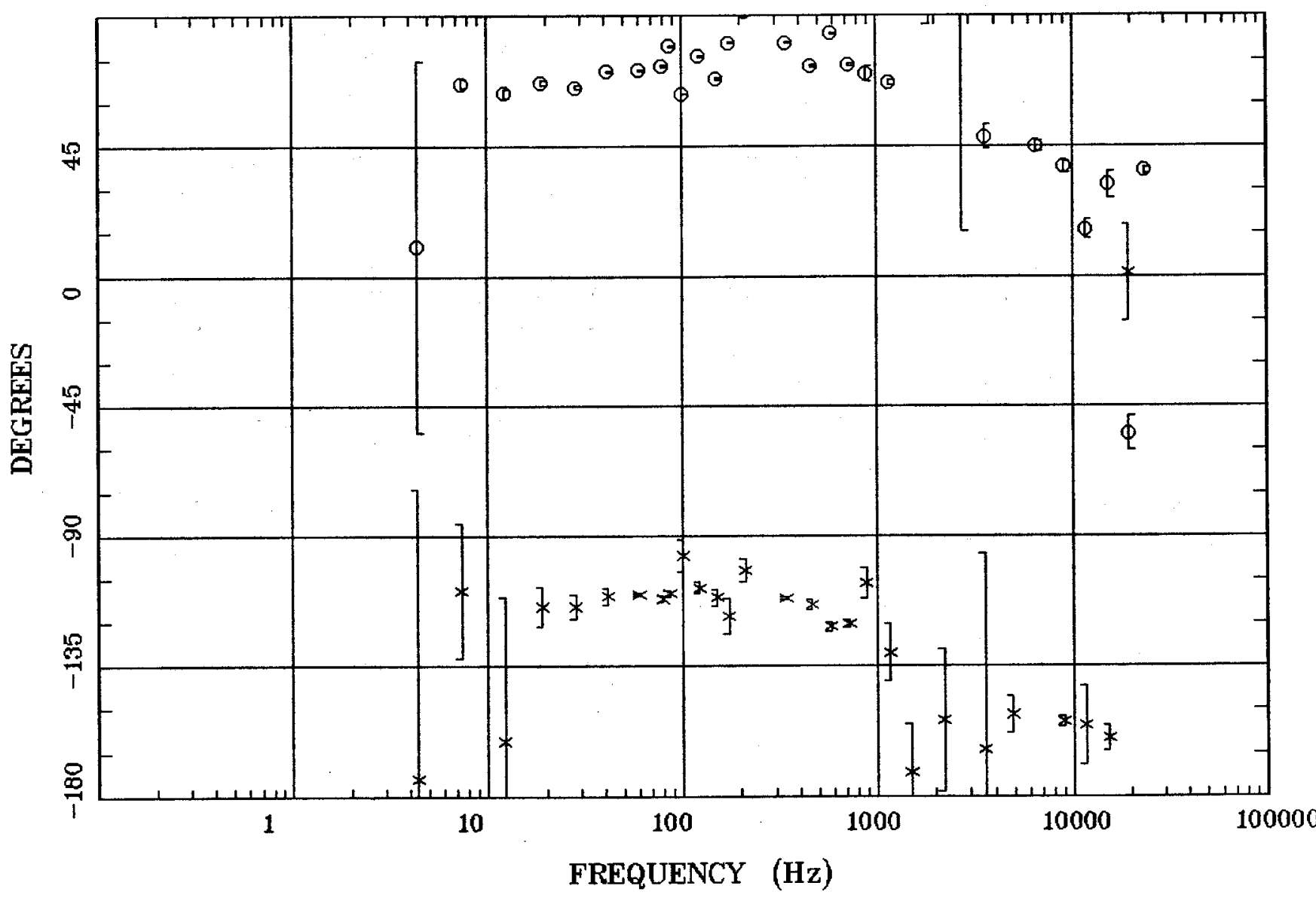

Client:

Remote: e-fld 90m east Acquired: 13:3 Jun 24, 2003 Survey Co:USGS
Rotation:

Filename: cp25.avg

Channels: Ch1 Ch2 Ch3 Ch4 Ch5 Ch6 Ch7 Plotted: 09:57 Jul 06, 2004

< EMI - ElectroMagnetic Instruments > 


\section{ROTATION ANGLE}

Santa Fe, NM

Station 25

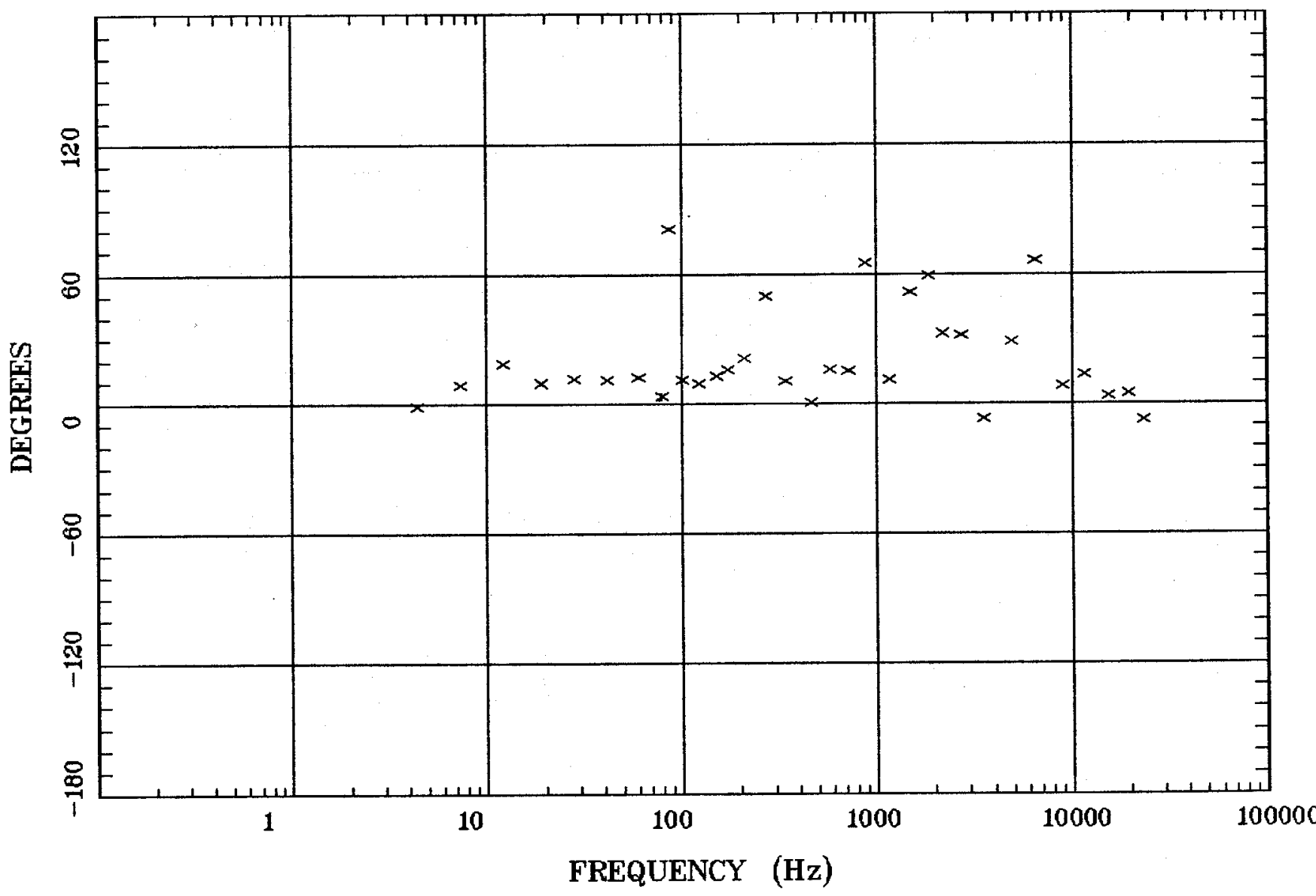

Client:

Remote: e-fld 90m east Acquired: 13:3 Jun 24, 2003 Survey Co:USGS
Rotation:

Filename: cp25.avg

Channels: Ch1 Ch2 Ch3 Ch4 Ch5 Ch6 Ch7

Plotted: 12:56 Jul 07, 2004

$<$ EMI - ElectroMagnetic Instruments > 


\section{IMPEDANCE SKEW}

Santa Fe, NM

Station 25

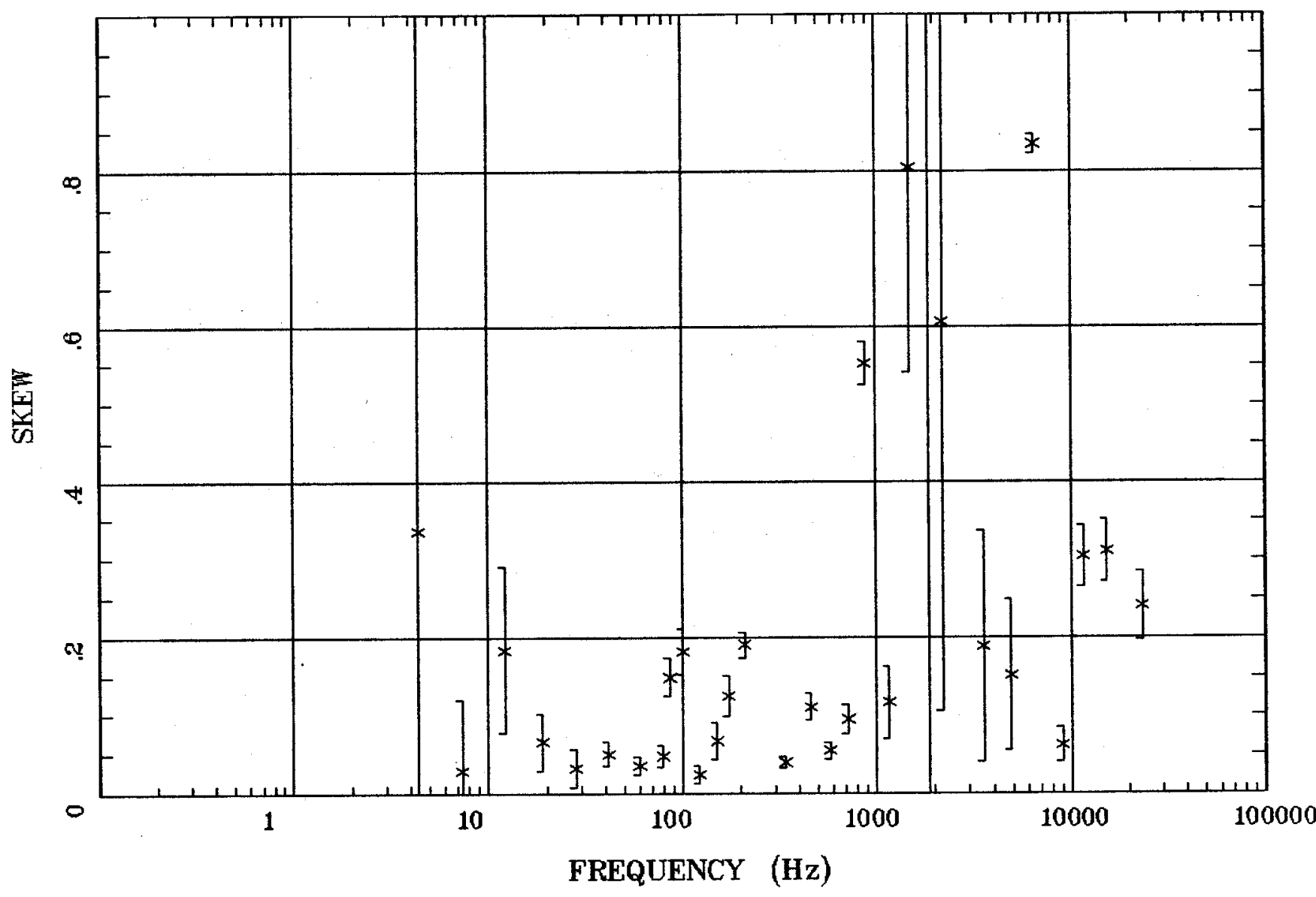

Client:

Remote: e-fld $90 \mathrm{~m}$ east Acquired: 13:3 Jun 24, 2003 Survey Co:USGS
Rotation:

Filename: cp25.avg

Channels: Ch1 Ch2 Ch3 Ch4 Ch5 Ch6 Ch7

Plotted: 09:57 Jul 06, 2004

< EMI - ElectroMagnetic Instruments > 
Station 25

E MULT Coh.

Santa Fe, NM

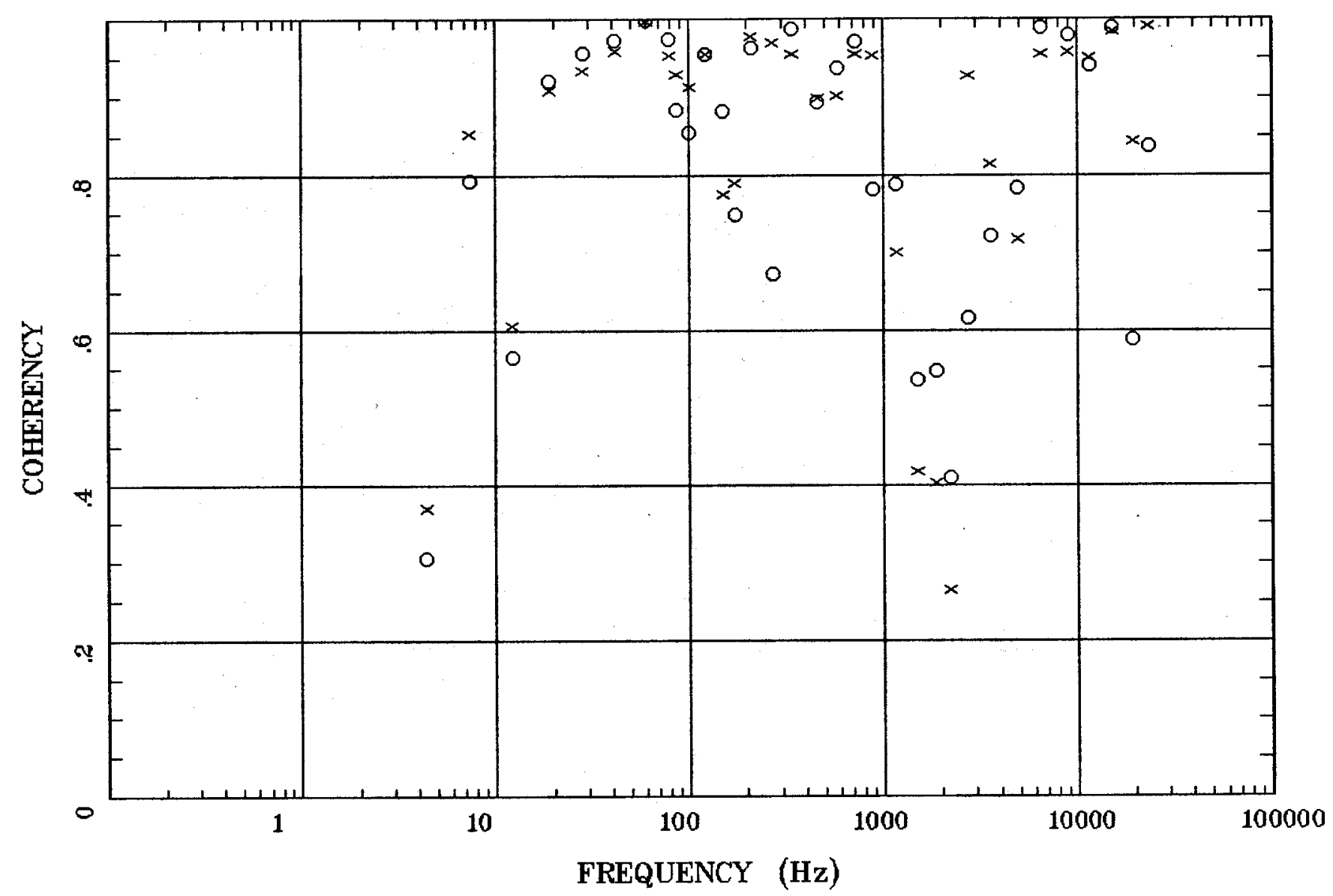

Client:

Remote: e-fld $90 \mathrm{~m}$ east

Acquired: 13:3 Jun 24, 2003

Survey Co:USGS

Rotation:

Filename: cp25.avg

Channels: Ch1 Ch2 Ch3 Ch4 Ch5 Ch6 Ch7

Plotted: 09:57 Jul 06, 2004

$<$ EMI - ElectroMagnetic Instruments > 


\section{POLAR PLOTS}

Santa Fe, NM

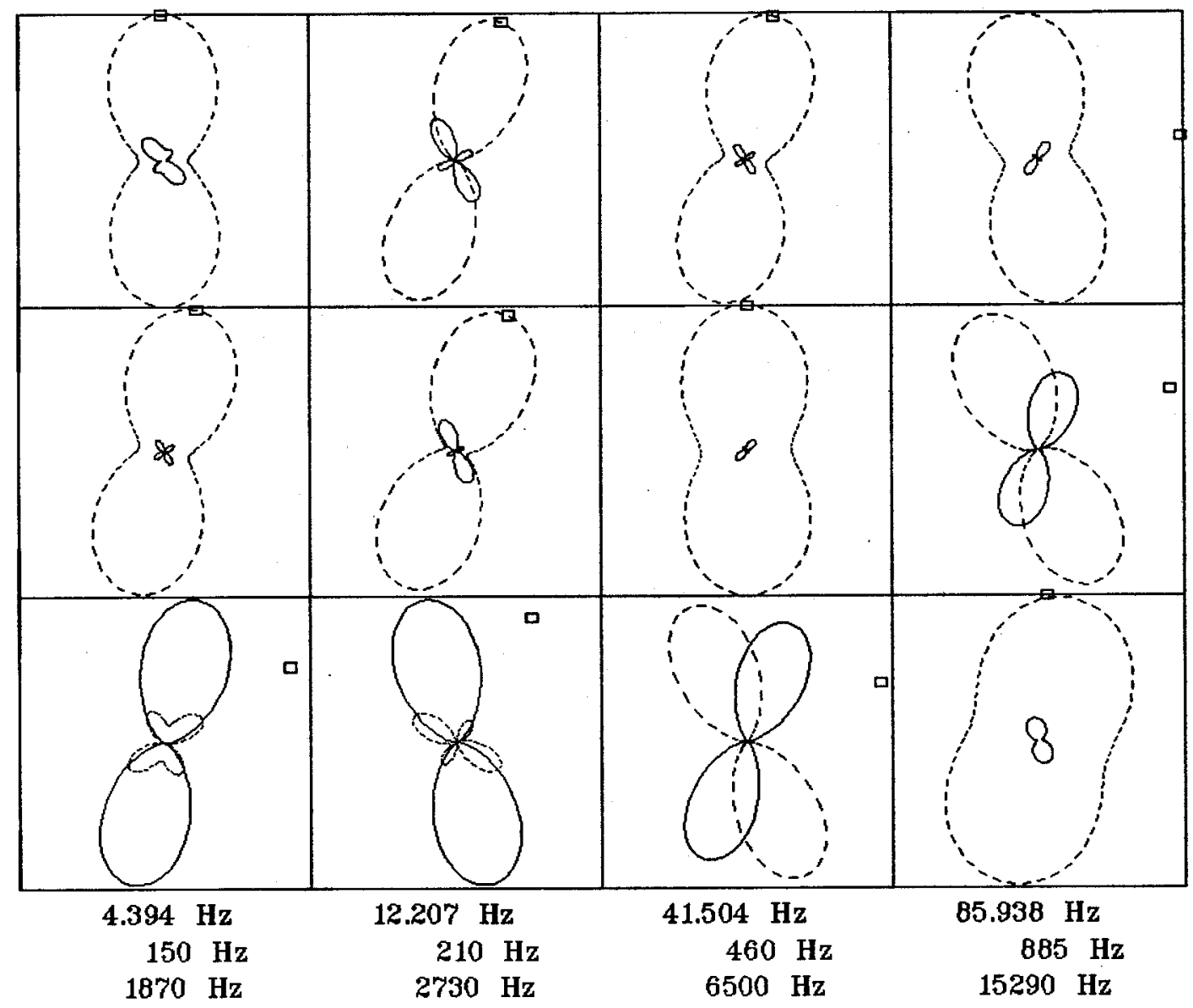

Rotation:

Client:

Remote: e-fld $90 \mathrm{~m}$ east

Acquired: 13:3 Jun 24, 2003 Survey Co:USGS
Filename: cp25.avg

Channels: Ch1 Ch2 Ch3 Ch4 Ch5 Ch6 Ch7

Plotted: 09:57 Jul 06, 2004

< EMI - ElectroMagnetic Instruments 


\section{TIPPER MAGNITUDE}

Santa Fe, NM

Station 25

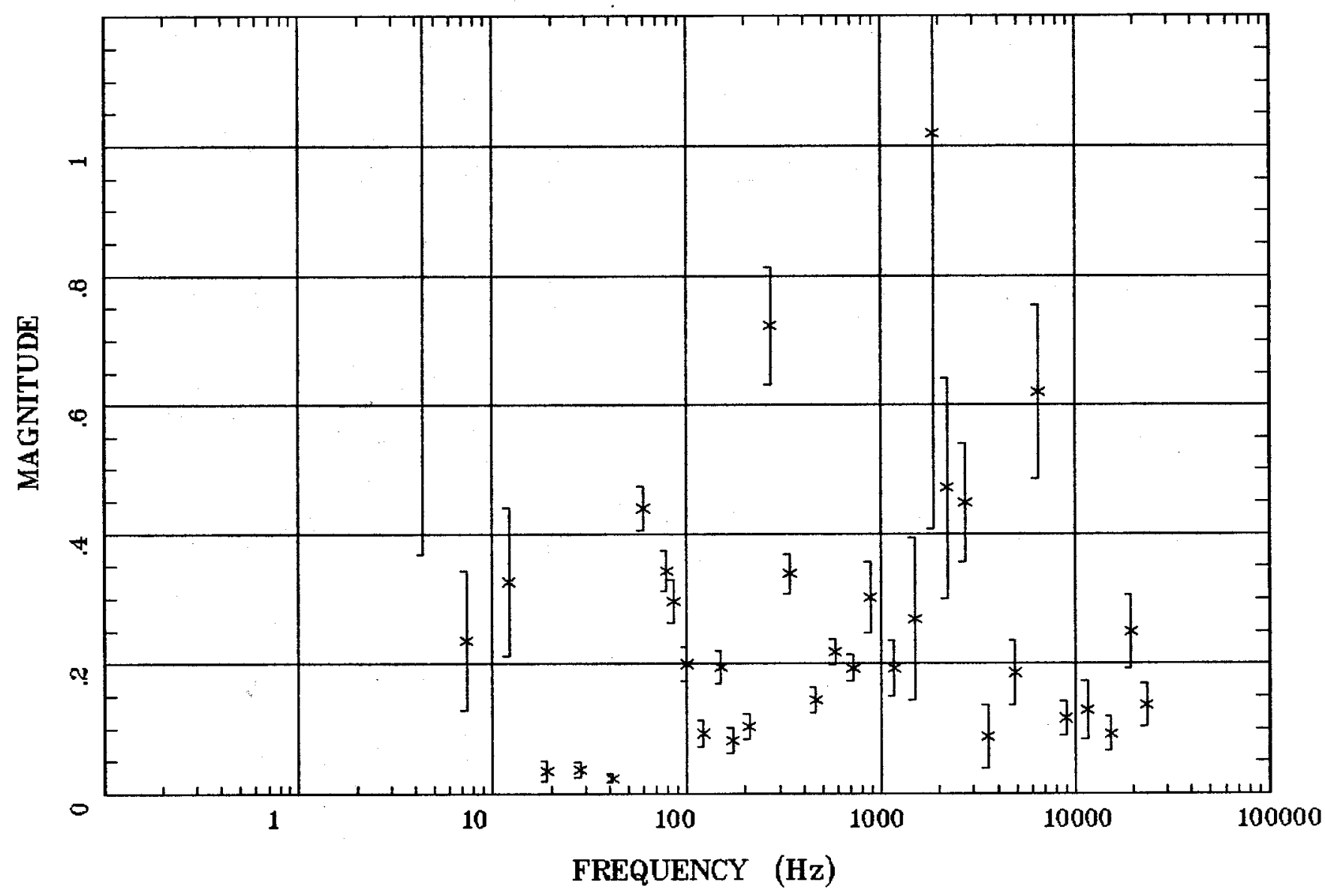

Client:

Remote: e-fld $90 \mathrm{~m}$ east

Acquired: 13:3 Jun 24, 2003

Survey Co:USGS
Rotation:

Filename: cp25.avg

Channels: Ch1 Ch2 Ch3 Ch4 Ch5 Ch6 Ch7

Plotted: 09:57 Jul 06, 2004

< EMI - ElectroMagnetic Instruments > 


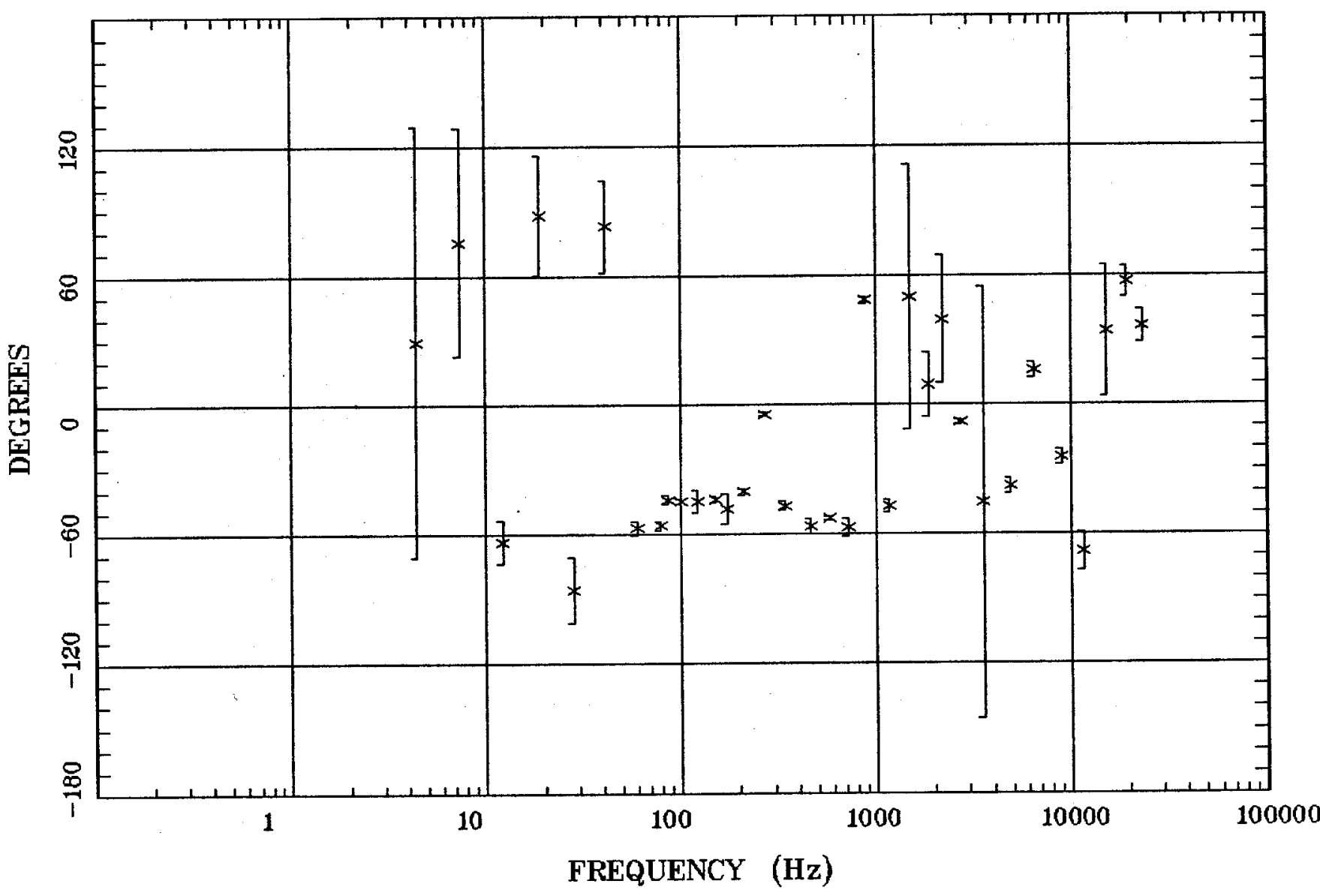

Client:

Remote: $e-f l d ~ 90 m$ east Acquired: 13:3 Jun 24, 2003 Survey Co:USGS
Rotation:

Filename: cp25.avg

Channels: Ch1 Ch2 Ch3 Ch4. Ch5 Ch6 Chr

Plotted: 09:57 Jul 06, 2004

< EMI - ElectroMagnetic Instruments > 


\section{HzHx.x Coh HzHy.o}

Santa Fe, NM

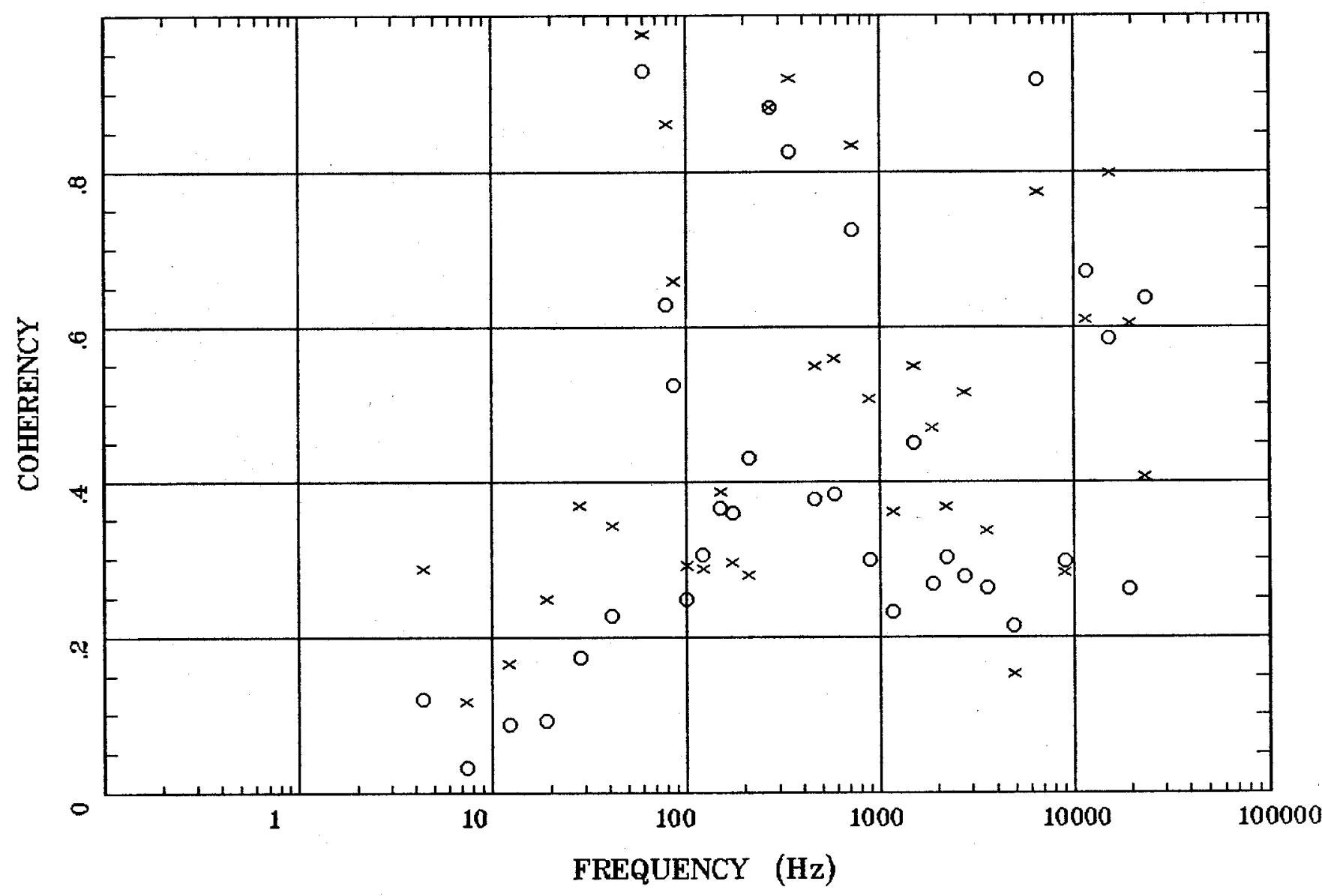

Client:

Remate: e-fld $90 \mathrm{~m}$ east Acquired: 13:3 Jun 24, 2003 Survey Ca:USGS
Rotation:

Filename: cp25.avg

Channels: Ch1 Ch2 Ch3 Ch4 Ch5 Ch6 Ch7 Plotted: 09:57 Jul 06, 2004

< EMI - ElectroMagnetic Instruments 


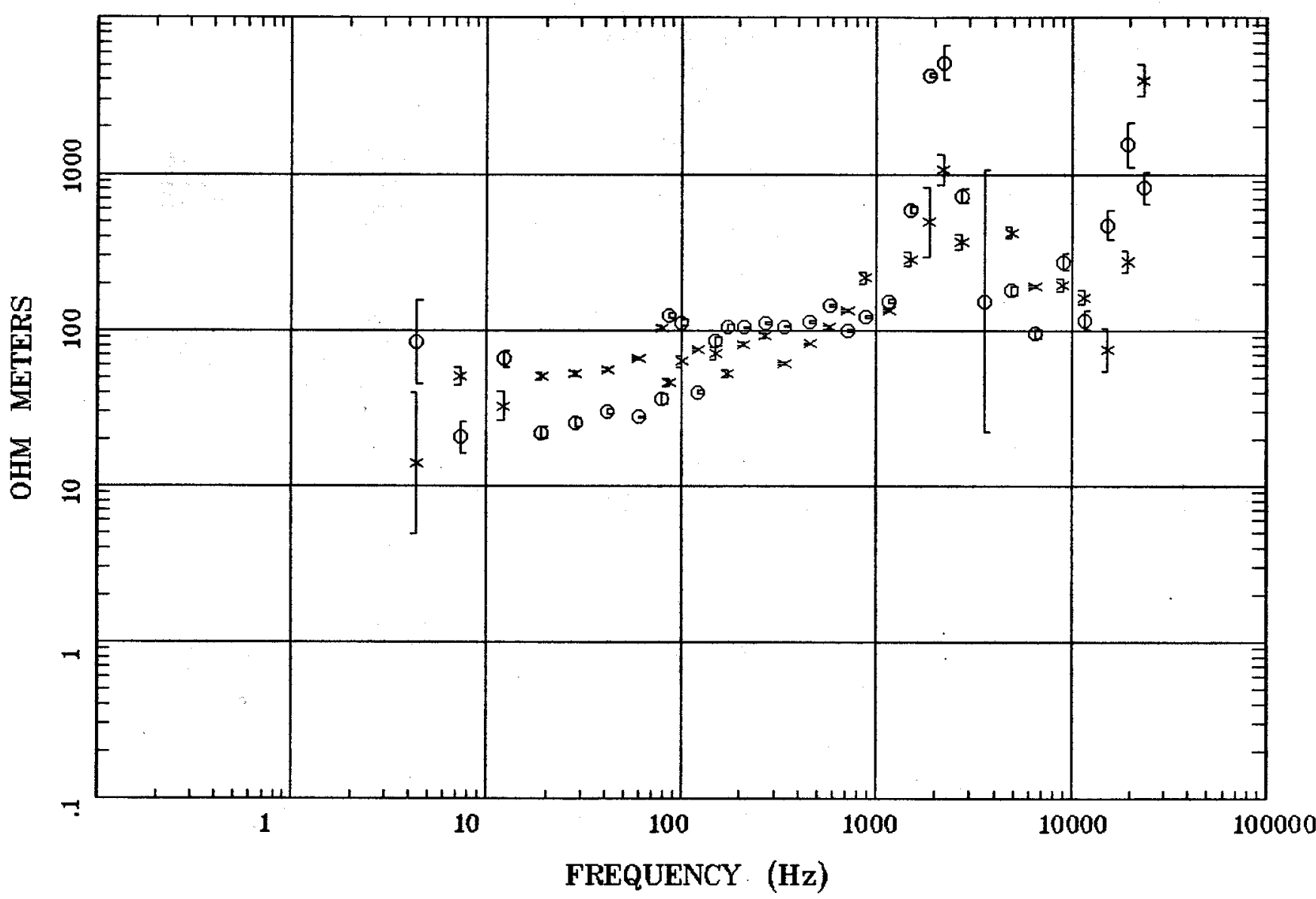

Client:

Remote: e-fld 64m east

Acquired: 14:4 Jun 25, 2003 Survey Co:USGS
Rotation:

Filename: cp26a.avg

Channels: Ch1 Ch2 Ch3 Ch4 Ch5 Ch6 Ch7 Plotted: 14:42 Jul 06, 2004

<EMI - ElectroMagnetic Instruments > 


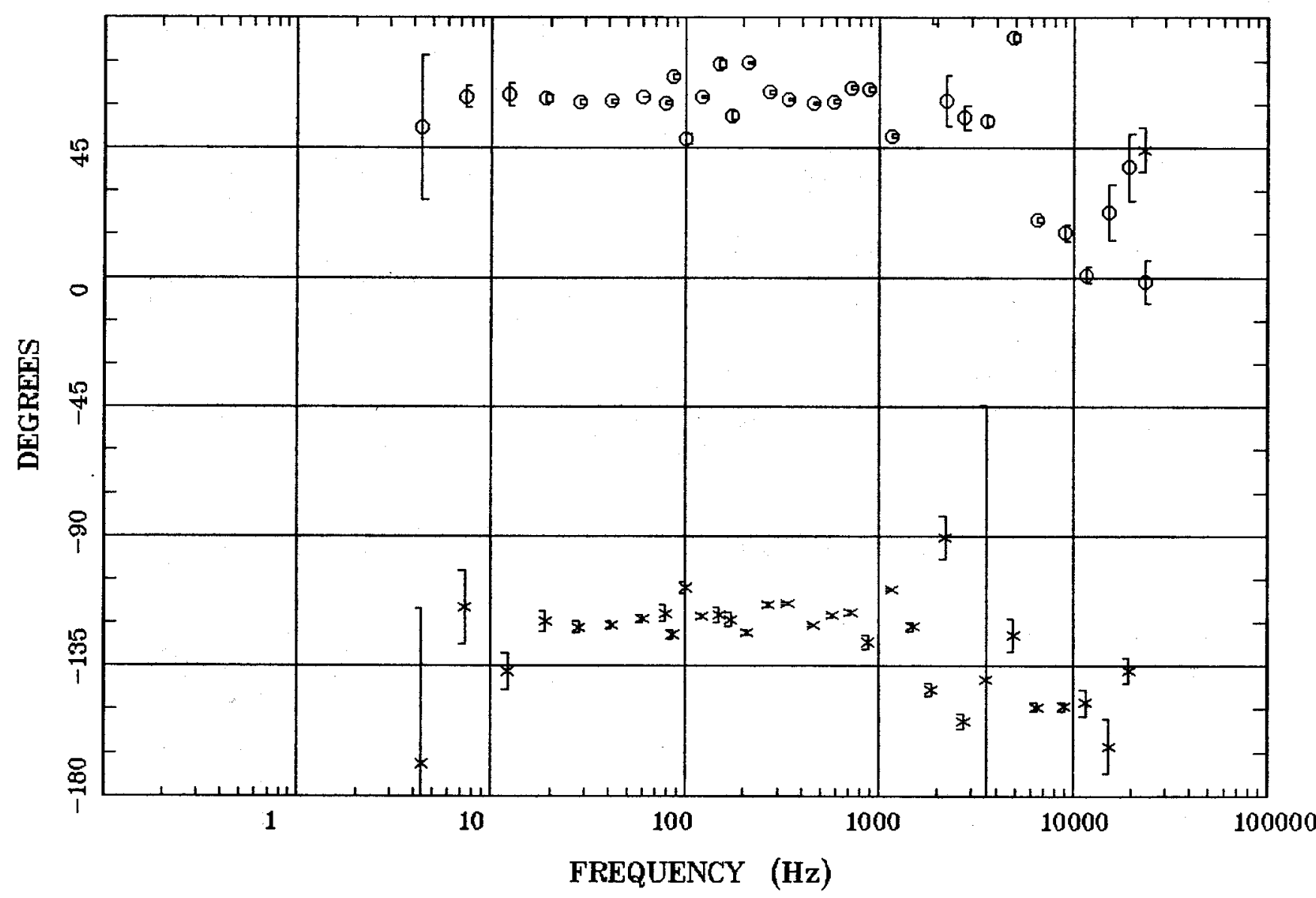

Client:

Remote: e-fld $64 \mathrm{~m}$ east Acquired: 14:4 Jun 25, 2003 Survey Co:USGS
Rotation:

Filename: cp26a.avg

Channels: Ch1 Ch2 Ch3 Ch4 Ch5 Ch6 Ch7 Plotted: 14:42 Jul 06, 2004

$<$ EMI - ElectroMagnetic Instruments > 


\section{ROTATION ANGLE}

Santa Fe, NM

Station 26

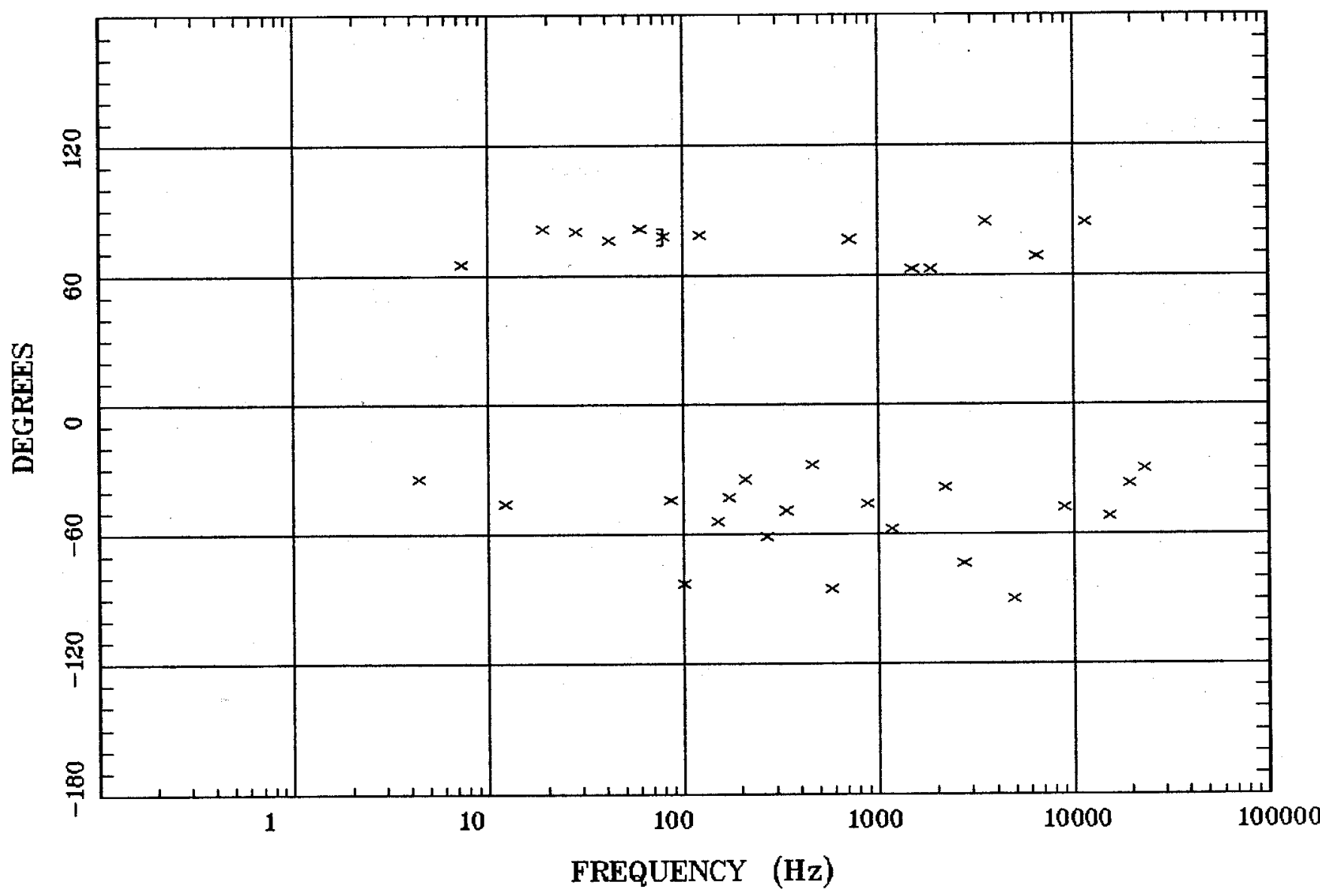

Client:

Remote: e-fld $64 \mathrm{~m}$ east Acquired: 14:4 Jun 25, 2003 Survey Co:USGS
Rotation:

Filename: cp26a.avg

Channels: Ch1 Ch2 Ch3 Ch4 Ch5 Ch6 Ch7

Plotted: 12:57 Jul 07, 2004

$<$ EMI - ElectroMagnetic Instruments > 


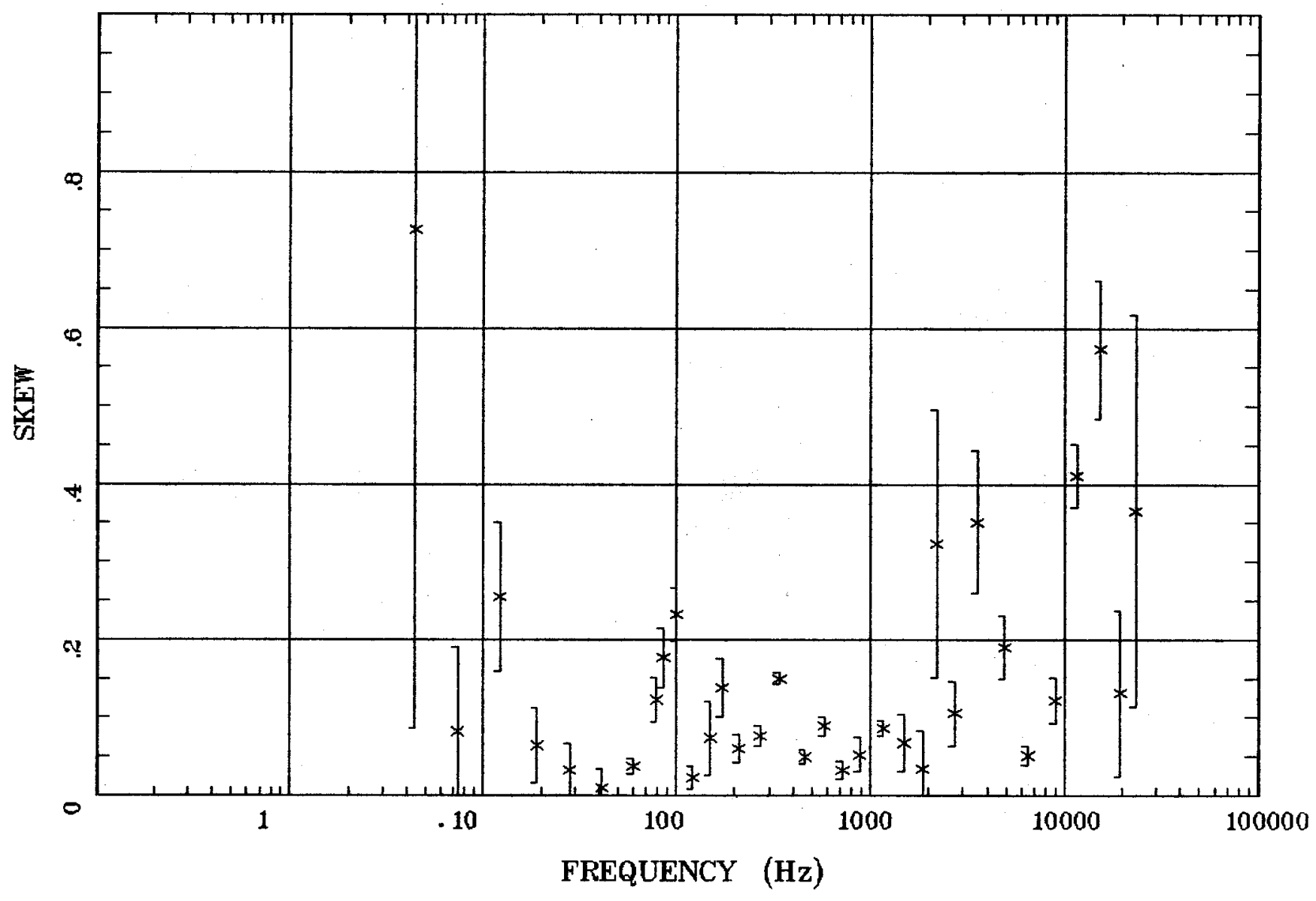

Client:

Remote: e-fld .64m east Acquired: 14:4 Jun 25, 2003 Survey Co:USGS
Rotation:

Filename: cp26a.avg

Channels: Ch1 Ch2 Ch3 Ch4 Ch5 Ch6 Ch7

Plotted: 14:42 Jul 06, 2004

< EMI - ElectroMagnetic Instruments > 
Station 26

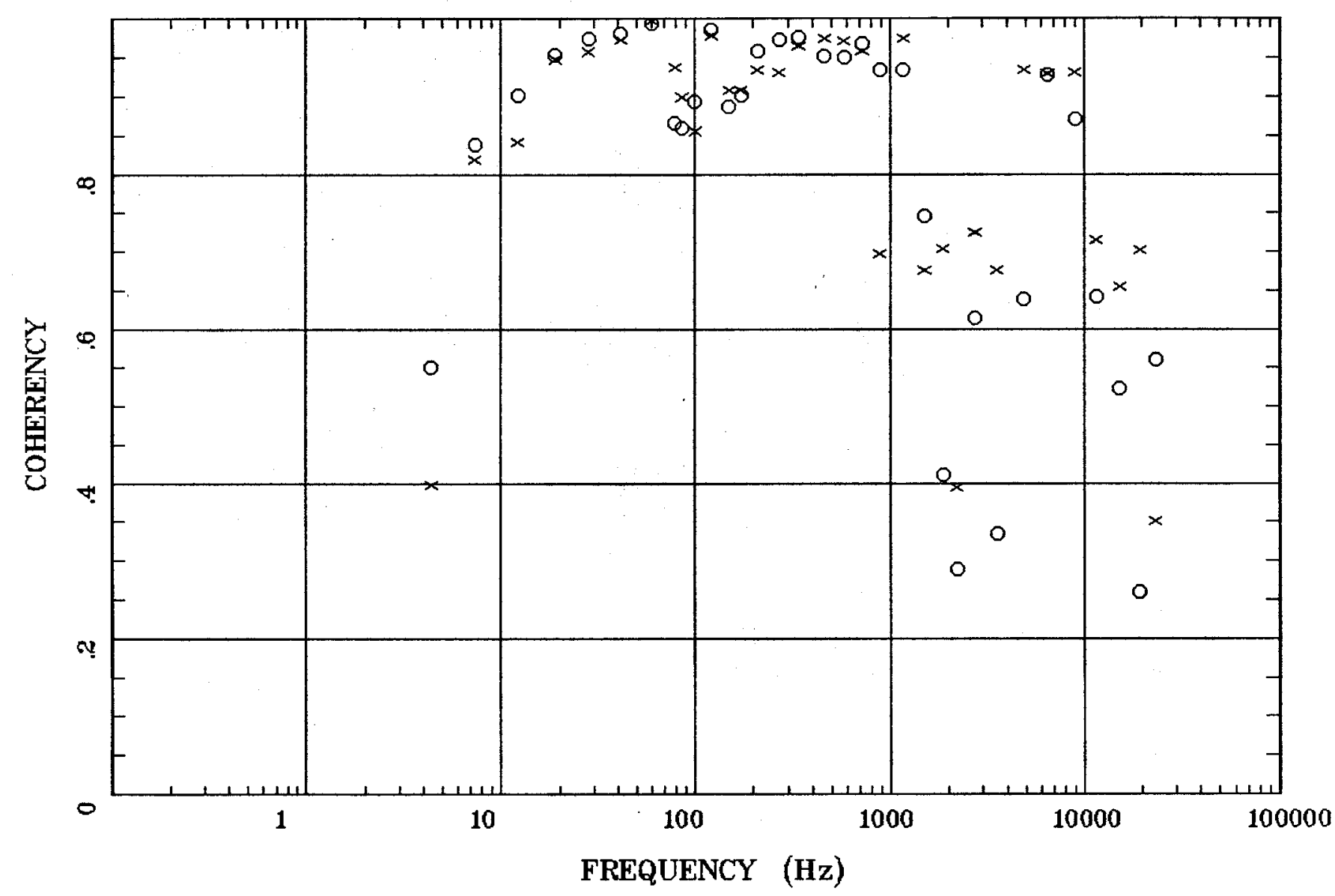

Client:

Remote: e-fld $64 \mathrm{~m}$ east

Acquired: 14:4 Jun 25, 2003

Survey Co:USGS
Rotation:

Filename: cp26a.avg

Channels: Ch1 Ch2 Ch3 Ch4 Ch5 Ch6 Ch7

Plotted: 14:42 Jul 06, 2004

$<$ EMI - ElectroMagnetic Instruments 


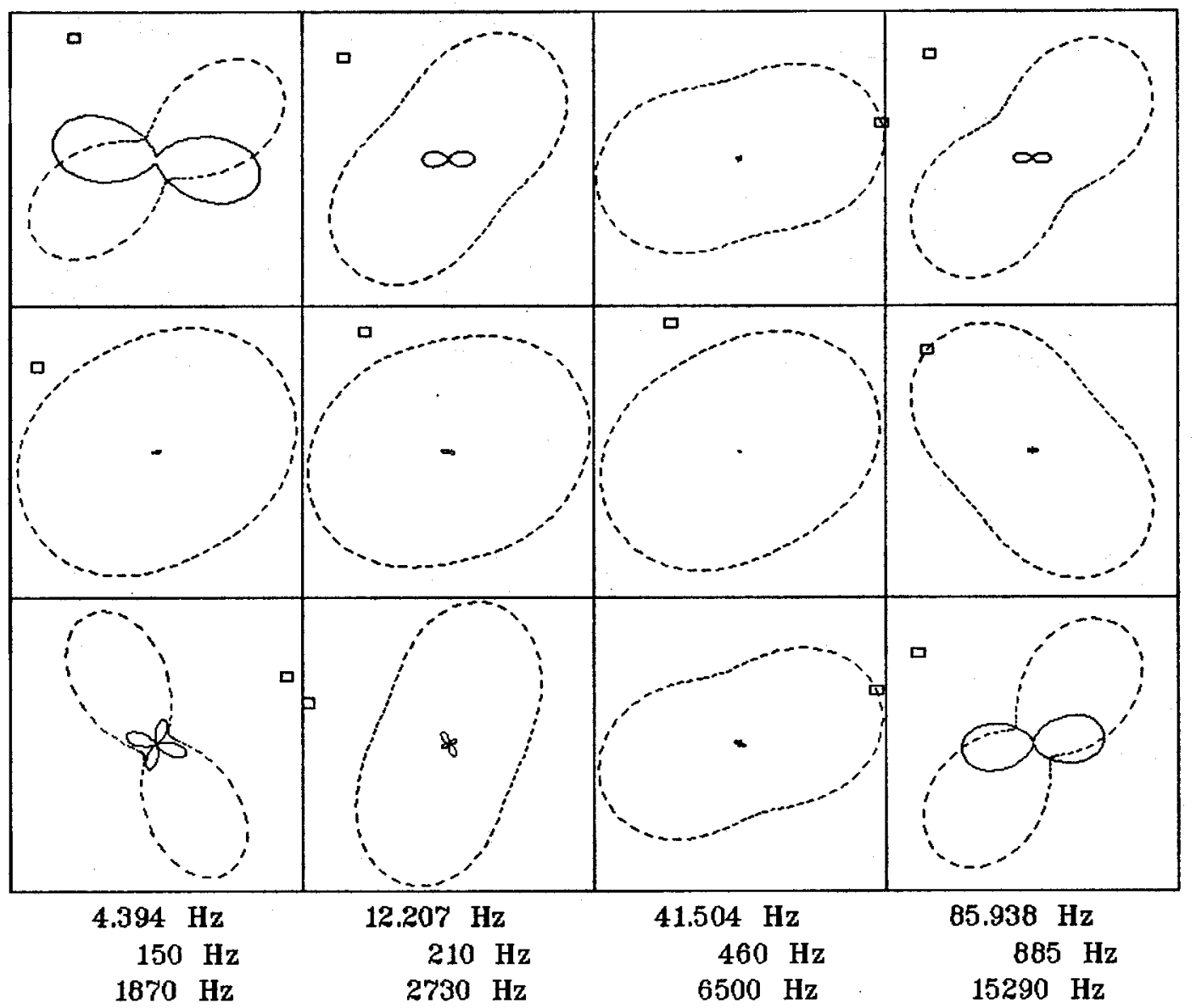

Rotation:

Client:

Remote: e-fld $64 \mathrm{~m}$ east

Acquired: 14:4 Jun 25, 2003 Survey Co:USGS
Filename: cp26a.avg

Channels: Ch1 Ch2 Ch3 Ch4 Ch5 Ch6 Ch7 Plotted: 14:42 Jul 06, 2004

< EMI - ElectroMagnetic Instruments > 


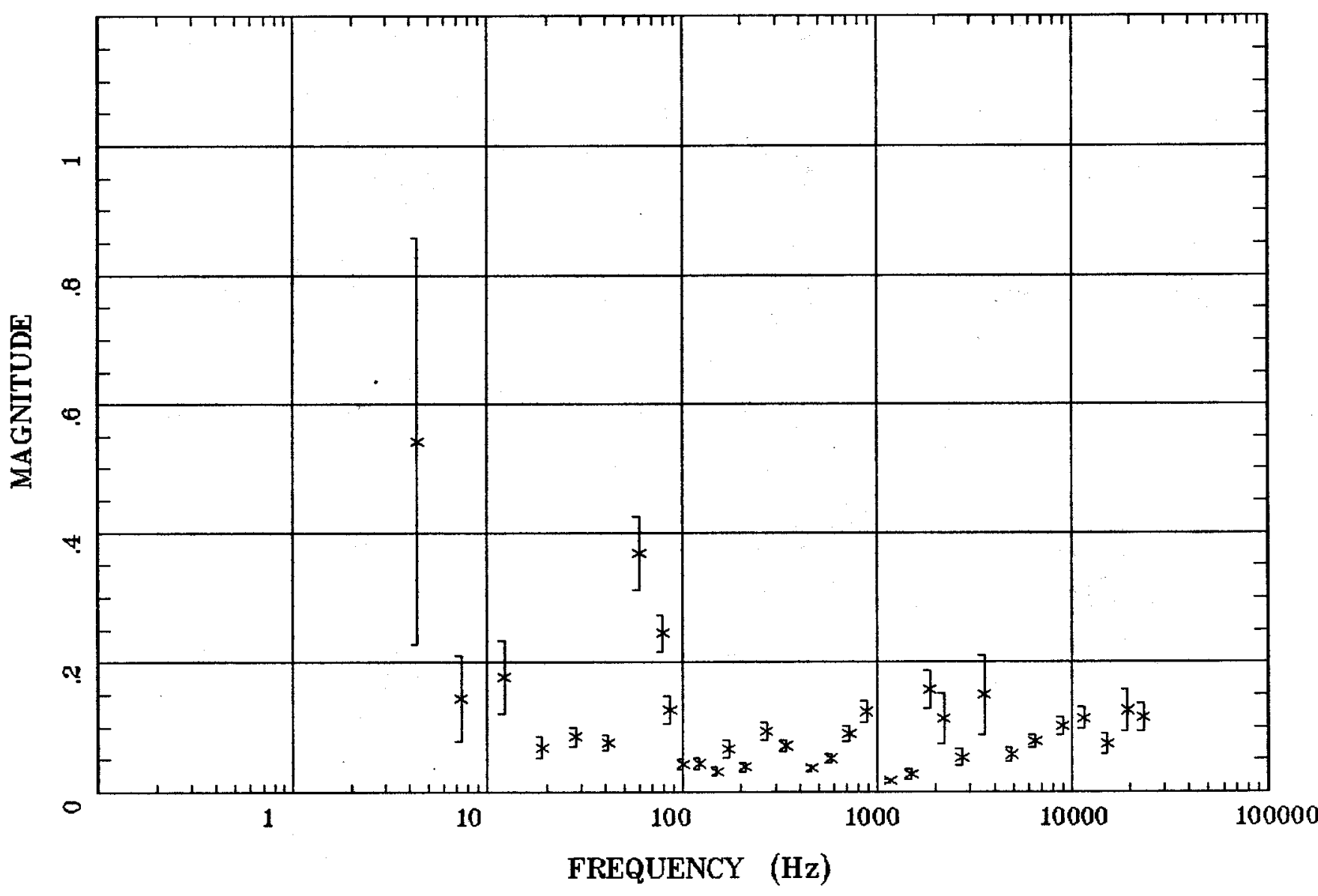

Client:

Remote: e-fld $64 \mathrm{~m}$ east

Acquired: 14:4 Jun 25, 2003

Survey Co:USGS
Rotation:

Filename: cp26a.avg

Channels: Ch1 Ch2 Ch3 Ch4 Ch5 Ch6 Ch7 Plotted: 14:42 Jul 06, 2004

$<$ EMI - ElectroMagnetic Instruments 


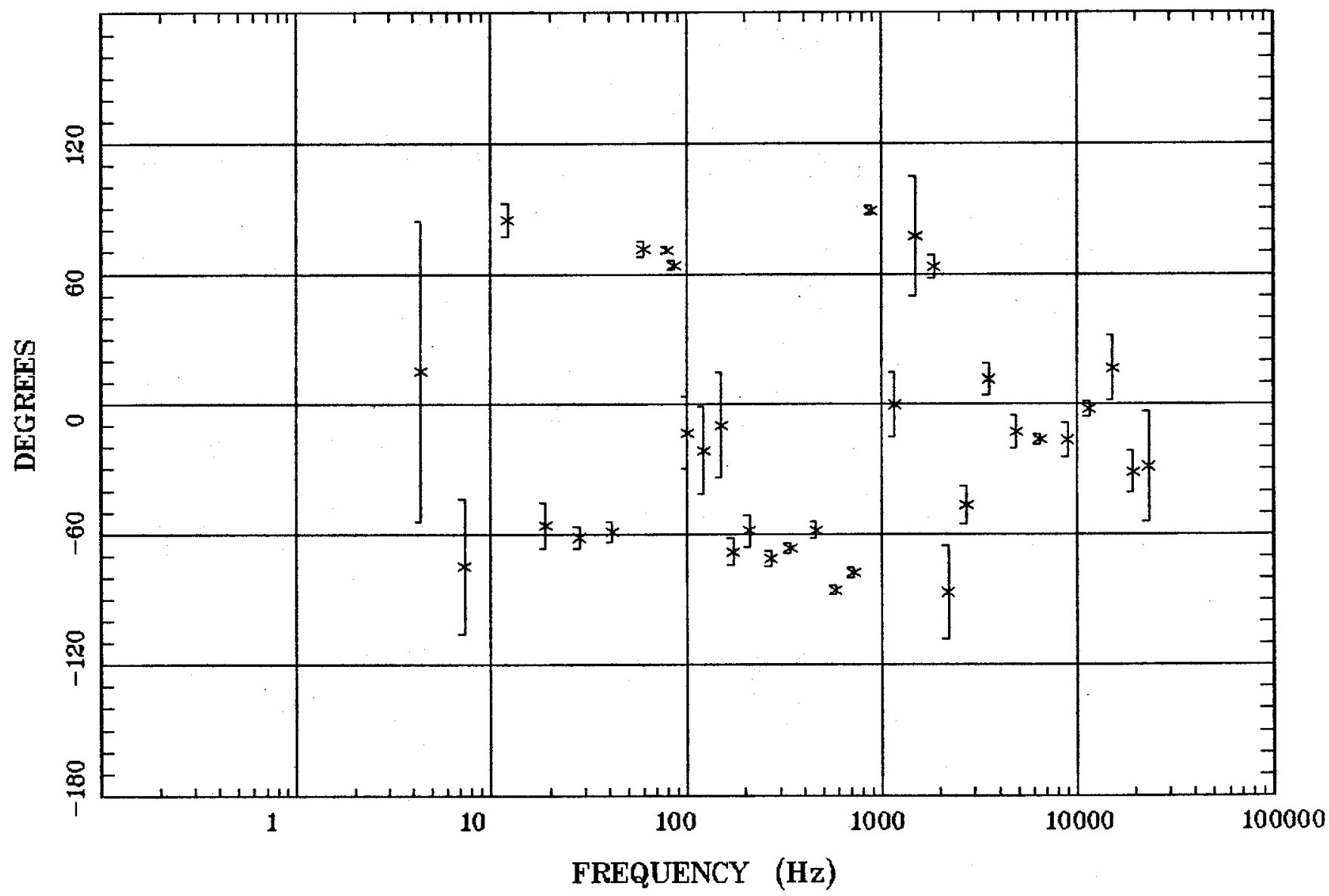

Client:

Remote: e-fld $64 \mathrm{~m}$ east

Acquired: 14:4 Jun 25, 2003

Survey Co:USGS
Rotation:

Filename: cp26a.avg

Channels: Ch1 Ch2 Ch3 Ch4 Ch5 Ch6 Ch7

Plotted: 14:42 Jul 06, 2004

$<$ EMI - ElectroMagnetic Instruments 


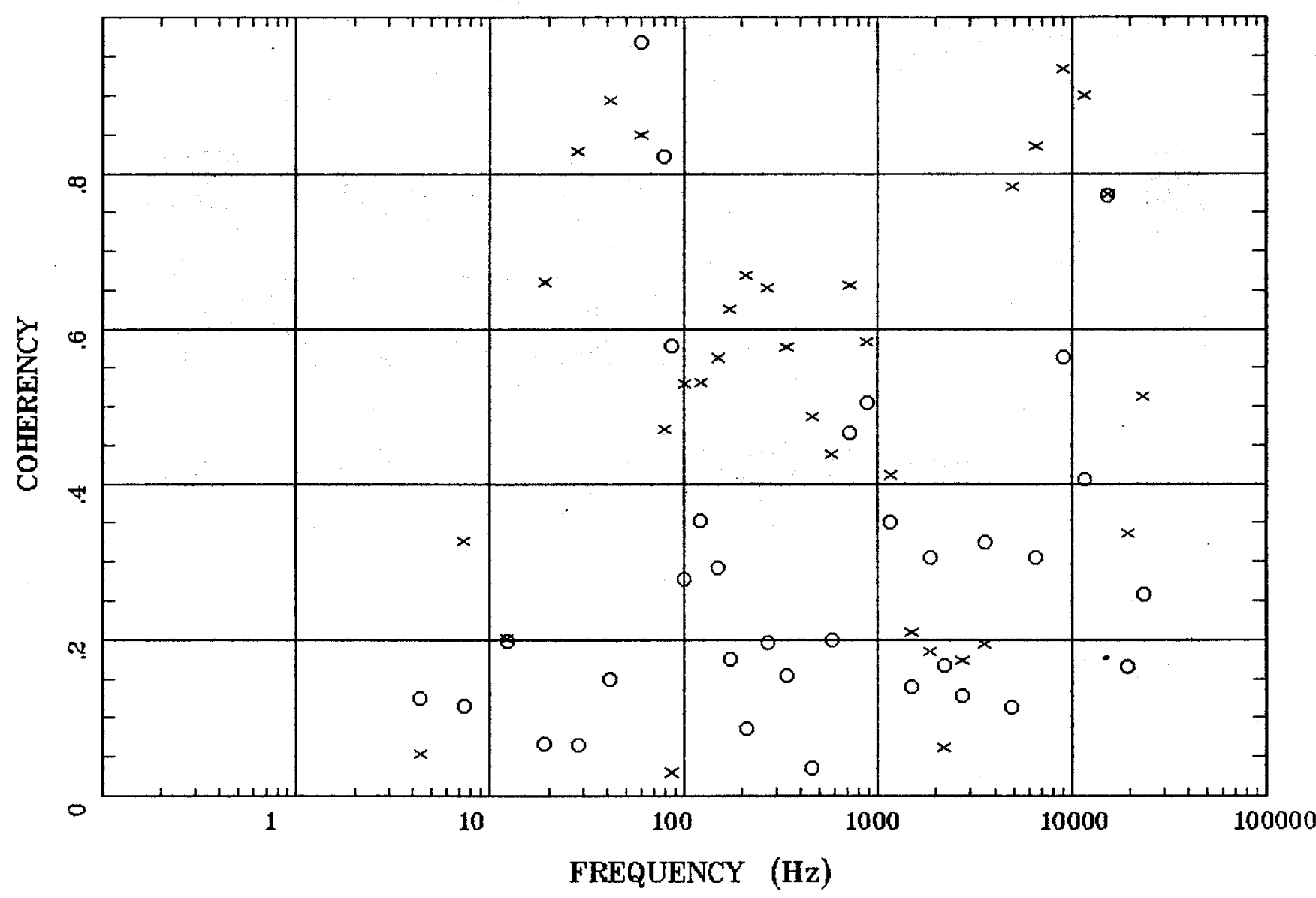

Client:

Remote: e-fld $64 \mathrm{~m}$ east Acquired: 14:4 Jun 25, 2003 Survey Co:USGS

\section{Rotation:}

Filename: cp26a.avg

Channels: Ch1 Ch2 Ch3 Ch4 Ch5 Ch6 Ch7 Plotted: 14:42 Jul 06, 2004

$<$ EMI - ElectroMagnetic Instruments 


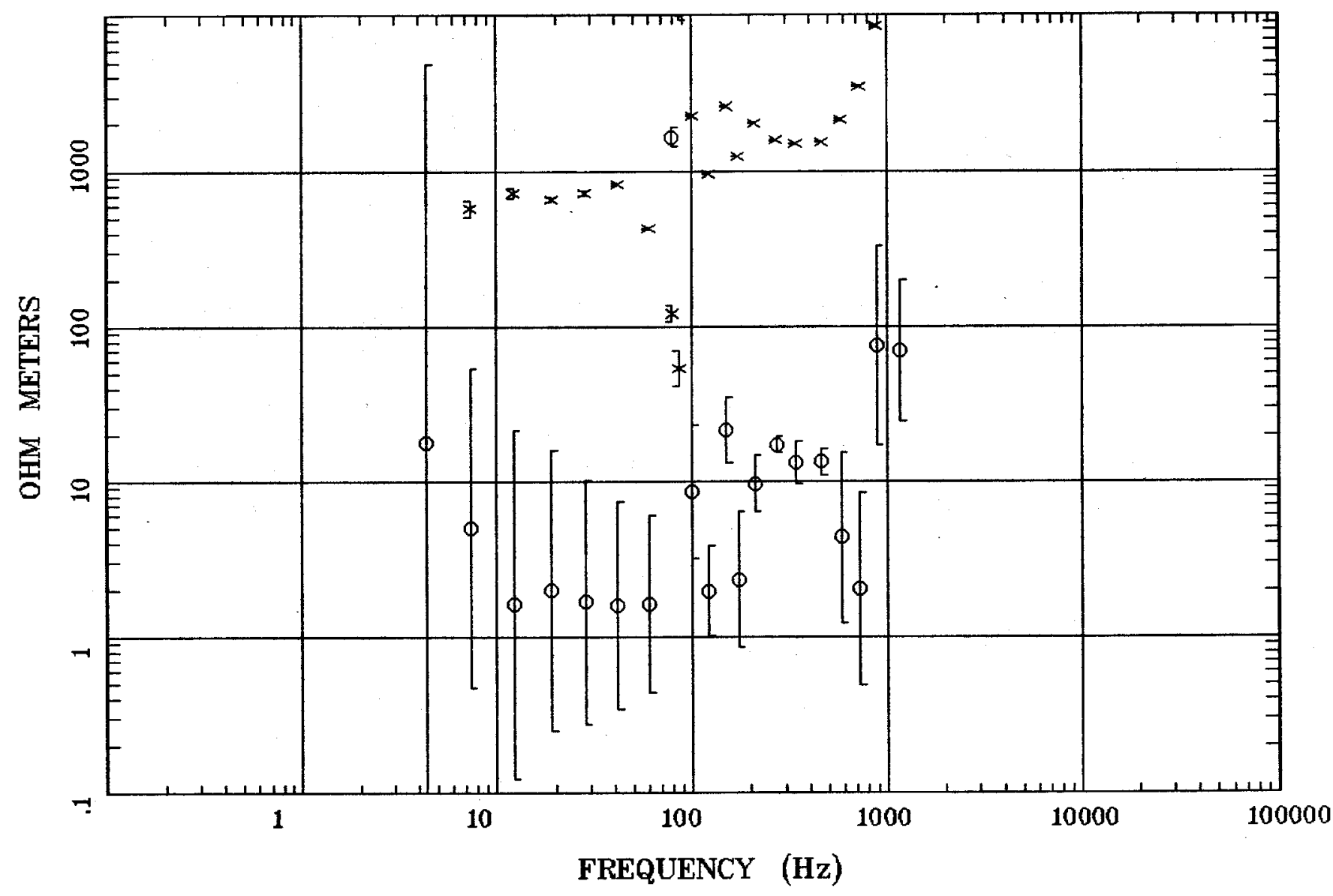

Client:

Remote: none

Acquired: 13:2 Jun 26, 2003 Survey Co:USGS
Rotation:

Filename: cp27.avg

Channels: Ch1 Ch2 Ch3 Ch4 Ch5 Ch1 Ch2 Plotted: 10:20 Jul 06, 2004

< EMI - ElectroMagnetic Instruments 


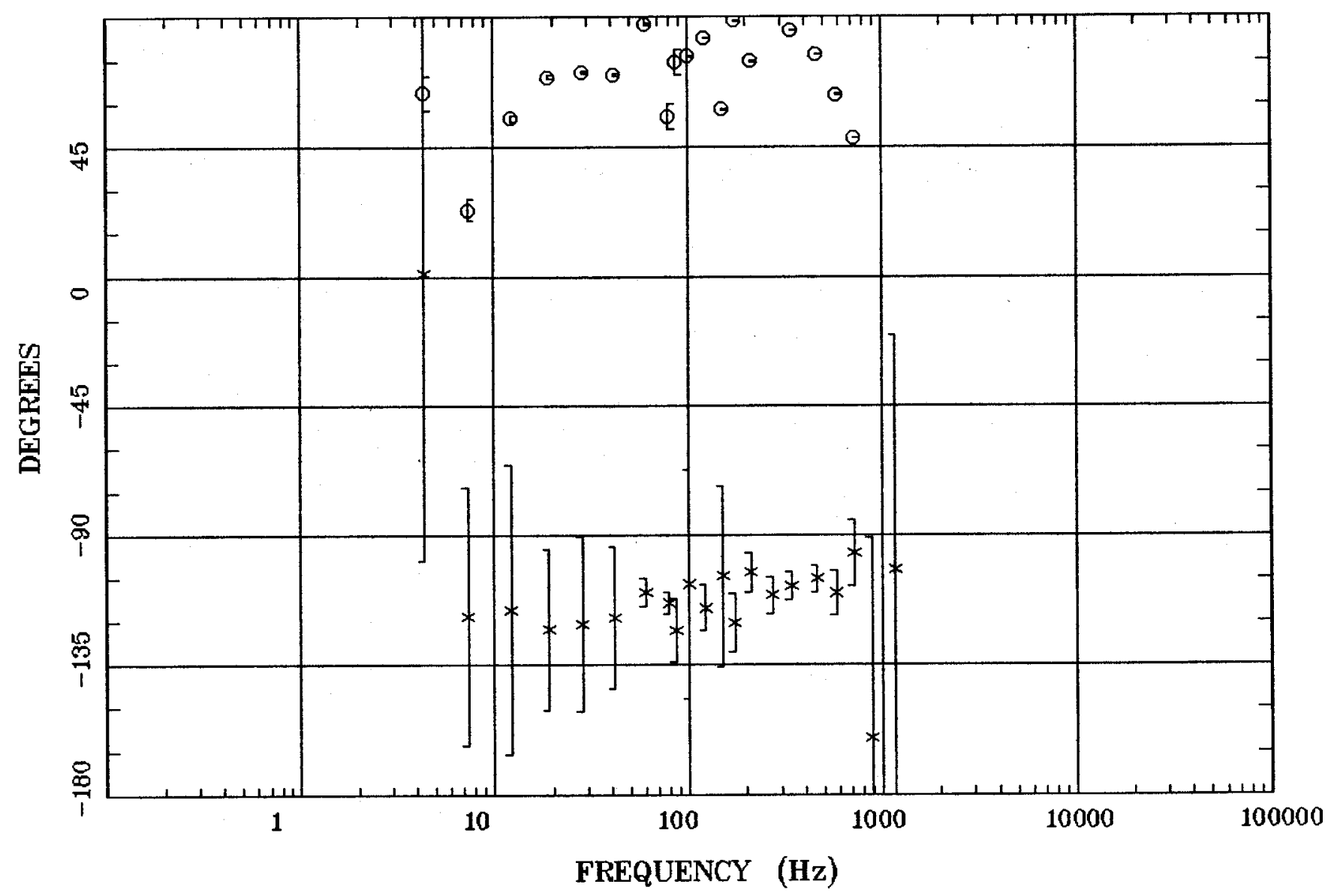

Client:

Remote: none

Acquired: 13:2 Jun 26, 2003

Survey Co:USGS
Rotation:

Filename: cp27.avg

Channels: Ch1 Ch2 Ch3 Ch4 Ch5 Ch1 Ch2 Plotted: 10:20 Jul 06, 2004

< EMI - ElectroMagnetic Instruments > 


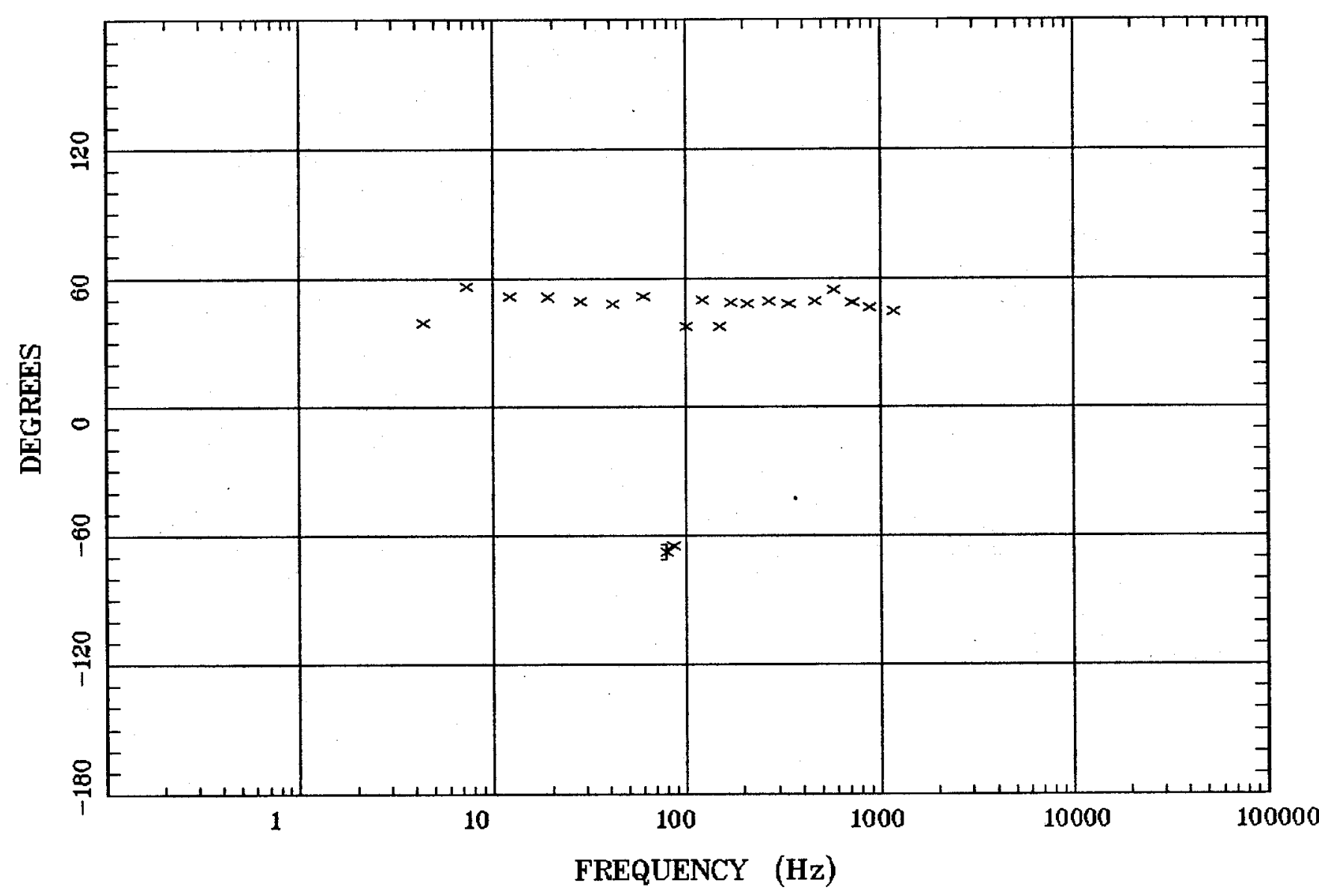

Client:

Remote: none

Acquired: 13:2 Jun 26, 2003

Survey Co:USGS
Rotation:

Filename: cp27a.avg

Channels: Ch1 Ch2 Ch3 Ch4 Ch5 Ch1 Ch2 Plotted: 12:58 Jul 07, 2004

< EMI - ElectroMagnetic Instruments > 


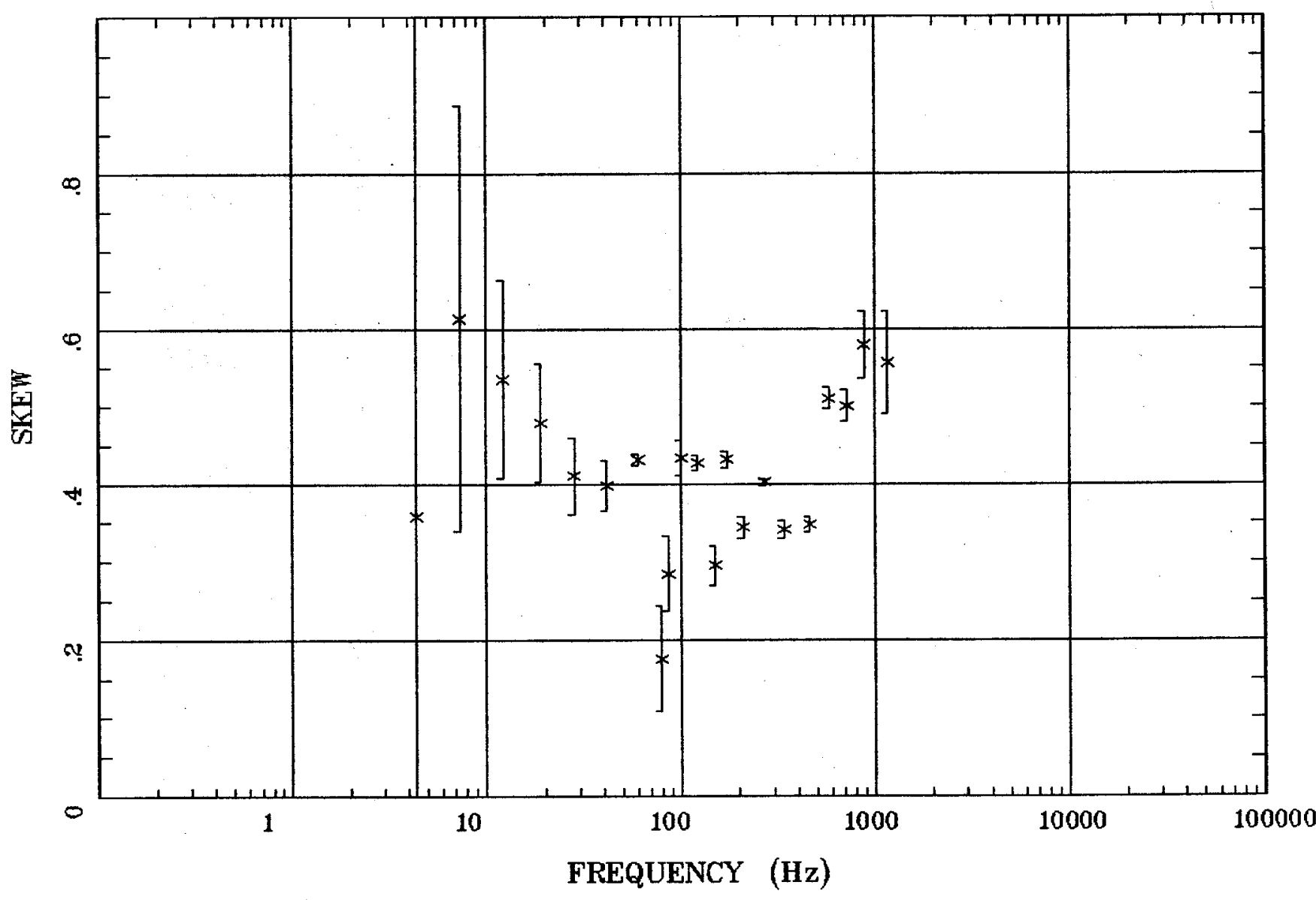

Client:

Remote: none

Acquired: 13:2 Jun 26, 2003

Survey Co:USGS
Rotation:

Filename: cp27.avg

Channels: Ch1 Ch2 Ch3 Ch4 Ch5 Ch1 Ch2 Plotted: 10:20 Jul 06, 2004

< EMI - ElectroMagnetic Instruments > 


\section{E MULT Coh.}

Santa Fe, NM

Station 27

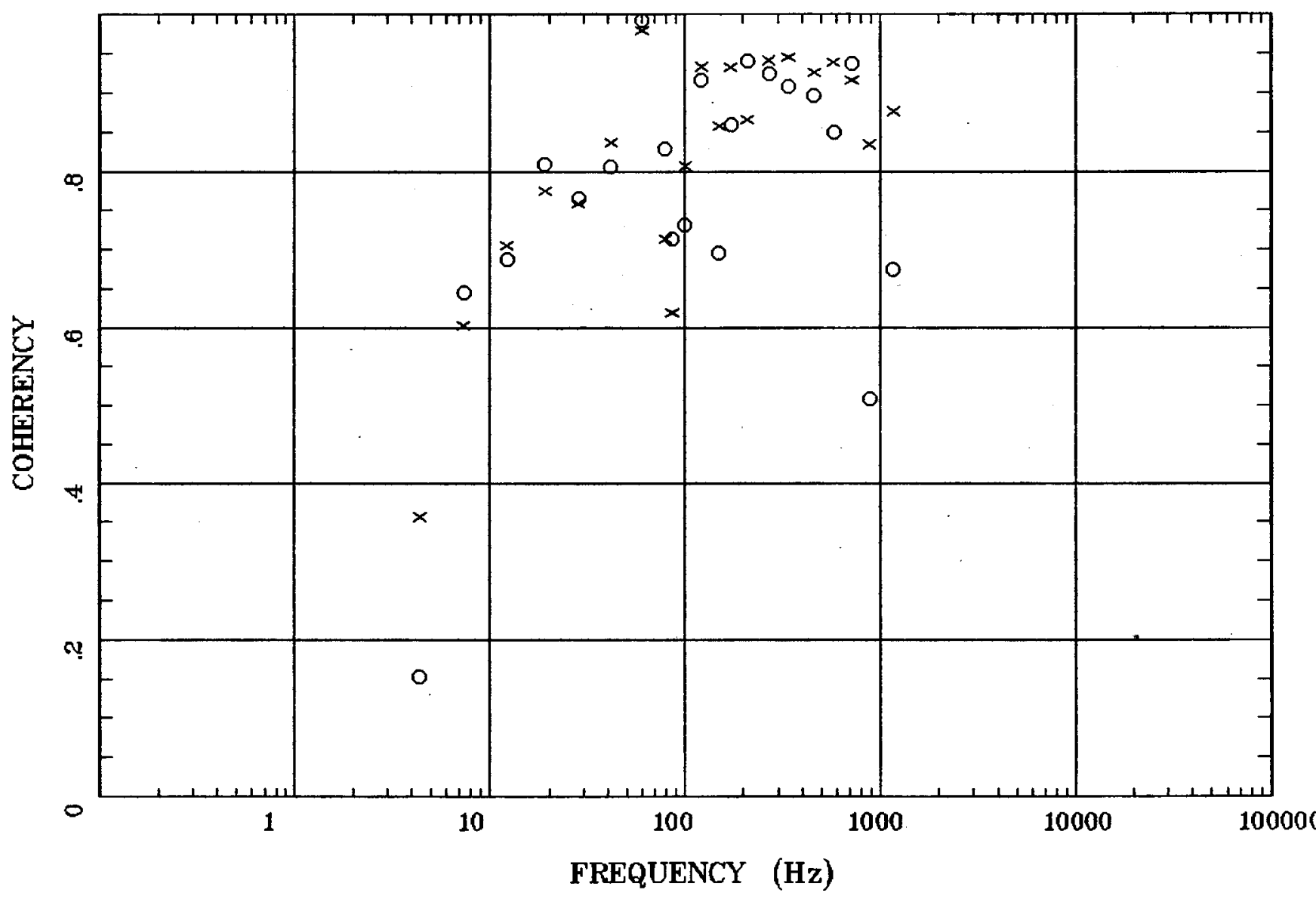

Client:

Remote: none

Acquired: 13:2 Jun 26, 2003

Survey Co:USGS
Rotation:

Filename: cp27.avg

Channels: Ch1 Ch2 Ch3 Ch4 Ch5 Ch1 Ch2

Plotted: 10:20 Jul 06, 2004

$<$ EMI - ElectroMagnetic Instruments > 


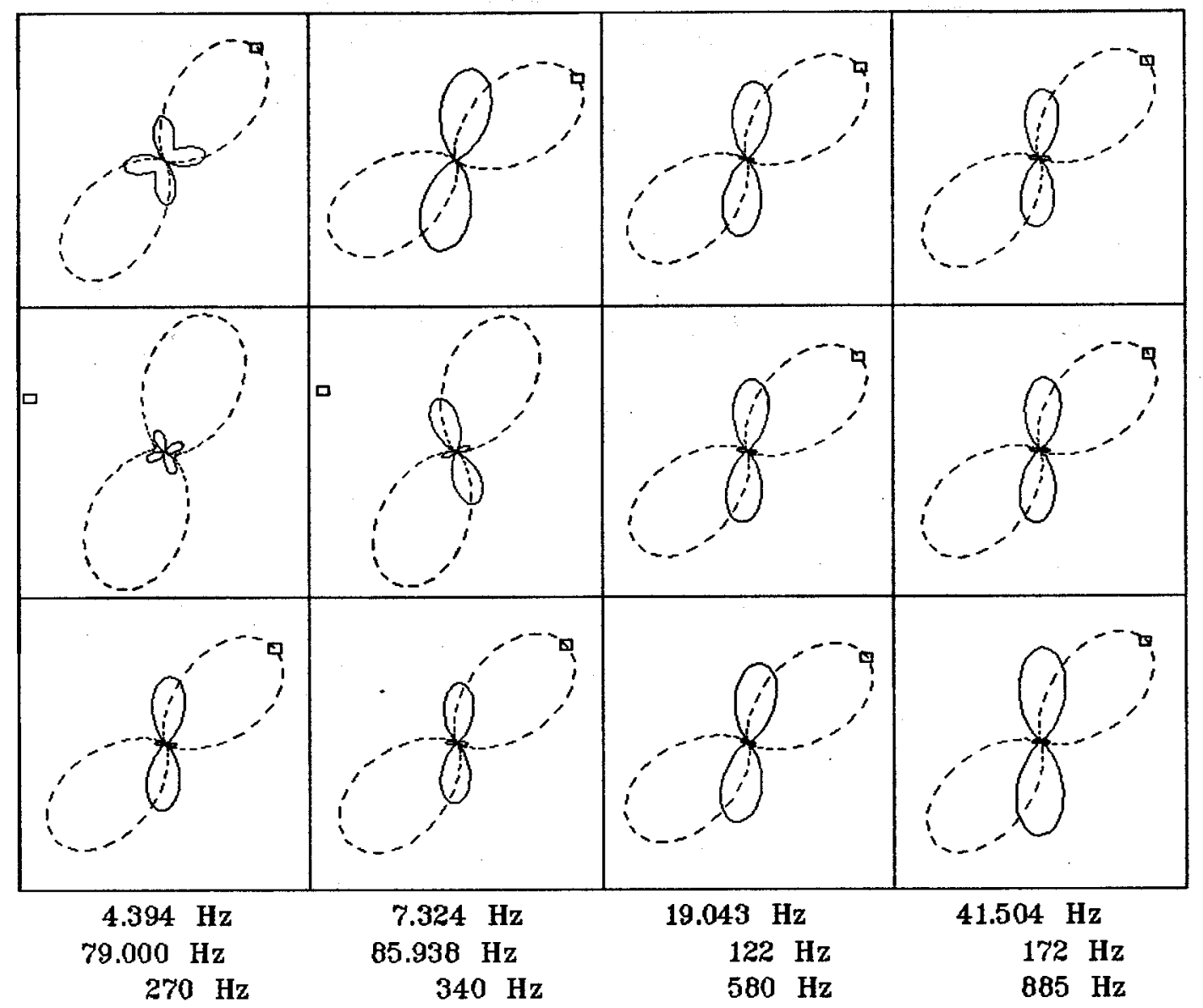

Client:

Remote: none

Acquired: 13:2 Jun 26, 2003 Survey Co:USGS
Rotation:

Filename: cp27.avg

Channels: Ch1 Ch2 Ch3 Ch4 Ch5 Ch1 Ch2 Plotted: 10:20 Jul 06, 2004

< EMI - ElectroMagnetic Instruments 


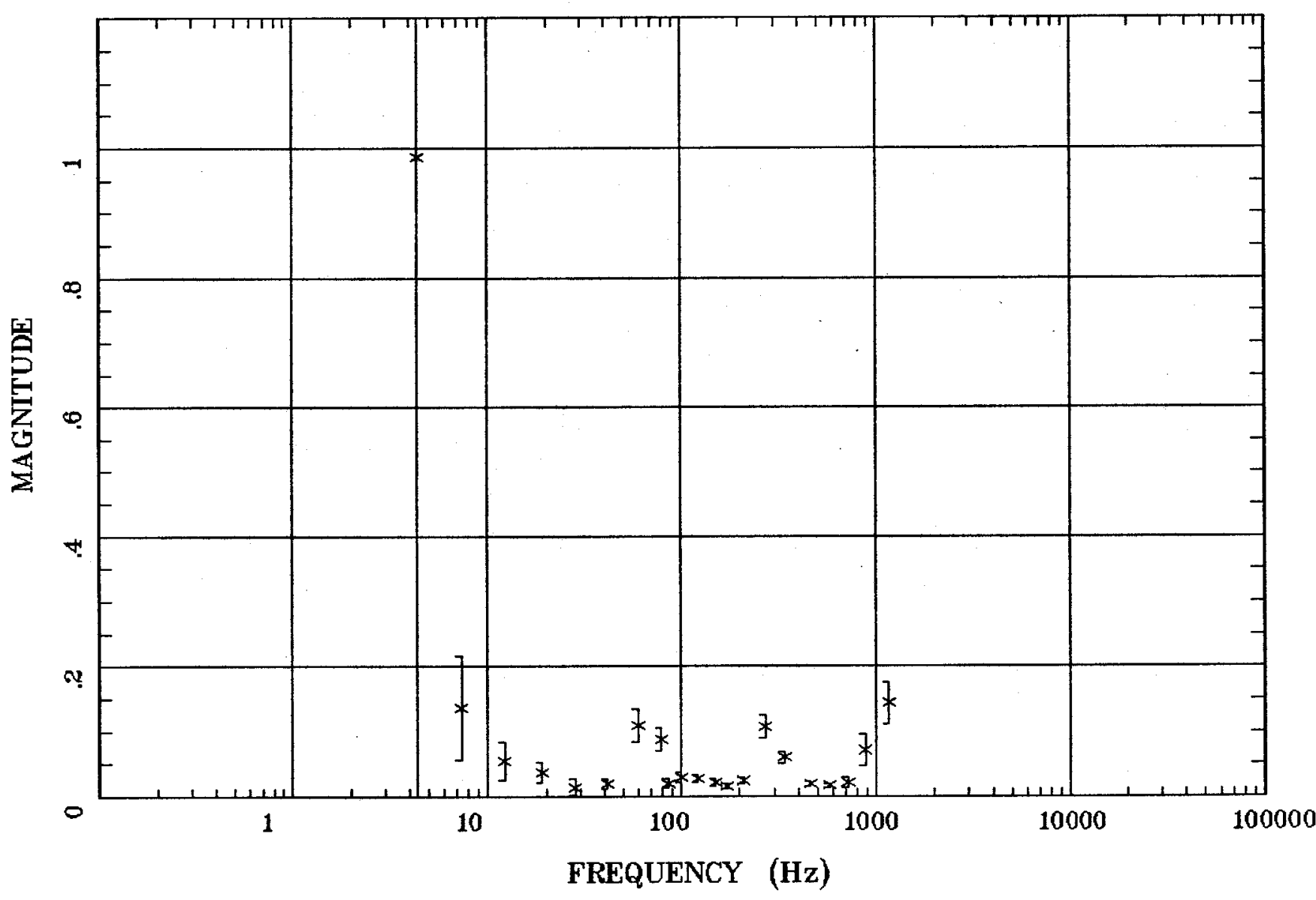

Client:

Remote: none

Acquired: 13:2 Jun 26, 2003

Survey Co:USGS
Rotation:

Filename: cp27.avg

Channels: Ch1 Ch2 Ch3 Ch4 Ch5 Ch1 Ch2

Plotted: 10:20 Jul 06, 2004

< EMI - ElectroMagnetic Instruments > 


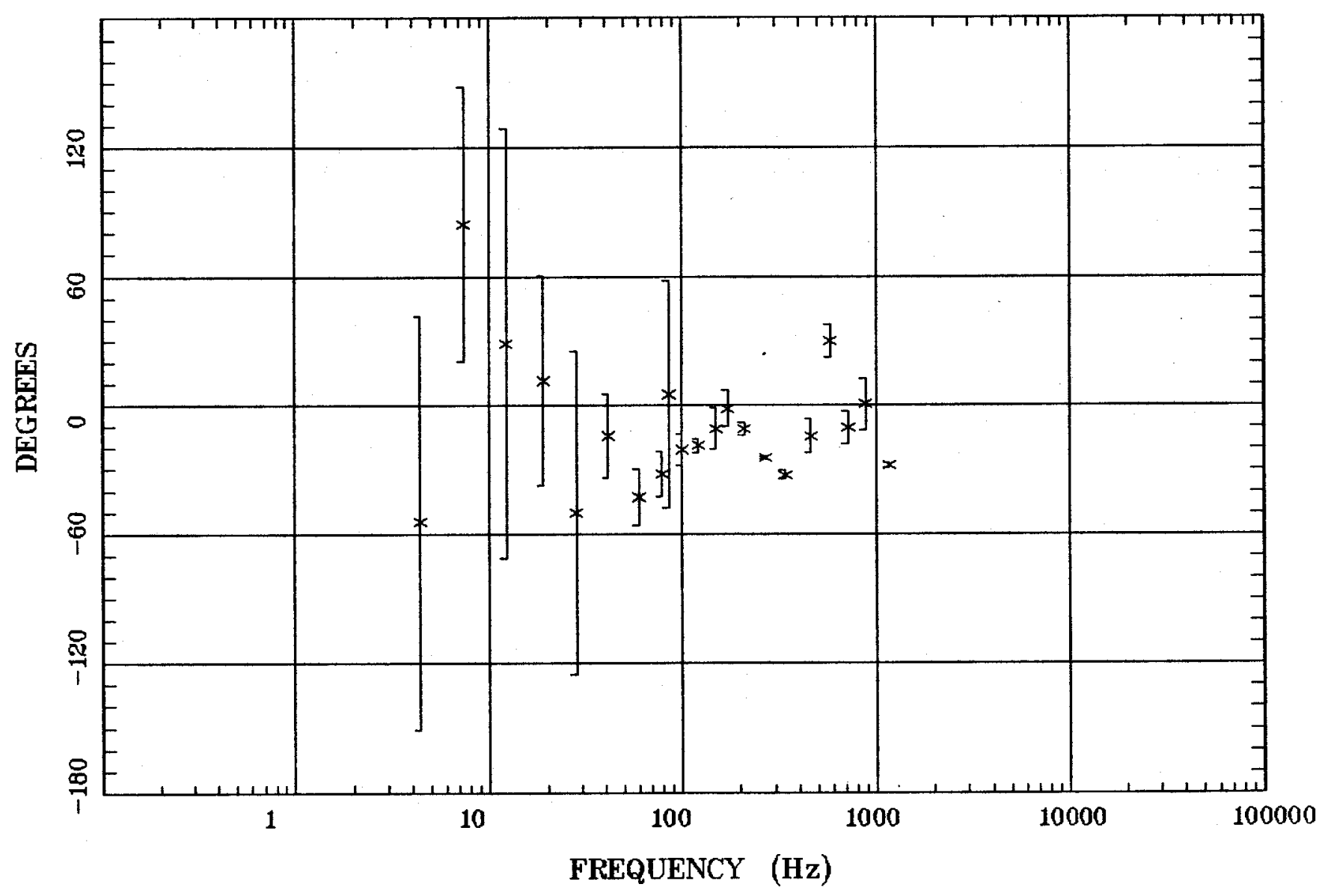

Client:

Remote: none

Acquired: 13:2 Jun 26, 2003

Survey Co:USGS
Rotation:

Filename: cp27.avg

Channels: Ch1 Ch2 Ch3 Ch4 Ch5 Ch1 Ch2

Plotted: 10:20 Jul 06, 2004

$<$ EMI - ElectroMagnetic Instruments 
HzHx.x Coh HzHy.o

Santa Fe, NM

Station 27

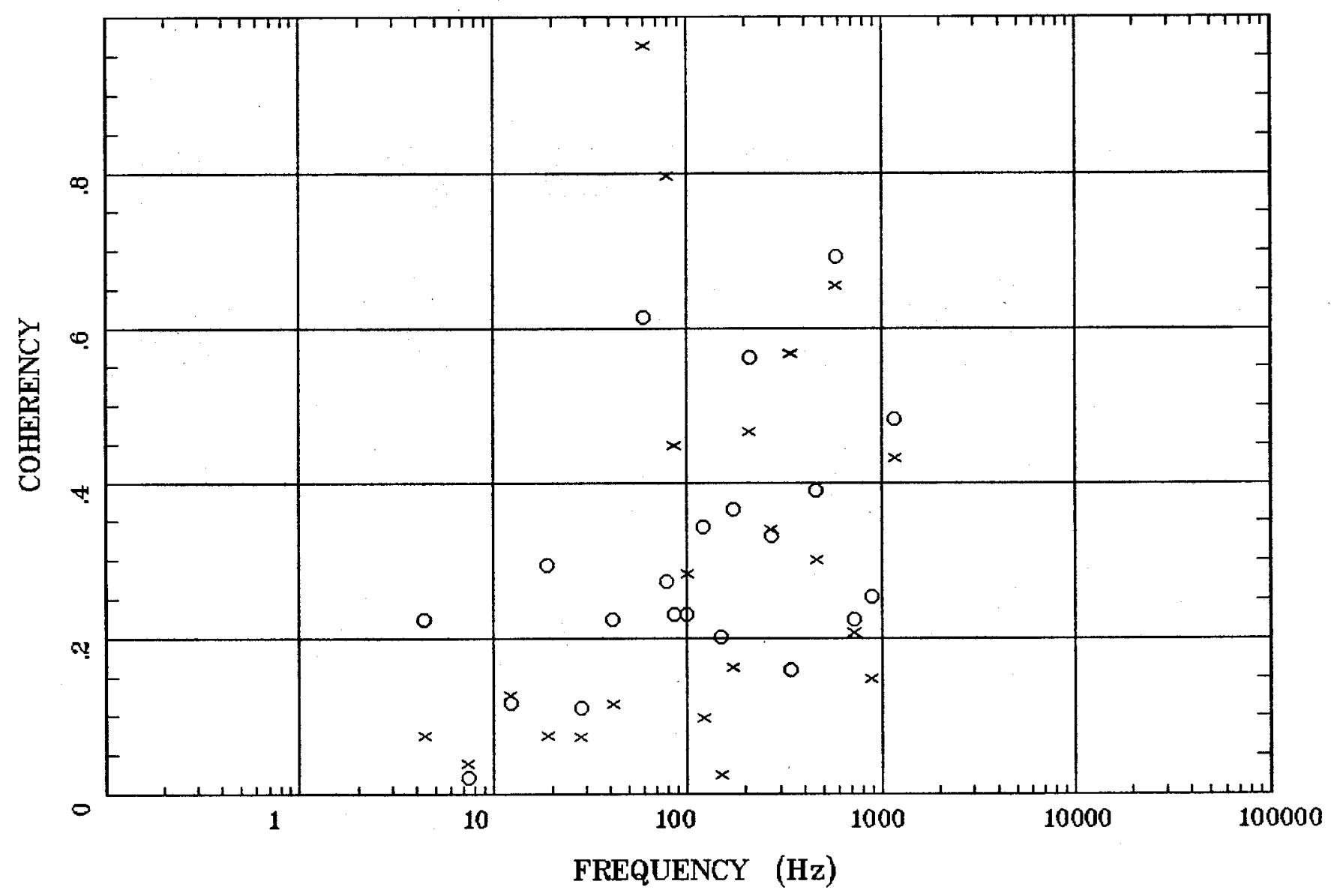

Client:

Remote: none

Acquired: 13:2 Jun 26, 2003 Survey Co:USGS

Rotation:

Filename: cp27.avg

Channels: Ch1 Ch2 Ch3 Ch4 Ch5 Ch1 Ch2

Plotted: 10:20 Jul 06, 2004

< EMI - ElectroMagnetic Instruments 


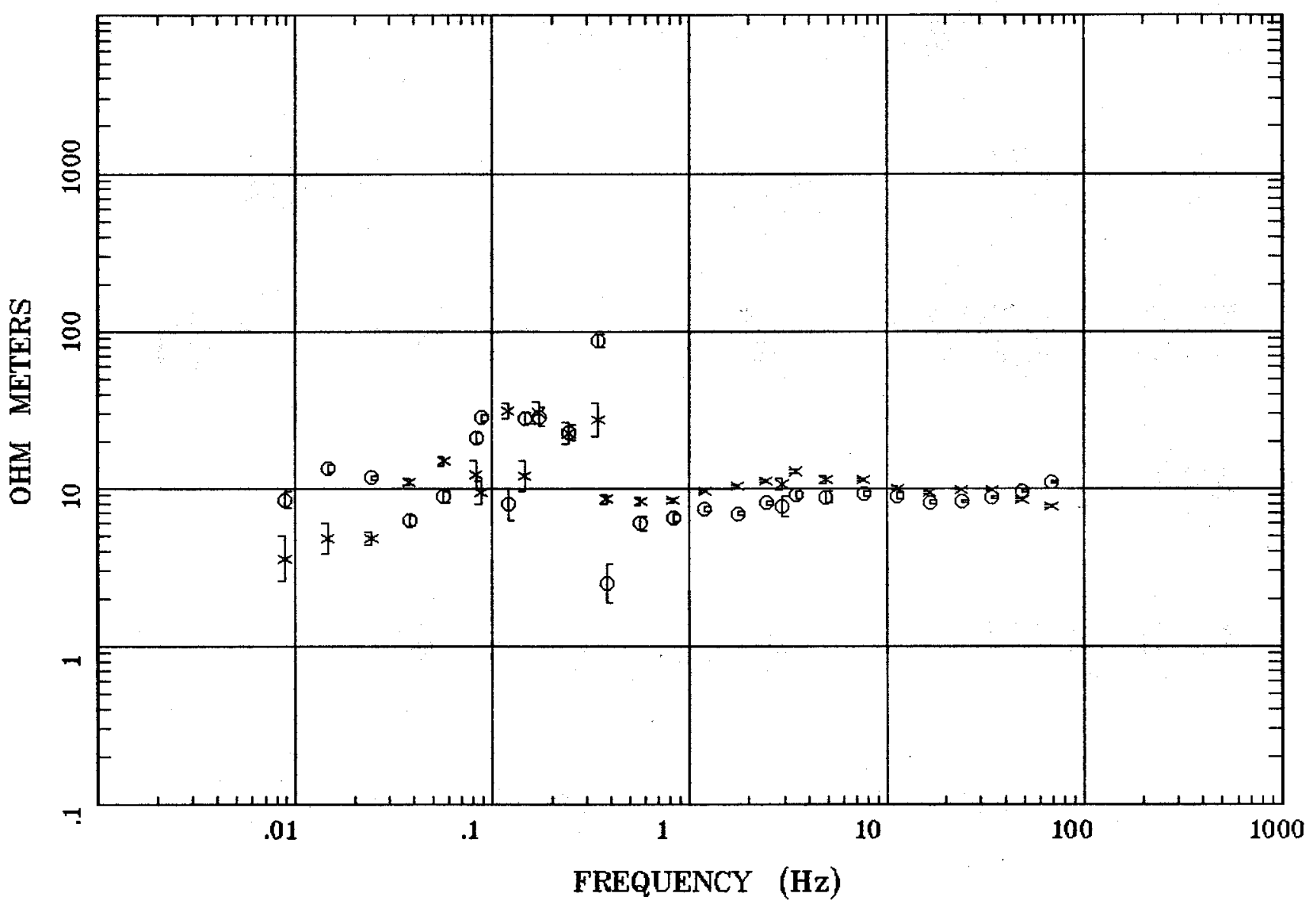

Client:

Remote: e-fld $95 \mathrm{~m}$ south Acquired: 12:3 Jun 17, 2003 Survey Co:USGS
Rotation:

Filename: cp 18m.avg

Channels: Ch1 Ch2 Ch3 Ch4 Ch5 Ch6 Ch7

Plotted: 08:41 Jul 07, 2004

< EMI - ElectroMagnetic Instruments 


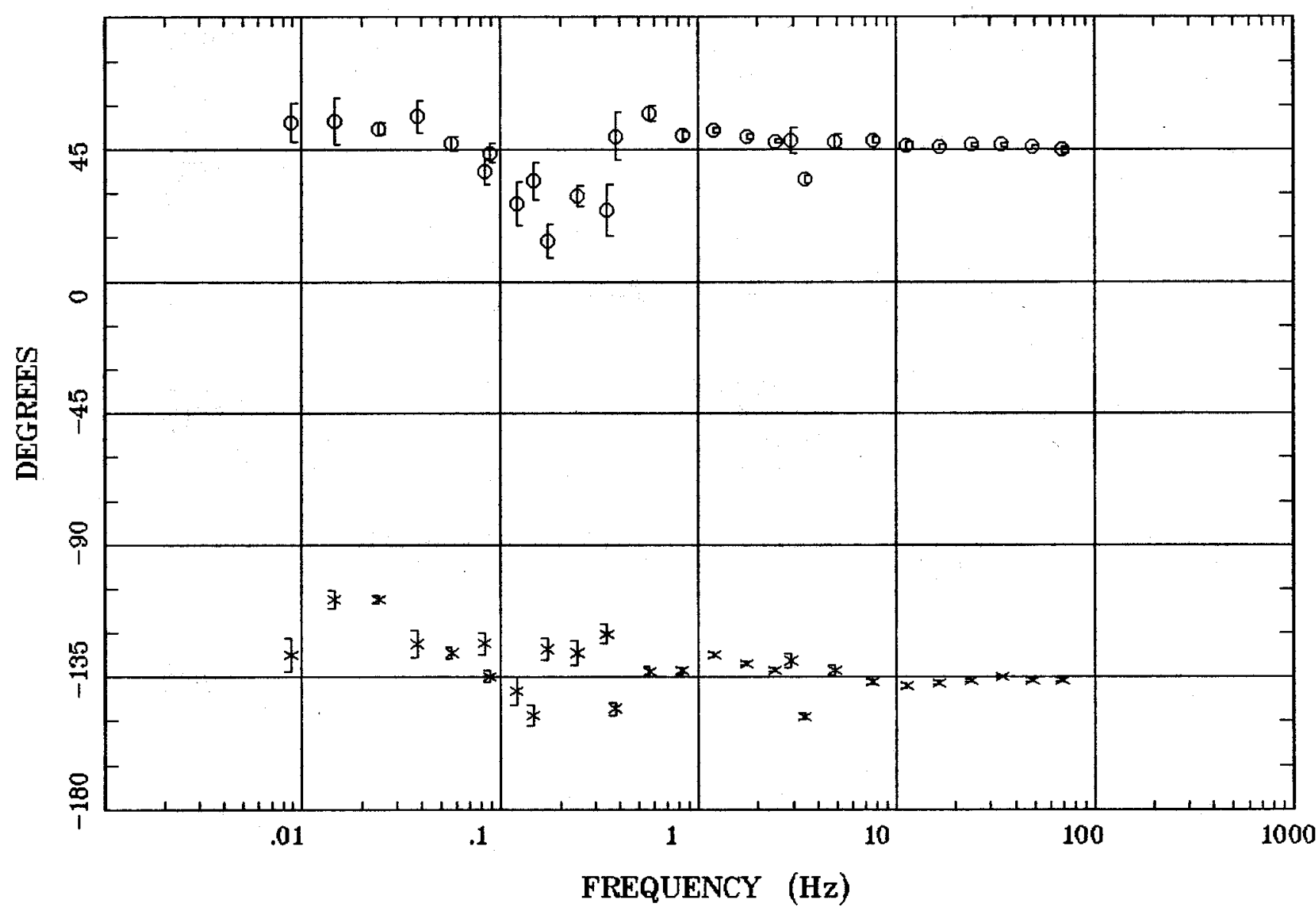

Client:

Remote: e-fld $95 \mathrm{~m}$ south Acquired: 12:3 Jun 17, 2003 Survey Co:USGS
Rotation:

Filename: cp $18 \mathrm{~m} . \mathrm{avg}$

Channels: Ch1 Ch2 Ch3 Ch4 Ch5 Ch6 Ch7

Plotted: 08:41 Jul 07, 2004

$<$ EMI - ElectroMagnetic Instruments 


\section{ROTATION ANGLE}

Santa Fe, NM

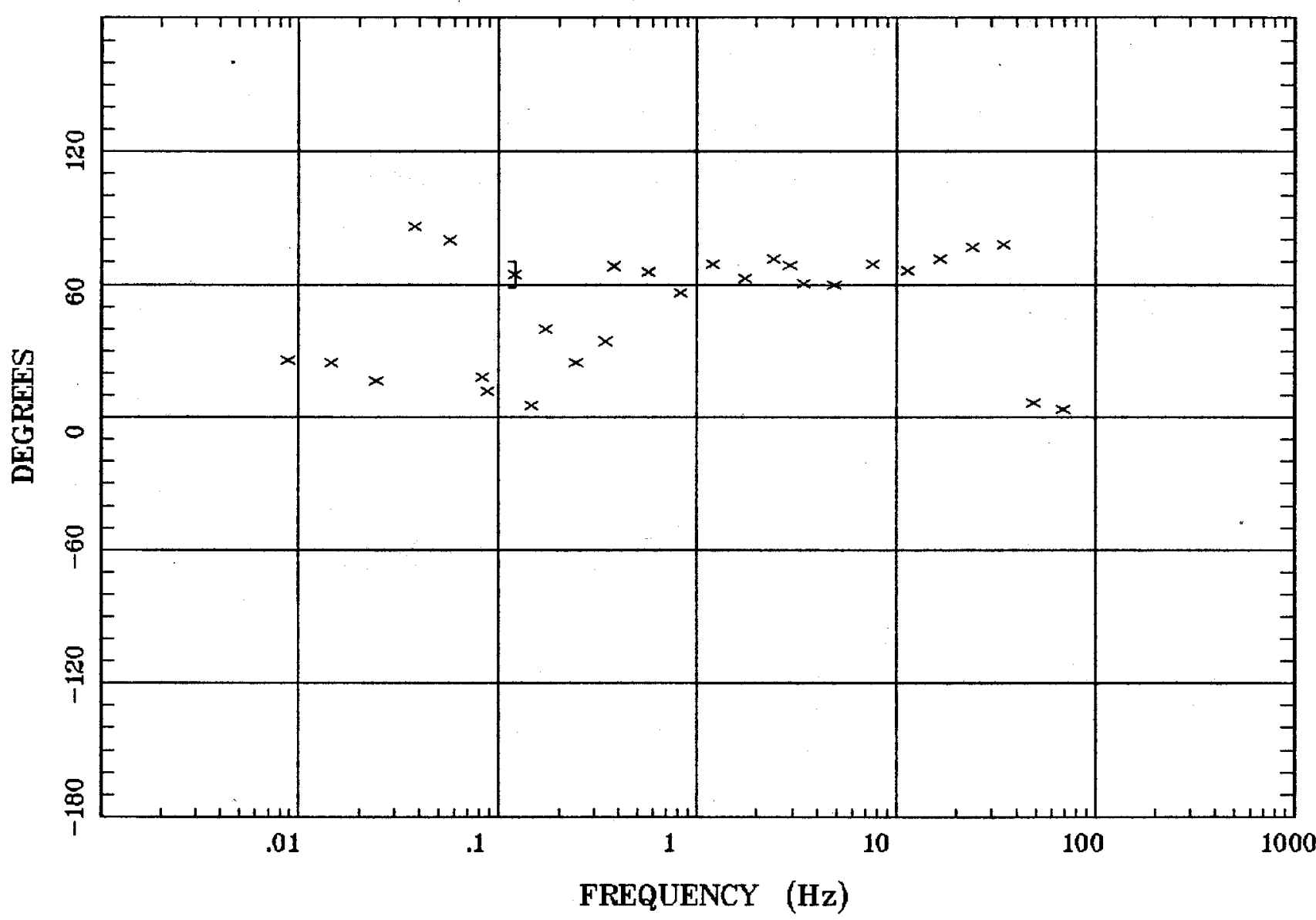

Client:

Remote: e-fld $95 \mathrm{~m}$ south Acquired: 12:3 Jun 17, 2003 Survey Co:USGS
Rotation:

Filename: cp 18m.avg

Channels: Ch1 Ch2 Ch3 ch4 Ch5 Ch6 Ch7 Plotted: 10:47 Jul 07, 2004

< EMI - ElectroMagnetic Instruments 


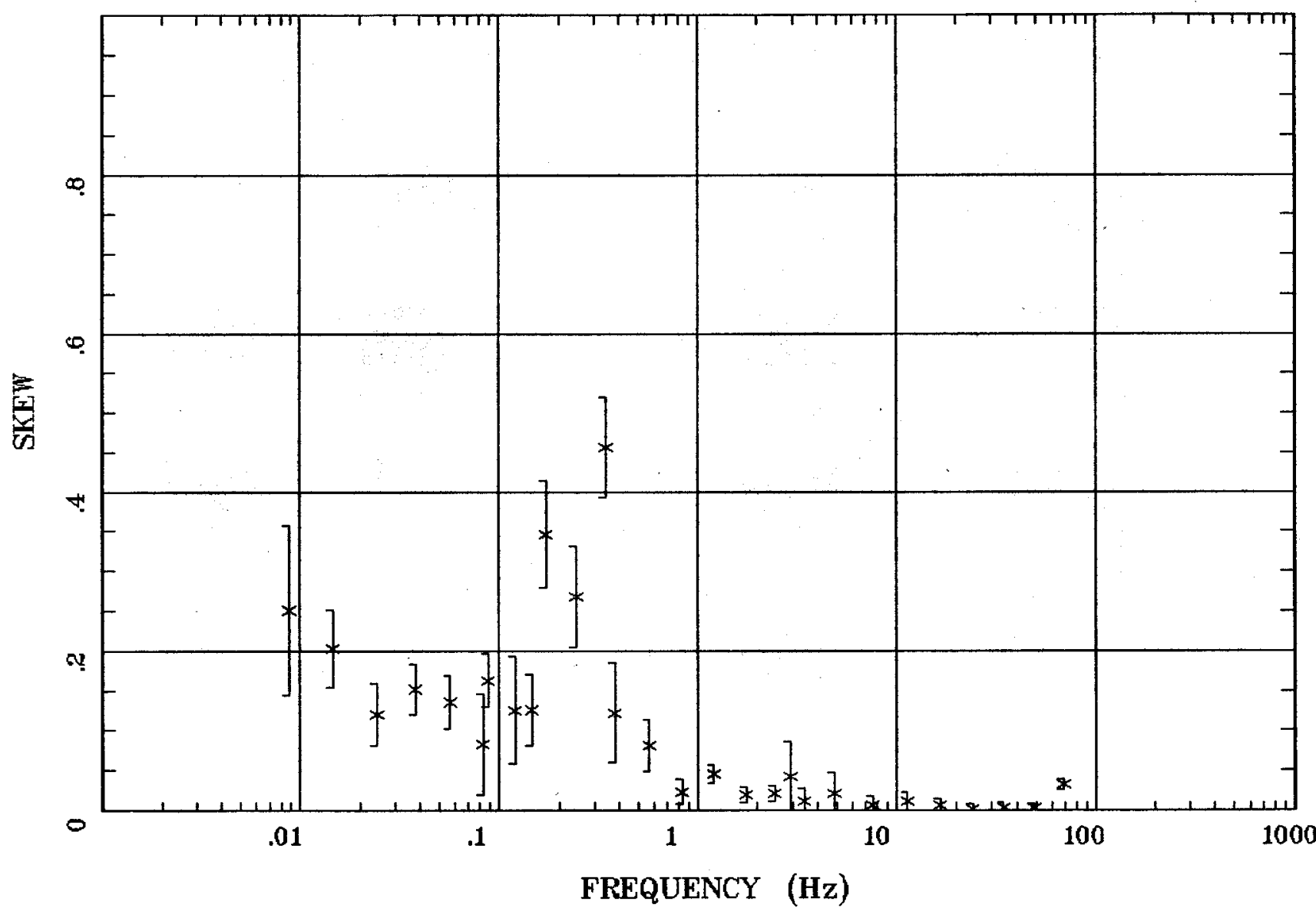

Client:

Remote: e-fld $95 \mathrm{~m}$ south Acquired: 12:3 Jun 17, 2003 Survey Co:USGS
Rotation:

Filename: cp 18m.avg

Channels: Ch1 Ch2 Ch3 Ch4 Ch5 Ch6 Ch7 Plotted: 08:41 Jul 07, 2004

< EMI - ElectroMagnetic Instruments 


\section{E MULT Coh.}

Santa Fe, NM

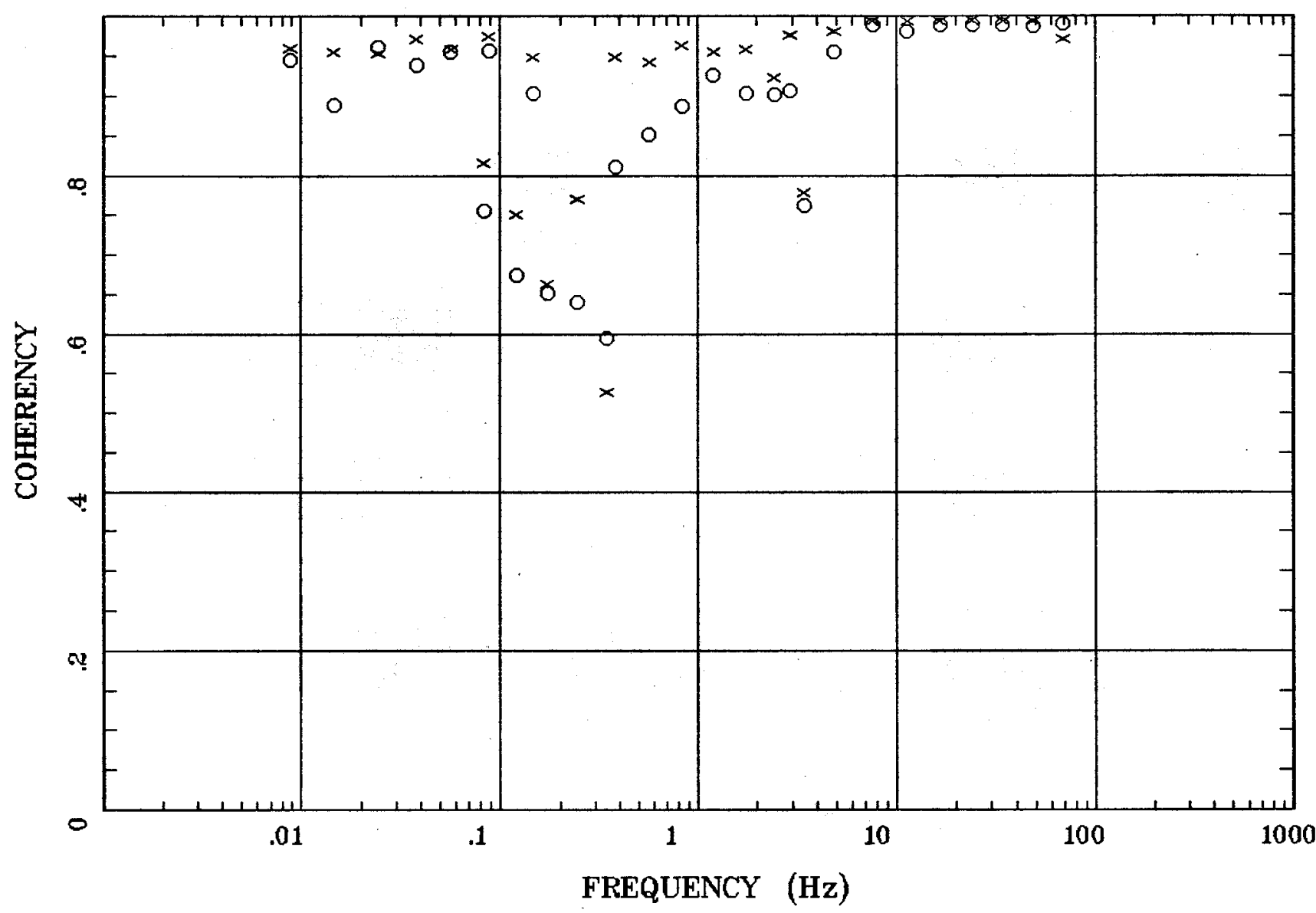

Client:

Remote: e-fld $95 \mathrm{~m}$ south Acquired: 12:3 Jun 17, 2003 Survey Co:USGS
Rotation:

Filename: cp18m.avg

Channels: Ch1 Ch2 Ch3 Ch4 Ch5 Ch6 Ch7

Plotted: 08:41 Jul 07, 2004

$<$ EMI - ElectroMagnetic Instruments 
Station 18

POLAR PLOTS

Santa Fe, NM

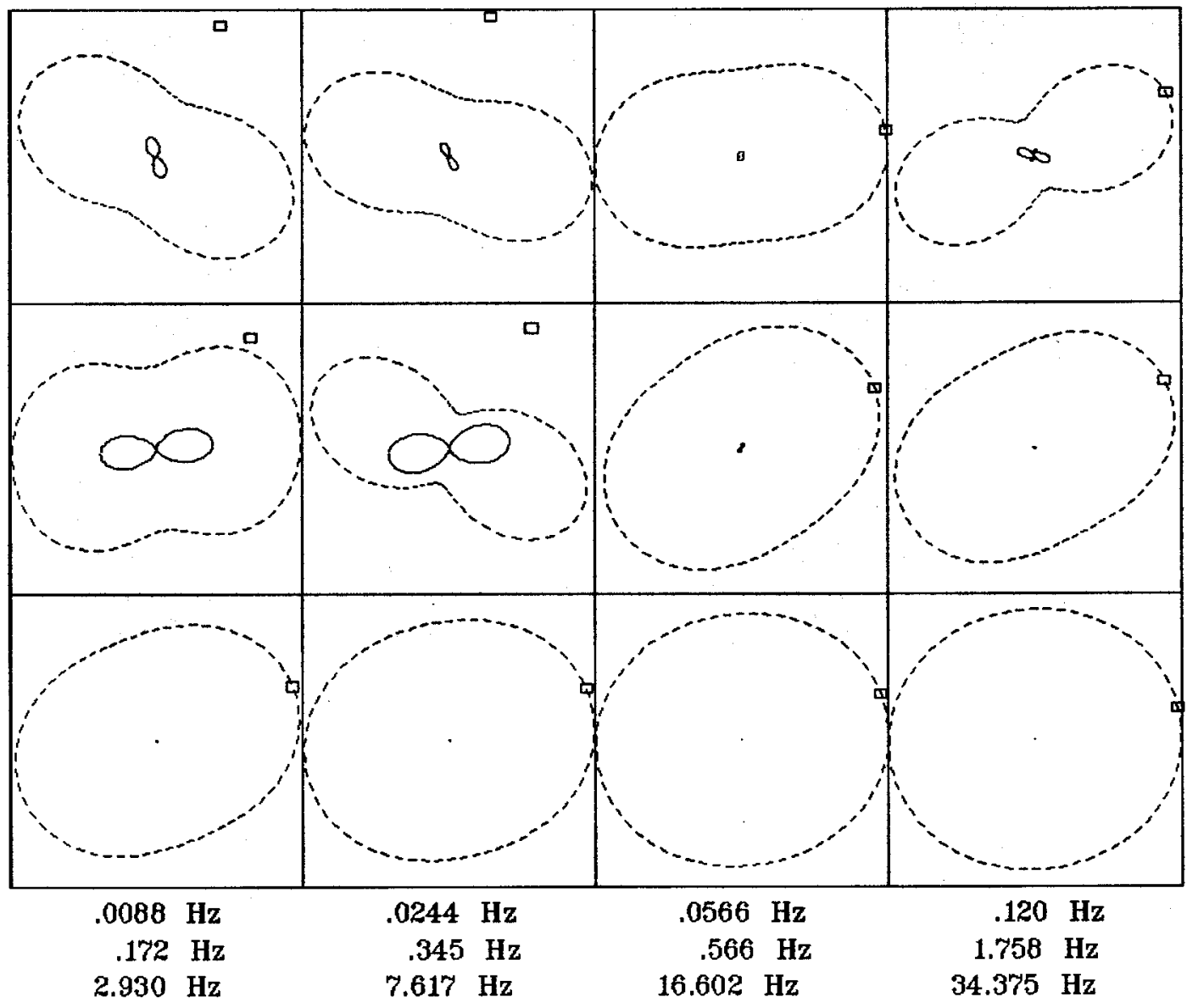

Rotation:

Client:

Remote: e-fld $95 \mathrm{~m}$ south Acquired: 12:3 Jun 17, 2003 Survey Co:USGS
Filename: cp 18m.avg

Channels: Ch1 Ch2 Ch3 Ch4 Ch5 Ch6 Ch7 Plotted: 08:41 Jul 07, 2004

$<$ EMI - ElectroMagnetic Instruments 


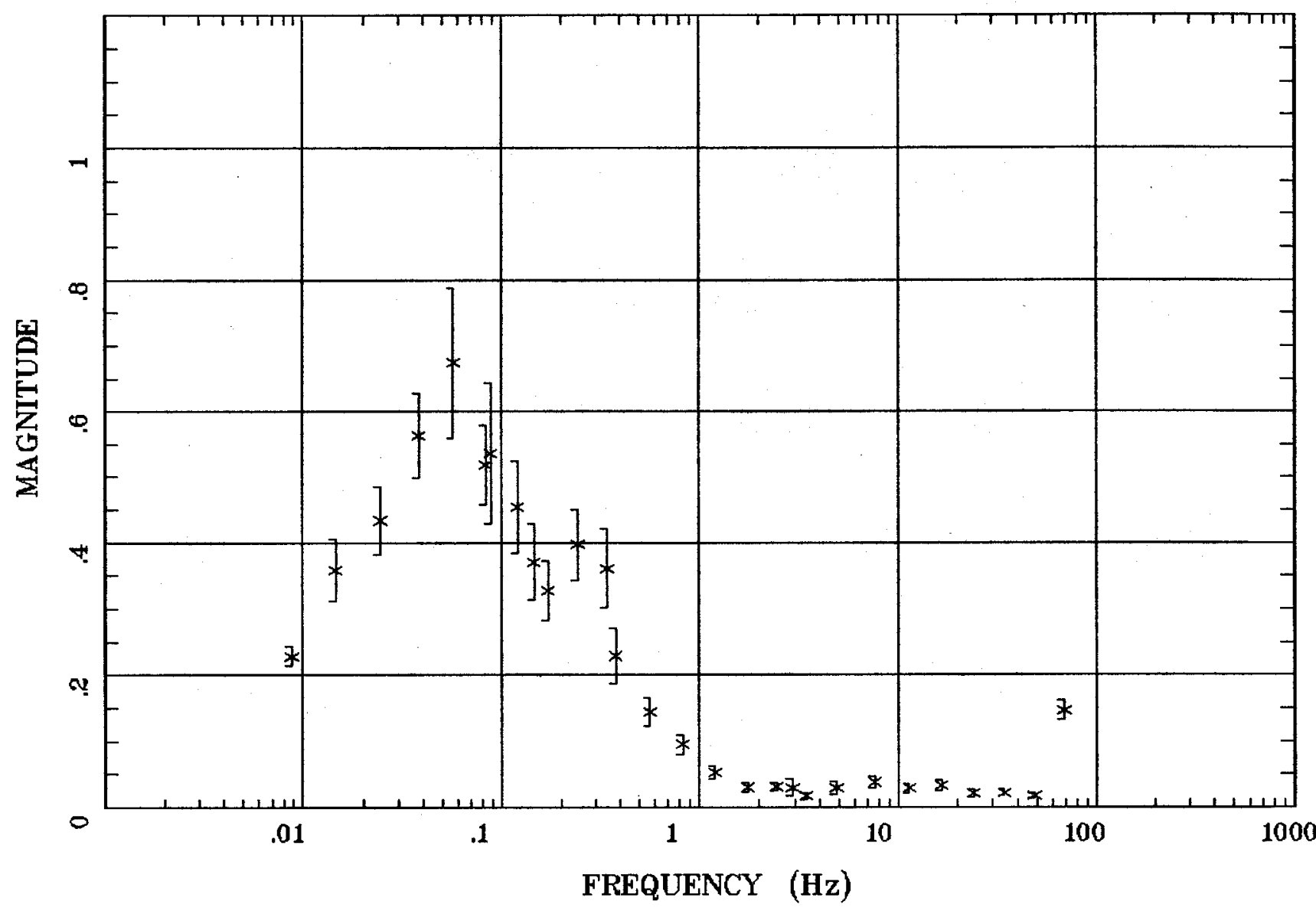

Client:

Remote: e-fld $95 \mathrm{~m}$ south Acquired: 12:3 Jun 17, 2003 Survey Co:USGS
Rotation:

Filename: cp18m.avg

Channels: Ch1 Ch2 Ch3 Ch4 Ch5 Ch6 Ch7 Plotted: 08:41 Jul 07, 2004

< EMI - ElectroMagnetic Instruments 


\section{Station 18}

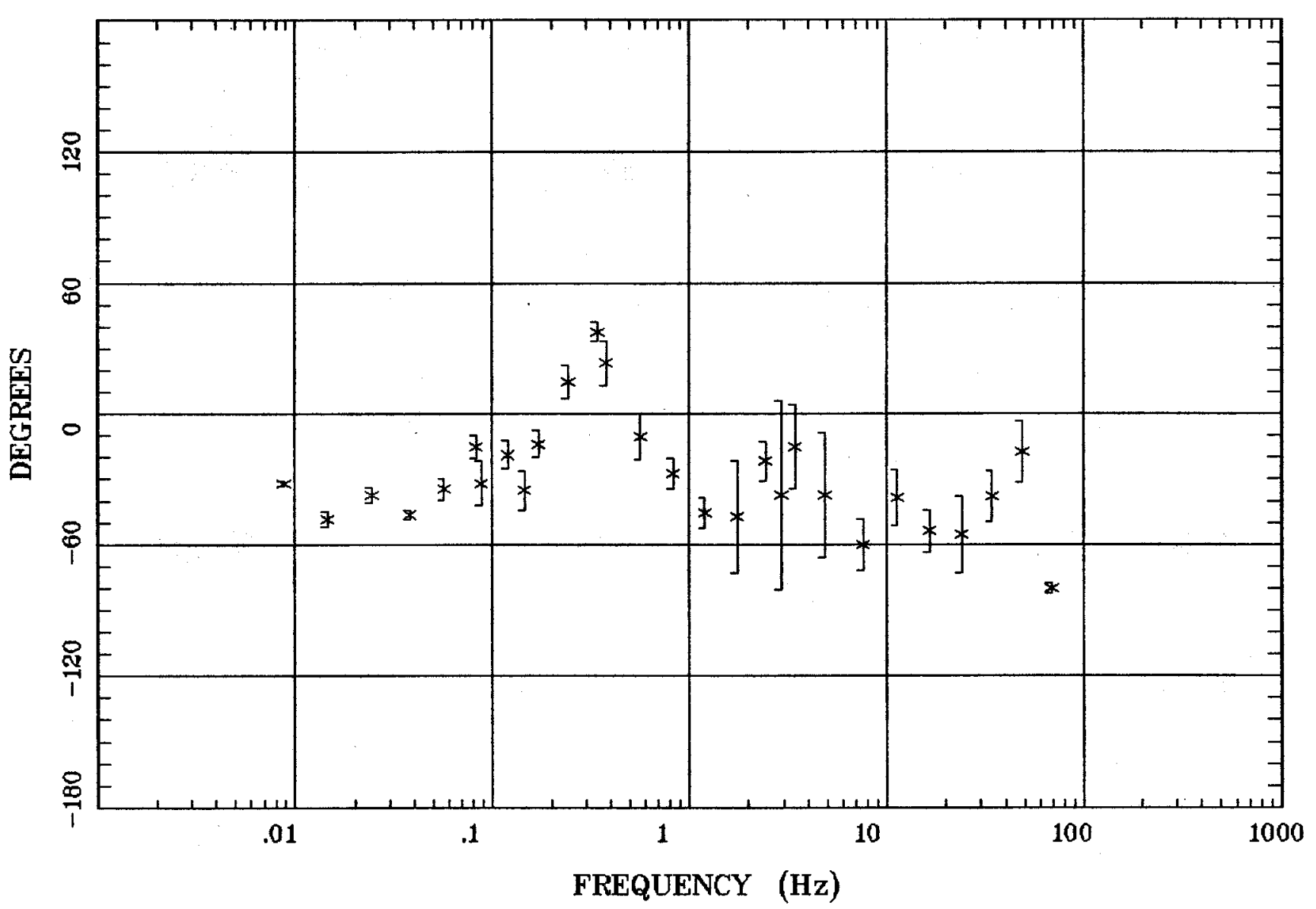

Client:

Remote: e-fld $95 \mathrm{~m}$ south

Acquired: 12:3 Jun 17, 2003

Survey Co:USGS
Rotation:

Filename: cp $18 \mathrm{~m} \cdot \mathrm{arg}$

Channels: Ch1 Ch2 Ch3 Ch4 Ch5 Ch6 Ch7

Plotted: 08:41 Jul 07, 2004

< EMI - ElectroMagnetic Instruments > 


\section{HzHx.x Coh HzHy.o}

Santa Fe, NM

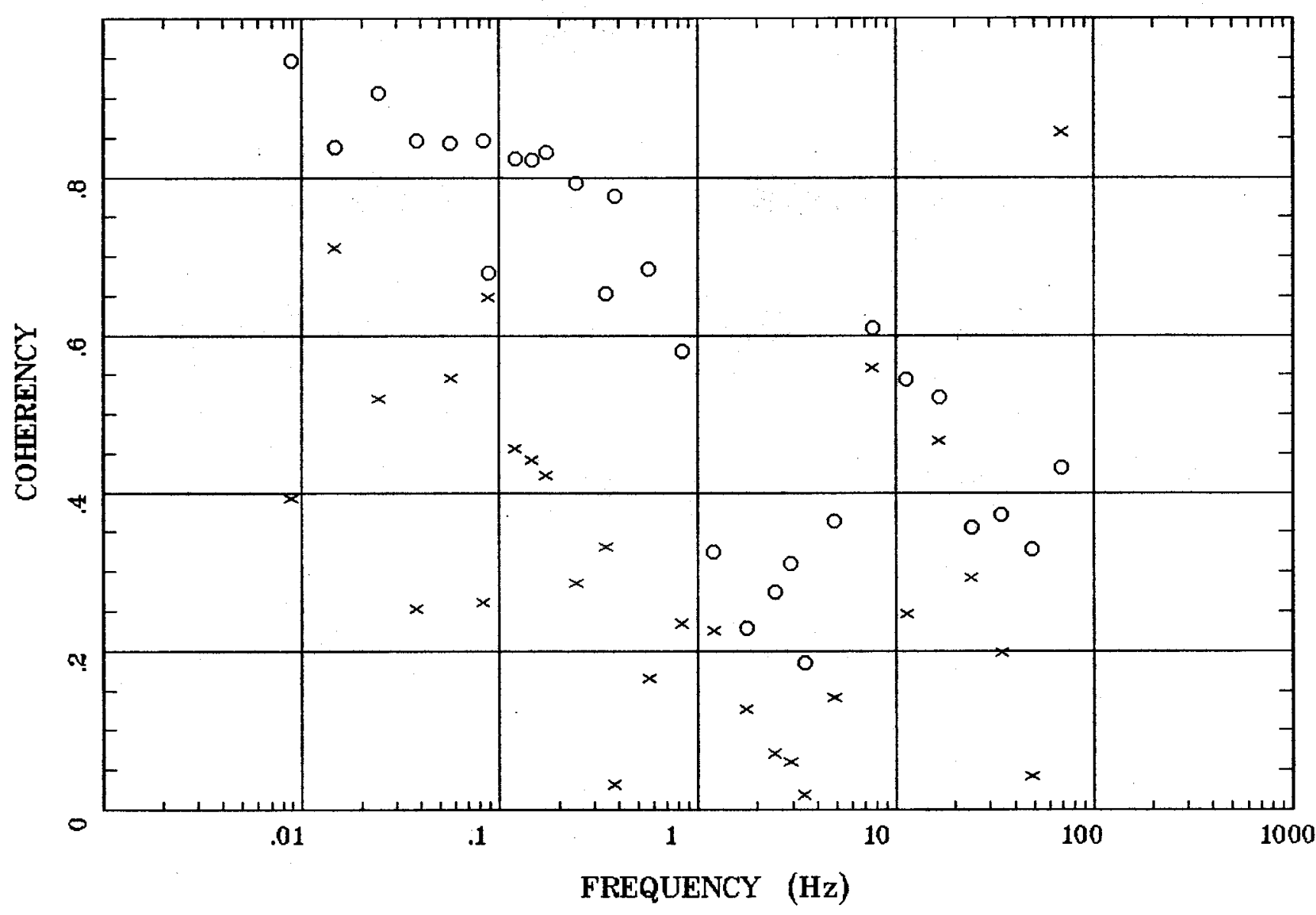

Client:

Remote: e-fld $95 \mathrm{~m}$ south Acquired: 12:3 Jun 17, 2003 Survey Co:USGS
Rotation:

Filename: cp $18 \mathrm{~m}$.avg

Channels: Ch1 Ch2 Ch3 ch4 ch5 Ch6 Ch7 Platted: 08:41 Jul 07, 2004

$<$ EMI - ElectroMagnetic Instruments 


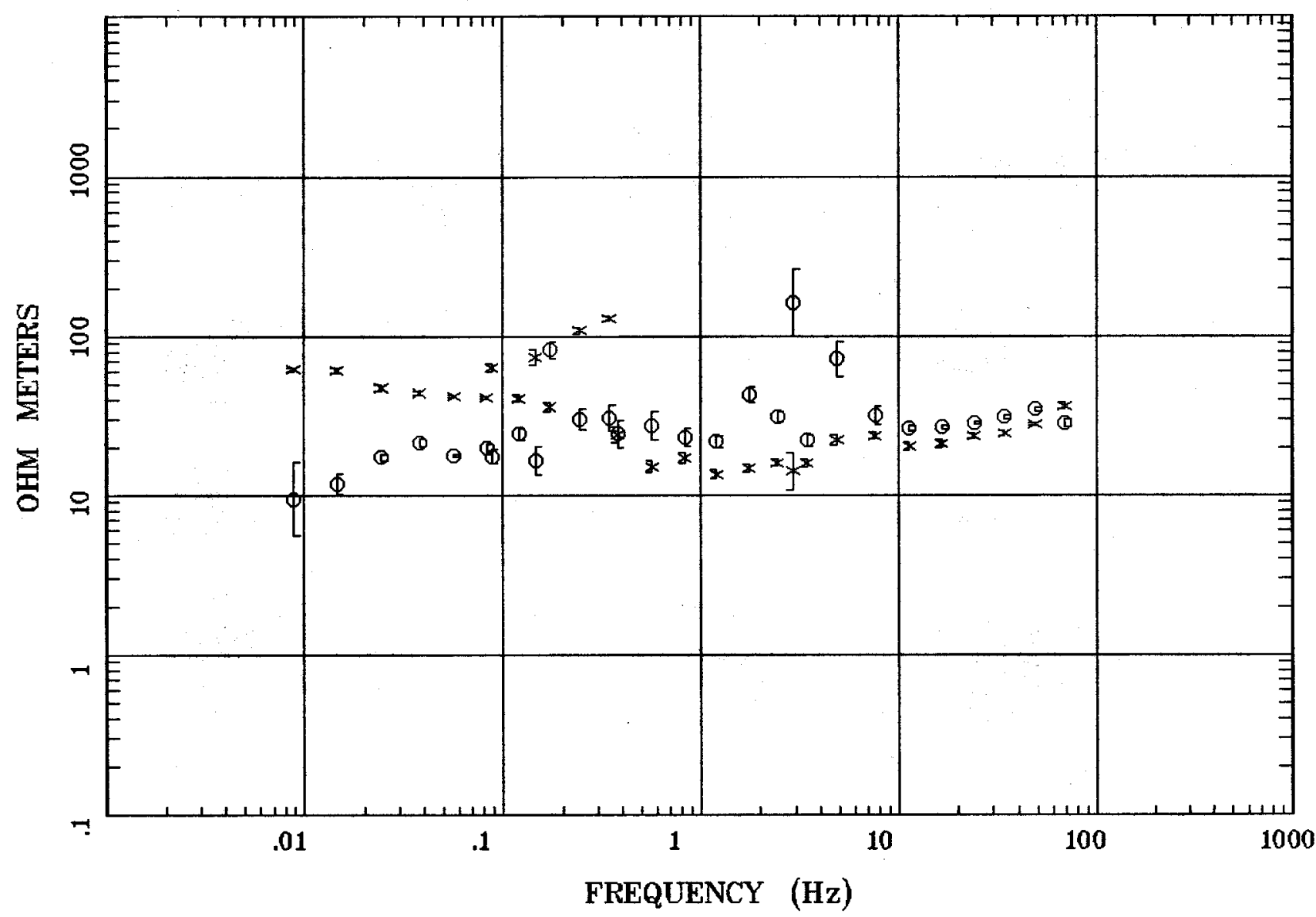

Client:

Remote: e-fld 95 m east Acquired: 11:5 Jun 18, 2003 Survey Co:USGS
Rotation:

Filename: cp $19 \mathrm{~m}$.avg

Channels: Ch1 Ch2 Ch3 Ch4 Ch5 Ch6 Ch7 Plotted: 08:45 Jul 07, 2004

$<$ EMI - ElectroMagnetic Instruments 


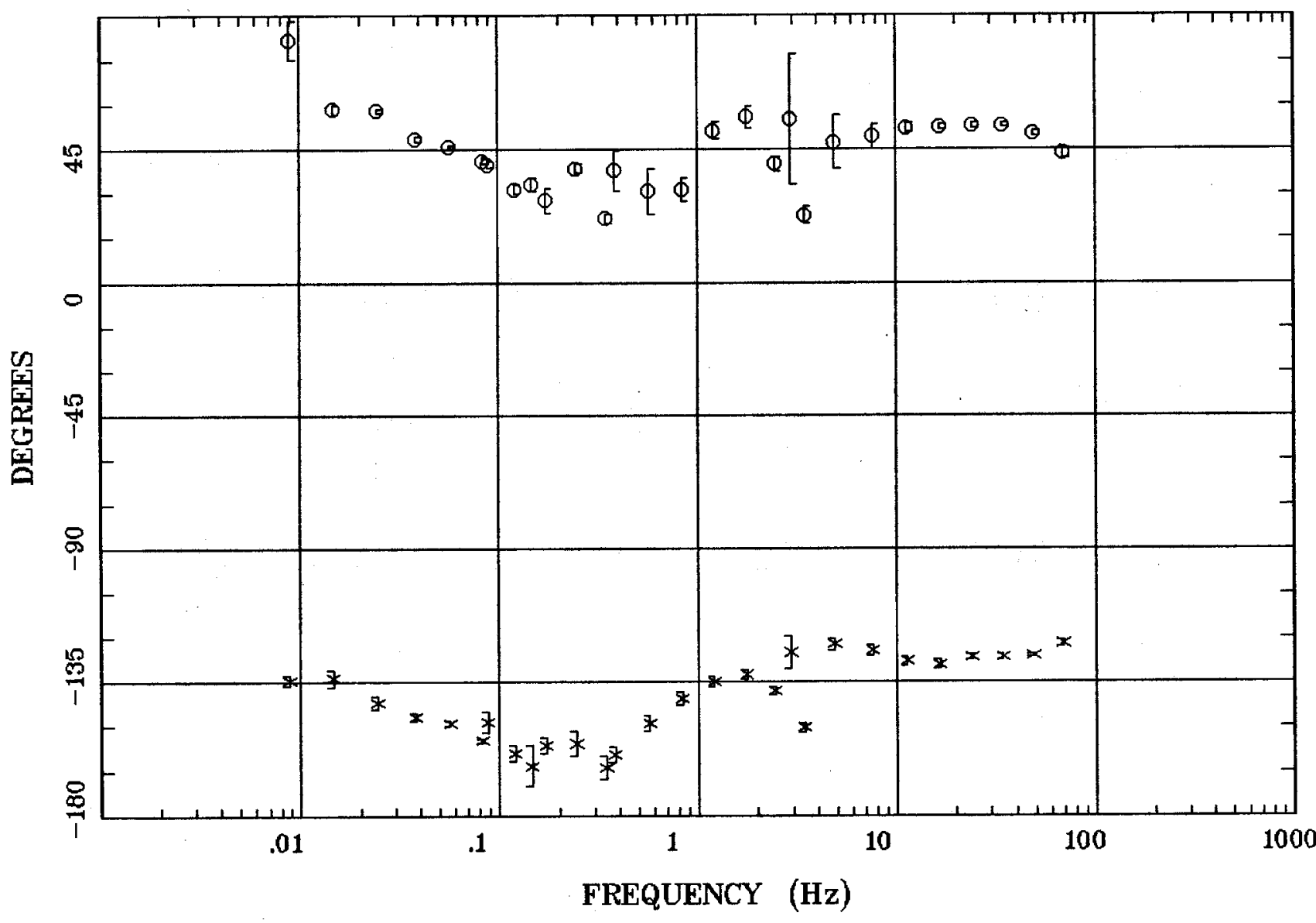

Client:

Remote: e-fld $95 \mathrm{~m}$ east Acquired: 11:5 Jun 18, 2003 Survey Co:USGS
Rotation:

Filename: cp $19 \mathrm{~m}$.avg

Channels: Ch1 Ch2 Ch3 Ch4 Ch5 Ch6 Ch7 Plotted: 08:45 Jul 07, 2004

$<$ EMI - ElectroMagnetic Instruments 


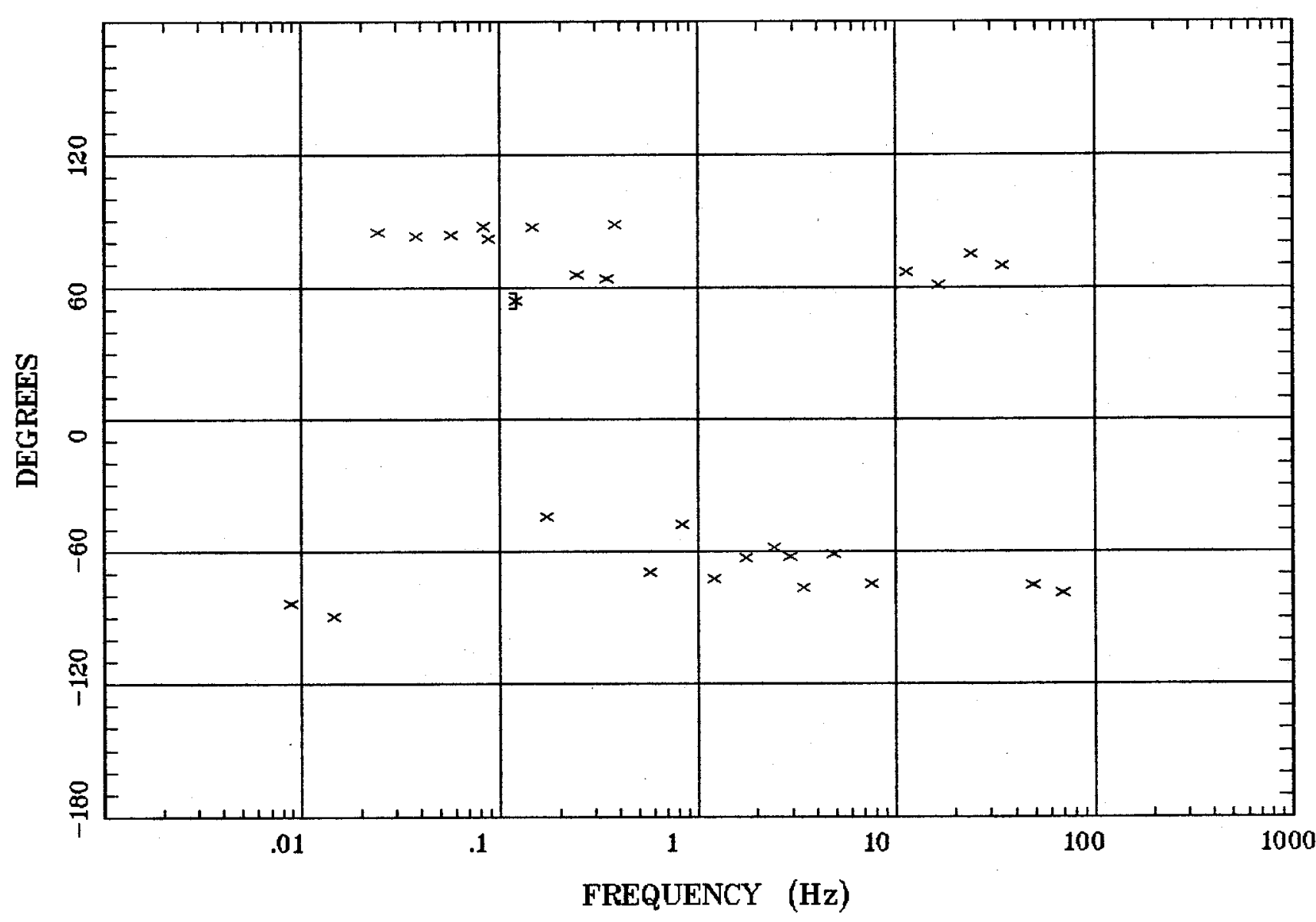

Client:

Remote: e-fld $95 \mathrm{~m}$ east Acquired: 11:5 Jun 18, 2003 Survey Co:USGS
Rotation:

Filename: cp $19 \mathrm{~m}$.avg

Channels: Ch1 Ch2 Ch3 Ch4 Ch5 Ch6 Ch7 Plotted: 10:46 Jul 07, 2004

$<$ EMI - ElectroMagnetic Instruments 


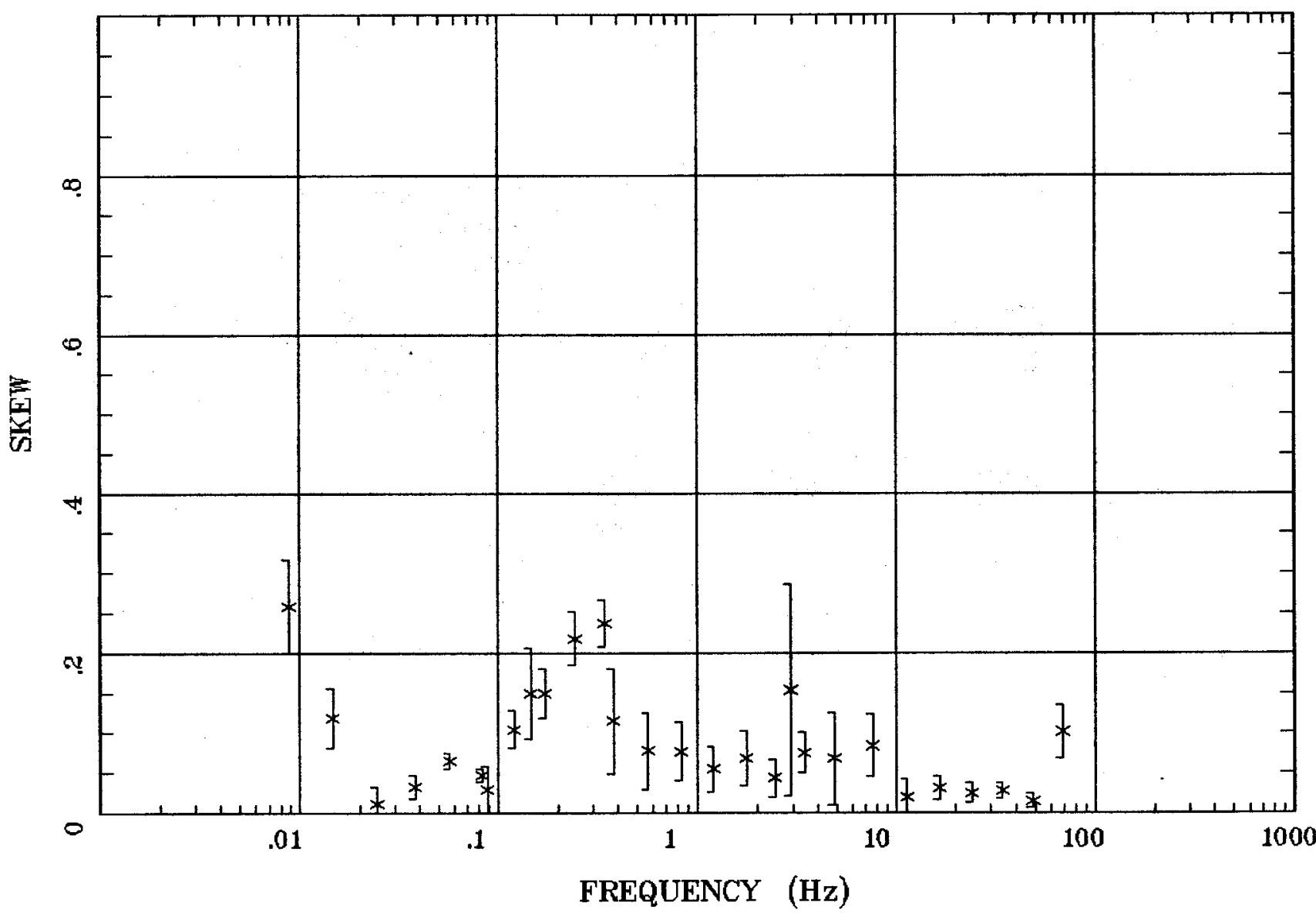

Client:

Remote: e-fld $95 \mathrm{~m}$ east

Acquired: 11:5 Jun 18, 2003 Survey Co:USGS
Rotation:

Filename: cp19m.avg

Channels: Ch1 Ch2 Ch3 Ch4 Ch5 Ch6 Ch7 Plotted: 08:46 Jul 07, 2004

< EMI - ElectroMagnetic Instruments 


\section{E MULT Coh.}

Santa Fe, NM

Station 19

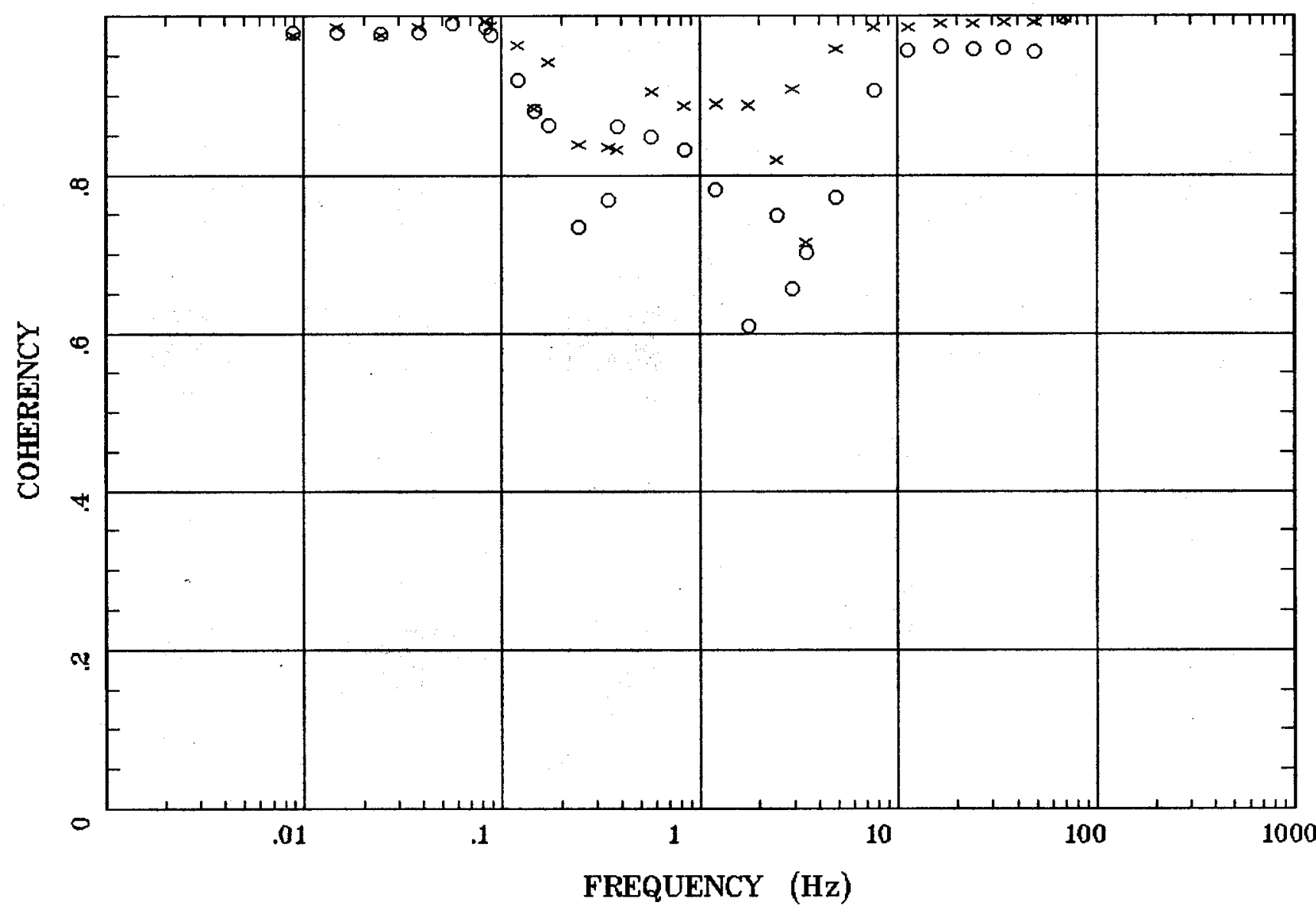

Client:

Remote: e-fld $95 \mathrm{~m}$ east

Acquired: 11:5 Jun 18, 2003 Survey Co:USGS
Rotation:

Filename: cp $19 \mathrm{~m}$.avg

Channels: Ch1.Ch2 Ch3 ch4 ch5 ch6 ch7 Plotted: 08:46 Jul 07, 2004

$<$ EMI - ElectroMagnetic Instruments 


\section{POLAR PLOTS}

Santa Fe, NM

Station 19

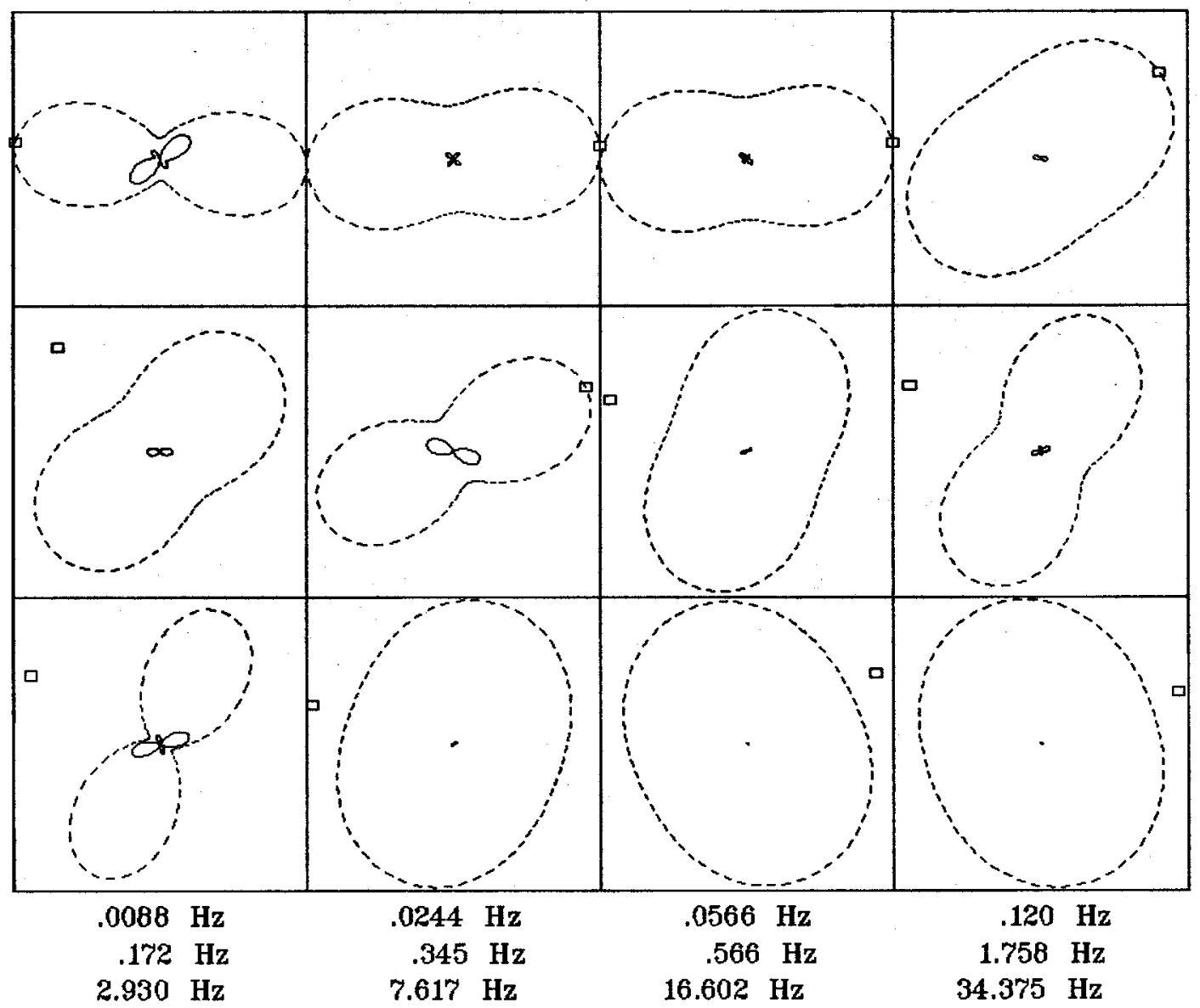

Client:

Remote: e-fld $95 \mathrm{~m}$ east Acquired: 11:5 Jun 18, 2003 Survey Co:USGS
Rotation:

Filename: cp $19 \mathrm{~m} . \mathrm{avg}$

Channels: Ch1 Ch2 Ch3 Ch4 Ch5 Ch6 Ch7 Plotted: 08:46 Jul 07, 2004

\& EMI - ElectroMagnetic Instruments 


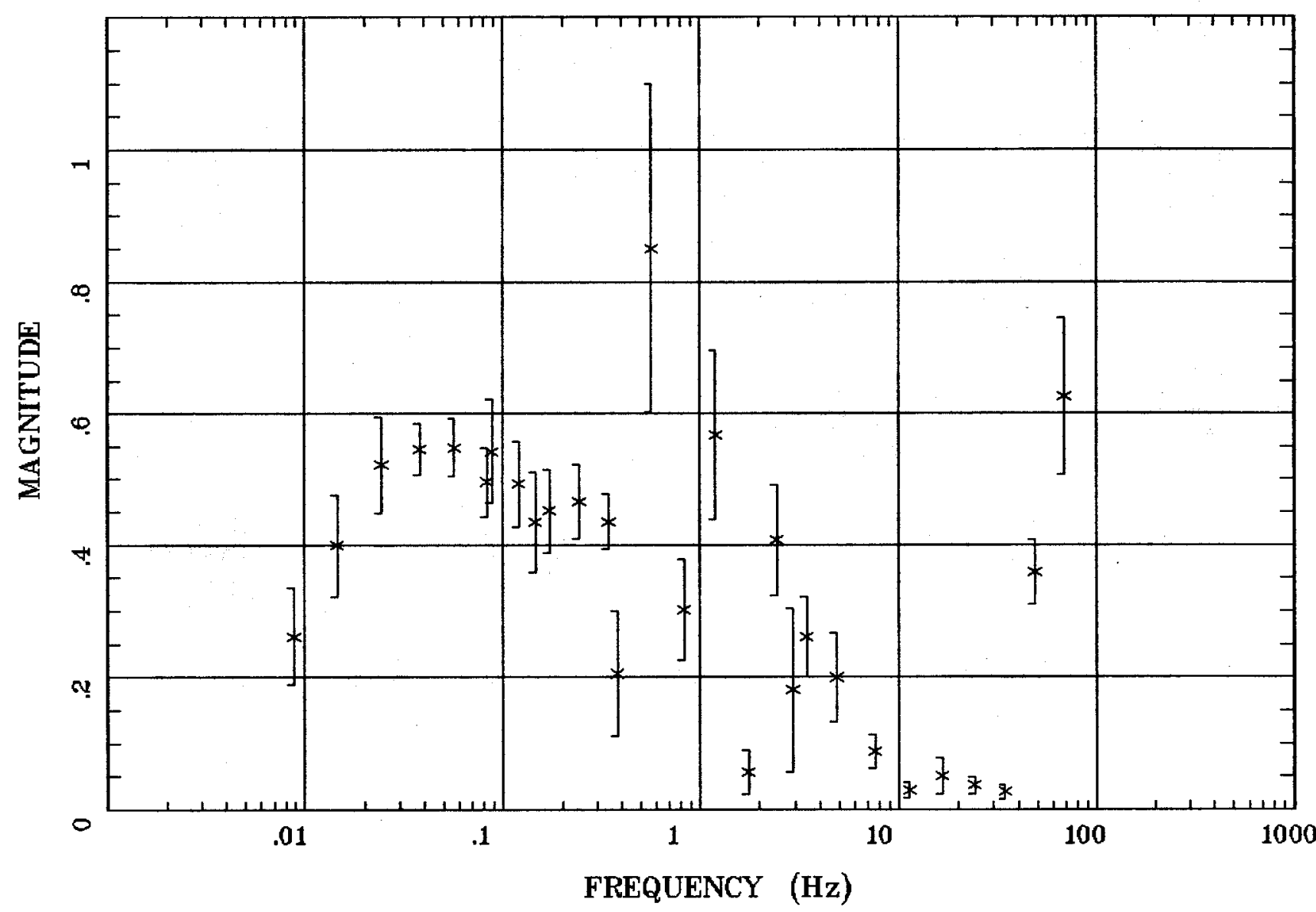

Client:

Remote: e-fld $95 \mathrm{~m}$ east Acquired: 11:5 Jun 18, 2003 Survey Co:USGS
Rotation:

Filename: cp $19 \mathrm{~m} . \mathrm{avg}$

Channels: Ch1 Ch2 Ch3 Ch4 Ch5 Ch6 Ch7 Plotted: 08:46 Jul 07, 2004

$<$ EMI - ElectroMagnetic Instruments 


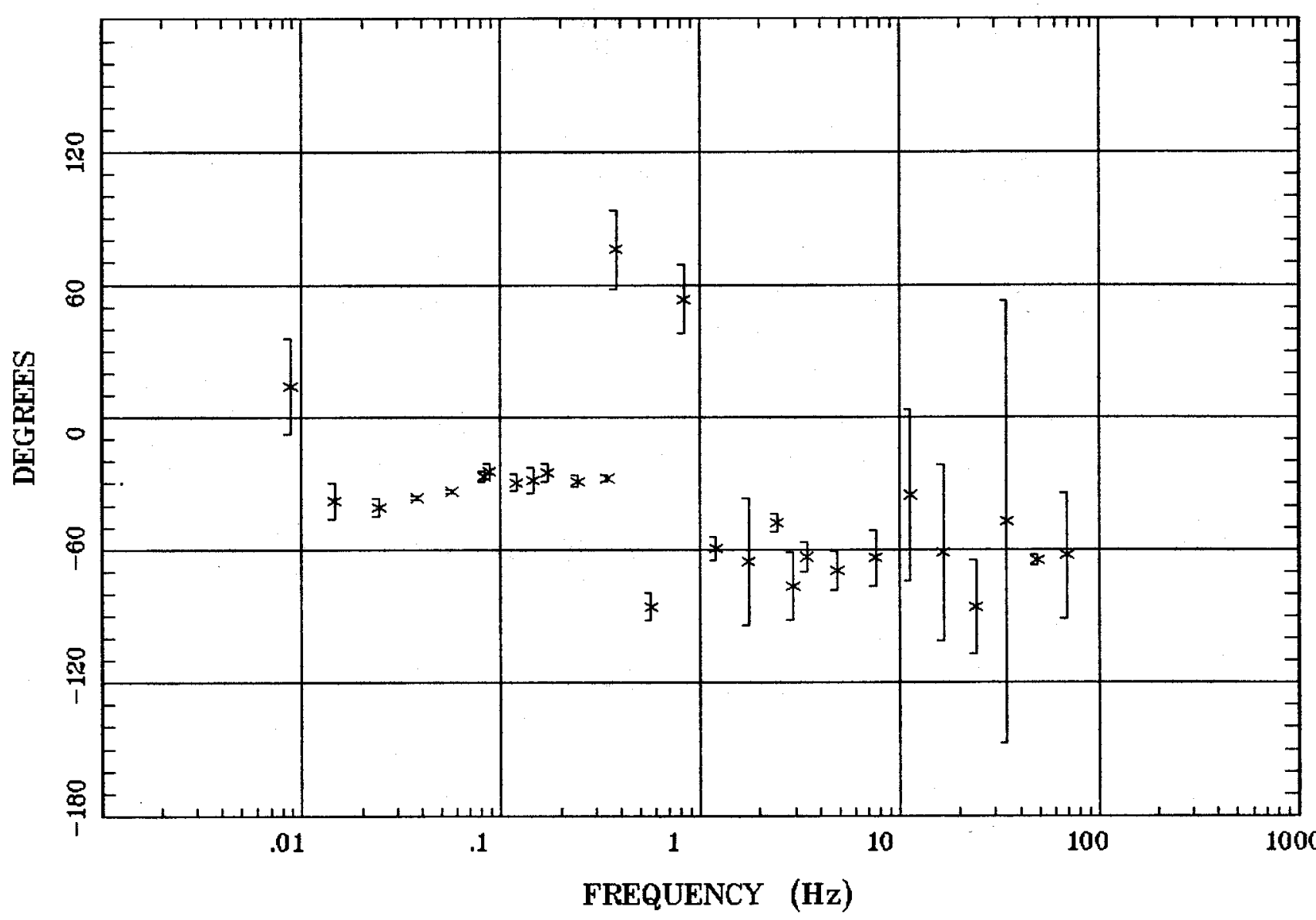

Client:

Remote: e-fld $95 \mathrm{~m}$ east

Acquired: 11:5 Jun 18, 2003 Survey Co:UsGS
Rotation:

Filename: cp $19 \mathrm{~m}$.avg

Channels: Ch1 Ch2 Ch3 Ch4 Ch5 Ch6 Ch7 Plotted: 08:46 Jul 07, 2004

< EMI - ElectroMagnetic Instruments 


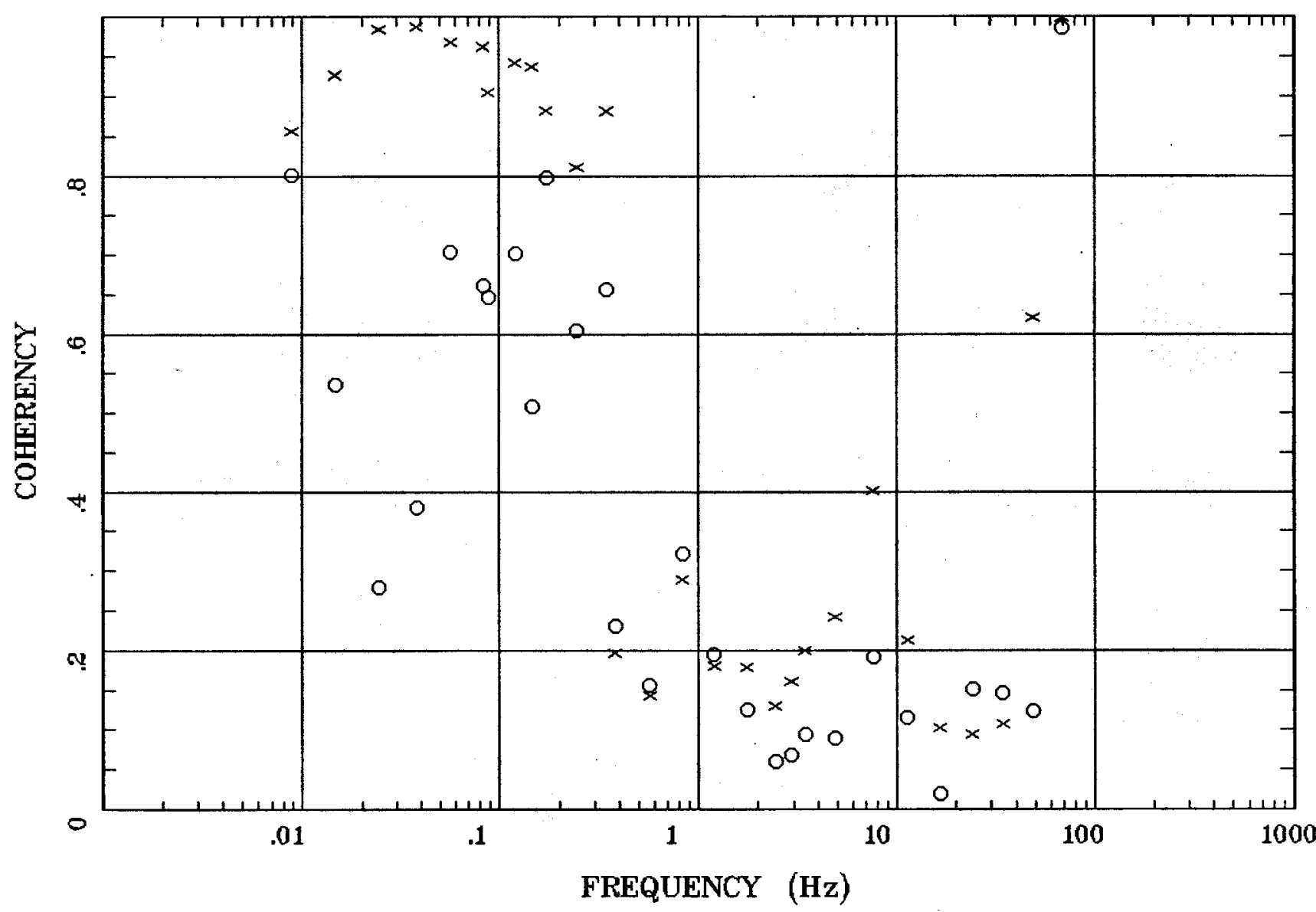

Client:

Remote: e-fld $95 \mathrm{~m}$ east Acquired: 11:5 Jun 18, 2003 Survey Co:USGS
Rotation:

Filename: cp $19 \mathrm{~m} . \mathrm{avg}$

Channels: Ch1 Ch2 Ch3 Ch4 Ch5 Ch6 Ch7 Plotted: 08:46 Jul 07, 2004

< EMI - ElectroMagnetic Instruments 


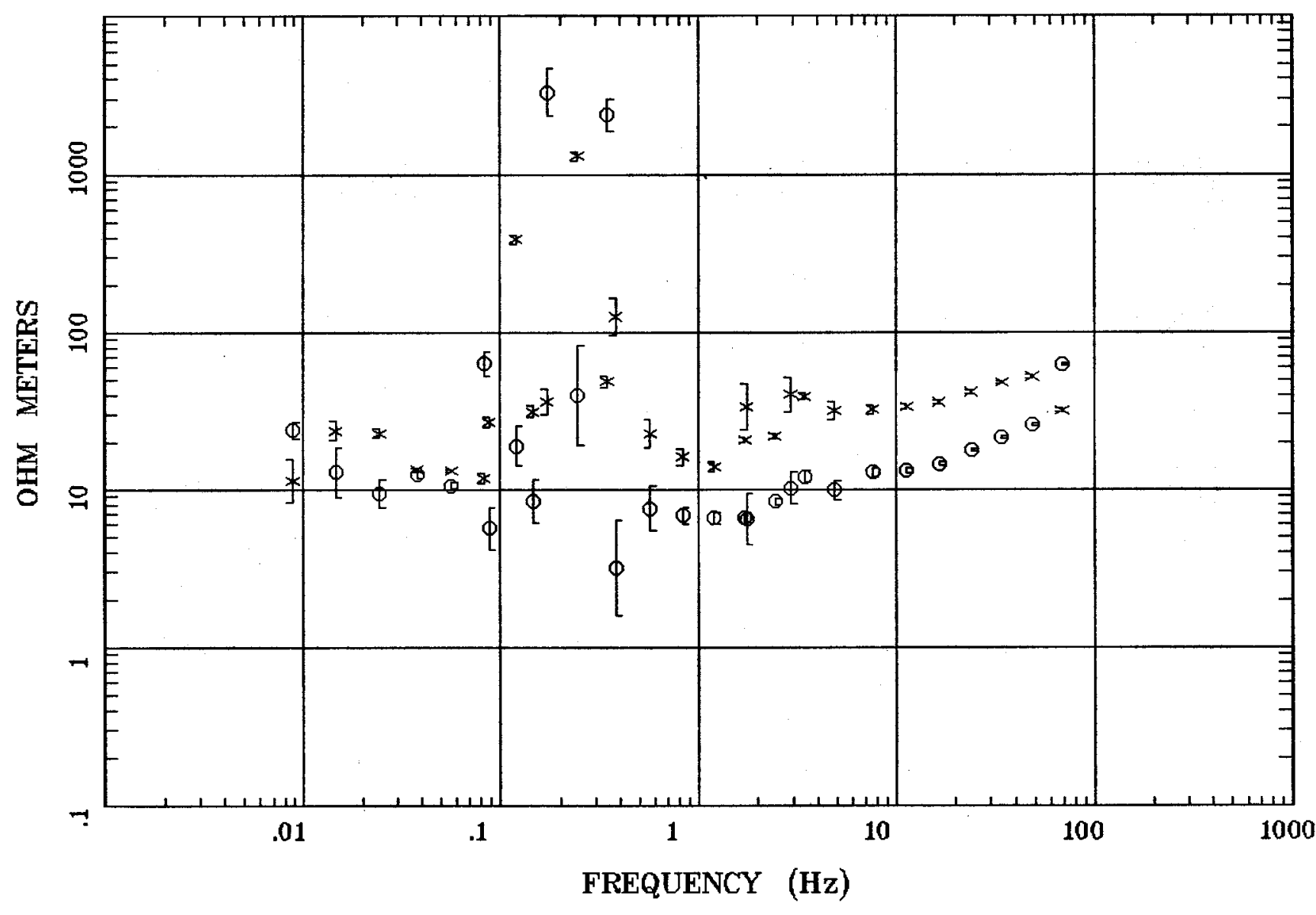

Client:

Remote: e-fld $95 \mathrm{~m}$ north Acquired: 11:2 Jun 19, 2003 Survey Co:USGS
Rotation:

Filename: cp20mall,avg

Channels: Ch1 Ch2 Ch3 Ch4 Ch5 Ch6 Ch7 Plotted: 08:47 Jul 07, 2004

$<$ EMI - ElectroMagnetic Instruments 


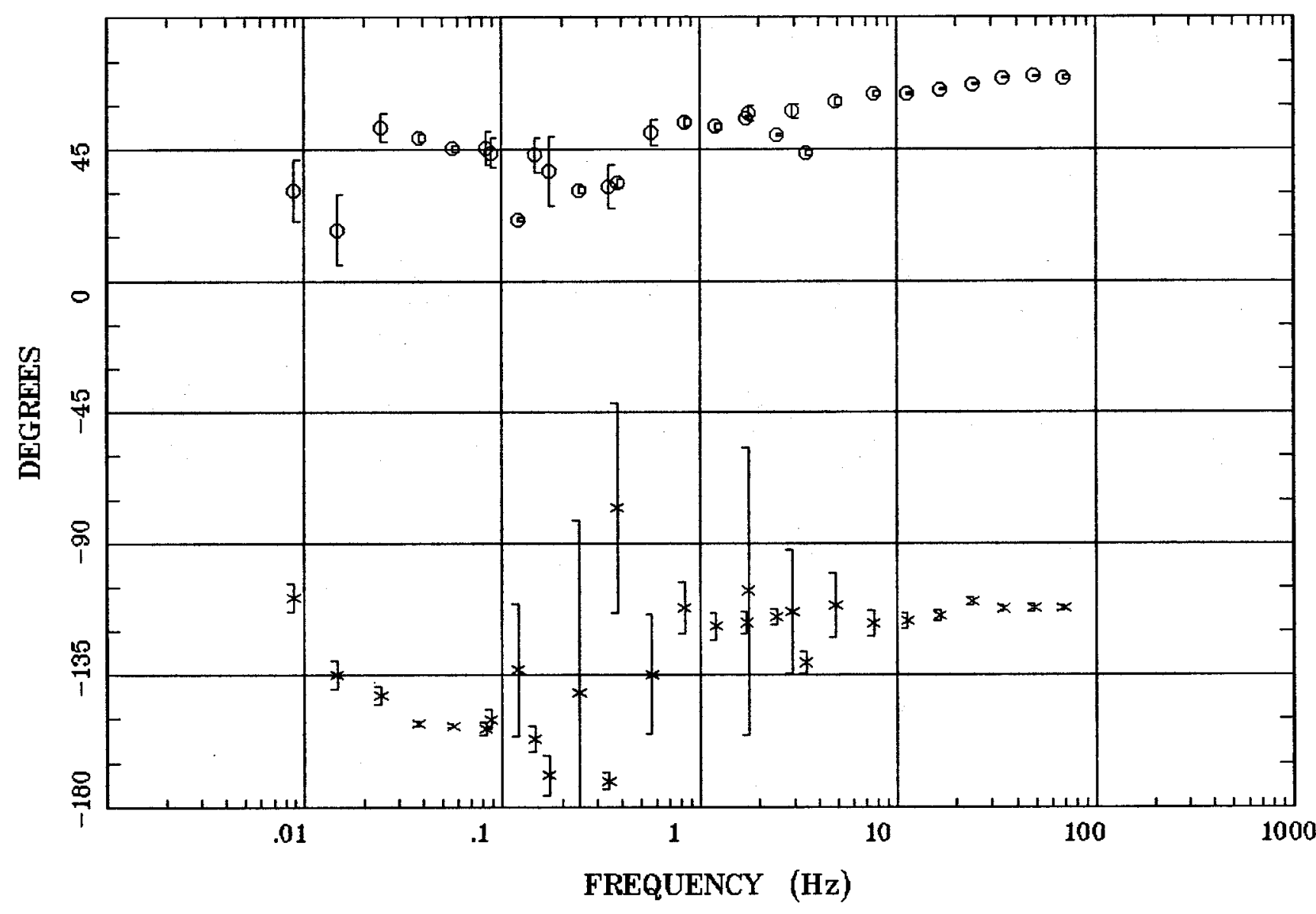

Client:

Remote: e-fld $95 \mathrm{~m}$ north Acquired: 11:2 Jun 19, 2003 Survey Co:USGS
Rotation:

Filename: cp20mall.avg Channels: Ch1 Ch2 Ch3 Ch4 Ch5 Ch6 Ch7 Plotted: 08:47 Jul 07, 2004

< EMI - ElectroMagnetic Instruments 


\section{ROTATION ANGLE}

Santa Fe, NM

Station 20

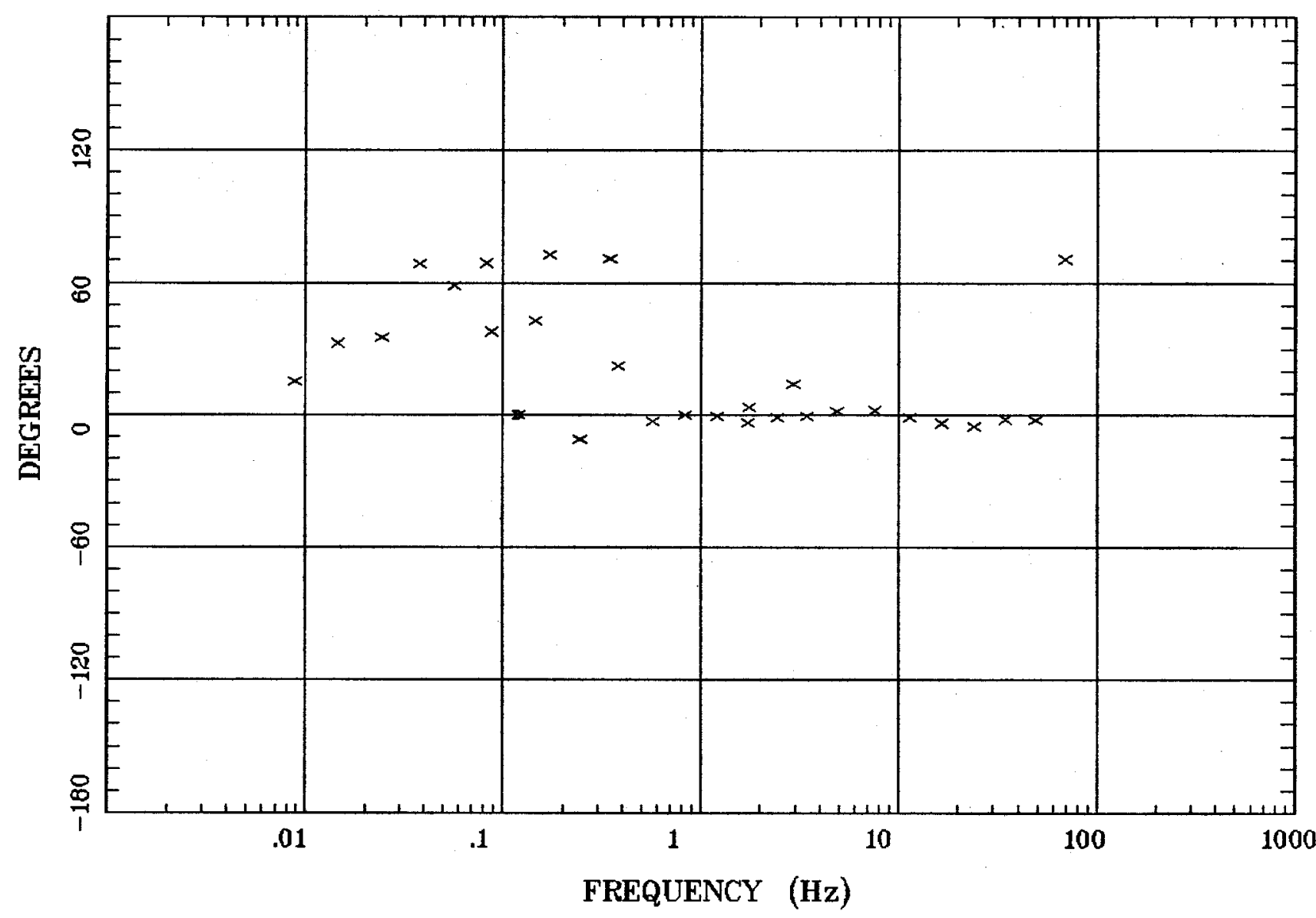

Client:

Remote: e-fld $95 \mathrm{~m}$ north Acquired: 11:2 Jun 19, 2003 Survey Co:USGS
Rotation:

Filename: cp20mall.avg Channels: Ch1 Ch2 Ch3 Ch4 Ch5 Ch6 Ch7 Plotted: 10:46 Jul 07, 2004

< EMI - ElectroMagnetic Instruments 


\section{IMPEDANCE SKEW}

Santa Fe, NM

Station 20

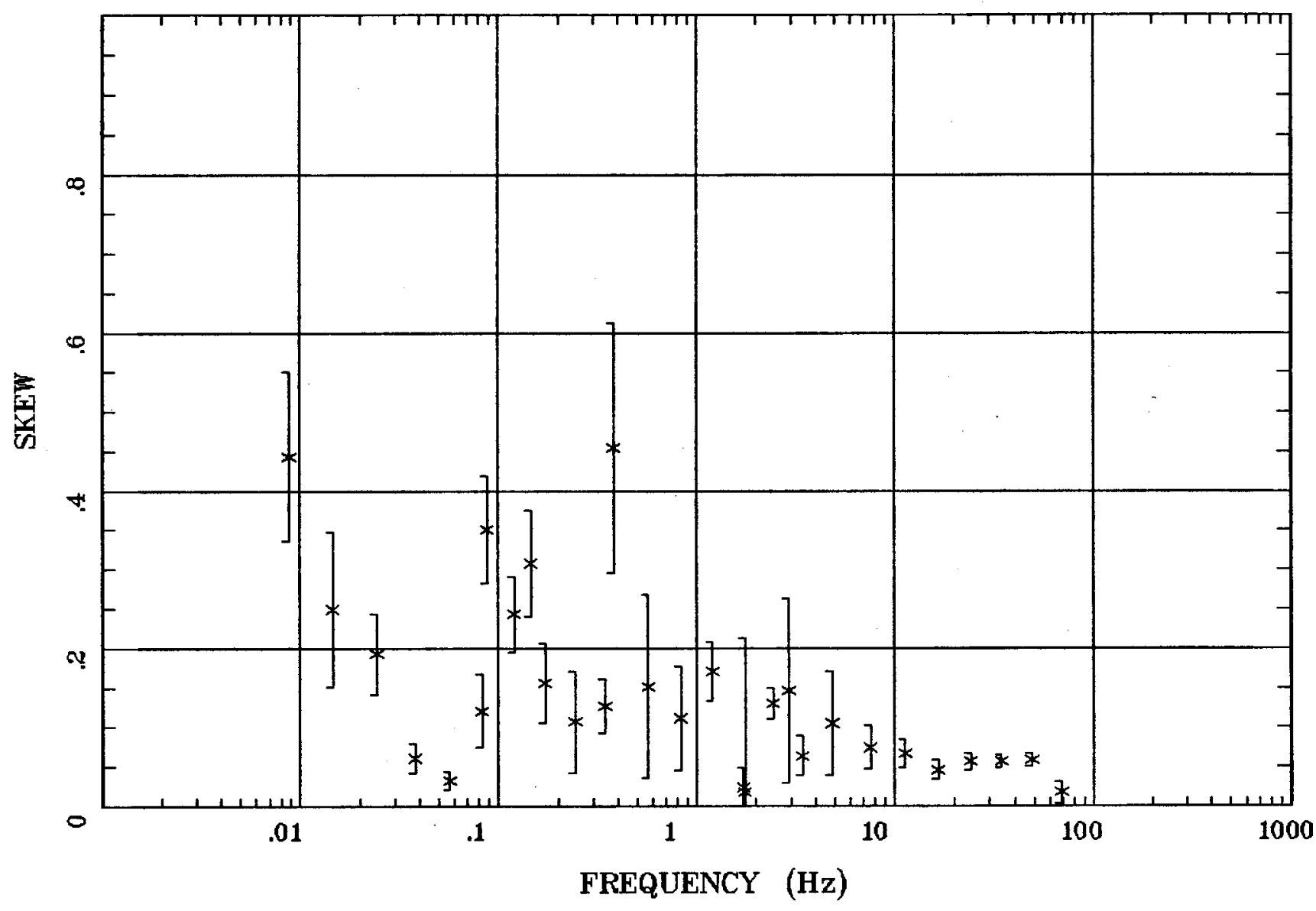

Client:

Remote: e-fld $95 \mathrm{~m}$ north Acquired: 11:2 Jun 19, 2003 Survey Co:USGS
Rotation:

Filename: cp20mall.avg

Channels: Ch1 Ch2 Ch3 Ch4 Ch5 Ch6 Ch7 Plotted: 08:47 Jul 07, 2004

< EMI - ElectroMagnetic Instruments 


\section{Station 20}

E MULT Coh.

Santa Fe, NM

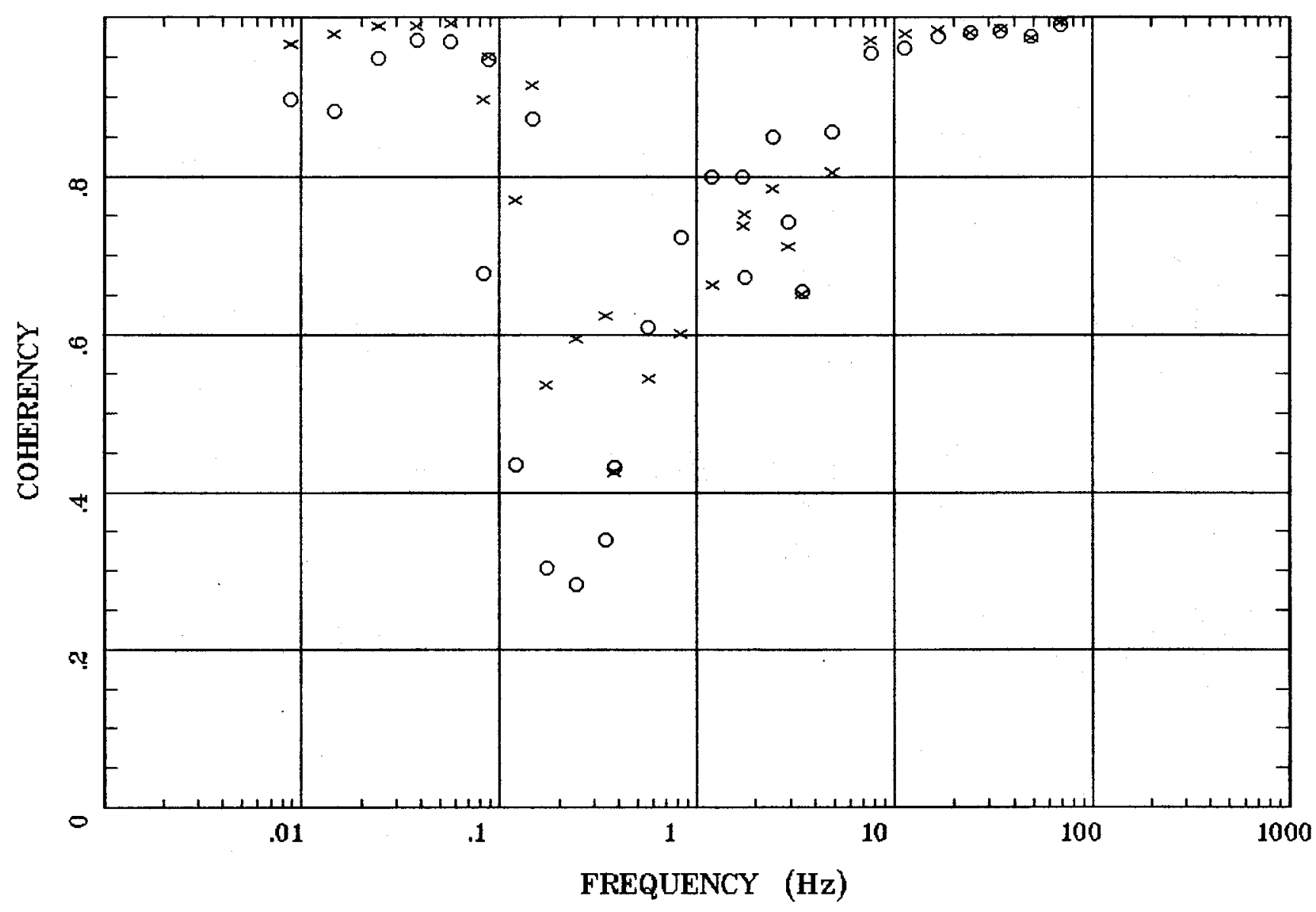

Client:

Remote: e-fld $95 \mathrm{~m}$ north Acquired: 11:2 Jun 19, 2003 Survey Co:USGS
Rotation:

Filename: cp20mall.avg

Channels: Ch1 Ch2 Ch3 Ch4 Ch5 Ch6 Ch7 Plotted: 08:47 Jul 07, 2004

< EMI - ElectroMagnetic Instruments > 


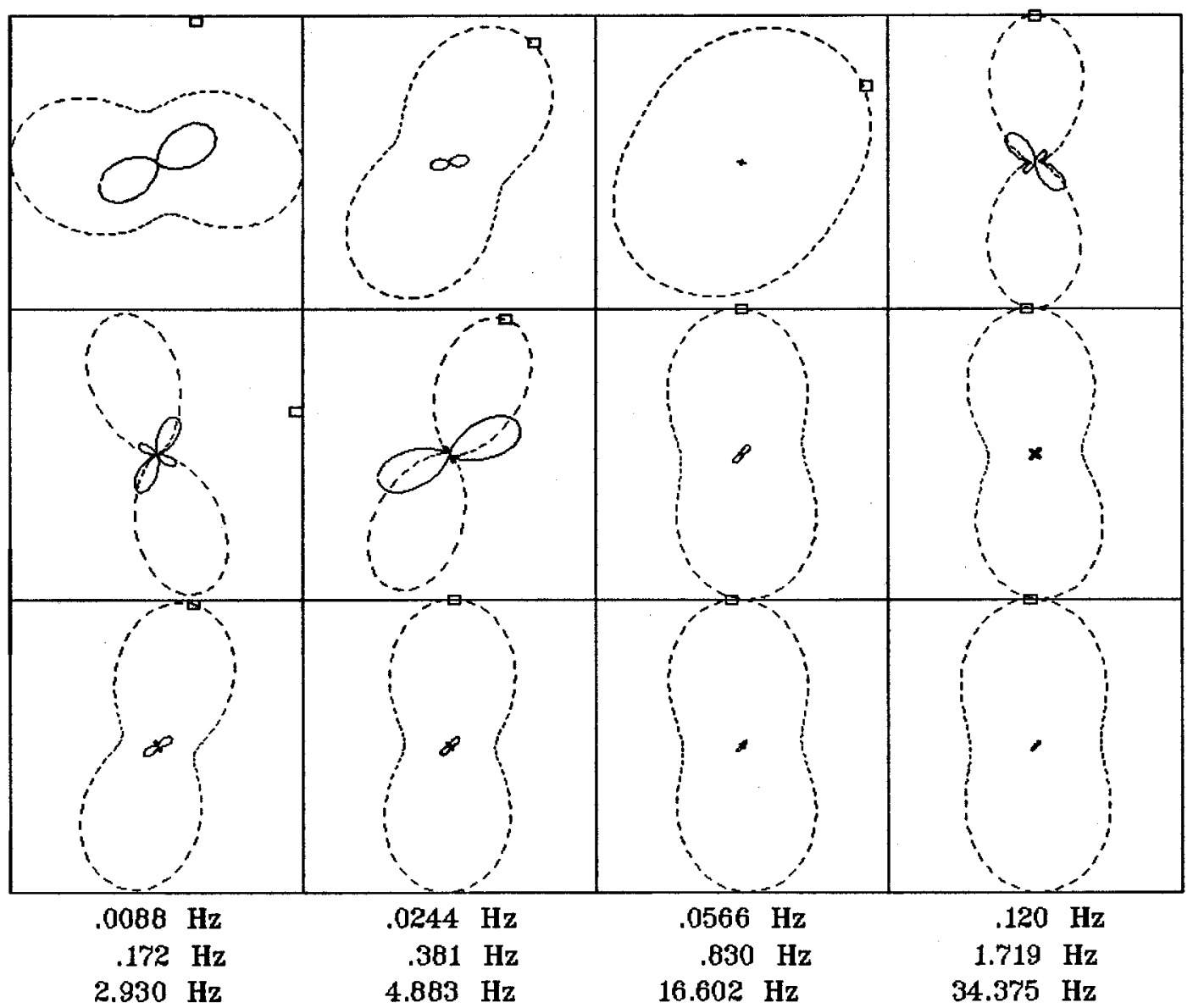

Rotation:

Client:

Remote: e-fld $95 \mathrm{~m}$ north Acquired: 11:2 Jun 19, 2003 Survey Co:USGS
Filename: cp20mall.avg

Channels: Ch1 Ch2 Ch3 Ch4 Ch5 Ch6 Ch7 Plotted: 08:47 Jul 07, 2004

< EMI - ElectroMagnetic Instruments > 


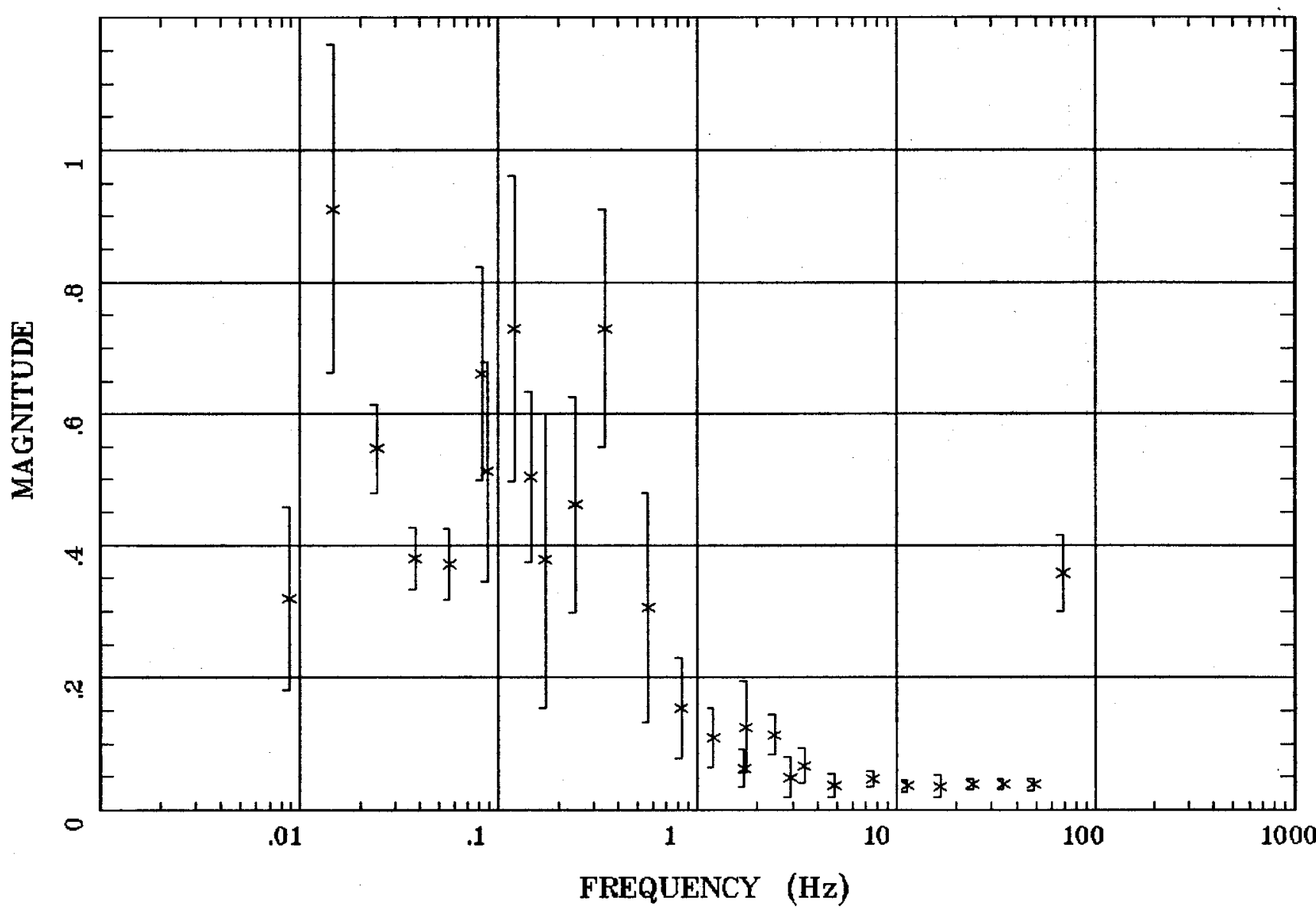

Client:

Remote: e-fld $95 \mathrm{~m}$ north Acquired: 11:2 Jun 19, 2003 Survey Co:USGS
Rotation:

Filename: cp20mall.avg

Channels: Ch1 Ch2 Ch3 Ch4 Ch5 Ch6 Ch7 Plotted: 08:47 Jul 07, 2004

< EMI - ElectroMagnetic Instruments 


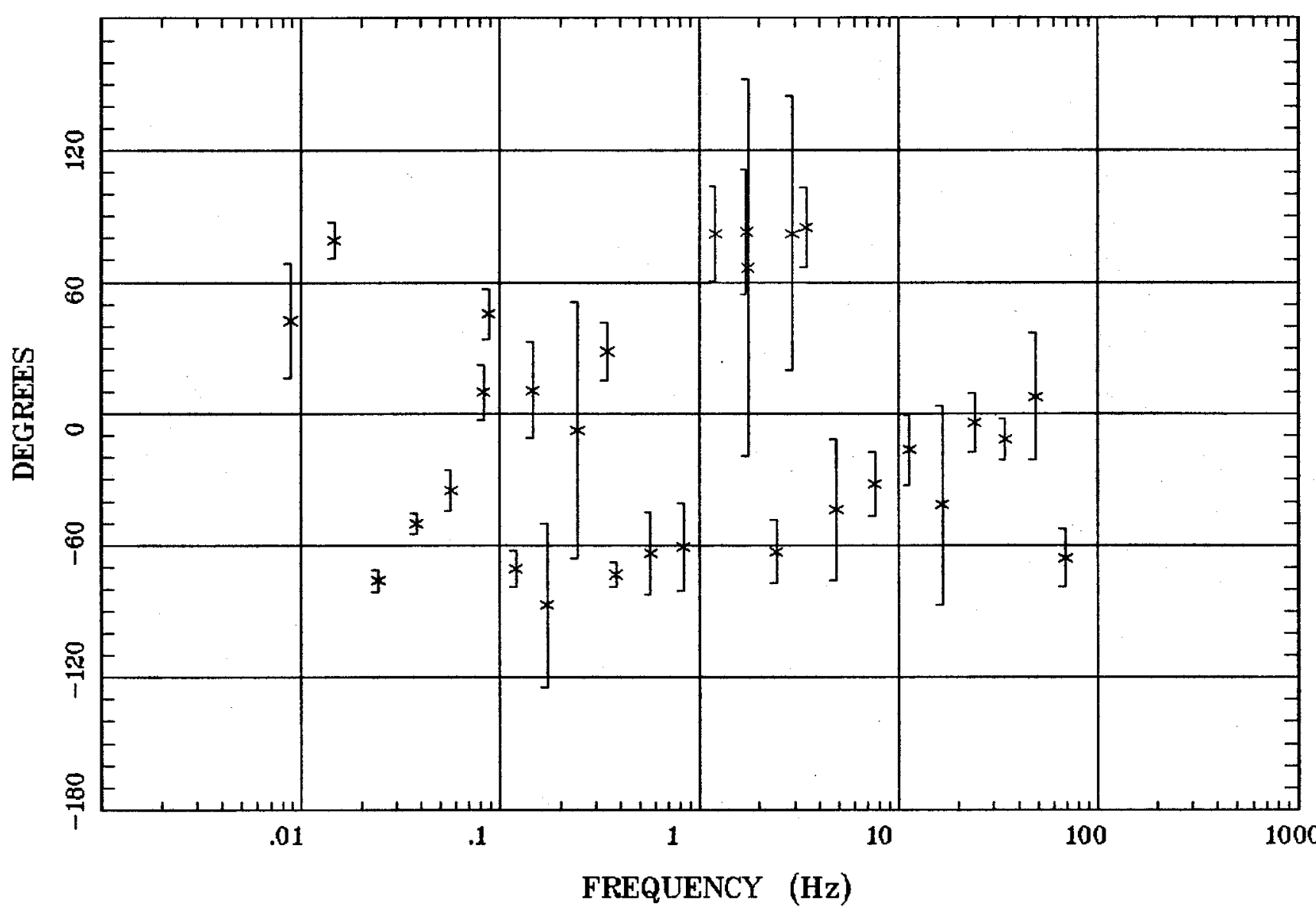

Client:

Remote: e-fld $95 \mathrm{~m}$ north Acquired: 11:2 Jun 19, 2003 Survey Co:USGS
Rotation:

Filename: cp20mall.avg Channels: Ch1 Ch2 Ch3 Ch4 Ch5 ch6 Ch7 Plotted: 08:47 Jul 07\%, 2004

$<$ EMI - ElectroMagnetic Instruments 


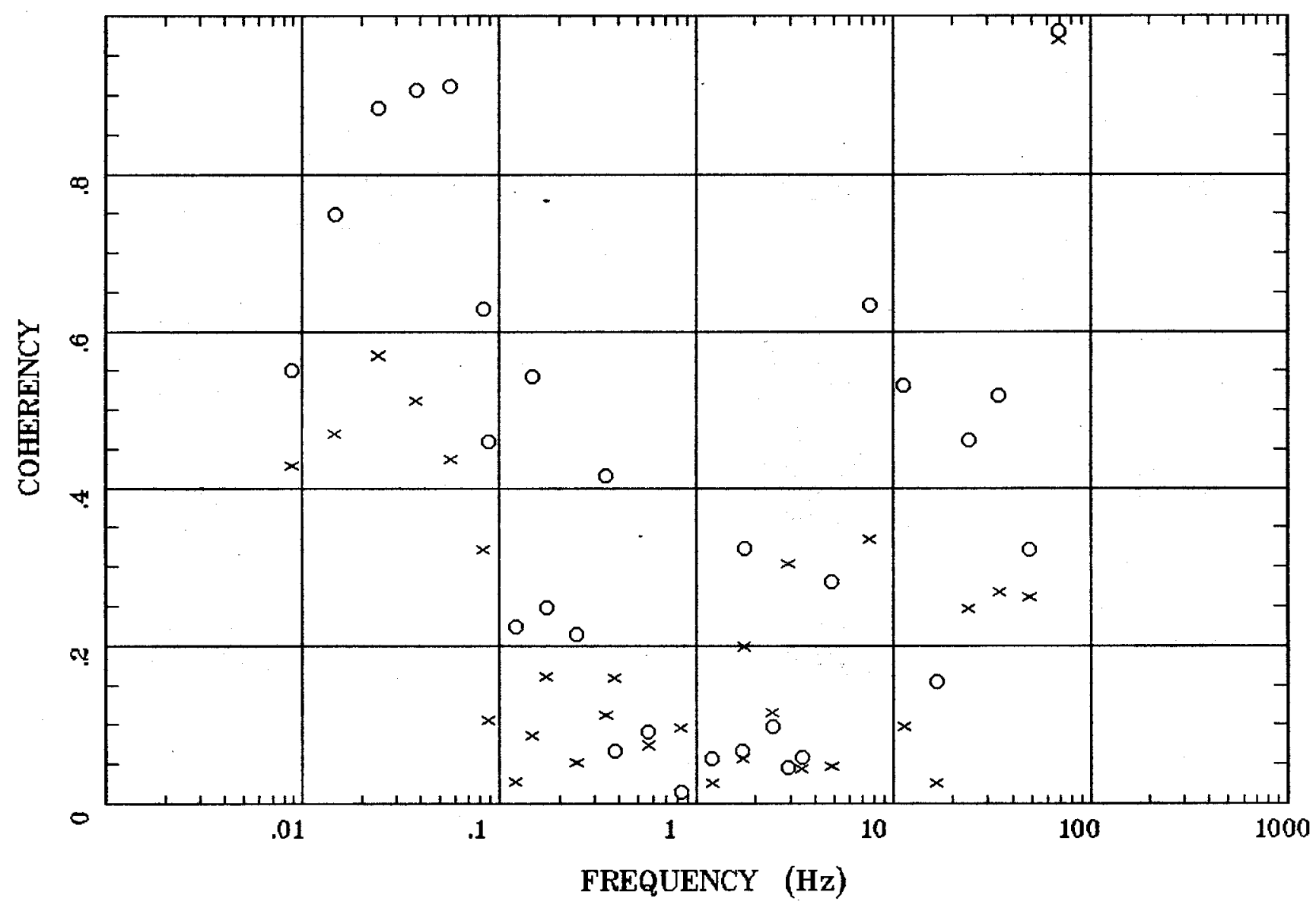

Client:

Remote: e-fld $95 \mathrm{~m}$ north Acquired: 11:2 Jun 19, 2003 Survey Co:USGS

\section{Rotation:}

Filename: cp20mall.avg

Channels: Ch1 Ch2 Ch3 Ch4 Ch5 Ch6 Ch7 Plotted: 08:47 Jul 07, 2004

< EMI - ElectroMagnetic Instruments > 


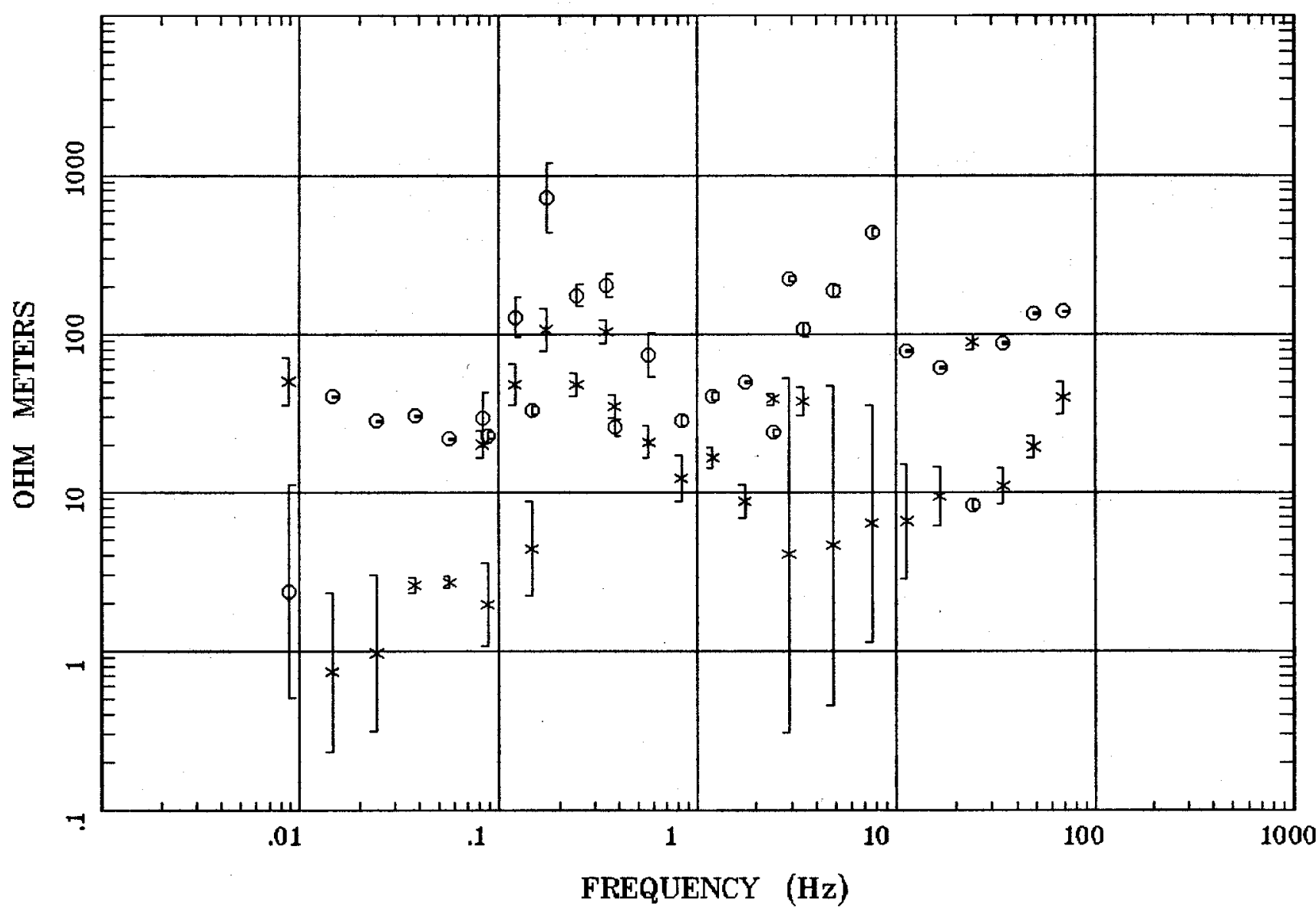

Client:

Remote: e-fld $95 \mathrm{~m}$ north Acquired: 11:4 Jun 20, 2003 Survey Co:USGS
Rotation:

Filename: cp21m.avg

Channels: Ch1 Ch2 Ch3 Ch4 Ch5 Ch6 Ch7 Plotted: 08:48 Jul 07, 2004

<EMI - ElectroMagnetic Instruments > 


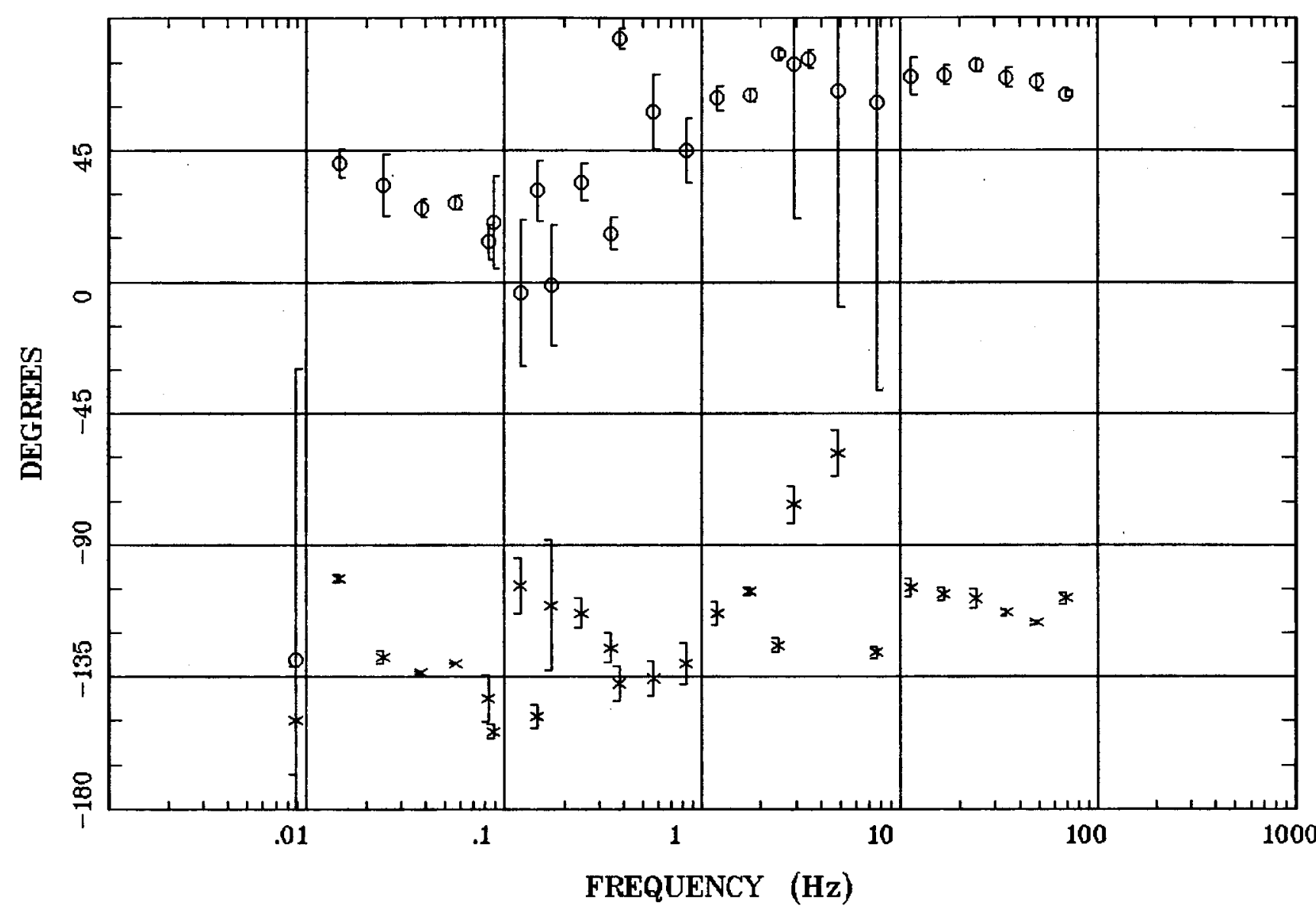

Client:

Remote: e-fld $95 \mathrm{~m}$ north Acquired: 11:4 Jun 20, 2003 Survey Co:USGS
Rotation:

Filename: cp21m.avg

Channels: Ch1 Ch2 Ch3 Ch4 Ch5 Ch6 Ch7 Plotted: 08:48 Jul 07, 2004

$<$ EMI - ElectroMagnetic Instruments > 
Station 21

ROTATION ANGLE

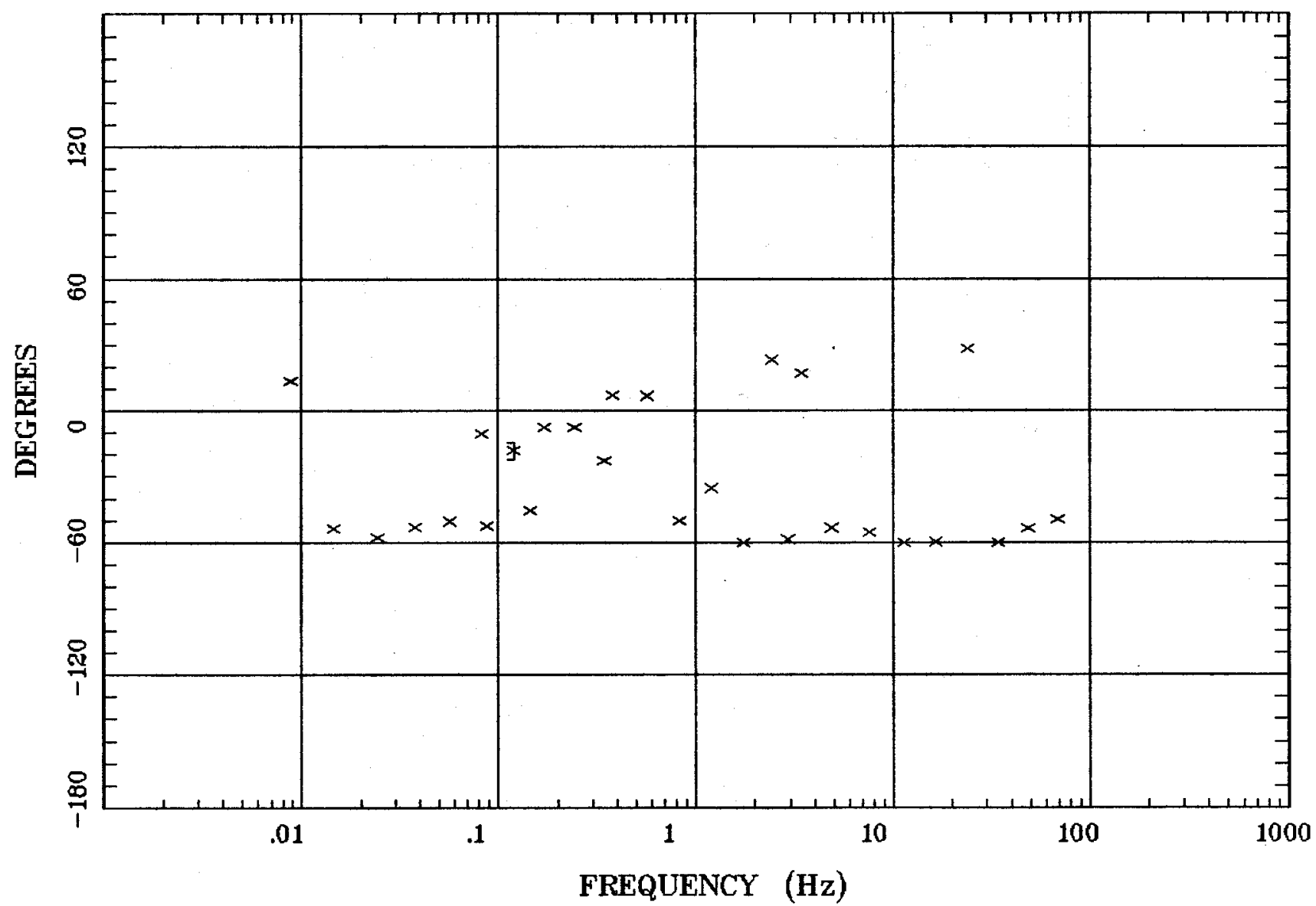

Client:

Remote: e-fld $95 \mathrm{~m}$ north

Acquired: 11:4 Jun 20, 2003

Survey Co:USGS
Santa Fe, NM

\section{Rotation:}

Filename: cp21m.avg

Channels: Ch1 Ch2 Ch3 ch4 Ch5 Ch6 Ch7

Plotted: 10:45 Jul 07, 2004

< EMI - ElectroMagnetic Instruments > 


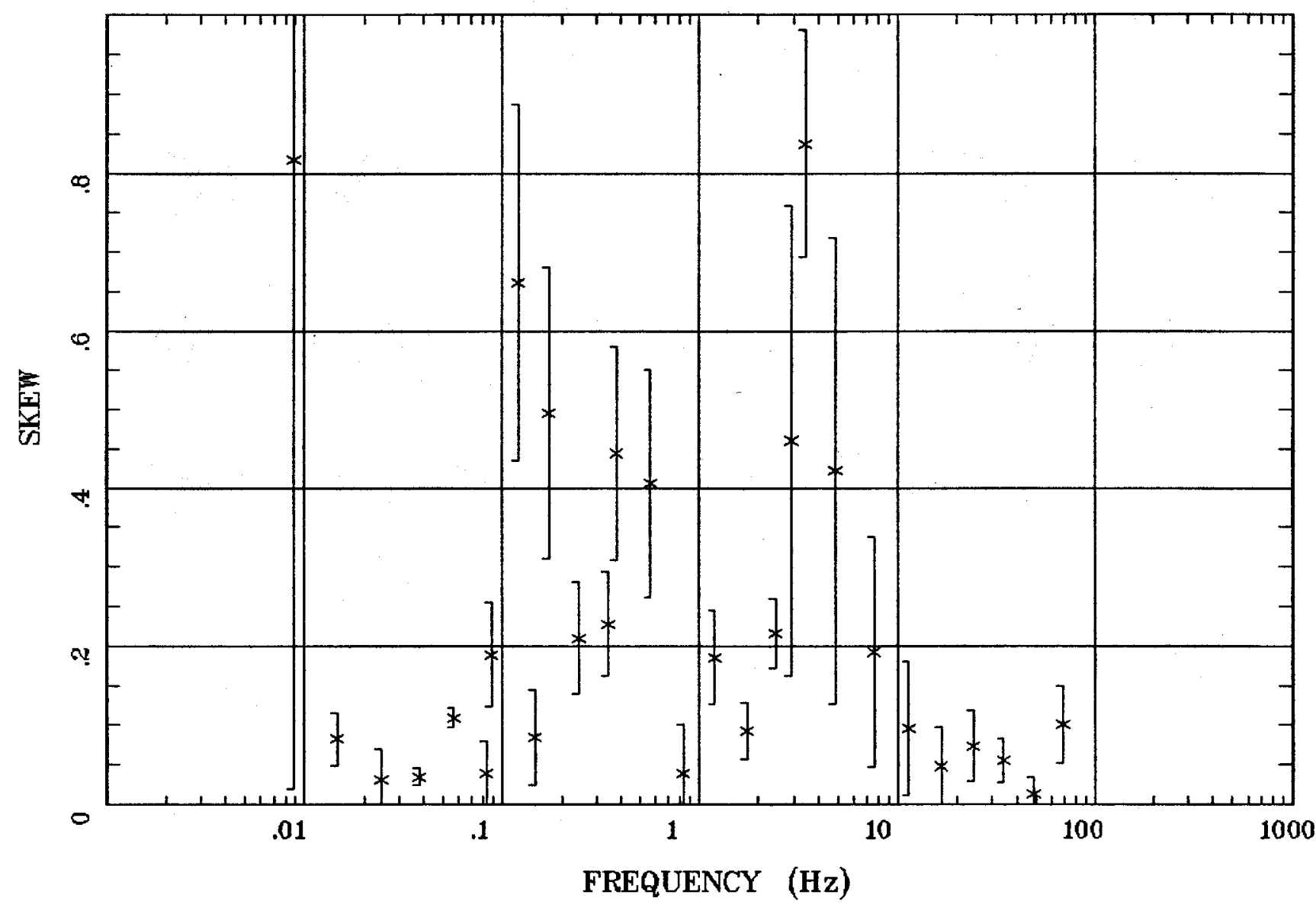

Client:

Remote: e-fld $95 \mathrm{~m}$ north

Acquired: 11:4 Jun 20, 2003 Survey Co:USGS
Rotation:

Filename: cp21m.avg

Channels: Ch1 Ch2 Ch3 Ch4 Ch5 Ch6 Ch7 Plotted: 08:48 Jul 07, 2004

< EMI - ElectroMagnetic Instruments > 


\section{E MULT Coh.}

Santa Fe, NM

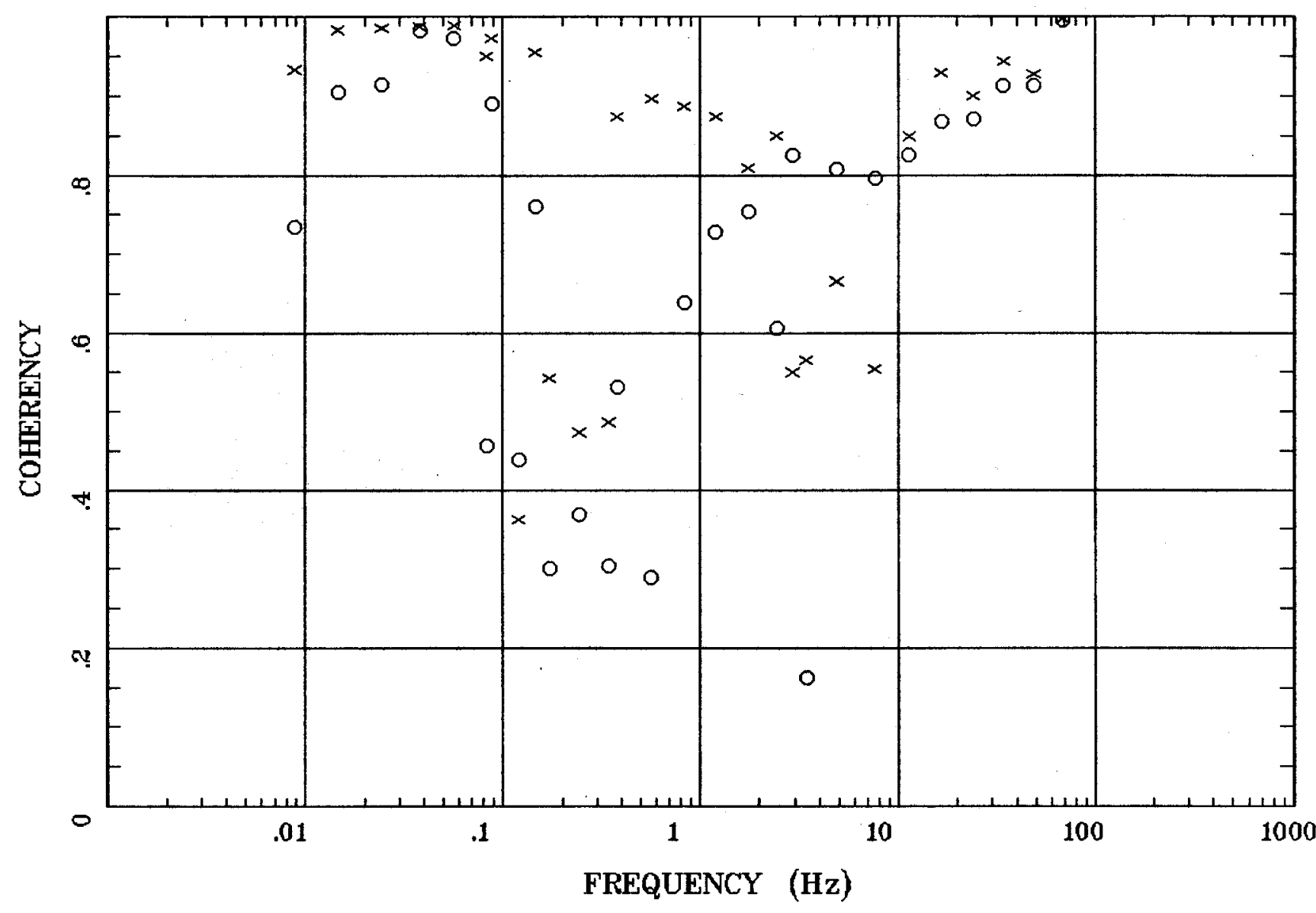

Client:

Remote: e-fld $95 \mathrm{~m}$ north Acquired: 11:4 Jun 20, 2003 Survey Co:USGS
Rotation:

Filename: cp21m.avg

Channels: Ch1 Ch2 Ch3 Ch4 Ch5 Ch6 Ch7 Plotted: 08:49 Jul 07, 2004

$<$ EMI - ElectroMagnetic Instruments > 


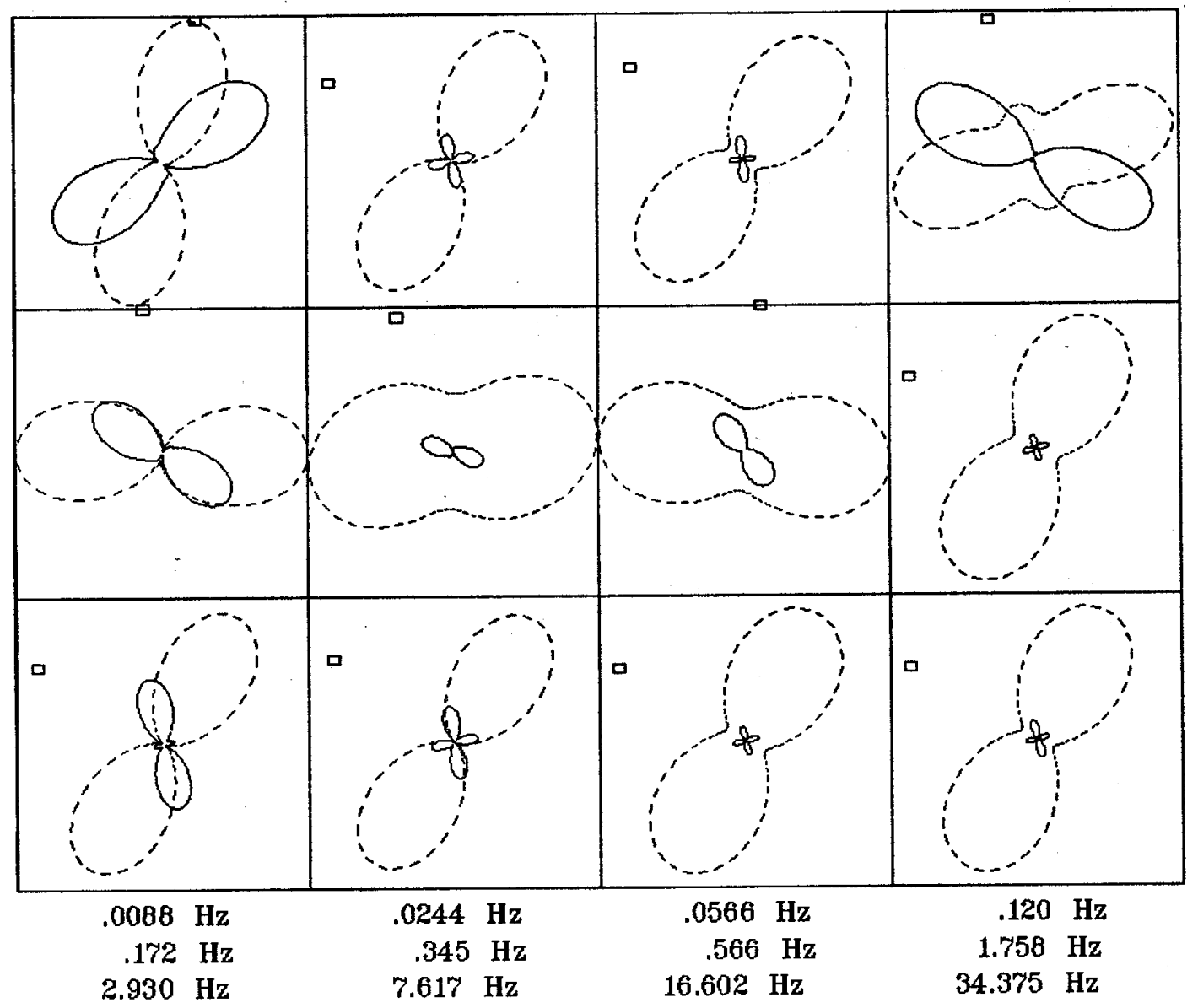

Rotation:

Client:

Remote: e-fld $95 \mathrm{~m}$ north Acquired: 11:4 Jun 20, 2003 Survey Co:USGS
Filename: cp21m.avg

Channels: Ch1 Ch2 Ch3 Ch4 Ch5 Ch6 Ch7 Plotted: 08:49 Jul 07, 2004

< EMI - ElectroMagnetic Instruments > 


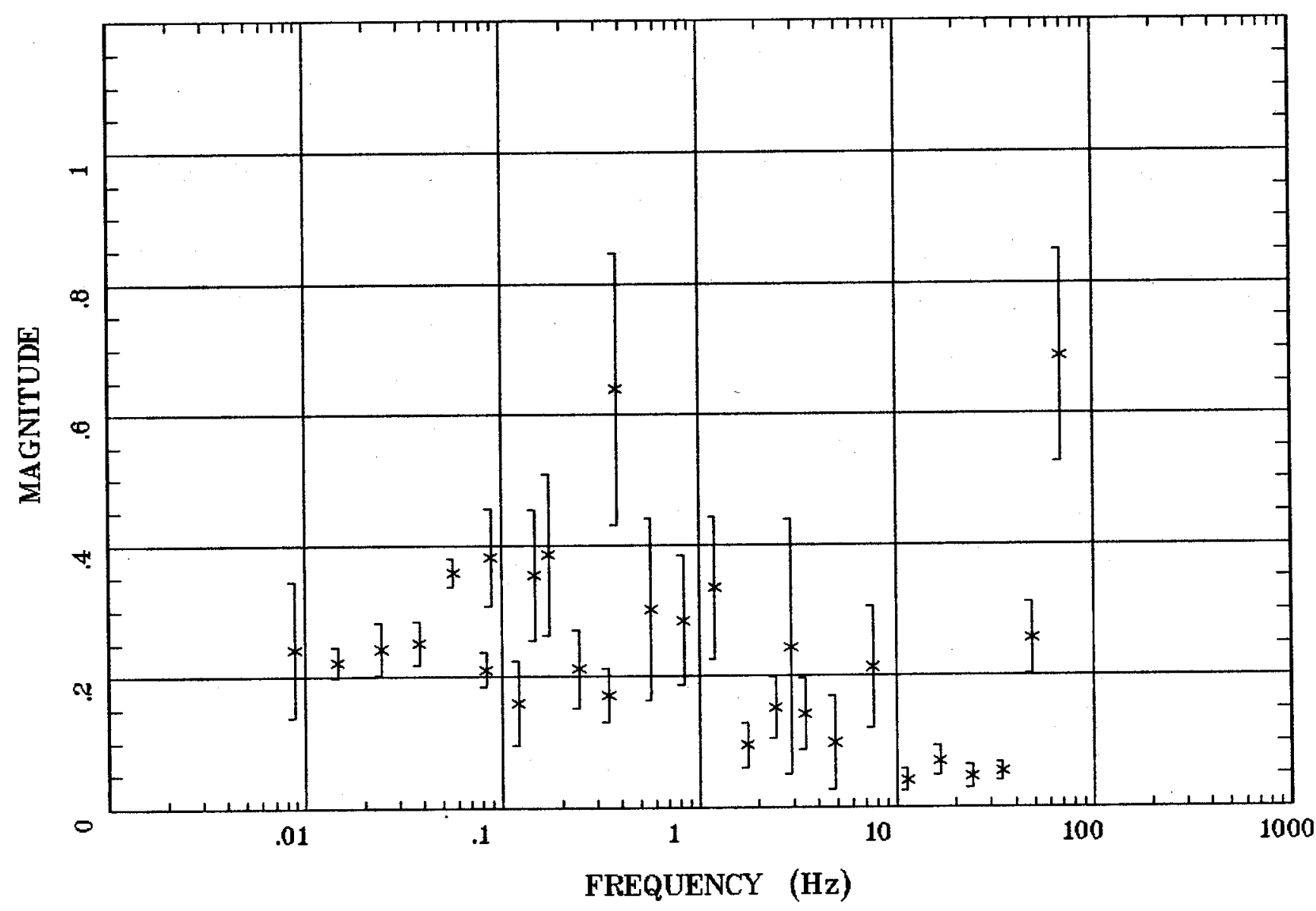

Client:

Remote: e-fld $95 \mathrm{~m}$ north Acquired: 11:4 Jun 20, 2003 Survey Co:USGS
Rotation:

Filename: cp21m.avg

Channels: Ch1 Ch2 Ch3 ch4 Ch5 Ch6 Chr Plotted: 08:49 Jul 07, 2004

$<$ EMI - ElectroMagnetic Instruments > 


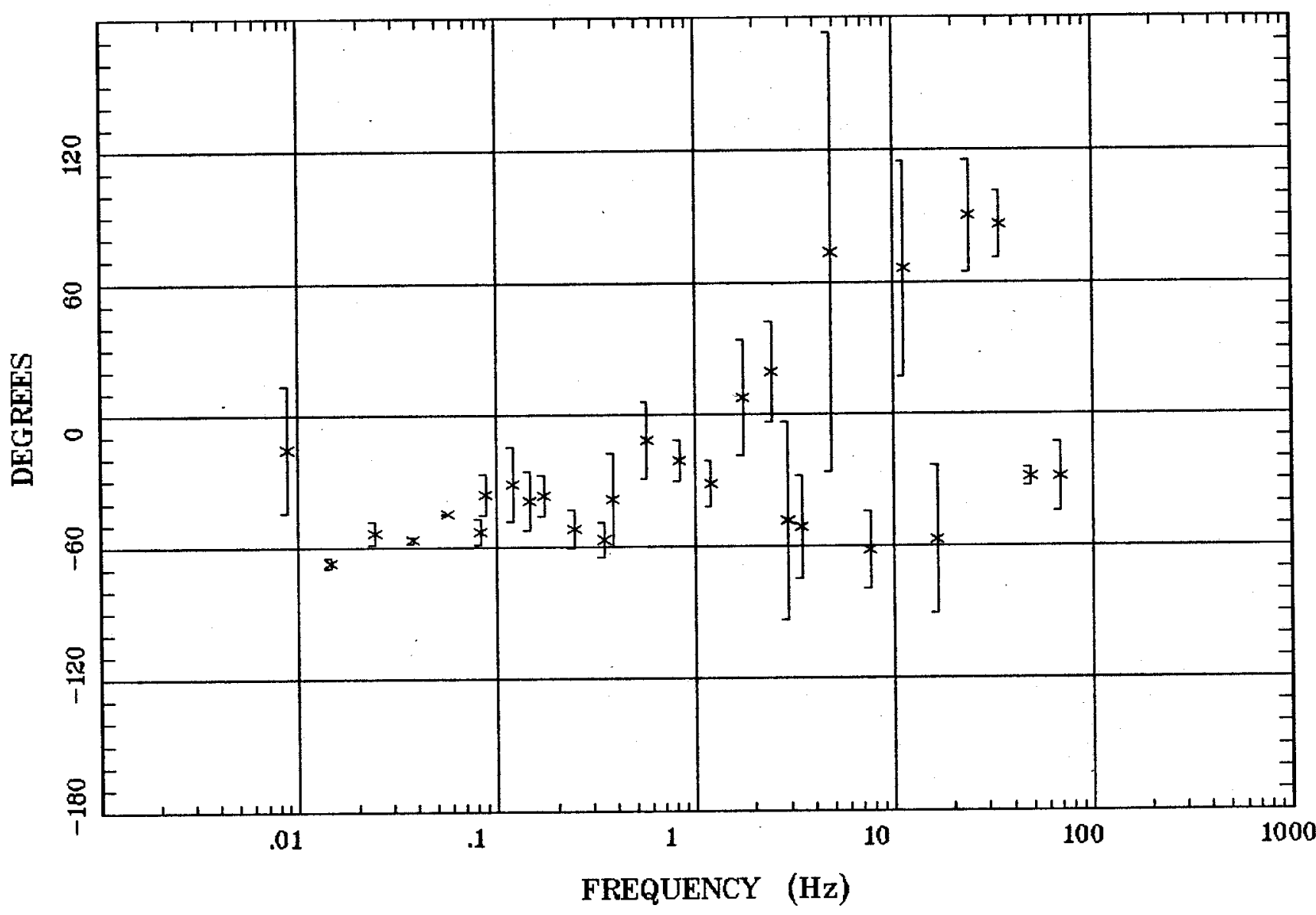

Client:

Remote: e-fld $95 \mathrm{~m}$ north Acquired: 11:4 Jun 20, 2003 Survey Co:USGS
Rotation:

Filename: cp21m.avg

Channels: Ch1 Ch2 Ch3 ch4 Ch5 Ch6 Ch7 Plotted: 08:49 Jul 07, 2004

$<$ EMI - ElectroMagnetic Instruments 


\section{HzHx.x Coh HzHy.o Santa Fe, NM}

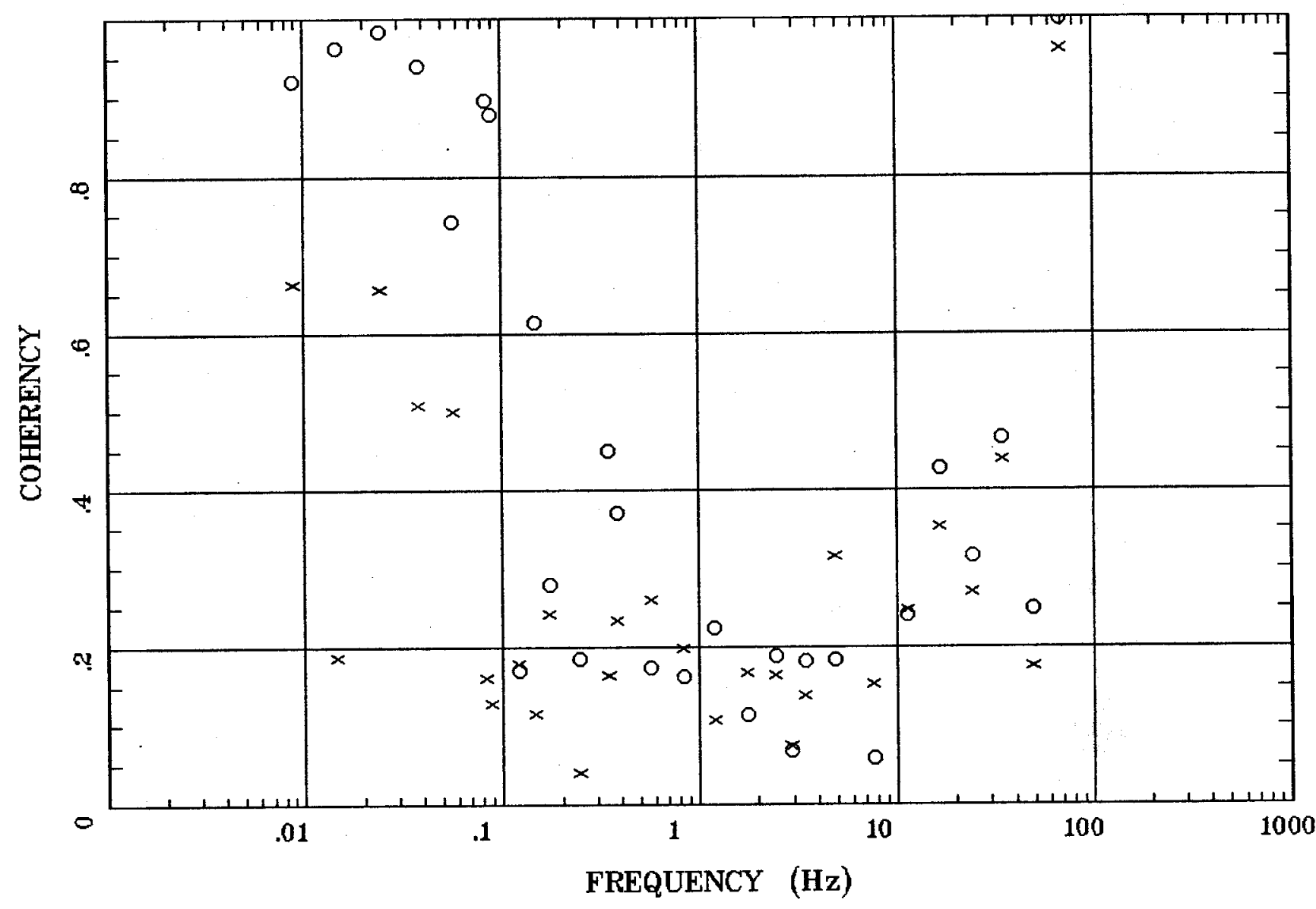

Client:

Remote: e-fld $95 \mathrm{~m}$ north Acquired: 11:4 Jun 20, 2003 Survey Co:USGS
Rotation:

Filename: cp21m.avg

Channels: Ch1 Ch2 Ch3 Ch4 Ch5 Ch6 Ch7 Plotted: 08:49 Jul 07, 2004

< EMI - ElectroMagnetic Instruments > 


\section{APPARENT RESISTIVITY}

Santa Fe, NM

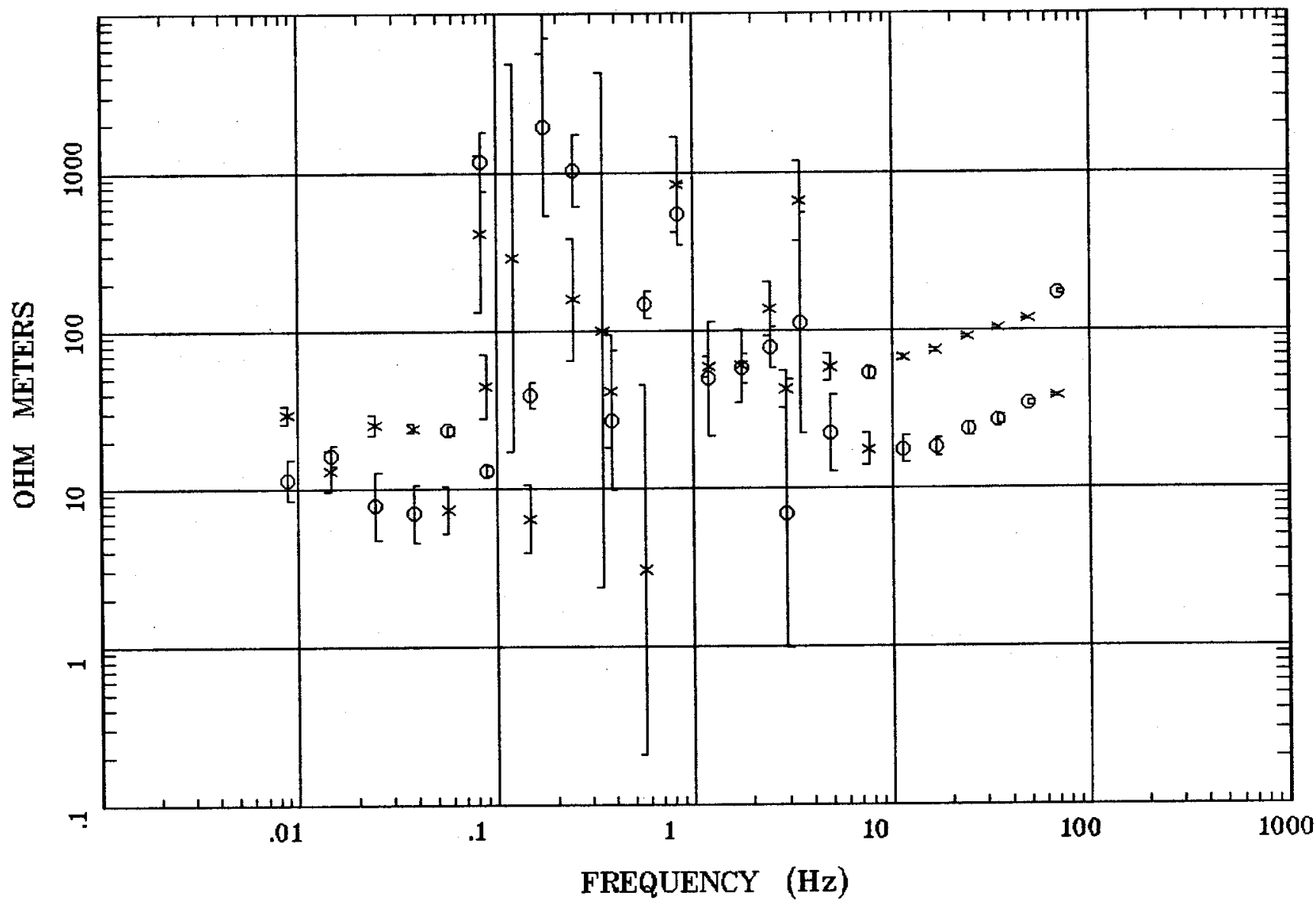

Client:

Remote: e-fld $95 \mathrm{~m}$ north Acquired: 11:4 Jun 21, 2003 Survey Co:USGS
Rotation:

Filename: cp22m.avg

Channels: Ch1 Ch2 Ch3 Ch4 Ch5 Ch6 Ch7 Plotted: 08:50 Jul 07, 2004

< EMI - ElectroMagnetic Instruments > 


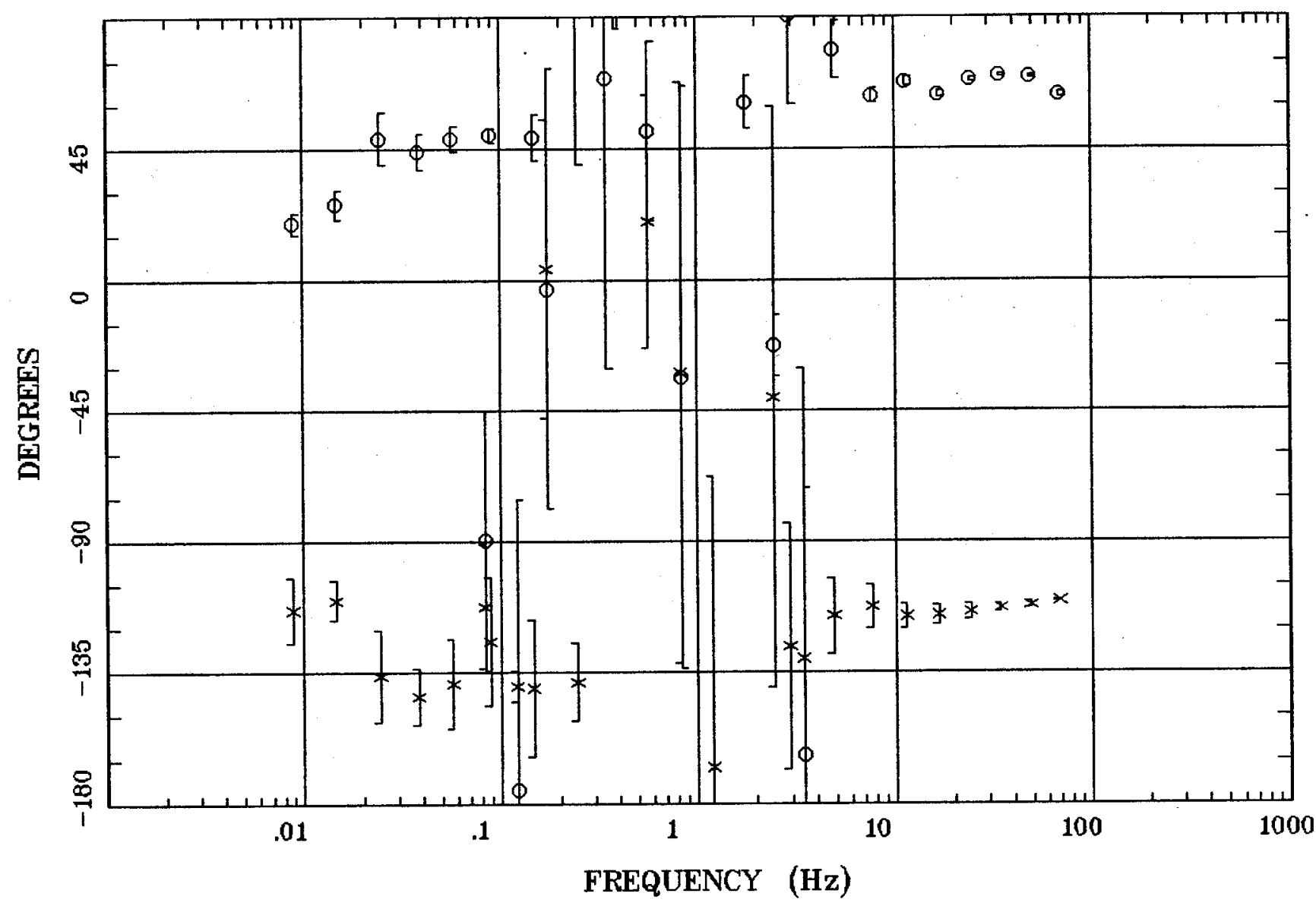

Client:

Remote: e-fld $95 \mathrm{~m}$ north Acquired: 11:4 Jun 21, 2003 Survey Co:USGS
Rotation:

Filename: cp22m.avg

Channels: Ch1 Ch2 Ch3 Ch4 Ch5 Ch6 Ch7 Plotted: 08:50 Jul 07, 2004

$<$ EMI - ElectroMagnetic Instruments > 


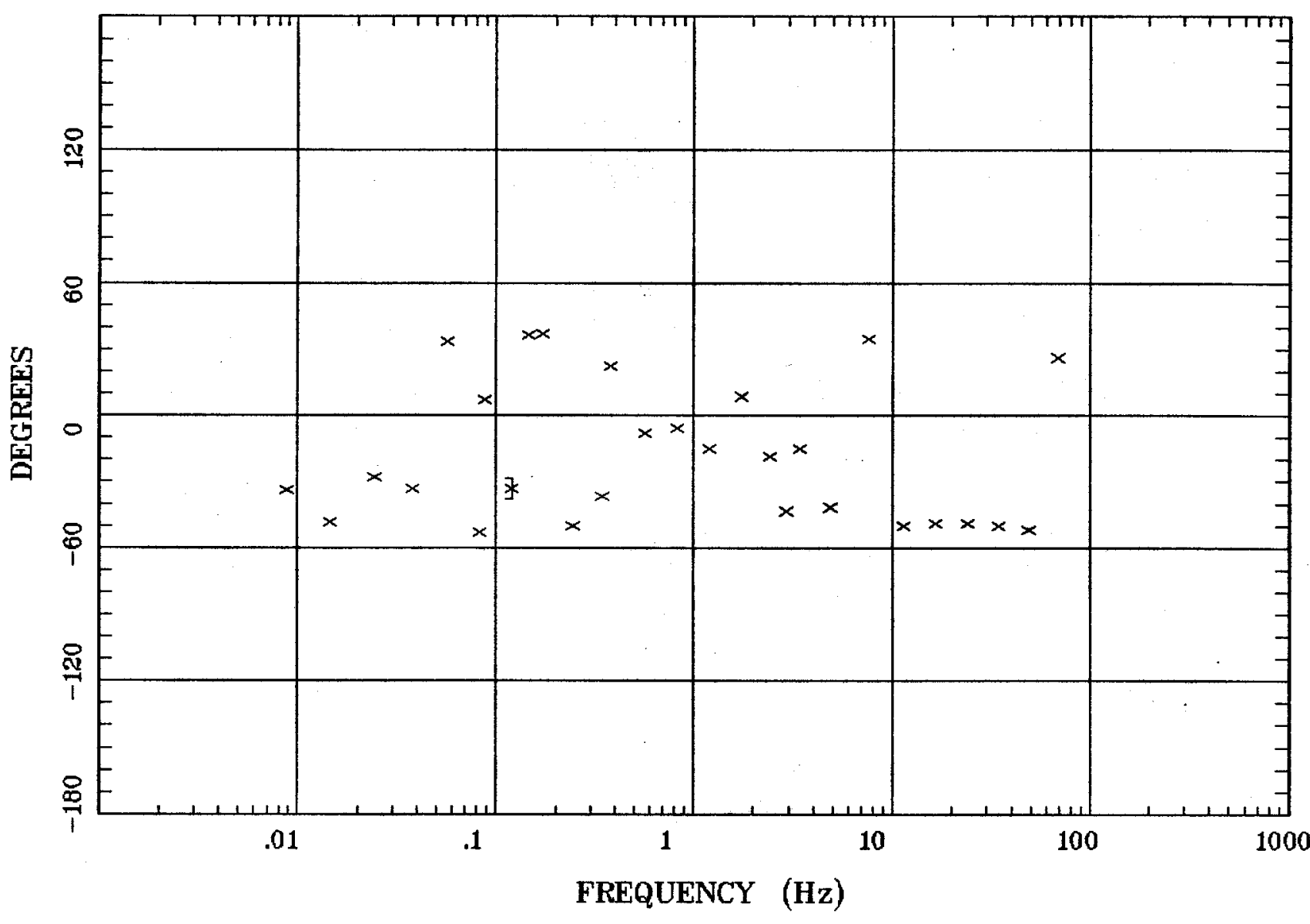

Client:

Remote: e-fld $95 \mathrm{~m}$ north Acquired: 11:4 Jun 21, 2003 Survey Co:USGS
Rotation:

Filename: cp22m.avg

Channels: Ch1 Ch2 Ch3 Ch4 Ch5 Ch6 Ch7 Plotted: 10:44 Jul 07, 2004

$<$ EMI - ElectroMagnetic Instruments 
IMPEDANCE SKEW

Santa Fe, NM

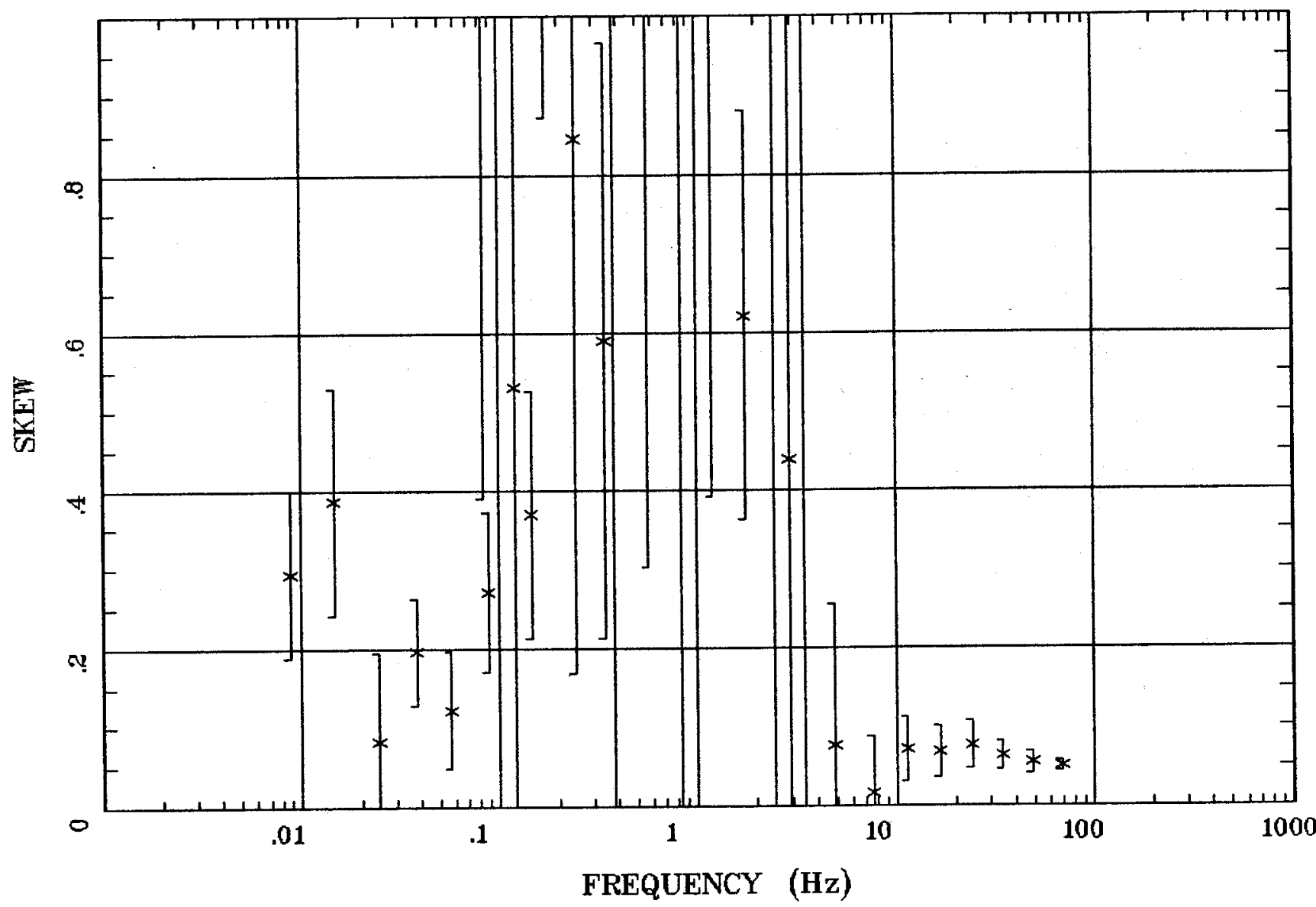

Client:

Remote: e-fld $95 \mathrm{~m}$ north Acquired: 11:4 Jun 21, 2003 Survey Ca:USGS
Rotation:

Filename: cp22m.avg

Channels: Ch1 Ch2 Ch3 Ch4 Ch5 Ch6 Ch7 Plotted: 08:50 Jul 07, 2004

< EMI - ElectroMagnetic Instruments 
E MULT Coh.

Santa Fe, NM

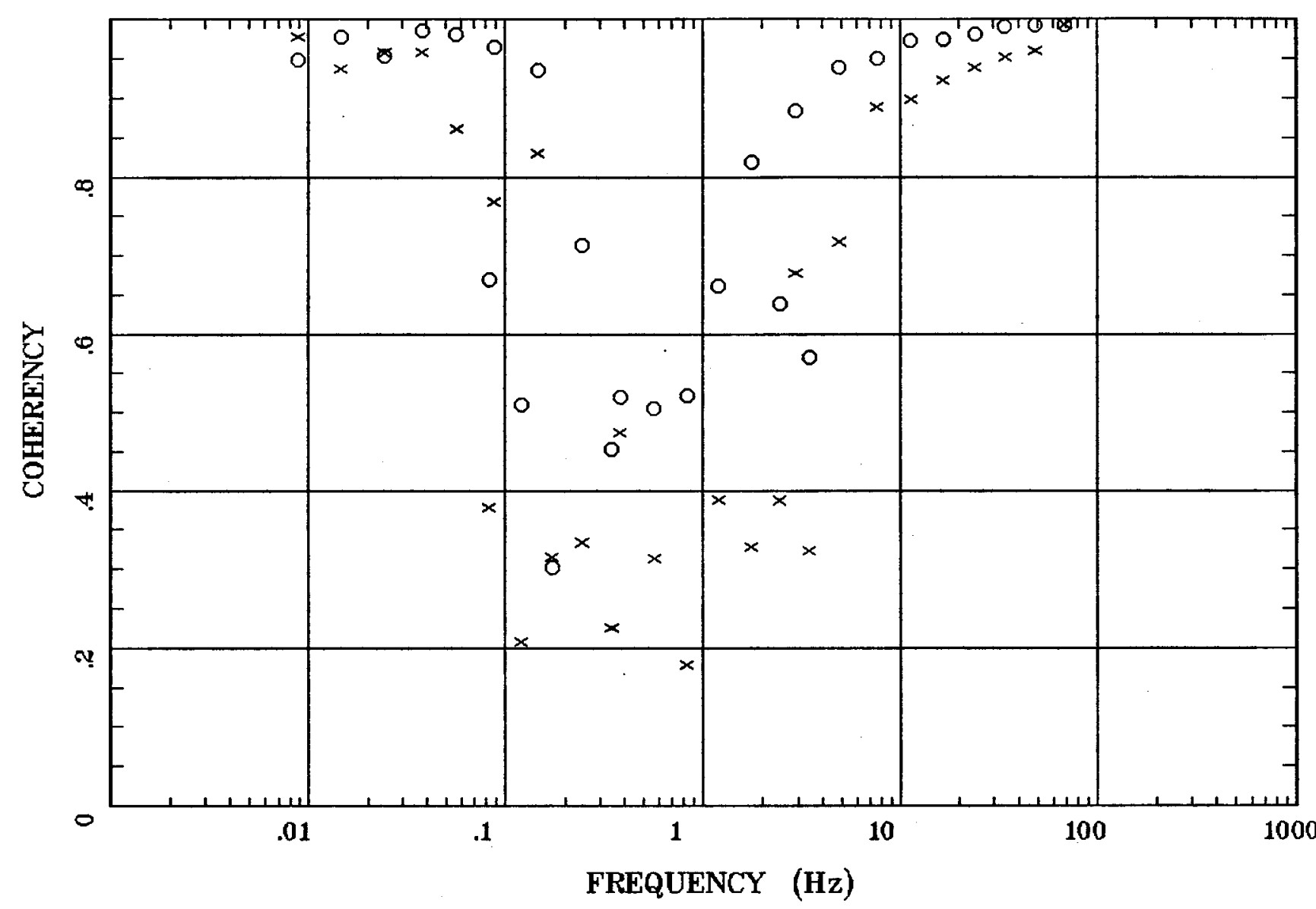

Client:

Remote: e-fld $95 \mathrm{~m}$ north Acquired: 11:4 Jun 21, 2003 Survey Co:USGS
Rotation:

Filename: cp22m.avg

Channels: Ch1 Ch2 Ch3 Ch4 Ch5 Ch6 Chr Plotted: 08:50 Jul 07, 2004

< EMI - ElectroMagnetic Instruments 


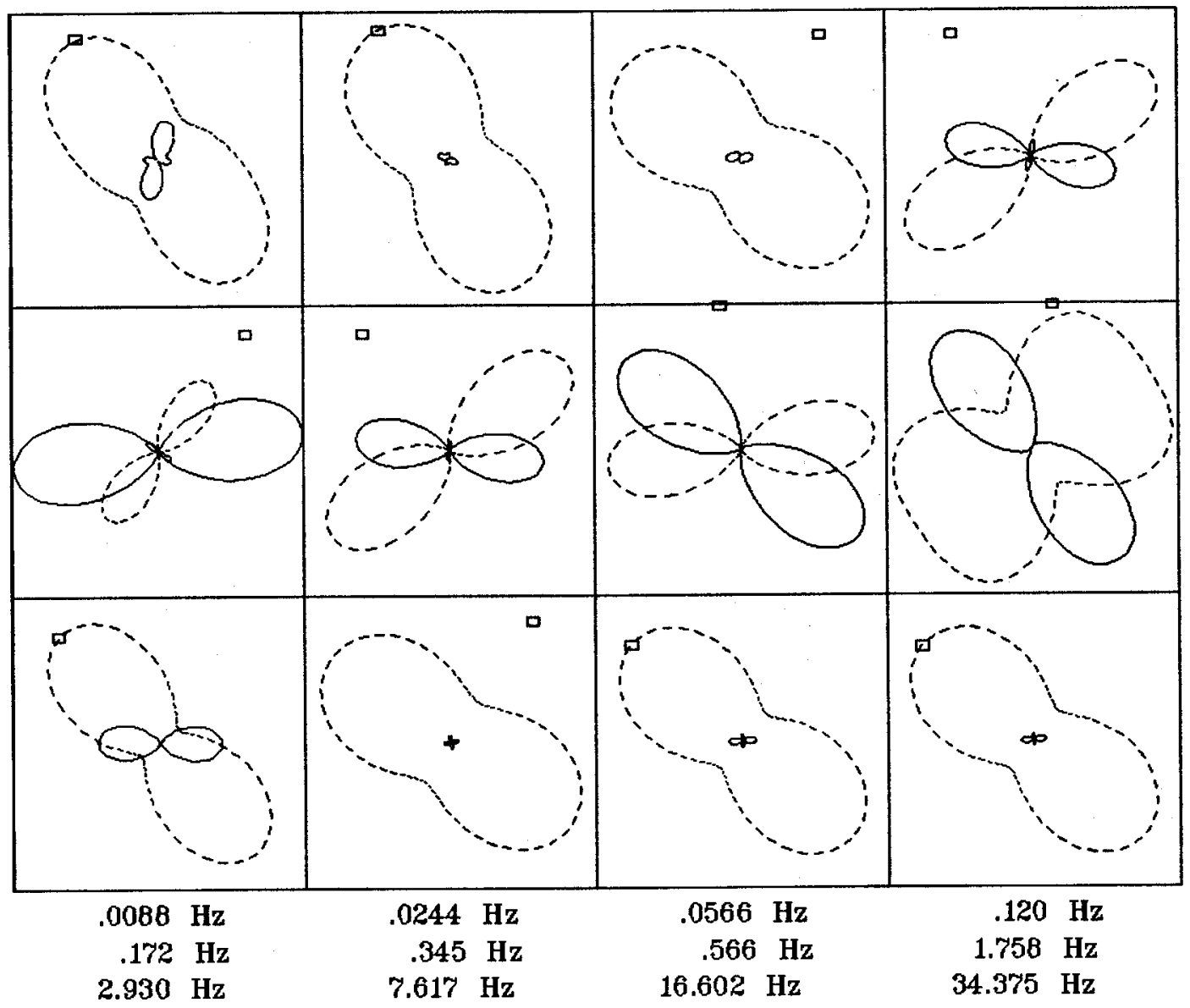

Client:

Remote: e-fld $95 \mathrm{~m}$ north Acquired: 11:4 Jun 21, 2003 Survey Co:USGS
Rotation:

Filename: cp22m.avg

Channels: Ch1 Ch2 Ch3 Ch4 Ch5 Ch6 Ch7 Plotted: 08:50 Jul 07, 2004

< EMI - ElectroMagnetic Instruments > 


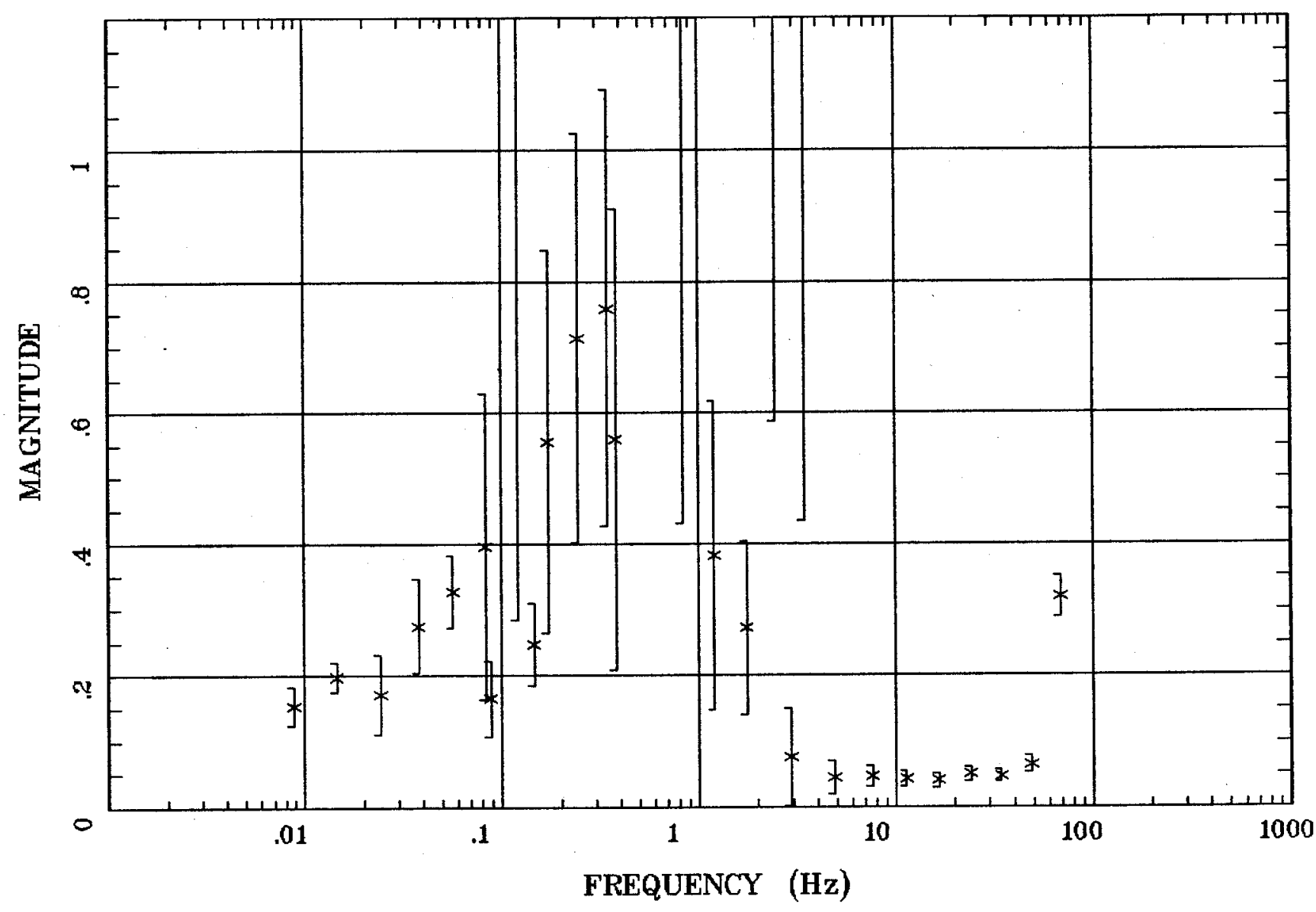

Client:

Remote: e-fld $95 \mathrm{~m}$ north Acquired: 11:4 Jun 21, 2003 Survey Co:USGS

\section{Rotation:}

Filename: cp22m.avg Channels: Ch1 Ch2 Ch3 Ch4 Ch5 Ch6 Ch7 Plotted: 08:50 Jul 07, 2004

$<$ EMI - ElectroMagnetic Instruments > 
TIPPER STRIKE

Santa Fe, NM

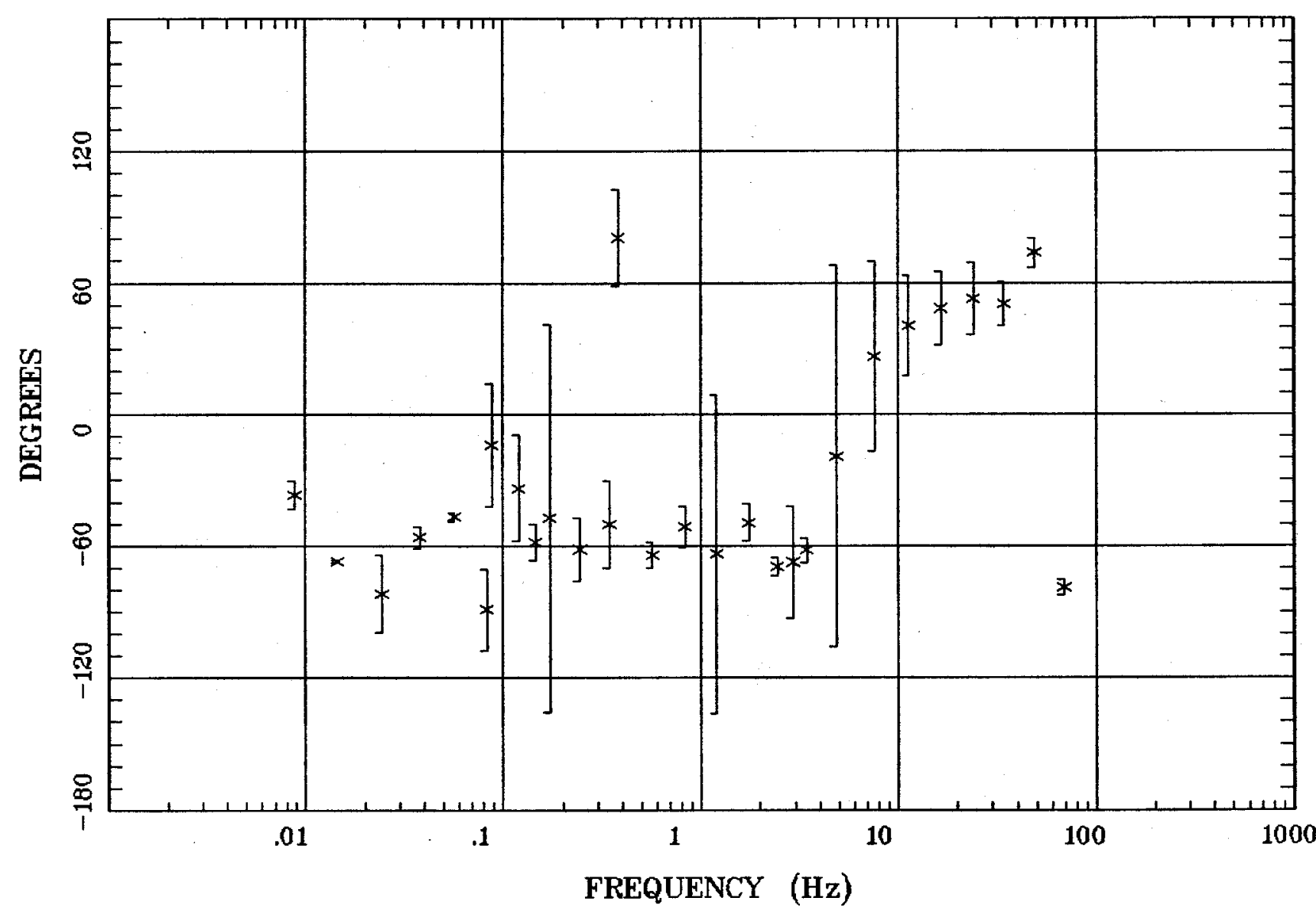

Client:

Remote: e-fld $95 \mathrm{~m}$ north Acquired: 11:4 Jun 21, 2003 Survey Co:USGS
Rotation:

Filename: cp22m.avg

Channels: Ch1 Ch2 Ch3 Ch4 Ch5 Ch6 Ch7

Plotted: 08:50 Jul 07, 2004

$<$ EMI - ElectroMagnetic Instruments 
Station 22

HzHx.x Coh HzHy.o

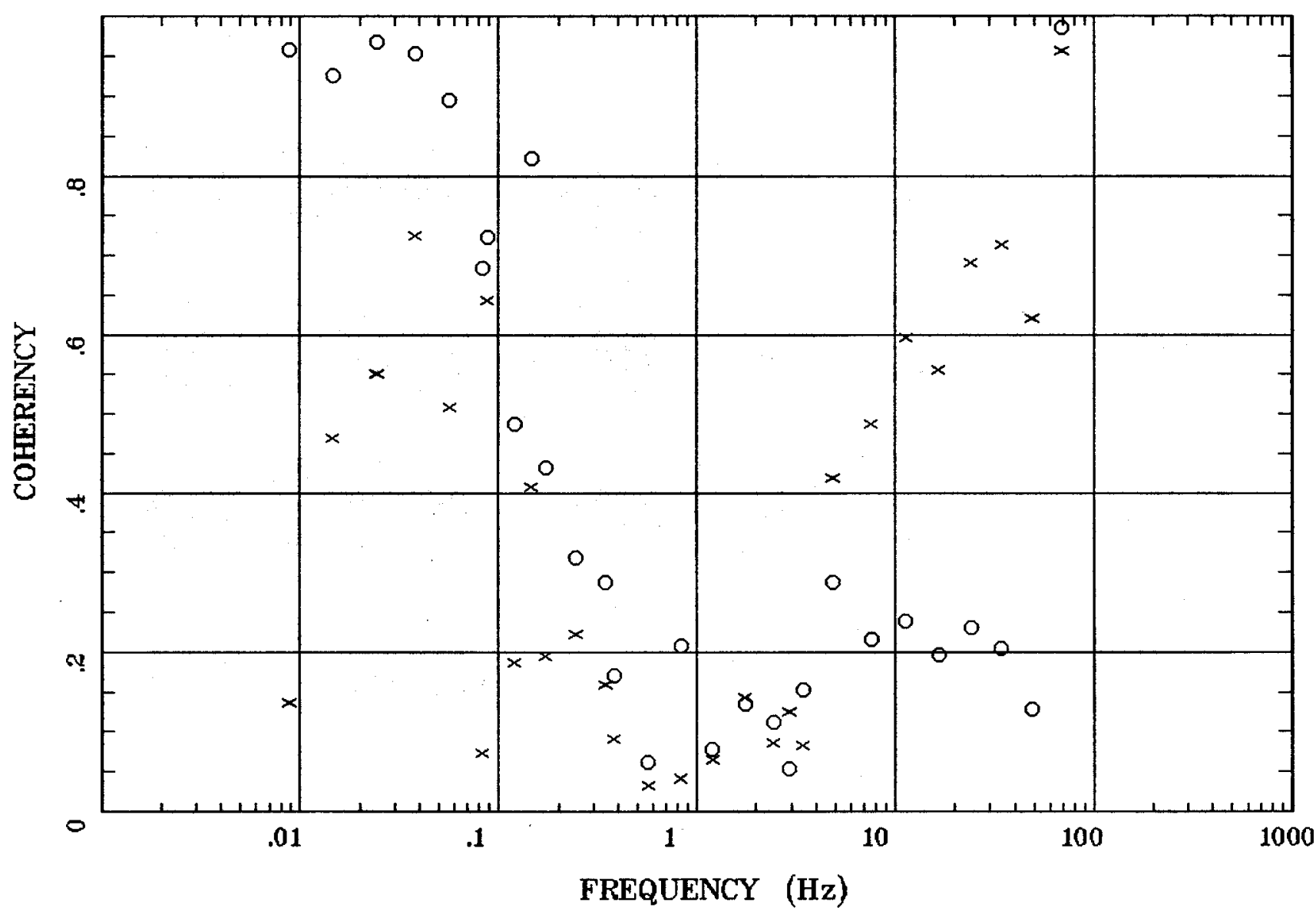

Client:

Remote: e-fld $95 \mathrm{~m}$ north Acquired: 11:4 Jun 21, 2003 Survey Co:USGS
Santa Fe, NM

$$
\text { 1000 }
$$

FREQUENCY (Hz)

\section{Rotation:}

Filename: cp22m.avg

Channels: Ch1 Ch2 Ch3 Ch4 Ch5 Ch6 Ch7

Plotted: 08:50 Jul 07, 2004

< EMI - ElectroMagnetic Instruments > 


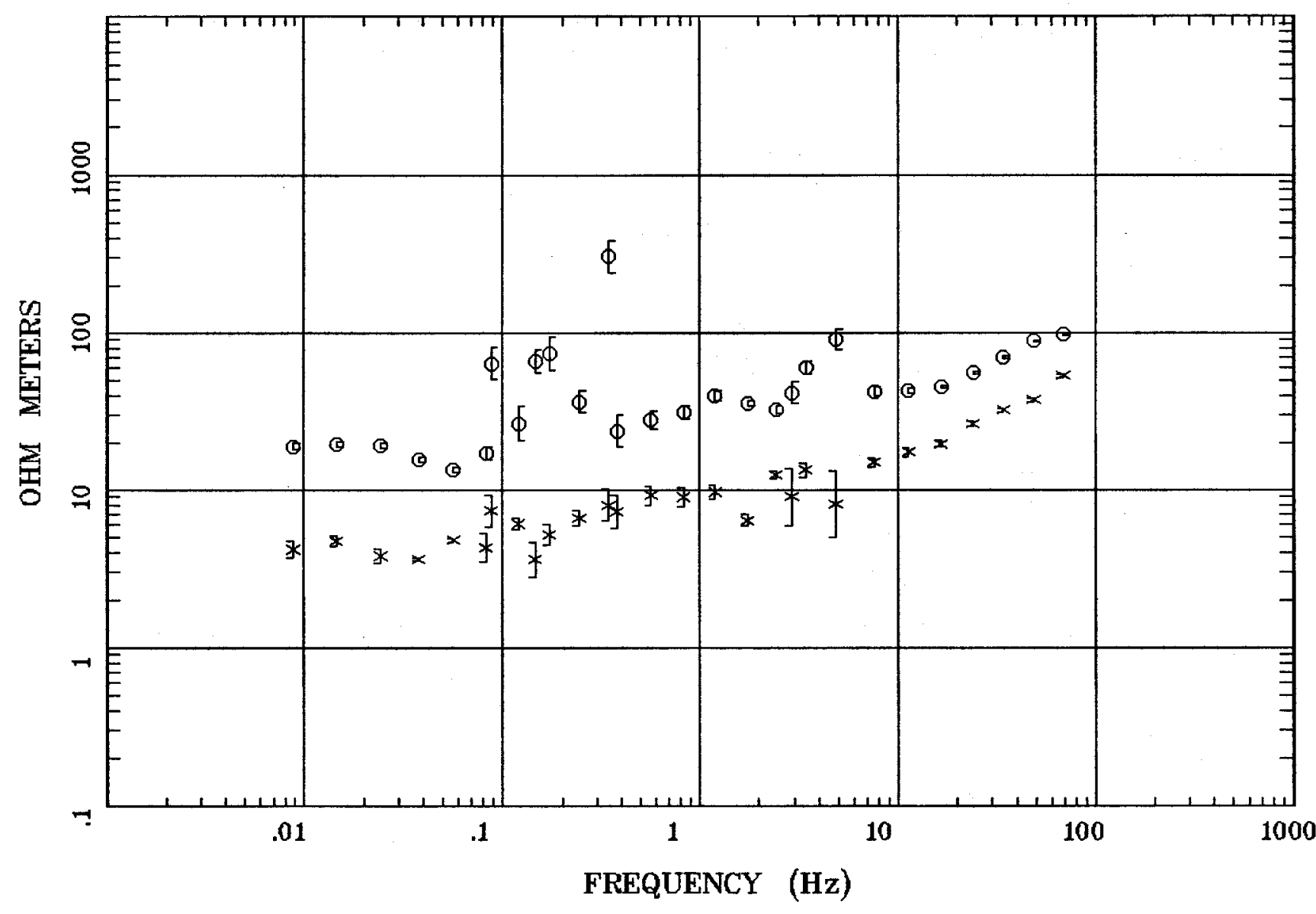

Client:

Remote: e-fld $70 \mathrm{~m}$ east

Acquired: 11:2 Jun 22, 2003 Survey Co:USGS
Rotation:

Filename: cp23m.avg

Channels: Ch1 Ch2 Ch3 Ch4 Ch5 Ch6 Ch7 Plotted: 08:51 Jul 07, 2004

$<$ EMI - ElectroMagnetic Instruments 


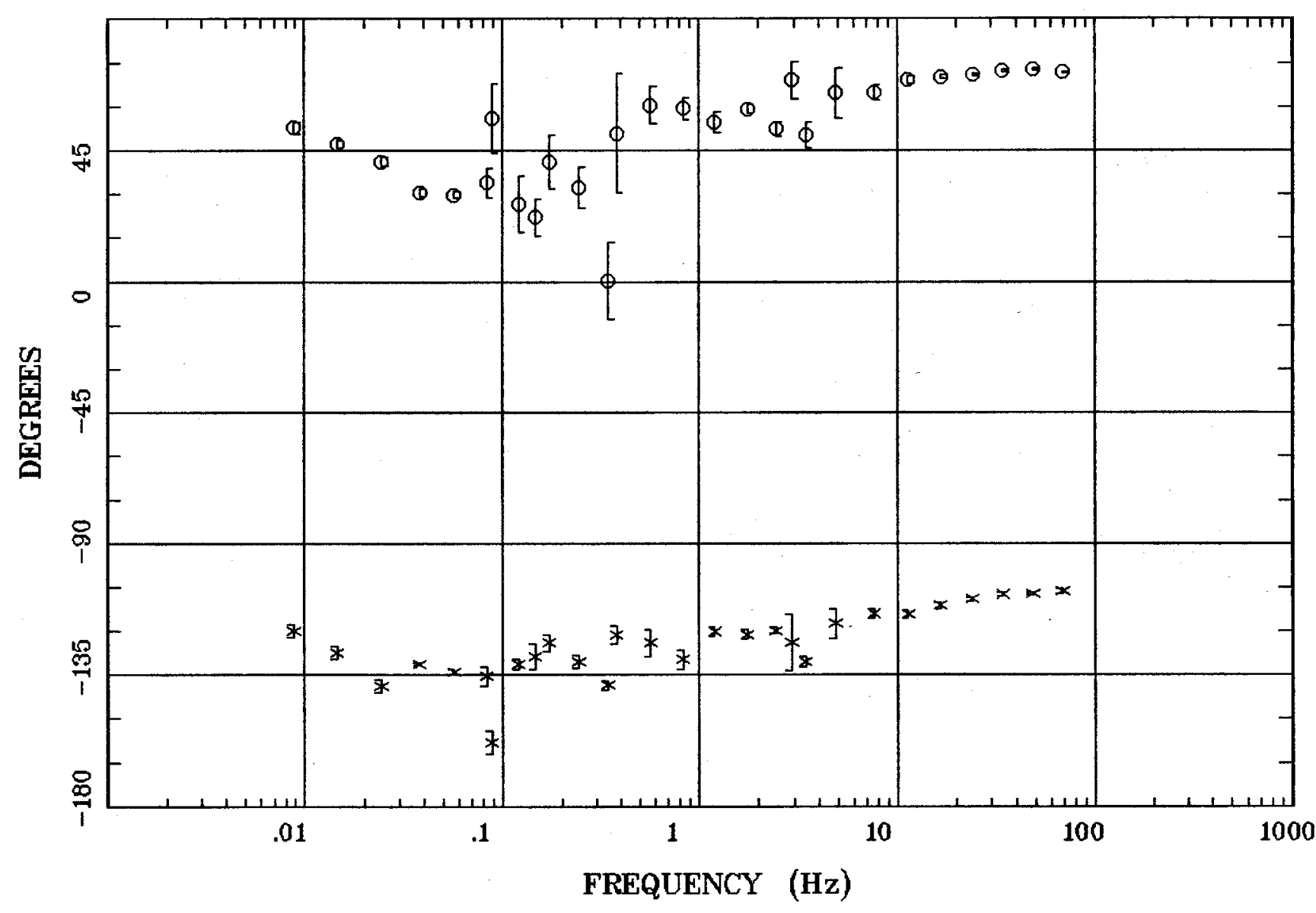

Client:

Remote: e-fld $70 \mathrm{~m}$ east

Acquired: 11:2 Jun 22, 2003 Survey Co:USGS
Rotation:

Filename: cp23m.avg

Channels: Ch1 Ch2 Ch3 Ch4 Ch5 Ch6 Ch7 Plotted: 08:51 Jul 07, 2004

$<$ EMI - ElectroMagnetic Instruments 
Station 23

ROTATION ANGLE

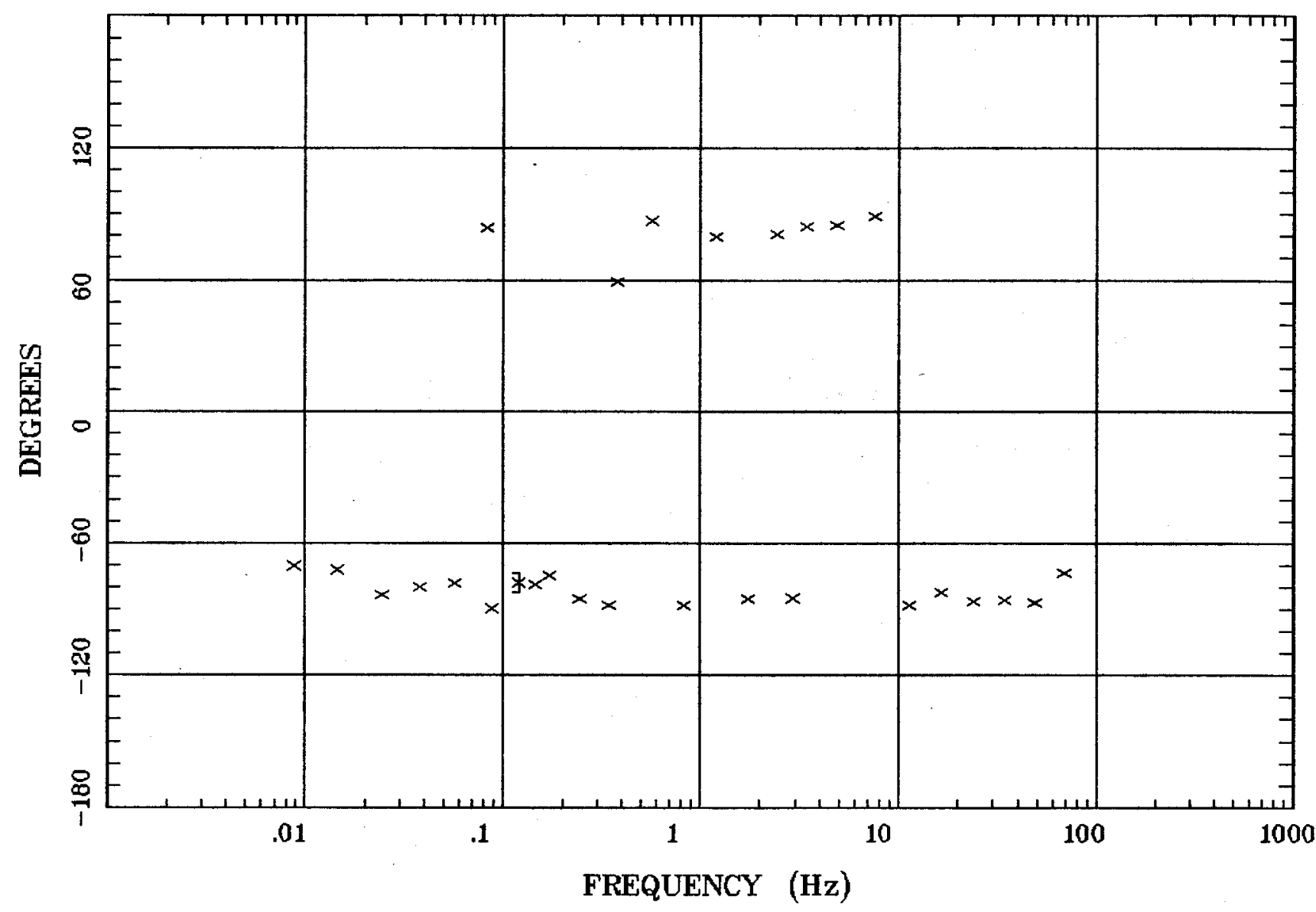

Client:

Remote: e-fld $70 \mathrm{~m}$ east

Acquired: 11:2 Jun 22, 2003

Survey Co:USGS
Santa Fe, NM

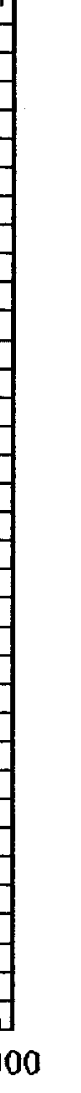




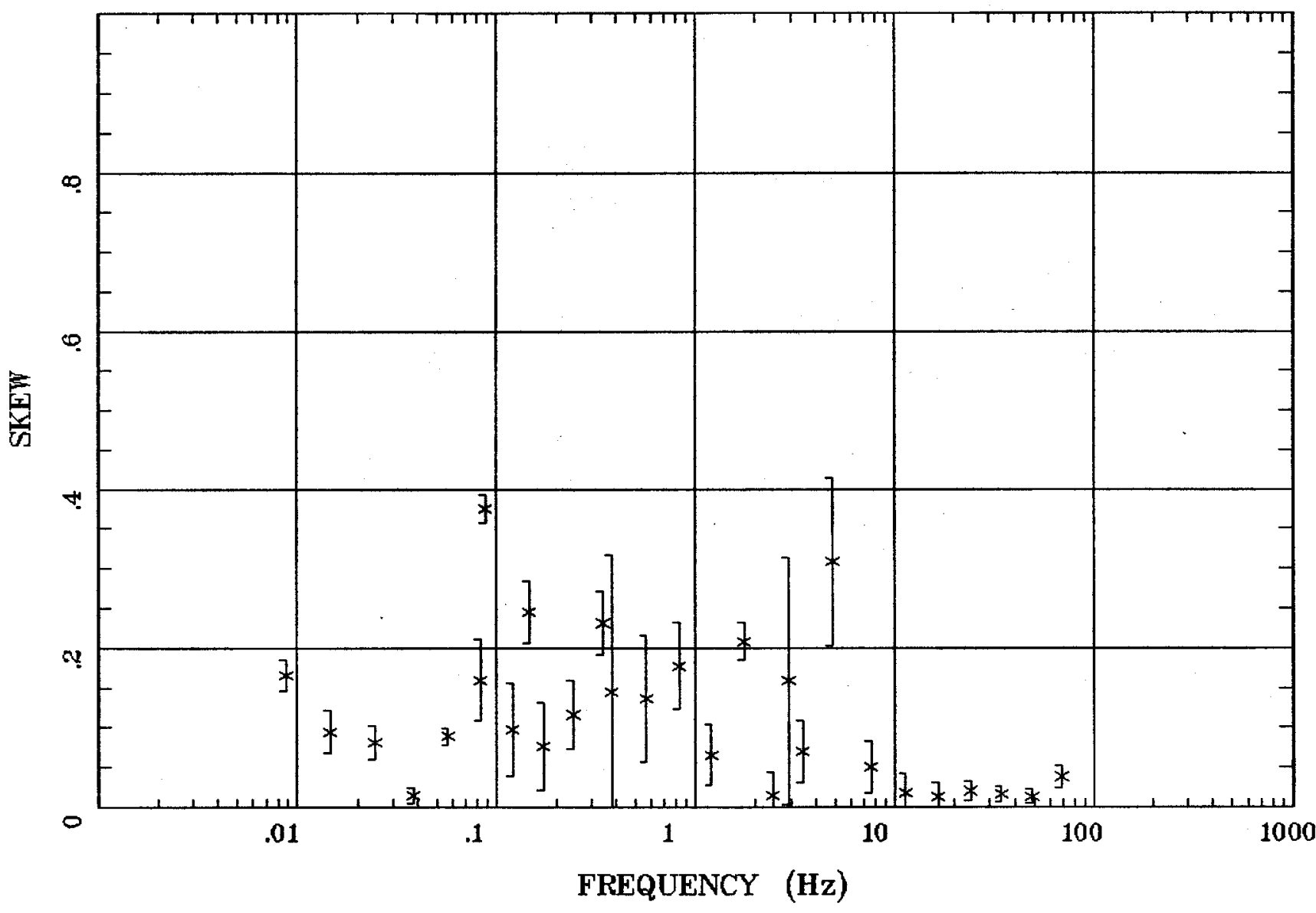

Client:

Remote: e-fld $70 \mathrm{~m}$ east

Acquired: 11:2 Jun 22, 2003 Survey Co:USGS
Rotation:

Filename: cp23m.avg

Channels: Ch1 Ch2 Ch3 Ch4 Ch5 Ch6 Chr Plotted: 08:51 Jul 07, 2004

$<$ EMI - ElectroMagnetic Instruments 
E MULT Coh.

Santa Fe, NM

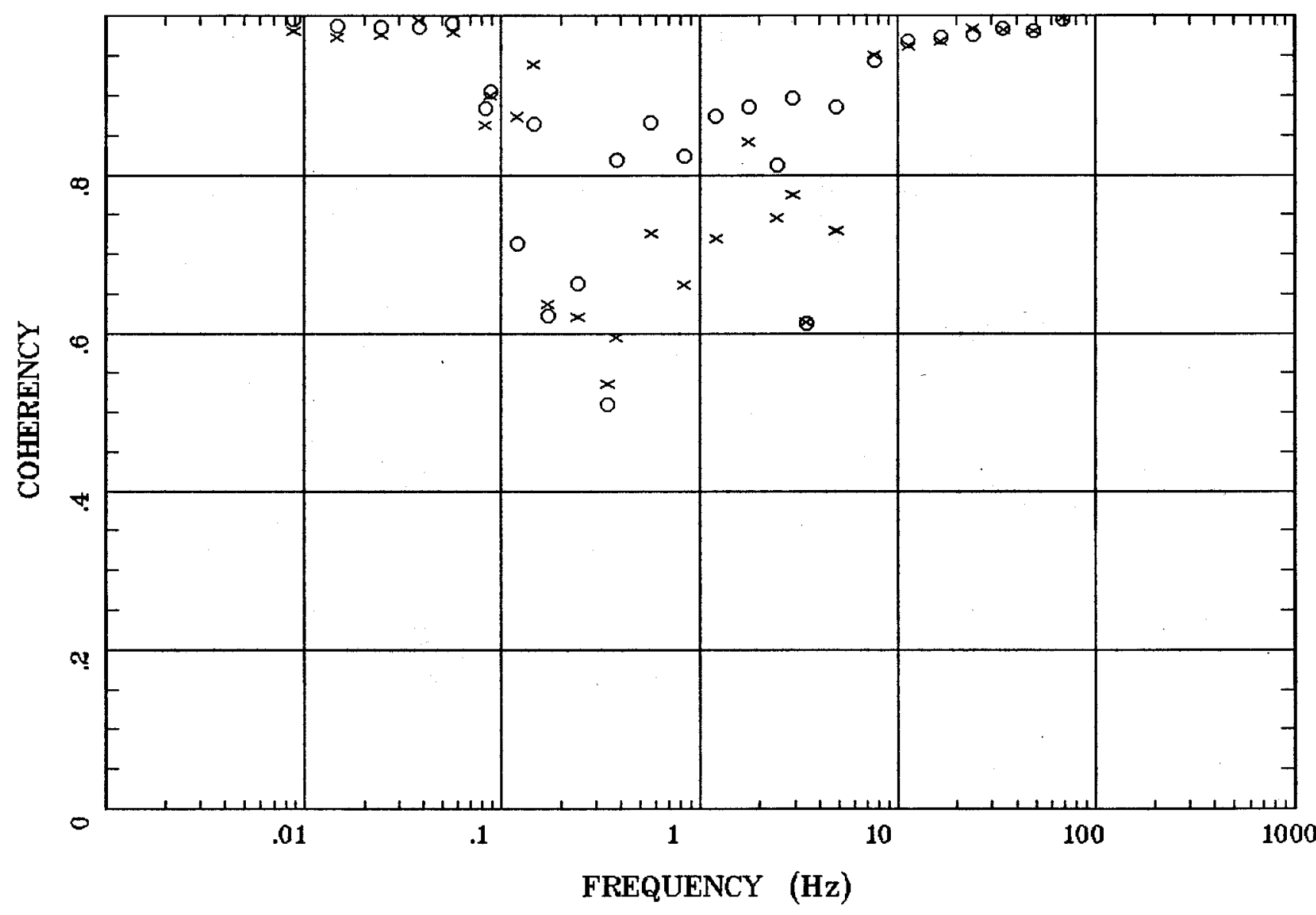

Client:

Remote: e-fld $70 \mathrm{~m}$ east Acquired: 11:2 Jun 22, 2003 Survey Co:USGS
Rotation:

Filename: cp23m.avg

Channels: Ch1 Ch2 Ch3 Ch4 Ch5 Ch6 Ch7 Plotted: 08:51 Jul 07, 2004

< EMI - ElectroMagnetic Instruments 


\section{POLAR PLOTS}

Santa Fe, NM

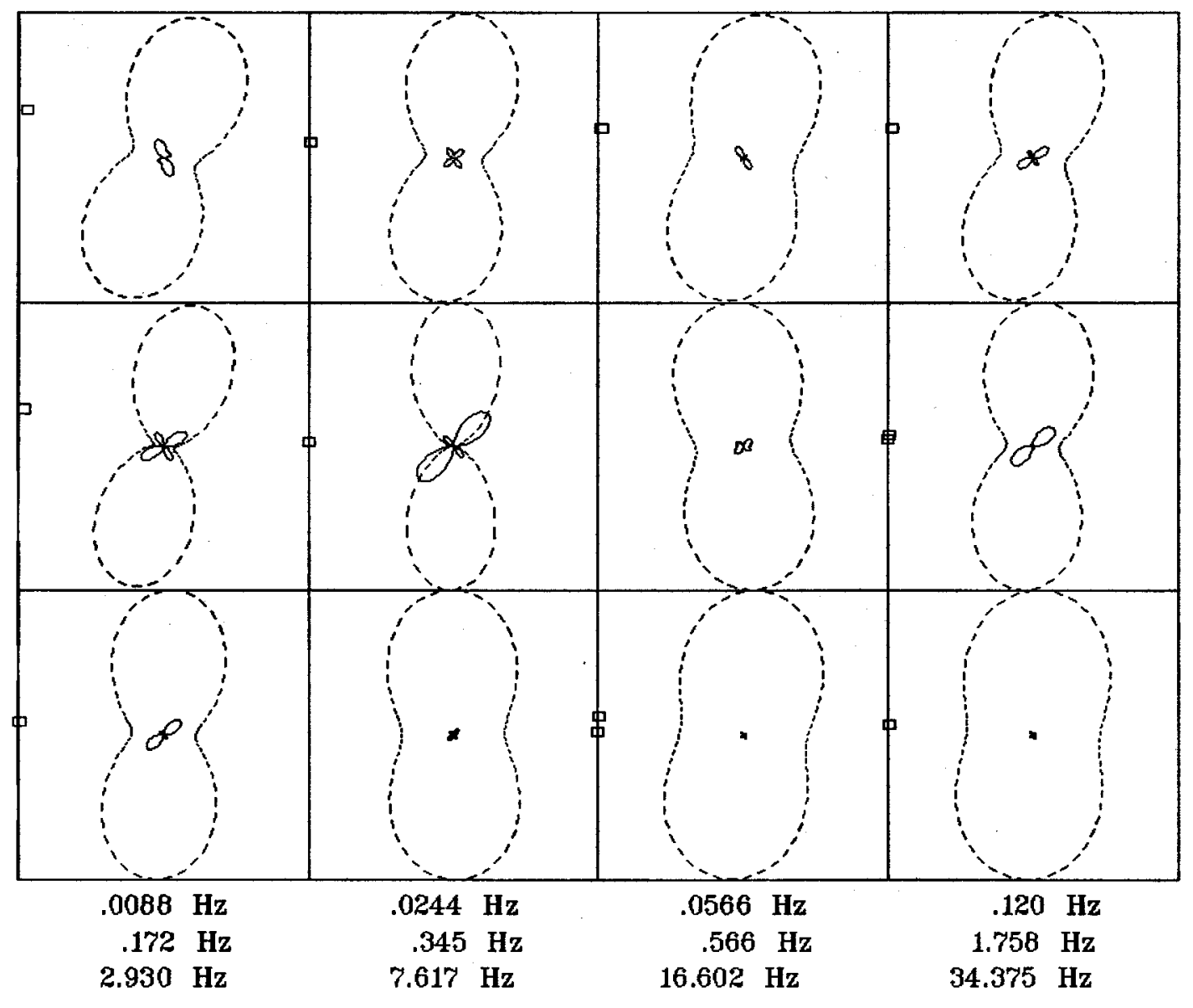

Rotation:

Client:

Remote: $e-f l d ~ 70 ~ m$ east

Acquired: 11:2 Jun 22, 2003

Filename: cp23m.avg

Channels: Ch1 Ch2 Ch3 Ch4 Ch5 Ch6 Ch7 Survey Co:USGS 


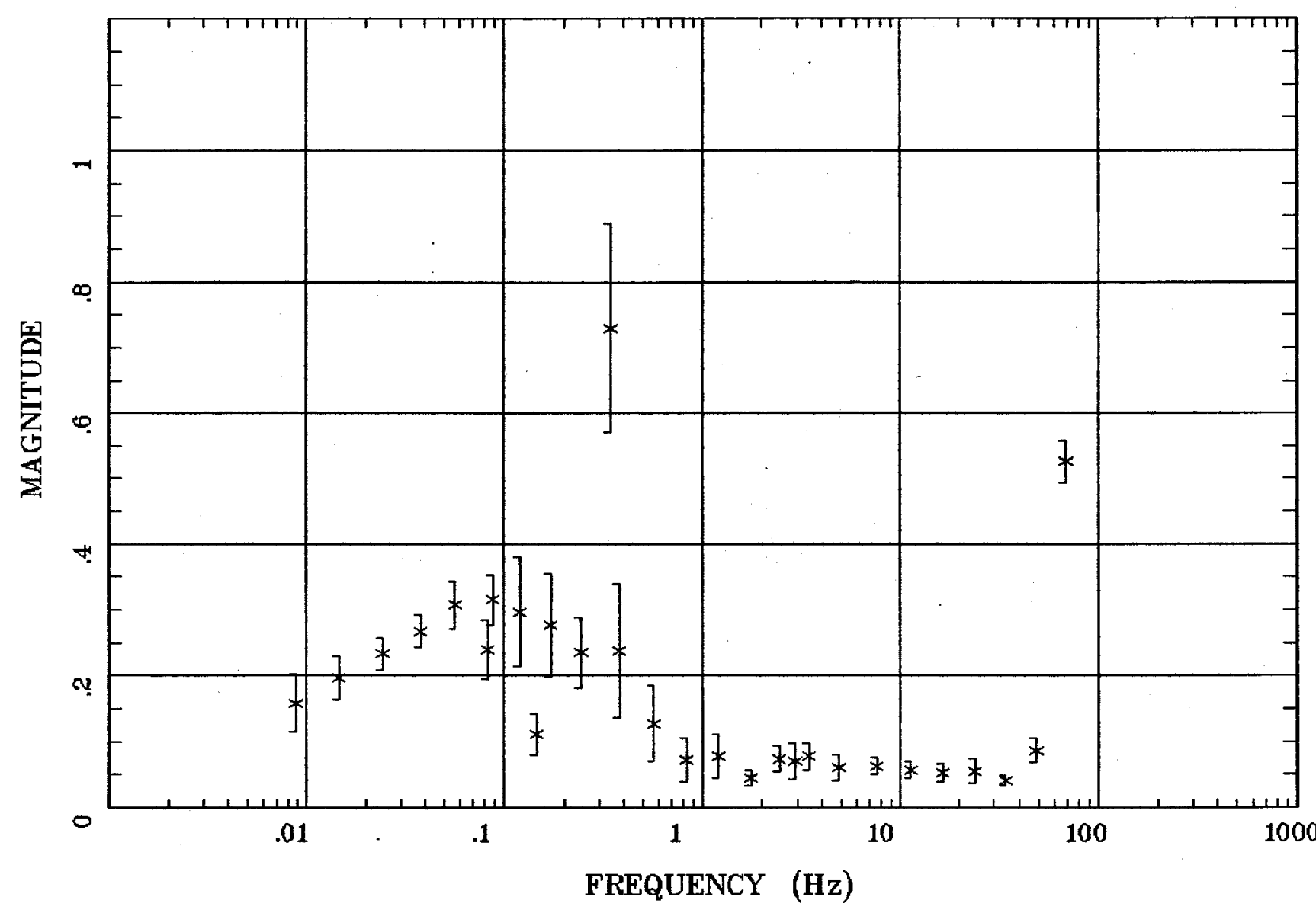

Client:

Remote: e-fld $70 \mathrm{~m}$ east Acquired: 11:2 Jun 22, 2003 Survey Co:USGS
Rotation:

Filename: cp23m.avg Channels: Ch1 Ch2 Ch3 Ch4 Ch5 Ch6 Ch7 Plotted: 08:51 Jul 07, 2004

$<$ EMI - ElectroMagnetic Instruments 


\section{Station 23}

TIPPER STRIKE

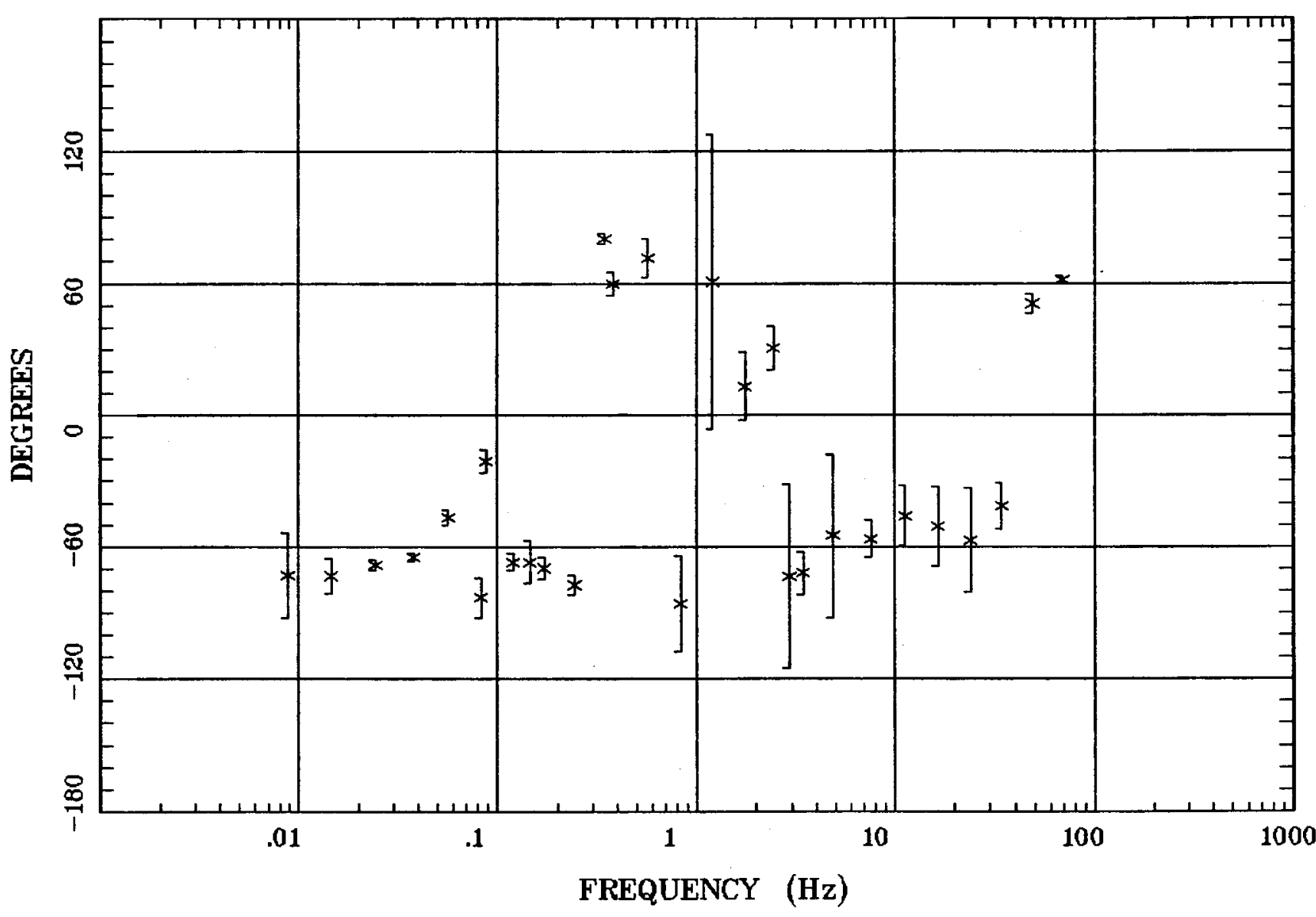

Client:

Remote: e-fld $70 \mathrm{~m}$ east

Acquired: 11:2 Jun 22, 2003

Survey Co:USGS
Rotation:

Filename: cp23m.avg

Channels: Ch1 Ch2 Ch3 Ch4 Ch5 Ch6 Ch7

Plotted: 08:51 Jul 07, 2004

< EMI - ElectroMagnetic Instruments 
HzHx.x Coh HzHy.o

Santa Fe, NM

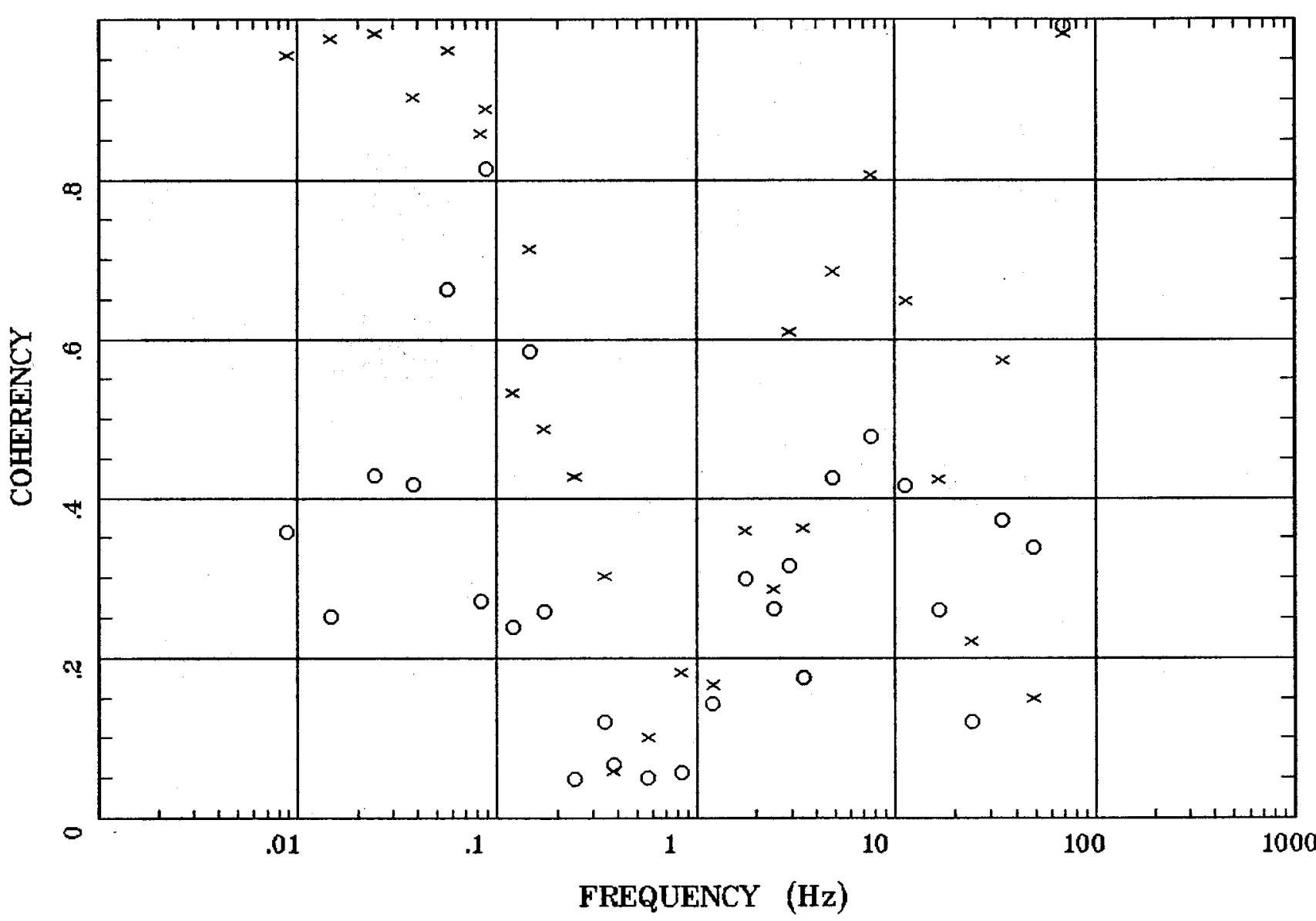

Client:

Remote: e-fld $70 \mathrm{~m}$ east Acquired: 11:2 Jun 22, 2003 Survey Co:USGS
Rotation:

Filename: cp23m.avg

Channels: Ch1 Ch2 Ch3 Ch4 Ch5 Ch6 Ch7 Plotted: 08:51 Jul 07, 2004

$<$ EMI - ElectroMagnetic Instruments 


\section{APPARENT RESISTIVITY}

\section{Santa Fe, NM}

Station 24

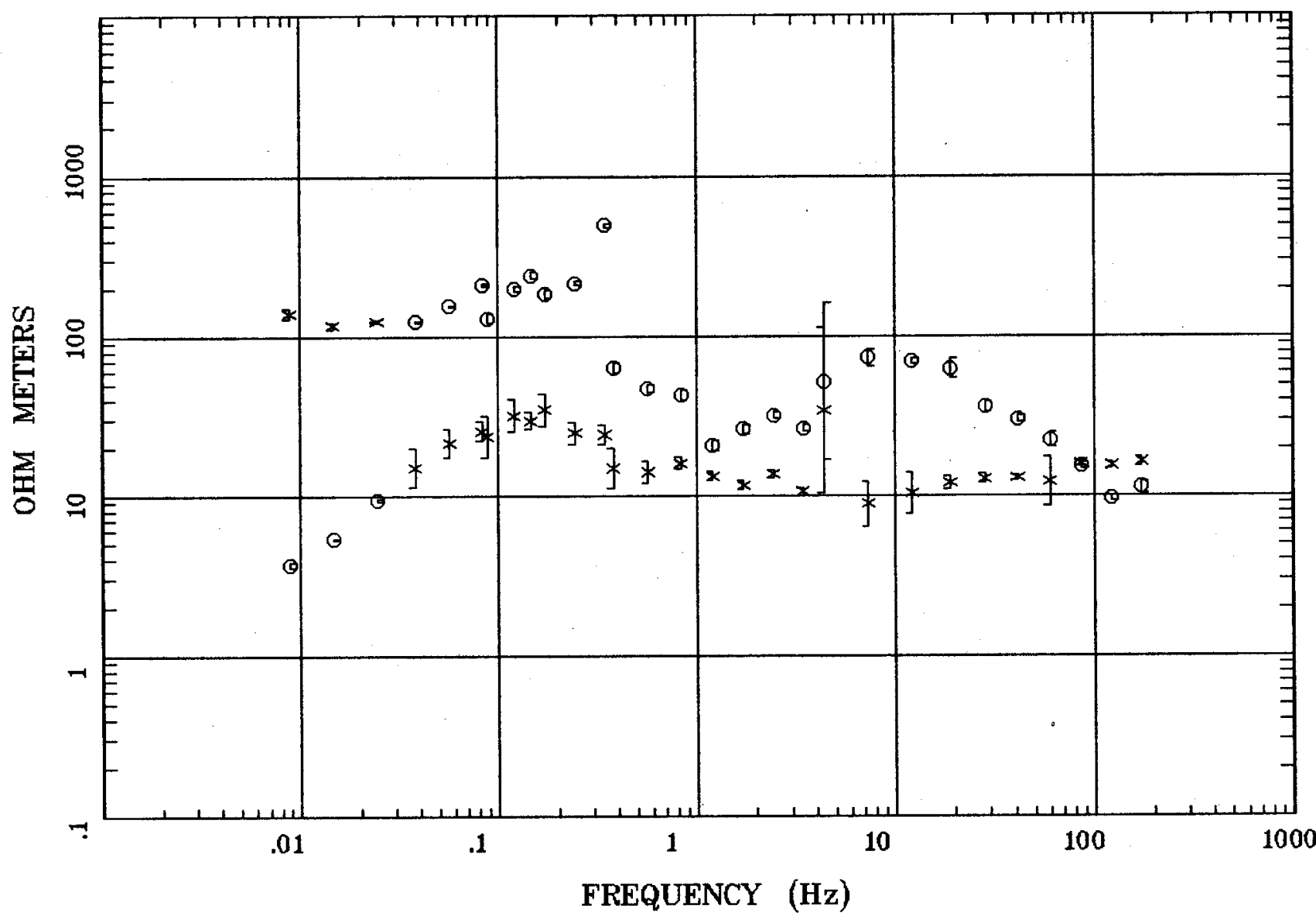

Client:

Remote: e-fld $80 \mathrm{~m}$ east Acquired: $10: 3$ Jun 23, 2003 Survey Co:USGS
Rotation:

Filename: cp 24m.avg

Channels: Ch1 Ch2 Ch3 Ch4 Ch5 Ch6 Ch7 Plotted: 14:53 Jul 27, 2004

< EMI - ElectroMagnetic Instruments 
Station 24

IMPEDANCE PHASE

Santa Fe, NM

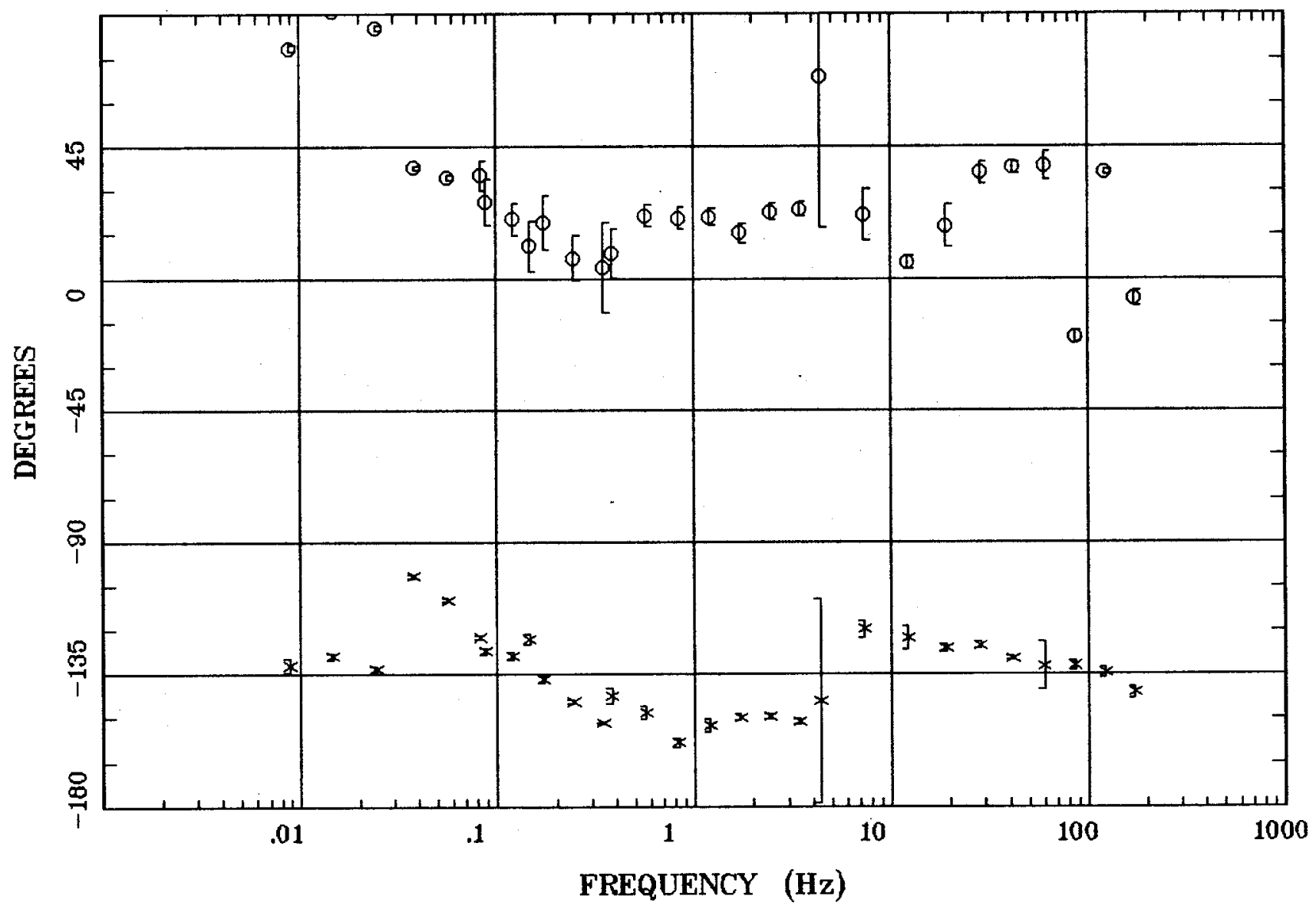

Client:

Remote: $\mathrm{e}-\mathrm{fld}$ 60m east

Acquired: 10:3 Jun 23, 2003

Survey Co:USGS
Rotation:

Filename: cp24m.avg

Channels: Ch1 Ch2 Ch3 Ch4 Ch5 Ch6 Chr

Plotted: 14:53 Jul 27, 2004

< EMI - ElectroMagnetic Instruments > 


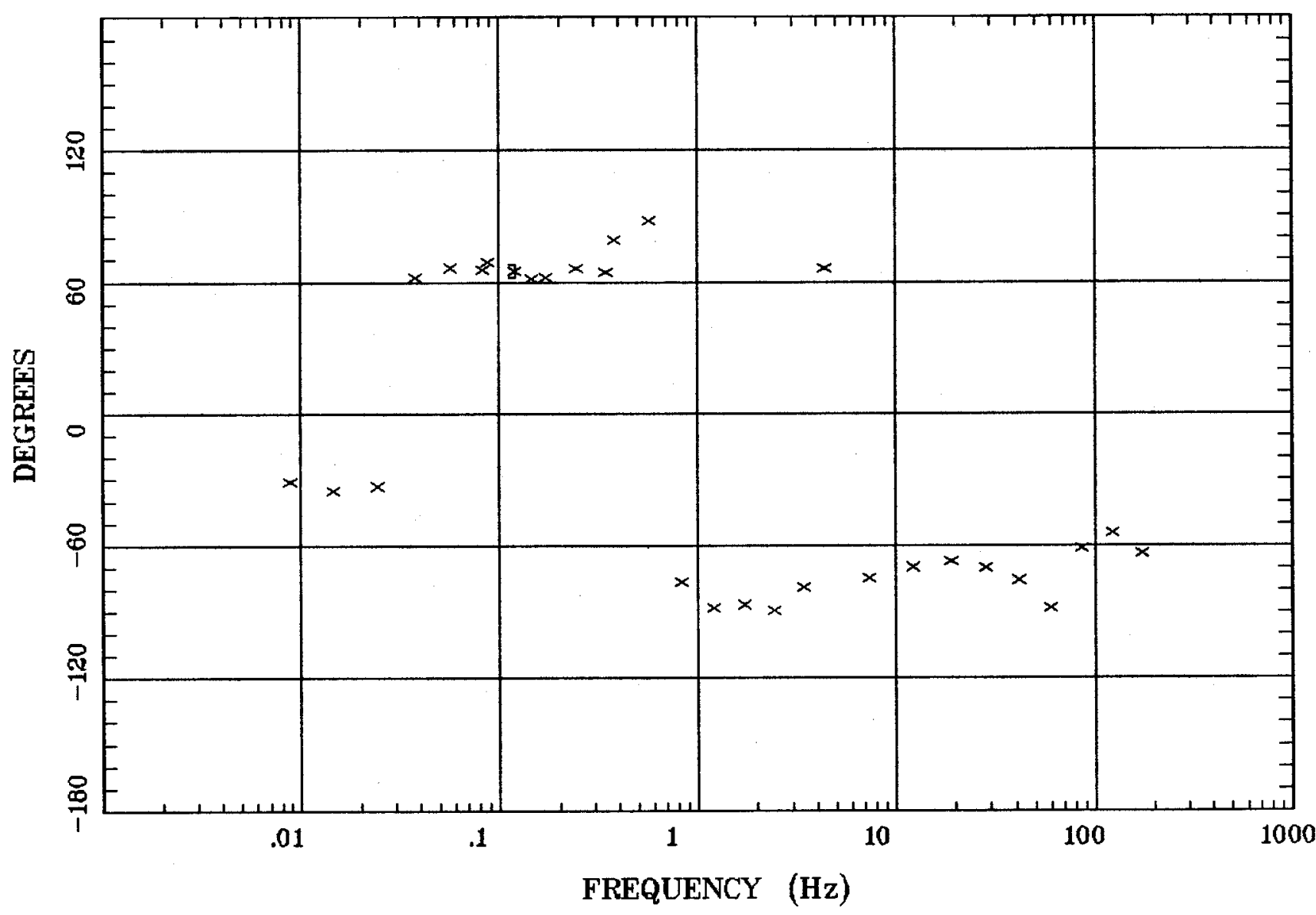

Client:

Remote: e-fld $80 \mathrm{~m}$ east Acquired: 10:3 Jun 23, 2003 Survey Co:USGS
Rotation:

Filename: cp24m.avg

Channels: Ch1 Ch2 Ch3 Ch4 Ch5 Ch6 Ch7

Plotted: 15:42 Jul 28, 2004

$<$ EMI - ElectroMagnetic Instruments 


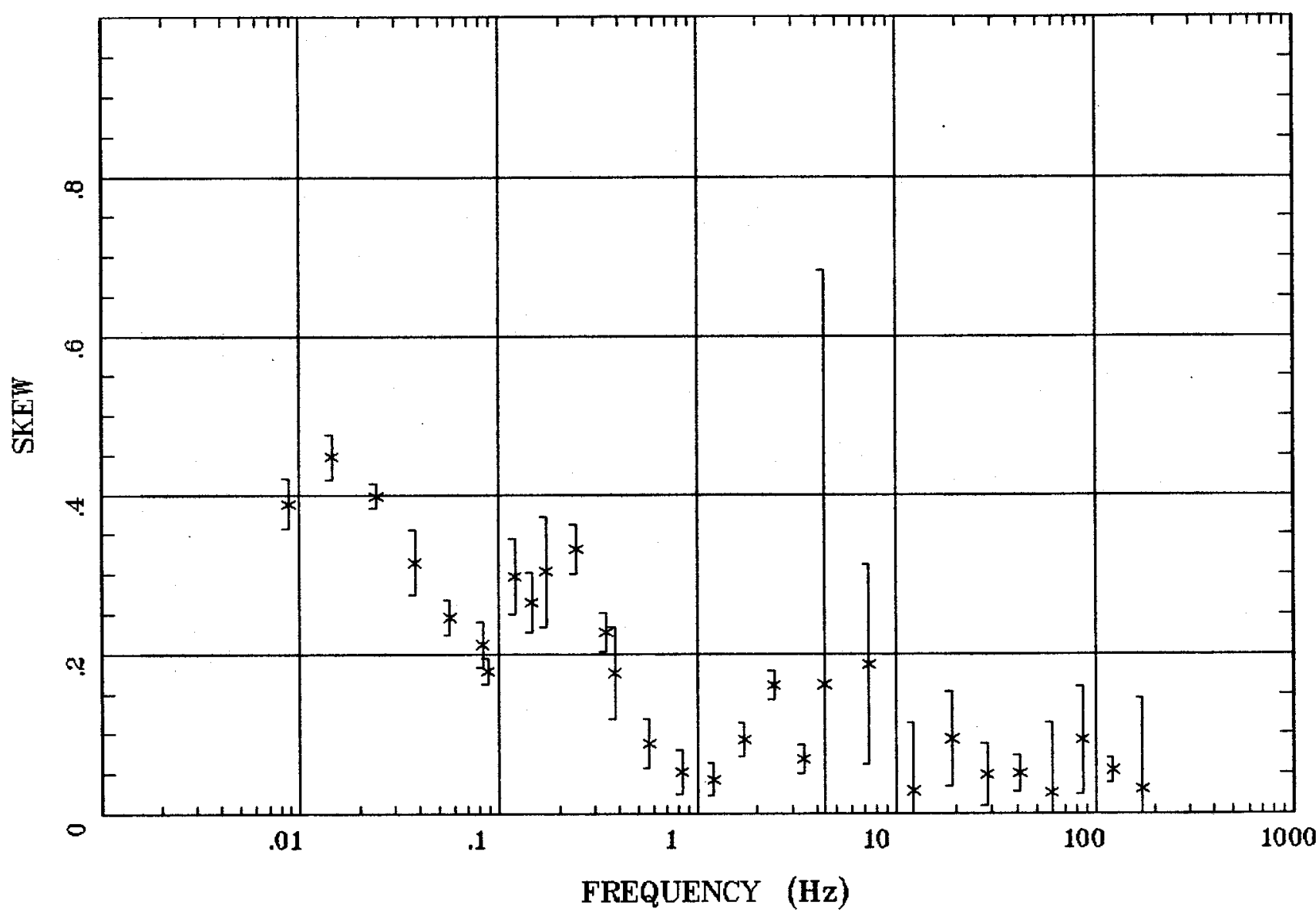

Client:

Remote: e-fld $80 \mathrm{~m}$ east Acquired: 10:3 Jun 23, 2003 Survey Co:USGS
Rotation:

Filename: cp24m.avg

Channels: Ch1 Ch2 Ch3 Ch4 Ch5 Ch6 Ch7

Plotted: 14:53 Jul 27, 2004

$<$ EMI - ElectroMagnetic Instruments 


\section{E MULT Coh.}

Santa Fe, NM

Station 24

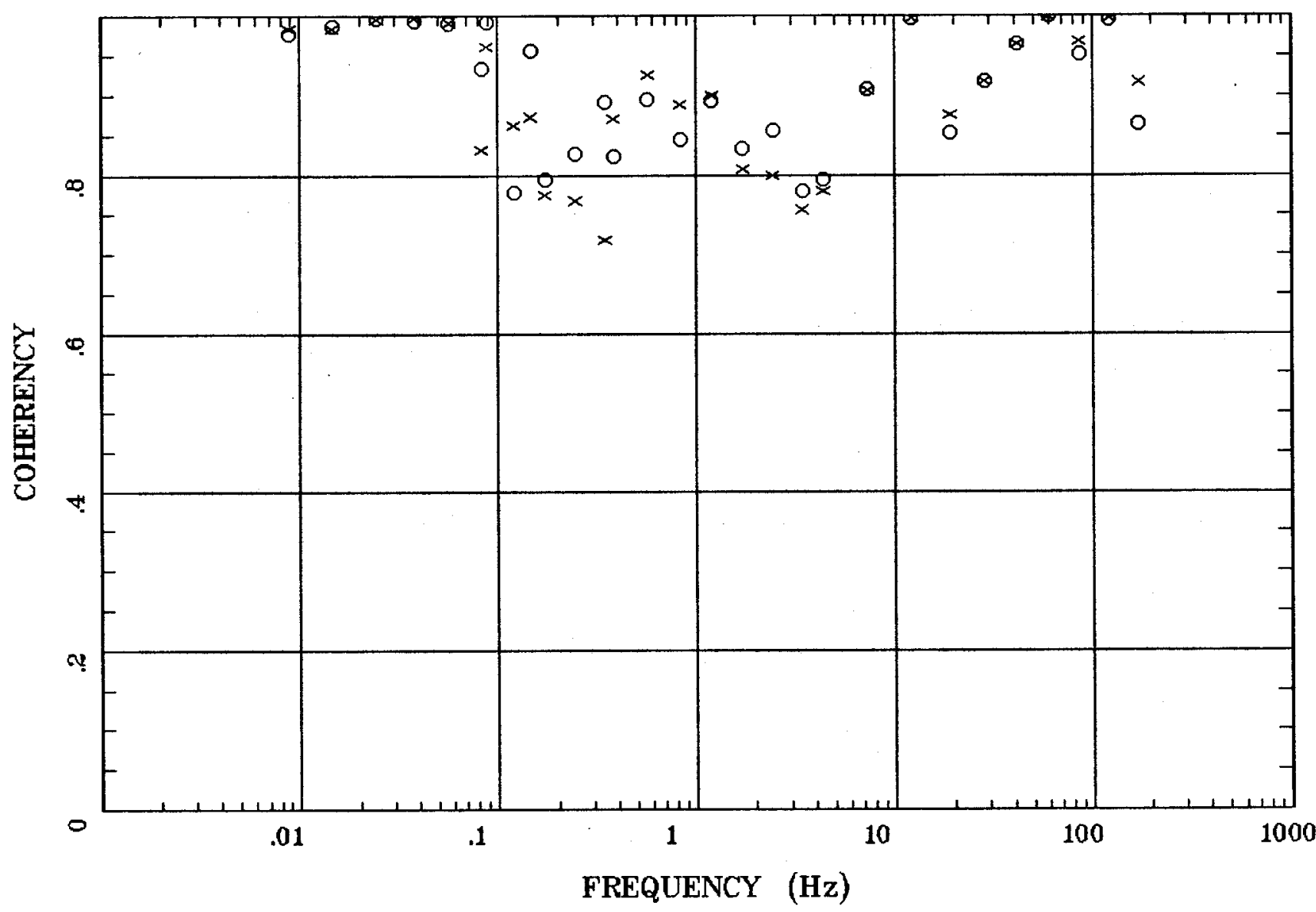

Client:

Remote: e-fld $80 \mathrm{~m}$ east Acquired: 10:3 Jun 23, 2003 Survey Co:USGS
Rotation:

Filename: cp24m.avg

Channels: Ch1 Ch2 Ch3 Ch4 Ch5 Ch6 Ch7 Plotted: 14:53 Jul 27, 2004

$<$ EMI - ElectroMagnetic Instruments 


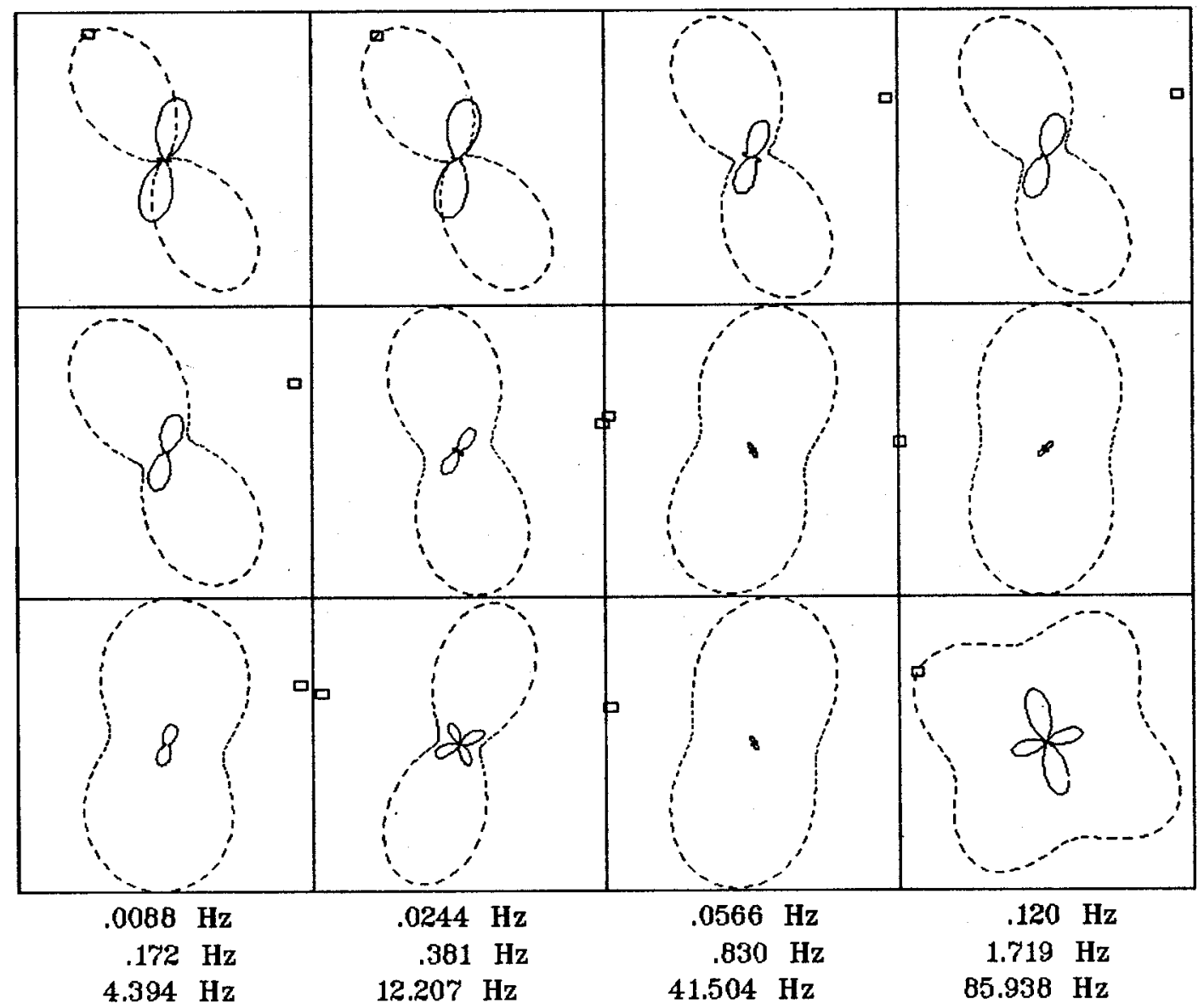

Rotation:

Client:

Remote: e-fld $80 \mathrm{~m}$ east Acquired: 10:3 Jun 23, 2003 Survey Co:USGS
Filename: cp24m.avg

Channels: Ch1 Ch2 Ch3 Ch4 Ch5 Ch6 Ch7 Plotted: 14:53 Jul 27, 2004

$<$ EMI - ElectroMagnetic Instruments 


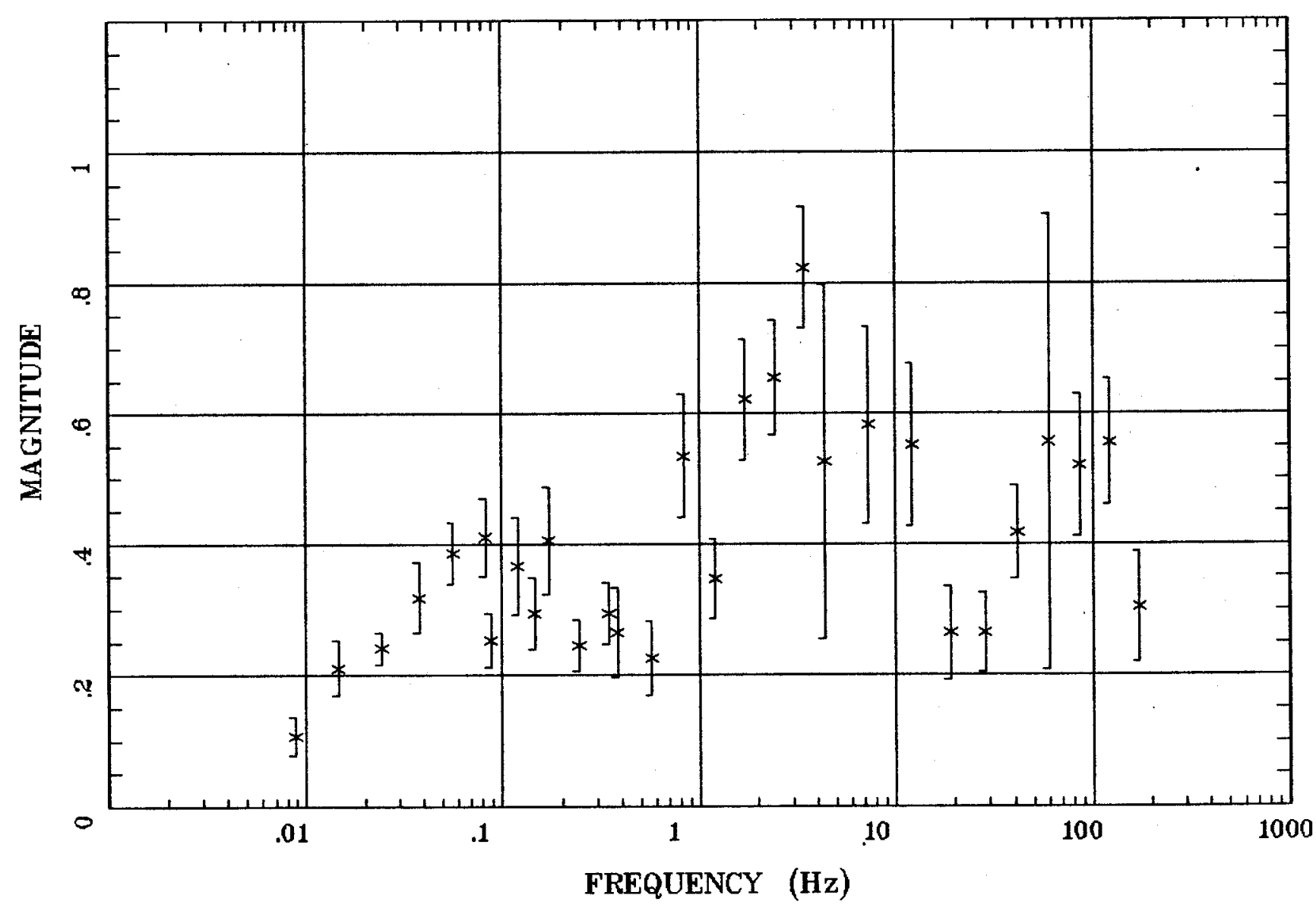

Client:

Remote: e-fld $80 \mathrm{~m}$ east

Acquired: 10:3 Jun 23, 2003

Survey Co:USGS
Rotation:

Filename: cp24m.avg

Channels: Ch1 Ch2 Ch3 Ch4 Ch5 Ch6 Ch7

Plotted: 14:53 Jul 27, 2004

$<$ EMI - ElectroMagnetic Instruments > 


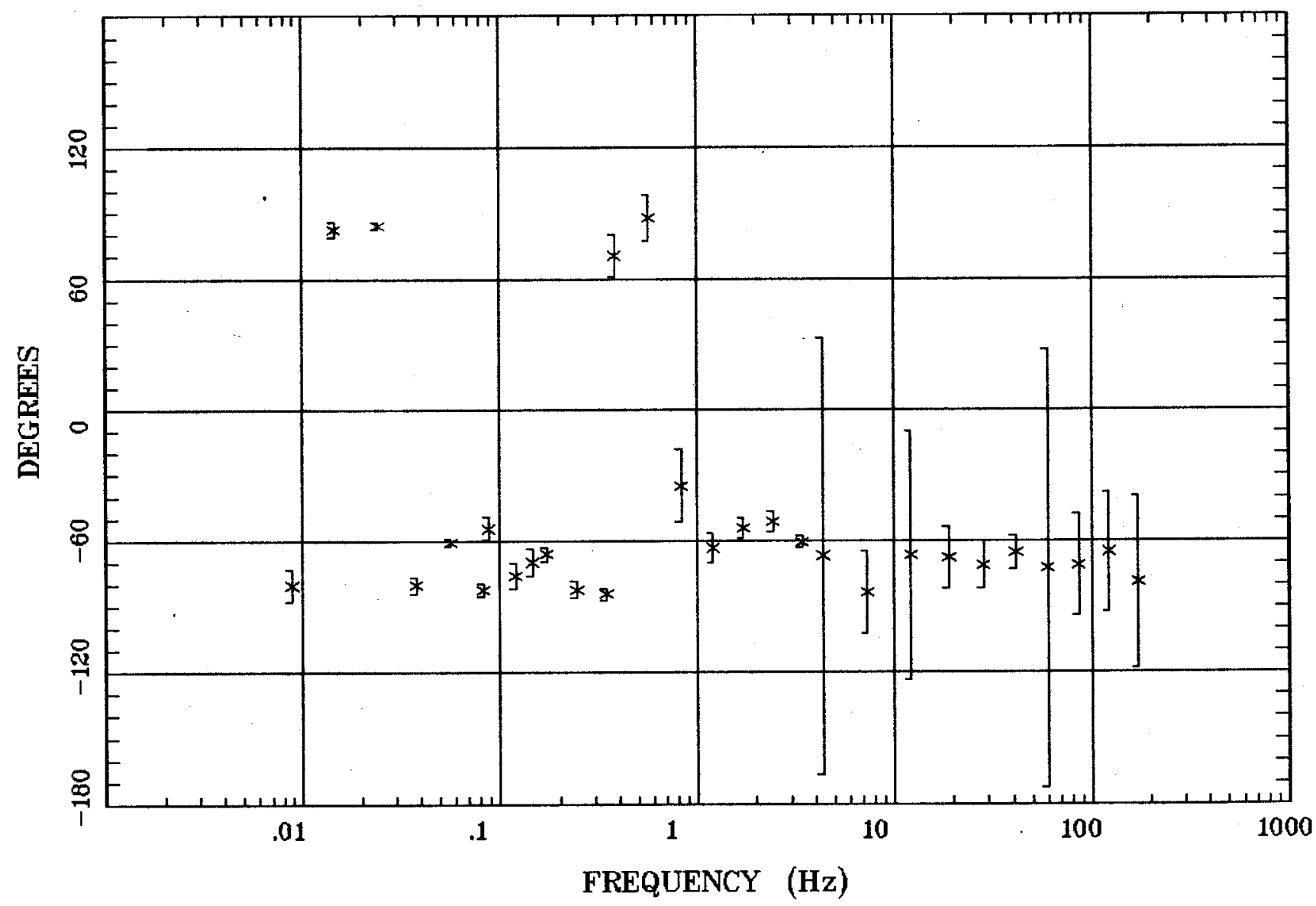

Client:

Remote: $\mathrm{e}-\mathrm{fld}$ $80 \mathrm{~m}$ east Acquired: 10:3 Jun 23, 2003 Survey Co:USGS
Rotation:

Filename: cp24m.avg

Channels: Ch1 Ch2 Ch3 Ch4 Ch5 Ch6 Ch7

Plotted: 14:53 Jul 27, 2004

< EMI - ElectroMagnetic Instruments 


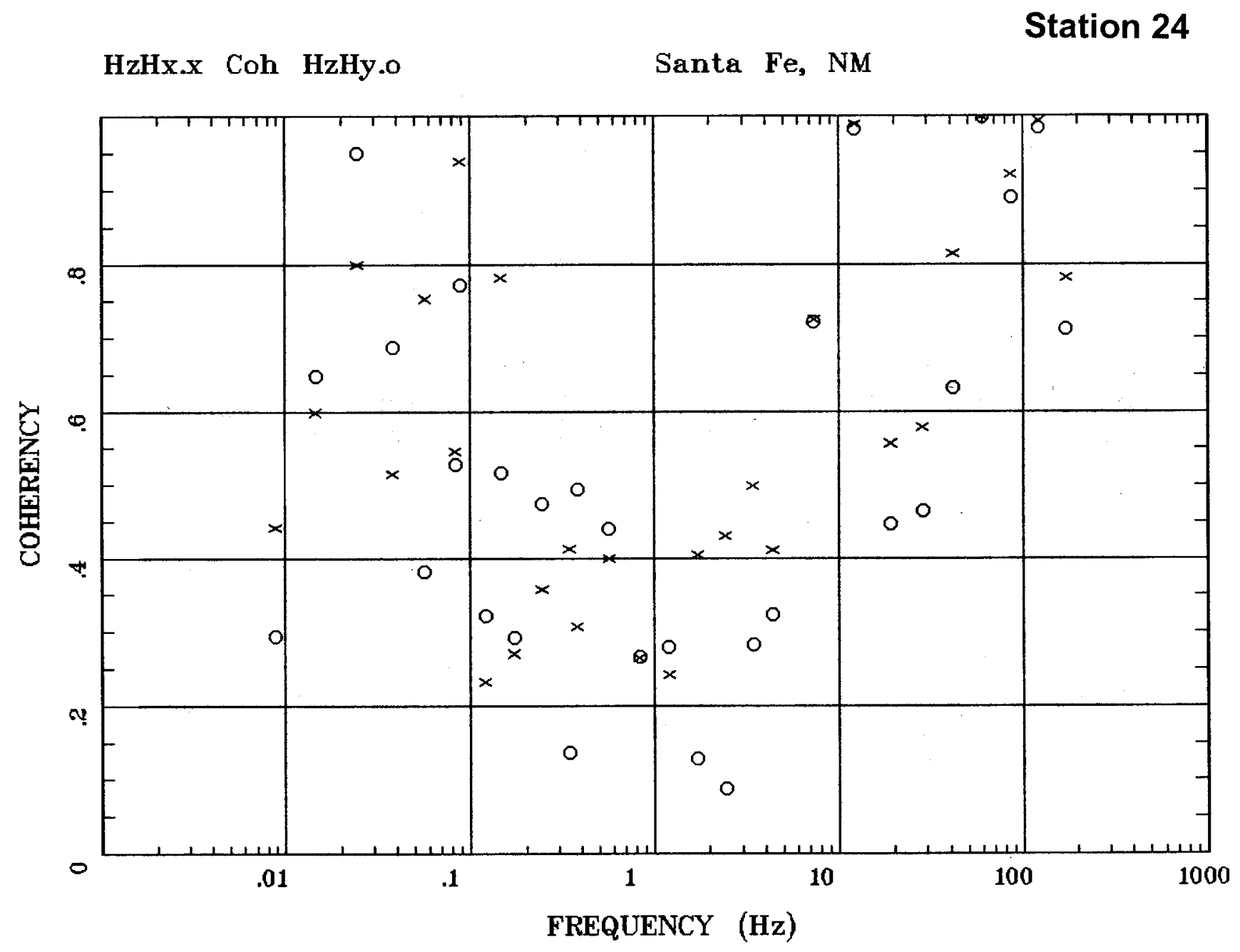

Client:

Remote: e-fld $80 \mathrm{~m}$ east

Acquired: 10:3 Jun 23, 2003 Survey Co:USGS
Rotation:

Filename: cp 24m.avg

Channels: Ch1 Ch2 Ch3 Ch4 Ch5 Ch6 Ch7 Plotted: 14:54 Jul 27, 2004

< EMI - ElectroMagnetic Instruments 


\section{APPARENT RESISTIVITY}

Santa Fe, NM

Station 25

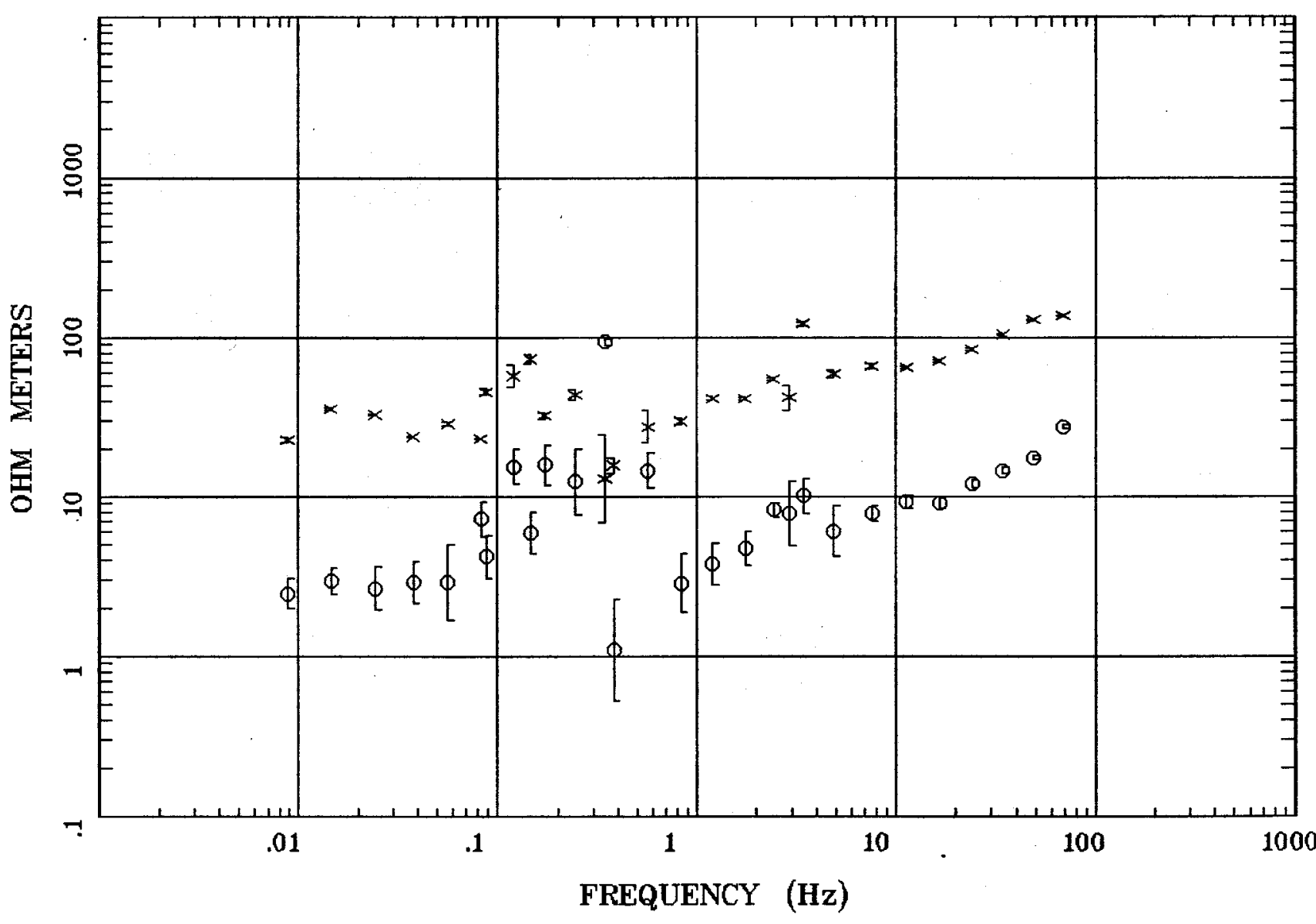

Client:

Remote: e-fld $90 \mathrm{~m}$ east

Acquired: 11:2 Jun 24, 2003

Survey Co:USGS
Rotation:

Filename: cp25m.avg

Channels: Ch1 Ch2 Ch3 Ch4 Ch5 Ch6 Chr Plotted: 08:55 Jul 07, 2004

$<$ EMI - ElectroMagnetic Instruments 


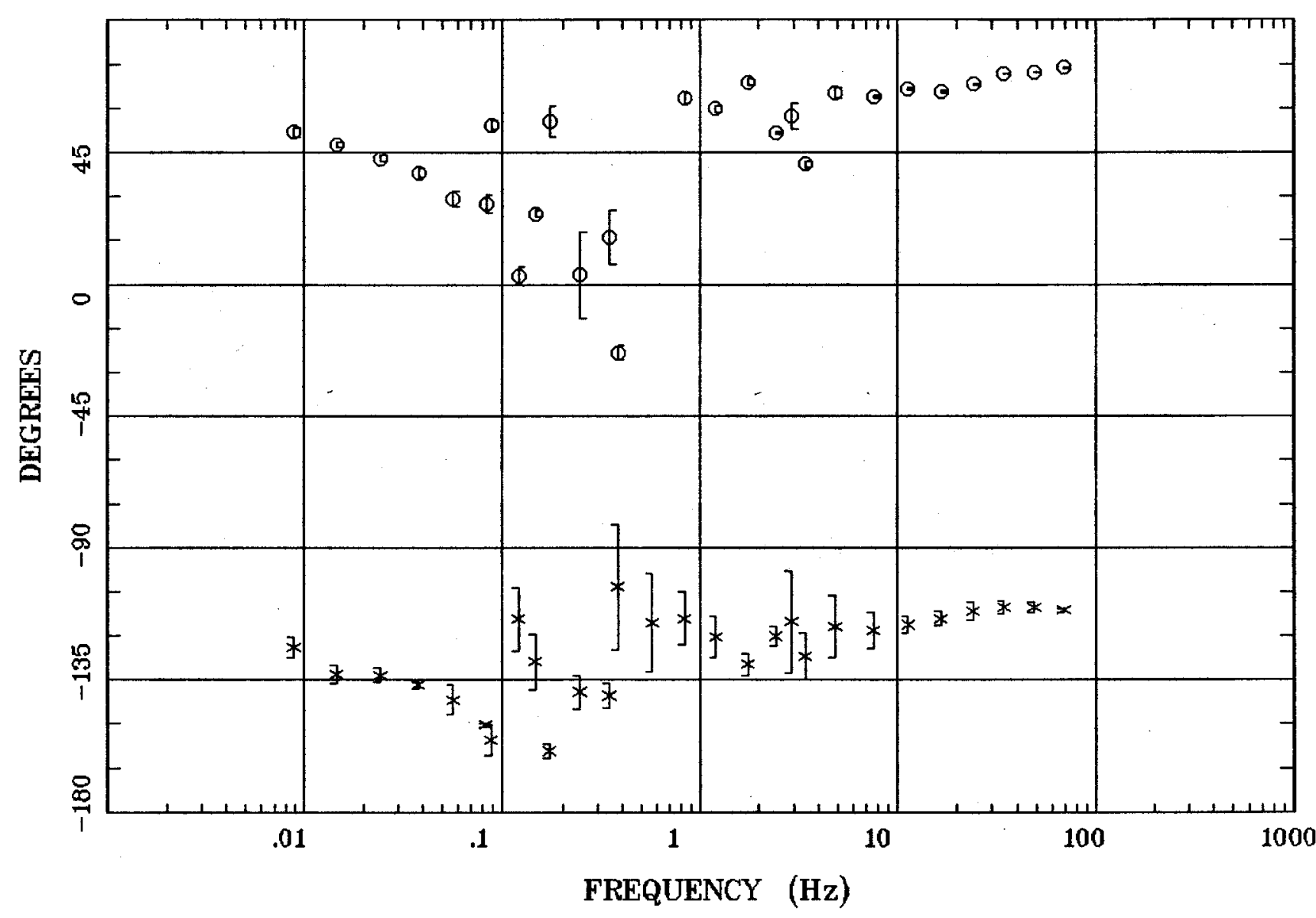

Client:

Remote: e-fld $90 \mathrm{~m}$ east Acquired: 11:2 Jun 24, 2003 Survey Co:USGS
Rotation:

Fileneme: cp25m.avg

Channels: Ch1 Ch2 Ch3 Ch4 Ch5 Ch6 Ch7 Plotted: 08:55 Jul 07, 2004

< EMI - ElectroMagnetic Instruments 


\section{ROTATION ANGLE}

Santa Fe, NM

Station 25

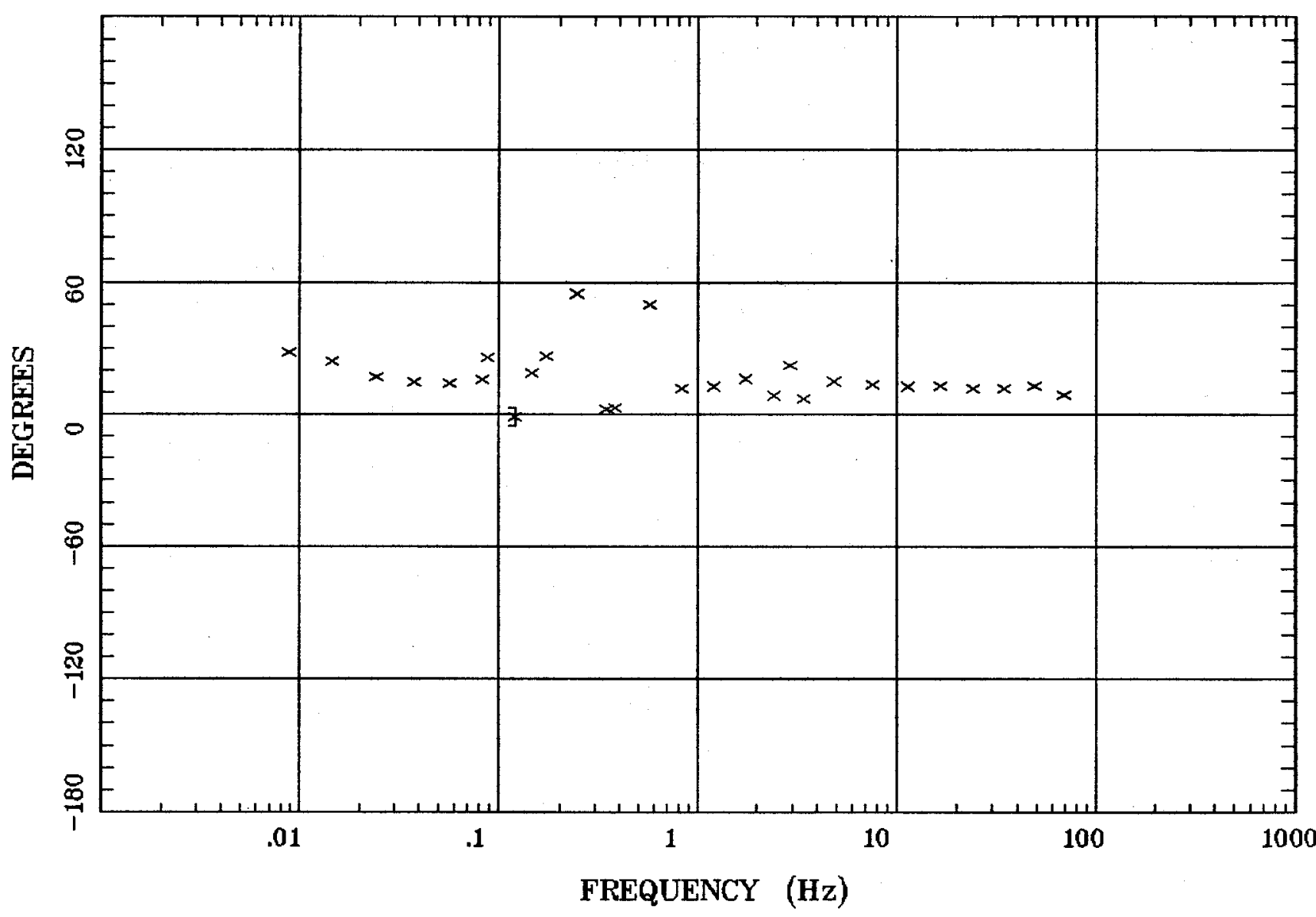

Client:

Remote: e-fld $90 \mathrm{~m}$ east

Acquired: 11:2 Jun 24, 2003

Survey Co:USGS
Rotation:

Filename: cp25m.avg

Channels: Ch1 Ch2 Ch3 Ch4 Ch5 Ch6 Ch7

Plotted: 10:22 Jul 07, 2004

$<$ EMI - ElectroMagnetic Instruments 


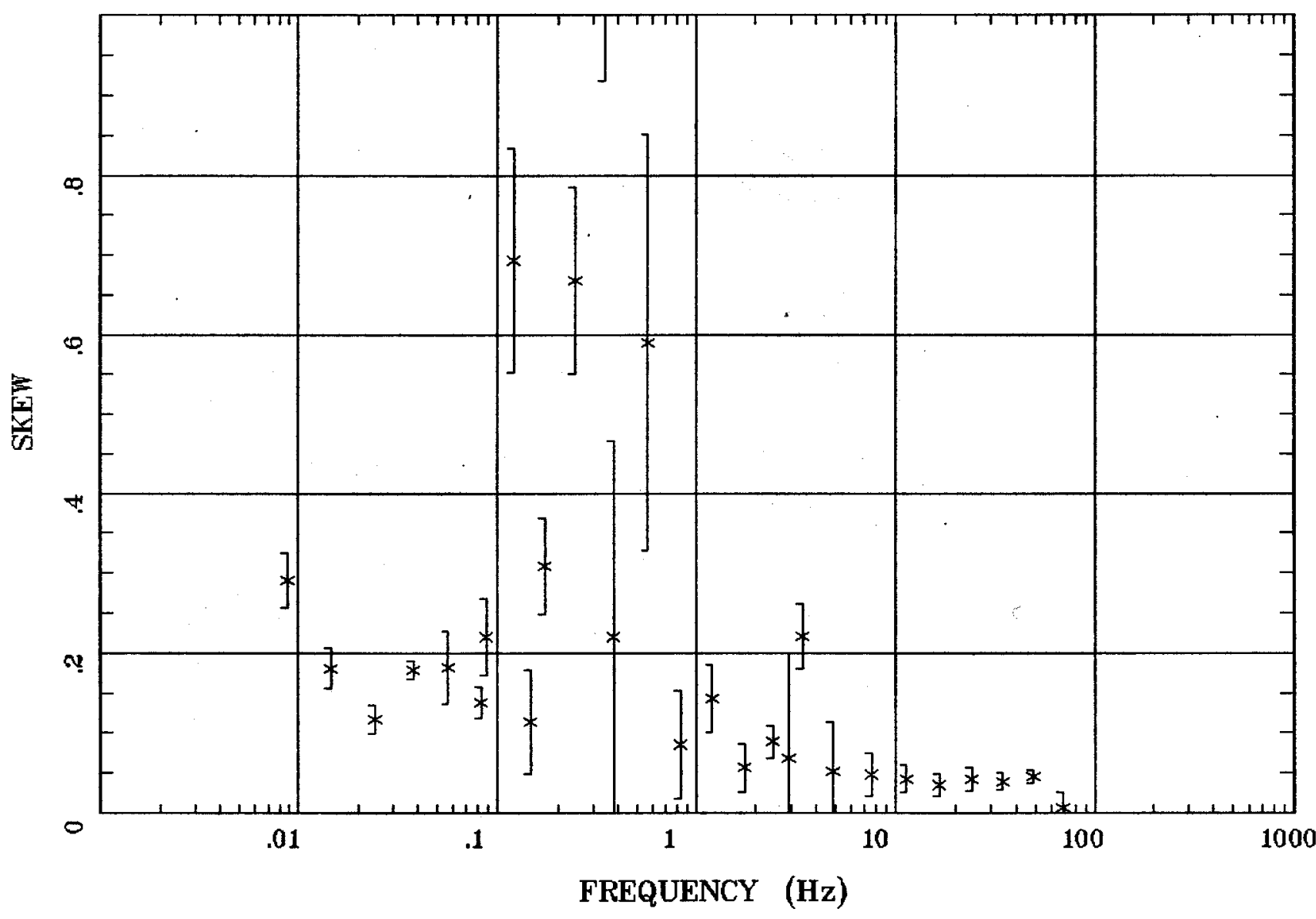

Client:

Remote: e-fld $90 \mathrm{~m}$ east

Acquired: 11:2 Jun 24, 2003 Survey Co:USGS
Rotation:

Filename: cp $25 \mathrm{~m}$.avg

Channels: Ch1 Ch2 Ch3 Ch4 Ch5 Ch6 Ch7 Plotted: 08:55 Jul 07, 2004

< EMI - ElectroMagnetic Instruments 


\section{E MULT Coh.}

Santa Fe, NM

Station 25

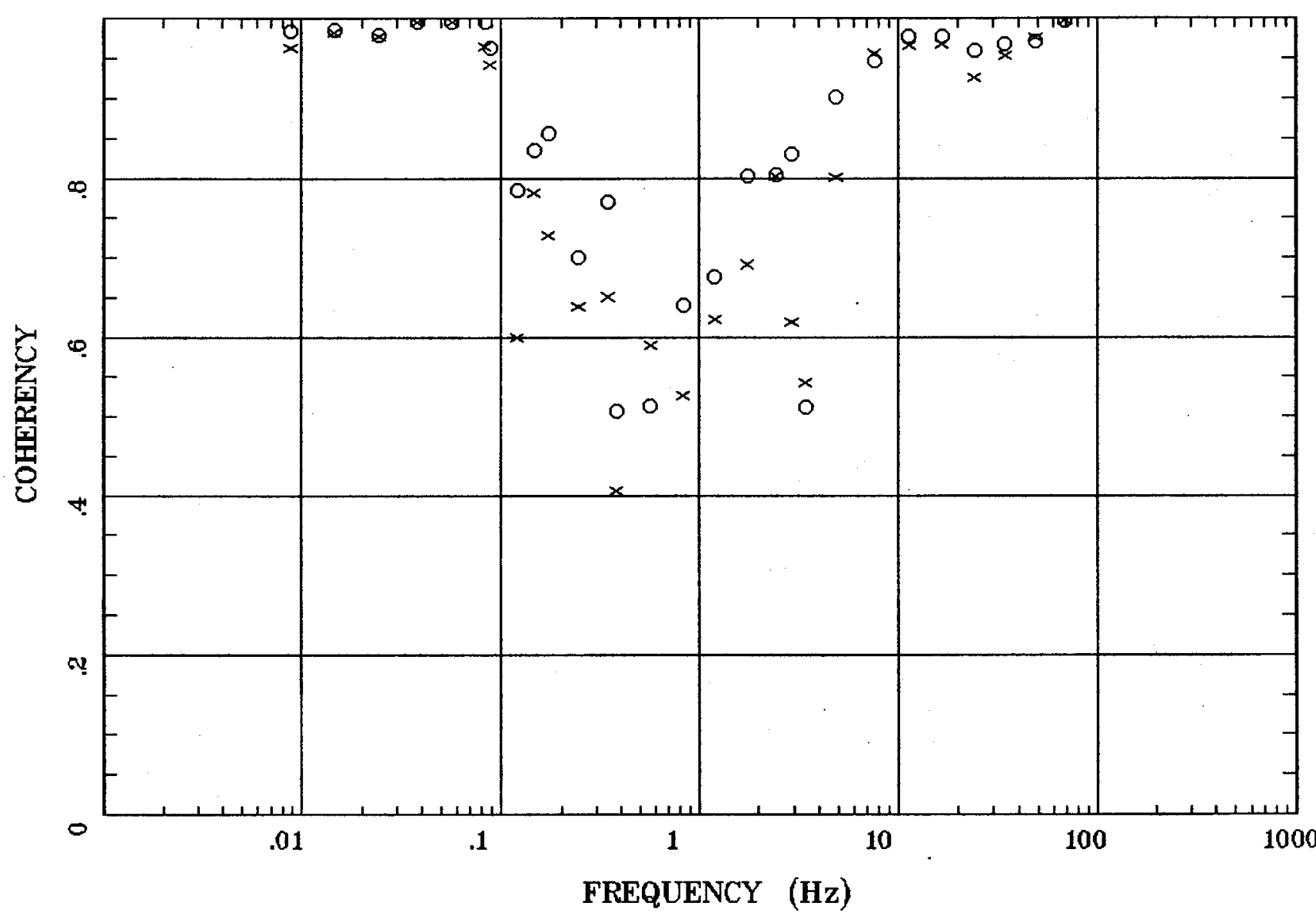

Client:

Remote: $e^{-f l d ~} 90 \mathrm{~m}$ east

Acquired: 11:2 Jun 24, 2003 Survey Co:USGS
Rotation:

Filename: cp 25m.avg

Channels: Ch1 Ch2 Ch3 ch4 Ch5 Ch6 ch7 Plotted: 08:55 Jul 07, 2004

$<$ EMI - ElectroMagnetic Instruments 


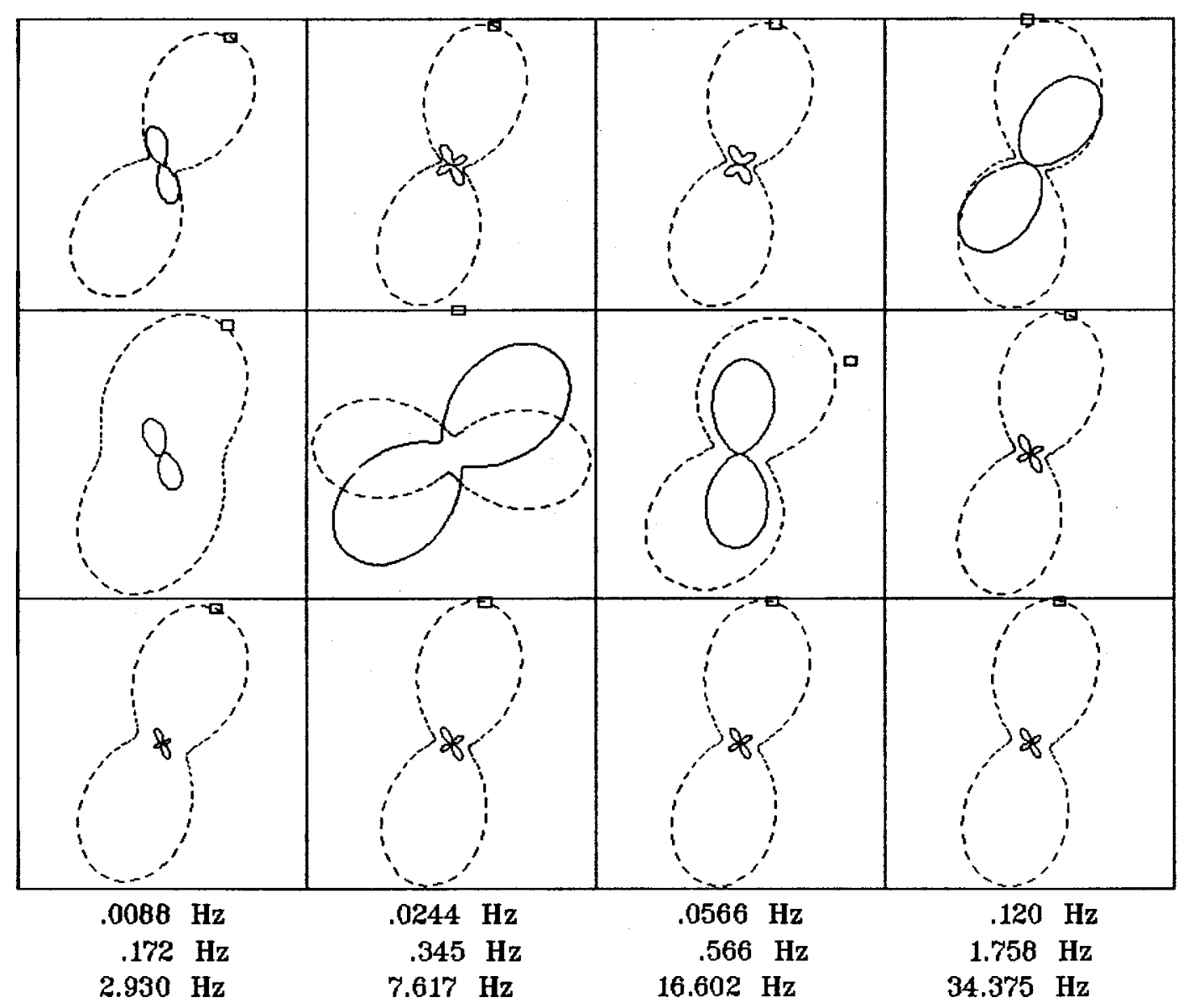

Rotation:

Client:

Remote: e-fld $90 \mathrm{~m}$ east Acquired: 11:2 Jun 24, 2003 Survey Co:USGS
Filename: cp25m.avg

Channels: Ch1 Ch2 Ch3 Ch4 Ch5 Ch6 Ch7 Plotted: 08:55 Jul 07, 2004

< EMI - ElectroMagnetic Instruments > 


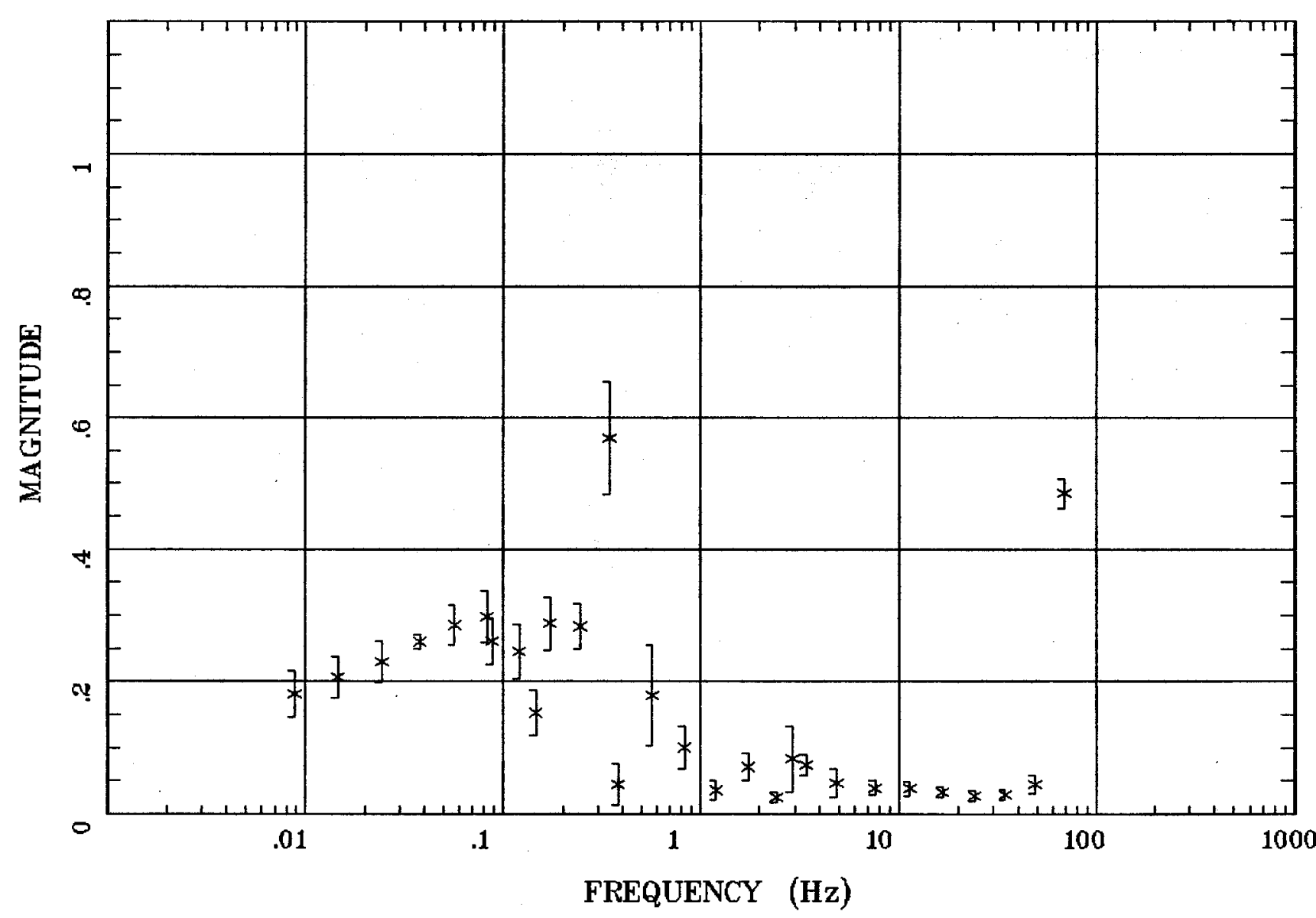

Client:

Remote: e-fld $90 \mathrm{~m}$ east

Acquired: 11:2 Jun 24, 2003 Survey Co:USGS
Rotation:

Filename: cp25m.avg

Channels: Ch1 ch2 Ch3 ch4 Ch5 Ch6 Ch7 Plotted: 08:55 Jul 07, 2004

< EMI - ElectroMagnetic Instruments 


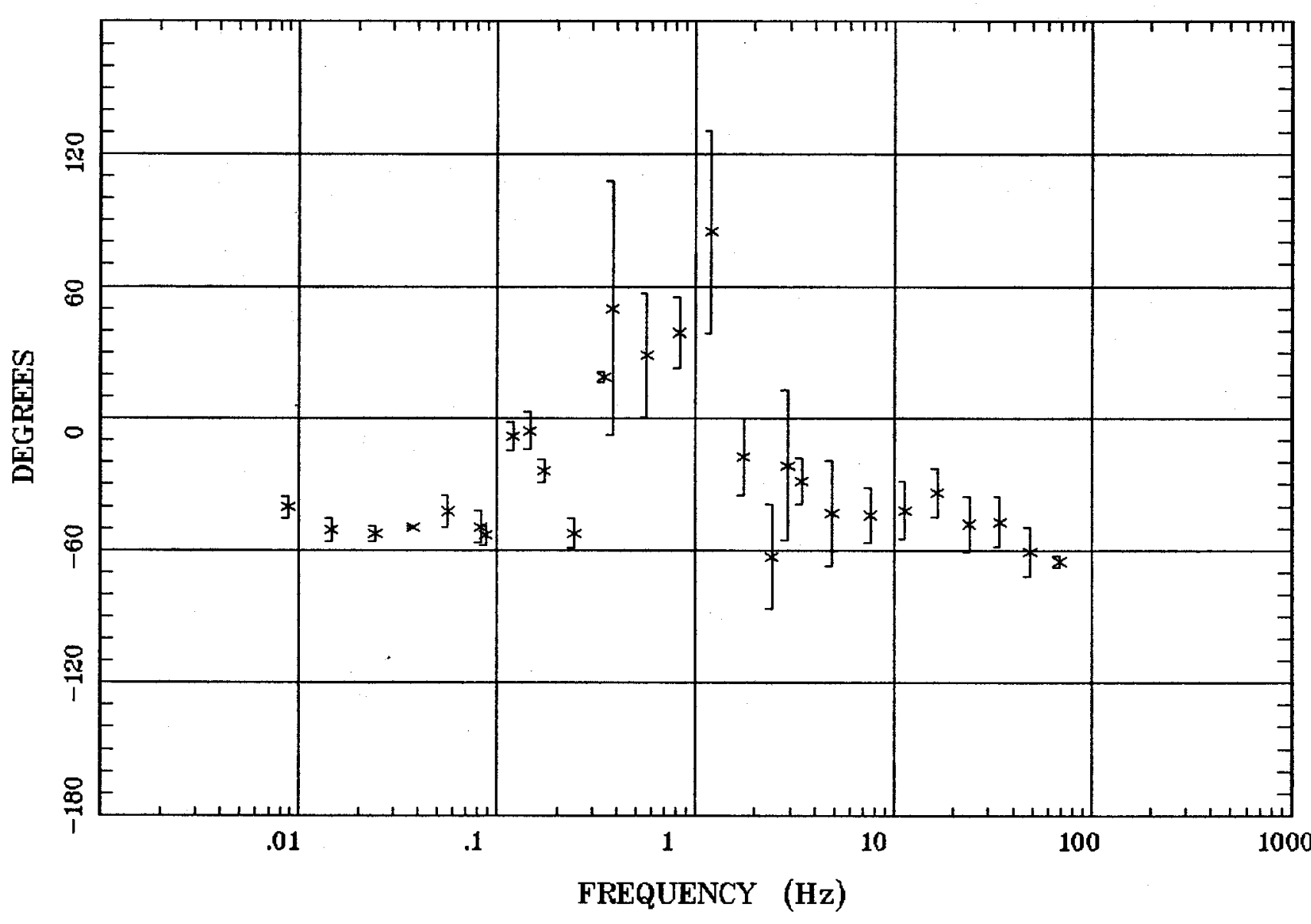

Client:

Remote: e-fld $90 \mathrm{~m}$ east Acquired: 11:2 Jun 24, 2003 Survey Co:USGS
Rotation:

Filename: cp25m.avg

Channels: Ch1 Ch2 Ch3 Ch4 Ch5 Ch6 Ch7 Plotted: 08:55 Jul 07, 2004

$<$ EMI - ElectroMagnetic Instruments 


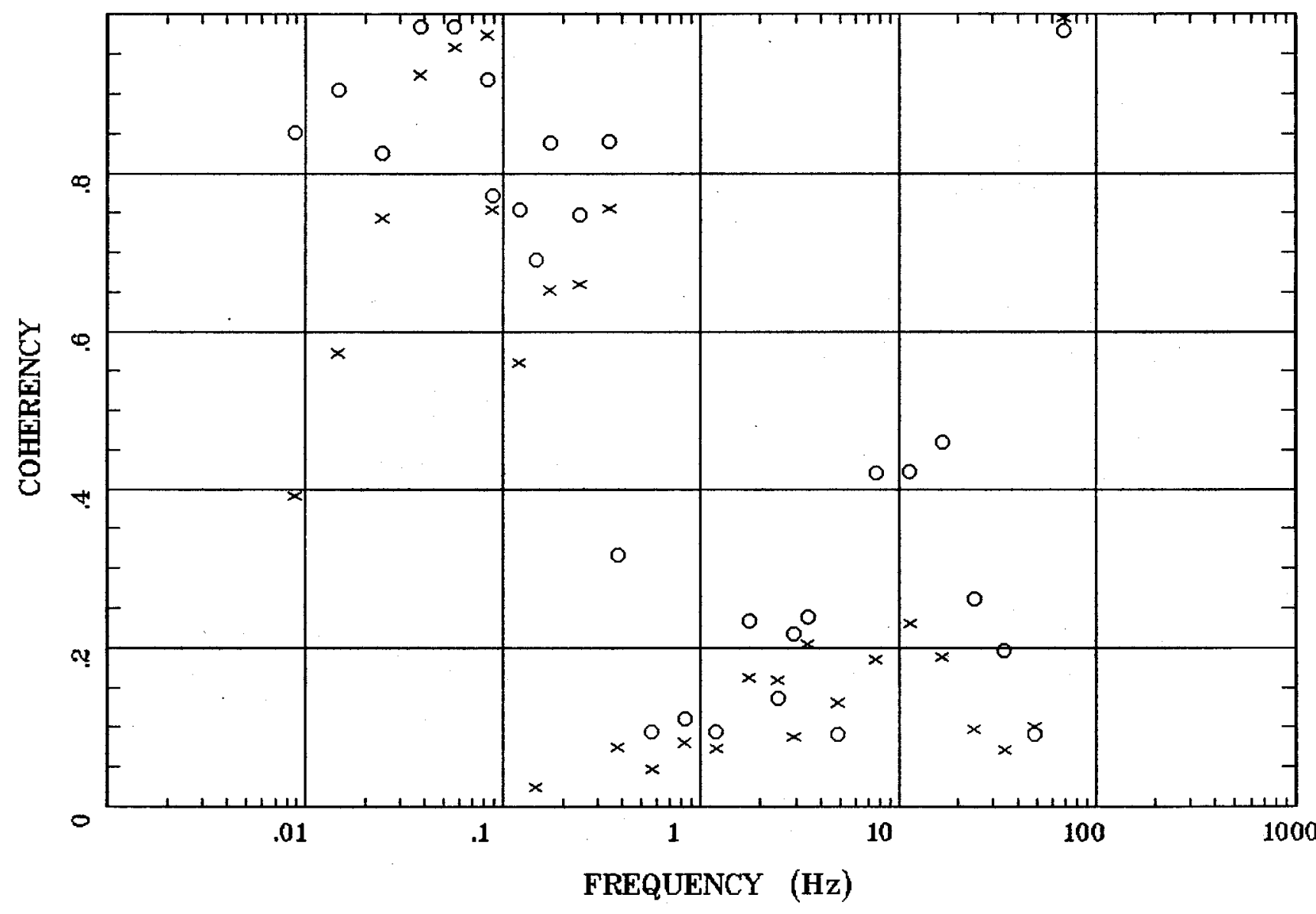

Client:

Remote: e-fld 90m east

Acquired: 11:2 Jun 24, 2003 Survey Co:USGS
Rotation:

Filename: cp25m.avg

Channels: Ch1 Ch2 Ch3 Ch4 Ch5 Ch6 Ch7 Plotted: 08:55 Jul 07, 2004

< EMI - ElectroMagnetic Instruments 


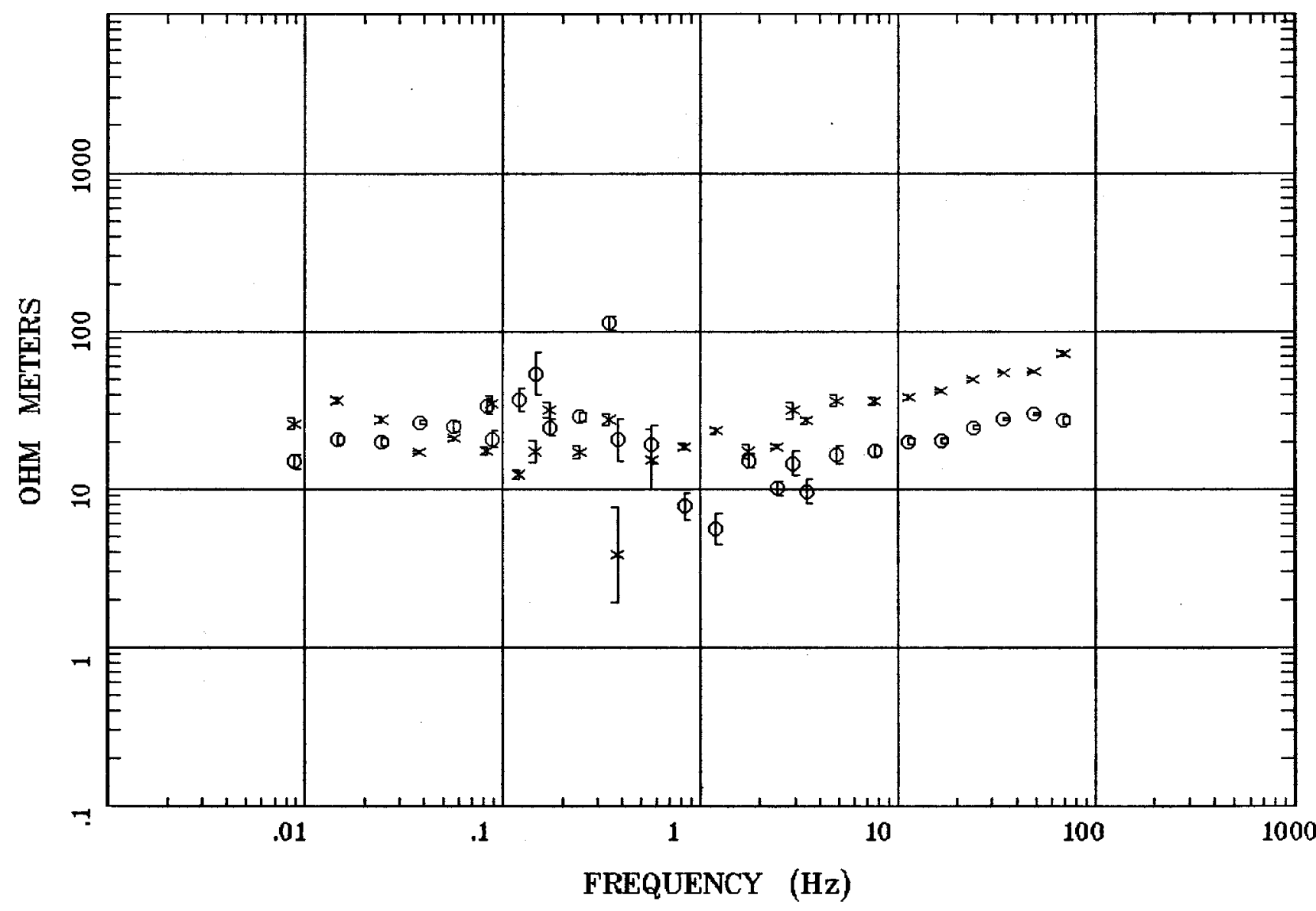

Client:

Remote: e-fld $64 \mathrm{~m}$ east

Acquired: 12:2 Jun 25, 2003

Survey Co:USGS
Rotation:

Filename: cp26m.avg

Channels: Ch1 Ch2 Ch3 Ch4 Ch5 Ch6 Ch7

Plotted: 08:56 Jul 07, 2004

$<$ EMI - ElectroMagnetic Instruments 


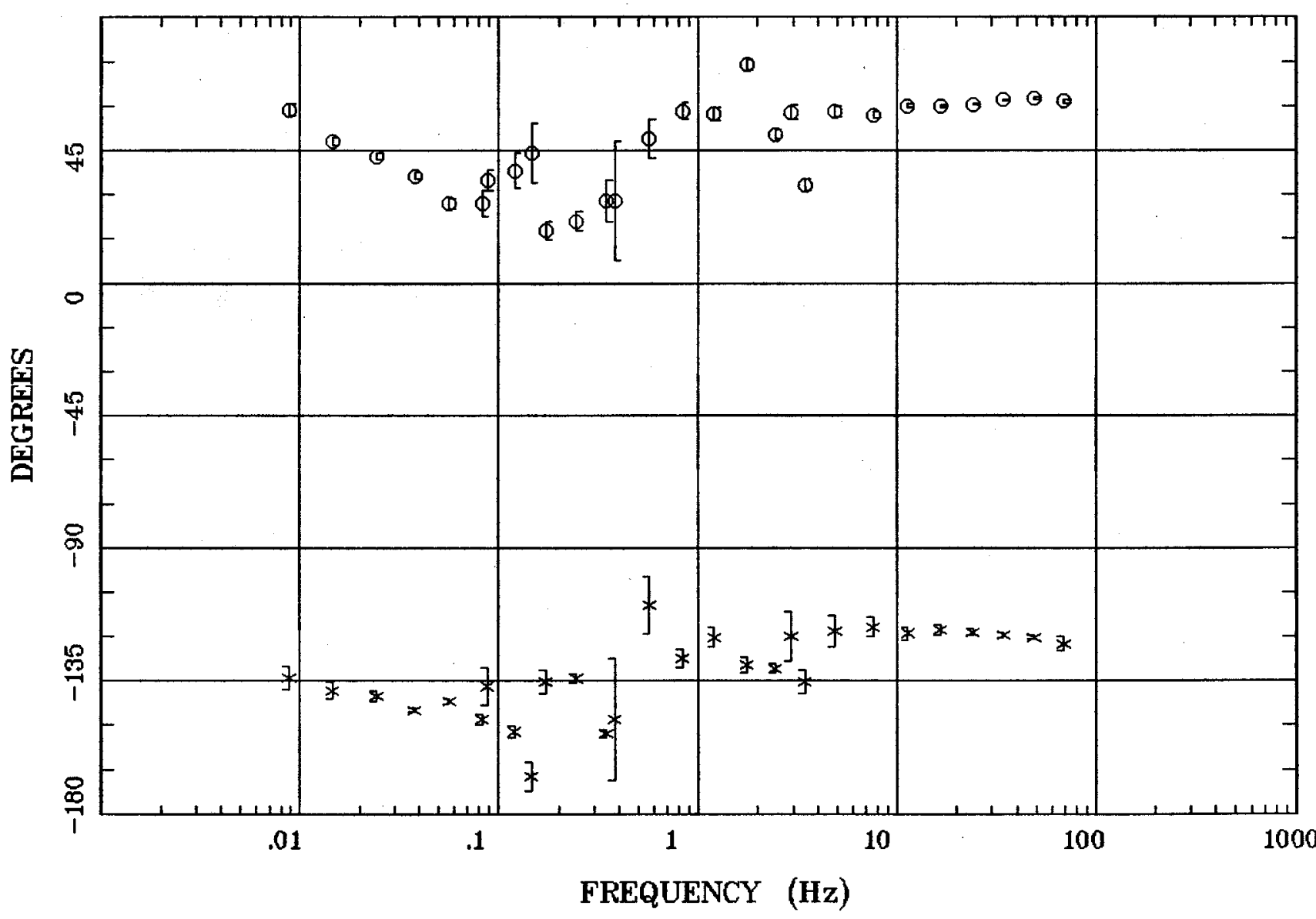

Client:

Remote: $\mathrm{e}-\mathrm{fld}$ 64m east Acquired: 12:2 Jun 25, 2003 Survey Co:USGS
Rotation:

Filename: cp26m.avg

Channels: Ch1 Ch2 Ch3 Ch4 Ch5 Ch6 Ch7 Plotted: 08:56 Jul 07, 2004

< EMI - ElectroMagnetic Instruments 


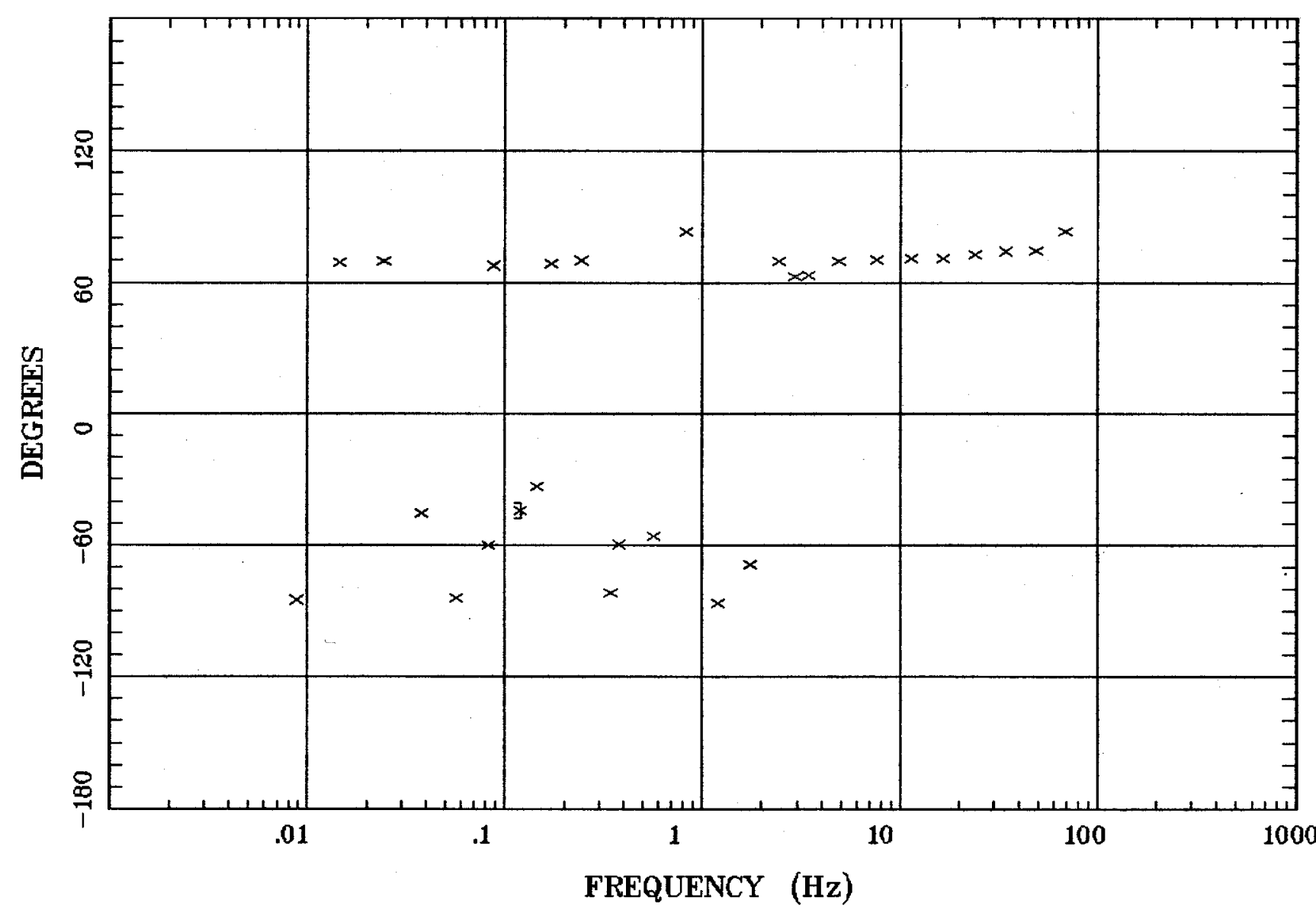

Client:

Remote: e-fld $64 \mathrm{~m}$ east Acquired: 12:2 Jun 25, 2003 Survey Co:USGS
Rotation:

Filename: cp26m.avg

Channels: Ch1 Ch2 Ch3 Ch4 Ch5 Ch6 Ch7 Plotted: 10:21 Jul 07, 2004

< EMI - ElectroMagnetic Instruments 


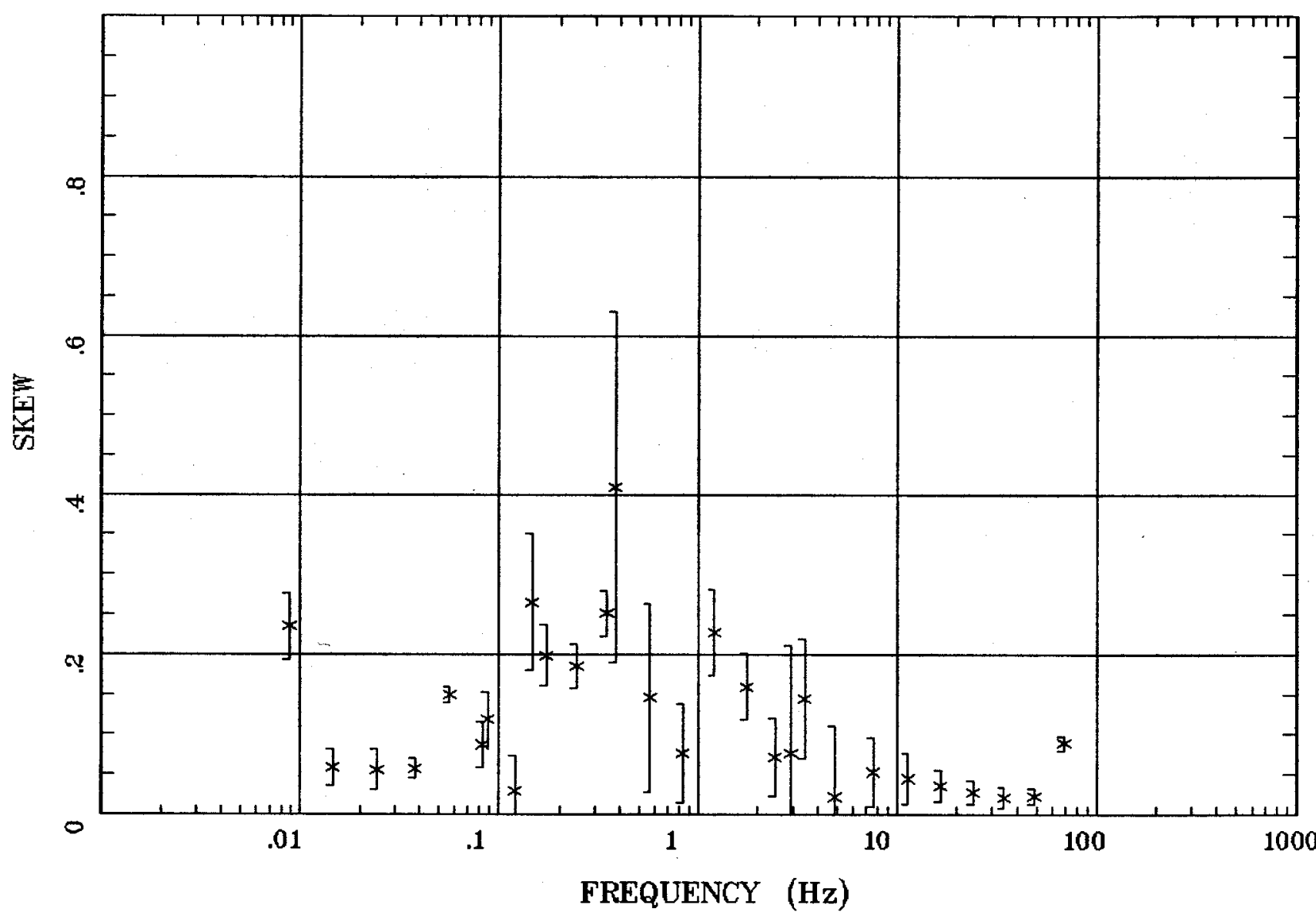

Client:

Remote: e-fld $64 \mathrm{~m}$ east

Acquired: 12:2 Jun 25, 2003

Survey Co:USGS
Rotation:

Filename: cp26m.avg

Channels: Ch1 Ch2 Ch3 Ch4 Ch5 Ch6 Ch7

Plotted: 08:56 Jul 07, 2004

$<$ EMI - ElectroMagnetic Instruments 


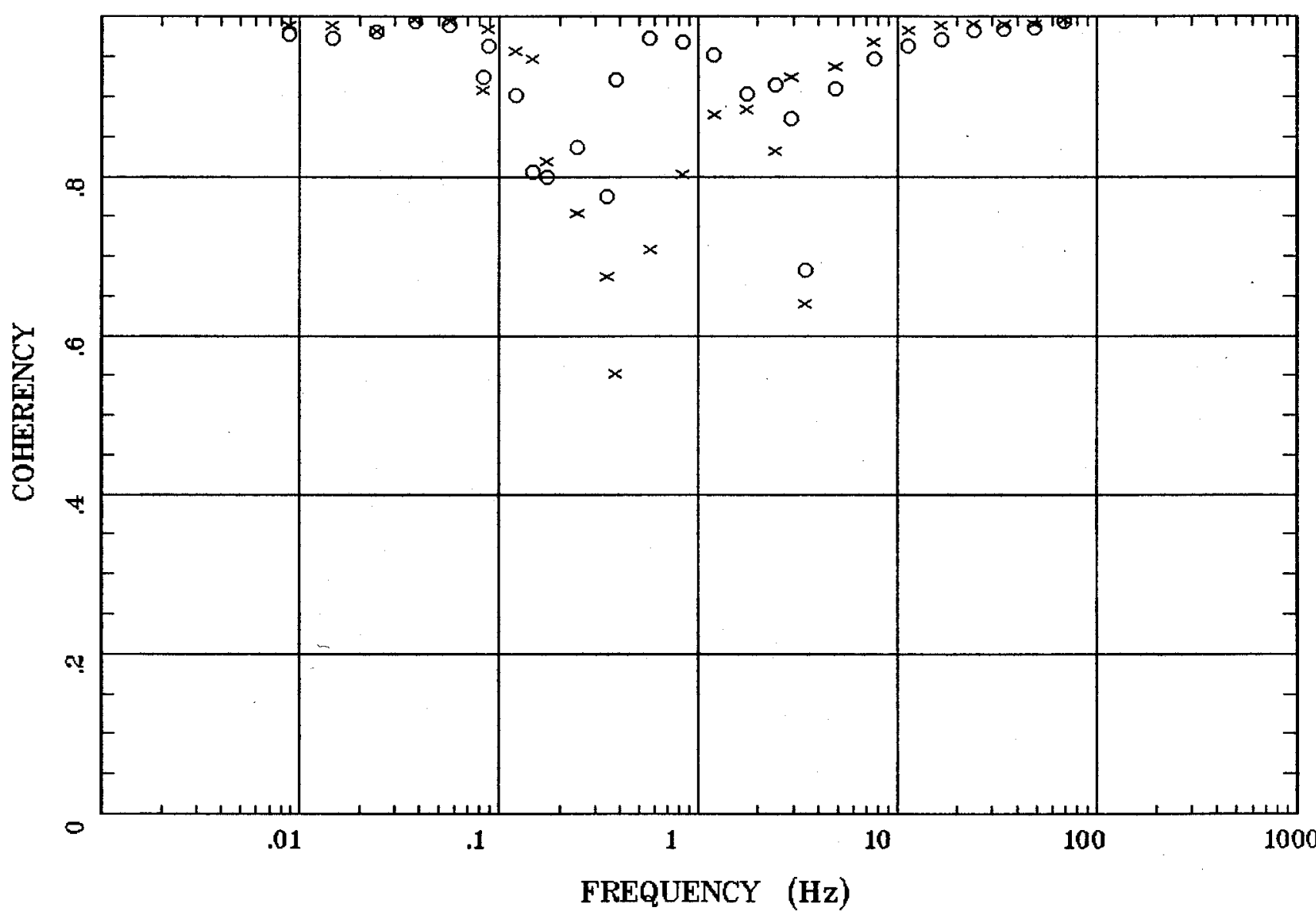

Client:

Remote: e-fld 64m east Acquired: 12:2 Jun 25, 2003 Survey Co:USGS
Rotation:

Filename: cp26m.avg

Channels: Ch1 Ch2 Ch3 Ch4 Ch5 Ch6 Ch7 Plotted: 08:56 Jul 07, 2004

< EMI - ElectroMagnetic Instruments 


\section{POLAR PLOTS}

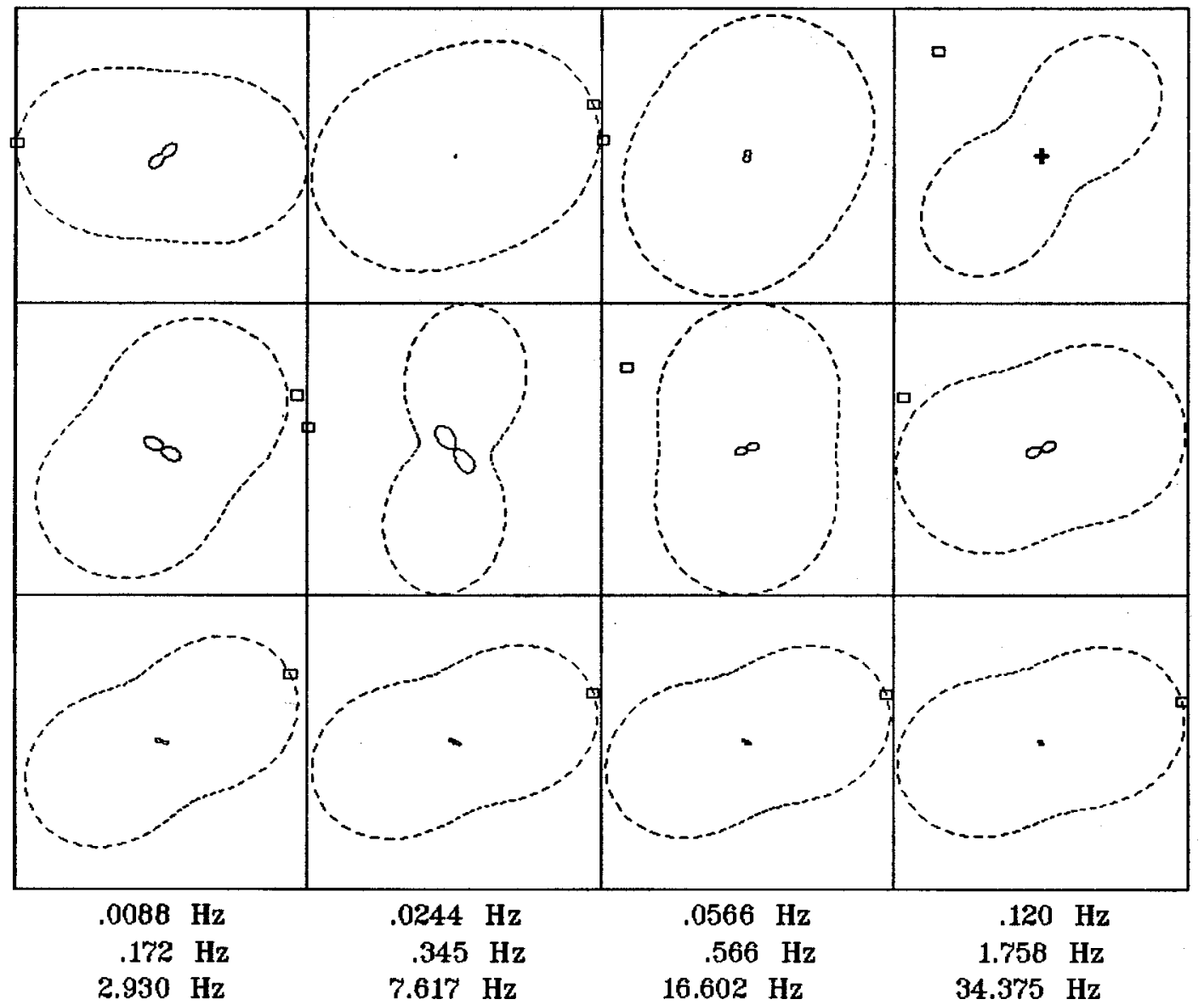

Client:

Remote: e-fld $64 \mathrm{~m}$ east Acquired: 12:2 Jun 25, 2003 Survey Co:USGS
Rotation:

Filename: cp26m.avg

Channels: Ch1 Ch2 Ch3 Ch4 Ch5 Ch6 Ch7 Plotted: 08:56 Jul 07, 2004

< EMI - ElectroMagnetic Instruments > 


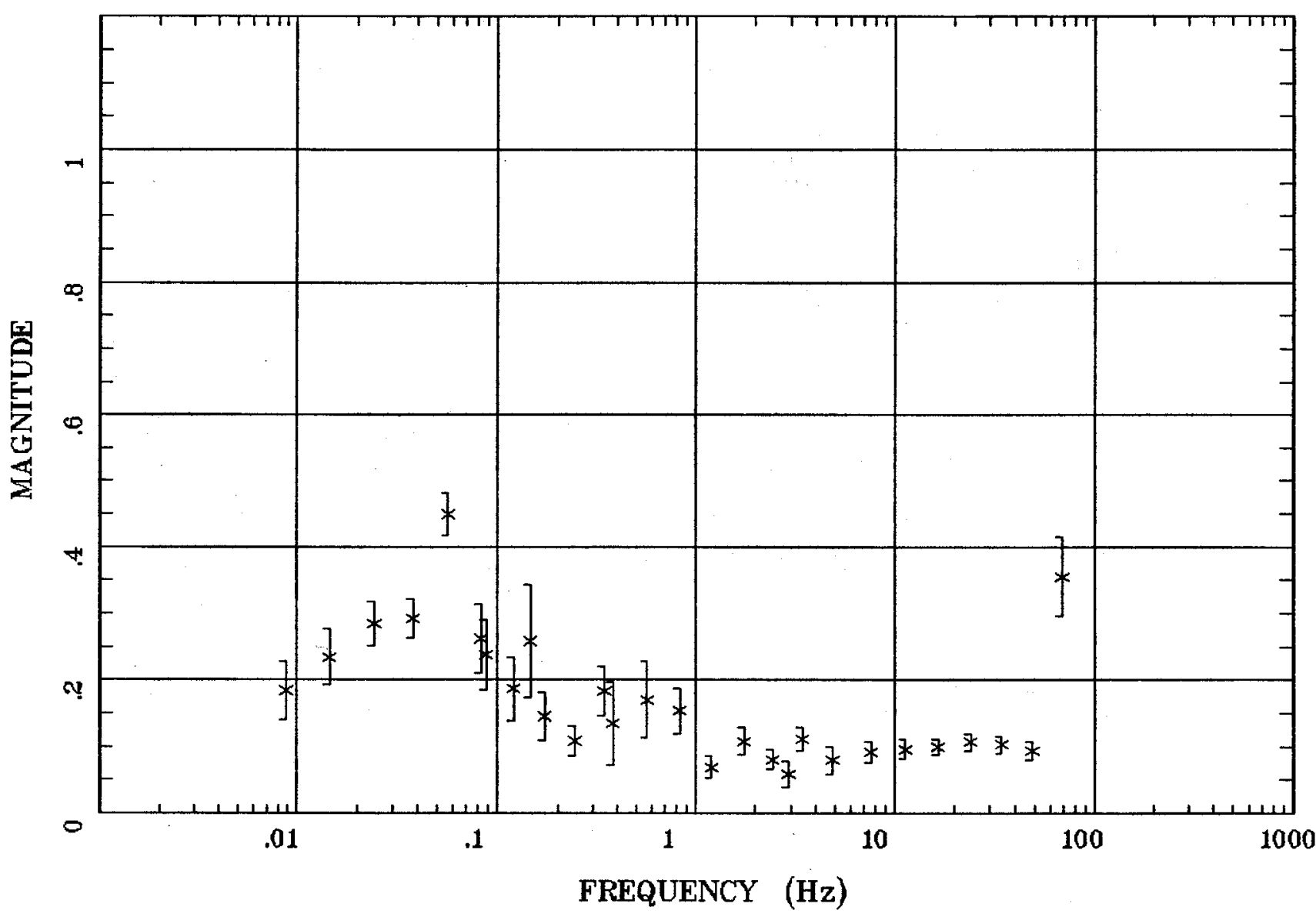

Client:

Remote: e-fld 64m east

Acquired: 12:2 Jun 25, 2003 Survey Co:USGS
Rotation:

Filename: cp26m.avg

Channels: Ch1 Ch2 Ch3 Ch4 Ch5 Ch6 Ch7 Plotted: 08:56 Jul 07, 2004

< EMI - ElectroMagnetic Instruments 


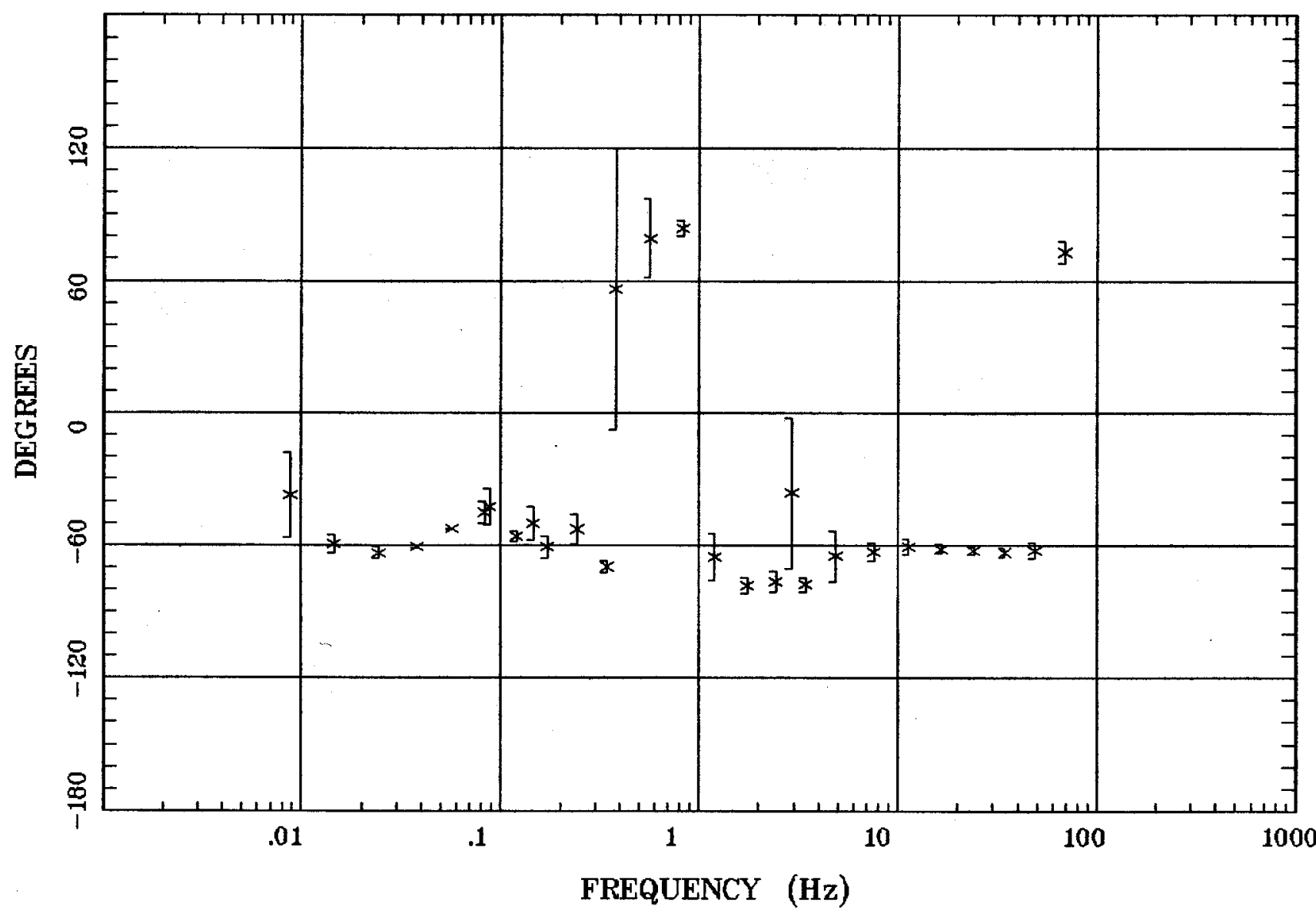

Client:

Remote: e-fld $64 \mathrm{~m}$ east

Acquired: 12:2 Jun 25, 2003

Survey Co:USGS
Rotation:

Filename: cp26m.avg

Channels: Ch1 Ch2 Ch3 Ch4 Ch5 Ch6 Ch7

Plotted: 08:56 Jul 07, 2004

$<$ EMI - ElectroMagnetic Instruments 


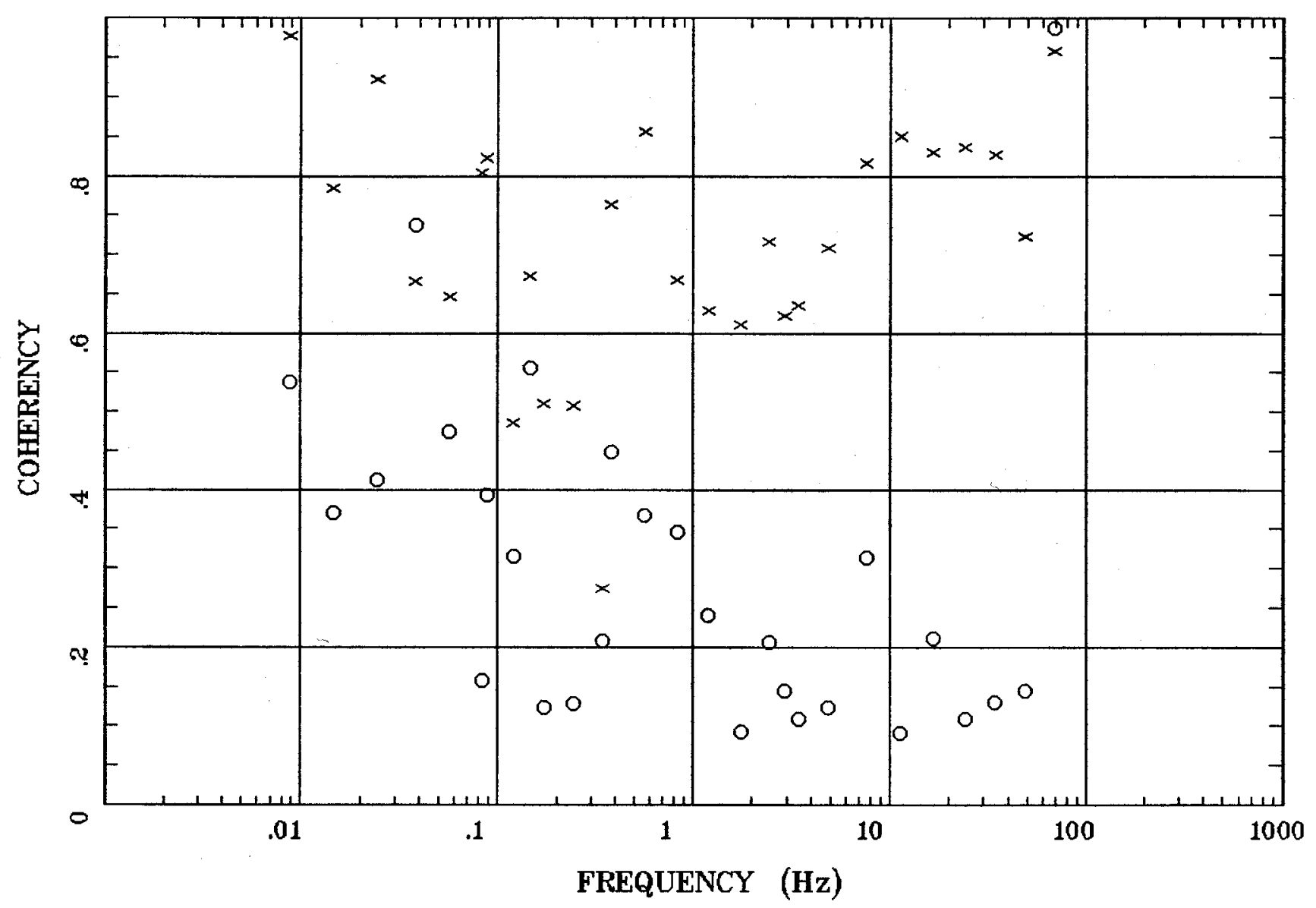

Client:

Remote: e-fld $64 \mathrm{~m}$ east

Acquired: 12:2 Jun 25, 2003 Survey Co:USGS
Rotation:

Filename: cp26m.avg

Channels: Ch1 Ch2 Ch3 Ch4 Ch5 Ch6 Ch7

Plotted: 08:56 Jul 07, 2004

< EMI - ElectroMagnetic Instruments 


\section{APPARENT RESISTIVITY}

Santa Fe, NM

Station 27

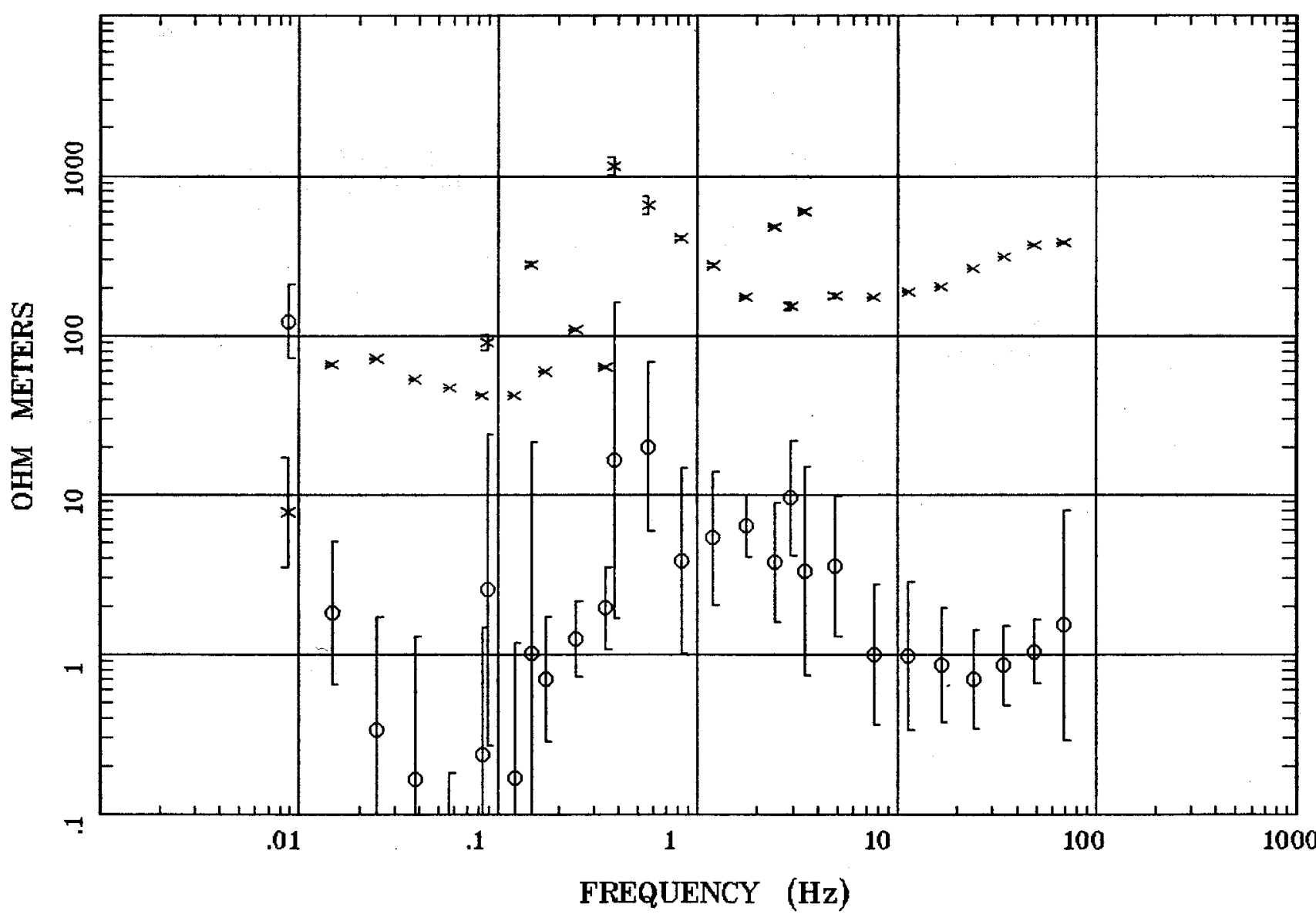

Client:

Remote: none

Acquired: 12:0 Jun 26, 2003

Survey Co:USGS
Rotation:

Filename: cp27m.evg

Channels: Ch1 Ch2 Ch3 Ch4 Ch5 Ch1 Ch2 Plotted: 09:00 Jul 07, 2004

< EMI - ElectroMagnetic Instruments 


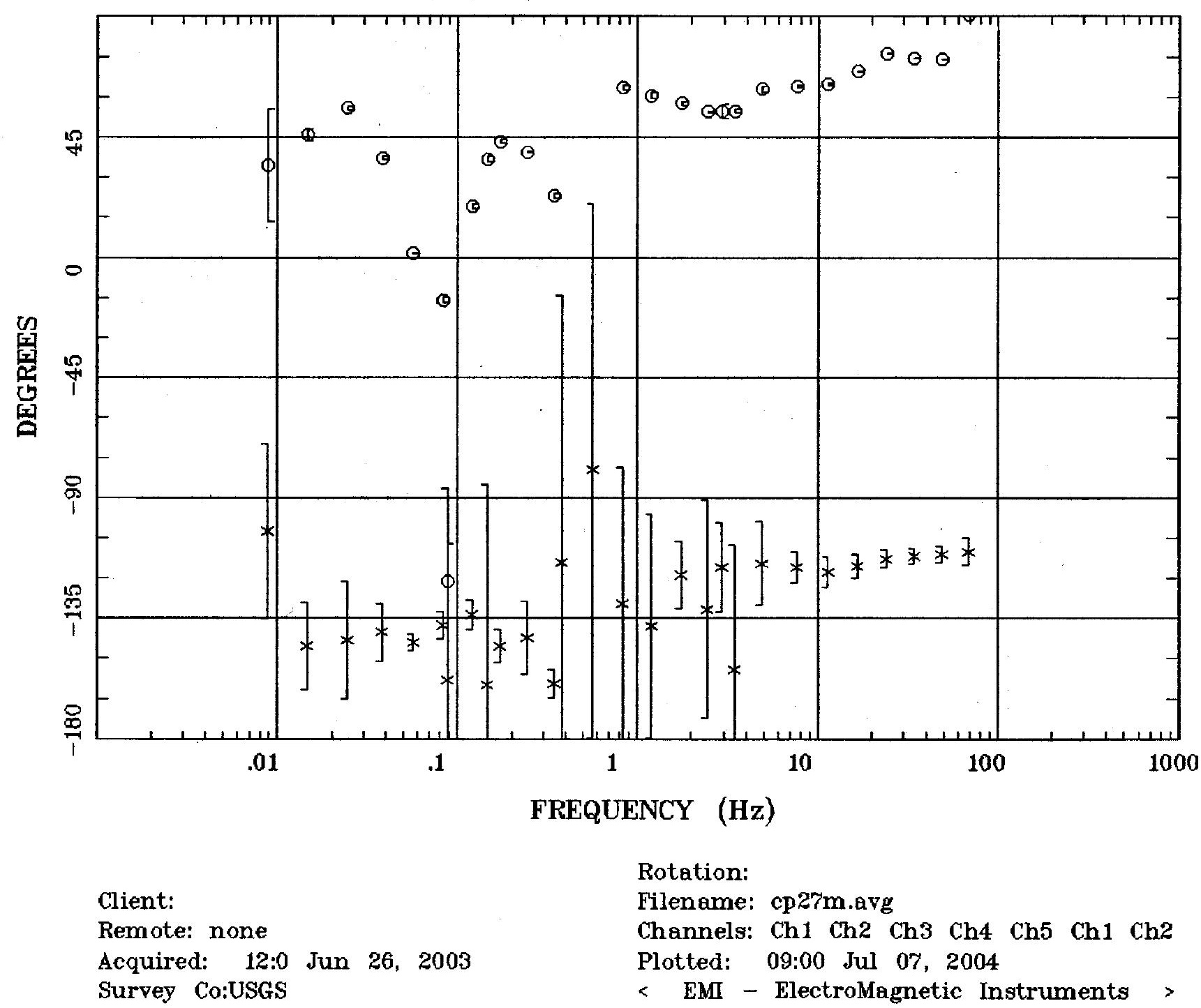




\section{ROTATION ANGLE}

Santa Fe, NM

Station 27

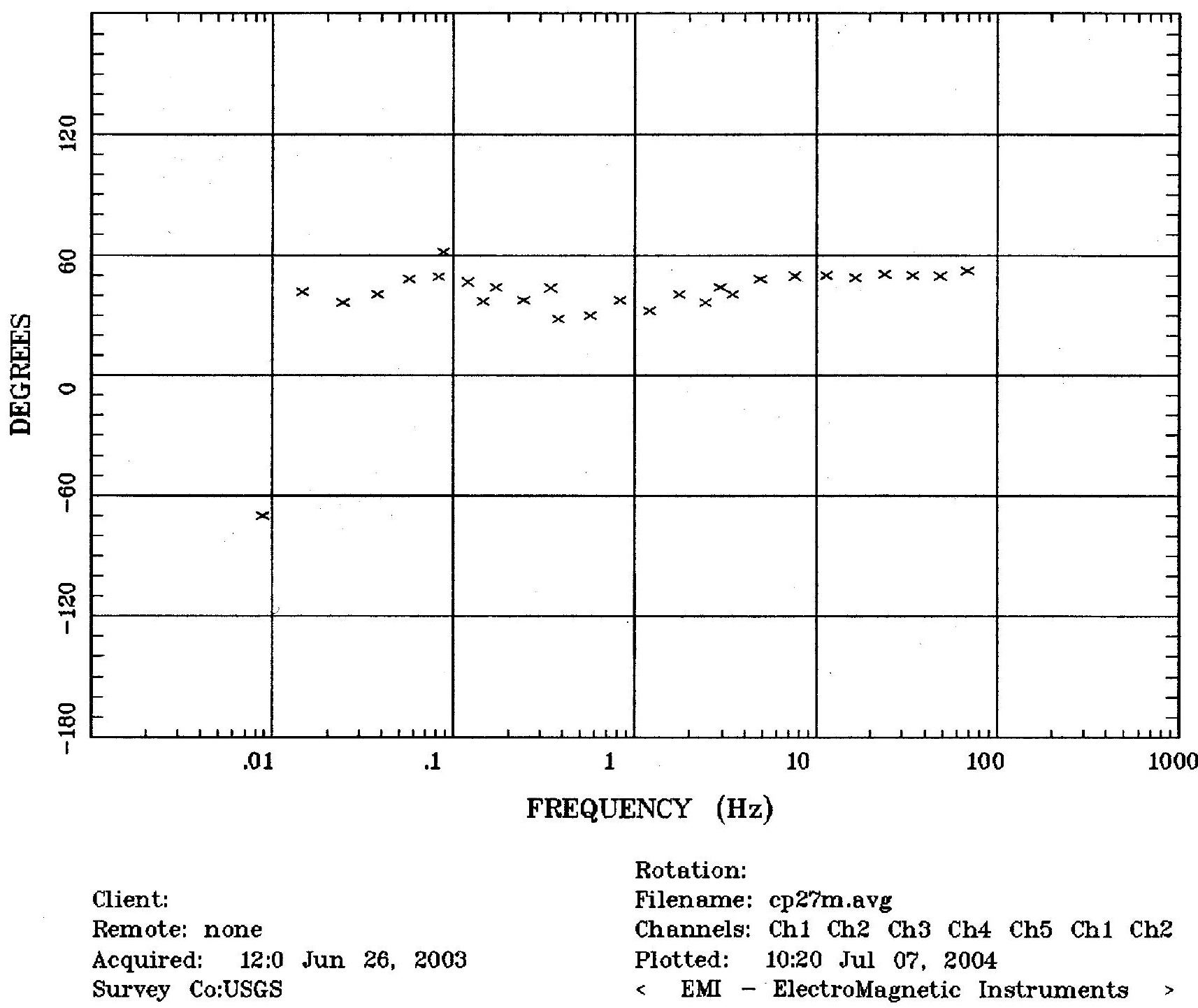




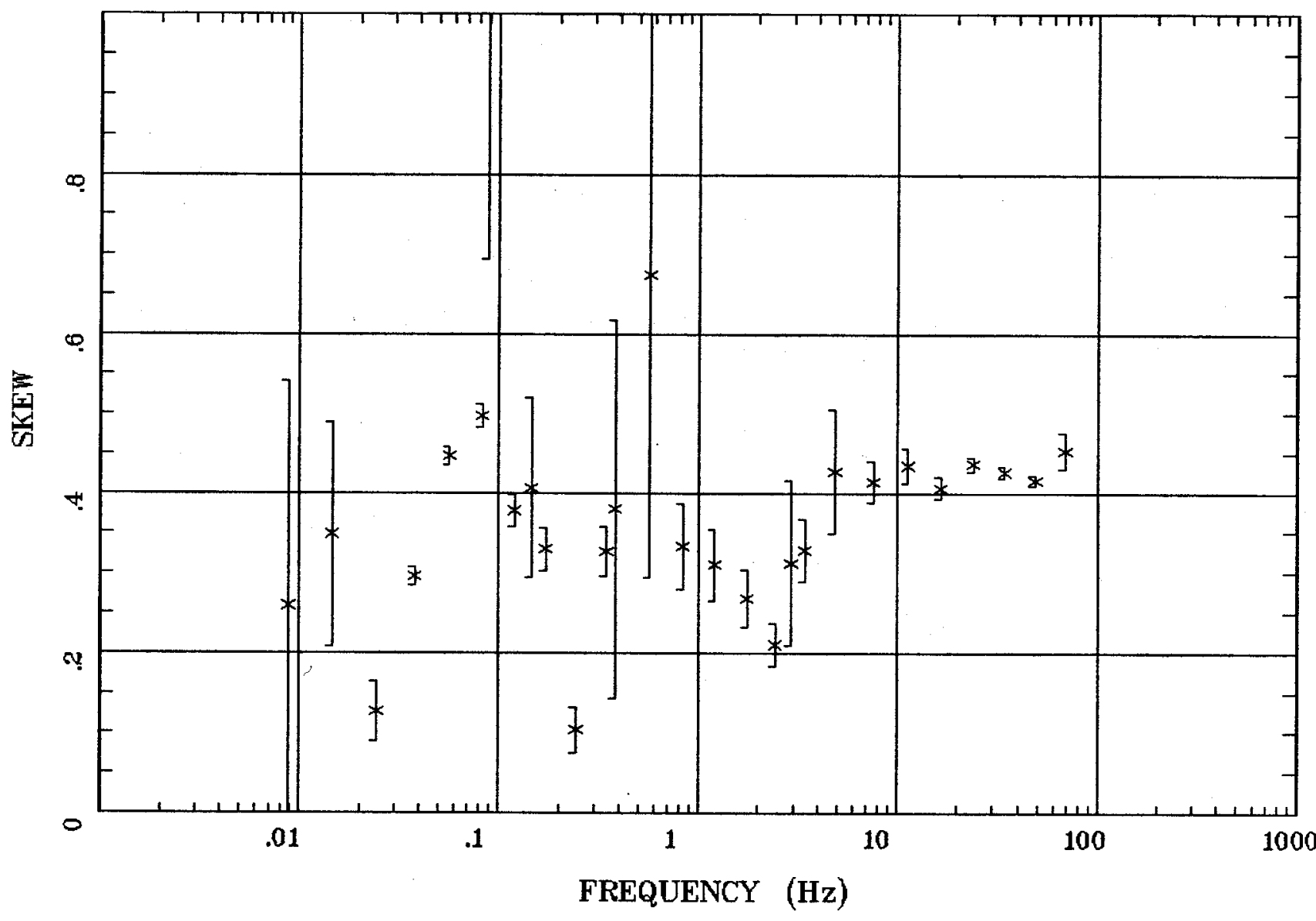

Client:

Remote: none

Acquired: 12:0 Jun 26, 2003 Survey Co:USGS
Rotation:

Filename: cp27m.avg

Channels: Ch1 Ch2 Ch3 Ch4 Ch5 Ch1 Ch2 Plotted: 09:00 Jul 07, 2004

$<$ EMI - ElectroMagnetic Instruments > 
E MULT Coh.

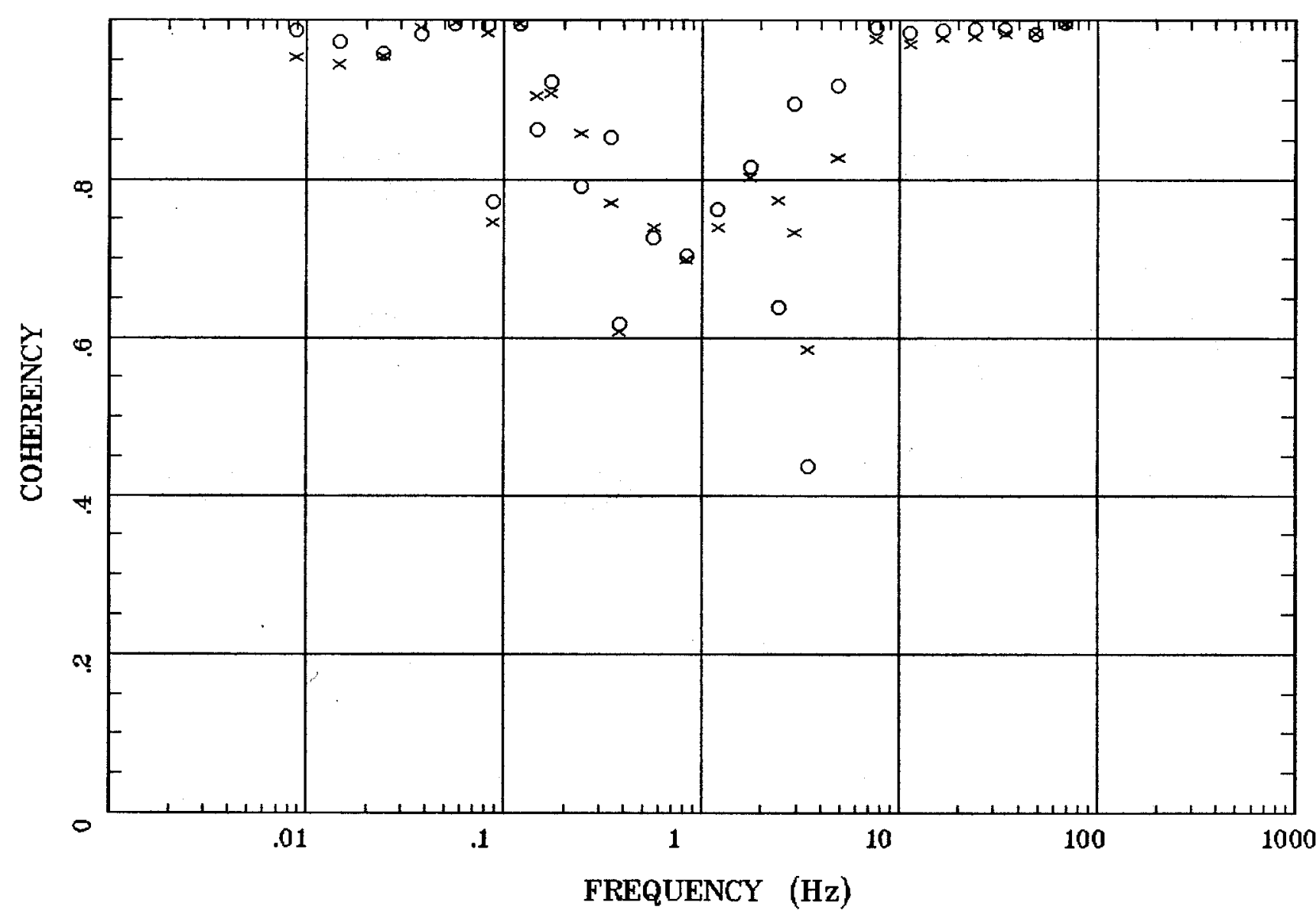

Client:

Remote: none

Acquired: 12:0 Jun 26, 2003

Survey Co:USGS
Rotation:

Filename: cp27m.avg

Channels: Ch1 Ch2 Ch3 Ch4 Ch5 Ch1 Ch2

Plotted: 09:00 Jul 07, 2004

$<$ EMI - ElectroMagnetic Instruments > 


\section{Station 27}

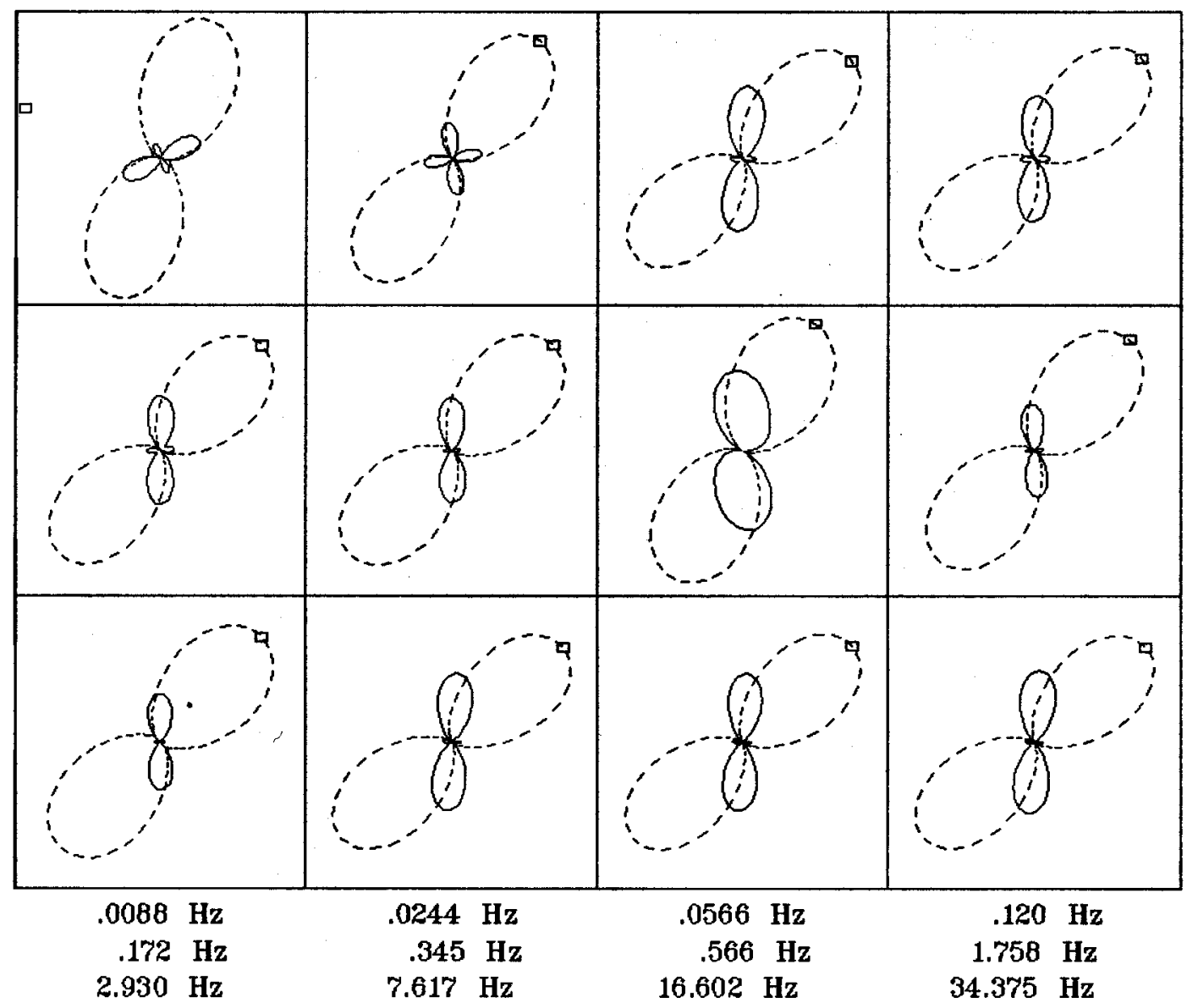

Client:

Rotation:

Remate: none

Filename: cparmavg

Channels: Ch1 Ch2 Ch3 Ch4 Ch5 Ch1 Ch2

Acquired: 12:0 Jun 26, 2003

Survey Co:USGS

Plotted: 09:00 Jul 07, 2004

< EMI - ElectroMagnetic Instruments > 
Station 27

TIPPER MAGNITUDE

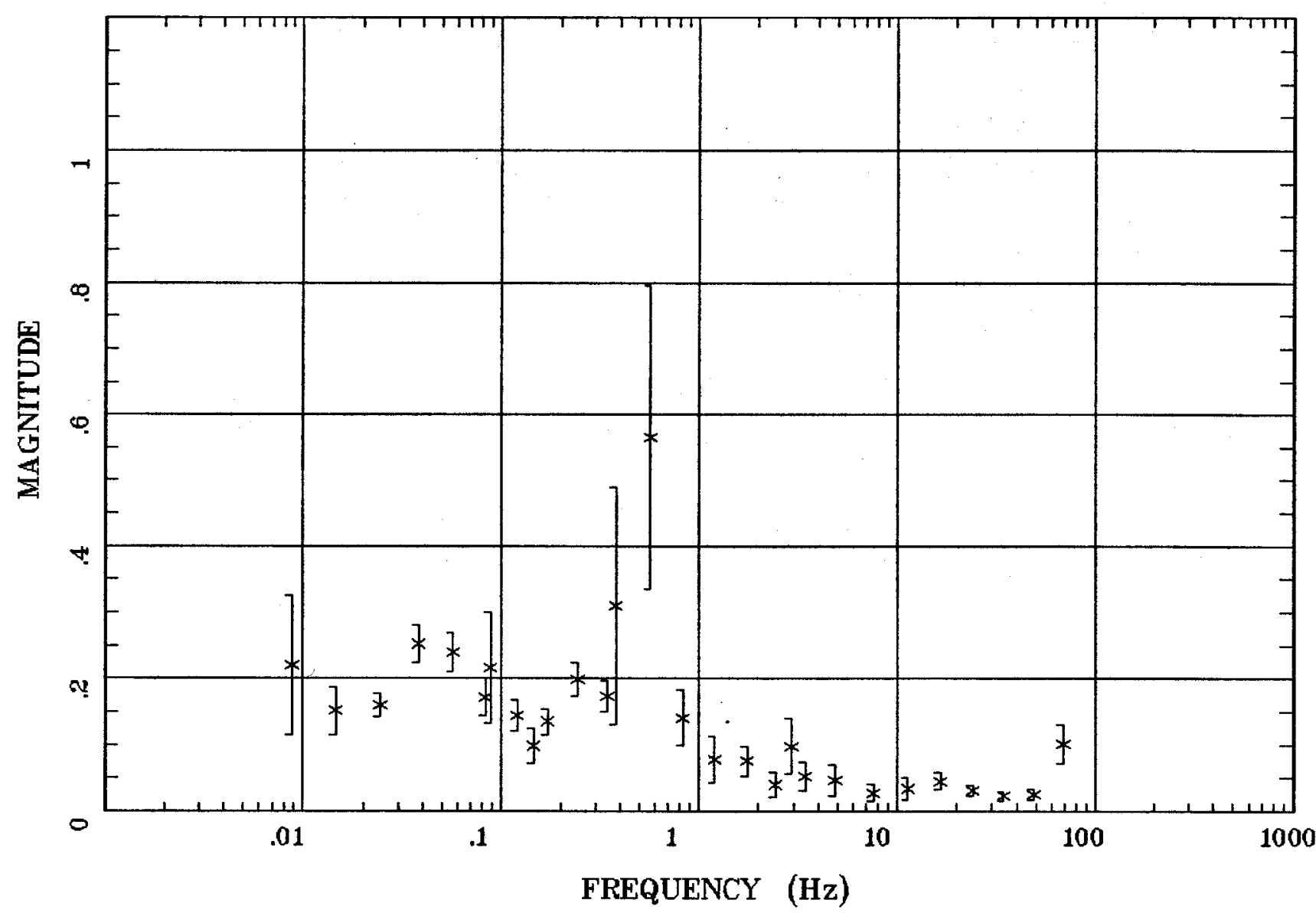

Client:

Remote: none

Acquired: 12:0 Jun 26, 2003

Survey Co:USGS
Rotation:

Filename: $\operatorname{cp} 27 \mathrm{~m}$. avg

Channels: Ch1 Ch2 Ch3 Ch4 Ch5 Ch1 Ch2

Plotted: 09:00 Jul 07, 2004

< EMI - ElectroMagnetic Instruments 


\section{TIPPER STRIKE}

Santa Fe, NM

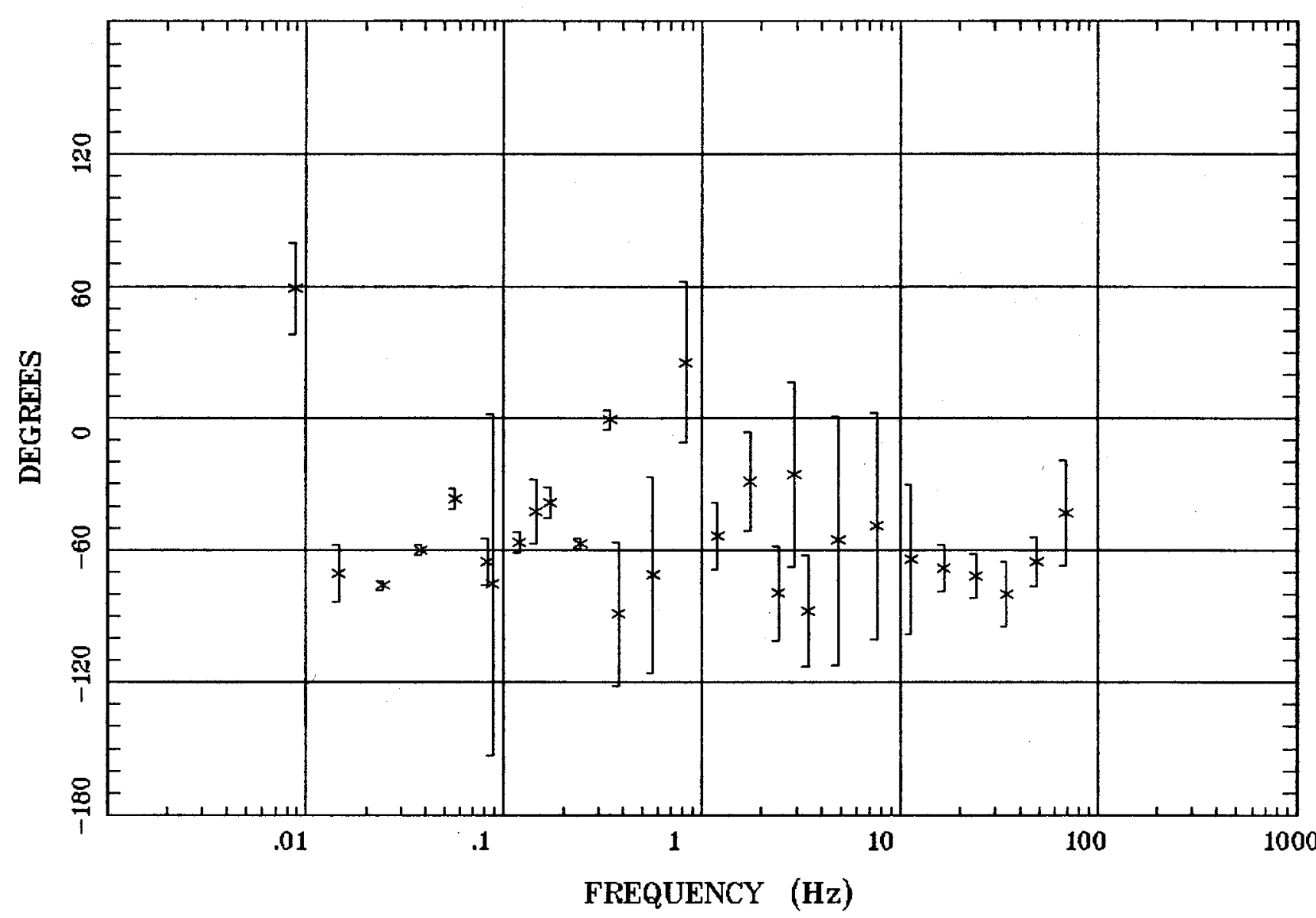

Client:

Remote: none

Acquired: 12:0 Jun 26, 2003

Survey Co:USGS
Rotation:

Filename: cp27m.avg

Channels: Ch1 Ch2 Ch3 ch4 Ch5 Ch1 Ch2 Plotted: 09:00 Jul 07, 2004

< EMI - ElectroMagnetic Instruments 


\section{Station 27}

HzHx.x Coh HzHy.o

Santa Fe, NM

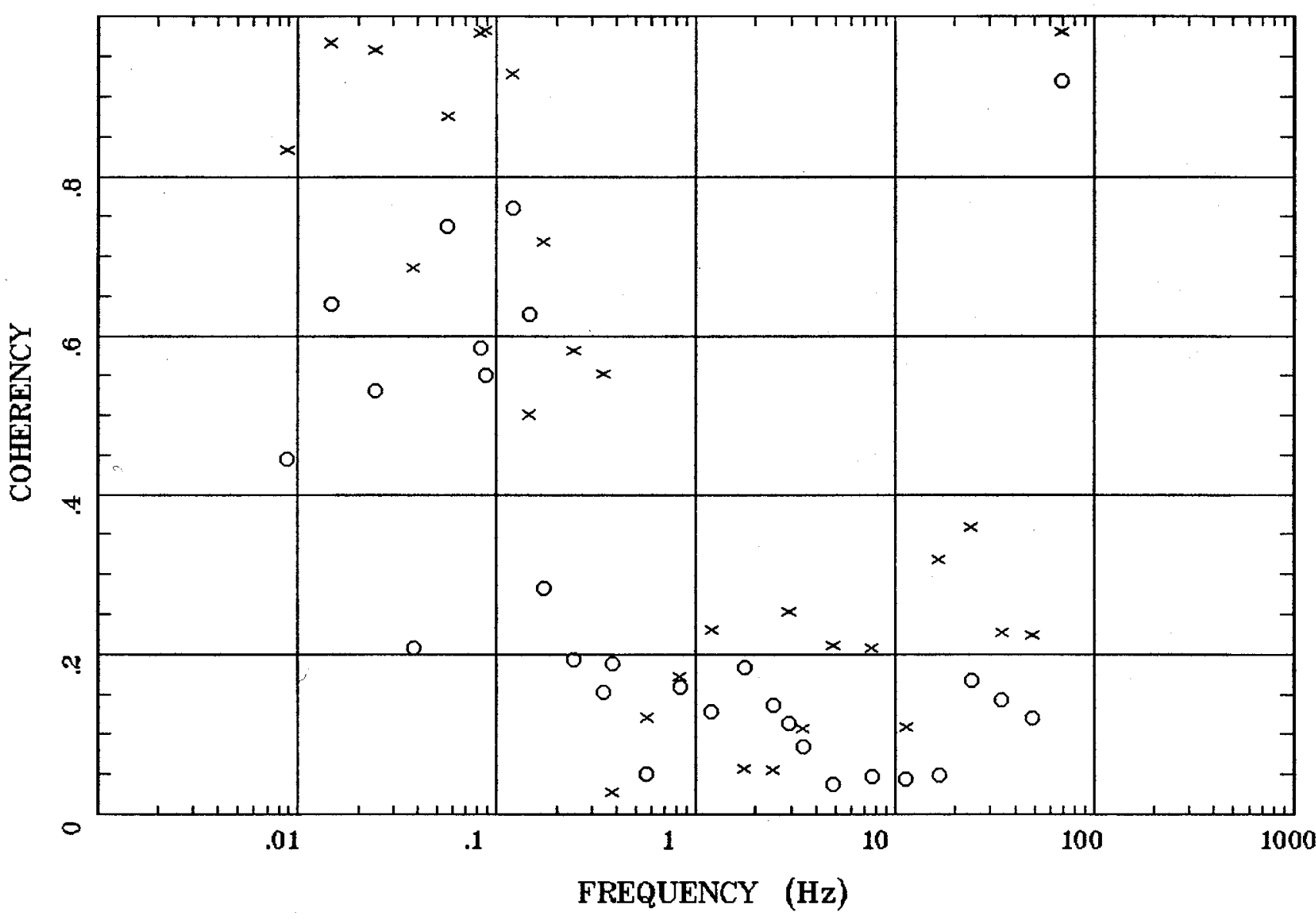

Client:

Remote: none

Acquired: $12: 0$ Jun 26, 2003

Survey Co:USGS
Rotation:

Filename: cparm.avg

Channels: Ch1 Ch2 Ch3 Ch4 Ch5 Ch1 Ch2

Plotted: 09:00 Jul 07, 2004

$<$ EMI - ElectroMagnetic Instruments 